\title{
Investigation of the Influence of Boundary Data Assignment on the Development of Multimodal Macro-level Collision Prediction Models (CPMs)
}

\author{
by \\ Ali Ihssian, BSc. Eng. \\ A thesis submitted to \\ the Faculty of Graduate Studies and Research \\ in partial fulfillment of \\ the requirements for the degree of \\ Master of Applied Science \\ Department of Civil and Environmental Engineering
Carleton University
Ottawa, Ontario
}

(C) Ali Ihssian 2014

The Master of Applied Science in Civil Engineering Program is a joint program with the University of Ottawa, administered by the OttawaCarleton Institute of Civil Engineering. 


\begin{abstract}
Developing reliable collision prediction models (CPMs) at the Traffic Analysis Zones (TAZs) aggregation level requires accurate assignment for boundary geocoded data between adjacent TAZs. Traffic Analysis Zones (TAZs) are spatial divisions within a region commonly used for traffic analysis purpose. The boundaries of TAZs are frequently set to match the centerline of major roadway segments. Collision counts data has shown that significant proportion of collisions occur frequently on these major roadways. Consequently, the way in which collisions, and also other geocoded data, along TAZs' boundaries are assigned into adjacent zones is of interest because it has direct impact on the prediction ability of macro-level CPMs. In this study, data for 422 TAZs from the City of Ottawa was used to develop macro-level CPMs. Geocoded data on TAZ's boundary were assigned between adjacent TAZs using ten different assignment methods. Negative binomial regression (NB) was applied to develop CPMs for total, nonfatal injury, property damage only (PDO), bike-involved, and pedestrian-involved collisions. Many explanatory variables expected to have an effect on the roadway safety performance at the TAZ's level were aggregated to the TAZ's level. These independent variables were categorized into four data categories including roadway characteristics, socio-economic and demographic characteristics, exposure, and Transportation Demand Management (TDM) variables. In addition, Zero-inflated regression was used to model fatal collisions as a function of Vehicle Kilometre Travelled (VKT) and total lane kilometre (TLKM). Results of the developed models show that different geocoded boundary data assignment methods do affect the accuracy of developed CPMs results significantly. It was found that allocating boundary data to TAZs evenly improved model
\end{abstract}


results significantly. The results also indicate that most of relevant independent variables have provided results consistent with previous studies. 


\section{Acknowledgments}

I would like to express my gratitude to my thesis supervisors Professor Y. Hassan and Professor K.Ismail for their continual guidance, endless assistance, and encouragement which helped me to realize the objectives of my research.

Sincere appreciation is extended to all my professors, fellow colleagues and friends, especially Abdu-allah Al-odah, for their help and encouragement.

Financial support by Libyan-North American Scholarship program is gratefully acknowledged.

Finally, I would like to express my deepest gratitude to my father, mother, family members, and my lovely fiancée Reem whom their love and support gave me hope for a better tomorrow. 


\section{Table of Contents}

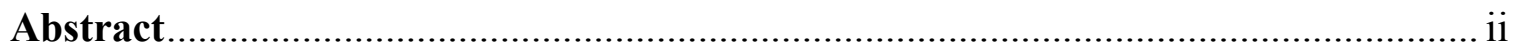

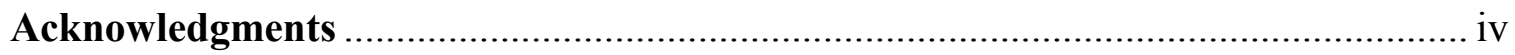

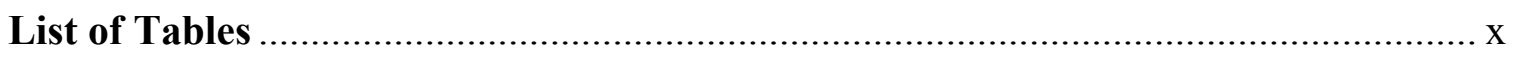

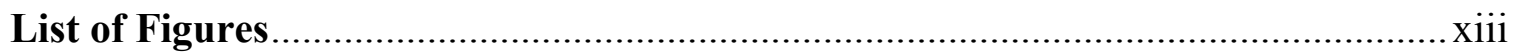

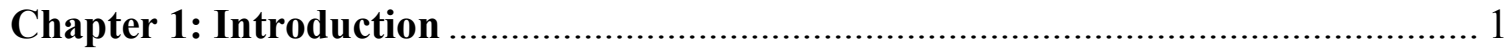

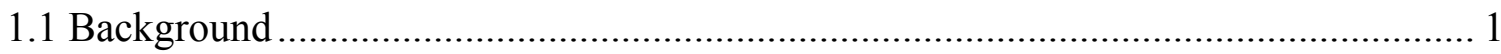

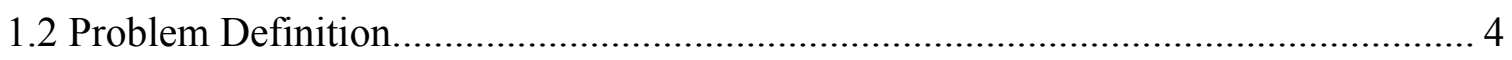

1.3 Objectives .......................................................................................................... 5

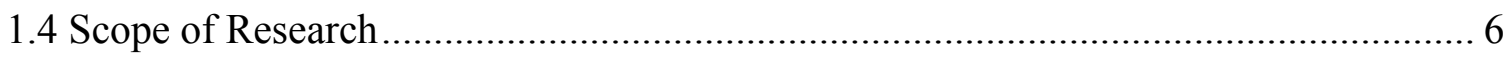

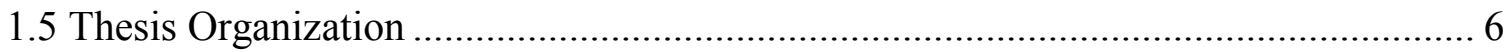

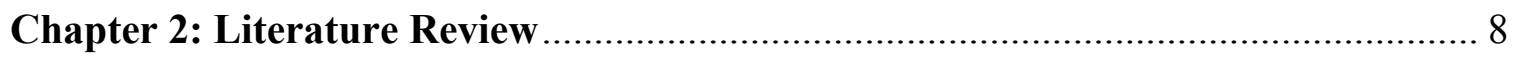

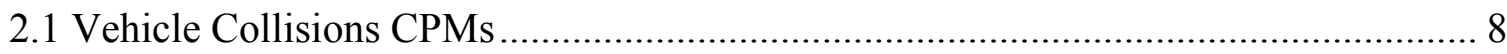

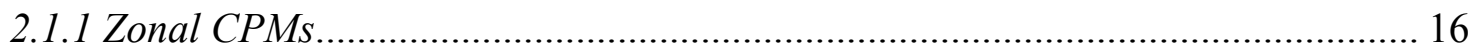

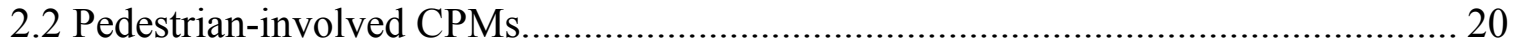

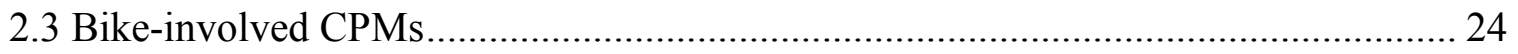

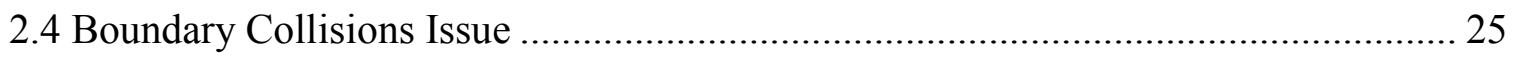

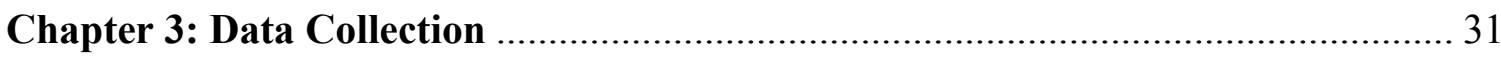

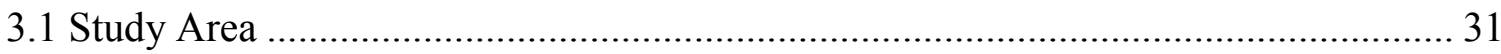

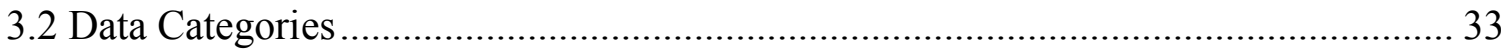




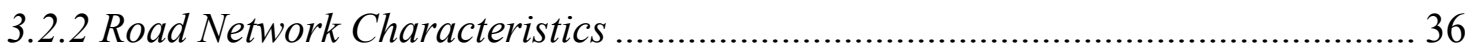

3.2.3 Traffic Exposure Variables ........................................................................ 39

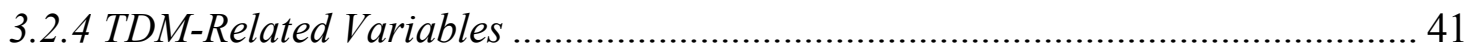

3.2.5 Demographic and Socio-Economic Variables ................................................. 42

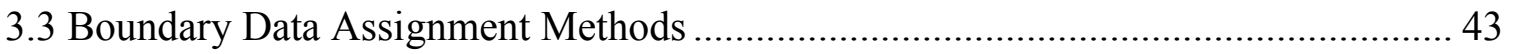

3.3.1 Data Points Assignment …………................................................................ 49

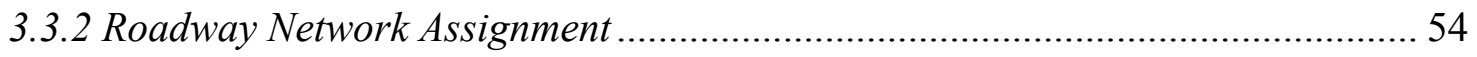

3.3.3 Exposure Variables Assignment ................................................................. 55

3.4 Spatial Analysis and Descriptive Statistics of Data ................................................ 56

3.4.1 Motor Vehicle Collisions ............................................................................. 56

3.4.2 Pedestrian-Involved and Bike-Involved Collisions ............................................ 61

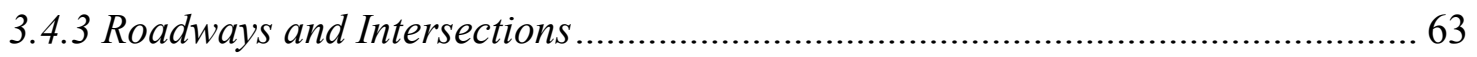

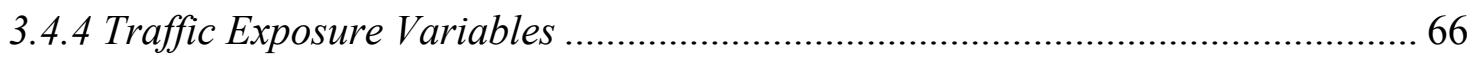

3.4.5 Demographic and Socio-Economic Characteristics .......................................... 67

Chapter 4: Statistical Modelling of Collision Data …………….................................. 71

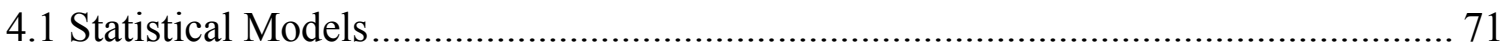

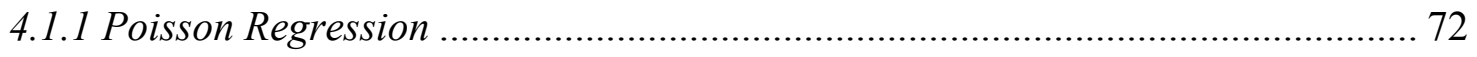

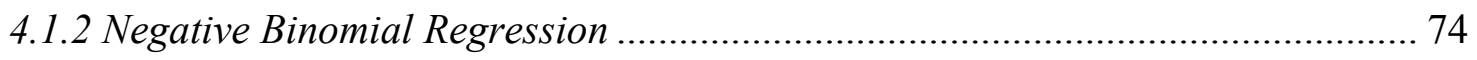

4.1.3 Akaike Information Criterion (AIC) …………............................................ 75 


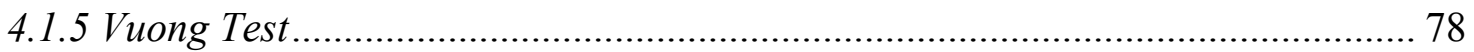

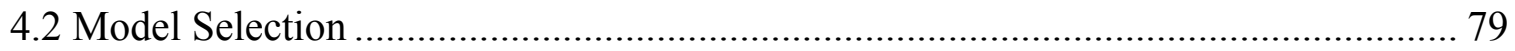

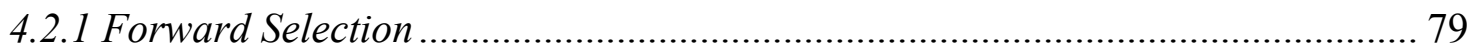

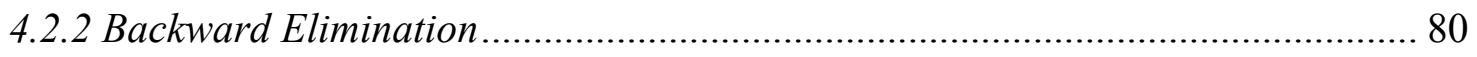

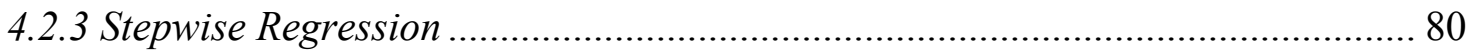

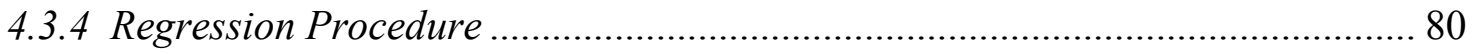

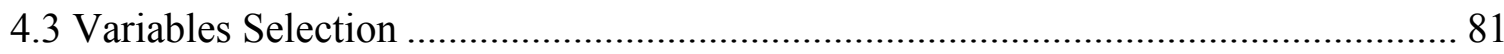

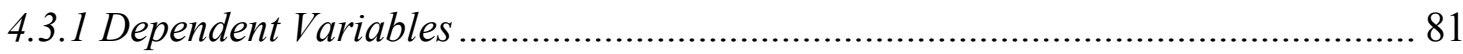

4.3.2 Explanatory Variables........................................................................... 82

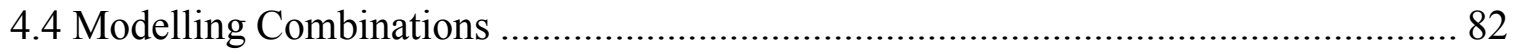

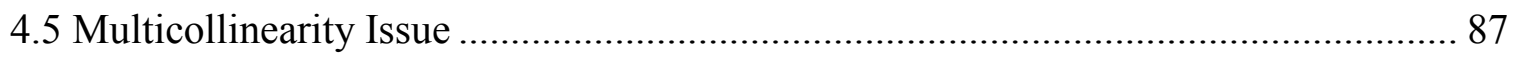

4.5.1 Exposure Variables Selection .................................................................... 89

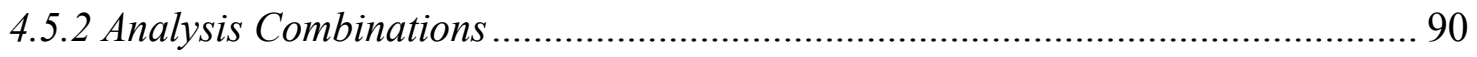

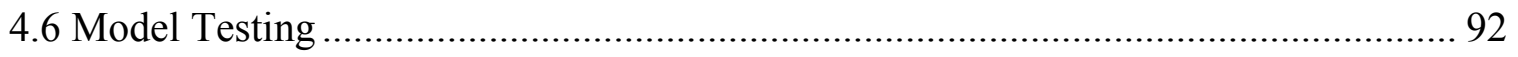

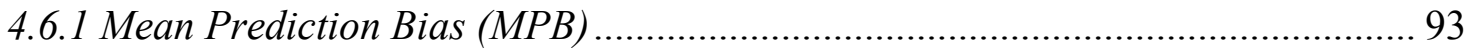

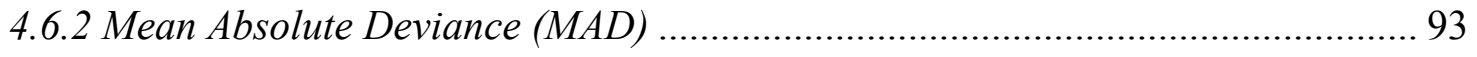

4.6.3 Mean Squared Predictive Error (MSPE) ..................................................... 94

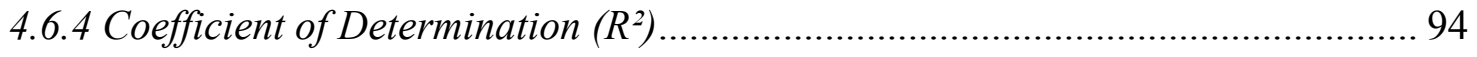

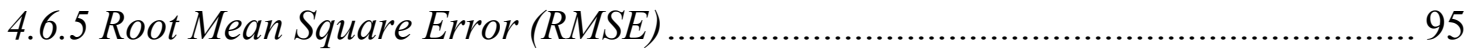




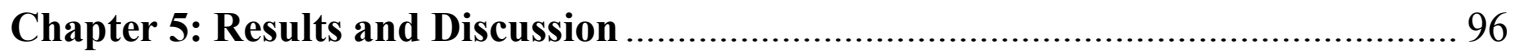

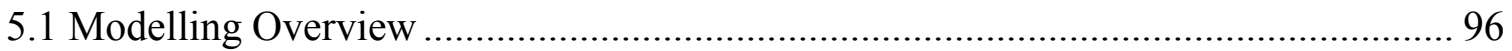

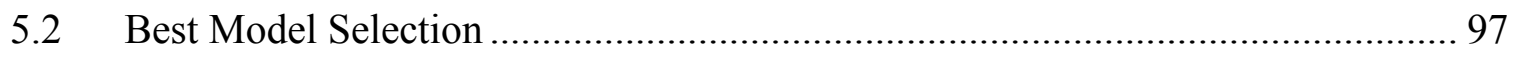

5.3 Comparing Boundary Assignment Methods............................................................. 99

5.3.1 Traffic exposure-related CPMs Ranking......................................................... 101

5.3.1 Roadway-related CPMs Ranking ............................................................... 105

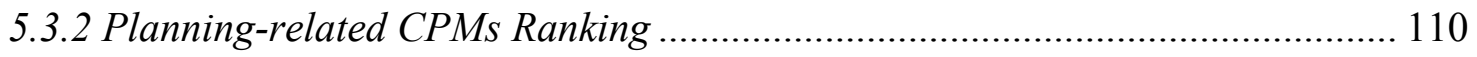

5.4 Best Exposure-related CPM........................................................................... 115

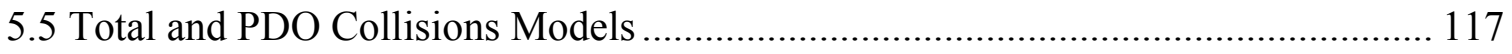

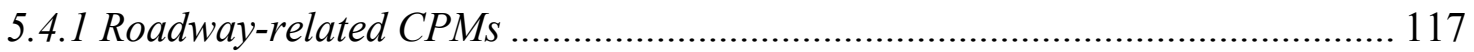

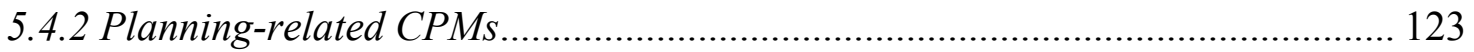

5.6 Non-fatal Injury Collisions Models .................................................................. 128

5.6.1 Roadway-related Non-fatal CPMs ........................................................... 128

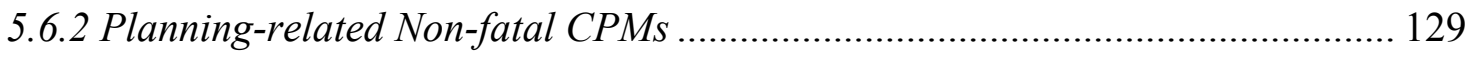

5.7 Bike-Involved Collision Models ........................................................................... 133

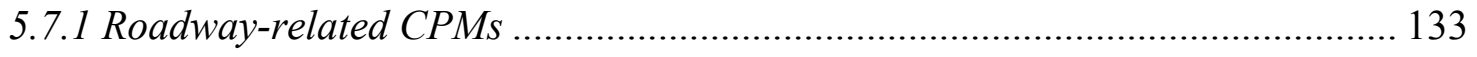

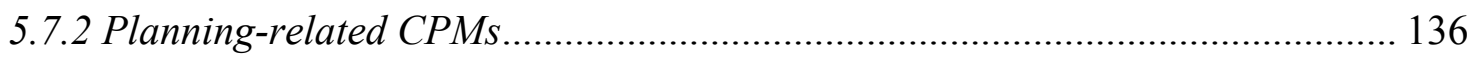

5.8 Pedestrian-Involved Collision Models.............................................................. 139

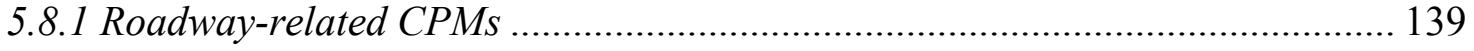




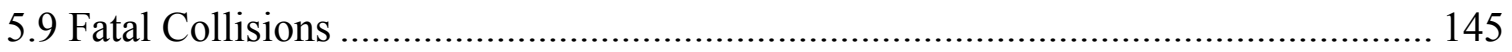

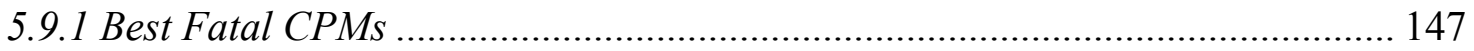

Chapter 6: Conclusions and Recommendations .................................................. 148

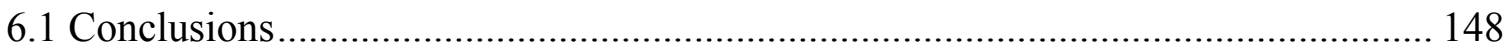

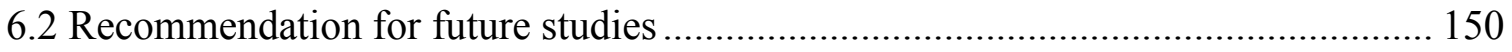

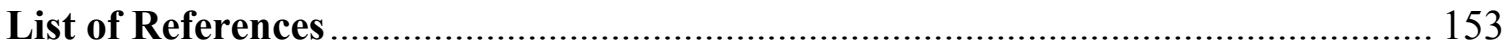

Appendix A: Spatial Analysis and Descriptive Statistics of Data............................. 160

Appendix B : Boundary Point Assignment Script and R Code.............................. 215

Appendix C: Independent Variables' Correlation Matrix and Analysis

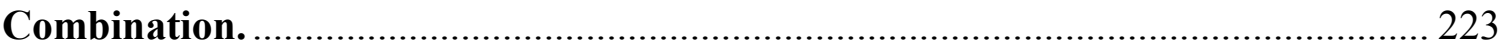

Appendix D : The Best Ten CPMs for Each of exposure-related, Roadway-relate, and Planning-related for Each Type of Collisions.................................................. 238 


\section{List of Tables}

Table 2.1Examples for Macro-level Data Categories....................................................... 9

Table 3.1 Boundary Collision Assignment Methods ...................................................... 47

Table 3.2 Descriptive Statistics of the Different Types of Collisions Within Ottawa's

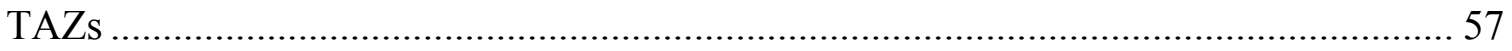

Table 3.3 Descriptive Statistics of the Roadways and Intersections Variables Within

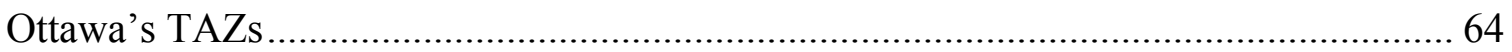

Table 3.4 Descriptive Statistics of Exposure Variables Within Ottawa's TAZs .............. 66

Table 3.5 Summary Statistics of Demographic and Socio-economic Variables Within

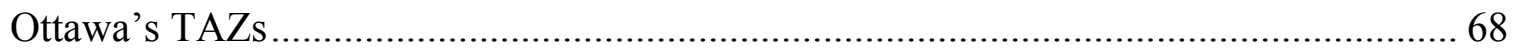

Table 4.1 All Candidate Explanatory Variables............................................................. 83

Table 4.2 All Candidate Exposure Variables combinations ........................................... 90

Table 4.3 Correlation Matrix for Exposure Variables ………........................................... 90

Table 4.4 Independent Variable Combinations .............................................................. 91

Table 5.1 Ranking Based on Goodness of Fit Measures for Total Collision Models

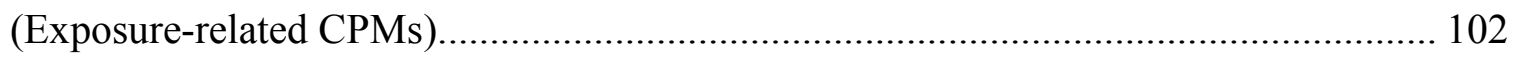

Table 5.2 Ranking Based on Goodness of Fit Measures for PDO Collision Models

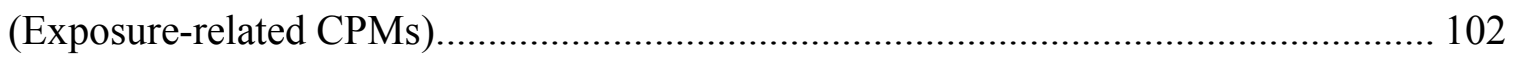

Table 5.3 Ranking Based on Goodness of Fit Measures for Non-fatal Injury CPMs

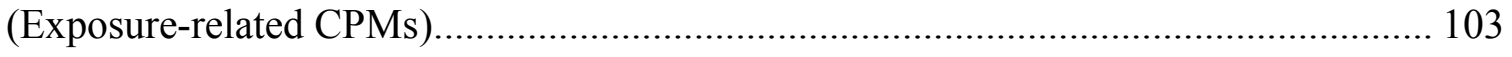

Table 5.4 Ranking Based on Goodness of Fit Measures for Bike-Involved CPMs

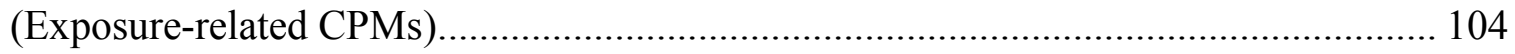


Table 5.5 Ranking Based on Goodness of Fit Measures for Pedestrian-Involved CPMs (Exposure-related CPMs) 104

Table 5.6 Ranking Based on Goodness of Fit Measures for Total CPMs (Roadwayrelated CPMs) 107

Table 5.7 Based on Goodness of Fit Measures for PDO CPMs (Roadway-related CPMs) 107

Table 5.8 Ranking Based on Goodness of Fit Measures for Non-fatal Injury CPMs (Roadway-related CPMs). 108

Table 5.9 Ranking Based on Goodness of Fit Measures for Bike-Involved CPMs (Roadway-related CPMs).

Table 5.10 Ranking Based on Goodness of Fit Measures for Pedestrian-Involved CPMs(Roadway-related CPMs) 110

Table 5.11 Ranking Based on Goodness of Fit Measures for Total CPMs (Planningrelated CPMs) 111

Table 5.12 Ranking Based on Goodness of Fit Measures for PDO CPMs (Planningrelated CPMs) 112

Table 5.13 Ranking Based on Goodness of Fit Measures for Non-fatal Injury CPMs (Planning-related CPMs) 112

Table 5.14 Ranking Based on Goodness of Fit Measures for Bike-Involved CPMs (Planning-related CPMs) 113

Table 5.15 Ranking Based on Goodness of Fit Measures for Pedestrian-Involved CPMs (Planning-related CPMs) 114

Table 5.16 Model Estimation Results for the Best Exposure-related CPMs. 116 
Table 5.17 Model Estimation Results of the Best Roadway-related CPMs for Total Collisions .....

Table 5.18 Model Estimation Results of the Best Roadway-related CPMs for PDO Collisions 122

Table 5.19 Model Estimation Results of the Best Planning-related CPMs for Total Collisions 126

Table 5.20 Model Estimation Results of the Best Planning-related CPMs for PDO Collisions 127

Table 5.21 Model Estimation Results of the Best Roadway-related CPMs for Non-fatal Injury 130

Table 5.22 Model Estimation Results of the Best Planning-related CPMs for Non-fatal Injury 131

Table 5.23 Model Estimation Results of the Best Roadway-related CPMs for Bikeinvolved Collisions 135

Table 5.24 Model Estimation Results of the Best Planning-related CPMs for Bikeinvolved Collisions 137

Table 5.25 Model Estimation Results of the Best Roadway-related CPMs for Pedestrianinvolved Collisions 141

Table 5.26 Model Estimation Results of the Best Planning-related CPMs for Pedestrianinvolved Collisions 144

Table 5.27 Ranking Based on Goodness of Fit Measures for Fatal CPMs 146

Table 5.28 Model Estimation Results for Fatal Collisions ...... 147 


\section{List of Figures}

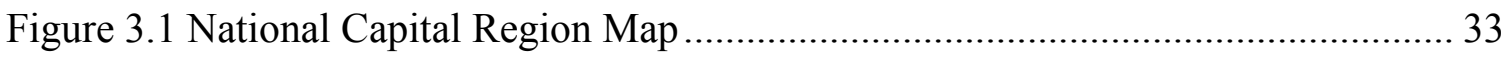

Figure 3.2 Roadway Network Map...................................................................... 38

Figure 3.3 Fully Controlled Access Highways Map..................................................... 39

Figure 3.4 A Boundary Roadway along with TAZ Boundaries ...................................... 44

Figure 3.5 Distance from a Point to a Line Segment ("d"=Perpendicular) ..................... 49

Figure 3.6 Distance from a Point to a Line Segment ("d"= To Closest Vertex) ............... 50

Figure 3.7 Data Points Distribution inside and around a TAZ ……….............................. 51

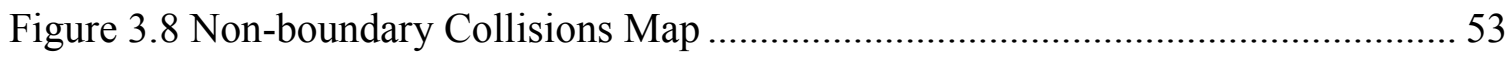

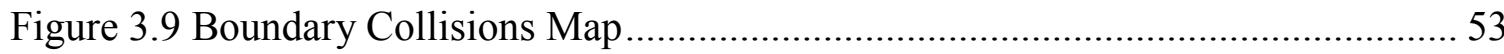

Figure 3.10 Spatial Distributions of Collision Density for Total Collisions in the City of

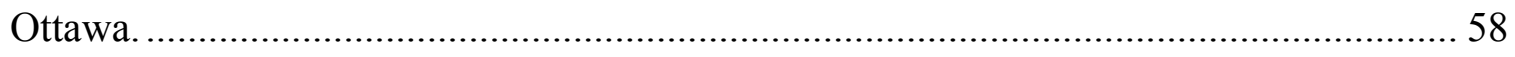

Figure 3.11 Spatial Distributions of Collision Density for Fatal Collisions in the City of

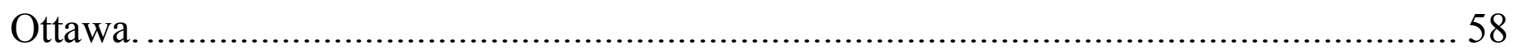

Figure 3.12 Spatial Distributions of Collision Density for Non-Fatal Injury Collisions in

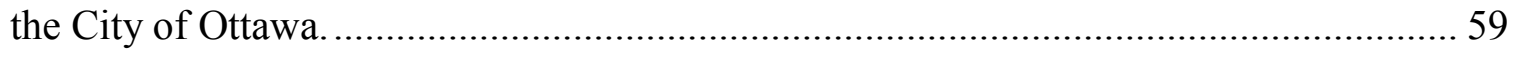

Figure 3.13 Spatial Distributions of Collision Density for PDO Collisions in the City of Ottawa

Figure 3.14 Histograms of Ottawa TAZ level Collision Frequencies. ............................. 60

Figure 3.15 Histograms of Ottawa TAZ level Collision Frequencies. .............................. 61

Figure 3.16 Spatial Distribution of the Density of Pedestrian-Involved Collisions......... 62

Figure 3.17 Spatial Distribution of the Density of Bike-Involved Collisions. ................. 62

Figure 3.18 Spatial Distributions of TLKM Density in the City of Ottawa..................... 65 
Figure 3.19 Spatial Distributions of Intersections Density in the City of Ottawa........... 65

Figure 3.20 Spatial Distributions of VKT Density in the City of Ottawa. ...................... 67

Figure 3. 21 Spatial Distributions of Population Density for the City of Ottawa............ 69

Figure 3.22 Spatial Distributions of Employment Density for the City of Ottawa. ....... 69

Figure 3.23 Spatial Distributions of Schools Density for the City of Ottawa. ............... 70

Figure 3.24 Spatial Distributions of Number of Households Density for the City of

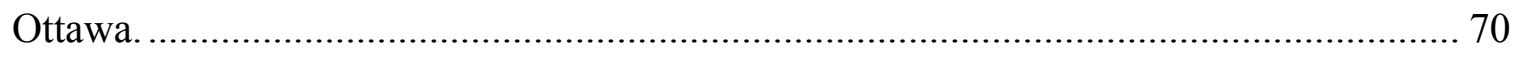

Figure 5.1 Selecting the best CPMs for Ranking.................................................. 99 


\section{Chapter 1: Introduction}

\subsection{Background}

Road traffic collisions and their associated human and economic losses have become one of the main problems facing the development of existing communities. The World Health Organization and World Bank have confirmed that road collisions are the eighth leading cause of death among the world's population (WHO, 2013). Annually, road collisions kill approximately 1.3 million people and lead to injury and disability of 20 to 50 million people worldwide (WHO, 2013). Most deaths happen among the age group of five to twenty nine years. By the year 2030, an increase in the proportion of deaths due to traffic collisions of approximately $80 \%$ is expected in countries with low and middle income. In addition, traffic deaths on the roads are expected to be the fifth leading cause of death unless urgent action is taken (WHO, 2013).

According to the Road Safety and Motor Vehicle Regulation division in Transport Canada, there are approximately 900,000 centreline kilometres of public roadways. Transport Canada's National Collision Database (NCDB, 2010) indicated that there were 2,000 fatal and 123,141 personal injury collisions occurred on these roadways in 2010. In these reported collisions, 2,227 persons died and a total of 170,629 injured victims suffered severity of injuries ranging from minimal to serious (seriously injured only were 11,226). Motor vehicle collision statistics show also that fatalities due to roadway collisions for young drivers and passengers constitute a relatively large proportion of all fatalities in Canada. The fatalities among the age group from 15 to 24 were 
approximately $23 \%$ of motor vehicle fatalities in 2010 . In addition, the same database (NCDB, 2010) indicated that rural areas are associated with more fatalities when compared to the urban areas, where $57 \%$ of fatal collisions occurred on rural roadways. The variation in fatal collisions between the rural and the urban areas could be attributed to the increase in traffic speed in the rural areas due to relatively low degree of congestion. On the other hand, injury collisions were reported to be more frequent in urban area when compared to rural area, where $73 \%$ of injury collisions were reported in urban areas. This is likely due to the higher traffic volumes experienced in urban areas.

Roadway collisions can be studied at two main aggregation levels, namely: microlevel and macro-level. The micro-level collision analysis is usually conducted to identify and evaluate the safety performance of specific entities or collision-prone locations (CPLs) such as specific highway segments or traffic intersections. Traditionally, this type of analysis is employed to develop road safety improvement programs. These programs mainly concentrate on the identification, analysis, and possible improvement of CPLs. Although these programs have been widely used, they have a major shortcoming, which is the significant collision history requirement before any initiative can be made. It is only recently that the transportation engineers have begun to pay close attention to the development of macro-level collision prediction models (CPMs). The macro-level CPMs are developed to relate the collision frequency at transportation planning levels such as census block groups and traffic analysis zones (TAZs) while considering different demographic, socioeconomic, traffic, and roadway characteristics. This type of collision models is used to study collision occurrence on a scale of groups of intersections in order to address the road safety before the problems would emerge. Such proactive road safety 
improvement tools can be used either to reduce the occurrence of collisions in new neighbourhoods at the planning stage or to effectively allocate resources to increase the traffic safety by focusing on municipal divisions or areas according to their collision reduction potential. Note this is done proactively in the sense that these communities may only exist in the future and consequently no collisions are observed yet.

Previous work on the study of macro-level collision prediction models generally emphasizes the addition of road safety to the planning process instead of focusing strictly on increasing capacity, mobility, and accommodating future travel demand. However, this goal is challenging due to the lack of traffic exposure data at this level of aggregation. At the same time, the challenge could come from the lack of appropriate models for evaluating the road safety during the planning process.

Development of transportation planning models is traditionally based on OriginDestination (O-D) surveys. The survey typically collects information about each household, its residents and their travel activities over a 24-hour period. The results of OD survey are usually entered along with transportation network map and trip pattern information into planning software such as Equilibrium Multimodal, Multimodal Equilibrium (EMME) (INRO, 1998). This software has been identified as one of the most powerful transportation planning software to generate traffic flows on a network links. The reliability of EMME planning models results depends on the accuracy of the survey and the size of the sample population interviewed in the survey. EMME is used by roadway safety professionals as a tool to model travel activities at the selected planning level, such as traffic analysis zone level (TAZ) Hadayeghi et al. (2003), when there is 
lack of the measured traffic exposure variables, e.g., Average Annual Daily Traffic (AADT) and modal splits, at the planning level.

\subsection{Problem Definition}

Several previous studies and surveys have identified that information collected for transportation planning purposes can be presented at the Traffic Analysis Zone (TAZ) level. TAZs are defined as a group of geographic units that are used to aggregate data used to develop conventional transportation planning models. TAZs are typically divided to meet the following criteria (Wei, 2010) :

1. TAZs should be of reasonably homogeneous land use.

2. TAZs should correspond with jurisdictional boundaries.

3. TAZs should be established based on trip assignments.

Several studies have been devoted to developing collision prediction models (CPMs) at the TAZ level. The aim of these studies was to relate the collision frequency to various zonal demographic, roadways network, land use, and traffic characteristics (Guevara, Washington, \& Oh, 1997; Hadayeghi, Shalaby, Persaud, \& Cheung, 2006; Lovegrove \& Sayed (2007); Sun \& Lovegrove 2013). A common challenge in the previous publications was the method of assigning boundary data (observations which lie at or are close to the boundaries of TAZs). Mostly, data attributes (collisions, roadway characteristics and exposure) in the previous studies were aggregated for each TAZ as they were geocoded. This problem arises because TAZ boundaries are frequently along arterial roads, which experience a relatively high rate of collisions due to the volume of traffic they carry. Accordingly, the presumption is that the characteristics of all adjacent 
TAZs can potentially influence the collision rate along a TAZ boundary. Therefore, the way in which geocoded data on TAZ boundaries is assigned to each TAZ may affect the macro-level CPM results.

The main focus of this thesis is to examine different techniques for the allocation of boundary data for development of reliable macro-level collision prediction models CPMs. Boundary data includes geocoded data which lies at or close to the TAZs boundaries

\subsection{Objectives}

Based on the motivation presented earlier, this thesis aims the following main objectives:

- To identify the influence of assigning TAZs' boundaries data such as collisions, roadway characteristics, exposure, and socio-economic on the prediction ability of the macro-level CPM results. The evaluation is based on aggregating the TAZs' boundary data to the adjacent TAZs using different assignment methods.

- To develop macro-level collision prediction models (CPMs) that can predict the frequency of the total collisions, severe collisions, collisions involving pedestrians, and collisions involving cyclists based on planning level inputs.

The findings of this research are expected to assist the transportation planners and roadway safety professionals to incorporate safety into the planning process. The thesis should aid in selecting the most appropriate methods for allocating the boundary data between TAZs and therefore developing reliable CPMs. These models can be used for identifying collision-prone TAZs or for classic observational before-and-after analysis. 
These models could also be used as a tool to explicitly consider the road safety at the planning stage of future developments.

\subsection{Scope of Research}

The scope of this study is limited to the City of Ottawa traffic analysis zones TAZs, in Ontario, Canada. A total of 422 TAZs are covered by this study, which can be considered as an adequate sample to establish collision prediction models that relate the collision frequencies to the variables related to TAZs. The City of Ottawa traffic zones cover urbanized and non-urbanized areas which represent enough variation in land use. All of this information could be helpful in capturing the safety performance for different scenarios.

\subsection{Thesis Organization}

This thesis consists of six chapters. Chapter 1 presents an introduction to the thesis. Chapter 2 contains the literature review of the different statistical techniques that have been proposed to develop macro-level CPMs. In addition, different contributing factors that influence the road safety performance at the planning levels are reviewed. Chapter 3 provides descriptive details for the data collection and preparation effort undertaken in this thesis. This includes data regarding the study area, collisions history, different socioeconomic factors, traffic exposure, transportation demand management (TDM) variables which are represented in the percentage of trips for each available transportation mode, and roadway characteristics. Moreover, Chapter 3 includes detailed information about the methods to treat TAZs' boundary data. Chapter 4 is devoted to the explanation of the modelling techniques that are used for developing CPMs with an acceptable goodness of 
fit. Chapter 5 summarizes the results of the modelling attempts. Finally, Chapter 6 provides a summary of the study findings with a list of recommendations for the future research 


\section{Chapter 2: Literature Review}

This chapter provides a review of previous studies that were conducted in order to evaluate the road safety performance at different levels of data aggregation. This chapter consists of four sections. The first section is devoted to review of previous studies regarding the development of motor vehicle Collision Prediction Models (CPMs). The second and third sections review published studies concerning pedestrian-involved and bike-involved collisions occurrence. Finally, the fourth section will shed the light on the issue of boundary data; observations which are located at the boundaries of the planning level units.

\subsection{Vehicle Collisions CPMs}

A considerable amount of academic work has been conducted to develop microlevel models to predict the collision frequencies on the transportation road networks. Typically, these studies have been used to evaluate the safety performance at specific sites, and thus given the term micro-level, such as Miaou \& Lord, 2003, horizontal curves, and other road segments ,e.g., Management et al., n.d.; Lord, 2000; Sayed, 2001. Recently, transportation engineers have started to pay more attention to the development of macro-level CPMs. Different approaches have been used previously to capture the effect of some potential contributing factors of different spatial units on the road traffic collisions. In this literature, the road traffic collision is modelled at various level of data aggregation, ranging from point events up to different area levels.

Many contributing factors have been used in modelling collisions at the planning level. G. R. Lovegrove \& Sayed, (2006) categorized factors that influence safety at traffic 
zone TAZ level into four categories, namely: roadway network characteristics, sociodemographic characteristics, exposure, and transportation demand management (TDM) variables. Various factors, falling under these four groups, were considered in pervious road safety studies. Typically, the first three categories could be quantified at the selected aggregation level, whereas GIS data is typically employed to obtain the network characteristics on these levels. Table 2.1 shows some examples for the data categories.

Table 2.1Examples for Macro-level Data Categories

\begin{tabular}{|c|l|}
\hline Category & \multicolumn{1}{|c|}{ Variables } \\
\hline \multirow{2}{*}{ Socio-Demographic } & Population density \\
& Number of households \\
& Number of employees \\
\hline Roadway Network & Intersection density per unit area \\
& Minor roadways lane-kilometres \\
& Major roadways lane-kilometres \\
\hline Exposure & Vehicle distance travelled (VKT) \\
& Total roadways lane kilometres (TLKM) \\
& Average congestion (VC) \\
& Number of drivers \\
& Number of commuter \\
& Modal splits (the share of different \\
& modes of transportation ) \\
\hline &
\end{tabular}

Levine et al. (1995) conducted one of the first collision analysis to describe spatial patterns in the records of motor vehicle collisions in City and County of Honolulu. In addition, collisions for every hour of the day, weekdays and weekends were analyzed. 
The relationships between all collisions, alcohol related, and the type of impact and various predictors including population and employment data were taken into consideration. Spatial software tools, including centre of gravity, standard distance deviation, the standard deviational ellipse, and the nearest neighbour index were developed for describing the degree of spatial concentration of collisions. The findings of this study showed that collisions vary spatially based on changes in traffic volume patterns. Moreover, employment centres experience a greater number of collisions than the residential areas. The author attributed this result to the higher traffic volume and more congestion at employment centres. Also, it was pointed out that concentration of collisions during the daytime on weekdays is higher than weekends' daytime; collisions at night time were found to be significantly different. With respect to the relationship between population and alcohol, it was stated that the severity of alcohol-related collisions is greater near the residential areas rather than in the more concentrated employment centres. This might be attributed to the travel speeds and night-time driving, higher concentration of teenagers, higher concentration of unemployed males and late night social drinking.

In a subsequent study, Levine et al.( 1995) investigated the relationship between activities which generate trips and the collision frequency in the City and County of Honolulu. A spatial lag model was used to explain how collisions vary spatially using aggregation level at small geographical areas. The authors argued that this type of models offers a very good benefit in that it relates the expected collisions not only to particular road segments or intersections, but also to the entire metropolitan area. Various covariates, including population, employment and some road characteristics were 
investigated and found to be statistically significant. The results of this study indicated that collisions vary significantly over the day due to the fluctuation of the trip generating activities.

Tarko et al.(1996) examined a sample of 21 collision categories that occurred in 92 counties in Indiana. Negative binomial regression was conducted for six-year collision data covering the period from 1988 to 1993 . The authors highlighted a very significant issue, which was the presence of multicollinearity among some of the potential covariates. They suggested using a stepwise regression procedure to overcome the models' evident over-fitting and to determine the most significant variables that explain a reasonable fraction of the variance in collision frequency. Among the considered variables, the Vehicle Miles Travelled (VMT) and the population density appeared as the most significant variables. It was also indicated that as the VMT increased, particularly on the urban roadways, the collisions within the counties would increase. Similar observation was noticed regarding the population density variable.

Jones et al. (1996) presented spatial point pattern analysis in the road traffic collisions. They attempted to set a spatial clustering on the residuals of a logit model using a K-function analysis. Age, road classification based on its speed, presence of pedal cyclist and motor cyclists were the indicators of this model. The results showed that the likelihood of death for casualties in road traffic collisions is largely associated with the taken explanatory variables.

Karlaftis \& Tarko (1998) considered the heterogeneity in collision modelling using panel data. A total of 92 Indiana counties were separated into three groups; urban, suburban, and rural areas. Models were developed for each group, as well as for the 
combination of all groups. The authors argued that in the combined model, which did not account for the variation in land use between the counties, the parameter coefficients were biased for the urban and suburban areas. Some variables lost their significance in the combined model due to the heterogeneity in the data. It was found that the total length of roadways within the counties was significant. Amoros et al. (2003) estimated models using the data on traffic collision for 8 counties in France. The interaction between the road type and the county was modelled using a Generalized Linear Model (GLM) assuming negative binomial error structure. Due to unavailability of data on property damage collisions, this study included only fatal and injury collisions. This allowed the development of two types of models. These are the incidence of road collisions (counts of collisions) and severity of road collisions. Severity, which was defined as the ratio between the counts of fatal collisions and the counts of injury collisions, was modelled using a logistic regression. Several infrastructure characteristics and demographic indicators were used as the independent variables. The forward selection method was used to obtain the most significant covariates for the models.

MacNab (2004) analyzed small-area variations in collisions and injury using the Bayesian spatial modelling technique. The study aimed at assessing the risk factors measured at group level. Various independent variables were considered in this study, which include socio-economic (age cohort), residential environment (parks and roads), medical services availability and utilisation crime rates and etc. This study showed that the collision injuries vary regionally in males aged $0-24$. In addition, it was found that the effect of socio-economic factors on collision injury is significant for males of all ages, especially in the areas with lower income. 
Noland \& Quddus (2004) used spatially disaggregate ward level data for England, where a ward is a division of a city or town used in England for administrative and representative purposes. Three models were developed using a negative binomial technique to predict the traffic fatalities, serious injuries and light injuries, collectively named traffic casualties. Four categories of explanatory variables were taken, including: land use types (employment and population density), road characteristics, e.g., number of roundabout and junction per meter square in area, and demographic characteristics, e.g., age cohorts. The outcome of this study showed that the land use and area deprivation (the percentage of persons under poverty) are strongly associated with the traffic casualties. The Level of fatalities is greater in the rural areas when compared to the urban areas. The authors attributed this finding to the reduced speeds, higher levels of congestion or possibly the lower design speeds of the roads in the urbanized areas. They reported an increase in the level of fatalities in areas with higher employment density and lower population density. Area deprivation was found to have a positive effect on the total traffic casualties. The effect of this variable was found to have a greater association with light injuries than other casualties. No statistical association of changes in traffic casualties is found for the number of nodes and roundabouts and the length of motorway per square meter of a ward.

The main focus of the study by Noland \& Oh (2004) was to examine the effect of the infrastructure and the demographic change on the traffic-related fatalities and collisions. The demographic data included in this study consisted of the population, income per capita and county age group as percent of the total population. Country-level data for the State of Illinois was used to develop two models using a negative binomial 
regression analysis.

Aguero-Valverde \& Jovanis (2006) examined how Fully Bayesian (FB) Hierarchical Models can be used to estimate the structured spatial and temporal effects. The outcomes of these models were compared to the traditional negative binomial models. Similarly to Noland and Oh (2004), county-level collisions data was used. Three data categories were used, including socioeconomic factors (e.g., age groups $0-14,15-$ 24, and over 64.), transportation-related factors (e.g., Federal Aid Miles of Road (\%) and Miles of Road Density $\left(\mathrm{mi} / \mathrm{mi}^{2}\right)$, environmental factors (e.g., mean total precipitation and mean total snowfall). For the data set under consideration, the results showed that the most significant variables in the negative binomial models are also significant in the FB models. Collision risk is found to be significantly increased in the counties with higher percentage of population under poverty level. A higher percentage of this population belongs to the age groups of $0-14,15-24$, and over 64 . In terms of the transportationrelated factors, the results showed that the increase in the road mileage and the road density within a county will lead to a significant increase in a collision risk.

El-Basyouny \& Sayed (2009) developed two models of collisions incorporating spatial components by using 281 urban road segments in Vancouver, British Columbia, Canada. The data was analyzed using the Gaussian conditional autoregressive (CAR) and the multiple membership (MM) models. The results of these models were compared with the traditional Poisson-Lognormal model. In addition, the authors aimed to investigate the effect of clustering segments within the same corridor on the spatial correlation. The model parameters were estimated in a Fully Bayesian framework using Markov Chain Monte Carlo sampling techniques. The variables used in these models were annual 
average daily traffic (AADT), the number of lanes between signals, the business land use, and the concentration of un-signalized intersections. It was stated that the CAR and MM models are more suited for estimating the heterogeneity and spatial correlation parameters. It was also concluded that the corridor variation was a major component of the total variability and that the spatial effects have been considerably reduced by the clustering segments within the same corridor.

Huang et al. (2010) developed county-level models for predicting the collision frequency by using a five-year (2003-2007) aggregated collision data. The macroscopic model used the roadway data from 67 counties for the state of Florida. A set of explanatory variables were considered in this study to explain collision variations between the counties. They are similar to the categories referenced in the aforementioned studies. In this article, the daily vehicle miles traveled (DVMT) and the population were considered to represent the exposure and were used in different models. The roadway traffic variables were: road density, which was based on different road classifications, intersection density, proportion of truck AADT over the total AADT, and the travel time to work. The latter variable represents the travel distance from the residence. In addition to various age cohorts, the population density was used to represent the sociodemographic factors. Median household income, percent of population under poverty line, and unemployment rate were used as the surrogate measures to reflect the area depravation level.

Huang et al. (2010) also tested the spatial auto-correlation of observed collisions among the adjacent counties using Moran's I test (Moran's I test has a value between -1 and +1 , a positive value suggests positive spatial correlation whereas a negative value 
indicates dispersion). The test results suggested that the spatial correlation between the counties is significant where all Moran's I values for all collisions types were positive. Then the estimated coefficients were obtained utilizing the Bayesian spatial model by specifying a conditional auto-regressive prior (CAR) model in order to realize the spatial autocorrelation. It was stated that a non-linear relationship between the collision rate and exposure, generally, results in a large increase in the rate of all collisions frequencies, which is associated directly with the increases of DVMT and the population. The findings demonstrated that controlling the DVMT and the population produced no significant variation in the safety effect of the risk factors on the collisions.

\subsubsection{Zonal CPMs}

Several previous academic works for the development of CPMs at TAZ's level are reviewed in this section. All of these studies have confirmed that an efficient strategy to ensure long term road network safety is to incorporate road safety analysis into the planning process.

Hadayeghi et al. (2003) presented a series of macro-level models for predicting of collision frequency using 1996 data aggregated for 463 TAZs in Toronto, Canada. The developed models were for total and severe (fatal and non-fatal injury) collisions. The collisions were related to different zonal socio-demographic, traffic demand, and network data variables. In this study, exposure data, including Vehicle Kilometres Travelled (VKT) and averages of congestion (VC) for each individual TAZ were generated using EMME/2 software. The VC in this study is measured for each link in the zone as the average of volume/capacity ratio. The aggregated zonal data did not include freeways infrastructure and their related traffic. Once all data were aggregated, negative binomial 
models were developed to estimate the model parameters. The findings of this study revealed that the collision occurrence at the TAZ level was associated significantly with the increase of intersection density, length of roadways, as well as number of households within TAZS. On the other hand, the results suggested a significant decrease in collision frequency as the increasing in average congestion. This study revealed some counterintuitive results where it indicates the possible reduction in collision frequency as the posted speed limit increases. The second section of this study was to explore spatial variations in the model relationships using a Geographically Weighted Regression (GWR) model. Taking into account the local variations in the parameter estimates from zone to zone was found to increase the prediction ability of the model by $17 \%$ as measured by the coefficient of determination $\left(\mathrm{R}^{2}\right)$. In the final remark, the authors suggested that different types of employment variables such as manufacturing, retail trade, and financial should be considered for the future works.

Using 859 TAZs' data from Tucson, Arizona, Guevara et al. (2004) developed a series of macro-level CPMs to estimate separately the number of total, severe (fatal, nonfatal injury) and PDO collisions. The Generalized Linear Modelling (GLM ) approach was applied in which a non-linear, exponential function, with negative binomial error distribution, using simultaneous equation and log-linear transformation techniques was used. The population density was used as a leading exposure measure in this study instead of using exposure measures such as VKT and AADT. Despite the separation of age groups $0-17$ (by law, they are not allowed to drive) from the total population, the population density was found misleading in developing CPMs due to its failure in achieving the required property for a CPM which is that the collision frequency is zero 
when there is no exposure. In other words, the developed CPMs failed to predict zero collisions in TAZs with zero exposure variables as the population density is the leading exposure measure.

.The work by Hadayeghi et al. (2003) was updated in a later publication in Hadayeghi et al. (2007). As indicated earlier, the collisions were related to different zonal socio-demographic, traffic demand, and network data variables. The only differences in Hadayeghi et al. (2007) were including more variables to explore the effects resulted from the variation in land use, employment and the existence of transit facilities. The number of included TAZs was different, where the safety at 481 TAZs was evaluated in the updated study. The results of this study showed an increase in number of collisions within Toronto's traffic zones as population density, unemployment, job density, and shortcut capacity, number of drivers, total commuters, and total commuter density increase.

Lovegrove has developed a series of studies in order to develop macro-level collision prediction models at community-based. Most of his studies were at the TAZ level. Lovegrove \& Sayed (2007) introduced guidelines that can be used by planners to consider the road safety explicitly in all planning stages. CPMs were also developed using GLM methods to generate collision prediction models that have negative binomial error structures. Models were developed for both urban and rural areas, and then ultimately these models were used in an Empirical-Bayes analysis of the data for use in a "Black-Spot Program". They proposed a method for determining "Collision Prone Zones" based on the macro-level CPMs. 
The work by Lovegrove (2008) was a continuation of the author's earlier work in attempt to develop model-use guidelines to perform a road safety evaluation. In this study, total collision frequency was modelled using measured and modelled (the output of transportation planning models, such as Emme/2 software) independent variables. Similar to Hadayeghi et al. (2003), limited-access highways were excluded from the analysis due to their unexpected impacts on road safety of individual neighbourhoods. Among the considered predictors, the averages of congestion (VC) were found to have the most significant effect on the CPMs results. CPMs were developed with and without the inclusion of VC. The results showed improvement in CPM estimates. The results also revealed that the collision frequency decreases when the VC decreases.

Abdel-Aty et al. (2011) used negative binomial regression in order to investigate the relationship between the collision frequency and the frequency and the type of trips attracted and produced at the TAZ's level. The paper was based on 1,349 TAZs of four counties in the state of Florida. A number of models were developed, using 2005-2006 collision data, to predict the total collisions, the severe (fatal and severe injury) collisions, the peak-hour collisions and the pedestrian- and -bike-related collisions per TAZ. Collisions predictors were categorized in two major categories: $(i)$ the roadway characteristics-related variables, which include the total roadway segment length within a TAZ with a posted speed limit and the total number of intersections, and (ii) the trip productions and attractions related variables which include home-based work productions/attractions, home-based social recreational productions/attractions, heavy truck productions/attractions and taxi productions/attractions. The study concluded that the total trip productions and the total trip attractions provide a better model fit for the 
total and the peak-hour collisions. In terms of severe collisions, the estimated results associated this type of collision to different trip-related variables.

Sun \& Lovegrove (2013) compared the safety influences of street patterns at the community level. Four network pattern including grid, culs-de-sac patterns, 3-way offset, and Dutch sustainable road safety (SRS) patterns were compared to the Fused Grid pattern. Their finding showed that the Fused Grid and 3-way offset pattern is better than the others in terms of safety performance.

The most recent work of zonal-level modelling of traffic collisions was developed by Wang, Jin, Abdel-Aty, Tremont, \& Chen, 2012. The authors investigated the relationship between collisions occurrence and the road network structure (meshed-ness coefficient) in addition to other different explanatory variables, including the demographic and the land use factors. The TAZ-based model was developed using a conditional autoregressive model (CAR). The finding of the study indicated that the meshed-ness coefficient was good to account for the nature of network patterns, in addition to linking the relationship to the zonal level collisions.

\subsection{Pedestrian-involved CPMs}

It is intuitive that the increase in population, together with the increase in traffic volume for motor vehicles, will lead to an increasing interaction between them, which in turn leads to an increase in pedestrian-involved collisions. Vehicle-pedestrian collisions are considered to be a major problem in traffic safety. According to the City of Ottawa database of 2007-2011, approximately 1,718 pedestrian-involved collisions were reported. Generally, most of the previous studies concerning the issue of pedestrian- 
involved collisions focused only on the influence of specific site characteristics, such as traffic volume at intersection or the road geometric. Only a few studies were performed to relate the pedestrian-involved collision occurrence to the planning characteristics. Some of these studies are included in this review.

Fontaine \& Gourlet (1997) examined fatal pedestrian collisions in French counties. A total of 1,289 pedestrians were reported to be killed in the collisions on the French roadways. In order to develop a model, several variables were considered, including age, sex, movements, change of transport mode and alcohol impairment. The findings of this study showed that the children and the seniors were the most vulnerable pedestrians.

(Aj, Rwg, Mjb, Bh, \& Cg, 1997) estimated the influence of a reduction in speed limit on the frequency fatal pedestrian-involved collisions in the Adelaide metropolitan area. The main observation in this study was that the fatal pedestrian-involved collisions significantly decreased when a small reduction in speed limit was applied. It was stated that in some cases the reduction of the travelling speed could also lead to avoid some pedestrian collisions because the drivers were able to take action and control their vehicles.

Al-Ghamdi (2002) collected pedestrian-involved collision data for Riyadh, Saudi Arabia. The attempt was to investigate the causes, characteristics, and the most common patterns of the pedestrian-involved collisions. The results of this study showed similarity with Fontaine \& Gourlet (1997) results, where the probability of collision involvement increases for the young and the old age groups. Also, the collision severity is greater among the elderly people. In addition, it was concluded that the divided roadways with 
relatively high speed limits and the residential streets were the most likely pedestrianinvolved collision hot spots.

Lascala et al. (2000) focused on the investigation of the pedestrian-involved collisions as a function of different demographic characteristics for the City of San Francisco. Pedestrian-involved collisions and environmental and demographic characteristics were mapped using a geographic information system GIS. Using ordinary least squares (OLS) regression model it was found that the probability of pedestrians involved in collisions is greater in the area with higher population, higher average daily traffic and larger number of cross-streets per kilometre roadway.

Zajac \& Ivan (2003) evaluated the impact of roadway and the area type features on the severity of injury of pedestrian-involved collisions in the rural Connecticut. The ordered Probit model was utilized to conduct the analysis. The study was specified only for pedestrians who were trying to cross the uncontrolled two-lane highways. The author identified seven different area types, including downtown, compact residential, village, downtown fringe, medium-density commercial, low-density commercial, and low-density residential. Among these area types, village and downtown fringe areas were associated with the most frequent pedestrian-involved collisions.

Lee \& Abdel-Aty (2005) applied log-linear regression to identify the group of driver- and pedestrian-related factors, as well as the traffic and environmental characteristics that are associated with pedestrian-involved collisions. Information on individual walking trips in the household travel survey was used to provide a logical measure for the pedestrian exposure. The study used several demographic factors including different age groups, vehicle type, roadway characteristics, location "rural or 
urban", traffic control-location interaction, lighting, drivers' alcohol/drug use, and alcohol-lighting interaction. The study results revealed that most of the considered variables had significant effect on the frequency and the severity of injury of the pedestrian collisions. It was also stated that the frequency of the pedestrian collisions is greater at the intersections with a high average traffic volume.

Wier et al. (2009) used the cross-sectional data for the City of San Francisco to study the effect of different census tract area-level characteristics on vehicle-pedestrian injury collisions (fatal and injury collisions). The ordinary least squares regression (OLS) was used to develop the models assuming that the pedestrian-involved collisions are normally distributed. The analysis was repeated using a negative binomial regression model, which resulted in very similar coefficients and standard errors. It was noted that the developed model can be used as a support tool for the development of the pedestrian injury prevention programs. It was concluded that the pedestrian-involved collisions are highly associated to the occurrence of pedestrian-involved collisions. In addition, some variables such as employee, resident populations, people living in poverty and proportion of people aged 65 or over had a significant impact on the of pedestrian-involved collision occurrence.

Obeng (2013) provided a descriptive study to determine the potential factors that could lead to an increase in the probability of a pedestrian being involved in traffic collisions on the trunk roads. In addition, the regional differences in the pedestrianinvolved collision data were assessed. It was concluded that $76 \%$ of vehicles involved in pedestrian collisions were light vehicles and pedestrian group between the ages of 14 and 46 are more likely to be involved in the traffic collisions. In terms of comparison of the 
pedestrian-involved collisions between regions, the obtained result showed that the regions with a greater number of vehicles were associated with a greater number of the pedestrian-involved collisions.

\subsection{Bike-involved CPMs}

Turner et al. (2006) conducted a study to develop CPMs using the pedestrianinvolved and the bike-involved collisions from three different municipalities in New Zealand. The study aimed mainly at developing CPMs collisions that occurred specifically at the signalized intersections, the roundabouts and the mid-blocks on the arterial routes. Also, the authors attempted to estimate the potential differences in the frequency of collisions and the collisions rates that have resulted from the changes in transportation mode choice.

Brüde \& Larsson (1993) used the least squares estimation method to develop models to predict the number of pedestrian-involved and bike-involved collision for 30 towns in Sweden. Cyclist exposure was based on the number of passing cyclists per average annual day and the approaching vehicles avenge annual daily traffic (AADT) for 377 intersections. The results of the analysis illustrated that the risk of involvement in a collision for a cyclists is higher when the vehicle traffic volume increases. It was also mentioned that there would be a reduction in the risk to cyclist as the cyclist volume increased.

Lovegrove \& Sayed (2006) applied a negative binomial regression analysis to develop several macro-level CPMs for the Greater Vancouver Regional District (GVRD) in $\mathrm{BC}$, Canada. Among the developed models, there was one specified for the prediction 
of the bike-involved collisions in the GVRD rural area. It was concluded that the probability of a cyclist involved in a collisions become significantly higher in the rural area when the bicycle mode share is increased.

Kim et al. (2010) used the data that collected from Honolulu in order to investigate the relation between the area characteristics and the various collision types. The independent variables include: population, land use, and accessibility measures such as road length, bus stops, and number of intersections. These variables were aggregated at census block group level. The Poisson and negative binomial were first applied to conduct the analysis, but they did not statistically fit the obtained data. Therefore, a binary logistic regression model was used as an alternative to estimate the model parameters. The findings of this study, regarding bike-involved collisions, revealed that the bike-involved collision occurrence significantly increased in an area with more accessibility variables. In addition, it was observed that there was a significant positive relationship between the bike-involved collisions and the job count and the number of people under poverty.

\subsection{Boundary Collisions Issue}

It is a common knowledge from previous studies that there is a common challenge with the roadway data being located on or near to TAZ boundaries. It is expected that this issue will affect the prediction ability of the macro-level CPMs. Fotheringham (2000)

studied spatial models and GIS and recognized that there was a possible inter-zonal influence in spatial data that is placed on or near the boundaries between the two spatial units. The inter-zonal influence is defined as the sharing effect of characteristics of 
adjacent spatial units on a phenomena occurring at their shared boundary. Many previous studies in road safety were built on the assumption that collisions at a specific TAZ were related directly to its characteristics. This assumption has been violated due to the fact that some collisions on roadways (mostly arterial) forming the TAZs boundaries might have been inflicted by the drivers from other TAZs. Consequently, the nature of these collisions is presumed to be a result of the overlapping characteristics of the adjacent TAZs. Some modelling attempts that were carried out to highlight this issue are reviewed in this section.

Guevara et al. (2004) developed several zonal CPMs. Two years of collision data between 1998-1999 in 859 TAZs in Tucson, Arizona were collected for the modelling attempts by use of the negative binomial regression model. In this research, the data attributes were assigned to each TAZ as they were geocoded in the ArcGIS software (Environmental Systems Resource Institute (ESRI)). During their data collection, two types of random errors were identified regarding the allocation boundary data between TAZs. It was found that some roadway segments were located exactly on the boundary lines of the TAZs. Guevara et al.(2004) assumed that there will be inter-zonal influence for such data, but the authors neglected this issue when they found that only $5 \%$ of their roadway segments are located on TAZs' boundary lines. The other error was with respect to data point that did not lie on the roadway segments, such as collisions and bus stops. It was assumed that the error due to the varying accuracy of measurements in the data points is random and it is thought to be small; therefore, it would not have a significant effect on their models' results.

Lovegrove \& Sayed (2006) presented the collision models using a negative 
binomial regression for urban and rural areas separately. In this study, a strong assumption was made by assuming existence of inter-zonal influence of the data located along zonal boundaries, but this study did not take any steps to check if this assumption will lead to a change in the final results of these models. It was pointed out that the geocoded collisions are of doubtful accuracy with error of $\pm 20 \mathrm{~m}$. Due to this error, it was assumed there will be overlapping of collision points with one or more other traffic zones. Despite all the above points the author ignored the possible inter-zonal influence and aggregated his data in the same way as was done in the previous paragraph.

Siddiqui \& Abdel-Aty (2012) explained the issue of boundary collisions in different way. They stated that aggregating the zone attributes, as they geocoded along its boundaries, leads to inaccuracies. Some variations were noticed in the nature of collisions within the TAZs boundaries. For example, it was found that boundary collisions formed $70 \%$ of the total collisions within Hillsborough and Pinellas Counties. Therefore, to investigate this issue, the authors (separated the data into) two sets of collisions. These are interior and exterior collisions. Boundary collisions were defined as collisions that occurred within $100 \mathrm{ft}$ buffer zone around the TAZs boundaries.

As stated earlier, the authors divided the collisions into two types, which are exterior and interior collisions. The latter was assumed to be effected only by the characteristics of the TAZ in which they are geographically located. On the other hand, exterior collisions of a specific TAZ (namely, TAZ $i$ ) are presumed to be impacted by the characteristics of the adjacent TAZs. To account for such influence, the characteristics of each neighbouring TAZs were weighted according to the boundary length that they shared with the TAZi . This assignment method allowed for preparing a set of explanatory 
variables that were used in developing the boundary collision prediction model of the $\mathrm{TAZ}_{i}$. It should be noted that only pedestrian collisions were considered in this study, yet the authors have stated that other types of collisions models may be used by following the same approach as for the pedestrian collisions for investigation of the boundary influence.

Wang et al.(2012) shed more light on the issue of the boundary collisions. They identified some problems associated with allocating the roadway segments and the collision data to their corresponding TAZs. The problem was represented in mismatching of the data that was obtained from different sources, which would usually lead to an incorrect allocation of collisions and other roadway component to the TAZs. To overcome this issue, all roads and TAZs boundaries that do not match were adjusted to correspond with TAZs boundary line by using ArcGIS software. Once the boundaries were adjusted, the collisions and all road features located on the boundaries located within a buffer zone of $60 \mathrm{~m}$ around the TAZs' boundaries were distributed equally between the adjacent TAZs. Although there was a treatment conducted in order to allocate the boundary data, it was not stated whether this treatments was effective in improving the models' results.

Wei (2010) was the most extensive study on this matter, which explored the interzonal influence for the geocoded collisions at the TAZs' boundaries. Five different methods of aggregating collisions that occur on the zone boundaries were examined:

- geo-processing methods (data attributes aggregated for each TAZ as they were geocoded in ArcGIS)

- Half-half method (equal portion of a collision is assigned to each TAZ forming the boundary) 
- One-to-one method (each TAZ forming the boundary is assigned one whole collision)

- VKT Proportion (each TAZ is assigned the collision in proportion with its VKT)

- TKLM Proportion (same as above, but with measured "total lane kilometre s")

In this study the boundary collisions were defined as the collisions that occurred within 20 meters of a zone boundary. The outcome of different models showed some interesting results that the models were similar from the geo-processing methods, halfhalf method and one-to-one method. Yet the VKT/TLKM models were to some extent different. However, this is to be expected, because the aggregation method is different.

"Collision-prone zones" (CPZs) were identified using each methodology, and the top zones were identified. The CPZs were ranked using twin-ranking criteria, Potential Collision Reduction (PCR) and Collision Risk Ratio (CRR) which were obtained based on empirical Bayes (EB) safety estimate. The EB in turn was obtained by combining the observed collisions and predicted collisions. It was determined that there was greatest similarity between the results of the geo-processing method and the half-half method and that the one-to-one method produced a significantly larger number of CPZs. The VKT and TLKM methods showed less similarity. Wei (2010) argues that because there are differences between the methods, collisions located near zone boundaries do have an inter-zonal influence and that the aggregation method impacts the CPM development. It was concluded that the half-half method is the one that should be recommended for the application because, when used in CPZ identification, it retains the highest similarity rate of the CPZs as identified (compared) with all of the other methods. Additionally, it is 
intuitive, since it allows for the influence of each zone in the collision without increasing the total number of collisions in the region being modelled.

A review of the literature shows that there is an inter-zonal influence of the spatial data located on or near zone boundaries and that most of the previous work in macrolevel CPMs has recognized this, but has not adequately justified the approaches taken to measure the effect of the inter-zonal influence. Typically, these are to ignore the effect of inter-zonal influence (i.e., just let the collisions be placed in the TAZ the GIS program assigns them to), or to assign the collisions on a "half-half" basis to each TAZ that forms the boundary. Wei (2010) points out this problem and then aggregated the boundary data to the TAZ aggregation level using different assignment methods. An important limitation in his study was that the boundary assignment methods were applied only to the total and severe (total of non-fatal and fatal collisions) collisions data and his models associated the collision frequency only to the exposure variables, e.g., VKT and TLKM. Therefore, this thesis expands the investigation regarding the inter-zonal influence in the boundary data to ensure their effect on the prediction ability of the macro-level collision prediction models (CPMs) for different types of collisions. Numerous TAZ's characteristics are used to develop a series of CPMs. The considered contributing factors and the different methods for assigning the boundary data are discussed in details in the following chapter. 


\section{Chapter 3: Data Collection}

This chapter describes the data collection effort and presents statistics of collected data. The collected data included various demographic and socioeconomic data for the study area as well as roadway characteristics, exposure, and collision data. The chapter is divided into four sections. The first section describes the study area and the geographic aggregation of data into traffic analysis zones (TAZs). The second section describes the different data categories used to support collision prediction models developed in subsequent chapters. The third section presents the procedure used for treating boundary data; observations which lie at the boundaries of the traffic analysis zones. The third section introduces also different assignment methods to allocate boundary data to different TAZs. Finally, the fourth section shows the spatial distribution and descriptive statistics for the used variables in this research.

\subsection{Study Area}

It is useful to outline the boundaries of the study area before discussing the details of the geographic aggregation of data. This helps to make the study effort focused and the data collection feasible. The study area is composed of all geographic units used by the City of Ottawa for traffic studies and socioeconomic data organization for which collision data was available. For the purpose of developing conventional transportation planning models, the concerned National Capital Region joint transportation planning committee (TRANS) has divided the National Capital Region, which covers the City of Ottawa and City of Gatineau, into a group of geographical units known as traffic analysis zones

(TAZs). TRANS is the main organization in National Capital Region that manages 
transportation studies and collects data for transportation planning. These TAZs vary according to their spatial size, ranging from large areas in the rural West, rural East, rural Southwest, and rural Southeast, to smaller areas closer to the central business districts (the Downtown area of Ottawa). In 2005, the number of traffic zones in the National Capital Region was 344 representing 20 districts. A district is defined as a division with a particular characteristic used sometimes by municipalities for administrative purposes. After conducting the 2005 Origin-Destination (O-D) survey, the TRANS committee reviewed the division system and increased the number of TAZs up to approximately 600. Because these $600 \mathrm{TAZs}$ cover both cities of Ottawa and Gatineau, collision data received from the City of Ottawa would cover only a subset of these TAZs, which fall in the City of Ottawa. Therefore, the total TAZs covered in this thesis are 422, which could be considered as an adequate sample to establish collision prediction models relating collision frequencies to TAZs related variables. As shown in Figure 3.1, the data is only available for the highlighted region. It should be noted that this map and all other maps presented in this thesis were projected using the projection coordinate system: Ontario MTM Zone 9, 78 to 75 deg West; NAD 83 datum. 


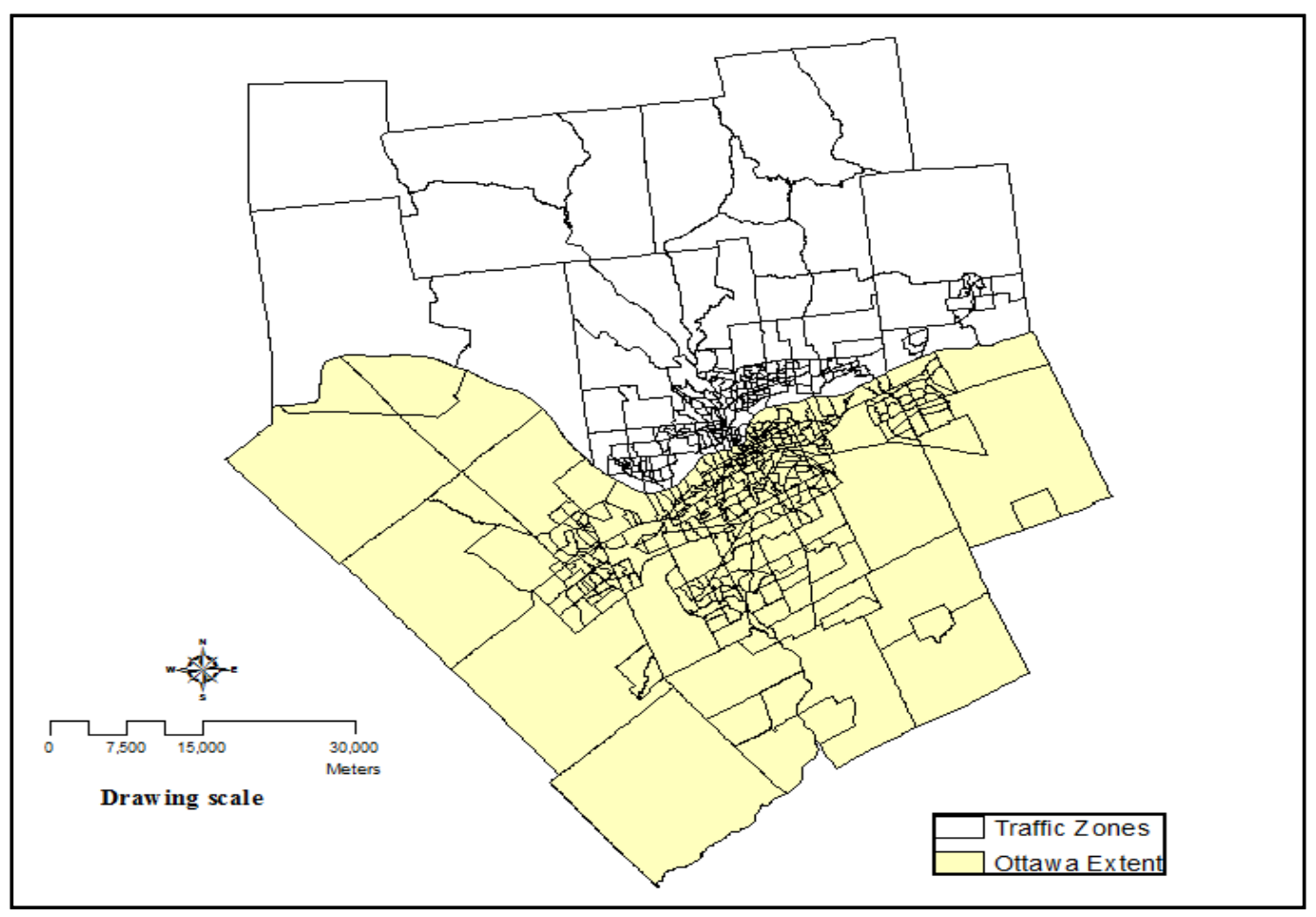

Figure 3.1 National Capital Region Map

\subsection{Data Categories}

As indicated earlier, one objective of this study is to analyze differences in traffic safety at the level of TAZs, i.e., where every TAZ provides a sample of collision observations. Many explanatory variables are considered in this study to investigate their effect on the safety performance at the TAZs' level. These variables are categorized as follows.

- Roadway characteristics.

- Traffic exposure.

- Socio-demographic characteristics.

- TDM-related (namely modal splits which are the share of different transportation modes). 
The mentioned variables are used as independent variables to predict the following response variables:

- Total collisions (including all types).

- Fatal collisions.

- Non-fatal injury collisions.

- Property damage only (PDO) collisions.

- Bike-involved collisions.

- Pedestrian-involved collisions.

The following sections are detailed information about how these different variables were extracted.

\subsubsection{Collision Data}

Collision data was provided for the whole study area by the City of Ottawa in a shapefile format readable by the Geographic Information Systems (ArcGIS) software. Each record in the shape file provided several attributes which include information about collision location, environmental condition (clear, rain, snow, fog, and others), light condition, traffic control, road characteristic, road surface type, road condition, road pavement marking, vehicle type, road jurisdiction, classification of collision, initial impact, vehicle manoeuvre, sequence of events, driver's sex, and vehicle damage. All data was collected for the calendar years 2007-2011 (five year period). Four severity levels were identified in the coded collisions which include fatal, non-fatal injury, property damage only (PDO), and non-reportable collisions. The Ministry of Transportation of Ontario (MTO) definitions for each of collision severity level classifications specified in this research are as follows: 
- Fatal: A collision whose outcome is the loss of life for at least one road user either at the scene or in a period of thirty days from the collision occurrence.

- Non-fatal injury: A collision whose outcome is at least bodily injury of one road user; no death is reported in such collision classification.

- PDO: A collision whose outcome is damage to property either vehicles or any others, e.g., government property, without any bodily injury or fatality. This level of collision severity is reported if the property damage excesses $1,000 \$$.

An initial evaluation of the City of Ottawa collision database was performed and it was found that the collision entries were recorded only for every vehicle involved in a collision. For example, if a collision involves two or more vehicles, each vehicle is coded in the database as one entry. In case when a collision involves a vehicle and a pedestrian or a bike, only the vehicle is coded as one entry in the collisions database. According to the data provided, the severity of a collision is typically the worst severity level of the collision. Since the objective of this research is to predict collision counts (as opposed to counts of persons involved in collisions), only one entry was used to represent each collision.

One of the early objectives of this study was to develop severity-based person (number of persons involved in collisions) macro-level CPMs. However, it was not possible to develop these CPMs as the current database does not include the total number of road users (including passengers) involved in a collision in addition to each road user's injury severity level if any. This however remains an interesting topic for future investigation.

The location of each reported collision within the study area was identified by the 
longitude and latitude coordinates. The collisions in the City of Ottawa's database are geocoded either at mid-blocks or intersections. This method of data geocoding allowed gathering the collisions to the selected aggregation level (TAZ's level).

It is noteworthy that the collision database contains collisions that occurred on freeways which pass through the City of Ottawa as well as collisions which occurred on other roadway classifications. However, collisions that occurred on freeways were excluded from the analysis due to the reasons explained in the following section. In addition, pedestrian-involved and bike-involved collisions could be identified from the collisions file by the given sequence of event attribute. This attribute defines all moveable and fixed objects involved in a collision, where a unique reference number (code) was given for each. For example, if a vehicle hit a bike and then turned towards a lighting pole on the side of a roadway, then all of these events were sequentially recorded in the received collisions file. Therefore, it was possible to identify collisions in which pedestrians and cyclists were involved.

\subsubsection{Road Network Characteristics}

In this study, the road network characteristics that are aggregated to the TAZ's level consist of the following.

- Total number of intersections.

- Total number of signalized intersections.

- Total number of non-signalized intersections.

- Total lane-kilometres length (TLKM).

- Total roadway segments length based on road classification. 
- Total roadways length with posted speeds from $40 \mathrm{~km} / \mathrm{h}$ to $100 \mathrm{~km} / \mathrm{h}$ (in 10 $\mathrm{km} / \mathrm{h}$ increments).

- Total number of bus stops.

- Total bike paths length.

Except for the roadway network and the bike paths network, all roadway characteristics were localized at points defined by their longitude and latitude coordinates. As such, these points may be at the boundary between two zones. Examining the distribution of boundary data (including inputs to the CPMs) is a major objective of this thesis.

The roadways characteristics were received in a shapefile format. Roadways network and bike paths files which included roadway and bike paths segments as polyline entities were received from the City of Ottawa in the form of geographic information system (GIS) shapefile as shown in Figure 3.2. The data include all the road segments for the calendar year of 2012. The road segments file was coded to contain detailed information about each road segment. This information includes road names, road classes based on their function, speed limit, neighbourhoods in which the road is located, and length of road segments.

Seven types of roadways classifications are identified inside Ottawa traffic zones. These are arterial, major collector, collector, local, city freeways, federal, and provincial highways. Before proceeding with any aggregation to the roadways network data, all freeways (Figure 3.3) which have fully controlled access have been removed from the database; whether they are managed at the provincial level or not. The freeways were excluded due to the unlikely dependence of collision occurrence on the characteristics of 
adjacent TAZs due to the large volume of long distance travel and full control of access (Hadayeghi et al., 2003; lavagrove, 2006). For this purpose, the collisions that occurred on those highways were not counted in data collection, the same rule was applied for the exposure variables which is the vehicle kilometre travelled (VKT) on these highways.

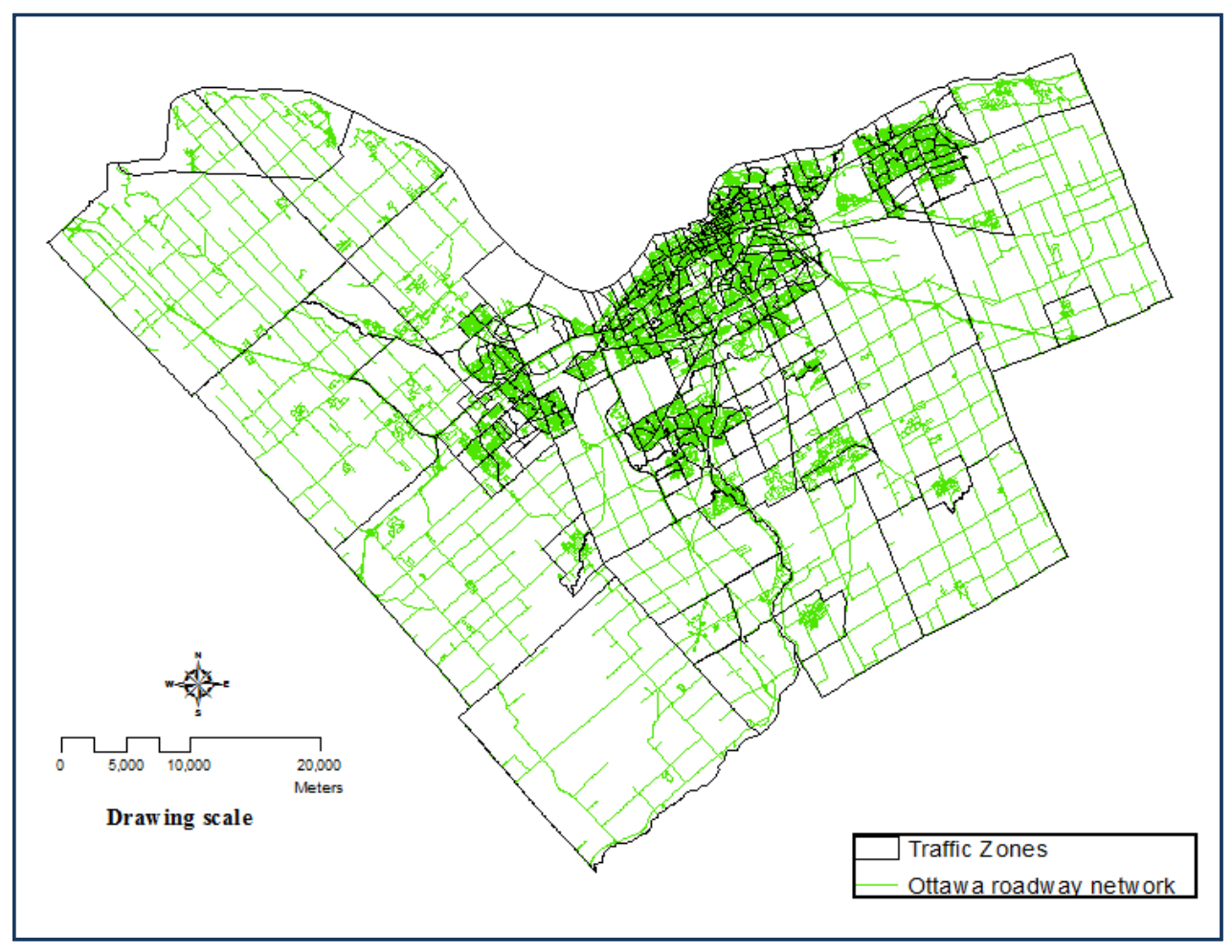

Figure 3.2 Roadway Network Map 


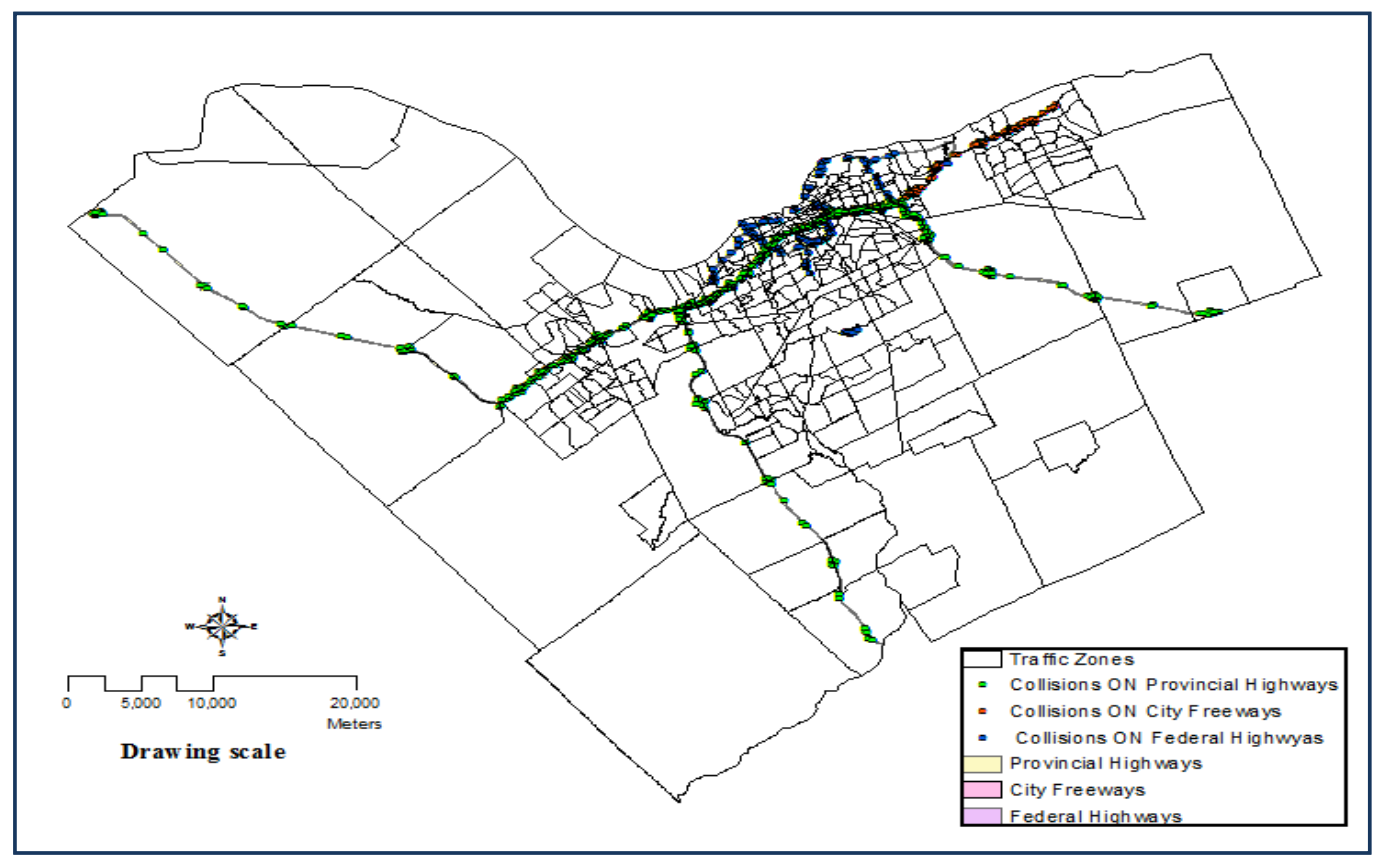

Figure 3.3 Fully Controlled Access Highways Map

\subsubsection{Traffic Exposure Variables}

The amount of travel activities which expose road users to the risk of collision, known as traffic exposure, was obtained for the entire study area from the City of Ottawa. Three types of traffic exposure variables identified in the literature are used in this study: [i] modelled vehicle kilometre traveled (VKT), [ii] trips produced, and [iii] trips attracted. The aggregated attracted and produced trips per TAZ were available for the morning peak period (6:30 - 8:59) in Excel spreadsheet format. The trip data for the selected TAZs was entered into the final input dataset in the format they were received. In addition to the mentioned exposure variables, the City of Ottawa database includes the average annual daily trips entering and exiting from the centroid of TAZs. The centroid connectors are used to represent the traffic on the local roadways network between the centroid of a TAZ and the major road network. Therefore, the total number of the trips "generated from" and "attracted to" the centroid of TAZs are considered also as traffic exposure 
variables. Two other variables are taken into account including the density of average annual trips entering and exiting from the centroid of TAZs per square meter for each TAZ.

The City's transportation planning department database contains both measured and modelled traffic volume data. Their survey for traffic counts does not cover all locations across the traffic zones. Consequently, the measured traffic volume results would not directly and sufficiently represent the traffic movement at the zonal level. Accordingly, it was decided to use the estimated traffic volume which was the final output of transportation planning models. The traffic volume on Ottawa's roadway network was the estimated result of a traffic assignment of the vehicle trips using the EMME/2 software (INRO,1998).

In order to develop the transportation planning models (precisely the travel demand forecast) at TAZ's level, the TRANS committee conducted the Origin-Destination (O-D) Survey in the fall of 2005 . The survey is a ten-week period survey conducted regularly every ten years in the National Capital Region on roughly 5\% of its population. The survey collected data regarding household characteristics (location, size, etc.), person data (age, gender, own a car or a public transit user, etc.), and trip characteristics (origin, destination, purpose, etc.) in order to provide a detailed information about trip patterns and travel choices. The collected data with the roadway network information for each TAZ were entered to EMME/2 in order to estimate the travel demand on the TAZ's links. This could be done by running the trip assignment in EMME/2 (TRANS 2008).

In order to validate their models reliability, TRANS had compared the modelled results with both reported and observed travel data which were obtained from the traffic 
counts at some locations for the year of survey. It was found that there are slight differences between the estimated travel demand and the reported one, where the models underestimated the auto trips with $(-1 \%$ and $-4 \%)$ during the $\mathrm{AM}$ and $\mathrm{PM}$ peaks, respectively (TRANS 2008). Since the estimated travel demand results are very close to the reported traffic counts on the TAZ's links, it was decided to use the estimated traffic volume in this study.

The output of EMME/2 software represents each roadway link as a pair of nodes. A node in a roadways network represents an intersection or a transit stop while a link can be defined as a directional connection between two nodes. Each of these nodes is identified by longitude and latitude coordinates. Traffic volume travelled between each pair of nodes is available for the morning peak period (6:30 - 8:59). Therefore, for the purpose of this study, the traffic volume was adjusted to represent AADT using a conversion factor of 5.6 which was provided by the City of Ottawa. To obtain the AADT on each link, the estimated traffic volume on each link was multiplied by 5.6. Furthermore, the VKT, which is used to represent exposure in this study, is calculated by multiplying the AADT volume travelling through each network link with its length

\subsubsection{TDM-Related Variables}

The Transportation Demand Management (TDM) can be defined as a group of strategies that support reducing vehicle travel by improving other transportation options (modes) such as biking, walking, public transit, and ridesharing. Modal split (also called the share of different transportation modes) is one key element in developing sustainable TDM strategies within a city or a province. Modal split is the percentage of total trips carried out by residents within TAZs using different transportation modes, including: 
motorized, non-motorized modes and pedestrian trips. The changes in the transportation mode choice are associated with changes in demographics, economic conditions, urban form and the transportation system.

For the purpose of this study, the modal split data for the City of Ottawa TAZs was received from the City of Ottawa transportation planning department. The aggregated modal spilt per TAZ is available for the morning peak period (6:30 - 8:59) in Excel spreadsheet format. In addition to internal trips and external inbound trips of a TAZ, the outbound trips are a part of the TAZs' modal split as well. The model was developed for different trip purposes including work or work-related, school, shopping, medical, and picking up or dropping off someone trips. The modal split data for the selected TAZs were entered into the final input dataset in the format they were received. It should be noted, in this study only the morning peak period modal split data was available, and it is assumed that the variation in modal split percentages during the day would not be significant. The modal split data includes the following:

- Modal Split Percentage Driver trips.

- Modal Split Percentage Passenger trips.

- Modal Split Percentage Transit trips.

- Modal Split Percentage Bike trips.

- Modal Split Percentage Pedestrians trips.

\subsubsection{Demographic and Socio-Economic Variables}

For the purpose of this research, some demographic and socio-economic variables pointed out in the literature were used. TAZ-level total employment, population, and number of households are used in the analysis. Furthermore, number of people in age 
groups between $0-4,5-14,15-24,25-44,45-64$, and older than 65 years old were extracted from the City of Ottawa database. As explained earlier, the data was based on the 2005 National Capital Region (TRANS) Travel Survey.

\subsection{Boundary Data Assignment Methods}

As indicated earlier, the problem of allocating TAZ's data that is coded on or near traffic zone boundaries was the major challenge in previous studies regarding the safety performance at the TAZ's level. In order to identify a data point (specific to data identified by a point location, e.g., collisions, roadway segment, or vehicle kilometre travelled belong geographically to which specific TAZ, different boundary assignment methods were utilized.

The different boundary assignment methods were utilized due to the fact that in some cases the traffic zones boundaries are set to match the centerline of roadway segments as shown in Figure 3.4. It is expected that collisions which occurred on these roadways would be affected by the characteristics of the adjacent traffic zones where these roadways act as a boundary. Typically, the TAZs', the collisions, and the roadway databases are created, coded and stored separately. This coding process sometimes produces imperfect data matching. Data used in this research had a considerable number of the roadways acting as nominal zonal boundaries. In the course of this study, it was found that boundary roadways do not match perfectly the TAZs' boundaries lines. In addition, many collisions were geocoded in a way that they do not precisely fall on the centerline of roadways segments. Due to these problems, it is assumed that extracting data attributes (collisions, roadway characteristics and exposure) for each TAZ as they 
were geocoded in ArcGIS would lead to unsatisfactory assignment for the data between TAZs. For instance, in some cases, some boundary roadways will be assigned to TAZs that have neither population nor any activities (TAZs in rural areas). Consequently, an appropriate method for assigning boundary data to TAZs needs to be developed. For this purpose, the following is the detailed description of the treatments performed for assigning the boundary data to the adjacent TAZs.
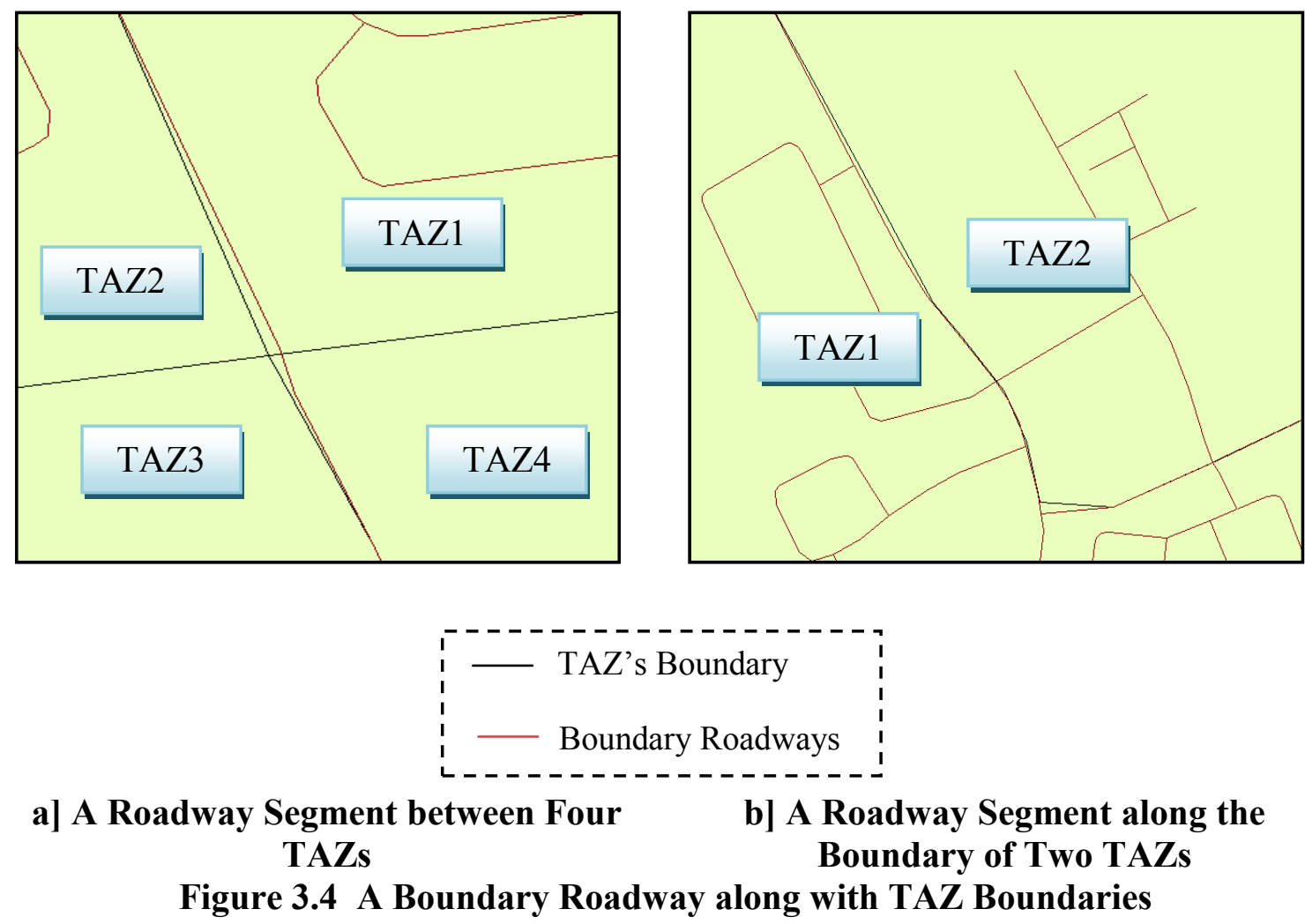

In order to check the sensitivity of collision prediction models to the procedure utilized for assigning boundary data to the traffic zones, six different methods of aggregating data were used. These methods are defined as follows:

- Equal-proportion-based method: boundary data is divided equally between adjacent TAZs, i.e., data on a boundary between two TAZs is divided between the two TAZs at half-half $(0.5: 0.5)$ ratio, and data lying on the boundary of three 
TAZs is considered to be a third of data for each TAZ, etc.

- Population-based method: boundary data is divided between adjacent TAZs in proportion to their population.

- Population-employment-based method: boundary data is divided between adjacent TAZs in proportion of the summation of population and employment inside TAZs.

- Total-lane-kilometre-based method: boundary data is divided between adjacent TAZs in proportion to their measured total lane kilometres (TLKM). The boundary TLKM is assigned to TAZs using the previous three assignment methods which are the equal-proportion-based, population-based, and population-employment-based. Therefore, three different ways of total lane kilometre based method are resulted. These ways are TLKM1, TLKM2, and TLKM3 as shown in Table 3.1.

- Vehicle-Kilometre-Travelled-based (VKT) method: boundary data are divided between adjacent TAZs in proportion to their modelled VKT. Similar to total lane kilometre based method, the boundary VKT is assigned to TAZs using three methods which are the equal-proportion-based, population-based, and population-employment based respectively. Therefore, three different ways of VKT based method are resulted. These ways are VKT1, VKT2, and VKT3 as shown in Table 3.1.

- Multiple-count-based method: all boundary data are assigned to all adjacent traffic zones, i.e., data on a boundary between two TAZs is assigned to both TAZs through double counting of boundary data. Compared to the equal- 
proportion-based method, this method allows multiple assignations of the same boundary data. In the multiple-count-based methods, a roadway segment on a boundary between two adjacent TAZs is fully considered in the total roadways length of each TAZ.

By using the different proposed boundary data assignment methods, thirteen datasets were created; named by alphabetic order from A to $M$ (Table 3.1). In addition to the data categories mentioned in the table, the socio-demographic data were added to each of these datasets.

- In the first three datasets $\mathrm{A}, \mathrm{B}$, and $\mathrm{C}$, it is clear that all boundary data were assigned in accordance to equal-proportion-based, population-based, and population-employment-based methods, respectively.

- In the datasets $\mathrm{D}, \mathrm{E}$, and $\mathrm{F}$, data points were divided between TAZs based on TLKM1, TLKM2, and TLKM3, respectively. Also, boundary roadways and boundary exposure in those datasets were allocated utilizing equal-proportionbased, population-based, and population-employment-based methods respectively.

- Data points in the datasets G, H, and I were divided between TAZs based on VKT1, VKT2, and VKT3, respectively. Also, boundary roadways and boundary exposure in those datasets were allocated utilizing equal-proportion-based, population-based, and population-employment-based methods respectively.

- In dataset $\mathrm{J}$, all boundary data were assigned to TAZs using the multiple-countbased methods. 
Table 3.1 Boundary Collision Assignment Methods

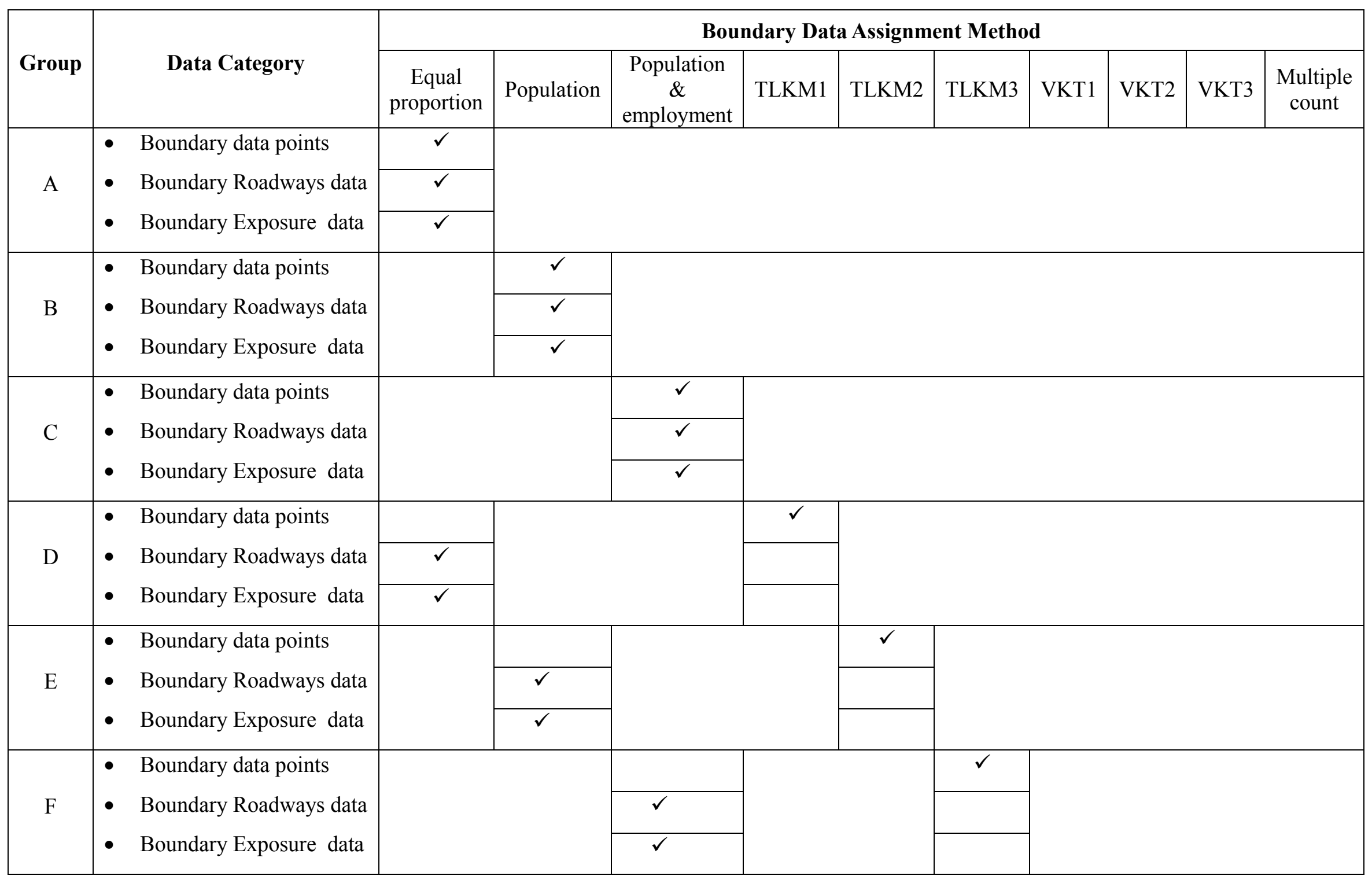


Table 3.1 Boundary Collision Assignment Methods (Cont')

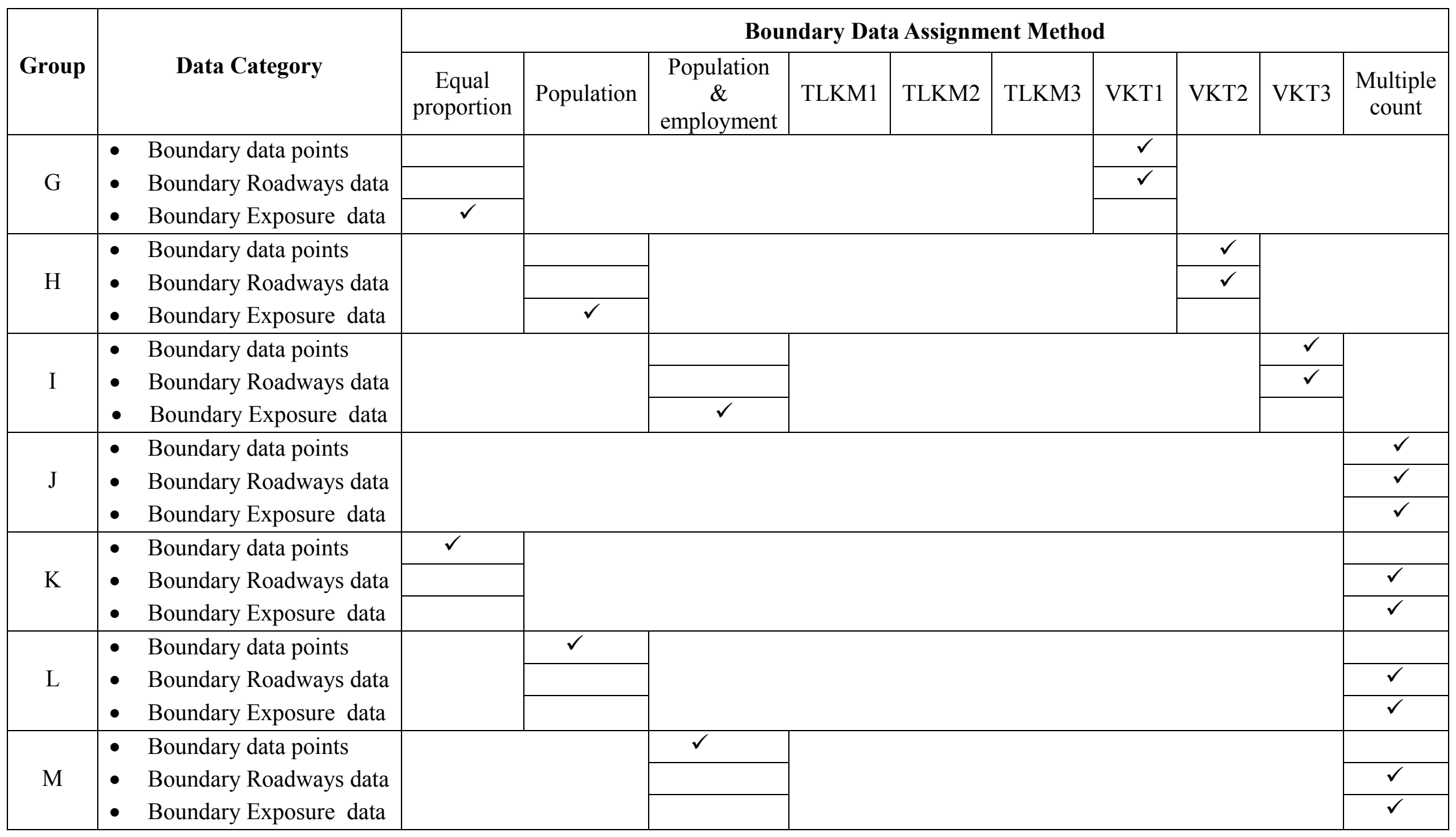


- Boundary data points in the datasets $\mathrm{K}, \mathrm{L}$, and $\mathrm{M}$ were allocated in order to equalproportion-based, population-based, and population-employment-based methods respectively, whereas roadways and exposure boundary data were assigned using the multiple-count-based methods.

\subsubsection{Data Points Assignment}

Aggregation of all collision types and other data which are localized as points (including total number of intersections, total number of signalized and non-signalized intersections, total number of bus stops, total number of schools) defined by their longitude and latitude coordinates was done using a specifically written code in MATLAB (Appendix B). In the code, a function (available online and was created by Alejandro Weinstein 2008) was used to measure the distance between each data point and its projections on the line segments enclosing all TAZs. A projection is defined as the point of intersection between the shortest straight line linking the data point to a segment line. Mathematically, the perpendicular is shortest distance from a point to a line segment (Figure 3.5). In the case that the perpendicular cannot be achieved between a point and a line segment, without extending this line, the shortest distance will be the distance from the point to the closest vertex of the line segment (Figure 3.6).

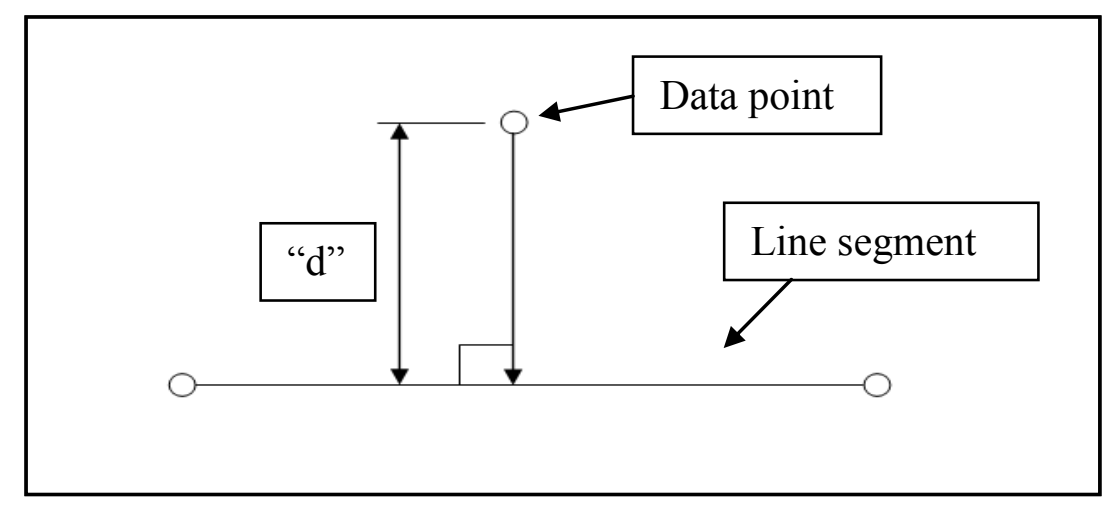

Figure 3.5 Distance from a Point to a Line Segment ("d"=Perpendicular) 


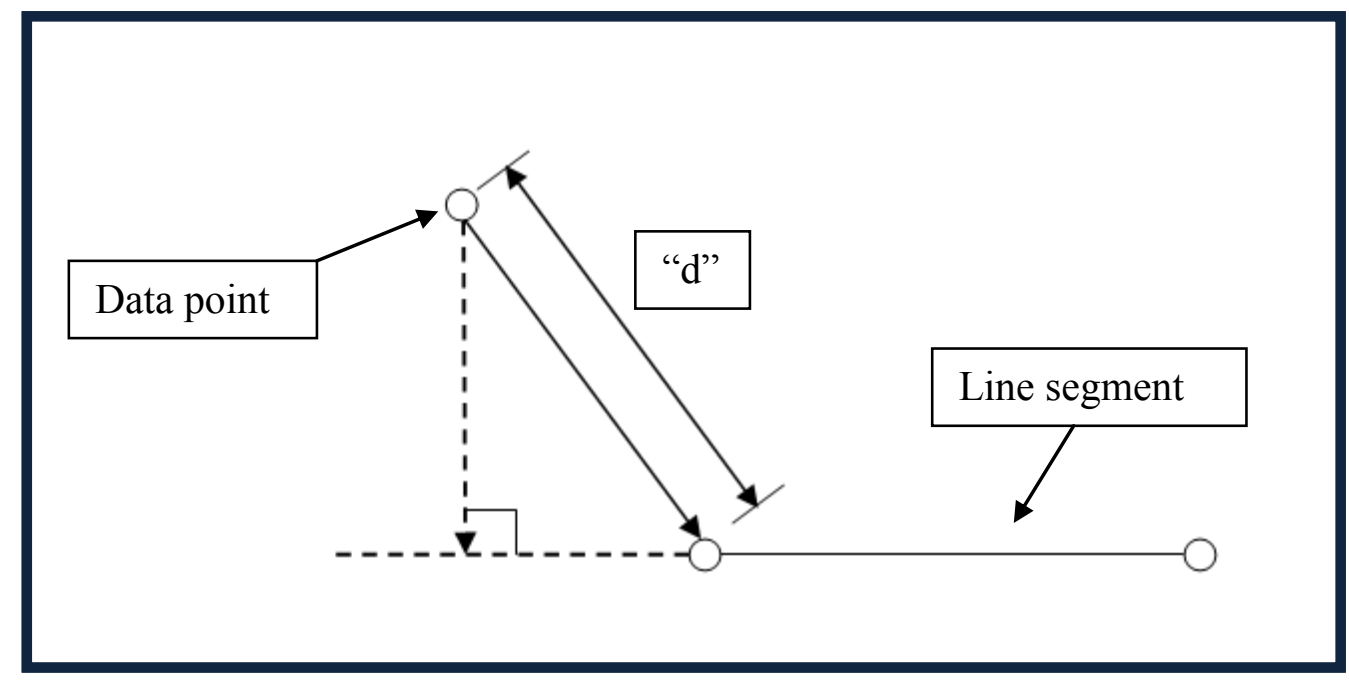

Figure 3.6 Distance from a Point to a Line Segment (“d"= To Closest Vertex)

Each of the City of Ottawa TAZs data are provided as a closed polygon whose vertices are specified by vectors $x$ and $y$. The shortest distance $d_{i j}$ from each data point $(i)$ to the closest line segment of each polygon $(j)$ is calculated in order to create a distance matrix between each data point and all polygons (TAZs). For a specific polygon, a point could be inside or outside. Therefore, any point that falls inside the polygon is given a negative sign by the coding method (see the code). On the other hand, any data point located outside the polygon is given a positive sign. This would eventually be used to designate the boundary and non-boundary points.

In the developed rectangular distance matrix, the number of rows in the distance matrix is the number of unique points over the study area. The columns of this matrix represent the number of TAZs (arranged according to their zone ID).

$$
\text { distance matrix }=\frac{\left[\begin{array}{ccc} 
\pm d_{11} & \cdots & \pm d_{1 n} \\
\vdots & \ddots & \vdots \\
\pm d_{m 1} & \cdots & \pm d_{m n}
\end{array}\right] \mid \text { Rows = Data points }}{\underset{\text { Columns }=\text { TAZs' number }}{\longrightarrow}}
$$


In order to differentiate the boundary points from non-boundary points $60 \mathrm{~m}$-wide $(30 \mathrm{~m}$ on each side) buffer zones around the polygons' line segments are considered (Figure 3.7) Therefore, points located within the buffer zone are considered as boundary points, whereas the remaining points are non-boundary. At a specific polygon, the boundary and non-boundary points in the matrix are determined as follows.

- If $\left(d_{i j}<-30\right)$, a point is considered a non-boundary data point (points inside a polygon).

- If ( $\left.-30 \leq d_{i j} \leq+30\right)$, a point is considered a boundary data point.

- If $\left(d_{i j}>+30\right)$, a point is outside (belongs to other polygon/s).

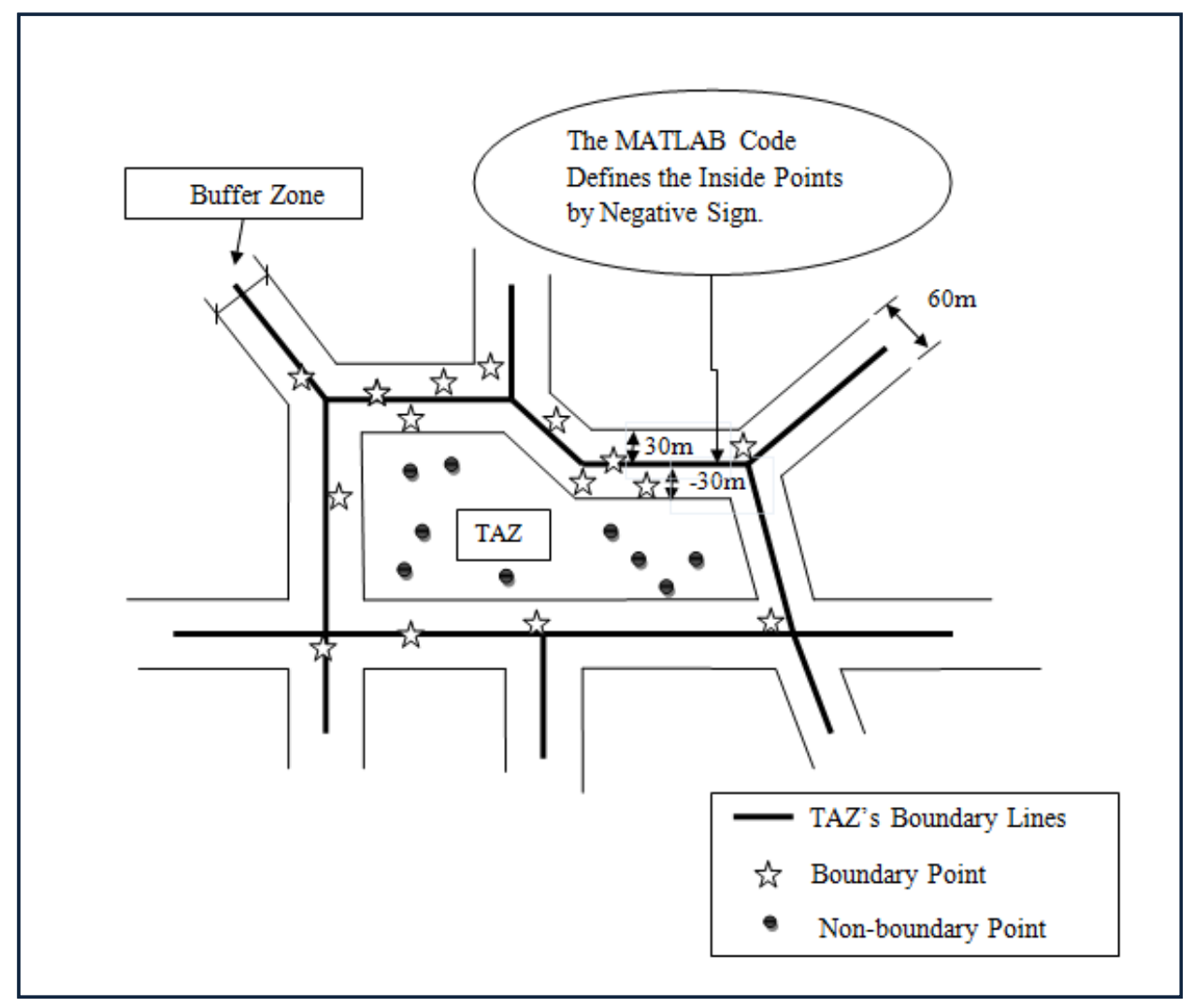

Figure 3.7 Data Points Distribution inside and around a TAZ 
In order to identify non-boundary points' matrix (points inside a polygon), all $d_{i j}$ values in the range $[-30, \infty)$ are replaced with zeros in the distance matrix, and all the remaining values, $d_{i j}$ values in the range $(-\infty,-30)$, are given a value equal to one. The sum of each column in the last matrix is finally used to represent the number of data points contained within each polygon (TAZ).

On the other hand, in order to obtain boundary points' matrix, all $d_{i j}$ values outside the range $[-30,+30]$ are replaced with zeros. After that the remaining values are given values equal to one. The boundary data points' matrix is then used to assign boundary data points between adjacent TAZs using the different assignment methods mentioned earlier. Figures 3.8 and 3.9 show non-boundary and boundary collision data points, respectively.

It is noteworthy that the adopted threshold distance of sixty meters around TAZs' boundaries is based on a study conducted by Ivan et al (2006) whose objective was to determine a procedure for assigning zone data to links in the context of a Geographic Information System (GIS). They concluded that applying a buffer zone of sixty meters was reasonable in the identification of links associated with each TAZ. This value was adopted also by Wang (2012) for developing macro-level model for safety assessments of road network structures. 


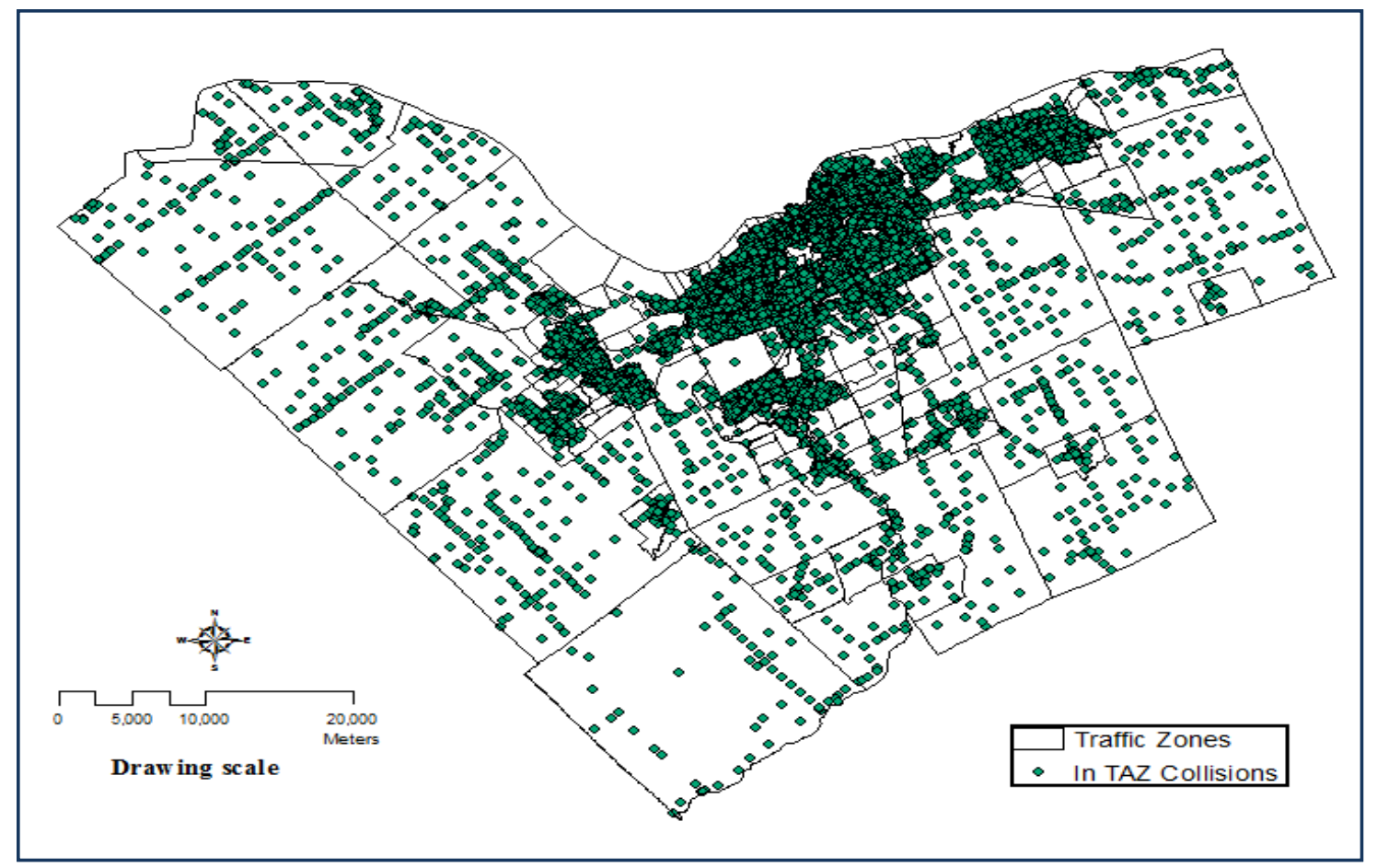

Figure 3.8 Non-boundary Collisions Map

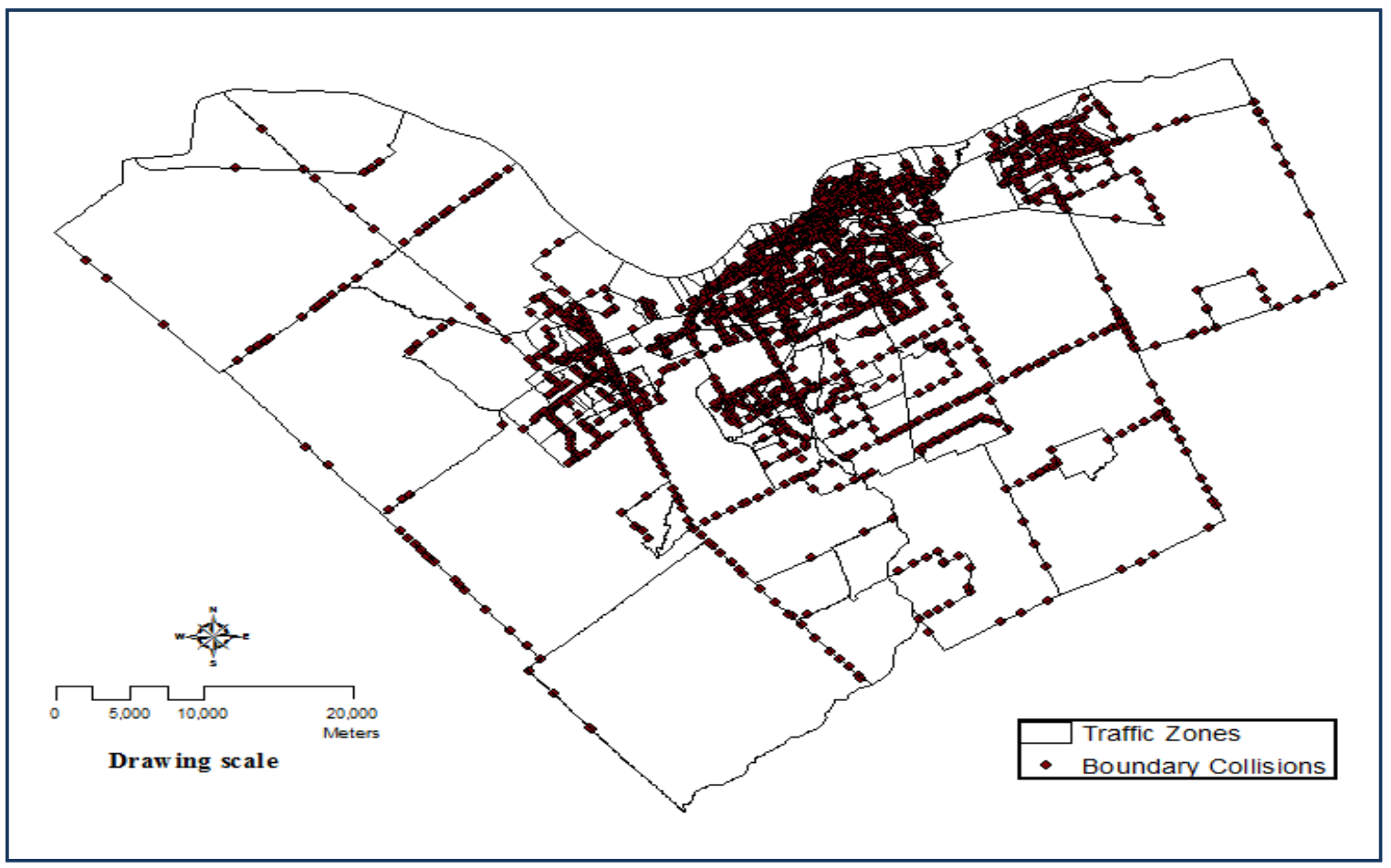

Figure 3.9 Boundary Collisions Map 


\subsubsection{Roadway Network Assignment}

Four roadways variables based on roadway classification were created for each TAZ. These are the total centreline lengths of arterial, local, collector, and major collector. Furthermore, to account for variations in collision occurrence according to posted speed limits, the total centerline lengths of roadways for speeds from $40 \mathrm{~km} / \mathrm{h}$ to $100 \mathrm{~km} / \mathrm{h}$ (in $10 \mathrm{~km} / \mathrm{h}$ increments) were formed for each TAZ. This produced seven roadways variables based on the posted speed limits.

Aggregating the roadway segments, either using roadway classification or posted speed limits, and bike paths length to the traffic zones was preformed through several steps. First, the roadway network shapefile was imported into the geographic information system software (ArcGIS). Separating non-boundary road segments required applying a thirty-meter buffer zone around the boundary of each TAZ. Once the buffer zones were created, the roadway segments that fall inside them were identified (the "identify" function in the analysis tool of the Arc Toolbox was used to identify where a road segment is geographically located). The use of this method resulted in splitting the City of Ottawa's original roadway network links between TAZs and buffer zones. Therefore, there was a need to get their recalculated length that lies within the buffer zone and length inside the TAZ as well. All boundary and non-boundary roadways segment lengths were recalculated using the "calculate geometry" function in ArcGIS. The total length of non-boundary (roadways lengths inside TAZs) roadway segments for each TAZ is then obtained. The boundary roadway segments (roadway segments within the buffer zones) were then separated in a new GIS layer.

To assign the boundary roadway segments to TAZs, the "Spatial Join" tool was used to assign boundary roadway segments into their respective TAZs (by joining GIS zones boundary 
shape layer to boundary roadway segments layer). To be able to easily assign each boundary roadway segment between adjacent TAZs, the joint-output attribute table was after that imported into MATLAB (using the code written in Appendix B) and boundary roadway segment matrix was created. In this matrix, each row indicates a length of one boundary roadway segment while the columns represent all TAZs arranged according to their zone ID. In other words, a cell in the matrix would correspond to the length of roadway segment that falls in a specific TAZ's boundary. Finally, the obtained matrix was utilized then to assign each boundary roadway segment to adjacent TAZs based on the different assignment methods shown in Table 3.1. It should be noted that this procedure of aggregating roadway network segments to TAZs was used for all previously mentioned roadway network variables as well as for bike paths network.

\subsubsection{Exposure Variables Assignment}

A special treatment process was carried out to aggregate the exposure variables at the zonal level. The data was treated according to the following steps.

- The Excel spreadsheet file received from the City's transportation planning department was first exported to ArcGIS.

- To relate the traffic volume traveling between each pair of nodes to their corresponding TAZ, each pair of geocoded points representing a link was connected to each other using "XY to line tool" of the data management tools in ArcGIS.

- As indicted in Section 3.2.3 the received data from the City of Ottawa transportation planning department includes the traffic volume traveling between each pair of nodes representing the roadway network in addition to the average annual daily trips entering and exiting from the centroid of TAZs. To avoid counting the average annual daily trips entering and exiting from the centroid of TAZs with traffic volumes traveled through 1 , 
the links connecting the centroid of each traffic zone were identified depending on their coded indices and then were removed. The centroid connectors are created to represent the traffic between the centroid of a TAZ and the major roadway network. The traffic on those links is used as another exposure measure and is included separately in the final dataset.

- The lengths of links representing roadway network were then divided between TAZs and buffer zones using the same procedure utilized to assign the roadway network to the TAZs. Once boundary and non-boundary links were identified, The VKT is calculated by multiplying AADT travelling within each link with its re-calculated length.

- Once the VKT was calculated, the arithmetic summation for the non-boundary VKT (VKT inside a TAZ) related to each TAZ was obtained.

- Finally, the boundary VKT matrix was created and the obtained matrix was utilized then to assign VKT to adjacent TAZs based on the different assignment methods shown in Table 3.1

\subsection{Spatial Analysis and Descriptive Statistics of Data}

\subsubsection{Motor Vehicle Collisions}

The statistical summary of all types of collisions identified in the City of Ottawa is shown in Table 3.2. This table displays the summary of collisions in which the boundary data are assigned based on the equal proportion assignment method. The summary obtained according to the other boundary assignment methods is shown in Appendix A. 
Table 3.2 Descriptive Statistics of the Different Types of Collisions Within Ottawa's TAZs

\begin{tabular}{|c|c|c|c|c|c|c|c|}
\hline Collisions & Observations & Total & Minimum & Maximum & Median & Mean & $\begin{array}{c}\text { Standard } \\
\text { deviation }\end{array}$ \\
\hline Total & 422 & 63978 & 0 & 759 & 130.50 & 151.61 & 113.26 \\
\hline Total PDO & 422 & 50776 & 0 & 598 & 102.50 & 120.32 & 89.59 \\
\hline $\begin{array}{c}\text { Total Non-Fatal } \\
\text { Injury }\end{array}$ & 422 & 12443 & 0 & 151 & 25.00 & 29.49 & 23.22 \\
\hline $\begin{array}{c}\text { Total Fatal } \\
\text { Total Bike- } \\
\text { involved }\end{array}$ & 422 & 117 & 0 & 5 & 0.00 & 0.34 & 0.71 \\
\hline $\begin{array}{c}\text { Total Pedestrian- } \\
\text { involved }\end{array}$ & 422 & 1760 & 0 & 35 & 2.50 & 4.17 & 4.82 \\
\hline
\end{tabular}

* Note: Boundary Data Assigned Using Equal-proportion-based assignment Method

It can be seen from Table 3.2, a total of 63,978 collisions occurred between 2007 and 2011 in the City of Ottawa. The percentages of severe collisions (collisions with fatalities and injuries) and Property Damage Only (PDO) are $20 \%$ and $80 \%$, respectively.

The spatial graphical distribution of the collisions is shown in Figures 3.10, 3.11, 3.12, and 3.13. These figures show the collisions per square meter $\left(\right.$ collisions $\left./ \mathrm{km}^{2}\right)$ for each TAZ. As expected, the highest densities for total collisions per area are noticed in the Downtown area and its surroundings. This increase in the density of collisions can be attributed to the increase in the traffic intensity (traffic volume per unit area) due to the existence of business activities in the core of the city and its surrounding areas as shown in Section (3.4.4).

It can be noted from Figure 3.11 that the density of fatal collisions varies substantially between TAZs. A total of 117 fatal collisions were identified within Ottawa's TAZs in the period from 2007 to 2011. Most rural TAZs are recognized with zero collisions. This is unexpected because drivers in rural areas tend to drive at high speeds. 


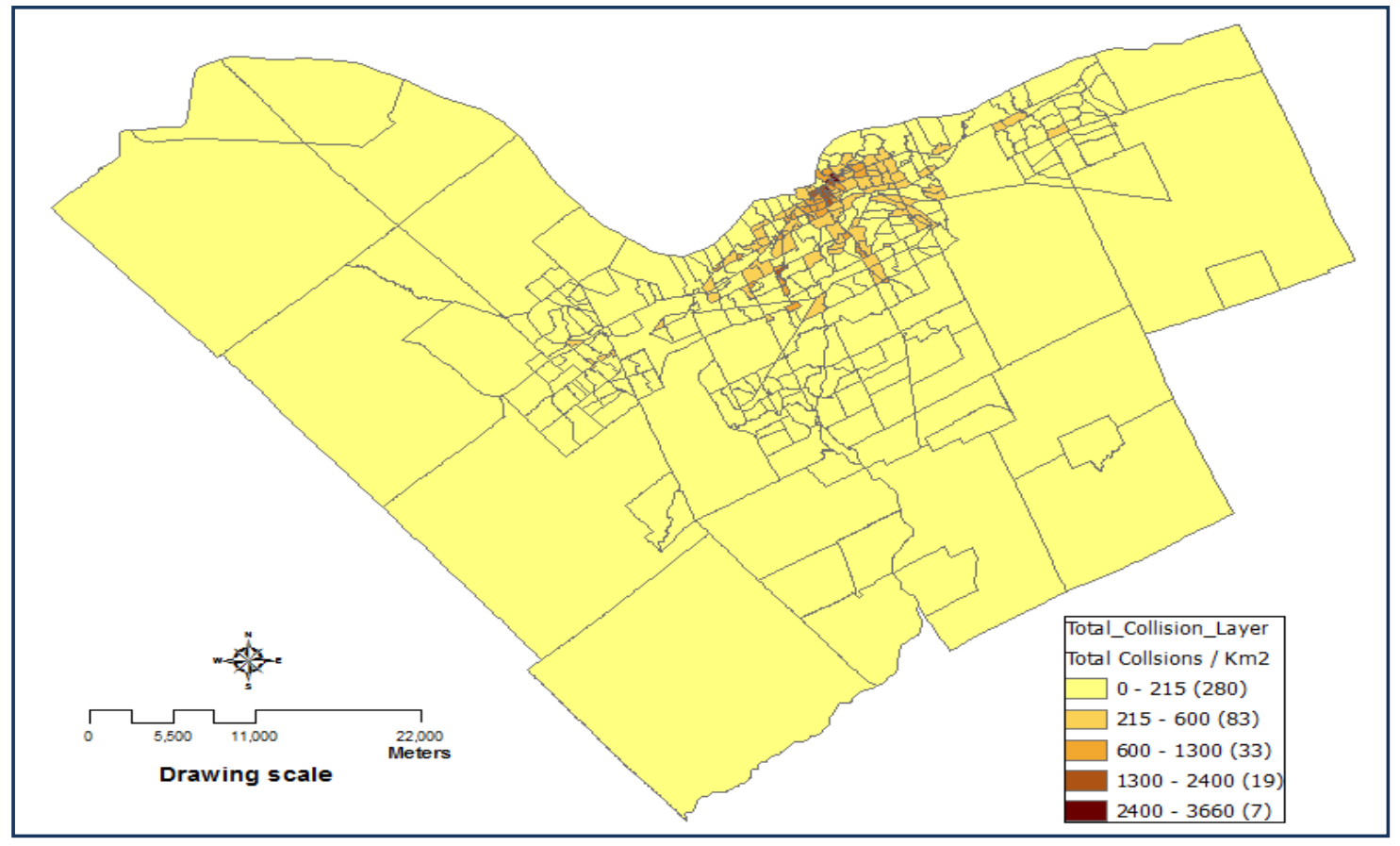

Figure 3.10 Spatial Distributions of Collision Density for Total Collisions in the City of Ottawa.

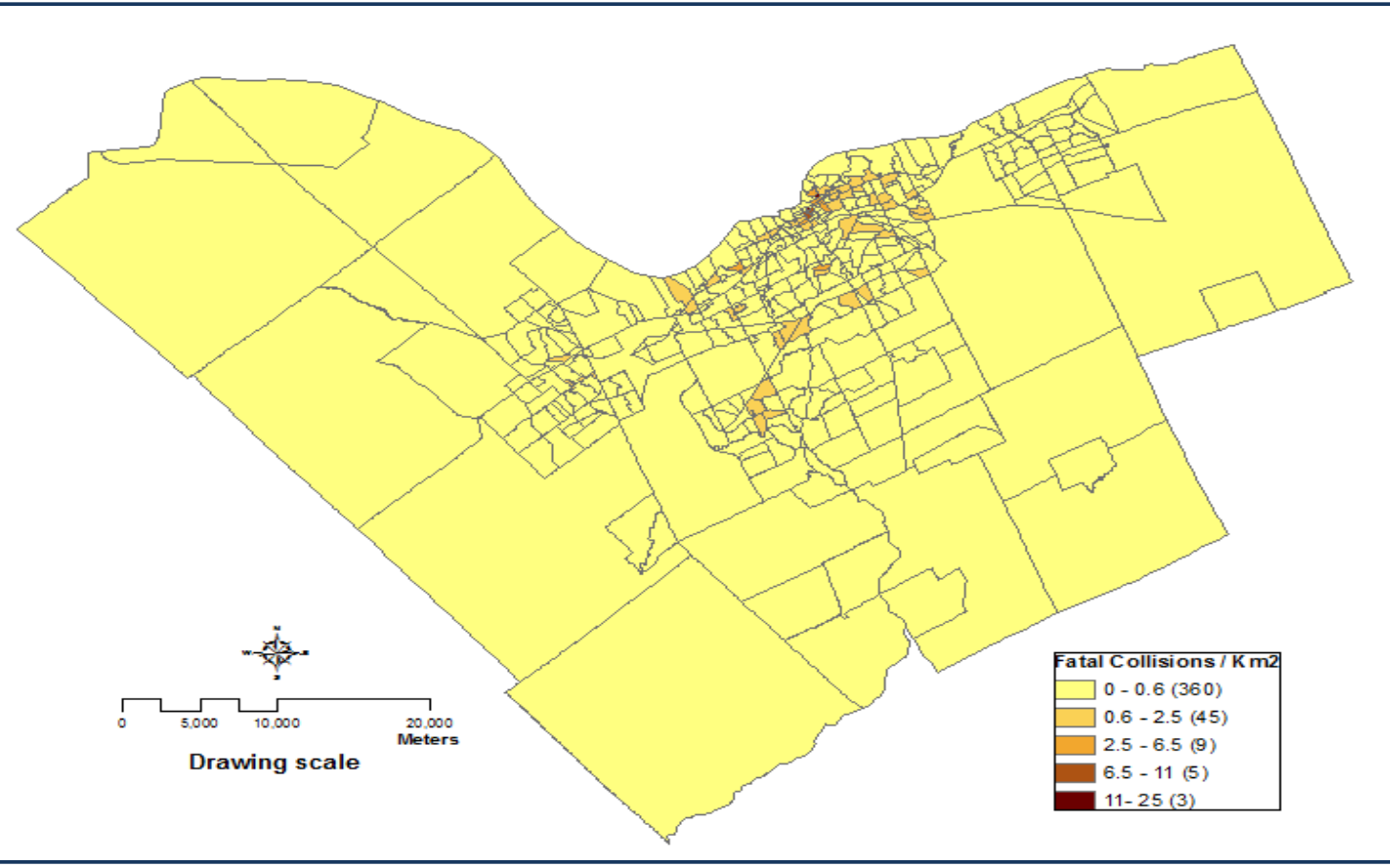

Figure 3.11 Spatial Distributions of Collision Density for Fatal Collisions in the City of Ottawa. 


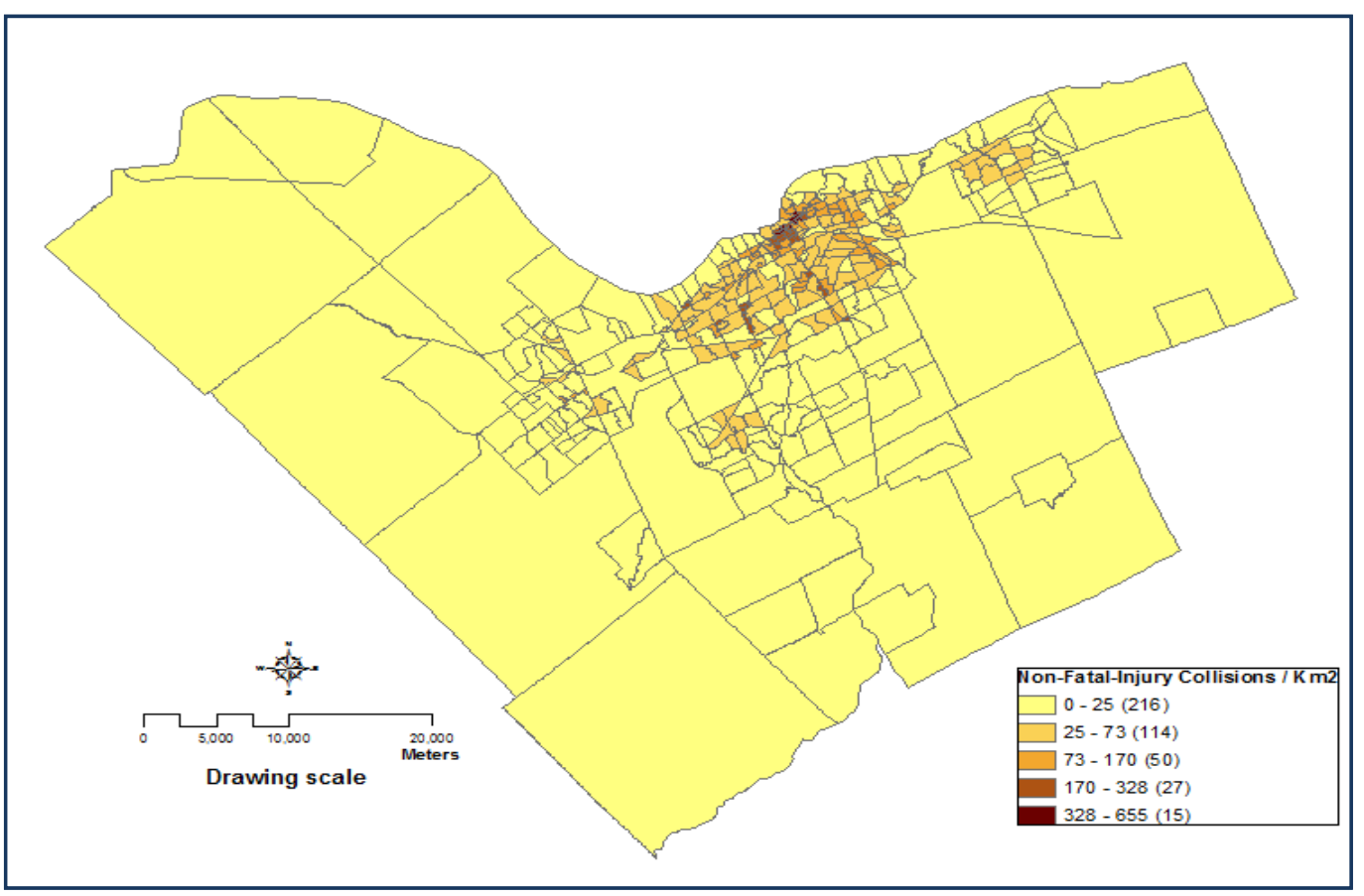

Figure 3.12 Spatial Distributions of Collision Density for Non-Fatal Injury Collisions in the City of Ottawa.

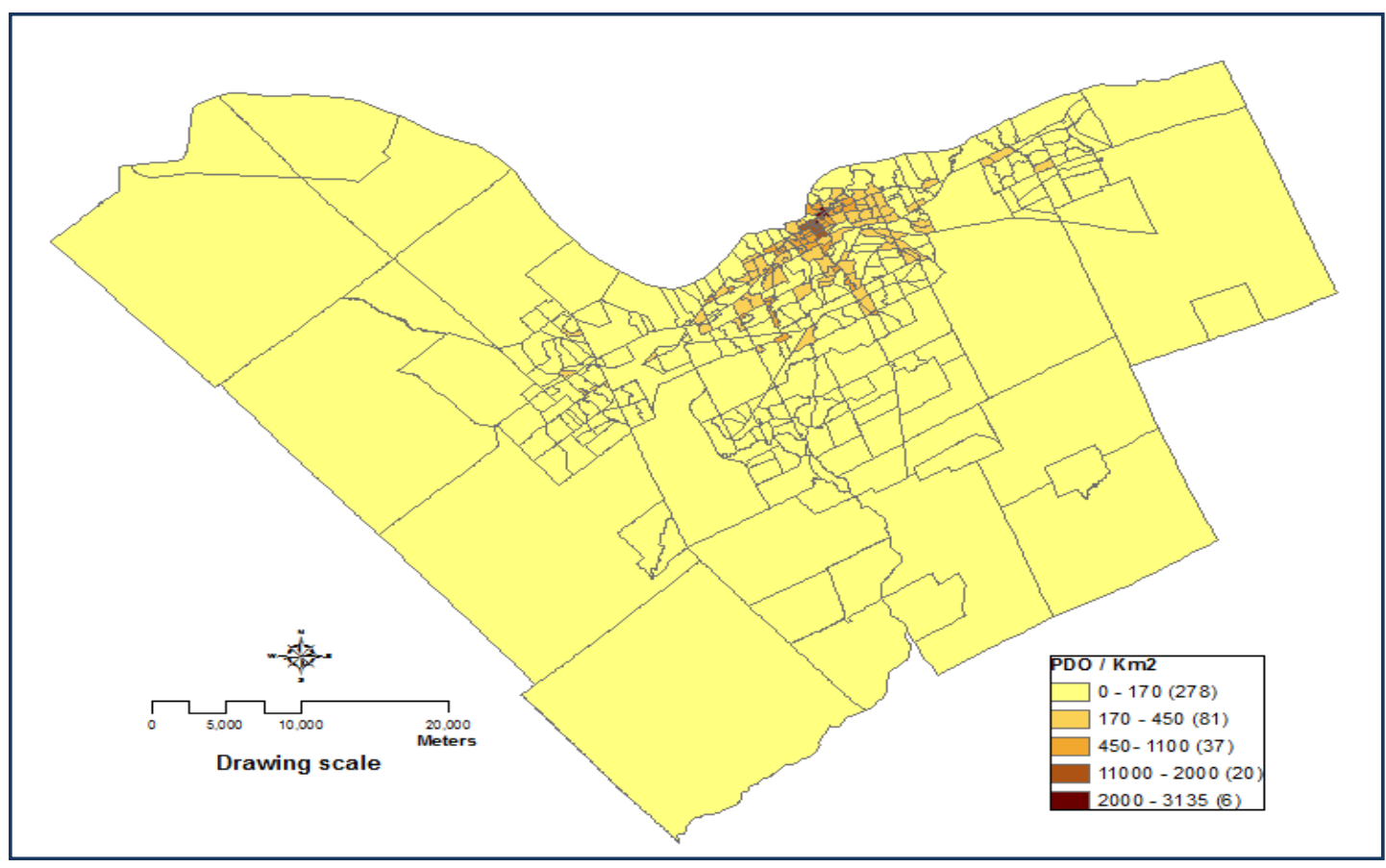

Figure 3.13 Spatial Distributions of Collision Density for PDO Collisions in the City of Ottawa. 
Figure 3.14 presents the histograms plot of total, non-fatal injury, fatal, and PDO collisions. It can be noted from the fatal collision histograms plot that the zero observations appear as the most frequent observation within the TAZs, suggesting that the zero inflated Poisson or negative binomial may be the most appropriate for modelling the fatal collisions. The histograms are highly skewed to the left for the total, non-fatal injury, and PDO collisions, as expected from count data, suggesting using the Poisson or negative binomial regression for developing the CPMs.

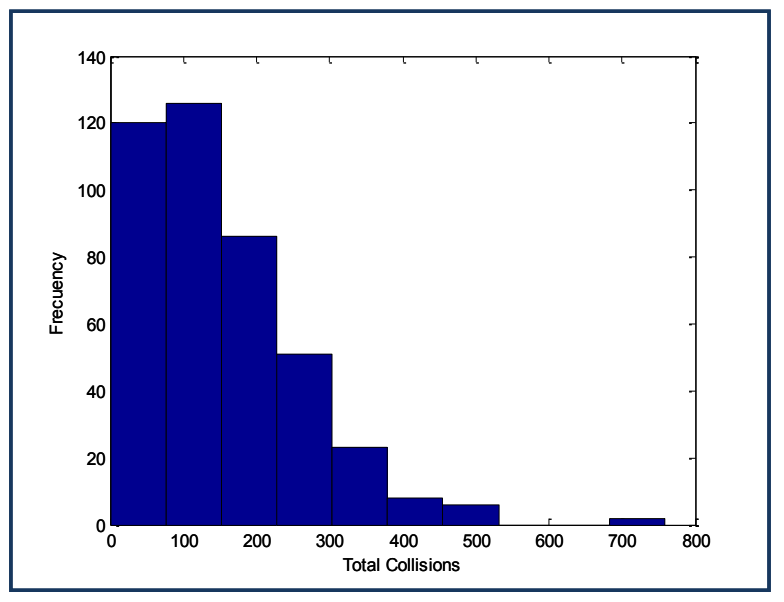

a ] Total Collisions Histogram

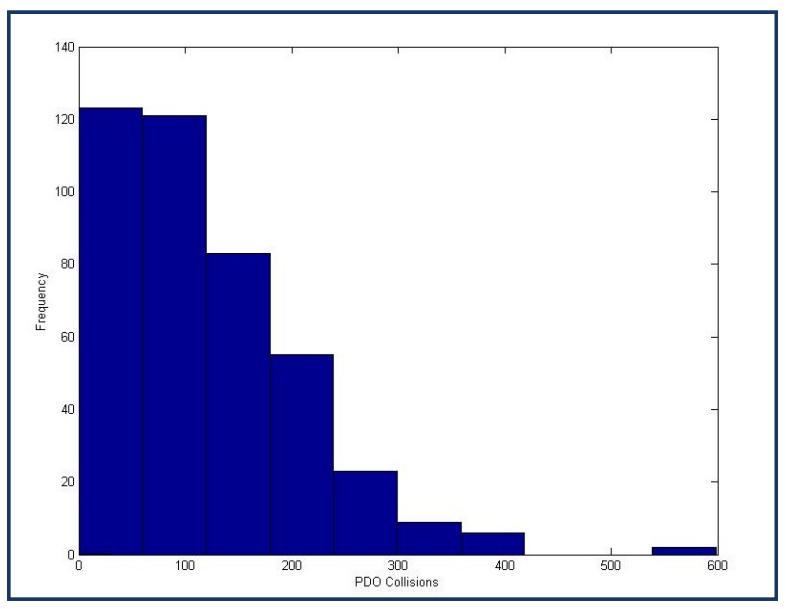

c ] PDO Collisions Histogram

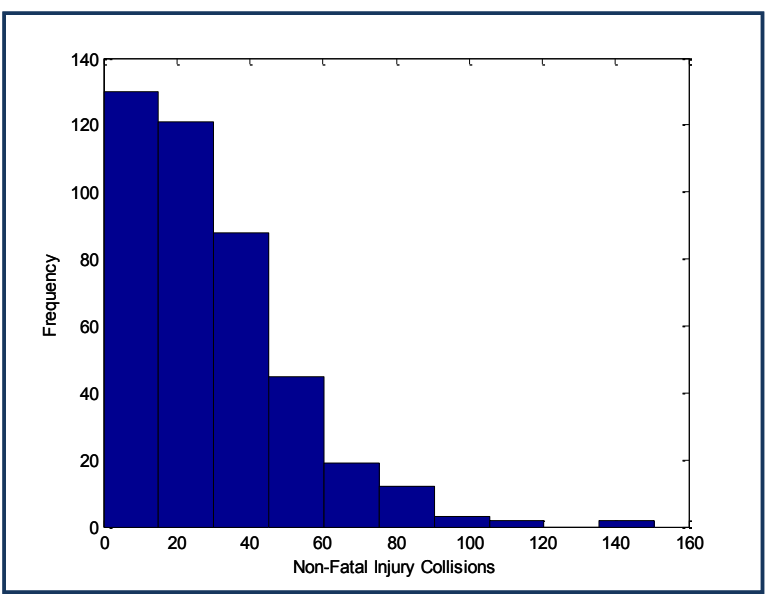

b ] Non-fatal Injury Collisions Histogram

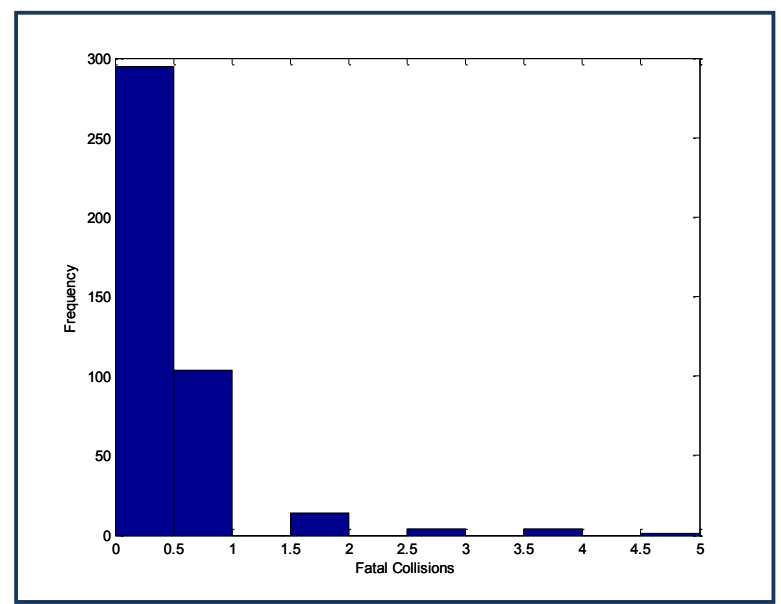

d ] Fatal Collisions Histogram

Figure 3.14 Histograms of Ottawa TAZ level Collision Frequencies. 


\subsubsection{Pedestrian-Involved and Bike-Involved Collisions}

The spatial distribution of pedestrians and cyclists involved in collisions is shown in Figures 3.16 and 3.17, respectively. It can be observed that the pedestrian and bike collisions occur more frequently in the downtown area. These observations may be attributed directly to higher pedestrian and bike activities and traffic volume in the core of the city. The density of these collisions declines to zero in some rural areas. It could be that the lack of pedestrian and bike paths and longer distances in those areas may discourage pedestrian and bike traffic. In addition, the population in those areas is less with different characteristics from other areas. It can be calculated from Table 3.2 that the bike-involved and pedestrian-involved collisions were and $1.40 \%$ and $2.75 \%$ from the total collisions, respectively.

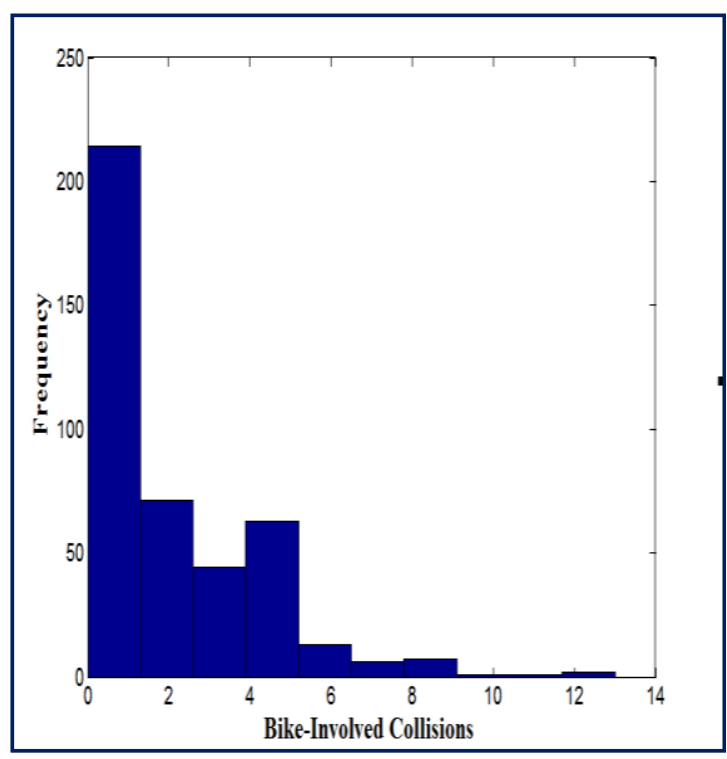

a ] Bike-Involved Collisions

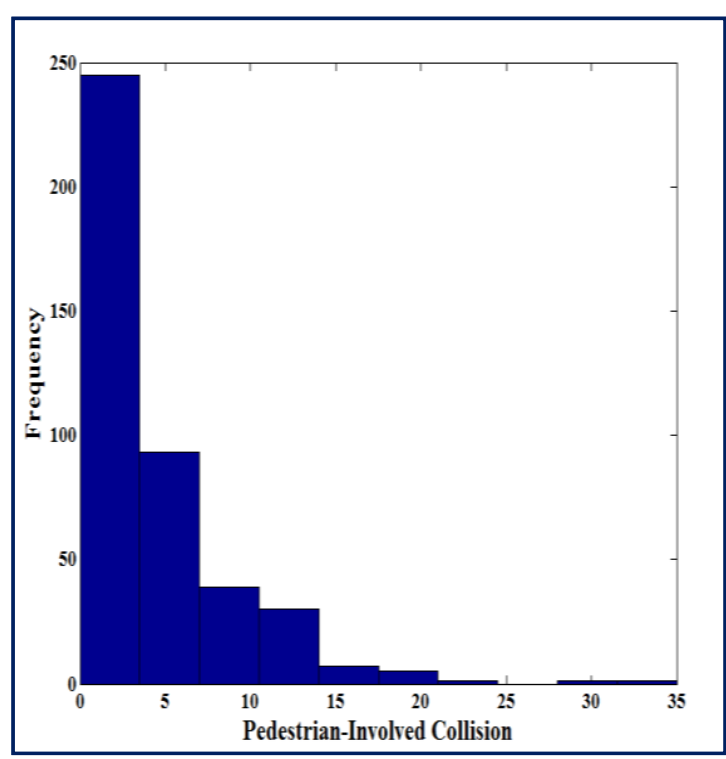

b ] Pedestrian-Involved Collisions

Figure 3.15 Histograms of Ottawa TAZ level Collision Frequencies. 


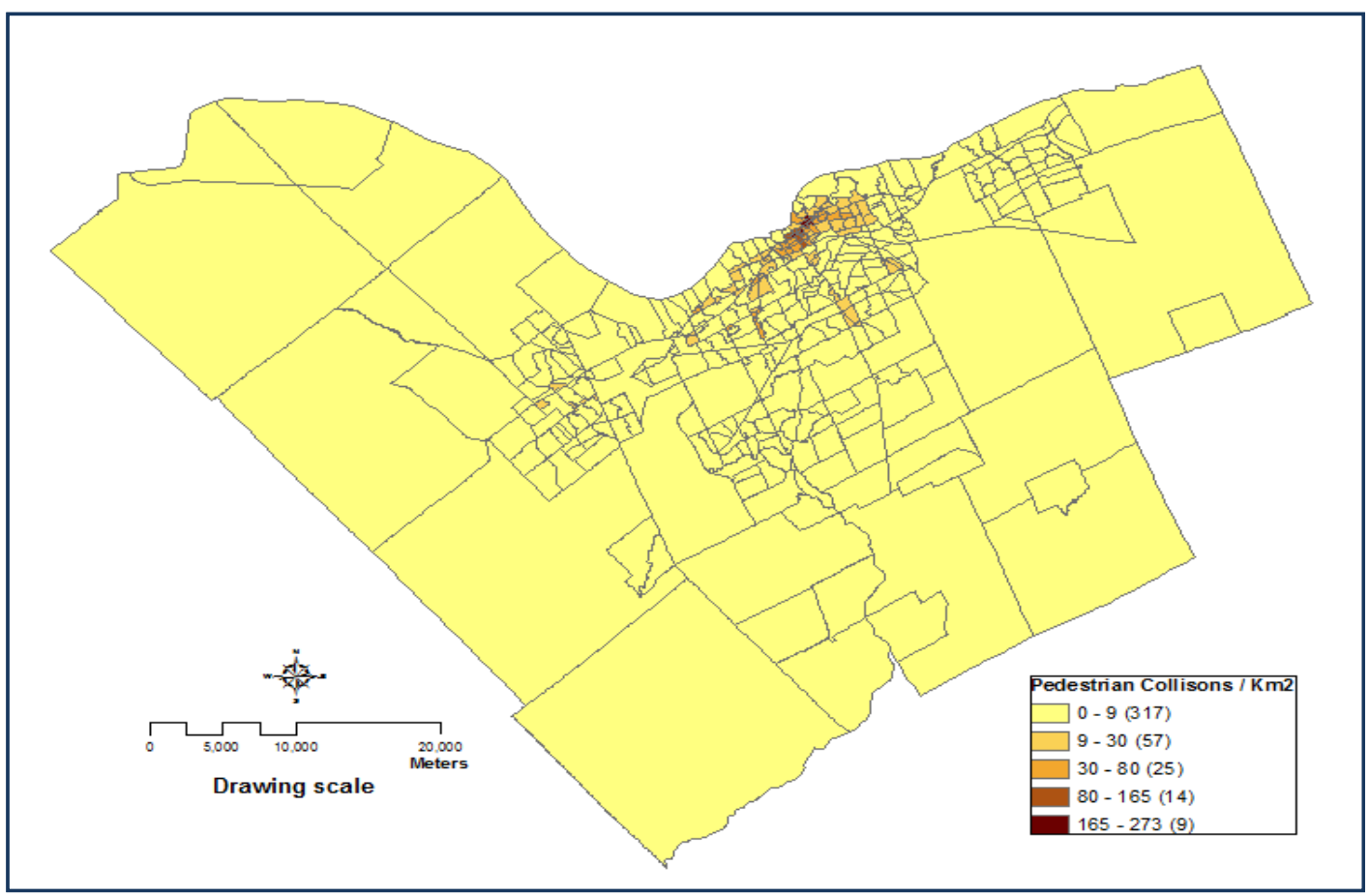

Figure 3.16 Spatial Distribution of the Density of Pedestrian-Involved Collisions.

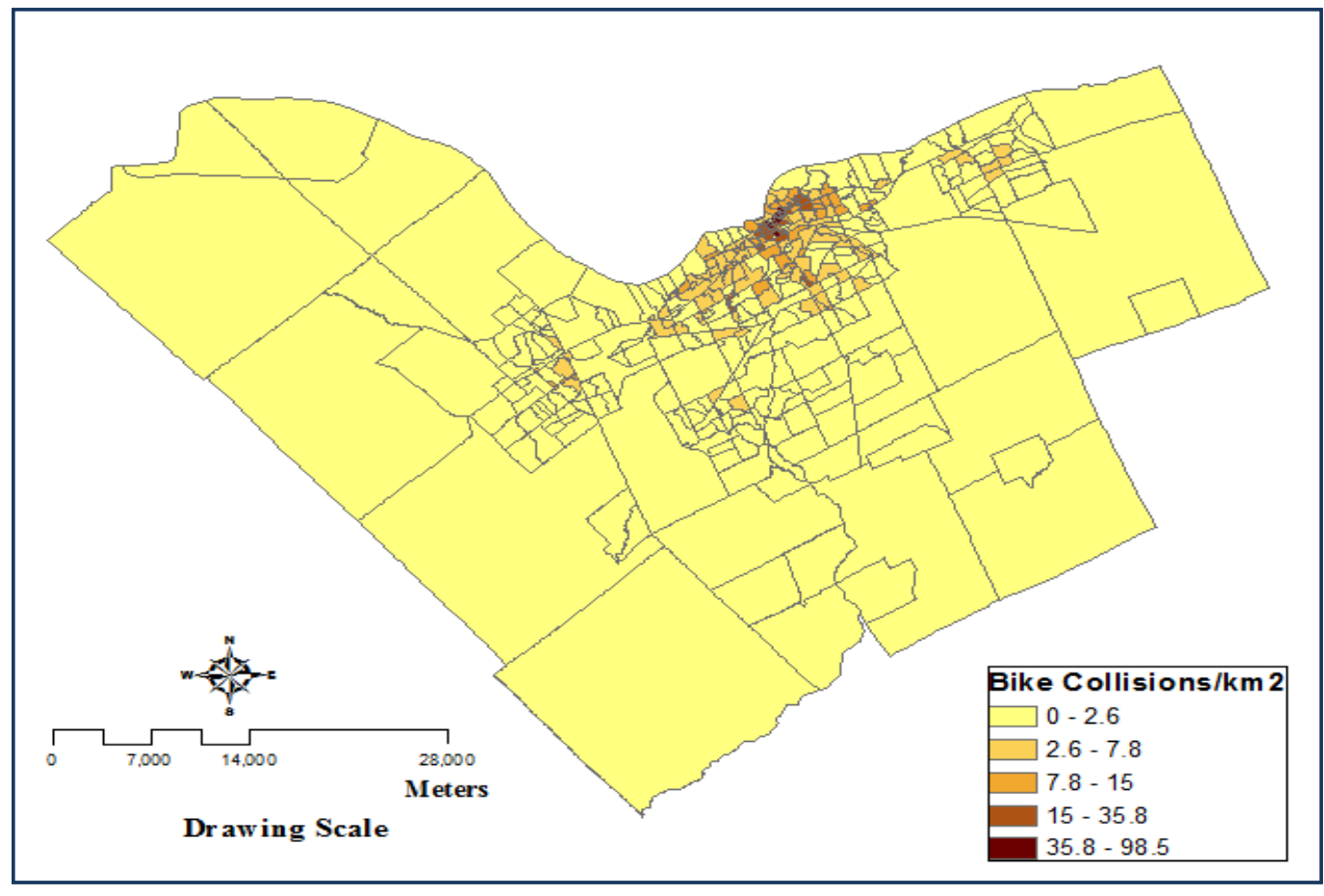

Figure 3.17 Spatial Distribution of the Density of Bike-Involved Collisions. 


\subsubsection{Roadways and Intersections}

The statistical summary of roadway segments length and intersections identified in the City is shown in Table 3.3. This table displays the summary of roadways and intersections in which the boundary data were assigned based on equal-proportion-based assignment method. The summary obtained according to the other methods is shown in Appendix (A).

Figures 3.18 and 3.19 show the roadway densities and intersection densities within the City of Ottawa TAZs, respectively. The spatial distribution of roadway indicates higher density closer to the downtown area and some suburban areas. This may indicate that TAZs with a relatively more roadways density may result in more collision occurrences.

With respect to the intersection density, a similar observation is noticed. Figure 3.19 shows declining number of intersections as the distance from the downtown increases. Normally, due to the traffic characteristics within the intersection, they are anticipated to have higher collision frequency. Hence, the traffic zones containing more intersections may have more collision occurrences than the others. 
Table 3.3 Descriptive Statistics of the Roadways and Intersections Variables Within Ottawa's TAZs

\begin{tabular}{|c|c|c|c|c|c|}
\hline Variables & Observation & Maximum & Minimum & Mean & $\begin{array}{l}\text { Standard } \\
\text { deviation }\end{array}$ \\
\hline $\begin{array}{c}\text { Total Arterial Road } \\
\text { Kilometres }(\mathrm{km}) \\
\end{array}$ & 422 & 75.23 & 0.00 & 3.01 & 8.13 \\
\hline $\begin{array}{c}\text { Total Collector Road } \\
\text { Kilometres }(\mathrm{km})\end{array}$ & 422 & 137.73 & 0.00 & 3.88 & 14.70 \\
\hline $\begin{array}{l}\text { Total local Road } \\
\text { Kilometres }\end{array}$ & 422 & 36.33 & 0.00 & 5.39 & 6.16 \\
\hline $\begin{array}{l}\text { Total Major Collector } \\
\text { Road Kilometres (km) }\end{array}$ & 422 & 4.98 & 0.00 & 0.18 & 0.45 \\
\hline $\begin{array}{l}\text { Total Roadways } \\
\text { Kilometres (km) }\end{array}$ & 422 & 229.20 & 0.00 & 12.46 & 25.88 \\
\hline $\begin{array}{l}\text { Length of Roadways with } \\
40 \mathrm{~km} / \mathrm{h} \text { Speed Limit }(\mathrm{km})\end{array}$ & 422 & 30.46 & 0.00 & 2.05 & 4.48 \\
\hline $\begin{array}{l}\text { Length of Roadways with } \\
50 \mathrm{~km} / \mathrm{h} \text { Speed Limit }(\mathrm{km})\end{array}$ & 422 & 40.34 & 0.00 & 4.68 & 5.73 \\
\hline $\begin{array}{l}\text { Length of Roadways with } \\
60 \mathrm{~km} / \mathrm{h} \text { Speed Limit }(\mathrm{km})\end{array}$ & 422 & 34.58 & 0.00 & 0.95 & 2.56 \\
\hline $\begin{array}{l}\text { Length of Roadways with } \\
70 \mathrm{~km} / \mathrm{h} \text { Speed Limit }(\mathrm{km})\end{array}$ & 422 & 19.34 & 0.00 & 0.21 & 1.13 \\
\hline $\begin{array}{l}\text { Length of Roadways with } \\
80 \mathrm{~km} / \mathrm{h} \text { Speed Limit }(\mathrm{km})\end{array}$ & 422 & 203.28 & 0.00 & 4.53 & 20.87 \\
\hline $\begin{array}{l}\text { Length of Roadways with } \\
90 \mathrm{~km} / \mathrm{h} \text { Speed Limit }(\mathrm{km})\end{array}$ & 422 & 10.23 & 0.00 & 0.03 & 0.50 \\
\hline $\begin{array}{c}\text { Length of Roadways with } \\
100 \mathrm{~km} / \mathrm{h} \text { Speed Limit } \\
(\mathrm{km})\end{array}$ & 422 & 0.16 & 0.00 & 0.00 & 0.01 \\
\hline $\begin{array}{l}\text { Total Number } \\
\text { Intersections }\end{array}$ & 422 & 182.30 & 0.00 & 33.41 & 30.60 \\
\hline $\begin{array}{l}\text { Total Number Signalized } \\
\text { Intersections }\end{array}$ & 422 & 17.50 & 0.00 & 2.63 & 2.01 \\
\hline $\begin{array}{c}\text { Total Number Non- } \\
\text { signalized Intersections }\end{array}$ & 422 & 181.80 & 0.00 & 30.78 & 30.43 \\
\hline Total Number of Bus Stops & 422 & 111.30 & 0.00 & 14.95 & 12.59 \\
\hline
\end{tabular}

* Note: Boundary Data Assigned Using Equal-proportion-based Assignment Method 


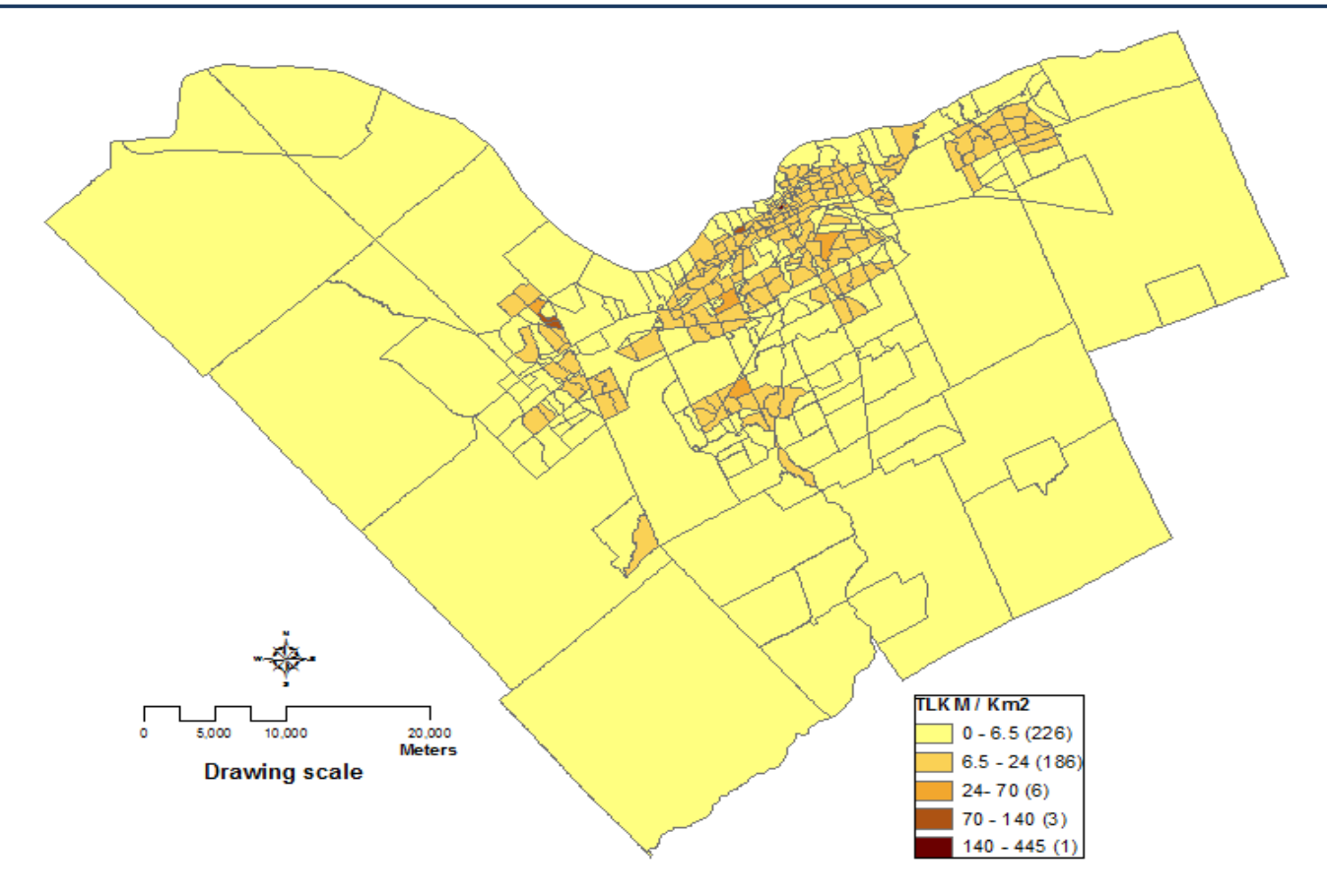

Figure 3.18 Spatial Distributions of TLKM Density in the City of Ottawa.

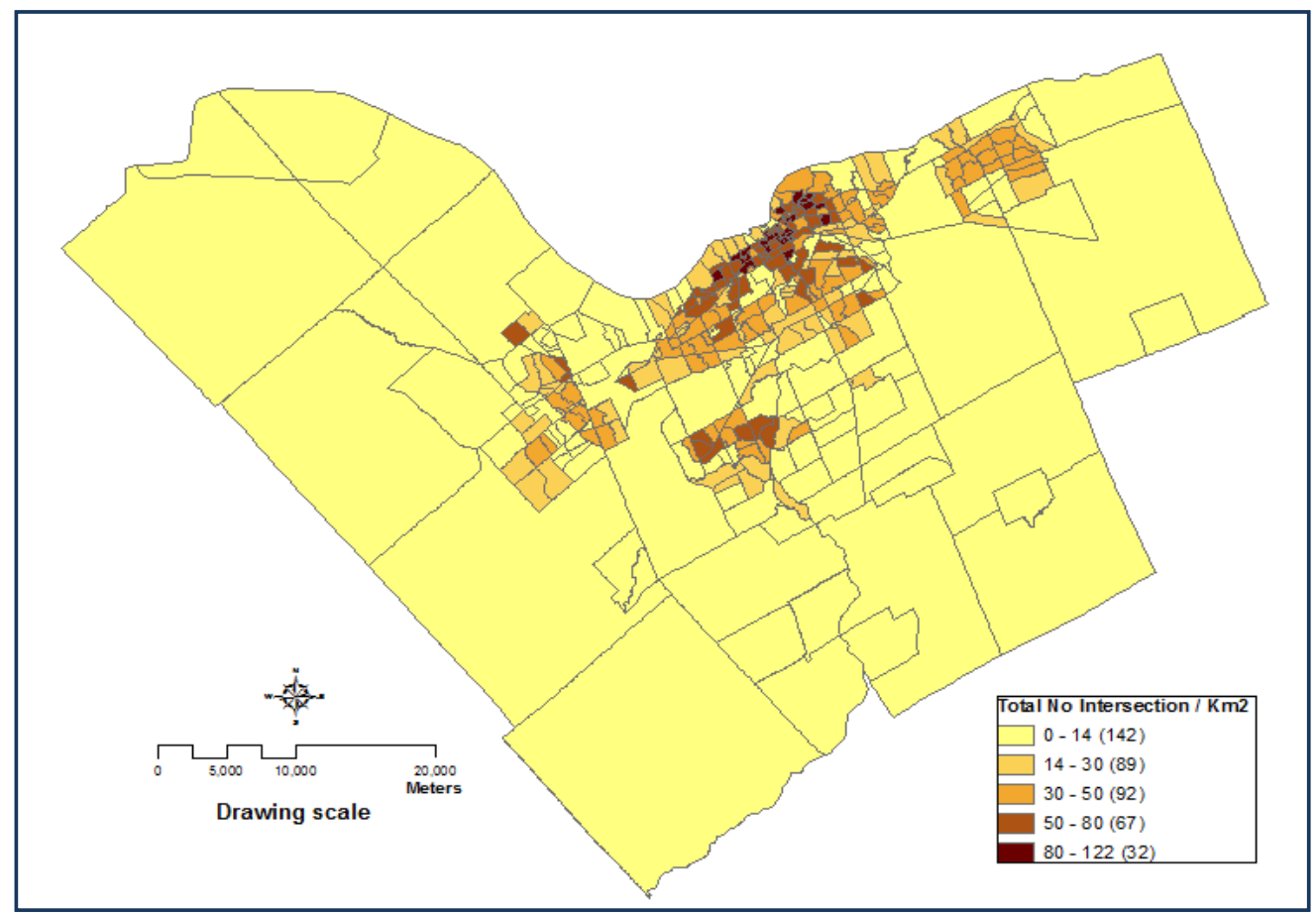

Figure 3.19 Spatial Distributions of Intersections Density in the City of Ottawa. 


\subsubsection{Traffic Exposure Variables}

The statistical summary of exposure variables identified in the City is shown in Table 3.4. This table displays the summary of exposure variables in which the boundary data are assigned based equal-proportion-based assignment method. The summary obtained according to the other methods is shown in Appendix A.

Table 3.4 Descriptive Statistics of Exposure Variables Within Ottawa's TAZs

\begin{tabular}{|c|c|c|c|c|c|}
\hline Variables & Observation & Minimum & Maximum & Mean & $\begin{array}{c}\text { Standard } \\
\text { Deviation }\end{array}$ \\
\hline $\begin{array}{c}\text { Vehicle Kilometre Traveled } \\
\text { (veh/km/day) }\end{array}$ & 422 & 508369.00 & 0.00 & 17269.81 & 46958.76 \\
\hline $\begin{array}{c}\text { Number of Annual Trips Entering } \\
\text { to Centroid of TAZs (veh/day) }\end{array}$ & 422 & 5997.60 & 5.60 & 1611.39 & 1304.69 \\
\hline $\begin{array}{c}\text { Number of Annual Trips Exiting } \\
\text { from Centroid of TAZs (veh/day) }\end{array}$ & 422 & 14240.80 & 16.80 & 1758.27 & 1700.22 \\
\hline Number of Trips Produced & 422 & 5053.28 & 0.00 & 1168.57 & 1146.02 \\
\hline Number of Trips Attracted & 422 & 13012.49 & 0.00 & 1212.78 & 1389.53 \\
\hline
\end{tabular}

* Note: Boundary Data Assigned Using Equal-proportion-based Assignment Method

Figure 3.20 shows the distribution VKT per traffic zones. The greatest densities are located around downtown area. This might indicate that the probability of being involved in a collision increases in the downtown area due to the expected traffic conflicts that result from higher interactions between roadway users. 


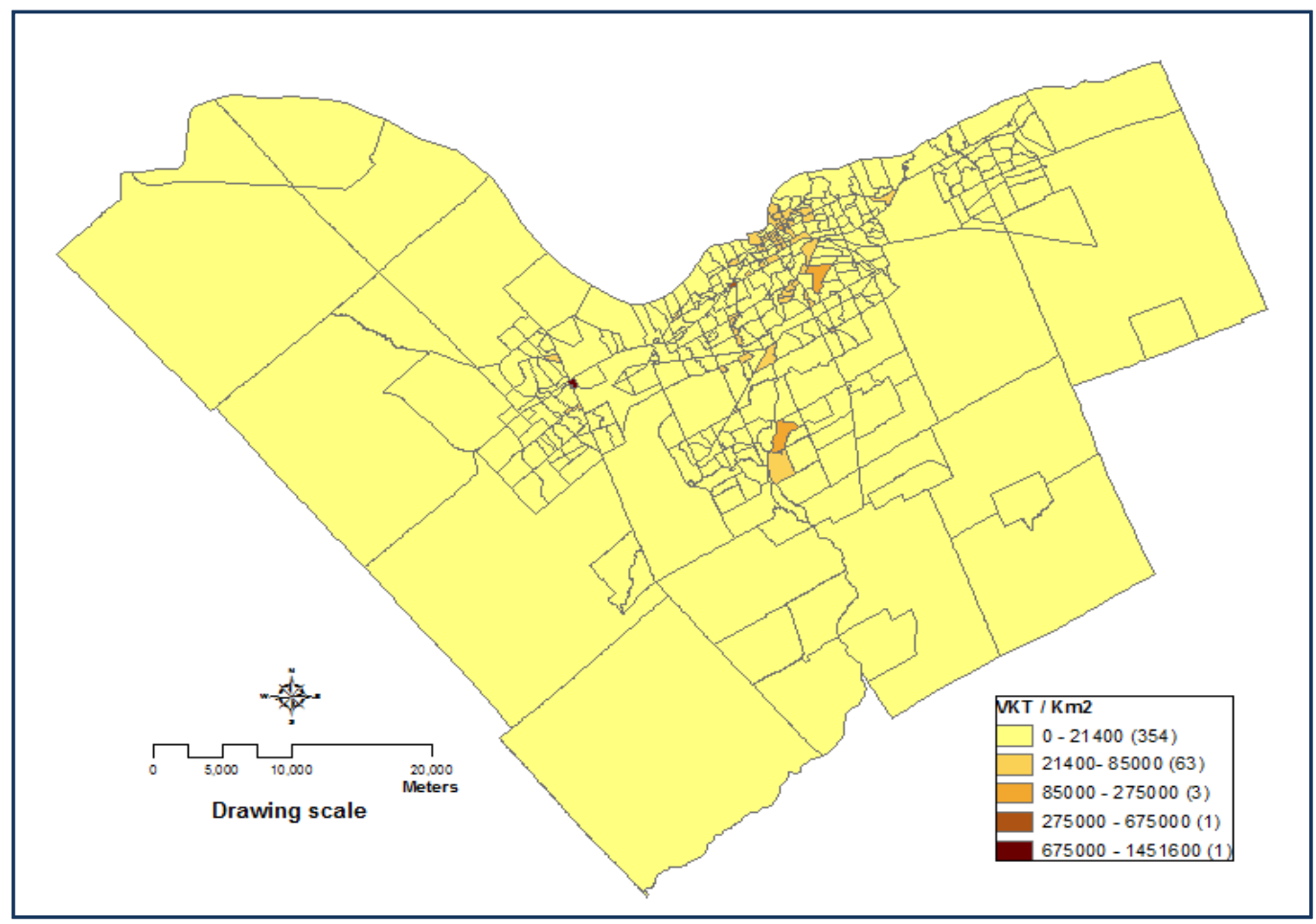

Figure 3.20 Spatial Distributions of VKT Density in the City of Ottawa.

\subsubsection{Demographic and Socio-Economic Characteristics}

The spatial distribution of the density of population, employment, schools, and number of households shows similar patterns to each other (Tables 3.21, 3.22, 3.23, and 3.24 respectively). The concentration of all of these demographic and socio-economic measures is greater in the downtown area and its surrounding areas. It is expected that the presence of all of these variables will increase the number of generated and attracted trips within that part of the city. The increase in the number of trips in turn is expected to contribute significantly to the increase of the probability of involving in a traffic collision. Descriptive statistics for these variables are summarized in Table 3.5. 
Table 3.5 Summary Statistics of Demographic and Socio-economic Variables Within Ottawa's TAZs

\begin{tabular}{|c|c|c|c|c|c|}
\hline Variables & Observation & Maximum & Minimum & Mean & $\begin{array}{c}\text { Standard } \\
\text { Deviation }\end{array}$ \\
\hline Number of Employment & 422 & 14448 & 0.00 & 1338.99 & 1902.66 \\
\hline Number of Population & 422 & 9591 & 0.00 & 2184.95 & 2148.70 \\
\hline $\begin{array}{c}\text { Number of Households } \\
\text { 0-4 years old }\end{array}$ & 422 & 4197 & 0.00 & 899.95 & 907.03 \\
\hline $\begin{array}{c}\text { Number of people between } \\
\text { 5-14 years old }\end{array}$ & 422 & 833 & 0.00 & 120.455 & 140.2603 \\
\hline $\begin{array}{c}\text { Number of people between } \\
\text { 15-24 years old }\end{array}$ & 422 & 1738 & 0.00 & 311.1588 & 336.4837 \\
\hline $\begin{array}{c}\text { Number of people between } \\
25-44 \text { years old }\end{array}$ & 422 & 2793 & 0.00 & 600.9739 & 607.1665 \\
\hline $\begin{array}{c}\text { Number of people between } \\
45-64 \text { years old }\end{array}$ & 422 & 2845 & 0.00 & 617.7559 & 622.3737 \\
\hline $\begin{array}{c}\text { Number of people between } \\
\text { Older than 65 years old }\end{array}$ & 422 & 2189 & 0.00 & 292.4384 & 338.8394 \\
\hline
\end{tabular}




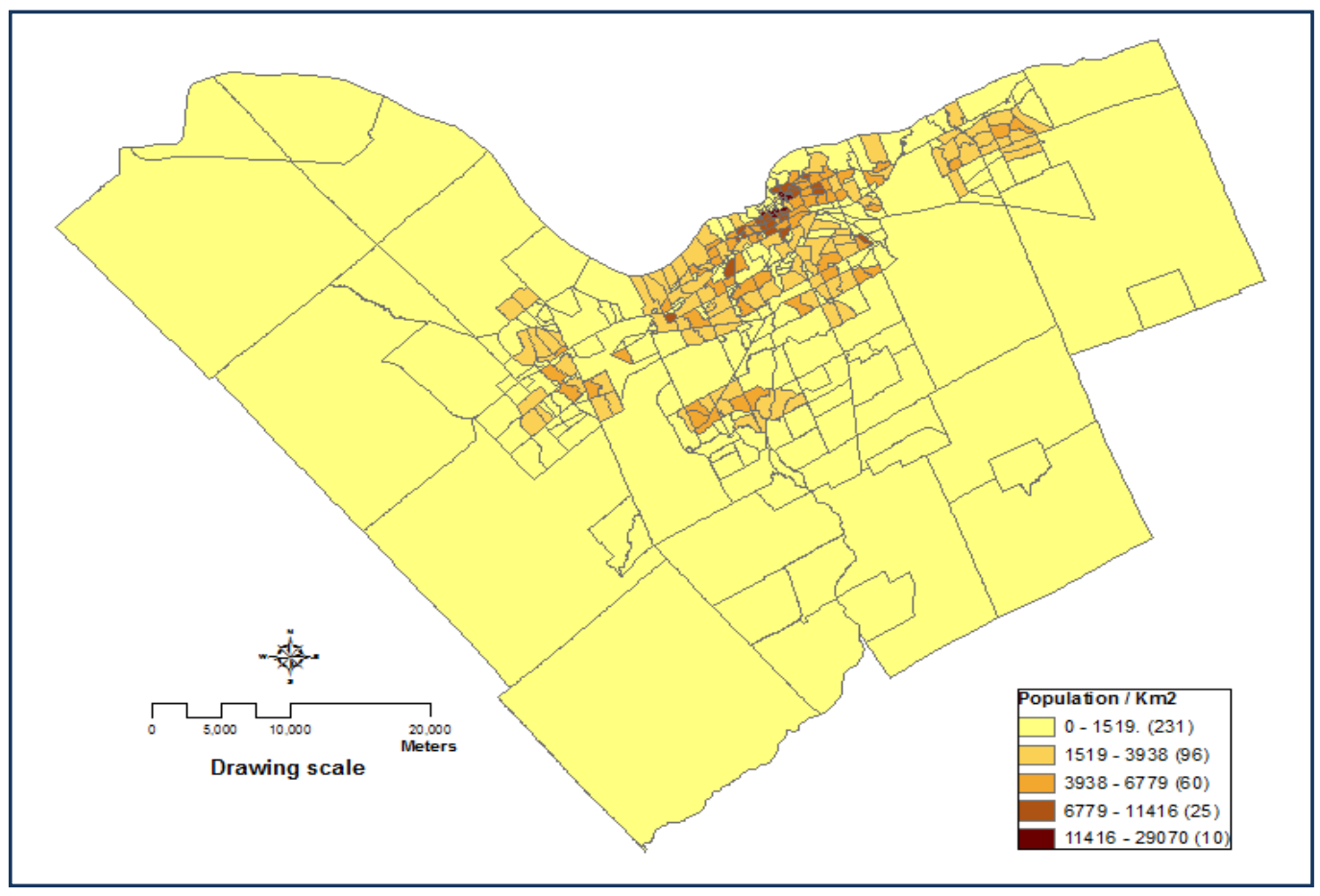

Figure 3. 21 Spatial Distributions of Population Density for the City of Ottawa.

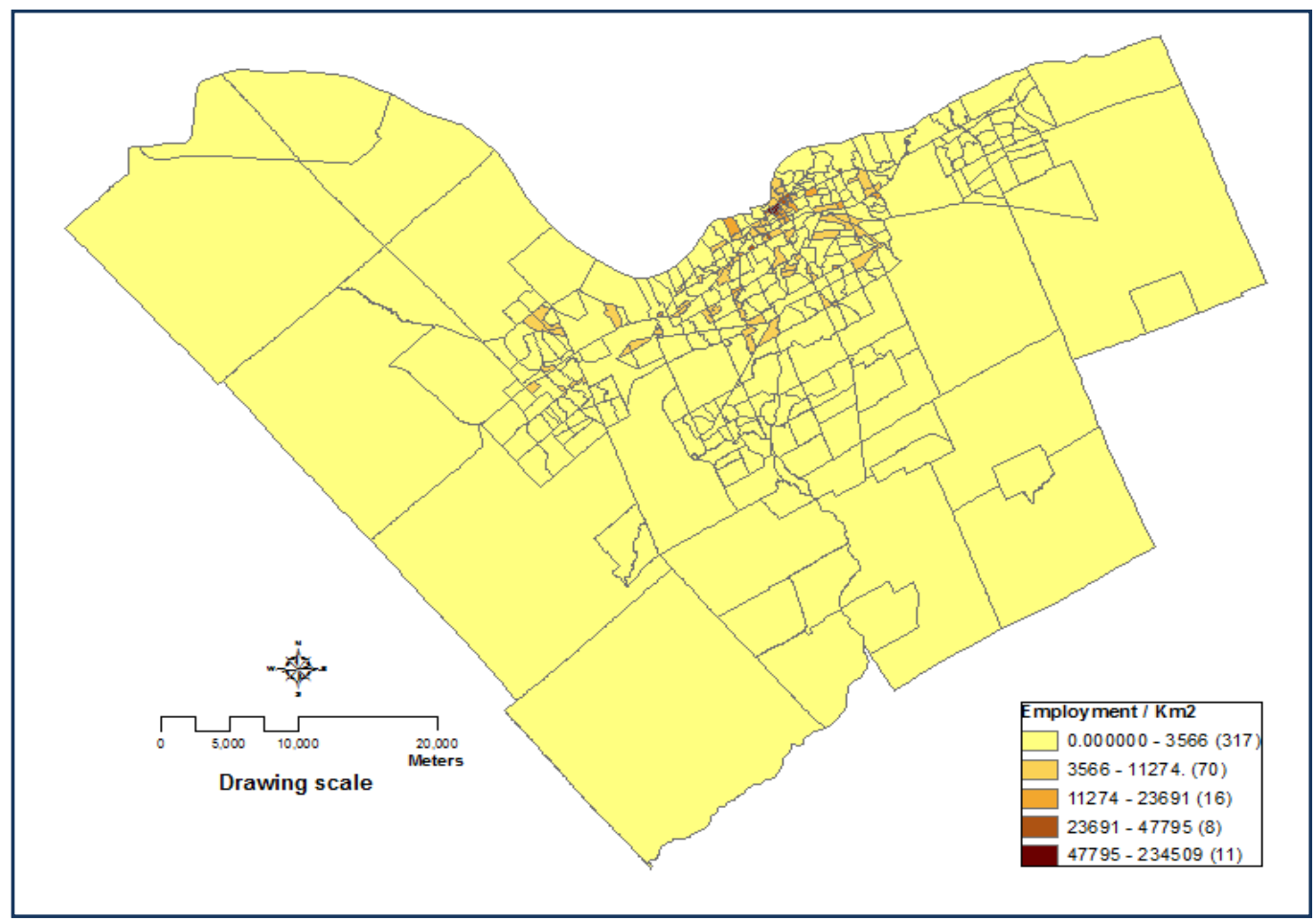

Figure 3.22 Spatial Distributions of Employment Density for the City of Ottawa. 


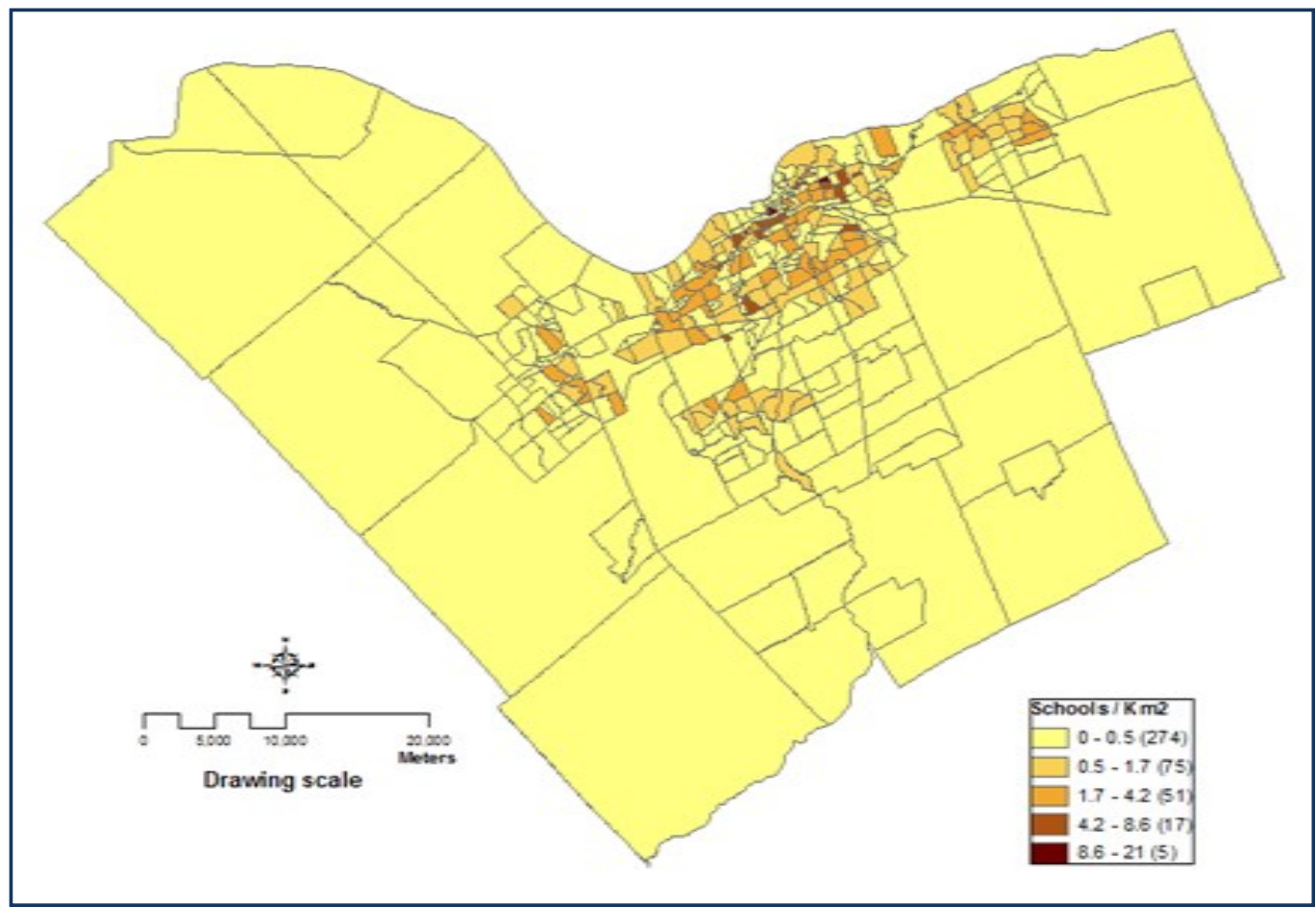

Figure 3.23 Spatial Distributions of Schools Density for the City of Ottawa.

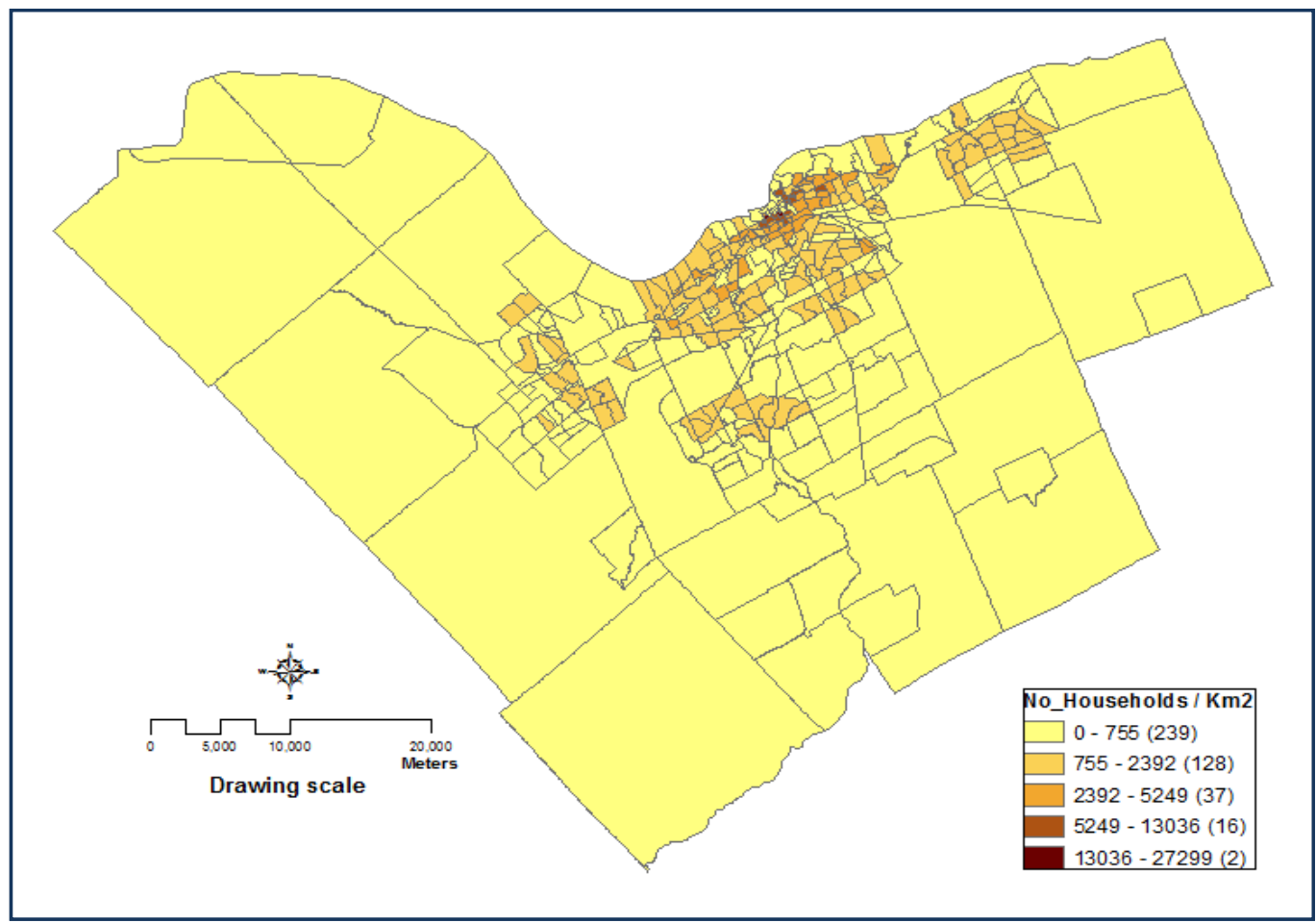

Figure 3.24 Spatial Distributions of Number of Households Density for the City of Ottawa. 


\section{Chapter 4: Statistical Modelling of Collision Data}

This chapter describes the modelling procedure followed to relate potential explanatory variables and collision counts. This chapter is divided into six sections. The first section presents an introduction to the common statistical techniques used for modelling collision frequency. The second section describes the regression procedure and the method on which the best subset of independent variables in explaining the variation in collision frequency was selected for the model attempts. The third section describes the various prepared dependent variables which are total, fatal, non-fatal injury, PDO, bike-involved and pedestrian-involved collisions. This section also presents the various independent variables prepared for the analysis. The forth displays the various modelling combinations used for model attempts development. Multicollinearity issue and its effect on the prediction ability of CPMs are highlighted in the fifth section. Finally, the sixth section provides the test measures for the goodness of fit for the developed models.

\subsection{Statistical Models}

Two major approaches of modeling techniques were identified from the reviewed studies. These approaches are the conventional univariate and multivariate models. The first approach allows modelling each collision frequency type separately (Aguero-Valverde \& Jovanis, 2006; Quddus, 2008; Trentacoste, 2000) while the second approach can be used to analyze simultaneously more than one type of collisions (Ma et al., 2008; Song et al. 2006; and ElBasyouny \& Sayed, 2009). As indicated earlier, the objective of this study is to investigate the effect of different assignment methods of allocating boundary data on the results of collision prediction models (CPMs). Therefore, the univariate approach was selected to develop the models. A number of univariate methods have been proposed to properly model collision data. 
The most common univariate analysis technique is traditional linear regression and Poisson regression and its extensions. Normally, collision data is non-negative, dispersed, and discrete (Mohamed E.A. Sarhan, 2004). Hence, using traditional linear regression for explaining the variation in collision data is considered inappropriate. The inappropriateness of the linear regression comes from that it can predict a negative value which is not meaningful in collision data. In addition, the linear regression does not have the capability to predict a concentrated proportion of specific count levels, e.g., zero collision occurrences. Alternatively, Poisson-based regression analysis has been widely suggested to model collision frequency (S.-P. Miaou, 1993).

The Poisson distribution is associated with one requirement that the mean of predicted collisions equals its variance. Sometimes, the variance of the collision frequency significantly exceeds its mean. Therefore, the data in this case is defined to be over-dispersed. In order to overcome the over-dispersion in collision data, numerous studies have suggested using negative binomial regression technique. Poisson and its extension such as negative binomial and zeroinflated are discussed in more details as follows.

\subsubsection{Poisson Regression}

In a Poisson regression model, the probability of having $y_{i}$ (where $y_{i}$ is a non-negative integer) collisions at $T A Z_{i}$ for the given time period is defined as follows:

$$
P\left(y_{i}\right)=\frac{\operatorname{Exp}\left(-\lambda_{i}\right) \lambda_{i}^{y_{i}}}{y_{i} !}
$$

where,

$$
\begin{aligned}
& P\left(y_{i}\right)=\text { The probability of } T A Z_{i} \text { having } y_{i} \text { collisions during a specific time period } \\
& \lambda_{i}=\text { The expected number of collisions (Poisson parameter) for } T A Z_{i} .
\end{aligned}
$$


The number of collisions can be estimated using Poisson regression by specifying the Poisson parameter $\lambda_{i}$ as function of various independent variables. The explanatory variables at aggregation TAZ's level might include various demographic characteristics, roadway network characteristics, or any other type of data that can be quantified and aggregated to the TAZ's level. The relationship between the expected number of collisions $E\left[y_{i}\right]$ which is equal to $\lambda_{i}$ and the specified predictors are commonly identified by the log-linear model as follows:

$$
E\left[y_{i}\right]=\lambda_{i}=E X P\left(\beta_{0 i}+\beta_{i} * X_{i}+\varepsilon_{i}\right)
$$

Equation 4.2

where,

$$
\begin{aligned}
& E\left[y_{i}\right]=\text { predicted collision frequency } \\
& \beta_{0 i}=\text { intercept } \\
& \beta_{i}=\text { vector parameter coefficients } \\
& X_{i}=\text { vector explanatory variables } \\
& \varepsilon_{i}=\text { random error term. }
\end{aligned}
$$

Applying Poisson regression for modelling collision counts has received some criticism. This criticism arose from that [i] in most cases the variance of collision data can be larger than the mean, which violates the basic assumption of the Poisson regression that the mean is equal to the variance, and [ii] it fails in recognizing that collision data may encompass preponderance of zero frequencies. These two inherent issues in Poisson regression have led to using negative binomial regression to overcome the first limitation and using zero-inflated regression to deal with the second limitation. 


\subsubsection{Negative Binomial Regression}

As mentioned in Section 4.1.1, a common limitation of the Poisson distribution is that the variance equals its mean of predicted collision $\left(E\left[y_{i}\right]=V A R\left[y_{i}\right]\right)$. If this property for Poisson distribution is not satisfied, then data is defined to be either under-dispersed when $\left(E\left[y_{i}\right]\right.$ $\left.>\operatorname{VAR}\left[y_{i}\right]\right)$ or over-dispersed when $\left(E\left[y_{i}\right]<V A R\left[y_{i}\right]\right)$. Collision data are classically found to exhibit over-dispersion. The over-dispersion in collision data is widely recognized to result from omitting some significant variables from the regression. It could be also due to uncertainty in exposure representatives and variables. Omitting some variables can be due to their unexpected influence on the safety performance or they cannot be quantified. To accommodate the overdispersion in collision data, Negative Binomial (NB) distribution has been widely used as an alternative of Poisson model (S.-P. Miaou, 1993). The NB models can be derived from Equation (4.3) as follows:

$$
E\left[y_{i}\right]=\lambda_{i}=\operatorname{EXP}\left(\beta_{0}+\left(\beta_{i} * X_{i}\right)+\varepsilon_{i}\right)
$$

Equation 4.3

where,

$\operatorname{EXP}\left(\varepsilon_{i}\right)$ Follows Gamma distribution with mean equal to 1 and variance equal to $\alpha$. The functional form of the Negative Binomial distribution is:

$$
P\left(y_{i} \mid \lambda_{i}, \alpha\right)=\frac{\Gamma\left((1 / \alpha)+y_{i}\right)}{\Gamma(1 / \alpha) y !}\left(\frac{1 / \alpha}{(1 / \alpha)+\lambda_{i}}\right)^{1 / \alpha}\left(\frac{\lambda_{i}}{(1 / \alpha)+\lambda_{i}}\right)^{y_{i}} \quad \text { Equation } 4.4
$$

where,

$$
\Gamma=\text { the Gamma function. }
$$

The added term to the variance $\alpha$ is known as over-dispersion parameter and it allows the estimated variance to vary from the mean as shown in Equation (4.5). As $\alpha$ approaches zero the 
negative binomial would approach the Poisson distribution.

$$
E\left[y_{i}\right]=\mu \quad \& \operatorname{VAR}\left[y_{i}\right]=\mu+\alpha \mu^{2}
$$

Equation 4.5

\subsubsection{Maximum Likelihood Estimation (MLE)}

Maximum likelihood estimation MLE has been identified as a fundamental tool in many inferential techniques in statistics. It was pointed out to be suitable for both Poisson as well as negative binomial techniques (Mohamed E.A. Sarhan, 2004; S.-P. Miaou, 1993). The likelihood function can be defined as shown in Equation 4.6 (Mohamed E.A. Sarhan, 2004).

$$
\log L(\beta)=\sum y_{i} \log \left(\mu_{i}\right)-\mu_{i}-\log \left(y_{i}\right)
$$

Equation 4.6

where,

$$
\begin{aligned}
& y_{i}=\text { the observed variable } \\
& \mu_{i}=\text { the variable mean }
\end{aligned}
$$

\subsubsection{Akaike Information Criterion (AIC)}

The test was first introduced by Akaike (1974). It has been widely used to evaluate the goodness of fit of different models. This measure can be defined as follows.

$$
A I C=-2 \ln (L L)+2 p
$$

Equation 4.7

where,

$$
L L=\log \text { likelihood of a fitted model obtained from Equation } 4.6
$$




$$
P=\text { number of model parameters }
$$

One of the powerful advantages of this measure is that there is no need for a critical value to be set for comparison. The criterion upon which the model selected is the lowest value of the AIC. In other words, the model with lowest AIC value is considered the best among the calibrated models.

\subsubsection{Zero-Inflated Poisson and Negative Binomial Regression}

Poisson regression models are usually considered to model collision count data. Nevertheless, collision count data can suffer from preponderance of zero counts. The overdispersion in collision data frequently appears when zero counts incidence becomes greater than what is typically anticipated for Poisson distribution. In analyzing collision data at TAZ's level, fatal collisions are rare and many TAZs have no fatal collision during the selected study period. The observed zeros at TAZ's level could arise from two different ways. First, there are limited travel activities within a TAZ boundary especially in rural areas. In addition, the total length of roadways passing through the TAZ is short and they are well-designed and operated. Second, the TAZ has not yet experienced any fatal collision because of the inherent rarity of this type of collisions. In order to deal with collision data containing zero-inflated counts, models such as zero-inflated Poisson (ZIP) and zero-inflated negative binomial (ZINB) regression have been proposed as an extension of a standard Poisson model. These models can help road safety planners to identify unsafe TAZs which have not yet experienced collisions in the selected study period because of randomness from safe TAZs with collision occurrence's probability being zero. A (ZIP) model presumes that the collision occurrence is independent and the model is defined by: 


$$
P\left(y_{i}\right)=\left\{\begin{array}{c}
\varphi+(1-\varphi) g\left(0 \mid X_{i}\right), \text { if }, \quad y_{i}=0 \\
(1-\varphi) g\left(y_{i} \mid X_{i}\right), \text { if }, y_{i}>0
\end{array}\right.
$$

where,

$$
\begin{aligned}
& g\left(y_{i} \mid X_{i}\right)=\text { the probability mass function of a Poisson or NB distribution } \\
& \varphi=\text { logistic function which could be computed as follows. }
\end{aligned}
$$

$$
\varphi_{i}=P\left(y_{i} \mid Z_{i}\right)=\frac{\exp \left(Z_{i}^{\prime} \theta\right)}{1+\exp \left(Z_{i}^{\prime} \theta\right)}
$$

where,

$$
\begin{aligned}
& Z_{i}=\text { the vector of zero-inflated variables } \\
& \theta=\text { the vector of zero-inflated parameters to be estimated }
\end{aligned}
$$

The mean and the variance for the zero-inflated Poisson ZIP model could be written as follows;

$$
\begin{gathered}
E\left(y_{i} \mid X_{i}, Z_{i}\right)=\lambda_{i}(1-\varphi) \\
\operatorname{VAR}\left(y_{i} \mid X_{i}, Z_{i}\right)=\lambda_{i}(1-\varphi)\left(1+\lambda_{i} \varphi_{i}\right)
\end{gathered}
$$

The mean and variance of a ZINB model could be written as follows;

$$
\begin{gathered}
E\left(y_{i} \mid X_{i}, Z_{i}\right)=\lambda_{i}(1-\varphi) \\
\operatorname{VAR}\left(y_{i} \mid X_{i}, Z_{i}\right)=\lambda_{i}(1-\varphi)\left(1+\lambda_{i}\left(\varphi_{i}+\frac{1}{k}\right)\right)
\end{gathered}
$$

where, 
$k=$ the dispersion parameter in the ZINB model

\subsubsection{Vuong Test}

Vuong statistics test was first proposed by Vuong (1989). It is mainly used to test the appropriateness of utilizing zero-inflated as an alternative of Poisson or negative binomial for modeling collision counts data with excessive zeroes. The test equation could be formulated as follows:

$$
V=\frac{\sqrt{n}\left[(1 / n) \sum_{i=1}^{n} m_{i}\right]}{\sqrt{(1 / n) \sum_{i=1}^{n}\left(m_{i}-\overline{m_{l}}\right)^{2}}}=\frac{\sqrt{n}(\bar{m})}{s_{m}}
$$

where,

$$
\begin{aligned}
& \bar{m}=\text { the mean and it is equal to }\left((1 / n) * \sum_{i=1}^{n} m_{i}\right) \\
& s_{m}=\text { slandered deviation } \\
& n=\text { sample size }
\end{aligned}
$$

Equation 4.14 is used for examining the non-nested hypothesis of a zero-inflated model against Poisson or negative binomial models. The selection of a model over another is based on the comparison of $V$ values of both models to the $V_{\text {critical }}$ which is set to be equal 1.96 for a $5 \%$ level of significance .If $V$ value is positive and significantly greater than $V_{\text {critical }}$, then the zeroinflated model would be selected to be better than the negative binomial or Poisson models. On the other hand, if $V$ value is negative and significantly greater than $V_{\text {critical }}$, then the negative binomial or Poisson would be considered to be better for modeling collision frequency. Values in between $[-1.96,1.96]$ would indicate that the test is inconclusive. 


\subsection{Model Selection}

In each model, the variables were tested for significance using stepwise regression procedure. The procedure was applied in order to determine the best subset of indicators in explaining the variation in collision frequency. The models were refined to retain only significant variables, and were tested for significance against each other by means of the AIC. The stepwise procedure includes two main variable selection procedures which are forward selection and backward elimination procedures. The description of the stepwise regression procedure will be better understood if the procedures of forward selection and backward elimination procedures are described first. The following is further explanation about these procedures.

\subsubsection{Forward Selection}

This method is based on entering the variables one at a time until the best regression model is found. This includes performing several steps. At first, the forward selection process begins with the best variable that explains the variation in the dependent variable. This variable should be the most significant (with smallest p-value at the pre-selected level of significance of $5 \%$ ) variable among all candidate variables for regression. Once the first variable is entered, Akaike Information Criterion (AIC) for the model is calculated. The second step is to choose the second most significant variable that, when entered into the regression, gives the largest decrease in AIC, in the presence of the first variable, over the AIC found when only including the first variable. If the second entered variable is found to cause an increase in AIC value, the variable is then dropped and another variable is added. The process continues until no more variables pass the criterion and the model with lowest AIC is obtained. 


\subsubsection{Backward Elimination}

With respect to the backward elimination, the process follows the same concept as forward selection except that it begins with all considered variables in the regression. Once the model is fitted including all variables, AIC is calculated. Then, the variable that is not significantly different from zero, at the pre-selected 5\% level of significance is eliminated and the model is refitted. AIC is calculated for the refitted model and the process is continued with dropping the least significant variable in the model until the model with the lowest AIC among all developed models is obtained.

\subsubsection{Stepwise Regression}

The stepwise regression procedure combines both of the previous procedures. This procedure can be considered as a modification of forward selection procedure with the same steps except that all variables are checked for significance in each step when another variable is entered to the regression. Therefore, at a step when a new variable has been entered into the regression model through a significant decrease in AIC, all the variables already in the model are checked for significance in the presence of the new variable. At this step, any variable/s found to have lost their significant at pre-selected 5\% level of significance is deleted. The procedure is continued until no more variables can be entered or deleted to the model.

\subsubsection{Regression Procedure}

After all of the desired input and output variables are created, regression analysis is performed. Collision data tends to have over-dispersion (variance greater than the mean) and is therefore best estimated using negative-binomial regression. The fatal collision data contains excessive zeroes, and therefore is modeled using zero-inflated regression model. The model coefficients and the overdispersion parameters are estimated by the Maximum Likelihood 
Estimation method using the R statistical computing language and related packages. In $\mathrm{R}$, the function "glm.nb" was used to relate any number of independent variables to a response variable. The regression analysis required installing multiple packages, which are "foreign", "MASS", and "ggplot2". In addition "zeroinfl" function in "pscl" package was used to model fatal collisions. The written code on $\mathrm{R}$ is shown in Appendix (B).

The last step after performing the parameter estimation is to extract the summary of each model. The output of the model contains the estimated parameter coefficients, standard error of the sampling distribution, log-likelihood value $L(\beta)$, Akaike Information Criterion (AIC), dispersion parameter (Theta). It should be noted that parameter (Theta) in R software is the inverse of dispersion parameter (alpha) which is represented by other statistical software such as SAS and SPSS [1]. Equation 4.3 represents the final models form.

\subsection{Variables Selection}

\subsubsection{Dependent Variables}

As mentioned in Chapter 3, the collision data was extracted for all traffic zones. The way by which the City of Ottawa stores its collision data allows for identifying three types of collision severity levels: fatal collisions, non-fatal injury collisions, and property damage only (PDO) collisions. In addition, the bike- and pedestrian- involved collisions within Ottawa traffic zones were determined. Furthermore, total collision frequency is taken as a dependent variable. In total, six dependent variables were formed and prepared for the analysis. 


\subsubsection{Explanatory Variables}

In developing collision prediction models (CPMs), it must be understood that the collision occurrence is the outcome of the complex interactions of numerous variables. The selection process of the explanatory variables is influenced by both data availability and logical consideration. Logically, the ability of a model in predicting collision occurrence increases as more independent variables are included in the model. As stated in Chapter Two, many predictors were used in the literature in order to capture the variation in collision frequencies at different planning levels. The candidate independent variables in general represent data that is easily available to the researcher. In this study, the explanatory variables were collected for 422 TAZs as explained in Chapter Three. The study utilized 40 explanatory variables, available at the city of Ottawa database for the TAZ's level of aggregation, related to four main categories. These are: exposure (related to travel demand), socio-demographics, transportation demand management - TDM (related to how the road system is used), and network (related to network characteristics). Table 4.1 summarizes all the explanatory variables that were included in the analysis

\subsection{Modelling Combinations}

As indicated in Chapter Three, thirteen explanatory variables' datasets using different boundary assignment methods were prepared for the analysis; named by alphabetic order from A to $\mathrm{M}$ (Table 3.1). For each of the thirteen datasets, a model was developed for each type of collision up to a total of six different models for six types of collisions. These types of collisions are: total, PDO, non-fatal injury, fatal, bike-involved, and pedestrian-involved. This produced a total of 78 model attempts (13 datasets $x 6$ collision types). 
Table 4.1 All Candidate Explanatory Variables

\begin{tabular}{|c|c|c|c|}
\hline NO & Category & Explanatory Variables & Description \\
\hline 1 & \multirow{17}{*}{ Network Characteristics } & No Bus stops & Number of bus stops \\
\hline 2 & & Tot-Int & Total Number intersections \\
\hline 3 & & Sig-Int & Number of signalized intersections \\
\hline 4 & & NonSig-Int & $\begin{array}{l}\text { Number of non-signalized } \\
\text { intersections }\end{array}$ \\
\hline 5 & & Artl & Length of arterial roadways (KM) \\
\hline 6 & & Colle & Length of collector roadways (KM) \\
\hline 7 & & Loc & Length of local roadways (KM) \\
\hline 8 & & Maj-Colle & $\begin{array}{l}\text { Length of Major collector roadways } \\
(\mathrm{KM})\end{array}$ \\
\hline 9 & & TLKM & $\begin{array}{c}\text { Total roadways Length per TAZ } \\
(\mathrm{KM})\end{array}$ \\
\hline 10 & & $40 . \mathrm{Km} / \mathrm{h}$ & $\begin{array}{l}\text { Length of roadways with } 40 \mathrm{~km} / \mathrm{h} \\
\text { speed limit (KM) }\end{array}$ \\
\hline 11 & & $50 . \mathrm{Km} / \mathrm{h}$ & $\begin{array}{l}\text { Length of roadways with } 50 \mathrm{~km} / \mathrm{h} \\
\text { speed limit }(\mathrm{KM})\end{array}$ \\
\hline 12 & & $60 . \mathrm{Km} / \mathrm{h}$ & $\begin{array}{l}\text { Length of roadways with } 60 \mathrm{~km} / \mathrm{h} \\
\text { speed limit }(\mathrm{KM})\end{array}$ \\
\hline 13 & & $70 . \mathrm{Km} / \mathrm{h}$ & $\begin{array}{l}\text { Length of roadways with } 70 \mathrm{~km} / \mathrm{h} \\
\text { speed limit (KM) }\end{array}$ \\
\hline 14 & & $80 . \mathrm{Km} / \mathrm{h}$ & $\begin{array}{l}\text { Length of roadways with } 80 \mathrm{~km} / \mathrm{h} \\
\text { speed limit }(\mathrm{KM})\end{array}$ \\
\hline 15 & & $90 . \mathrm{Km} / \mathrm{h}$ & $\begin{array}{l}\text { Length of roadways with } 90 \mathrm{~km} / \mathrm{h} \\
\text { speed limit (KM) }\end{array}$ \\
\hline 16 & & $100 . \mathrm{Km} / \mathrm{h}$ & $\begin{array}{l}\text { Length of roadways with } 100 \mathrm{~km} / \mathrm{h} \\
\text { speed limit }(\mathrm{KM})\end{array}$ \\
\hline 17 & & Bike-Path & $\begin{array}{l}\text { Length of bike paths within each } \\
\text { zone (KM) }\end{array}$ \\
\hline 18 & \multirow{5}{*}{ Modal Split } & MS-Driver & $\begin{array}{c}\text { Percentage of driver trips per traffic } \\
\text { zone }(\%)\end{array}$ \\
\hline 19 & & MS-Passenger & $\begin{array}{l}\text { Percentage of Passenger trips per } \\
\text { traffic zone }(\%)\end{array}$ \\
\hline 20 & & MS-Transit & $\begin{array}{l}\text { Percentage of Transit trips per traffic } \\
\text { zone }(\%)\end{array}$ \\
\hline 21 & & MS-Walk & $\begin{array}{c}\text { Percentage of Walk trips per traffic } \\
\text { zone }(\%)\end{array}$ \\
\hline 22 & & MS-Bike & $\begin{array}{c}\text { Percentage of Bike trips per traffic } \\
\text { zone }(\%)\end{array}$ \\
\hline
\end{tabular}


Table 4.1 All Candidate Explanatory Variables (continued)

\begin{tabular}{|c|c|c|c|}
\hline No & Category & Explanatory variables & Description \\
\hline 23 & \multirow{7}{*}{ Traffic Exposure } & Tot-VKT & $\begin{array}{l}\text { Vehicle kilometre travelled } \\
(\mathrm{Veh} / \mathrm{KM} / \mathrm{d})\end{array}$ \\
\hline 24 & & Trips-Out-Center & $\begin{array}{l}\text { Average annual daily trips exiting } \\
\text { directly from the centroid of traffic } \\
\text { zones }(V e h / d)\end{array}$ \\
\hline 25 & & Trips-Enter-Center & $\begin{array}{l}\text { Average annual daily trips entering } \\
\text { directly to the centroid of traffic } \\
\text { zones (Veh/d) }\end{array}$ \\
\hline 26 & & Trips-Produced & $\begin{array}{l}\text { Total number of trips produced per } \\
\text { traffic zone }\end{array}$ \\
\hline 27 & & Trips-Attracted & $\begin{array}{c}\text { Total number of trips attracted per } \\
\text { traffic zone }\end{array}$ \\
\hline 28 & & $\begin{array}{l}\text { Density- Trips-Out- } \\
\text { Center }\end{array}$ & $\begin{array}{c}\text { Density per square kilometre of } \\
\text { total average annual daily trips } \\
\text { exiting from the centroid of traffic } \\
\text { zones }\end{array}$ \\
\hline 29 & & $\begin{array}{l}\text { Density- Trips-Enter- } \\
\text { Center }\end{array}$ & $\begin{array}{c}\text { Density per square kilometre of } \\
\text { total average annual daily trips } \\
\text { entering from the centroid of traffic } \\
\text { zones }\end{array}$ \\
\hline 30 & \multirow{5}{*}{ Socio-Demographics } & Employment & $\begin{array}{l}\text { Total number of employment per } \\
\text { traffic zone }\end{array}$ \\
\hline 31 & & Population & $\begin{array}{l}\text { Total number of population per } \\
\text { traffic zone }\end{array}$ \\
\hline 32 & & No.Households & $\begin{array}{l}\text { Total number of households per } \\
\text { traffic zone }\end{array}$ \\
\hline 33 & & No.Schools & Number of Schools \\
\hline 34 & & Area & The area of each TAZ $\left(\mathrm{km}^{2}\right)$ \\
\hline 35 & Socio-demographics & Age.0-4 & $\begin{array}{l}\text { Total number of population from } 0 \\
\text { to } 4 \text { years per traffic zone }\end{array}$ \\
\hline 36 & & Age.5-14 & $\begin{array}{c}\text { Total number of population from } 5 \\
\text { to } 14 \text { years per traffic zone }\end{array}$ \\
\hline 37 & & Age.15-24 & $\begin{array}{l}\text { Total number of population from } 15 \\
\text { to } 24 \text { years per traffic zone }\end{array}$ \\
\hline 38 & & Age.25-44 & $\begin{array}{c}\text { Total number of population from } 25 \\
\text { to } 44 \text { years per traffic zone }\end{array}$ \\
\hline 39 & & Age.45-64 & $\begin{array}{l}\text { Total number of population from } 45 \\
\text { to } 64 \text { years per traffic zone }\end{array}$ \\
\hline 40 & & Age $>65$ & $\begin{array}{l}\text { Total number of population older } \\
\text { than } 65 \text { years per traffic zone }\end{array}$ \\
\hline
\end{tabular}


Each of these attempts involved using different formats and combinations of the independent variables. First, some independent variables represent count data, e.g., number of bus stops in a zone. This group of independent variables was labeled $C$, in three combinations as follows:

- Combination C1: countable data representing physical attributes only (network variables). For example, the number of intersections in a zone.

- Combination $C 2$ : countable data representing planning variables only. For example, population within a zone.

- Combination $C 3$ : the union of the previous two combinations (group $C 1$ and $C 2$ ).

Second, certain independent variables were taken as densities inside a TAZ. Densities are represented in this model as a value of a variable per square kilometres. The density items consisted of the network variables as well as employment and population data. The combinations of density variables, labeled $D$, were as follows:

- Combination D1: densities of physical attributes only "network variables".

- Combination D2: densities of planning variables only.

- Combination D3: the union of the previous two models (group D1 and D2)

It should be taken in consideration that some variables could not be readily converted to (or represented as) densities (such as modal split percentages). While not ideal, it was decided to still include them in these combinations (D2 and D3) because they have a continuous nature. Therefore, they were kept in the model during the density analysis. 
The variables within the previously described six combinations of independent variables are described as follows (for details, see Table 4.1);

- Combination C1: Variables 1-17,23-29.

- Combination $C 2$ : Variables $18-40$.

- Combination C3: Variables 1-40.

- Combination D1: Variables 1-17 (densities), and 23-27 (densities).

- Combination D2: Variables 18-22, 23 - 27 (densities), and 30-40(densities)

- Combination D3: 1 - 17 (densities), 18 -22, $23-27$ (densities), and 30-40 (densities).

It can be seen that each combination of independent variables contains all exposure variables. The exposure variables are the essential elements in all developed models due to the fact that the collision incidences are essentially the result of the intersections of traffic movement within TAZs. Therefore, the exposure variables are expected to be significant factors affecting the collision occurrence. Beside the vehicle kilometre travelled (VKT), other exposure measures are added including trips produced and/or attracted to TAZs, and the count and density of total average annual trips directly entering to or exiting from the centroid of each TAZ.

In summary, for each of the thirteen datasets (A to M), 78 model attempts were developed for each type of collisions, which are total, PDO, non-fatal injury, fatal, bike-involved, and pedestrian-involved using the previously mentioned independent variables combinations. Most of these model attempts which included all variables simultaneously ended up with unreliable results due to the presence of multicollinearity issue between the independent variables in each combination. The multicollinearity issue is discussed in more details in the following section. 


\subsection{Multicollinearity Issue}

Multicollinearity is one of the common statistical issues which occurs when two or more independent variables in a statistical model are highly correlated. This is explained by the fact that highly correlated variables may deliver redundant information about the dependent variable. By exploratory examination of the data, the multicollinearity mostly comes from two sources. The first source is formed by a variable that has been derived from other variables, e.g., vehicle kilometre travelled $(\mathrm{VKT})=$ total $\mathrm{AADT} * \mathrm{TLKM}$, and regression includes all three exposure measures. The second source is to include variables which have remarkable similarity, e.g., population in two different age groups treated as two different variables.

The presence of multicollinearity between independent variables usually misleads the computation of a statistical model results. The effect of the multicollinearity is commonly responsible for increasing the standard error of estimates, where the multicollinearity and the standard errors are changing proportionally (the greater the multicollinearity, the greater the standard errors). Therefore, the prediction ability of estimated model will be reduced.

As mentioned previously, the attention in this thesis is to develop CPMs using all possible available information, e.g., all independent variables combinations, and identify the significant variables which have the most influence on collisions occurrence. The modeling procedure is based on the stepwise regression method, which in sequence adds or drops variables based on the possible improvement in the model fit. Using stepwise regression must result in model that includes only significant variables at a level of significance of $5 \%$.

All modelling attempts developed based on the variables combinations mentioned in Section 4.4 failed. The algorithm in $\mathrm{R}$ packages could not converge and failed to provide 
reliable models' parameter estimates. In most of the model attempts, some variables' estimated coefficients were statistically insignificant. In addition, significant and insignificant variables were retained in the same model. This is considered as an evidence of the presence of multicollinearity. The other evidence is the changes in the signs of independent variables from model to another, e.g. switches from positive to negative.

One of the best ways to avoid the mathematical limitations resulting from the presence of multicollinearity on the regression results is to avoid using the highly correlated independent variables in one model. For this purpose, a correlation matrix between the independent variables was developed. From the matrix, if two independent variables are highly correlated (correlation coefficient set to be equal to or more than 0.65$)$, the variables will not be used in the same model. Then, the best model would be selected based on the possible improvement provided by each variable in the model fit.

Because of encountered multicollinearity issues, it was not possible to use the previously mentioned variable combinations presented in Section 4.4. Therefore, it was decided to relate collisions in each prepared dataset (A to $\mathrm{M}$ ) to TAZs variables according to the following variable combinations;

- $\quad$ Traffic exposure-related CPMs

- Combination I: Exposure variables only.

- Roadways-related CPMs.

- Combination II: Roadway characteristics, where the roadway classified based on roadway classes, and exposure variables. 
- Combination III: Roadway characteristics, where the roadway classified based on posted speed limit, and exposure variables.

- Planning-related CPMs

- Combination $I V$ : Modal split variables and exposure variables.

- Combination V: Modal split variables and socio-demographic characteristics variables.

- Combination VI: Modal split variables only.

\subsubsection{Exposure Variables Selection}

As indicted in the previous section, exposure measures are essential in developing CPMs. They are added into regression in all models developed. In total, eight zonal exposure measures are collected including total vehicle kilometre travelled (VKT), total lane kilometre (TLKM), average annually trips enter to or generated from the centroid of each TAZ, and trips produced and generated for each TAZ. Using all of these exposure measures in one model to predict collision frequency is expected to lead to unreliable estimates because some of these exposure measures are highly correlated as shown in Table 4.3. Therefore, the statistical correlation between the exposure variables is considered to determine the exposure measures combinations that can be used together in one model.

From this matrix, it can be seen that some exposure variables are highly correlated. For instance, the total trips generated from a TAZ's centroid is highly correlated with total trips produced (correlation coefficient $=0.90)$. Also, Trips attracted and total trips entering to a TAZ's centroid are correlated (correlation coefficient $=0.65$ ). Therefore, to avoid the issue of model's non-convergence, these correlated exposure measures were not used together in one model in 
regression. Based on correlation matrix, two exposure variables combinations are identified for the analysis as can be seen in Table 4.2.

Table 4.2 All Candidate Exposure Variables combinations

\begin{tabular}{|c|c|}
\hline Combination & Exposure Variables \\
\hline i & $\begin{array}{c}\text { VKT + Number of Trips Out Centroid + Number of Trips Enter Centroid + } \\
\text { Density of Trips out Centroid + Density of Trips enter Centroid + TLKM }\end{array}$ \\
\hline ii & $\begin{array}{c}\text { VKT + Trips Produced + Trips Attracted + Density of Trips out Centroid + } \\
\text { Density of Trips enter Centroid + TLKM }\end{array}$ \\
\hline
\end{tabular}

Table 4.3 Correlation Matrix for Exposure Variables

\begin{tabular}{|c|c|c|c|c|c|c|c|c|}
\hline & $\frac{5}{2}$ & 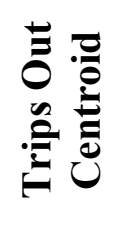 & 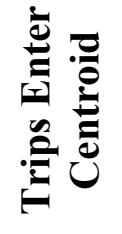 & 莺 & 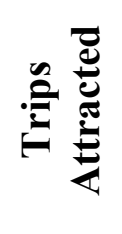 & 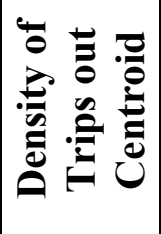 & 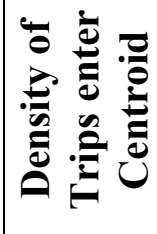 & $\frac{\sum}{Z}$ \\
\hline VKT & 1.00 & & & & & & & \\
\hline Trips Out Centroid & 0.17 & 1.00 & & & & & & \\
\hline Trips Enter Centroid & -0.01 & -0.11 & 1.00 & & & & & \\
\hline Trips Produced & 0.12 & 0.90 & -0.20 & 1.00 & & & & \\
\hline Trips Attracted & -0.04 & 0.11 & 0.65 & 0.11 & 1.00 & & & \\
\hline Density of Trips out Centroid & -0.16 & 0.01 & 0.23 & 0.04 & 0.41 & 1.00 & & \\
\hline Density of Trips enter Centroid & -0.09 & -0.25 & 0.36 & -0.24 & 0.32 & 0.55 & 1.00 & \\
\hline TLKM & 0.47 & 0.39 & -0.11 & 0.23 & -0.06 & -0.21 & -0.14 & 1.00 \\
\hline
\end{tabular}

\subsubsection{Analysis Combinations}

For each of the variable combinations presented earlier, highly correlated variables were not used in the same model attempt. This required dividing each variable combination into several sub-combinations. These sub-combinations were created using two different exposure 
combinations, i.e., exposure combination $i$ and exposure combination ii, Table 4.3. Correlation matrix along with sub-combinations identified within each variables combination prepared for the analysis are shown in Appendix C. The model attempts were developed for each subcombination using two different independent variables formats namely count data (labelled C) and density of the independent variables (labeled D). The numbers of sub-variables combinations that consider the count and density formats of independent variables within each variables combination are shown in Table 4.4.

\section{Table 4.4 Independent Variable Combinations}

\begin{tabular}{|l|l|}
\hline \multicolumn{1}{|c|}{ Count data combination } & \multicolumn{1}{c|}{ Density data combination } \\
\hline Combination $C$ - $I: 2$ sub-combinations & Combination $D-I: 2$ sub-combinations \\
Combination $C$ - $I I: 14$ sub-combinations & Combination $D-I I: 16$ sub-combinations \\
Combination $C-I V: 2$ sub-combinations & Combination $D$ - $I I I: 6$ sub-combinations \\
Combination $C-V: 20$ sub-combinations & Combination $D-I V: 2$ sub-combinations \\
Combination $C-I: 1$ sub-combinations & Combination $D-V: 8$ sub-combinations \\
\hline
\end{tabular}

A total of 51 and 34 model attempts were developed for count and density of independent variables, respectively, for each of the thirteen datasets (A to M). These model attempts were developed for each type of collisions which are total, PDO, non-fatal injury, fatal, bike-involved, and pedestrian-involved collisions. However, only the best models in terms of explaining the variation in collisions frequency was selected for each combination. This produced 11 best models (6 for count data and 5 for density data) for each of the thirteen datasets (A to M). 
Eventually, a total of $78(6 \times 13)$ and $67(5 \times 13)$ best selected model attempts for count and density of independent variables, respectively, were candidate for comparison.

In order to ensure applicability according to the availability of TAZs' level data, the best obtained model attempts will be compared to each other based on the type of the data included in each model attempts which are:

- Exposure-related CPMs (combinations C-I and D-I, (2x13=26 model attempts))

- Roadways-related CPMs (combinations C-II ,C-III, D-II , and D-III, (4x13=52 model attempts ))

- Planning-related CPMs (combinations C-IV,C-V,C-VI,D-IV, and D-V, (5x13=65 model attempts))

These model comparisons will be discussed in more details in Chapter five in Section 5.2. It is noteworthy that the number of population age group $0-4,5-14,15-24,24-64$, and older than 65 years old were highly correlated. To avoid the mathematical limitation formed by the multicollinearity when using these variables together in one model, the percentage of each population age group was employed instead of their count number. However, the percentage age group 5-14 and 15-24 remained highly correlated. These two age groups were combined together and entered to the analysis as one variable.

\subsection{Model Testing}

Many methods of assigning boundary data are used to build the collision models. To be able to determine which method is the best among these methods, as well as to determine which 
set of variables is the best in explaining the variation in collision frequency, several statistical test measures are used herein to evaluate the goodness of the different models.

\subsubsection{Mean Prediction Bias (MPB)}

This method was recommended by Oh et al. (2003), who used the MPB to measure the magnitude and direction of the average model bias. The $M P B$ is the sum of the differences between the observed and predicted collisions, divided by the number of TAZs. The MPB could be formulated as follows:

$$
M P B=\frac{1}{n} \sum_{i=1}^{n}\left(\hat{y}-y_{i}\right)
$$

where,

$$
\begin{aligned}
& \mathrm{n}=\text { the sample size } \\
& \hat{y}=\text { Predicted collisions at TAZ } i \\
& y_{i}=\text { Observed collisions at TAZ } i
\end{aligned}
$$

The result of this measure can be positive or negative. The $M P B$ value is negative when the model under-predicts collision frequency, whereas if the $M P B$ is positive, the model overpredicts collision frequency. The accuracy of CPMs increases when the absolute value of $M P B$ decreases.

\subsubsection{Mean Absolute Deviance (MAD)}

The $M A D$ is the average difference in prediction of the model in an absolute format. The model with $M A D$ closest to the zero is considered the best. The $M A D$ is calculated using the following formula. 


$$
M A D=\frac{1}{n} \sum_{i=1}^{n}\left|\hat{y}-y_{i}\right|
$$

where,

$\mathrm{n}=$ The sample size,

$\hat{y}=$ Predicted collisions at $T A Z i$

$y_{i}=$ Observed collisions at $T A Z i$

\subsubsection{Mean Squared Predictive Error (MSPE)}

The MSPE is utilized to assess the error associated with the prediction. The model with MSPE closest to 0 is considered the best. The MSPE could be formulated as follows:

$$
M S P E=\frac{1}{n} \sum_{i=1}^{n}\left(\hat{y}-y_{i}\right)^{2}
$$

\subsubsection{Coefficient of Determination $\left(R^{2}\right)$}

The $R^{2}$ can be calculated using the following formula. The model with highest $R^{2}$ is considered the best;

$$
R^{2}=\left(\frac{\sum\left(\left(y_{i}-\overline{y_{l}}\right)(\hat{y}-\overline{\hat{y}})\right)}{\sqrt{\left(\sum\left(y_{i}-\bar{y}_{l}\right)^{2}\right) *\left(\sum(\hat{y}-\overline{\hat{y}})^{2}\right)}}\right)^{2}
$$

where,

$\bar{y}_{l}=$ The mean of observed collisions.

$\overline{\hat{y}}=$ The mean of predicted collisions. 
$R^{2}$ is expressed as a value between zero and one. If $R^{2}$ value is equal to one, it indicates a perfect fit, and therefore, a very reliable CPM. On the other hand, if $R^{2}$ is equal to zero, this means that the model fails to accurately model the dataset.

\subsubsection{Root Mean Square Error (RMSE)}

It is also called the standrad devation of the errors. RMSE is mainly used to masure the spread of the data around the regression line and it is computed as follows.

$$
R M S E=\sqrt{\frac{\sum\left(y_{i}-\hat{y}\right)^{2}}{n}}
$$

A RMSE of zero means that the model predicted the observed values with perfect accuracy.

\subsubsection{Nash-Sutcliffe efficiency (E)}

This method was recommended by Nash and Sutcliffe (1970). The efficiency $E$ is defined as one minus the sum of the absolute squared difference between the observed and predicted values. The $\mathrm{E}$ ranges between 1 (perfect fit) and $-\infty$, the model with $\mathrm{E}$ equal 1 is considered the best. $E$ is computed as follows.

$$
E=1-\left(\frac{\sum_{i=1}^{n}\left(\hat{y}-y_{i}\right)^{2}}{\sum_{i=1}^{n}\left(\hat{y}-\bar{y}_{l}\right)^{2}}\right)
$$




\section{Chapter 5: Results and Discussion}

This chapter is divided into nine main sections. The first section includes an overview of the developed models described in Chapter 4. The second section presents the measures according to which the developed models are evaluated. The third section shows a comparison between different boundary data assignment methods along with the selection of the best developed models. The sections from fourth to ninth discuss the zonal collision modelling of each type of collisions. It should be noted that only the first three best ranked models among the developed model attempts for each combination of independent variables are presented in this chapter. The remaining model attempts are shown in Appendix (D).

\subsection{Modelling Overview}

A series of macro-level CPMs were developed for each of collision types which are: total, non-fatal injury, property damage only (PDO), pedestrian-involved and bike-involved collisions. Note that fatal collisions were treated differently as discussed later in Section 5.9. By using different boundary data assignment methods, thirteen datasets, named by alphabetic order from A to $\mathrm{M}$, were prepared for the analysis as discussed in Section 3.3. Ten of these datasets represent the different utilized assignment methods whereas the last three are a combination of the first, second and third methods with the tenth method as previously shown in Table 3.1. For each of the thirteen datasets, a model was developed for each type of collision up to a total of six different models for six types of collisions (i.e., total, PDO, non-fatal injury, fatal, bike-involved, and pedestrian-involved). All six models are developed separately for network, planning, and the union of network and planning variable combinations. In addition, further models were 
developed taking into account densities of certain variables inside the zone as explained in Section 4.4. This produced a total of 78 model attempts.

Developing acceptable CPMs using these variable combinations was not possible since a high multicollinearity exists between many independent variables in each combination. This problem required creating new combinations of variables as shown in Section 4.5. To avoid including highly correlated variables in the same model, each of these combinations were divided into sub-combinations as explained in Section 4.5. Therefore, several model attempts were developed for each combination as explained in Section 4.5.2. Only the best models in terms of explaining the variation in collisions frequency was selected for each combination presented in Table 4.4. This produced 11 best models ( 6 for count data and 5 for density data) for each type of collisions and each of the thirteen datasets (A to M) as shown in Section 4.5.2.

Eventually, for each type of collisions, a total of $78(6 \times 13)$ and $67(5 \times 13)$ best selected model for count and density of independent variables, respectively, were candidates for model ranking. Each candidate model was given abbreviation that consists of three characters where the first character refers to the dataset while the second and third characters refer to the formats and the combination of the independent variables respectively. For instance, the model attempt A-C-I expresses that; A: dataset A; $\mathrm{C}$ : count data format; I: the first variable combination, while the

model attempt B-D-IV expresses that B: dataset B; D: density data format; IV: the fourth variable combination.

\subsection{Best Model Selection}

The modeling procedure is based on the stepwise regression method, which in sequence adds or drops variables based on the possible improvement in the model fit. In each model 
attempt, the variables are tested for significance. Those variables found to be significant at the pre-selected level of significance of $5 \%$ are retained for the next iteration of the models. In this way, models with the lowest AIC are chosen to represent the best models as explained in Section 4.2 .

The models were compared statistically to identify the best method for assigning boundary data. Model within each set of collision type were tested against each other to determine the best fitted among them. This was done using the mean absolute deviance $(M A D)$, mean squared predictive error $(M S P E)$, and root mean square error (RMSE), in which the lowest value amongst competitor model attempts is considered the best model. In addition, Nash-Sutcliffe coefficient $(E)$ and coefficient of determination $\left(R^{2}\right)$, in which the highest value amongst competitor model attempts is considered the best, were used. The model attempts were ranked based on the above mentioned goodness of fit measures to identify the best model attempt. For each prepared dataset (A to M), the models selected for the ranking process were obtained based on the diagram shown in Figure 5.1.

In the ranking process, the model with lowest $M A D$ was given the highest ranking which is 1. This was also applied for $M S P E$, and $R M S E$ goodness of fit measures. In terms of $E$ and $R^{2}$, the model with highest value was given the highest ranking which is 1 . After ranking the models according to the goodness of fit measures from the highest to the lowest, the total ranking for each model was obtained. Total ranking was based on the summation of individual goodness of fit measures ranking. The ranking results for the best fitted model attempts were discussed in details in the following sections. 


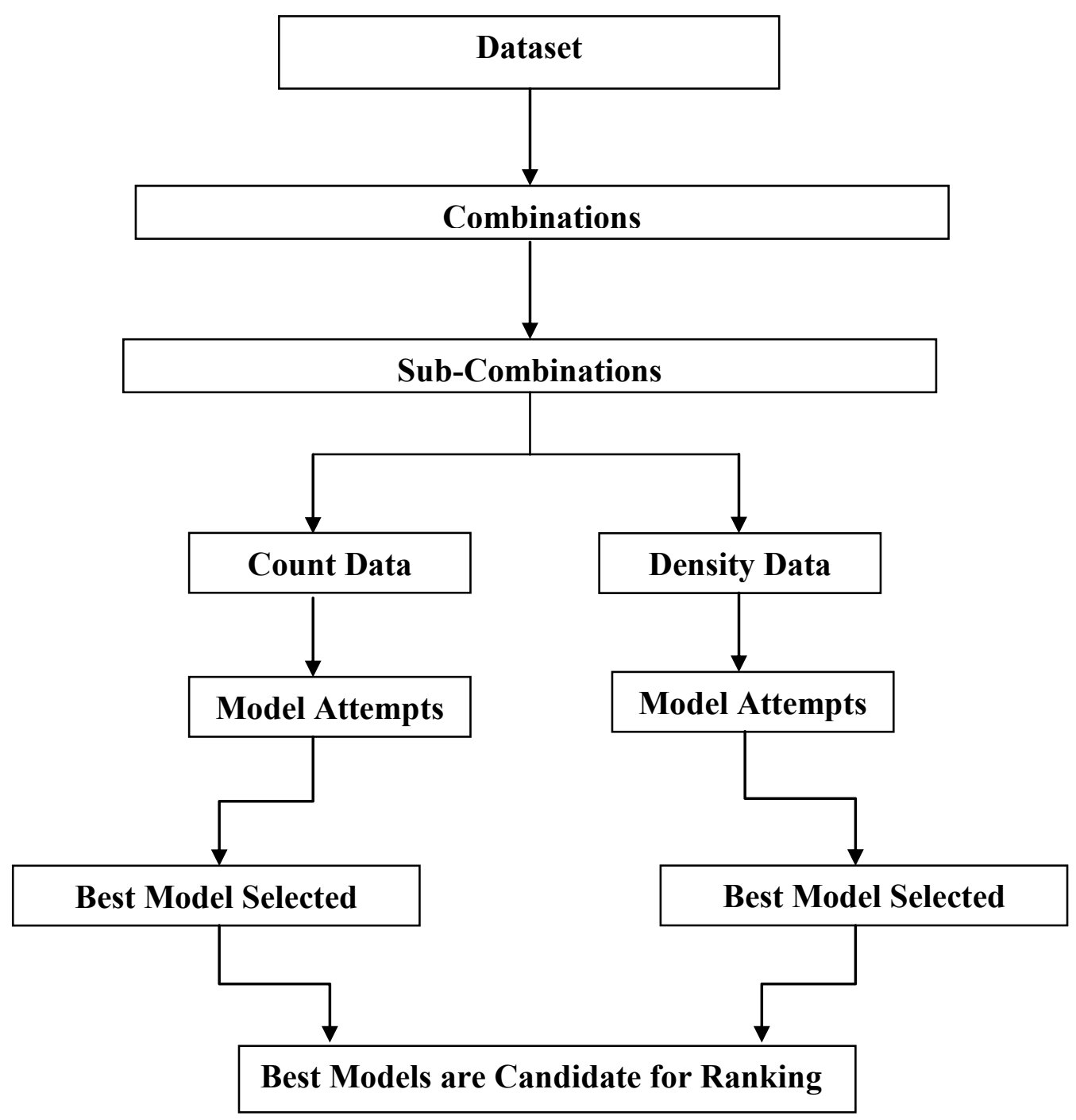

Figure 5.1 Selecting the best CPMs for Ranking

\subsection{Comparing Boundary Assignment Methods}

In order to ensure applicability according to the availability of TAZ's level data, the comparison of different boundary data assignment methods at TAZ's level of aggregation is based on the type of the data included in each model. As indicated in Chapter 4, separate macrolevel CPMs were developed for;

- Exposure-related CPMs (combinations C-I and D-I, (2x13=26 model attempts))

- Roadways-related CPMs (combinations C-II ,C-III, D-II , and D-III,(4x13=52 models)) 
- Planning-related CPMs (combinations C-IV,C-V,C-VI,D-IV, and D-V, (5x13=65 models))

As indicted in Section 4.4, exposure variables are expected to be leading factors in traffic collisions occurrence because of their dominating prediction influence on collision data. Therefore, the first comparison was between the traffic exposure-related models for each type of collisions. Second comparison will be between models that were developed using roadwayrelated and exposure variables. Comparison of these models specifically is expected to show clearly and significantly the impact of different methods of allocating boundary data on the prediction ability of CPMs at the TAZ's level. This is because most of the variables (dependent and independent) in each roadway-related developed model contained boundary data which was assigned to adjacent TAZs using a specific assignment method.

The effect of the various boundary data assignment methods on the models that were developed considering planning and exposure variables is expected to be minimal since only the collision and VKT data was treated among all considered variables in the models. However, the comparison between planning-related and exposure variables model attempts was considered to examine the effect of the boundary data assignment methods on the models developed. It should be noted herein that the planning variables in the planning-related and exposure models are obtained from the City of Ottawa database and included in the regression as they were received without performing any boundary data treatment (see Sections 3.2.4 and 3.2.5). Finally, to determine which developed model best predicts collisions frequency among all model, the comparison of all developed models (roadway related, planning-related, and exposure related CPMs) is performed as well. The following is the ranking results for all model combinations for each type of collisions. It is worth mentioning that the ranking tables in the following section 
include only the best ten models for each collision type. It should be noted that all best fitted models could be practically used for safety performance assessment as all the model inputs are available in the City of Ottawa database.

\subsubsection{Traffic exposure-related CPMs Ranking}

Twenty six $(2 \times 13)$ models for each of total, PDO, non-fatal injury, bike-involved, and pedestrian-involved collisions were qualified for ranking using the TAZ exposure variables. These exposure-related models depend mainly on independent variables describing the TAZs' traffic exposure (combination I, see Section 4.5). The ranking results for the best traffic exposure-related CPMs and their relative goodness of fit measures for total, PDO, non-fatal injury collisions were shown in Tables 5.1, 5.2, and 5.3, respectively. The dispersion parameters $(\theta)$ were in the range of 0.73 and 2.75 which indicates that the collision data was quite overdispersed relative to the Poisson distribution and therefore using the negative binomial regression for modelling was justified. For total, PDO, non-fatal injury collisions, mean prediction bias $(M P B)$ value for the vast majority of best models was positive which indicates that the model over-predicts collisions on average. Also, it was noticed that the model $A-C-I$ that to relate the counted variables to the number of collisions was superior for all of the models for total, PDO, and non-fatal injury collisions. This result indicates that the equal-proportion-based assignment method was the best among all other utilized assignment methods. The results showed also that model that were developed using the population-based (model L-D-I) and populationemployment-based (model M-D-I) assignment methods came in ranking after the equalproportion-based assignment method, and they were better than different TLKM-based and VKT-based assignment methods. 
Table 5.1 Ranking Based on Goodness of Fit Measures for Total Collision Models (Exposure-related CPMs)

\begin{tabular}{|c|c|c|c|c|c|c|c|c|}
\hline Models & MPB & MAD & MSPE & RMSE & $\mathbf{E}$ & $\mathbf{R}^{\mathbf{2}}$ & $\begin{array}{c}\text { Total } \\
\text { Ranking }\end{array}$ & Theta \\
\hline A-C-I & 7.82 & 75.29 & 15360.46 & 123.94 & -0.20 & 0.22 & 18 & 2.08 \\
\hline K-D-I & -0.22 & 80.28 & 11937.19 & 109.26 & 0.07 & 0.07 & 18 & 1.62 \\
\hline L-D-I & 2.76 & 95.27 & 16165.92 & 127.15 & 0.14 & 0.17 & 19 & 0.73 \\
\hline M-D-I & 2.07 & 90.53 & 14659.75 & 121.08 & 0.06 & 0.09 & 23 & 1.06 \\
\hline A-D-I & -0.72 & 83.17 & 12186.85 & 110.39 & 0.05 & 0.05 & 29 & 1.56 \\
\hline D-D-I & -1.78 & 101.23 & 17762.35 & 133.28 & 0.06 & 0.06 & 39 & 0.92 \\
\hline E-C-I & 27.16 & 104.30 & 67824.15 & 260.43 & -2.58 & 0.15 & 40 & 0.96 \\
\hline D-C-I & 27.16 & 104.30 & 67824.15 & 260.43 & -2.58 & 0.15 & 41 & 0.96 \\
\hline C-C-I & 31.05 & 97.56 & 79023.91 & 281.11 & -4.05 & 0.13 & 44 & 1.56 \\
\hline J-D-I & 2.55 & 138.10 & 36445.61 & 190.91 & -0.01 & 0.08 & 48 & 1.71 \\
\hline
\end{tabular}

Table 5.2 Ranking Based on Goodness of Fit Measures for PDO Collision Models (Exposure-related CPMs)

\begin{tabular}{|c|c|c|c|c|c|c|c|c|}
\hline Models & MPB & MAD & MSPE & RMSE & E & $\mathbf{R}^{\mathbf{2}}$ & $\begin{array}{c}\text { Total } \\
\text { Ranking }\end{array}$ & Theta \\
\hline A-C-I & 5.91 & 58.75 & 9375.52 & 96.83 & -0.17 & 0.23 & 18 & 2.15 \\
\hline K-D-I & -0.26 & 63.29 & 7400.28 & 86.02 & 0.08 & 0.08 & 18 & 1.65 \\
\hline L-D-I & 2.15 & 75.23 & 10056.33 & 100.28 & 0.15 & 0.17 & 19 & 0.76 \\
\hline M-D-I & 1.68 & 71.47 & 9141.66 & 95.61 & 0.07 & 0.09 & 23 & 1.09 \\
\hline A-D-I & -0.61 & 65.77 & 7605.03 & 87.21 & 0.05 & 0.05 & 30 & 1.59 \\
\hline E-C-I & 20.37 & 80.85 & 38287.38 & 195.67 & -2.23 & 0.15 & 37 & 1.01 \\
\hline D-D-I & -1.48 & 79.90 & 11012.41 & 104.94 & 0.06 & 0.06 & 39 & 0.93 \\
\hline C-C-I & 24.37 & 76.44 & 51176.63 & 226.22 & -4.22 & 0.12 & 42 & 1.63 \\
\hline D-C-I & 22.16 & 86.55 & 52975.76 & 230.16 & -3.52 & 0.12 & 47 & 1.17 \\
\hline J-D-I & 1.79 & 107.66 & 22136.29 & 148.78 & 0.00 & 0.09 & 48 & 1.74 \\
\hline
\end{tabular}


Table 5.3 Ranking Based on Goodness of Fit Measures for Non-fatal Injury CPMs (Exposure-related CPMs)

\begin{tabular}{|c|c|c|c|c|c|c|c|c|}
\hline Models & MPB & MAD & MSPE & RMSE & $\mathbf{E}$ & $\mathbf{R}^{\mathbf{2}}$ & $\begin{array}{c}\text { Total } \\
\text { Ranking }\end{array}$ & Theta \\
\hline A-C-I & 1.48 & 15.73 & 634.92 & 25.20 & -0.18 & 0.21 & 19 & 2.13 \\
\hline L-D-I & 0.22 & 19.14 & 662.15 & 25.73 & 0.14 & 0.15 & 22 & 0.84 \\
\hline M-D-I & 0.22 & 18.28 & 606.66 & 24.63 & 0.06 & 0.07 & 24 & 1.17 \\
\hline K-D-I & 0.10 & 16.83 & 520.12 & 22.81 & 0.03 & 0.05 & 25 & 1.65 \\
\hline A-D-I & -0.09 & 17.02 & 519.83 & 22.80 & 0.03 & 0.03 & 26 & 1.58 \\
\hline D-D-I & -0.19 & 20.44 & 760.42 & 27.58 & 0.05 & 0.05 & 37 & 0.97 \\
\hline C-C-I & 4.65 & 18.93 & 1862.28 & 43.15 & -1.88 & 0.18 & 39 & 1.82 \\
\hline B-C-I & 5.85 & 21.75 & 2805.03 & 52.96 & -2.63 & 0.14 & 53 & 1.08 \\
\hline E-C-I & 5.00 & 21.43 & 2873.16 & 53.60 & -2.65 & 0.15 & 53 & 1.13 \\
\hline K-C-I & 8.26 & 21.17 & 7816.68 & 88.41 & -13.53 & 0.23 & 53 & 2.76 \\
\hline
\end{tabular}

Bike-involved and pedestrian-involved collisions model attempts ranking results are presented in Tables 5.4 and 5.5 respectively. The ranking results for bike-involved and pedestrian-involved models confirmed that the equal-proportion based method is the best for allocating boundary data at TAZ's level. In Each of Tables 4.5 and 5.5 it can be seen that most of the best models have coefficient of determination $\left(R^{2}\right)$ values equal almost to 0 except for the first three best model. This weak relationship might indicate that exposure-related variables only were not the best TAZ's level indicators in explaining variations in bike-involved and pedestrian-involved collisions. 
Table 5.4 Ranking Based on Goodness of Fit Measures for Bike-Involved CPMs (Exposurerelated CPMs)

\begin{tabular}{|c|c|c|c|c|c|c|c|c|}
\hline Models & MPB & MAD & MSPE & RMSE & $\mathbf{E}$ & $\mathbf{R}^{2}$ & $\begin{array}{c}\text { Total } \\
\text { Ranking }\end{array}$ & Theta \\
\hline K-D-I & 0.05 & 1.40 & 4.12 & 2.03 & 0.12 & 0.19 & 6 & 3.49 \\
\hline M-D-I & 0.08 & 1.49 & 4.77 & 2.18 & 0.07 & 0.18 & 11 & 2.34 \\
\hline L-D-I & 0.15 & 1.57 & 5.97 & 2.44 & 0.00 & 0.21 & 13 & 1.80 \\
\hline A-C-I & 0.19 & 1.64 & 18.52 & 4.30 & -2.94 & 0.02 & 22 & 2.45 \\
\hline A-D-I & 0.23 & 1.73 & 26.32 & 5.13 & -4.60 & 0.01 & 32 & 2.21 \\
\hline K-C-I & 0.24 & 1.66 & 28.23 & 5.31 & -5.01 & 0.01 & 32 & 2.67 \\
\hline J-D-I & 0.27 & 2.50 & 20.73 & 4.55 & -0.46 & 0.12 & 35 & 1.93 \\
\hline G-C-I & 0.27 & 1.79 & 32.72 & 5.72 & -5.03 & 0.01 & 38 & 1.56 \\
\hline I-C-I & 0.37 & 1.90 & 56.37 & 7.51 & -9.16 & 0.01 & 44 & 1.51 \\
\hline D-C-I & 0.39 & 1.92 & 62.97 & 7.94 & -10.25 & 0.01 & 55 & 1.52 \\
\hline
\end{tabular}

Table 5.5 Ranking Based on Goodness of Fit Measures for Pedestrian-Involved CPMs (Exposure-related CPMs)

\begin{tabular}{|c|c|c|c|c|c|c|c|c|}
\hline Models & MPB & MAD & MSPE & RMSE & $\mathbf{E}$ & $\mathbf{R}^{2}$ & $\begin{array}{c}\text { Total } \\
\text { Ranking }\end{array}$ & Theta \\
\hline L-D-I & 0.49 & 3.17 & 28.90 & 5.38 & 0.08 & 0.31 & 32 & 0.98 \\
\hline M-D-I & 0.47 & 3.06 & 33.10 & 5.75 & -0.27 & 0.23 & 33 & 1.27 \\
\hline K-D-I & 0.51 & 2.98 & 61.92 & 7.87 & -1.67 & 0.12 & 34 & 1.95 \\
\hline J-D-I & 2.17 & 6.33 & 955.80 & 30.92 & -10.11 & 0.04 & 41 & 1.34 \\
\hline A-D-I & 4.51 & 7.35 & 7153.58 & 84.58 & -308.06 & 0.01 & 46 & 1.46 \\
\hline A-C-I & 6.50 & 9.33 & 16735.04 & 129.36 & -722.01 & 0.01 & 54 & 1.61 \\
\hline K-C-I & 8.40 & 11.20 & 28343.38 & 168.35 & -1223.53 & 0.01 & 57 & 1.66 \\
\hline I-C-I & 22.10 & 24.96 & 196778.55 & 443.60 & -7096.77 & 0.01 & 59 & 1.09 \\
\hline G-C-I & 16.06 & 18.92 & 103560.25 & 321.81 & -3882.75 & 0.01 & 60 & 1.10 \\
\hline C-C-I & 29.76 & 32.60 & 359336.53 & 599.45 & -13760.04 & 0.01 & 61 & 1.28 \\
\hline
\end{tabular}




\subsubsection{Roadway-related CPMs Ranking}

Fifty two $(4 \times 13)$ models for each of total, PDO, non-fatal injury, bike-involved, and pedestrian-involved collisions were qualified for ranking using the TAZ roadway network variables. These network-based models depend mainly on explanatory variables describing the TAZ roadway network and exposure (models II and III, see Section 4.5). The ranking results for roadway-related CPMs and their relative goodness of fit measures for total, PDO, non-fatal injury collisions are shown in Tables 5.6, 5.7, and 5.8, respectively. The dispersion parameters ( $\theta)$ were in the range of 0.8 and 1.75 which indicates that the data are quite over-dispersed relative to the Poisson distribution and therefore using the negative binomial regression for modelling was justified. For total, PDO, non-fatal injury collisions, mean prediction bias (MPB) value for the vast majority of best models was positive which indicated that the model overpredicts collisions on average. Also, it was noticed that the models that attempted to relate the counted variables to the number of collisions are not as successful as the models that used density variables.

Generally, the model attempts developed based on datasets $A$ and $K$ were recognized always among the highest ranked developed models. The goodness of fit measures for the models obtained based on these two datasets were the closest to each other. In the dataset $A$ all boundary data was assigned based on the equal-proportion-based ratio while in the dataset $K$ only the boundary points (collisions and roadways characteristics localized as points) were assigned according to the equal-proportion-based whereas the boundary roadway lengths and VKT data are assigned to TAZs based on the multiple-count-based method. These results indicate that boundary collisions are influenced by the characteristics of adjacent TAZs and it is better to allocate the boundary data points between TAZs evenly. These can be considered as to be logical 
since any collision occurring on the boundary of adjacent TAZs can be seen to belong to all adjacent TAZs. In an intuitive view, assigning boundary roadways and VKT equally into adjacent TAZs without increasing the total might be better than multiple counting them since they lead to obtain similar results.

In each of these collision types (total, PDO, and non-fatal injury), the comparison of all roadway-related models indicated small variation between the best fitted models in terms of their goodness of fit measures. For total and PDO CPMs, the ranking process showed that model $M$ $D-I I$, which was developed based on dataset $M$, was the highest ranked CPM. Furthermore, this model was among the first six best models for non-fatal injury CPMs. In this model, the collisions data was assigned to TAZs using the population-employment based assignment method whereas the boundary roadway lengths and exposure data was assigned to TAZs based on the multiple-count-based methods. These results imply that the population-employment-based assignment method for assigning boundary data which lies as points was the best among all utilized assignment methods while it is better to assign the boundary roadway lengths and exposure data to TAZs based on the multiple counted based methods. However, using population-employment-based assignment method for allocating boundary data points might not be practical; especially the equal-proportion-based assignment method ended up with similar results. This method may not be preferred for developing CPMs using roadway-related variables mainly for the reason that it requires extra effort to assemble employment and the population data which could be time consuming; or worse in some cases this data may not be available. Therefore, since the equal-proportion-based assignment method gave similar results to population-employment-based assignment method, it is better to divide the boundary data evenly between TAZs. 
Table 5.6 Ranking Based on Goodness of Fit Measures for Total CPMs (Roadway-related CPMs)

\begin{tabular}{|c|c|c|c|c|c|c|c|c|}
\hline Models & MPB & MAD & MSPE & RMSE & $\mathbf{E}$ & $\mathbf{R}^{2}$ & $\begin{array}{c}\text { Total } \\
\text { Ranking }\end{array}$ & Theta \\
\hline M-D-III & 2.16 & 85.40 & 13299.43 & 115.32 & 0.15 & 0.17 & 26 & 1.09 \\
\hline A-D-III & 0.38 & 77.83 & 11221.57 & 105.93 & 0.12 & 0.13 & 28 & 1.67 \\
\hline K-D-II & 0.30 & 77.34 & 11266.68 & 106.14 & 0.12 & 0.12 & 31 & 1.67 \\
\hline L-D-III & 7.53 & 89.91 & 17113.85 & 130.82 & 0.09 & 0.23 & 43 & 0.80 \\
\hline M-D-II & 1.50 & 87.29 & 13864.66 & 117.75 & 0.11 & 0.13 & 45 & 1.06 \\
\hline A-D-II & -0.31 & 78.83 & 11452.24 & 107.02 & 0.11 & 0.11 & 47 & 1.61 \\
\hline D-D-II & 0.34 & 93.08 & 16256.73 & 127.50 & 0.14 & 0.14 & 48 & 0.98 \\
\hline K-D-III & 1.27 & 77.71 & 11877.98 & 108.99 & 0.07 & 0.11 & 48 & 1.71 \\
\hline D-D-III & 2.68 & 92.76 & 16362.45 & 127.92 & 0.13 & 0.14 & 49 & 1.01 \\
\hline C-D-III & 7.77 & 87.56 & 15978.10 & 126.40 & -0.02 & 0.15 & 50 & 1.17 \\
\hline
\end{tabular}

Table 5.7 Based on Goodness of Fit Measures for PDO CPMs (Roadway-related CPMs)

\begin{tabular}{|c|c|c|c|c|c|c|c|c|}
\hline Models & MPB & MAD & MSPE & RMSE & $\mathbf{E}$ & $\mathbf{R}^{2}$ & Ranking & Theta \\
\hline M-D-III & 1.69 & 67.21 & 8246.63 & 90.81 & 0.16 & 0.18 & 24 & 1.12 \\
\hline A-D-III & 0.21 & 61.41 & 6957.52 & 83.41 & 0.13 & 0.14 & 26 & 1.71 \\
\hline K-D-II & 0.25 & 61.06 & 7005.85 & 83.70 & 0.13 & 0.13 & 31 & 1.71 \\
\hline K-D-III & 1.12 & 60.69 & 7227.18 & 85.01 & 0.10 & 0.13 & 41 & 1.75 \\
\hline A-D-III & -0.24 & 62.09 & 7071.31 & 84.09 & 0.12 & 0.12 & 45 & 1.66 \\
\hline C-D-III & 6.60 & 69.28 & 9362.44 & 96.76 & 0.05 & 0.17 & 45 & 1.24 \\
\hline D-D-II & 0.27 & 73.38 & 9996.15 & 99.98 & 0.15 & 0.15 & 45 & 1.00 \\
\hline L-D-III & 5.82 & 72.43 & 10515.89 & 102.55 & 0.11 & 0.21 & 46 & 0.83 \\
\hline M-D-II & 1.05 & 69.29 & 8611.47 & 92.80 & 0.12 & 0.13 & 46 & 1.08 \\
\hline D-D-III & 2.16 & 73.25 & 10142.51 & 100.71 & 0.14 & 0.14 & 51 & 1.03 \\
\hline
\end{tabular}

It was noticed that the model attempt $L-D-I I$ (Table 5.6) came in ranking after the models developed based on $A, K$, and $M$ datasets. The boundary data points in dataset $L$ were assigned to TAZs in proportion with its population whereas the roadways and exposure data was assigned using multiple-count-based assignment method. Population-based assignment method for allocating boundary data to adjacent TAZs was found to be the fourth best assignment method. 
This may indicate that the boundary collisions are somewhat associated with population of adjacent TAZs. However, this method might not be preferred because of the loss of boundary data between adjacent TAZs with zero population. In other words, some boundary data is lost during the assignment process when there are adjacent TAZs with zero population. There might be solution for this problem that the data between zero populated TAZs is assigned to TAZs using equal-proportion-based method. This solution was not used for the model attempts in this thesis. It is expected that following this procedure might lead to improvements for CPMs which have variables assigned based on population-based boundary assignment method.

For total, PDO, and non-fatal injury collisions, VKT-based, TKLM-based, and populationbased assignment methods showed lower ranking when compared to the equal-proportion-based and multiple-count-based assignment methods. This indicates that collisions on TAZ's boundaries were not associated much with the weight of VKT, TKLM and population within each TAZ.

Table 5.8 Ranking Based on Goodness of Fit Measures for Non-fatal Injury CPMs (Roadway-related CPMs)

\begin{tabular}{|c|c|c|c|c|c|c|c|c|}
\hline Models & MPB & MAD & MSPE & RMSE & $\mathbf{E}$ & $\mathbf{R}^{2}$ & $\begin{array}{c}\text { Total } \\
\text { Ranking }\end{array}$ & Theta \\
\hline K-D-II & 0.01 & 15.99 & 484.15 & 22.00 & 0.10 & 0.10 & 34 & 1.71 \\
\hline A-D-III & 0.10 & 16.11 & 485.07 & 22.02 & 0.10 & 0.10 & 39 & 1.69 \\
\hline K-D-III & 0.21 & 16.04 & 499.91 & 22.36 & 0.07 & 0.10 & 45 & 1.75 \\
\hline C-D-III & 1.37 & 17.26 & 640.12 & 25.30 & 0.01 & 0.15 & 46 & 1.38 \\
\hline L-D-III & 1.63 & 18.59 & 762.12 & 27.61 & 0.01 & 0.19 & 52 & 0.95 \\
\hline M-D-III & 0.26 & 17.24 & 561.66 & 23.70 & 0.13 & 0.07 & 56 & 1.22 \\
\hline L-D-II & 1.49 & 18.84 & 756.77 & 27.51 & 0.02 & 0.15 & 57 & 0.93 \\
\hline F-D-III & 1.72 & 18.61 & 774.53 & 27.83 & -0.01 & 0.16 & 59 & 1.15 \\
\hline M-D-II & 0.20 & 18.03 & 597.35 & 24.44 & 0.08 & 0.09 & 61 & 1.17 \\
\hline A-D-II & -0.05 & 16.58 & 507.53 & 22.53 & 0.06 & 0.06 & 63 & 1.61 \\
\hline
\end{tabular}


Bike-involved and pedestrian-involved collisions CPMs ranking results are presented in Tables 5.9 and 5.10 respectively. The models ranking for bike-involved collisions revealed that variation in the boundary data assignment methods does not lead to noticeable difference in terms of the models' goodness of fit. However, equal-proportion-based and multiple-count-based assignment methods led to produce the best fitted bike-involved CPMs. Again, models developed based on $A$ and $K$ datasets were superior for all of the models. This confirms that boundary data is better assigned in accordance with equal-proportion-based or multiple-count based methods. The only difference between bike-involved CPMs and total, PDO, and non-fatal collisions models was that the models that attempted to relate the density variables to the number of bike-involved collisions were not as successful as the models that used counted variables. On the other hand, taking into consideration density of independent variables led to obtain best models for pedestrian-involved collisions.

Table 5.9 Ranking Based on Goodness of Fit Measures for Bike-Involved CPMs (Roadwayrelated CPMs)

\begin{tabular}{|c|c|c|c|c|c|c|c|c|}
\hline Models & MPB & MAD & MSPE & RMSE & $\mathbf{E}$ & $\mathbf{R}^{\mathbf{2}}$ & $\begin{array}{c}\text { Total } \\
\text { Ranking }\end{array}$ & Theta \\
\hline K-C-III & 0.03 & 1.21 & 2.97 & 1.72 & 0.37 & 0.39 & 6 & 9.40 \\
\hline A-C-III & 0.03 & 1.23 & 3.09 & 1.76 & 0.34 & 0.37 & 13 & 8.23 \\
\hline M-C-III & 0.05 & 1.29 & 3.60 & 1.90 & 0.30 & 0.34 & 20 & 5.00 \\
\hline L-C-III & 0.10 & 1.31 & 4.18 & 2.04 & 0.30 & 0.40 & 35 & 3.68 \\
\hline G-C-II & 0.13 & 1.33 & 5.91 & 2.43 & -0.09 & 0.29 & 36 & 4.61 \\
\hline K-D-III & 0.03 & 1.33 & 3.61 & 1.90 & 0.23 & 0.26 & 53 & 4.54 \\
\hline B-C-III & 0.11 & 1.34 & 4.45 & 2.11 & 0.25 & 0.38 & 60 & 3.51 \\
\hline C-C-III & 0.08 & 1.31 & 4.21 & 2.05 & 0.18 & 0.33 & 63 & 4.95 \\
\hline M-D-III & 0.06 & 1.38 & 4.00 & 2.00 & 0.22 & 0.27 & 70 & 3.29 \\
\hline A-D-III & 0.03 & 1.35 & 3.67 & 1.92 & 0.22 & 0.24 & 72 & 3.92 \\
\hline
\end{tabular}


Table 5.10 Ranking Based on Goodness of Fit Measures for Pedestrian-Involved CPMs(Roadway-related CPMs)

\begin{tabular}{|c|c|c|c|c|c|c|c|c|}
\hline Models & MPB & MAD & MSPE & RMSE & E & RSQ & $\begin{array}{c}\text { Total } \\
\text { Ranking }\end{array}$ & Theta \\
\hline A-D-III & 0.22 & 2.67 & 17.92 & 4.23 & 0.23 & 0.34 & 19 & 2.14 \\
\hline M-D-III & 0.39 & 2.79 & 21.93 & 4.68 & 0.16 & 0.38 & 23 & 1.60 \\
\hline G-D-II & 0.44 & 2.86 & 21.95 & 4.68 & 0.18 & 0.41 & 24 & 1.53 \\
\hline A-D-II & 0.26 & 2.74 & 17.98 & 4.24 & 0.22 & 0.33 & 27 & 1.98 \\
\hline K-D-II & 0.30 & 2.68 & 19.65 & 4.43 & 0.15 & 0.34 & 29 & 2.15 \\
\hline G-D-III & 0.45 & 2.90 & 23.03 & 4.80 & 0.14 & 0.42 & 30 & 1.56 \\
\hline C-D-II & 0.55 & 2.95 & 26.90 & 5.19 & -0.03 & 0.45 & 36 & 1.64 \\
\hline H-D-II & 0.58 & 3.01 & 28.63 & 5.35 & 0.01 & 0.37 & 44 & 1.45 \\
\hline H-D-III & 0.56 & 3.07 & 29.30 & 5.41 & -0.02 & 0.37 & 53 & 1.48 \\
\hline D-D-II & 0.57 & 3.07 & 28.68 & 5.36 & -0.03 & 0.33 & 63 & 1.39 \\
\hline
\end{tabular}

As can be seen, the results of the bike-involved and pedestrian-involved collisions models do indicate goodness of fit improvements over the other types of collisions (total, PDO, non-fatal injury). In Table 5.9 and 5.10, the $\mathrm{R}^{2}$ values range from of $24-40 \%$ and $33-45 \%$ indicating that the roadways-related models explain a reasonable fraction of the variance in bikeinvolved and pedestrian-involved collisions.

\subsubsection{Planning-related CPMs Ranking}

Sixty five $\left(5^{*} 13\right)$ models for each of total, PDO, non-fatal injury, bike-involved, and pedestrian-involved collisions were qualified for ranking using the TAZ planning variables. These planning-based models depend mainly on explanatory variables describing the TAZ socioeconomic, modal split, and exposure (models $I V, V$, and $V I$, see Section 4.5). The ranking results for planning-related CPMs and their relative goodness of fit measures for total, PDO, non-fatal injury collisions are shown in Tables 5.11, 5.12, and 5.13, respectively. The dispersion parameters $(\theta)$ were in the range of 1.01 and 2.5 which indicates that the data was quite over- 
dispersed relative to the Poisson distribution and therefore using the negative binomial regression for modelling was again justified. The ranking results for PDO and non-fatal injury CPMs show that the best ten PDO and non-fatal injury CPMs were similar to the best ten total CPMs. Here as well, results showed superiority for models developed based on datasets $A$ and $K$ compared to other developed models in this thesis. This confirms the previous results for the roadway-related models that boundary collision data must be allocated to adjacent TAZs using equal-proportionbased method and assigning other exposure variables in either equal-proportion-based assignment method or multiple-count-based assignment method.

Table 5.11 Ranking Based on Goodness of Fit Measures for Total CPMs (Planning-related CPMs)

\begin{tabular}{|c|c|c|c|c|c|c|c|c|}
\hline Models & MPB & MAD & MSPE & RMSE & $\mathbf{E}$ & $\mathbf{R}^{2}$ & $\begin{array}{c}\text { Total } \\
\text { Ranking }\end{array}$ & Theta \\
\hline K-D-V & 2.39 & 70.37 & 9942.59 & 99.71 & 0.22 & 0.28 & 11 & 2.16 \\
\hline A-C-V & 3.80 & 68.15 & 10357.29 & 101.77 & 0.19 & 0.31 & 12 & 2.25 \\
\hline L-D-V & 3.83 & 78.18 & 12609.77 & 112.29 & 0.33 & 0.37 & 21 & 1.01 \\
\hline M-D-V & 2.74 & 80.49 & 12521.11 & 111.90 & 0.20 & 0.25 & 32 & 1.26 \\
\hline A-C-IV & 5.04 & 72.31 & 12767.06 & 112.99 & 0.00 & 0.24 & 50 & 2.15 \\
\hline K-D-IV & 1.40 & 77.81 & 11605.22 & 107.73 & 0.09 & 0.11 & 53 & 1.76 \\
\hline D-D-V & 2.30 & 88.59 & 15314.20 & 123.75 & 0.19 & 0.23 & 56 & 1.13 \\
\hline A-D-IV & 1.47 & 79.83 & 12020.82 & 109.64 & 0.06 & 0.08 & 61 & 1.71 \\
\hline A-D-IV & 1.47 & 79.83 & 12020.82 & 109.64 & 0.06 & 0.08 & 66 & 1.71 \\
\hline M-D-IV & 3.24 & 88.31 & 14212.45 & 119.22 & 0.09 & 0.11 & 77 & 1.13 \\
\hline
\end{tabular}


Table 5.12 Ranking Based on Goodness of Fit Measures for PDO CPMs (Planning-related CPMs)

\begin{tabular}{|c|c|c|c|c|c|c|c|c|}
\hline Models & MPB & MAD & MSPE & RMSE & $\mathbf{E}$ & $\mathbf{R}^{\mathbf{2}}$ & $\begin{array}{c}\text { Total } \\
\text { Ranking }\end{array}$ & Theta \\
\hline K-D-V & 1.82 & 55.71 & 6189.57 & 78.67 & 0.23 & 0.28 & 11 & 2.21 \\
\hline A-C-V & 3.45 & 51.84 & 6429.76 & 80.19 & 0.20 & 0.34 & 15 & 2.49 \\
\hline L-D-V & 2.88 & 61.70 & 7868.72 & 88.71 & 0.33 & 0.37 & 24 & 1.06 \\
\hline A-D-V & 0.78 & 57.59 & 6252.54 & 79.07 & 0.22 & 0.24 & 25 & 1.99 \\
\hline A-C-IV & 3.74 & 56.86 & 7650.26 & 87.47 & 0.04 & 0.25 & 38 & 2.21 \\
\hline M-D-V & 2.04 & 63.56 & 7805.56 & 88.35 & 0.20 & 0.25 & 39 & 1.29 \\
\hline K-D-IV & 1.13 & 61.59 & 7238.72 & 85.08 & 0.10 & 0.11 & 58 & 1.80 \\
\hline D-D-V & 1.82 & 70.17 & 9525.08 & 97.60 & 0.19 & 0.23 & 61 & 1.16 \\
\hline A-D-IV & 0.87 & 62.82 & 7406.38 & 86.06 & 0.08 & 0.09 & 66 & 1.75 \\
\hline M-D-IV & 2.54 & 69.87 & 8879.26 & 94.23 & 0.09 & 0.11 & 81 & 1.16 \\
\hline
\end{tabular}

Table 5.13 Ranking Based on Goodness of Fit Measures for Non-fatal Injury CPMs (Planning-related CPMs)

\begin{tabular}{|c|c|c|c|c|c|c|c|c|}
\hline Models & MPB & MAD & MSPE & RMSE & $\mathbf{E}$ & $\mathbf{R}^{2}$ & $\begin{array}{c}\text { Total } \\
\text { Ranking }\end{array}$ & Theta \\
\hline K-D-V & 0.58 & 14.51 & 426.38 & 20.65 & 0.21 & 0.27 & 14 & 2.26 \\
\hline A-C-V & 0.84 & 14.01 & 456.70 & 21.37 & 0.15 & 0.30 & 17 & 2.45 \\
\hline A-D-V & 0.18 & 14.76 & 421.48 & 20.53 & 0.22 & 0.23 & 22 & 2.01 \\
\hline L-D-V & 0.63 & 16.25 & 520.65 & 22.82 & 0.33 & 0.35 & 22 & 1.25 \\
\hline M-D-V & 0.68 & 16.60 & 524.49 & 22.90 & 0.19 & 0.24 & 39 & 1.44 \\
\hline D-D-V & 0.49 & 17.96 & 646.01 & 25.42 & 0.19 & 0.23 & 56 & 1.23 \\
\hline B-C-V & 2.47 & 15.48 & 771.12 & 27.77 & 0.00 & 0.37 & 57 & 1.62 \\
\hline K-D-IV & 0.17 & 15.88 & 486.16 & 22.05 & 0.10 & 0.10 & 58 & 1.80 \\
\hline A-D-IV & 0.23 & 16.37 & 511.11 & 22.61 & 0.05 & 0.06 & 70 & 1.73 \\
\hline A-C-IV & 1.16 & 15.39 & 638.72 & 25.27 & -0.19 & 0.20 & 76 & 2.22 \\
\hline
\end{tabular}

It is found that the model attempt $K-D-V$ is the best fitted model for total, PDO, and nonfatal injury, bike-involved, and pedestrian-involved collisions. It can be seen also that the models in which boundary collisions data was assigned based population-based assignment method (datasets $B$ and $L$ ) were among the best ten models. In the dataset $B$ collisions and exposure data 
was assigned based the population-based assignment method while in the dataset $L$ only the collision data was assigned according to the population-based assignment method whereas the exposure data was assigned to TAZs based on the multiple-count based method. These results may indicate that collision occurrence is associated with the population of adjacent TAZs. However, this method provided less accuracy than the equal-proportion-based and multiplecount-based assignment methods.

For all best fitted CPMs developed considering planning variables, mean prediction bias (MPB) value for the vast majority of best models was positive which indicates that the models over-predicts collisions on average. Also, it is noticed that the models that attempted to relate the counted variables to the number of collisions were not as successful as the models that used density variables.

Table 5.14 Ranking Based on Goodness of Fit Measures for Bike-Involved CPMs (Planning-related CPMs)

\begin{tabular}{|c|c|c|c|c|c|c|c|c|}
\hline Models & MPB & MAD & MSPE & RMSE & $\mathbf{E}$ & $\mathbf{R}^{2}$ & $\begin{array}{c}\text { Total } \\
\text { Ranking }\end{array}$ & Theta \\
\hline L-C-V & 0.03 & 1.28 & 3.54 & 1.88 & 0.40 & 0.42 & 17 & 4.02 \\
\hline K-D-V & 0.01 & 1.28 & 3.16 & 1.78 & 0.33 & 0.33 & 18 & 5.74 \\
\hline B-C-V & 0.03 & 1.30 & 3.58 & 1.89 & 0.40 & 0.41 & 28 & 3.75 \\
\hline K-D-IV & 0.02 & 1.28 & 3.25 & 1.80 & 0.31 & 0.32 & 29 & 5.45 \\
\hline M-D-V & 0.03 & 1.31 & 3.48 & 1.87 & 0.32 & 0.33 & 34 & 3.94 \\
\hline A-C-V & 0.02 & 1.29 & 3.25 & 1.80 & 0.31 & 0.32 & 35 & 5.54 \\
\hline L-D-V & 0.03 & 1.34 & 3.78 & 1.94 & 0.37 & 0.38 & 45 & 2.99 \\
\hline M-C-V & 0.05 & 1.29 & 3.65 & 1.91 & 0.29 & 0.34 & 46 & 4.84 \\
\hline K-C-V & 0.03 & 1.29 & 3.46 & 1.86 & 0.26 & 0.30 & 50 & 5.87 \\
\hline C-C-V & 0.05 & 1.29 & 3.65 & 1.91 & 0.29 & 0.34 & 51 & 4.85 \\
\hline
\end{tabular}


As can be seen in Table 5.14 and 5.15 the $\mathrm{R}^{2}$ values range from of $30-42 \%$ and $30-49 \%$ for bike-involved and pedestrian-involved receptively indicating that the planning-related models explain a reasonable fraction of the variance in bike-involved and pedestrian-involved collisions. Tables 5.6, 5.7, and 5.8 showed that planning-related CPMs showed better goodness of fit measures when compared to roadway-related and exposure-related CPMs. This indicates that the planning variables were better explaining the variation in traffic collisions at the TAZs' level.

Table 5.15 Ranking Based on Goodness of Fit Measures for Pedestrian-Involved CPMs (Planning-related CPMs)

\begin{tabular}{|c|c|c|c|c|c|c|c|c|}
\hline Models & MPB & MAD & MSPE & RMSE & $\mathbf{E}$ & $\mathbf{R}^{2}$ & Ranking & Theta \\
\hline K-D-V & 0.12 & 2.34 & 12.62 & 3.55 & 0.45 & 0.49 & 6 & 3.27 \\
\hline K-D-IV & 0.18 & 2.42 & 14.22 & 3.77 & 0.39 & 0.47 & 14 & 3.04 \\
\hline L-D-V & 0.14 & 2.56 & 18.50 & 4.30 & 0.41 & 0.46 & 25 & 1.69 \\
\hline M-D-V & 0.20 & 2.62 & 16.31 & 4.04 & 0.38 & 0.43 & 25 & 1.88 \\
\hline A-D-V & 0.15 & 2.59 & 15.96 & 4.00 & 0.31 & 0.37 & 26 & 2.36 \\
\hline A-C-VI & 0.26 & 2.79 & 17.68 & 4.21 & 0.24 & 0.33 & 39 & 1.94 \\
\hline M-D-IV & 0.31 & 2.72 & 20.35 & 4.51 & 0.22 & 0.38 & 40 & 1.81 \\
\hline K-C-VI & 0.26 & 2.79 & 17.68 & 4.21 & 0.24 & 0.33 & 44 & 1.94 \\
\hline C-C-VI & 0.41 & 3.09 & 22.96 & 4.79 & 0.12 & 0.30 & 69 & 1.28 \\
\hline G-C-VI & 0.35 & 3.11 & 22.28 & 4.72 & 0.16 & 0.29 & 71 & 1.16 \\
\hline
\end{tabular}

In summary, it is better for a roadway safety practitioner using only one assignment method to allocate boundary data between adjacent TAZs. Since the equal-proportion-based assignment method (dataset $A$ and $K$ ) led to obtain the best fitted zonal CPMs for different collisions types, therefore this method is best recommended for application. It is the easiest way of all proposed methods because there only need to spilt boundary data equally between adjacent TAZs. 


\subsection{Best Exposure-related CPM}

The regression results of the first best traffic exposure-related models for total, PDO, non-fatal injury, bike-involved, and pedestrian-involved collisions are presented in Table 5.16. For total, PDO, and non-fatal injury collisions the ranking results showed superiority for the model $A-C-I$ in which collisions and VKT boundary data were assigned according to equalproportion-based assignment compared to other developed models in this thesis. Since the best three models for each collision type revealed almost similar results, only the best fitted model for each collision type is displayed in Table 5.16.

The models reveal that collision frequency increases with the increase in the following explanatory variables: vehicle kilometre travelled (VKT), total lane kilometre (TLKM), number and density of average annual trips exiting from the centroid of TAZs, and the number of average annual trips entering to TAZs centroid. However, the density of average annual trips entering to TAZs centroid is found to be inversely related to collision frequency.

As expected, the relationship between collisions and VKT was statistically significant at a level of significance of 5\%. The positive coefficient of the VKT in the models indicates that as the VKT increases, the number of total, PDO, and non-fatal injury collisions increase. The explanation is that as the VKT increases within a TAZ, exposure of vehicle traffic and their number of conflicts would increase, which in turn increases the probability of being involved in a collision.

For bike involved and pedestrian-involved collisions the result showed superiority for models $K-D-I$ and $L-D-I$, respectively, compared to other developed models in this thesis. These two models showed similar result to the best exposure-related CPMs for other types of collisions. 
Table 5.16 Model Estimation Results for the Best Exposure-related CPMs

\begin{tabular}{|c|c|c|c|c|c|c|c|c|c|c|}
\hline \multirow{3}{*}{$\begin{array}{c}\text { Models } \\
\text { Variable }\end{array}$} & \multicolumn{2}{|c|}{ Total CPM } & \multicolumn{2}{|c|}{ PDO CPM } & \multicolumn{2}{|c|}{$\begin{array}{c}\text { Non-fatal Injury } \\
\text { CPM }\end{array}$} & \multicolumn{2}{|c|}{ Bike-involved CPM } & \multicolumn{2}{|c|}{ Bike-involved CPM } \\
\hline & \multicolumn{2}{|c|}{ Model $A-C-I$} & \multicolumn{2}{|c|}{ Model $A-C-I$} & \multicolumn{2}{|c|}{ Model $A-C-I$} & \multicolumn{2}{|c|}{ Model $K-D-I$} & \multicolumn{2}{|c|}{ Model $L-D-I$} \\
\hline & Estimate & $p$-value & Estimate & $p$-value & Estimate & $p$-value & Estimate & $p$-value & Estimate & $p$-value \\
\hline (Intercept) & 4.06 & $<2 \mathrm{E}-16$ & $3.81 \mathrm{E}+00$ & $<2 \mathrm{E}-16$ & $2.51 \mathrm{E}+00$ & $<2 \mathrm{E}-16$ & $-9.23 \mathrm{E}-02$ & 0.2567 & $-1.77 \mathrm{E}-01$ & 0.0915 \\
\hline VKT & $3.97 \mathrm{E}-06$ & $1.32 \mathrm{E}-06$ & $3.76 \mathrm{E}-06$ & $3.20 \mathrm{E}-06$ & 4.32E-06 & $1.22 \mathrm{E}-07$ & $5.18 \mathrm{E}-06$ & 0.0326 & $-7.87 \mathrm{E}-06$ & 0.0179 \\
\hline TLKM & 0.00385 & 0.0183 & $3.93 \mathrm{E}-03$ & 0.0143 & $3.91 \mathrm{E}-03$ & 0.0169 & $8.47 \mathrm{E}-02$ & $<2 \mathrm{E}-16$ & $1.15 \mathrm{E}-01$ & $<2 \mathrm{E}-16$ \\
\hline Trips Out Centroid & $1.85 \mathrm{E}-04$ & $1.11 \mathrm{E}-09$ & $1.91 \mathrm{E}-04$ & $1.92 \mathrm{E}-10$ & $1.47 \mathrm{E}-04$ & $3.46 \mathrm{E}-06$ & - & - & - & - \\
\hline $\begin{array}{l}\text { Trips Enter } \\
\text { Centroid }\end{array}$ & $1.68 \mathrm{E}-04$ & $6.94 \mathrm{E}-15$ & $1.67 \mathrm{E}-04$ & $3.90 \mathrm{E}-15$ & $1.70 \mathrm{E}-04$ & $1.09 \mathrm{E}-14$ & - & - & - & - \\
\hline $\begin{array}{l}\text { Density of Trips } \\
\text { Enter Center }\end{array}$ & $-1.3 \mathrm{E}-05$ & $5.72 \mathrm{E}-07$ & $-1.36 \mathrm{E}-05$ & 5.87E-07 & $-1.61 \mathrm{E}-05$ & $1.17 \mathrm{E}-05$ & $-6.45 \mathrm{E}-05$ & 2.71E-09 & $-4.82 \mathrm{E}-05$ & $3.52 \mathrm{E}-11$ \\
\hline $\begin{array}{c}\text { Density of Trips } \\
\text { Out Center }\end{array}$ & $1.10 \mathrm{E}-04$ & $3.62 \mathrm{E}-10$ & $1.14 \mathrm{E}-04$ & $7.75 \mathrm{E}-11$ & $1.01 \mathrm{E}-04$ & $8.48 \mathrm{E}-07$ & - & - & $2.38 \mathrm{E}-04$ & $2.40 \mathrm{E}-07$ \\
\hline Trips Attracted & - & - & - & - & - & - & $3.13 \mathrm{E}-05$ & $1.02 \mathrm{E}-05$ & - & - \\
\hline Trips Produced & - & - & - & - & - & - & $-4.41 E-05$ & 0.0829 & - & - \\
\hline
\end{tabular}




\subsection{Total and PDO Collisions Models}

\subsubsection{Roadway-related CPMs}

The regression results of the best three roadway-related CPMs for total PDO collisions are presented in Table 5.17 and 5.18, respectively. Interestingly, the CPMs' results of roadwayrelated variables for total and PDO collisions showed similarity regarding variables affecting them. They vary in their variables' coefficient values due to the difference in their number. The variables' coefficient values of total collisions models were bigger because the number of total collisions is bigger. As shown the model M-D-III was the best fitted models in both collisions types. The model retained 8 independent variables while assessing the association with roadwayrelated and exposure variables. In the model, density of total number of intersection within a TAZ was found positively related to total and PDO collisions at a level of significance 5\%. Total number of intersections included both signalized and non-signalized intersections. Nonsignalized intersections are usually placed in location with low AADT, possibly at low speed roadways with fewer collisions. While signalized intersections are placed in locations with high AADT and possibly collision prone locations. Therefore, the total number of intersections coefficient in the model depends on the mixture of signalized and non-signalized intersections, respectively. Traffic control signals were consistently determined to be a significant variable in the analysis. Whether the models considered their number or density, there was a positive relationship between the presence of signals and the occurrence of total collisions. This is an expected result as traffic signals are usually located at intersections where there are large conflicting volumes of traffic. These intersections, despite the intersection control device, may have more collisions than low volume intersections. In micro-level CPMs, the AADT of an 
intersection is a common variable in determining the expected number of collisions. In the macro-level, a high number of traffic signals may indicate high traffic volume. Therefore, the number of traffic signals can be an indicator of collision frequency at the TAZ's level (as the density of signalized intersections within a TAZ increases, collision frequency in turn increases). Additionally, there are higher numbers of traffic signals in downtown TAZs than rural areas (Figure 3.19), where pedestrians, cyclists and vehicles densities are higher.

Local roadways tend to have lower speeds than other roadway classes, thus it is expected that they are not associated with total and PDO collisions. Lower speeds could be an important factor in reducing collisions as there is more time to react at lower speeds Furthermore, slight vehicle collisions which could happen at low speed local roadways (with a damage less than $1000 \$$ ) were unavailable in the City of Ottawa database, which in turn may affect the collision model coefficients of local roadways. Surprisingly, the model $K-D-I I$ for both total and PDO collisions revealed that TAZs with more density of local roadways tend not to be safer than TAZs with less or no local roadways. From the data, local roadways form roughly $45 \%$ of the total lane kilometres within Ottawa's TAZs. This indicates that this class of roadways is widely used particularly in the urban TAZs where there is relatively high degree of congestion. This could be possible illustration for increasing total and PDO collisions in TAZs with higher density of local roadway class.

Furthermore, the density of roadways with $70 \mathrm{~km} / \mathrm{h}$ speed limit was significant variable in the model $A-D-I I I$ for total collisions. It was inversely related to total collisions (negative coefficient). This can indicate that as the density of roadways with $70 \mathrm{~km} / \mathrm{h}$ speed limit in a TAZ increases, the expected number of total and PDO collisions decrease. This could be attributed to that these roadway classes usually exist in rural TAZs where there is relatively low degree of 
congestion. Due to this the collisions on these roadways are expected to be less while the level of fatalities is expected to be higher compared to roadways with lower posted speed limits.

In the model $M-D-I I I$, The density of roadway lengths with 40 and $50 \mathrm{~km} / \mathrm{h}$ speed limits were positively associated with total and PDO collisions. This indicates that as the density of kilometres of roadways with 40 and $50 \mathrm{~km} / \mathrm{h}$ speed limits in a TAZ increases, exposure of vehicle traffic would increase, which in turn increases the probability of being involved in a collision. The model $A-D-I I I$ indicated conflicting results in terms of these two roadways variables. Moreover, model $A-D-I I I$ showed that TAZs with higher density of roadways with a posted speed limit of $100 \mathrm{~km} / \mathrm{h}$ are expected to have high number of collisions. Roadways with a posted speed of $100 \mathrm{~km} / \mathrm{h}$ may not be the best indicator of total and PDO collisions since only two TAZs had roadway/s with a posted speed limit of $100 \mathrm{~km} / \mathrm{h}$. However, this result can indicate a strong statistical relationship between speed and roadway safety performance at TAZs level.

Intuitively, increased total vehicle kilometre traveled (VKT) and total lane kilometre (TLKM) within a TAZ would increase the probability of being involved in a collision. The three best total and PDO CPMs showed conflicting results with respect to total VKT and TLKM. These variables were significant in all best fitted roadway-related models but the sign of the variables differs. The model $A-D-I I I$ showed a negative relationship between total and PDO collision frequency and density of VKT, and a positive relationship with the density of TLKM. The sign of VKT and TLKM respectively was opposite in the model $M-D-I I I$ and $K$-D-II. As it can be seen from Figure 3.20 that VKT is dependent on the land use and it is expected to have a positive effect on the total and PDO collisions; however, different combination of AADT and roadway lengths and different boundary assignment method could play an important role in 
determining the sign of VKT coefficient. For instance, at some locations AADT could be low while roadway lengths are high or vice versa. Also, both AADT and roadway lengths could be either high or low. The main concept in regression analysis is that the coefficients represent the mean change in the dependent variable for one unit of change in the independent variable while holding other dependent variables in the model constant. Therefore, the model $A-D-I I I$ showed a decrease in total and PDO collisions as the VKT within a TAZ increase while holding other dependent variable in the model constant. Total lane kilometre was positively related to total collision frequency at the level of significance of $5 \%$ in the model $A-D-I I I$. This finding is reasonable since increasing total lane kilometre within a TAZ increases exposure, which in turns increases collision frequency.

Bus stops are thought to be collision-prone locations. The reason is the interaction between buses and motor vehicles at bus stop points particularly in the downtown areas, where they may share the traffic lanes. Vehicle-bus conflicts may occur at bus stop locations while a bus is stopping and the following vehicle is trying to make lane change maneuver or having inadequate stopping gap (uncomfortable deceleration). Conflicts also could happen while a bus is departing from a bus stop to a shared lane which is already occupied by a vehicle. Surprisingly, bus stops within the City of Ottawa TAZs were determined to be a significant variable in all best fitted models for the total and PDO collisions.

Bike paths in the models include the mixture of marked paths (bike lanes on the side roadways) and separate paths from the roadway. The marked bike paths identify a clear separation between vehicle traffic and cyclist flow. In the model $A-D-I I I$ and $K-D-I I$, it was found that increasing the length of bike paths within a TAZ increases total and PDO collisions. In summary, more bike lanes length indicates less safety performance within a TAZ. 
Table 5.17 Model Estimation Results of the Best Roadway-related CPMs for Total Collisions

\begin{tabular}{|c|c|c|c|c|c|c|}
\hline \multirow{2}{*}{$\frac{\text { Models }}{\text { Variable }}$} & \multicolumn{2}{|c|}{ Model $M-D-I I I$} & \multicolumn{2}{|c|}{ Model $A-D-I I I$} & \multicolumn{2}{|c|}{ Model $K-D-I I I$} \\
\hline & Estimate & $p$-value & Estimate & $p$-value & Estimate & $p$-value \\
\hline (Intercept) & $4.72 \mathrm{E}+00$ & $<2 \mathrm{E}-16$ & $4.68 \mathrm{E}+00$ & $<2 \mathrm{E}-16$ & $4.76 \mathrm{E}+00$ & $<2 \mathrm{E}-16$ \\
\hline Total Number of Intersections & $8.23 \mathrm{E}-03$ & 0.002186 & - & - & - & - \\
\hline Signalized Intersections & - & - & $1.69 \mathrm{E}-02$ & $2.06 \mathrm{E}-05$ & - & - \\
\hline Non-signalized Intersections & - & - & $6.35 \mathrm{E}-03$ & 0.000483 & - & - \\
\hline Total Arterial Road Kilometres & - & - & - & - & - & - \\
\hline Total Collector Road Kilometres & - & - & - & - & - & - \\
\hline Total Major Collector Road Kilometres & - & - & - & - & - & - \\
\hline Total Local Road Kilometres & - & - & - & - & $9.03 \mathrm{E}-02$ & $6.43 \mathrm{E}-05$ \\
\hline Total 40 km/h Road Kilometres & $1.14 \mathrm{E}-01$ & 0.002389 & $-9.40 \mathrm{E}-02$ & 0.015627 & - & - \\
\hline Total 50 km/h Road Kilometres & $7.15 \mathrm{E}-02$ & 0.012871 & $-1.10 \mathrm{E}-01$ & 0.001594 & - & - \\
\hline Total 60 km/h Road Kilometres & - & - & - & - & - & - \\
\hline Total 70 km/h Road Kilometres & - & - & $-2.75 \mathrm{E}-01$ & 0.03545 & - & - \\
\hline Total 80 km/h Road Kilometres & - & - & - & - & - & - \\
\hline Total 90 km/h Road Kilometres & - & - & - & - & - & - \\
\hline Total 100 km/h Road Kilometres & $9.83 \mathrm{E}+02$ & 0.022663 & $1.33 \mathrm{E}+03$ & 0.016064 & - & - \\
\hline Total Bike Paths Kilometres & - & - & $2.32 \mathrm{E}-02$ & 0.034158 & $1.97 \mathrm{E}-02$ & 0.06686 \\
\hline VKT & $1.30 \mathrm{E}-05$ & 0.000108 & $-1.56 \mathrm{E}-06$ & 0.002389 & $1.25 \mathrm{E}-05$ & $2.54 \mathrm{E}-06$ \\
\hline TLKM & $-1.06 \mathrm{E}-01$ & 0.003101 & $1.09 \mathrm{E}-01$ & 0.001929 & $-5.36 \mathrm{E}-02$ & 0.00453 \\
\hline Density of Trips Enter Center & $-2.43 \mathrm{E}-05$ & $6.35 \mathrm{E}-07$ & $-1.41 \mathrm{E}-05$ & $9.90 \mathrm{E}-07$ & $-1.52 \mathrm{E}-05$ & $1.95 \mathrm{E}-06$ \\
\hline Density of Trips Out Center & $9.41 \mathrm{E}-05$ & 0.009585 & - & - & - & - \\
\hline Trips Attracted & - & - & - & - & $9.02 \mathrm{E}-06$ & 0.02033 \\
\hline Trips Produced & - & - & - & - & - & - \\
\hline
\end{tabular}


Table 5.18 Model Estimation Results of the Best Roadway-related CPMs for PDO Collisions

\begin{tabular}{|c|c|c|c|c|c|c|}
\hline \multirow{2}{*}{$\begin{array}{c}\text { PDO Models } \\
\text { Variable }\end{array}$} & \multicolumn{2}{|c|}{ Model $M-D-I I I$} & \multicolumn{2}{|c|}{ Model $A-D-I I I$} & \multicolumn{2}{|c|}{ Model $K-D-I I I$} \\
\hline & Estimate & $p$-value & Estimate & $p$-value & Estimate & $p$-value \\
\hline (Intercept) & $4.48 \mathrm{E}+00$ & $<2 \mathrm{E}-16$ & $4.45 \mathrm{E}+00$ & $<2 \mathrm{E}-16$ & $4.52 \mathrm{E}+00$ & $<2 \mathrm{E}-16$ \\
\hline Total Number of Intersections & $8.62 \mathrm{E}-03$ & 0.001219 & - & - & - & - \\
\hline Signalized Intersections & - & - & $1.68 \mathrm{E}-02$ & $2.26 \mathrm{E}-05$ & - & - \\
\hline Non-signalized Intersections & - & - & $6.79 \mathrm{E}-03$ & 0.000165 & - & - \\
\hline Total Arterial Road Kilometres & - & - & - & - & - & - \\
\hline Total Collector Road Kilometres & - & - & - & - & - & - \\
\hline Total Major Collector Road Kilometres & - & - & - & - & - & - \\
\hline Total Local Road Kilometres & - & - & - & - & $9.03 \mathrm{E}-02$ & $5.49 \mathrm{E}-05$ \\
\hline Total 40 km/h Road Kilometres & $1.16 \mathrm{E}-01$ & 0.00182 & $-8.51 \mathrm{E}-02$ & 0.026991 & - & - \\
\hline Total 50 km/h Road Kilometres & $7.36 \mathrm{E}-02$ & 0.009567 & $-1.02 \mathrm{E}-01$ & 0.003267 & - & - \\
\hline Total 60 km/h Road Kilometres & - & - & - & - & - & - \\
\hline Total 70 km/h Road Kilometres & - & - & $-2.78 \mathrm{E}-01$ & 0.031896 & - & - \\
\hline Total 80 km/h Road Kilometres & - & - & - & - & - & - \\
\hline Total 90 km/h Road Kilometres & - & - & - & - & - & - \\
\hline Total 100 km/h Road Kilometres & $9.59 \mathrm{E}+02$ & 0.024103 & $1.30 \mathrm{E}+03$ & 0.01697 & - & - \\
\hline Total Bike Paths Kilometres & - & - & $2.26 \mathrm{E}-02$ & 0.037758 & $1.94 \mathrm{E}-02$ & 0.0671 \\
\hline VKT & $1.26 \mathrm{E}-05$ & 0.000136 & $-1.46 \mathrm{E}-06$ & 0.004391 & $1.19 \mathrm{E}-05$ & $5.96 \mathrm{E}-06$ \\
\hline TLKM & $-1.07 \mathrm{E}-01$ & 0.002436 & $1.00 \mathrm{E}-01$ & 0.003923 & $-5.07 \mathrm{E}-02$ & 0.0067 \\
\hline Density of Trips Enter Center & $-2.39 \mathrm{E}-05$ & $1.44 \mathrm{E}-06$ & $-1.42 \mathrm{E}-05$ & $1.24 \mathrm{E}-06$ & $-1.50 \mathrm{E}-05$ & $3.27 \mathrm{E}-06$ \\
\hline Density of Trips Out Center & $9.15 \mathrm{E}-05$ & 0.011381 & - & - & - & - \\
\hline Trips Attracted & - & - & - & - & $8.49 \mathrm{E}-06$ & 0.0281 \\
\hline Trips Produced & - & - & - & - & - & - \\
\hline
\end{tabular}


Consistent results are obtained with regard to the density of average annual trips entering to the centroid of TAZs. It was found to have an inverse relationship with total and PDO collisions in all best fitted total and PDO CPMs. As the density of trips enter to the centroid of a TAZ increases, exposure of vehicle traffic would increase, which in turn increases the probability of being involved in a collision. It could be that the more urbanized TAZs (especially small TAZs) experience better traffic regulation than rural TAZs, which may result in an inverse relationship between collision frequency and the density of trips entering into the centroid in all models.

\subsubsection{Planning-related CPMs}

Table 5.19 and 5.20 present the best fitted CPMs for total and PDO collisions estimation results, respectively. In each table, the first three models $(K-D-V, A-C-V$ and $L-D-V)$ are developed considering planning variables in addition to exposure variables. These three models respectively were the best fitted CPMs for total and PDO collisions in all developed models. All input independent variables in these three models were the same, and obtained from the City of Ottawa database and included in the regression as they are received, except for total VKT in which boundary data was assigned using either equal-proportion-based assignment method (model $A-C-V$ ) or multiple-count-based assignment method (models $K-D-V$ and $L-D-V$ ). Collisions data in these models was assigned using equal-proportion-based assignment method for models $K-D-V$ and $A-C-V$ whereas collisions data in the model $L-D-V$ was assigned using population-based assignment method. The difference between models $K-D-V$ and $L-D-V$ and model $A-C-V$ is that counted exposure variables were considered in the model $A-C-V$ whereas the density of exposure variables were considered in models $K-D-V$ and $L-D-V$. Interestingly, the models' results of planning-related and exposure variables for total and PDO collisions showed 
similarity regarding variables affecting them. They vary in their variables' coefficient values due to the difference in their number. The variables' coefficient values of total collisions models were higher because the number of total collisions is higher.

As indicated earlier, model $K-D-V$ was the best fitted model. The model retains 10 independent variables where collision frequency was assessed in association with planning and exposure variables. It can be clearly seen that all independent variables in the model were significant at level of significance of $5 \%$. The model reveals that total collision frequency increases with the increase in the following explanatory variables: percentage of driver, passenger, transit, walking, and biking trips within TAZs, area of TAZs, percentage of age groups of 25-64 and 65 and above, and total VKT. However, the density of trips enter centroid of TAZs was found to be inversely related to total collision frequency.

As driver trips within a TAZ increase, it is expected that collisions would increase. In the model the modal split of driver trips was found to have high significance with a positive relation with collisions. The model also showed that the modal spilt of walking, transit, and passenger had a positive relationship with total collision as they affect exposure. It is intuitive that more walking activities would result in high exposure of pedestrians to vehicle traffic (such as crossing roadways). Total and PDO collisions tend to increase as the walking modal split increases.

Models $A-C-V$ and $L-D-V$ results revealed that total lane kilometre was positively related to total collision frequency at the level of significance of $5 \%$. This finding is reasonable and confirmed the intuitive expectations that the more travel, drivers, or roadway kilometres, the higher exposure, which in turns increases the probability of being involved in a collision.

It is found that TAZ's area was significant at $5 \%$ level of significance with a positive relation with total and PDO collisions. It could be argued that bigger TAZs (especially in the 
urban areas) would have larger communities (schools, shopping malls and other attractions...), which in turn may result in a high exposure and high level of vehicle conflicts, which may result in more collision occurrence. Also, the trips' length in big areas could be longer. This finding is reasonable since increasing total lane kilometre within a TAZ indicates increases exposure, which in turns increases collision frequency as explained earlier.

All best planning-related models revealed percentage of population age group 25-64 as a significant age group at the level of significance of 5\% affecting collisions. As the number of people in this age group within a TAZ increases, total and PDO collision frequency increases. This could be intuitive since most of drivers are from this age group. In the models $K-D-V$ and $L-D-V$, the percentage of population age group above 65 was found significant and have a positive relation with total and PDO collisions. This result is expected since people under this population age group are expected to have slower reaction time (weather as drivers or pedestrians) compared to other population groups. Therefore, as the percentage of population age group above 65 within a TAZ increases, the total and PDO collisions would increase. Conflicting results were obtained with respect to the percentage of population age group $0-4$, the total and PDO collisions models A-C-V indicate a decrease in collision frequency within a TAZ as the percentage of population age group 0-4 increase while PDO model $L-D-V$ indicated an increase in collision frequency within a TAZ as the percentage of population age group 0-4 increase. 
Table 5.19 Model Estimation Results of the Best Planning-related CPMs for Total Collisions

\begin{tabular}{|c|c|c|c|c|c|c|}
\hline \multirow{2}{*}{$\begin{array}{c}\text { Models } \\
\text { Variable }\end{array}$} & \multicolumn{2}{|c|}{ Model $K-D-V$} & \multicolumn{2}{|c|}{ Model $A-C-V$} & \multicolumn{2}{|c|}{ Model $L-D-V$} \\
\hline & Estimate & $p$-value & Estimate & $p$-value & Estimate & $p$-value \\
\hline (Intercept) & $2.56 \mathrm{E}+00$ & $<2 \mathrm{E}-16$ & $2.73 \mathrm{E}+00$ & $<2 \mathrm{E}-16$ & $2.43 \mathrm{E}-01$ & 0.52209 \\
\hline MS. Driver & $1.19 \mathrm{E}-02$ & $7.90 \mathrm{E}-06$ & $1.28 \mathrm{E}-02$ & $1.18 \mathrm{E}-06$ & $2.18 \mathrm{E}-02$ & $3.74 \mathrm{E}-07$ \\
\hline MS. Passenger & $3.49 \mathrm{E}-02$ & $7.41 \mathrm{E}-10$ & $2.74 \mathrm{E}-02$ & $1.28 \mathrm{E}-06$ & 4.87E-02 & $5.24 \mathrm{E}-09$ \\
\hline MS. Transit & $2.93 \mathrm{E}-02$ & $2.34 \mathrm{E}-10$ & $2.24 \mathrm{E}-02$ & $7.85 \mathrm{E}-06$ & $4.47 \mathrm{E}-02$ & $3.38 \mathrm{E}-09$ \\
\hline MS. Walk & $1.25 \mathrm{E}-02$ & 0.01081 & $1.93 \mathrm{E}-02$ & $5.36 \mathrm{E}-05$ & $2.16 \mathrm{E}-02$ & 0.006096 \\
\hline MS. Bike & $4.36 \mathrm{E}-02$ & 0.00853 & $4.16 \mathrm{E}-02$ & 0.01126 & - & - \\
\hline School & - & - & - & - & - & - \\
\hline Employment & - & - & - & - & $-1.14 \mathrm{E}-05$ & $1.52 \mathrm{E}-05$ \\
\hline Area & $8.50 \mathrm{E}-03$ & $7.05 \mathrm{E}-12$ & - & - & $9.24 \mathrm{E}-03$ & $4.65 \mathrm{E}-07$ \\
\hline Population & - & - & $6.51 \mathrm{E}-05$ & 0.00178 & - & - \\
\hline Age $0-4$ & - & - & $-3.01 \mathrm{E}-02$ & 0.06805 & - & - \\
\hline Age 5-24 & - & - & $-9.60 \mathrm{E}-03$ & 0.00458 & $1.66 \mathrm{E}-02$ & 0.000304 \\
\hline Age $25-64$ & $5.83 \mathrm{E}-03$ & 0.00146 & $9.66 \mathrm{E}-03$ & $5.08 \mathrm{E}-06$ & $1.82 \mathrm{E}-02$ & $1.24 \mathrm{E}-09$ \\
\hline Age above 65 & $1.24 \mathrm{E}-02$ & 0.02069 & - & - & $2.67 \mathrm{E}-02$ & 0.000679 \\
\hline VKT & $1.18 \mathrm{E}-05$ & $5.03 \mathrm{E}-14$ & $3.28 \mathrm{E}-06$ & $3.52 \mathrm{E}-05$ & $5.55 \mathrm{E}-06$ & 0.050196 \\
\hline TLKM & - & - & $5.23 \mathrm{E}-03$ & 0.00086 & $2.54 \mathrm{E}-02$ & 0.034198 \\
\hline Trips Out Centroid & - & - & - & - & - & - \\
\hline Trips Enter Centroid & - & - & - & - & - & - \\
\hline Density of Trips Enter Center & $-1.73 \mathrm{E}-05$ & $4.86 \mathrm{E}-12$ & $-1.03 \mathrm{E}-05$ & $7.13 \mathrm{E}-06$ & - & - \\
\hline Density of Trips Out Center & - & - & - & - & - & - \\
\hline Trips Attracted & - & - & $1.89 \mathrm{E}-04$ & $1.75 \mathrm{E}-10$ & - & - \\
\hline Trips Produced & - & - & - & - & - & - \\
\hline
\end{tabular}


Table 5.20 Model Estimation Results of the Best Planning-related CPMs for PDO Collisions

\begin{tabular}{|c|c|c|c|c|c|c|}
\hline Models & \multicolumn{2}{|c|}{ Model $K-D-V$} & \multicolumn{2}{|c|}{ Model $A-C-V$} & \multicolumn{2}{|c|}{ Model $L-D-V$} \\
\hline Variable & Estimate & $p$-value & Estimate & $p$-value & Estimate & $p$-value \\
\hline (Intercept) & $2.38 \mathrm{E}+00$ & $<2 \mathrm{E}-16$ & $2.58 \mathrm{E}+00$ & $<2 \mathrm{E}-16$ & $2.08 \mathrm{E}-02$ & $<2 \mathrm{E}-16$ \\
\hline MS. Driver & $1.14 \mathrm{E}-02$ & $2.03 \mathrm{E}-05$ & $7.84 \mathrm{E}-03$ & 0.00248 & $2.20 \mathrm{E}-02$ & $3.98 \mathrm{E}-07$ \\
\hline MS. Passenger & $3.47 \mathrm{E}-02$ & $7.32 \mathrm{E}-10$ & $2.80 \mathrm{E}-02$ & $3.41 \mathrm{E}-07$ & $4.86 \mathrm{E}-02$ & $3.75 \mathrm{E}-09$ \\
\hline MS. Transit & $2.78 \mathrm{E}-02$ & $1.59 \mathrm{E}-09$ & $2.10 \mathrm{E}-02$ & $3.15 \mathrm{E}-06$ & $4.28 \mathrm{E}-02$ & $9.79 \mathrm{E}-09$ \\
\hline MS. Walk & $1.35 \mathrm{E}-02$ & 0.005522 & $1.88 \mathrm{E}-02$ & $3.96 \mathrm{E}-05$ & $2.26 \mathrm{E}-02$ & 0.003657 \\
\hline MS. Bike & $4.56 \mathrm{E}-02$ & 0.005517 & $4.86 \mathrm{E}-02$ & 0.00196 & - & - \\
\hline School & - & - & $5.72 \mathrm{E}-02$ & 0.07659 & - & - \\
\hline Employment & - & - & $1.51 \mathrm{E}-04$ & $6.86 \mathrm{E}-14$ & $-1.13 \mathrm{E}-05$ & $1.33 \mathrm{E}-05$ \\
\hline Area & $8.36 \mathrm{E}-03$ & $9.65 \mathrm{E}-12$ & - & - & $9.14 \mathrm{E}-03$ & $3.21 \mathrm{E}-07$ \\
\hline Population & - & - & - & - & - & - \\
\hline Age 0-4 & - & - & $-3.78 \mathrm{E}-02$ & 0.01416 & $1.71 \mathrm{E}-02$ & 0.000136 \\
\hline Age 5-24 & - & - & - & - & $1.79 \mathrm{E}-02$ & $9.80 \mathrm{E}-10$ \\
\hline Age 25-64 & $5.98 \mathrm{E}-03$ & 0.000989 & $7.55 \mathrm{E}-03$ & $7.66 \mathrm{E}-05$ & $2.58 \mathrm{E}-02$ & 0.000776 \\
\hline Age above 65 & $1.20 \mathrm{E}-02$ & 0.024154 & - & - & - & - \\
\hline VKT & $1.16 \mathrm{E}-05$ & $1.14 \mathrm{E}-13$ & $3.13 \mathrm{E}-06$ & $3.30 \mathrm{E}-05$ & $4.86 \mathrm{E}-06$ & 0.079563 \\
\hline TLKM & - & - & $4.09 \mathrm{E}-03$ & 0.00756 & $2.82 \mathrm{E}-02$ & 0.016355 \\
\hline Trips Out Centroid & - & - & - & - & - & - \\
\hline Trips Enter Centroid & - & - & - & - & - & - \\
\hline Density of Trips Enter Center & $-1.73 \mathrm{E}-05$ & $7.84 \mathrm{E}-12$ & $-1.09 \mathrm{E}-05$ & $2.42 \mathrm{E}-06$ & - & - \\
\hline Density of Trips Out Center & - & - & $1.61 \mathrm{E}-04$ & $6.05 \mathrm{E}-06$ & - & - \\
\hline Trips Attracted & - & - & - & - & - & - \\
\hline Trips Produced & - & - & - & - & - & - \\
\hline
\end{tabular}


Population and employment (person counts) are expected to be significant variables in explaining the variation in collision frequency at TAZs' level. It is expected as more population within a TAZ should result in more trips, thus more exposure to conflicts or collisions. It was found that collisions occurrence within a TAZ was associated with increased number of employment within the TAZ. As the number of employment increases the collision frequency increases, the density of employment within a TAZ was found inversely related to collisions, which mean that the small TAZs with higher employment density indicate better safety performance than bigger TAZs with less employment. The model $A-C-V$ retained number of population as a significant variable. It was found that TAZs with higher number population experience relatively more collisions frequency.

The three best planning-related total and PDO CPMs showed consistent results with respect to total VKT and total lane kilometre (TLKM). As explained in the roadway-related CPMs, these findings are intuitive since increasing of both VKT and TLKM increases exposure, which in turns expected to increases collision frequency. Similar to roadway-related CPMs, the density of trips enter to the centroid of TAZs was significant and inversely related to collision.

\subsection{Non-fatal Injury Collisions Models}

\subsubsection{Roadway-related Non-fatal CPMs}

The complete estimation results for the final non-fatal injury collision negative binomial best fitted models are shown in Table 5.21. The model $(K-D-I I)$ for non-fatal injury collisions retained five variables, among which local roadways kilometres, bike paths, VKT, TLKM, density of trips enter TAZs centroid, and trips attracted to TAZs. Factors effecting non-fatal injury are almost similar to the factors effecting total and PDO collisions. For all three beat non- 
fatal injury collisions, all of the effecting factors were explained previously in the discussion of total and PDO collisions.

\subsubsection{Planning-related Non-fatal CPMs}

Table 5.22 present the best fitted CPMs for non-fatal injury collisions estimation results. Same as total and PDO models, the models $K-D-V, A-C-V$ and $L-D-V$ are the best fitted models. These models are developed considering planning and exposure variables. The best fitted model $K-D-V$ for non-fatal injury collisions retained nine variables, among which percentage of driver, passenger, transit, and walking trips within TAZs, area of TAZs, percentage of age groups of 2564 and 65 and above, and VKT. Density of trips inter to centroid of TAZs has an inverse relationship with non-fatal injury collisions. The model L-D-V retained almost the same variables as the model K-D-V.

The modal split of walking was found to have the highest significance in the non-fatal injury collisions model compared to other modal split variables. It is intuitive that more walking activities would result in higher exposure of pedestrians to vehicle traffic (such as crossing roadways). Non-fatal injury collisions tend to increase as the walking modal split increases. Similarly, it is very reasonable that the other modal split variables would have a positive significant relationship with pedestrian-involved collisions, since the walking part is required to reach the other modes of transportation (vehicle or public transport). Total VKT was found positively related to the non-fatal collisions at a level of significance of 5\%. As the VKT of vehicles increases, exposure of vehicle traffic would increase, which in turn increases the probability of being involved in non-fatal injury collision. 
Table 5.21 Model Estimation Results of the Best Roadway-related CPMs for Non-fatal Injury

\begin{tabular}{|c|c|c|c|c|c|c|}
\hline \multirow{2}{*}{$\begin{array}{c}\text { Models } \\
\text { Variable }\end{array}$} & \multicolumn{2}{|c|}{ Model $K-D-I I$} & \multicolumn{2}{|c|}{ Model $A-D-I I I$} & \multicolumn{2}{|c|}{ Model K:D-III } \\
\hline & Estimate & $p$-value & Estimate & $p$-value & Estimate & $p$-value \\
\hline (Intercept) & $3.19 \mathrm{E}+00$ & $<2 \mathrm{E}-16$ & $3.06 \mathrm{E}+00$ & $<2 \mathrm{E}-16$ & $3.25 \mathrm{E}+00$ & $<2 \mathrm{E}-16$ \\
\hline Total Number of Intersections & - & - & $6.31 \mathrm{E}-03$ & 0.000107 & - & - \\
\hline Signalized Intersections & - & - & - & - & - & - \\
\hline Non-signalized Intersections & - & - & - & - & - & - \\
\hline Total Arterial Road Kilometres & - & - & - & - & - & - \\
\hline Total Collector Road Kilometres & - & - & - & - & - & - \\
\hline Total Major Collector Road Kilometres & - & - & - & - & - & - \\
\hline Total Local Road Kilometres & $8.65 \mathrm{E}-02$ & 0.000194 & - & - & - & - \\
\hline Total 40 km/h Road Kilometres & - & - & $-1.38 \mathrm{E}-01$ & 0.000545 & $1.70 \mathrm{E}-01$ & 0.000177 \\
\hline Total 50 km/h Road Kilometres & - & - & $-1.46 \mathrm{E}-01$ & $4.82 \mathrm{E}-05$ & $1.39 \mathrm{E}-01$ & 0.001042 \\
\hline Total 60 km/h Road Kilometres & - & - & - & - & 9.81E-02 & 0.0319 \\
\hline Total 70 km/h Road Kilometres & - & - & $-2.59 \mathrm{E}-01$ & 0.054633 & - & - \\
\hline Total 80 km/h Road Kilometres & - & - & - & - & - & - \\
\hline Total 90 km/h Road Kilometres & - & - & - & - & - & - \\
\hline Total 100 km/h Road Kilometres & - & - & $1.40 \mathrm{E}+03$ & 0.011064 & $9.48 \mathrm{E}+02$ & 0.005793 \\
\hline Total Bike Paths Kilometres & $2.01 \mathrm{E}-02$ & 0.061316 & $2.56 \mathrm{E}-02$ & 0.020731 & $1.75 \mathrm{E}-02$ & 0.01002 \\
\hline VKT & $1.47 \mathrm{E}-05$ & $5.16 \mathrm{E}-08$ & $-1.71 \mathrm{E}-06$ & 0.005277 & $1.33 \mathrm{E}-05$ & $2.62 \mathrm{E}-07$ \\
\hline TLKM & $-6.35 \mathrm{E}-02$ & 0.00108 & $1.45 \mathrm{E}-01$ & $5.54 \mathrm{E}-05$ & $-1.49 \mathrm{E}-01$ & 0.001127 \\
\hline Density of Trips Enter Center & $-1.81 \mathrm{E}-05$ & $3.49 \mathrm{E}-05$ & $-1.54 \mathrm{E}-05$ & $1.07 \mathrm{E}-04$ & $-1.81 \mathrm{E}-05$ & $9.80 \mathrm{E}-09$ \\
\hline Density of Trips Out Center & - & - & - & - & - & - \\
\hline Trips Attracted & $1.18 \mathrm{E}-05$ & 0.005613 & $7.83 \mathrm{E}-06$ & 0.039639 & - & - \\
\hline Trips Produced & - & - & - & - & - & - \\
\hline
\end{tabular}


Table 5.22 Model Estimation Results of the Best Planning-related CPMs for Non-fatal Injury

\begin{tabular}{|c|c|c|c|c|c|c|}
\hline \multirow{2}{*}{$\begin{array}{c}\text { Models } \\
\text { Variable }\end{array}$} & \multicolumn{2}{|c|}{ Model $K-D-V$} & \multicolumn{2}{|c|}{ Model $A-C-V$} & \multicolumn{2}{|c|}{ Model $A-D-V$} \\
\hline & Estimate & $p$-value & Estimate & $p$-value & Estimate & $p$-value \\
\hline (Intercept) & $1.07 \mathrm{E}+00$ & $3.42 \mathrm{E}-06$ & $1.09 \mathrm{E}+00$ & $4.49 \mathrm{E}-05$ & $1.05 \mathrm{E}+00$ & 0.000209 \\
\hline MS. Driver & $1.13 \mathrm{E}-02$ & $1.20 \mathrm{E}-05$ & $1.07 \mathrm{E}-02$ & 0.00049 & $1.58 \mathrm{E}-02$ & $7.35 \mathrm{E}-07$ \\
\hline MS. Passenger & $3.03 \mathrm{E}-02$ & $1.48 \mathrm{E}-07$ & $2.86 \mathrm{E}-02$ & $1.87 \mathrm{E}-06$ & $3.31 \mathrm{E}-02$ & $1.31 \mathrm{E}-07$ \\
\hline MS. Transit & $3.46 \mathrm{E}-02$ & $1.44 \mathrm{E}-12$ & $3.12 \mathrm{E}-02$ & $5.46 \mathrm{E}-10$ & $4.03 \mathrm{E}-02$ & $4.16 \mathrm{E}-15$ \\
\hline MS. Walk & - & - & $1.47 \mathrm{E}-02$ & 0.003201 & $1.83 \mathrm{E}-02$ & 0.000605 \\
\hline MS. Bike & $3.51 \mathrm{E}-02$ & 0.03268 & $3.18 \mathrm{E}-02$ & 0.052733 & $2.96 \mathrm{E}-02$ & 0.095568 \\
\hline School & - & - & $7.84 \mathrm{E}-02$ & 0.019163 & - & - \\
\hline Employment & - & - & $1.46 \mathrm{E}-04$ & $1.85 \mathrm{E}-11$ & - & - \\
\hline Area & $8.64 \mathrm{E}-03$ & $1.24 \mathrm{E}-12$ & - & - & $7.29 \mathrm{E}-03$ & $1.80 \mathrm{E}-08$ \\
\hline Population & - & - & - & - & - & - \\
\hline Age 0-4 & - & - & $-3.95 \mathrm{E}-02$ & 0.015092 & $-2.70 \mathrm{E}-02$ & 0.111159 \\
\hline Age 5-24 & - & - & - & - & - & - \\
\hline Age 25-64 & $5.55 \mathrm{E}-03$ & 0.00198 & $6.91 \mathrm{E}-03$ & 0.000512 & $5.01 \mathrm{E}-03$ & 0.044387 \\
\hline Age above 65 & $1.38 \mathrm{E}-02$ & 0.01025 & - & - & $1.05 \mathrm{E}-02$ & 0.068949 \\
\hline VKT & $1.36 \mathrm{E}-05$ & $<2 \mathrm{E}-16$ & $3.38 \mathrm{E}-06$ & $1.07 \mathrm{E}-05$ & - & - \\
\hline TLKM & - & - & $4.76 \mathrm{E}-03$ & 0.002416 & - & - \\
\hline Trips Out Centroid & - & - & $1.04 \mathrm{E}-04$ & 0.004938 & - & - \\
\hline Trips Enter Centroid & - & - & - & - & - & - \\
\hline Density of Trips Enter Center & $-2.04 \mathrm{E}-05$ & $6.82 \mathrm{E}-11$ & $-1.44 \mathrm{E}-05$ & $2.22 \mathrm{E}-06$ & $-1.09 \mathrm{E}-05$ & $2.03 \mathrm{E}-05$ \\
\hline Density of Trips Out Center & - & - & - & - & - & - \\
\hline Trips Attracted & - & - & - & - & - & - \\
\hline Trips Produced & - & - & - & - & - & - \\
\hline
\end{tabular}


The model $A-C-V$ showed that total number of schools positively affects non-fatal injury collisions in. It is expected that more schools within a TAZ would result in higher pedestrian activates; thus more pedestrian exposure to vehicle traffic, which in turns increases the probability of non-fatal injury collisions. The model $A-C-V$ also indicted that total lane and number of employment are positively related to non-fatal injury collision frequency at the level of significance of $5 \%$. This finding is reasonable since increasing total lane kilometre within a TAZ increases exposure, which in turns increases collision frequency as explained in total collision models. Moreover, employment centres experience a greater number of collisions than the residential areas. The increase in non-fatal collisions within TAZs with high number of employment could be attributed to the higher traffic volume and more congestion at employment in urban areas.

Population age groups have been recognized as significant factors in collisions frequency. The best three fitted non-fatal injury collisions models show consistent results with respect of the percentage of age group 25-64. As the percentage of this age group within a TAZ increase, nonfatal injury collisions increase. It is expected that as the percentage of age group 5-24 increases, the non-fatal injury collision frequency within a TAZ increases. Surprisingly, this variable was not retained in any of the best three fitted models. With regard to the elderly population (age 65 and above), the results show that an increase in the percentage of elderly population within a TAZ leads to increase the non-fatal injury collisions. This finding is expected since elderly population have slower reaction time compared to other groups. 


\subsection{Bike-Involved Collision Models}

\subsubsection{Roadway-related CPMs}

The best three fitted bike-involved CPMs together with their statistic summary are presented in Table 5.23. Model results revealed intuitive relationships between bike-involved collisions and explanatory variables. The model $A-C-I I I$ and $M-C-I I I$ appeared that more bus stops within a TAZ would generate more conflicts between bikes and buses. Furthermore, at some locations, (where the bike lanes are on the right side of the roadway) when a bus stops, cyclists may use the motor vehicle lane to overpass the bus resulting in a potential bike-vehicle conflict particularly at heavy traffic conditions. Similarly, increased number or density of signalized intersections within a TAZ would result in higher number of bike-involved collisions. This is an intuitive expected result since the traffic characteristics at intersections, e.g., left movement at green through, right movement at red ..., are complex which generate more conflicts. Furthermore, the speed differential between bikes and other vehicles may lead to higher bike-involved collisions frequency and severity

In all best fitted roadway-related models for the bike-involved collisions, it was found that (TLKM) and vehicle kilometre travelled (VKT) were not the leading exposure variables in bikeinvolved collisions occurrence when considering only the roadways-related variables. These variables are dropped through the stepwise regression because they did not meet the $5 \%$ level of significance. This may be counterintuitive because as the total roadway length within a TAZ increases, exposure of cyclist to vehicle traffic is expected to increase. Therefore, conflicts would likely increase. However, consistent results were obtained in all best models with respect to the roadways length with different speed limits. Roadways with speed limits of 40,50 and $60 \mathrm{~km} / \mathrm{h}$ most likely exist on areas, e.g., local areas, with high bike-vehicle interaction. Lower speeds 
could be an important factor in reducing bike-involved collisions as there is more time to react at lower speeds. However, it was found that these variables would have a positive relation with bike-involved collisions. It could be that high traffic volumes on these roadways play an important role in bike-vehicle conflicts.

From the data, it is known that only roughly 25 and $40 \%$ of the TAZs in Ottawa actually have any roadways where the speed limit is 70 and $80 \mathrm{~km} / \mathrm{h}$, respectively. This indicates that 70 and $80 \mathrm{~km} / \mathrm{h}$ speed limits are used sparingly and therefore are likely only in TAZs that have suburban arterial roadways build to a modern standard, or that have rural roadways that operate with reduced speed limits (expected would be $90 \mathrm{~km} / \mathrm{h}$ ). These conditions exist in many of the areas that are already not experiencing any pedestrian or cyclist collisions. Furthermore, the characteristics of a TAZ that does not have either these suburban facilities or a rural nature are those of an urban TAZ. In urban TAZs, one is unlikely to find 70 and $80 \mathrm{~km} / \mathrm{h}$ speed limits, but is likely to find a lot of pedestrian and cyclist activity. The presence of $70,80 \mathrm{~km} / \mathrm{h}$ speed limits roadways in a TAZ were an indicator of safety for cyclists. Roadways with a posted speed limit of $90 \mathrm{~km} / \mathrm{h}$ are expected to have high bikes collisions, if bikes are allowed to use these roadways. As discussed earlier the speed differential between bikes and other vehicles is significantly high. However, roadways with a posted speed of $90 \mathrm{~km} / \mathrm{h}$ were found negatively associated with bikeinvolved collisions. The roadways with posted speed limits $90 \mathrm{~km} / \mathrm{h}$ may not be the best indicator of bike-involved collisions since only four TAZs had roadway/s with a posted speed limit of $90 \mathrm{~km} / \mathrm{h}$ and the bike modal split in these TAZs is roughly between 0 to $1 \%$. 
Table 5.23 Model Estimation Results of the Best Roadway-related CPMs for Bike-involved Collisions

\begin{tabular}{|c|c|c|c|c|c|c|}
\hline \multirow{2}{*}{$\begin{array}{c}\text { Bike Models } \\
\text { Variable }\end{array}$} & \multicolumn{2}{|c|}{ Model $K-C-I I I$} & \multicolumn{2}{|c|}{ Model $A-C-I I$} & \multicolumn{2}{|c|}{ Model $K-C-I I I$} \\
\hline & Estimate & $p$-value & Estimate & $p$-value & Estimate & $p$-value \\
\hline (Intercept) & $-4.02 \mathrm{E}-01$ & $2.31 \mathrm{E}-05$ & $-4.59 \mathrm{E}-01$ & $9.57 \mathrm{E}-07$ & $-6.47 \mathrm{E}-01$ & $6.02 \mathrm{E}-09$ \\
\hline Total Number of Intersections & - & - & - & - & - & - \\
\hline Signalized Intersections & $2.43 \mathrm{E}-01$ & $<2 \mathrm{E}-16$ & $2.39 \mathrm{E}-01$ & $<2 \mathrm{E}-16$ & $1.68 \mathrm{E}-01$ & $<2 \mathrm{E}-16$ \\
\hline Non-signalized Intersections & - & - & - & - & $-5.88 \mathrm{E}-03$ & 0.092771 \\
\hline Total Number of Bus Stops & - & - & $1.12 \mathrm{E}-02$ & 0.00517 & $8.42 \mathrm{E}-03$ & 0.068183 \\
\hline Total Arterial Road Kilometres & - & - & - & - & - & - \\
\hline Total Collector Road Kilometres & - & - & - & - & - & - \\
\hline $\begin{array}{c}\text { Total Major Collector Road } \\
\text { Kilometres }\end{array}$ & - & - & - & - & - & - \\
\hline Total Local Road Kilometres & - & - & - & - & - & - \\
\hline Total 40 km/h Road Kilometres & $3.07 \mathrm{E}-02$ & 0.0068 & - & - & $3.13 \mathrm{E}-02$ & 0.0505 \\
\hline Total 50 km/h Road Kilometres & $4.56 \mathrm{E}-02$ & $2.63 \mathrm{E}-06$ & $1.26 \mathrm{E}-02$ & 0.13025 & $5.17 \mathrm{E}-02$ & 0.001347 \\
\hline Total 60 km/h Road Kilometres & $3.46 \mathrm{E}-02$ & 0.09213 & - & - & - & - \\
\hline Total 70 km/h Road Kilometres & $-2.30 \mathrm{E}-01$ & $4.55 \mathrm{E}-05$ & $-4.15 \mathrm{E}-01$ & $3.24 \mathrm{E}-07$ & $-2.53 \mathrm{E}-01$ & 0.000128 \\
\hline Total 80 km/h Road Kilometres & $-5.91 \mathrm{E}-03$ & 0.08306 & - & - & - & - \\
\hline Total 90 km/h Road Kilometres & $-5.28 \mathrm{E}-01$ & 0.18706 & $-8.29 \mathrm{E}-01$ & 0.46237 & $-3.54 \mathrm{E}-01$ & 0.111407 \\
\hline Total $100 \mathrm{~km} / \mathrm{h}$ Road Kilometres & - & - & - & - & - & - \\
\hline Total Bike Paths Kilometres & - & - & - & - & - & - \\
\hline $\mathrm{VKT}$ & - & - & - & - & - & - \\
\hline TLKM & - & - & - & - & - & - \\
\hline Trips Out Centroid & $-1.14 \mathrm{E}-04$ & 0.03071 & - & - & - & - \\
\hline Trips Enter Centroid & - & - & - & - & $1.07 \mathrm{E}-04$ & $7.67 \mathrm{E}-05$ \\
\hline Density of Trips Enter Center & $-1.48 \mathrm{E}-05$ & 0.00282 & $-1.24 \mathrm{E}-05$ & 0.00783 & $-1.24 \mathrm{E}-05$ & 0.017498 \\
\hline Density of Trips Out Center & $1.33 \mathrm{E}-04$ & $2.00 \mathrm{E}-08$ & $1.22 \mathrm{E}-04$ & 4.10E-08 & $1.46 \mathrm{E}-04$ & $5.42 \mathrm{E}-09$ \\
\hline
\end{tabular}




\subsubsection{Planning-related CPMs}

The regression results of the best planning-based CPMs for bike-involved collisions are presented in Table 5.24. Models' results revealed that all variables are statistically significant at a level of significance of $5 \%$. The model results also revealed that bike-involved collisions increase as employment and population increase. This is could be expected since some employment chose biking as their mean of transportation to get to workplaces.

In the developed model schools were found to be significant at a level of significance slightly higher than 5\%. It is expected that presence school close enough to residential area would encourage people to commute using bicycles. Therefore, more schools in a TAZ would result in higher pedestrian and biking activates; thus more cyclist exposure to vehicle traffic. It was found that the total lane kilometres has a positive relationship with the bike-involved collisions occurrence in model K-D-V. This makes an intuitive sense because as the total roadway length within a TAZ increases, exposure of cyclist to vehicle traffic increases. Therefore, conflicts would likely increase.

The modal split of biking was found to have a high significance in the bike-involved collisions model. It is intuitive that more biking activities would result in higher exposure of cyclists to vehicle traffic. TAZs with more biking activates tend to have more collisions. Similarly, it is very reasonable that the other modal split variables would have a positive significant relationship with bike-involved collisions, since the biking part is sometimes preferred to reach the other modes of transportation (vehicle or public transport). 
Table 5.24 Model Estimation Results of the Best Planning-related CPMs for Bike-involved Collisions

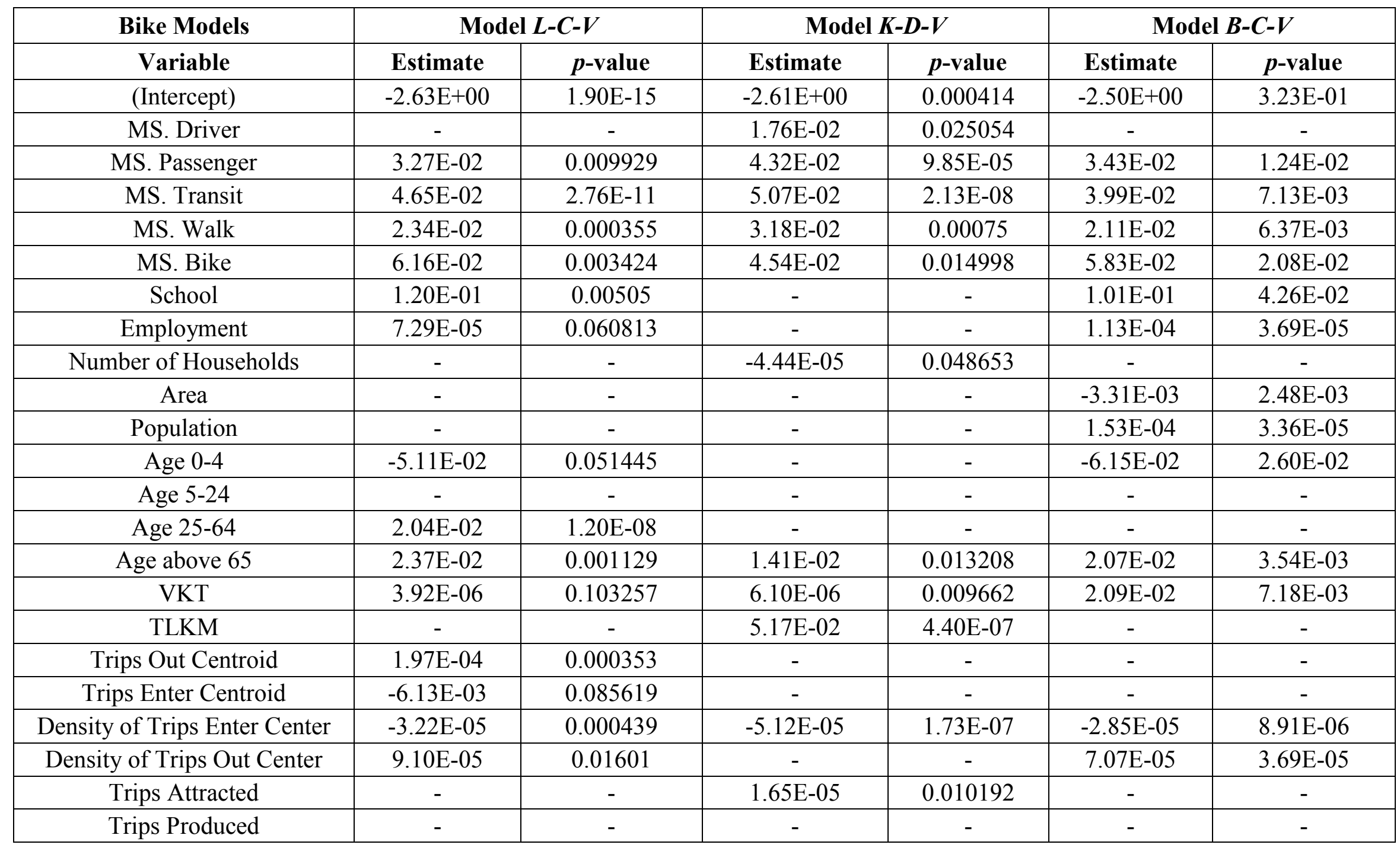


The bicycle is used by many households for short trips to shops and in some case for leisure purposes. For some people, biking is a common way for going to work. However, the model K-D-V revealed that as the number of households increase within a TAZ the number of bike-involved collisions would decrease. This might be reasonable since the percentage of bike trips varies between 0 to $17.5 \%$ of the total trips within each TAZ. The 2005 Origin-Destination Survey by TRANS Canada indicates that only $1.3 \%$ of all daily trips in the National Capital Region are made by bicycle, which indicates that people within the city of Ottawa TAZs tend to use other modes of transportation than bikes.

As indicted earlier, population age groups have been widely recognized as a significant factors in collision occurrence. The best fitted CPMs results showed that an increase in the percentage of elderly population within a TAZ leads to increase the bike-involved collisions. This finding is expected since elderly population have slower reaction time compared to other groups. Also, increase the percentage of population age group 0-4 would lead decrease the bikeinvolved collisions within a TAZ. This result could be intuitive since people within this age group do not bike. The percentage age group 25-64 was found to be positively related to bikeinvolved collisions in model L-C-V. As expected the coefficients of age groups of 25-64 would be less than the other groups older than 65 because of these groups' faster reaction time compared to other groups.

As expected, the model indicates that as the VKT increases, so does the number of bikeinvolved collisions. As explained earlier, this result in accord with intuition that as the VKT within a TAZ increases, exposure of cyclist to vehicle traffic is expected to increase. Therefore, conflicts would likely increase. Average annual trips entering to the centroid of TAZs were consistently determined to be a significant variable in the analysis. Whether the models 
considered their number or density, there was a negative relationship between the average annual trips entering to the centroid and bike-involved collisions. On the contrary, bike-involved collisions are associated positively with the average annual trips exiting from the centroid of a TAZ. This may indicate that drivers from other TAZs mostly use the arterials or major collectors where there is less bike activates.

\subsection{Pedestrian-Involved Collision Models}

\subsubsection{Roadway-related CPMs}

The best three fitted pedestrian-involved CPMs together with their statistic summary are presented in Table 5.25. In models $A-D-I I I$ and $M-D-I I I$ Bus stops were found to have a positive significant relationship with pedestrian-involved collisions. It is intuitive that pedestrian and transit users activities would increase at bus stop locations, which may increase conflicts between pedestrians and vehicles. Also, this variable may be used to indicate the level of violation that may occur at bus stop locations. Similarly, as the density of signalized and nonsignalized intersection increases the probability of a pedestrian being involved in a collision increases. For example, a conflict with crossing pedestrians at a signalized intersection may occur when a vehicle performs a right turn at green.

Roadways with speed limits of 40 and $50 \mathrm{~km} / \mathrm{h}$ most likely exist on areas, e.g., local areas, with high pedestrian-vehicle interaction. Lower speeds could be an important factor in reducing bike-involved collisions as there is more time to react at lower speeds. However, it was found that these variables would have a positive relation with bike-involved collisions. It could be that the traffic intensity on these roadways plays an important role in bike-vehicle conflicts. Another result was that the numbers of kilometres of roadways with a 60 and $70 \mathrm{~km} / \mathrm{h}$ speed 
limit were a strongly significant variable in the model developed. They were positively related to pedestrian-involved collisions. Roadway with speed limits of $60,70 \mathrm{~km} / \mathrm{h}$. This indicates that as the number of kilometres of roadways with a 60 and $70 \mathrm{~km} / \mathrm{h}$ speed limit in a TAZ increases, the expected number of pedestrian-involved collisions decrease. It is expected that such roadway would experience low pedestrian activities. From the data, roughly $7.65 \%$ and $22 \%$ of the TAZs in Ottawa have roadways where the speed limit is 60 and $70 \mathrm{~km} / \mathrm{h}$, respectively. This indicates that 60 and $70 \mathrm{~km} / \mathrm{h}$ speed limits are used carefully and therefore are likely only in TAZs that have suburban arterial roads. In urban TAZs, one is unlikely to find 60 and $70 \mathrm{~km} / \mathrm{h}$ speed limits, but is likely to find a lot of pedestrian and cyclist activity.

Marked bike paths on the side of roadways may reduce conflicts between bikes and pedestrian flow. A bike path indicates a clear separation between bike routes and pedestrian routes. It was found that as the length of bike paths increase, pedestrian involved collisions increase. It may be that the bike-vehicles conflicts plays important role in increasing pedestrianinvolved collisions. For example, a bike-vehicles conflict may occur at a signalized intersection while pedestrians waiting for green light. The vehicle or the bike may hit the pedestrians after the collisions between the vehicle and bike happened.

Model G-D-II revealed that major collector roadways inversely related to pedestrianinvolved collisions. Such roadway may not be designed for pedestrian activities. Also, major collector roadways may not be the best indicator of pedestrian-involved collisions since they only form $1.5 \%$ of the total roadways in Ottawa TAZs. Collector roadways are found to have positive relationship with pedestrian-involved collisions. This might be due to their heavy pedestrian and vehicle traffic. 
Table 5.25 Model Estimation Results of the Best Roadway-related CPMs for Pedestrian-involved Collisions

\begin{tabular}{|c|c|c|c|c|c|c|}
\hline \multirow{2}{*}{$\begin{array}{c}\text { Models } \\
\text { Variable }\end{array}$} & \multicolumn{2}{|c|}{ Model $A-D-I I I$} & \multicolumn{2}{|c|}{ Model M-D-III } & \multicolumn{2}{|c|}{ Model $G-D-I I$} \\
\hline & Estimate & $p$-value & Estimate & $p$-value & Estimate & $p$-value \\
\hline (Intercept) & $3.73 \mathrm{E}-01$ & 0.000474 & $3.35 \mathrm{E}-01$ & 0.004393 & $-5.40 \mathrm{E}-02$ & 0.58425 \\
\hline Total Number of Intersections & $1.60 \mathrm{E}-02$ & 4.79E-12 & - & - & $1.83 \mathrm{E}-02$ & $3.72 \mathrm{E}-11$ \\
\hline Signalized Intersections & - & - & $9.41 \mathrm{E}-03$ & 0.007778 & $1.83 \mathrm{E}-02$ & $3.72 \mathrm{E}-11$ \\
\hline Non-signalized Intersections & - & - & $1.34 \mathrm{E}-02$ & $3.27 \mathrm{E}-05$ & - & - \\
\hline Total Number of Bus Stops & $1.19 \mathrm{E}-02$ & 0.000725 & - & - & $2.53 \mathrm{E}-02$ & $2.90 \mathrm{E}-09$ \\
\hline Total Arterial Road Kilometres & - & - & - & - & - & - \\
\hline Total Collector Road Kilometres & - & - & - & - & $1.02 \mathrm{E}-01$ & 0.07866 \\
\hline $\begin{array}{l}\text { Total Major Collector Road } \\
\text { Kilometres }\end{array}$ & - & - & - & - & $-2.35 \mathrm{E}-01$ & 0.03988 \\
\hline Total Local Road Kilometres & - & - & - & - & - & - \\
\hline Total 40 km/h Road Kilometres & $4.37 \mathrm{E}-01$ & 0.000407 & $5.09 \mathrm{E}-01$ & $1.07 \mathrm{E}-07$ & - & - \\
\hline Total 50 km/h Road Kilometres & 4.19E-01 & 0.000684 & $4.72 \mathrm{E}-01$ & $4.47 \mathrm{E}-07$ & - & - \\
\hline Total 60 km/h Road Kilometres & $4.73 \mathrm{E}-01$ & 0.000156 & $4.06 \mathrm{E}-01$ & $1.69 \mathrm{E}-05$ & - & - \\
\hline Total 70 km/h Road Kilometres & $2.75 \mathrm{E}-01$ & 0.177961 & $3.16 \mathrm{E}-01$ & 0.02382 & - & - \\
\hline Total 80 km/h Road Kilometres & - & - & - & - & - & - \\
\hline Total 90 km/h Road Kilometres & - & - & - & - & - & - \\
\hline Total $100 \mathrm{~km} / \mathrm{h}$ Road Kilometres & - & - & - & - & - & - \\
\hline Total Bike Paths Kilometres & $3.15 \mathrm{E}-02$ & 0.001893 & $3.22 \mathrm{E}-02$ & 0.000789 & $8.89 \mathrm{E}-03$ & 0.06234 \\
\hline VKT & - & - & $1.14 \mathrm{E}-05$ & 0.001532 & - & - \\
\hline TLKM & $-4.21 \mathrm{E}-01$ & 0.000639 & $-4.45 \mathrm{E}-01$ & $4.43 \mathrm{E}-06$ & $1.58 \mathrm{E}-02$ & 0.03346 \\
\hline Trips Attracted & $6.07 \mathrm{E}-05$ & 0.009025 & - & - & $1.42 \mathrm{E}-05$ & 0.04325 \\
\hline Trips Produced & $6.52 \mathrm{E}-06$ & 0.139319 & - & - & - & - \\
\hline Density of Trips Enter Center & $-1.23 \mathrm{E}-05$ & 0.022498 & $-9.25 \mathrm{E}-06$ & 0.034449 & $-2.61 \mathrm{E}-05$ & 0.00609 \\
\hline Density of Trips Out Center & - & - & - & - & - & - \\
\hline
\end{tabular}


Trips attracted and produced from other TAZs were found to be significant at a level of significance of 5\%. They have an inverse relationship with pedestrian-involved collisions which indicates that the trips attracted and produced usually use major roadways that involve low pedestrian activities or low pedestrian-vehicle interaction. Density of trips entering centroid of TAZs is negatively related to pedestrian-involved collisions. As the density of trips entering centroid increase, pedestrian-involved collisions decrease.

\subsubsection{Planning-related CPMs}

The regression results of the best planning-based CPMs for bike-involved collisions are presented in Table 5.26. Models' results revealed that all variables are statistically significant at a level of significance of $5 \%$. The modal split of walking was found to have a high significance in the pedestrian-involved collisions model. It is intuitive that more walking activities would result in higher exposure of pedestrians to vehicle traffic (such as crossing roadways). Pedestrianinvolved collisions tend to increase as the walking modal split increases. Similarly, it is very reasonable that the other modal split variables would have a positive significant relationship with pedestrian-involved collisions, since the walking part is required to reach the other modes of transportation (vehicle or public transport).

More schools in a TAZ would result in higher pedestrian activates; thus more pedestrian exposure to vehicle traffic. It is expected that high pedestrian (students, teachers and parents) roadway crossing activates to take place nearby schools. In all best fitted planning-related CPMs (Table 5.26) for the pedestrian-involved collisions, it was found that presence of schools is not significant factor. This variable was dropped through the stepwise regression because they did not meet the $5 \%$ level of significance. 
All best models revealed that increased the percentage of population age groups $0-4$ and older than 65 were associated with an increased number of pedestrian-involved collisions. The second best model showed all population age groups are significant. This result may indicate a homogenous mixture of all groups within the pedestrian activities in TAZs. Density of average annual trips exiting from centroid and trips produced from TAZs were found significant at a level of significance of 5\%. It was found that they have an inverse relationship with pedestrianinvolved collisions which indicates that the trips produced usually use major roadways that involve low pedestrian activities or low pedestrian-vehicle interaction.

It was found also that TAZ's area is significant with a negative relationship with pedestrian involved collisions. It could be argued that bigger TAZs would have large communities (schools, shopping malls and other attractions...), which in turn have a high exposure of pedestrians to vehicle traffic resulting in more pedestrian-collisions. But this was not the case where as the area of a TAZ increase, the probability of pedestrian being involved in a collision decrease. As total vehicle kilometre traveled (VKT), and TLKM increase, bike-involved collisions increase. Mostly only serious bike-involved collisions which happened at rural areas, where vehicles speeds are higher compared to urban areas, are reported. 
Table 5.26 Model Estimation Results of the Best Planning-related CPMs for Pedestrian-involved Collisions

\begin{tabular}{|c|c|c|c|c|c|c|}
\hline \multirow{2}{*}{$\begin{array}{c}\text { Models } \\
\text { Variable }\end{array}$} & \multicolumn{2}{|c|}{ Model $K-D-V$} & \multicolumn{2}{|c|}{ Model $K-D-I V$} & \multicolumn{2}{|c|}{ Model $L-D-V$} \\
\hline & Estimate & $p$-value & Estimate & $p$-value & Estimate & $p$-value \\
\hline (Intercept) & $-2.63 \mathrm{E}+00$ & 0.000338 & $-2.14 \mathrm{E}+00$ & 0.000518 & $-2.68 \mathrm{E}+00$ & $<2 \mathrm{E}-16$ \\
\hline MS. Driver & $1.38 \mathrm{E}-02$ & 0.071986 & $1.12 \mathrm{E}-02$ & 0.094886 & - & - \\
\hline MS. Passenger & $6.16 \mathrm{E}-02$ & $4.02 \mathrm{E}-09$ & $6.13 \mathrm{E}-02$ & $1.98 \mathrm{E}-10$ & $4.97 \mathrm{E}-02$ & $4.43 \mathrm{E}-06$ \\
\hline MS. Transit & $6.10 \mathrm{E}-02$ & $4.37 \mathrm{E}-12$ & $6.01 \mathrm{E}-02$ & $8.93 \mathrm{E}-14$ & $5.68 \mathrm{E}-02$ & $5.62 \mathrm{E}-15$ \\
\hline MS. Walk & $3.82 \mathrm{E}-02$ & $9.50 \mathrm{E}-06$ & $4.10 \mathrm{E}-02$ & $6.65 \mathrm{E}-07$ & $2.40 \mathrm{E}-02$ & 0.000371 \\
\hline MS. Bike & $2.90 \mathrm{E}-02$ & 0.119566 & - & - & - & - \\
\hline School & - & - & - & - & - & - \\
\hline Employment & - & - & - & - & $-1.14 \mathrm{E}-05$ & $3.06 \mathrm{E}-06$ \\
\hline Number of Households & - & - & - & - & - & - \\
\hline Area & $-3.83 \mathrm{E}-03$ & 0.156191 & & & $-4.03 \mathrm{E}-03$ & 0.191883 \\
\hline Population & - & - & - & - & - & - \\
\hline Age $0-4$ & $3.77 \mathrm{E}-02$ & 0.025258 & - & - & $4.86 \mathrm{E}-02$ & 0.060101 \\
\hline Age 5-24 & - & - & - & - & $8.01 \mathrm{E}-03$ & 0.079825 \\
\hline Age $25-64$ & - & - & - & - & $1.08 \mathrm{E}-02$ & 0.003165 \\
\hline Age above 65 & $1.90 \mathrm{E}-02$ & 0.000485 & - & - & $3.40 \mathrm{E}-02$ & $8.30 \mathrm{E}-06$ \\
\hline VKT & $8.59 \mathrm{E}-06$ & 0.000139 & $5.77 \mathrm{E}-06$ & 0.007559 & & \\
\hline TLKM & $4.55 \mathrm{E}-02$ & $8.63 \mathrm{E}-07$ & $5.82 \mathrm{E}-02$ & 9.33E-09 & $7.52 \mathrm{E}-02$ & 4.94E-16 \\
\hline Trips Out Centroid & - & - & - & - & - & - \\
\hline Trips Enter Centroid & - & - & - & - & - & - \\
\hline Density of Trips Enter Center & $-2.03 \mathrm{E}-05$ & 4.88E-08 & $-3.09 \mathrm{E}-05$ & $9.61 \mathrm{E}-11$ & - & - \\
\hline Density of Trips Out Center & - & - & $5.67 \mathrm{E}-05$ & 0.061954 & - & - \\
\hline Trips Attracted & - & - & - & - & - & - \\
\hline Trips Produced & - & - & $-4.69 \mathrm{E}-05$ & 0.049924 & - & - \\
\hline
\end{tabular}




\subsection{Fatal Collisions}

The fatal collision frequencies distribution histogram (Figure 3.14) clearly illustrates that there are excessive zeros (over 75\%) in the collision data. Therefore, it strongly supports that the collision data does not follow the normal distribution. Therefore, the ZINB models are suitable to the collision count data regression analyses. There was an attempt to relate fatal collisions to all possible variables' combination identified in Section 4.5, but this was it was not possible due to very much information to explain rare events. The logarithm in $\mathrm{R}$ could not estimate the parameter estimate of the variables effecting fatal collisions. Therefore, it was decided to relate fatal collisions only to logarithmic transformation of two zonal level exposure variables which are VKT and TLKM. ZINB was applied to estimate the parameter coefficient in all datasets. The ZINB results were compared negative binomial results through voung test. As indicated in Section 4.1.6 a level of significance is usually set in the Vuong test for model comparisons. As explained at level of significance of $5 \%$, the $V_{\text {critical }}$ is set to be equal 1.96. A value greater than 1.96 for the Vuong test favours the ZINB model whereas a value less than -1.96 the negative binomial (NB) is considered to be better for modeling collision frequency, and a value between 1.96 and -1.96 indicate that the test is inconclusive. As shown in Table 5.27, the Vuong test statistic was significant and the values between ZINB and NB for the best ten developed models is more than $V_{\text {critical }}$ confirming that the ZINB model is better than the NB for modeling zero count data such as fatal collisions. 
Table 5.27 Ranking Based on Goodness of Fit Measures for Fatal CPMs

\begin{tabular}{|c|c|c|c|c|c|c|c|c|c|c|}
\hline Model & MPB & MAD & MSPE & RMSE & $\mathbf{E}$ & $\mathbf{R}^{2}$ & $\begin{array}{c}\text { Total } \\
\text { Ranking }\end{array}$ & Vuong & $p$-value & $\begin{array}{c}\text { Preferred } \\
\text { Model }\end{array}$ \\
\hline $\mathrm{L}-\mathrm{C}$ & $-3.74 \mathrm{E}-03$ & 0.33 & 0.28 & 0.53 & 0.37 & 0.38 & 13 & 1.81 & $3.50 \mathrm{E}-02$ & $\mathrm{ZINB}>\mathrm{NB}$ \\
\hline M-C & $-2.44 \mathrm{E}-03$ & 0.34 & 0.28 & 0.53 & 0.38 & 0.38 & 14 & 1.95 & $2.57 \mathrm{E}-02$ & $\mathrm{ZINB}>\mathrm{NB}$ \\
\hline $\mathrm{I}-\mathrm{C}$ & $-3.05 \mathrm{E}-03$ & 0.33 & 0.30 & 0.55 & 0.37 & 0.38 & 21 & 3.28 & $5.18 \mathrm{E}-04$ & $\mathrm{ZINB}>\mathrm{NB}$ \\
\hline $\mathrm{F}-\mathrm{C}$ & $-3.11 \mathrm{E}-03$ & 0.35 & 0.31 & 0.55 & 0.38 & 0.39 & 25 & 2.69 & $3.56 \mathrm{E}-03$ & $\mathrm{ZINB}>\mathrm{NB}$ \\
\hline D-C & $-3.21 \mathrm{E}-03$ & 0.35 & 0.31 & 0.56 & 0.38 & 0.39 & 26 & 2.21 & $1.37 \mathrm{E}-02$ & $\mathrm{ZINB}>\mathrm{NB}$ \\
\hline C-C & $-2.60 \mathrm{E}-03$ & 0.34 & 0.29 & 0.54 & 0.35 & 0.36 & 28 & 2.55 & $5.36 \mathrm{E}-03$ & $\mathrm{ZINB}>\mathrm{NB}$ \\
\hline G-C & $-3.04 \mathrm{E}-03$ & 0.34 & 0.31 & 0.55 & 0.37 & 0.38 & 28 & 2.92 & $1.77 \mathrm{E}-03$ & $\mathrm{ZINB}>\mathrm{NB}$ \\
\hline $\mathrm{B}-\mathrm{C}$ & $-5.58 \mathrm{E}-03$ & 0.34 & 0.30 & 0.55 & 0.34 & 0.36 & 31 & 2.52 & $5.93 \mathrm{E}-03$ & $\mathrm{ZINB}>\mathrm{NB}$ \\
\hline $\mathrm{K}-\mathrm{C}$ & $6.39 \mathrm{E}-05$ & 0.41 & 0.32 & 0.57 & 0.36 & 0.37 & 43 & 2.09 & $1.85 \mathrm{E}-02$ & $\mathrm{ZINB}>\mathrm{NB}$ \\
\hline E-C & $-3.68 \mathrm{E}-03$ & 0.35 & 0.32 & 0.57 & 0.35 & 0.36 & 47 & 2.08 & $1.86 \mathrm{E}-02$ & $\mathrm{ZINB}>\mathrm{NB}$ \\
\hline
\end{tabular}


for all best fitted fatal CPMs developed, mean prediction bias (MPB) value for the vast majority of best models was negative which indicates that the models under-predicts collisions on average. Also, it is noticed that the models that attempted to relate the density variables to the number of collisions are not as successful as the models that used counted variables. The following is the discussion of the first three best fitted fatal CPMs.

\subsubsection{Best Fatal CPMs}

The best three fitted fatal CPMs together with their statistic summary are presented in Table 5.28. Model results reveal intuitive relationships between fatal collisions and the considered explanatory variables.

Table 5.28 Model Estimation Results for Fatal Collisions

\begin{tabular}{|c|c|c|c|c|c|c|}
\hline Fatal CPM & \multicolumn{2}{|c|}{ Model $\boldsymbol{M}$ - $\boldsymbol{C}$} & \multicolumn{2}{c|}{ Model $\boldsymbol{L}$ - } & \multicolumn{2}{c|}{ Model $\boldsymbol{I}$ - $\boldsymbol{C}$} \\
\hline Variables & Estimate & $\boldsymbol{p}$-value & Estimate & $\boldsymbol{p}$-value & Estimate & $\boldsymbol{p}$-value \\
\hline (Intercept) & -5.7249 & 0.00956 & -4.1768 & 0.05268 & -4.712 & 0.0158 \\
\hline VKT & 0.3434 & 0.14564 & 0.1574 & 0.50159 & 0.311 & 0.1528 \\
\hline TLKM & 0.4823 & 0.0018 & 0.6191 & 0.00011 & 0.3509 & 0.0293 \\
\hline
\end{tabular}

In all best models, it is found that $\log \mathrm{VKT}$ is not significant at $5 \%$, yet significant at a level of significance of $10 \%$ with a positive relation with fatal collisions. It could be that most of fatal collisions occurred in rural areas where the traffic speed increases due to relatively low degree of congestion. The log of TLKM found to be the most significant factor in fatal collision occurrences. It is found positively effecting the fatal collisions. As the TLKM increases within a TAZ, exposure of vehicle traffic increase, which in turns increases the probability of being involved in a fatal collision. 


\section{Chapter 6: Conclusions and Recommendations}

\subsection{Conclusions}

A significant amount of academic work has gone into the production and use of microlevel collision prediction models as part of reactive road safety improvement programs. It is only more recently that transportation engineers have begun looking into the potential of macrolevel collision prediction models, used either to reduce the incidence of collisions in new neighbourhoods at the planning stage, or to allocate resources effectively by ranking areas of a municipality according to their collision reduction potential. The common challenge in the previous publications of macro-level collisions analysis was how to properly assign traffic analysis zone boundary data between TAZs.

This research effort attempted to identify the influence of assigning TAZs' boundaries data such as collisions, roadway characteristics, traffic exposure, and socio-economic characteristics on the prediction ability of the macro-level CPM results. Ten assignment methods for boundary geocoded data were proposed, including: equal-proportion-based, populationbased, population-employment-based, TLKM1-based, TLKM2-based, TLKM3-based, VKT1based, VKT2-based, VKT3-based, and multiple-count-based methods. Geocoded boundary data such as collisions, roadways, exposure variables were assigned to TAZs using ten assignment methods. Thirteen datasets were prepared for the collisions analyses. Ten of the datasets were used in order to represent the various utilized boundary assignment methods, while the rest of the datasets were a combination between equal-proportion-based, population-based, populationemployment-based with multiple-count-based methods. 
Models for predicting the frequency of collisions in 422 traffic analysis zones (TAZs) in the City of Ottawa were developed. Using a wide variety of planning, traffic exposure, and network data that was provided by the City, a negative-binomial regression analysis was conducted on strategic subsets of the data. The independent variables were arranged in eleven different combinations according to the three data categories; exposure-related, roadway-related, and planning-related. These independent variables were used to predict six numbers of collisions such as total, PDO, fatal, non-fatal injury, bike-involved, pedestrian involved collisions. From the results of all models that were developed, the following conclusions are drawn:

- The ranking process showed superiority for the developed models based on equalproportion-based assignment method compared to other boundary data assignment methods. Therefore, this method is the most recommended for assigning TAZ's collision and other boundary data between adjacent TAZs to obtain CPMs. This finding is considered to be logical since a collision at a boundary of TAZs can be seen to belong to all adjacent TAZs.

- The findings indicated also multiple-count-based assignment to be the second best assignment method for assigning roadway and exposure boundary data.

- Planning-related variables were better than the roadway-related and exposure-related variables in predicting collisions frequency at the TAZ's level.

- The goodness of fit measures for exposure-related CPMs indicated that relating the different types of collisions at TAZ's level of aggregation to the exposure variables only did not lead to acceptable CPMs.

- Some variables appeared prominently in the models; 
- The models identified a positive correlation between the number of traffic signals in a TAZ and the number of different types of collisions.

- Bus stops within a TAZ were found to generate more conflicts between bikes and buses as well as between buses and pedestrian. This finding indicated the level of violation that may occur at bus stop locations.

- The sign of VKT and TLKM varied in the best developed models based on roadway-related variables for total and PDO. However, the best planning-related and exposure-related CPMs revealed intuitive results regarding to these two variables indicating that increased total VKT and total TLKM within a TAZ would increase the probability of being involved in a collision.

- For the modal split data from the planning side was what proved most useful, although the employment and population data also remained significant in some models.

- A negative correlation was found between the numbers of kilometers of roadway posted at a $70 \mathrm{~km} / \mathrm{h}$ speed limit and the number of total, PDO, non-fatal injury, and bike-involved collisions. This variable was positively correlated with pedestrian-involved collisions.

\subsection{Recommendation for future studies}

Based on the finding of this research study, the following are recommended as a future work in the area of developing collisions prediction model at the planning level such as traffic analysis zone level. 
- It is recommended to include more data and increase the TAZs' sample to provide wider variation of land use and improve the models prediction ability. The study area could be expanded to include all National Capital Region TAZs.

- Hot spot analysis was not conducted in this thesis study, therefore, it is recommended to identify and rank Collision Prone Zone (CPZ) using the different proposed data combination and boundary assignment methods.

- Using different boundary data assignment methods can lead to different distributions for collisions and independent variables. Therefore, outlier analysis using negative binomial regression technique is recommended to test the effect of outlier collision count values on the prediction ability of CPMs. This outlier analysis might lead to removing TAZs with very low collisions frequencies from the data. Also, it is recommended to attempt to develop models after splitting the data along the lines of urban and rural.

- The statistical modelling in this study involved using only conventional univariate approach. Therefore, it is recommended to use multivariate models and the different spatial analysis techniques to develop CPMs for different types of collisions. Using such modelling techniques is expected to improve overall prediction ability of CPMs because of their capabilities in accommodating correlation between independent variables.

- It is recommended to identify the best subset of independent variables to be included in the model using techniques such as factor analysis and principal component analysis. These types of statistical analysis allow for bringing the highly correlated variables together under lower number of independent variables. 
- It is expected that the bike-involved and pedestrian-involved collisions are more related to bike and pedestrian activities, respectively, within TAZs. Thus, using direct exposure measures for pedestrian and bike such as pedestrian flow and bike flow is expected to lead to better result for bike-involved and pedestrian-involved collisions. 


\section{List of References}

Abdel-Aty, M., Siddiqui, C., Huang, H., \& Wang, X. (2011). Integrating Trip and Roadway Characteristics to Manage Safety in Traffic Analysis Zones. Transportation Research Record: Journal of the Transportation Research Board, 2213(-1), 20-28. doi: $10.3141 / 2213-04$

Alejandro Weinstein, M. (2008).Distance from a point to polygon. Retrieved from http://www.mathworks.com/matlabcentral/fileexchange/19398-distance-from-a-pointpolygon/content/p_poly_dist.m.

Aguero-Valverde, J., \& Jovanis, P. P. (2006). Spatial analysis of fatal and injury crashes in Pennsylvania. Accident; Analysis and Prevention, 38(3), 618-625. doi:10.1016/j.aap.2005.12.006

Aj, M., Rwg, A., Mjb, F., Bh, L., \& Cg, B. (1997). Vehicle Travel Speeds and The Incidence of Fatal Pedestrian Collisions. Accident Analysis and Prevention, 29, 667-674.

Al-Ghamdi, A. S. (2002). Pedestrian-vehicle Crashes and Analytical Techniques for Stratified Contingency Tables. Accident; Analysis and Prevention, 34(2), 205-514.

Amoros, E., Martin, J. L., \& Laumon, B. (2003). Comparison of road crashes incidence and severity between some French counties. Accident; Analysis and Prevention, 35(4), 537-47.

Andrzej P. Tarko, Kumares C. Sinha, \& O. F. (1996). Methodology for Identifying Highway Safety Problem Areas. Transportation Research Record, 1542, 49-53. 
Brüde, U., \& Larsson, J. (1993). Models for Predicting Accidents at Junctions Where Pedestrians and Cyclists are Involved. How Well Do They Fit? Accident; Analysis and Prevention, 25(5), 499-509.

El-Basyouny, K., \& Sayed, T. (2009). Urban Arterial Accident Prediction Models with Spatial Effects. Transportation Research Record: Journal of the Transportation Research Board, 2102(-1), 27-33. doi:10.3141/2102-04

Fontaine, H., \& Gourlet, Y. (1997). Fatal Pedesrtian Accidents in France: A Typological Analysis. Accident Analysis \& Prevention, 29(3), 303-312.

Guevara, F. L. De, Washington, S. P., \& Oh, J. (1997). Applied in Tucson , Arizona. Transportation Research Record: Journal of the Transportation Research Board, No. 1897, TRB, National Research Council, Washington, D.C., 2004, Pp. 191-199.

Guevara, F. L. De, Washington, S. P., \& Oh, J. (2004). Forecasting Crashes at the Planning Level Simultaneous Negative Binomial Crash Model Applied in Tucson, Arizona. Transportation Research Record 1897, 191-199.

Hadayeghi, A., Shalaby, A. S., \& Persaud, B. N. (2003). Macrolevel Accident Prediction Models for Evaluating Safety of Urban Transportation Systems. Transportation Research Record, 1840(03), 87-95.

Hadayeghi, A., Shalaby, A. S., Persaud, B. N., \& Cheung, C. (2006). Temporal transferability and updating of zonal level accident prediction models. Accident; Analysis and Prevention, 38(3), 579-89. doi:10.1016/j.aap.2005.12.003 
Huang, H., Abdel-Aty, M. a., \& Darwiche, A. L. (2010). County-Level Crash Risk Analysis in Florida. Transportation Research Record: Journal of the Transportation Research Board, 2148(-1), 27-37. doi:10.3141/2148-04

Jones, a P., Langford, I. H., \& Bentham, G. (1996). The application of K-function analysis to the geographical distribution of road traffic accident outcomes in Norfolk, England. Social Science \& Medicine (1982), 42(6), 879-885.

Karlaftis, M. G., \& Tarko, a P. (1998). Heterogeneity considerations in accident modeling. Accident; Analysis and Prevention, 30(4), 425-33.

Kim, K., Pant, P., \& Yamashita, E. (2010). Accidents and Accessibility Measuring Influences of Demographic and Land Use Variables in Honolulu, Hawaii. Transportation Research Record: Journal of the Transportation Research Board, 2147(-1), 9-17. doi:10.3141/214702

Lascala, E. a, Gerber, D., \& Gruenewald, P. J. (2000). Demographic and Environmental Correlates of Pedestrian Injury Collisions: a Spatial Analysis. Accident; Analysis and Prevention, 32(5), 651-685.

Lee, C., \& Abdel-Aty, M. (2005). Comprehensive Analysis of Vehicle-Pedestrian Crashes at Intersections in Florida. Accident; Analysis and Prevention, 37(4), 775-786. doi:10.1016/j.aap.2005.03.019

Levine, N. E. D., Nitz, L. H., \& Kim, K. E. (1995). Spatial Analysis of Honolulu Motor Vehicle Crashes:I.Spatial Patterns. Accident Analysis \& Prevention, 27(5), 663-674. 
Levine, N., Kim, K. E., \& Nitz, L. H. (1995). Spatial analysis of Honolulu motor vehicle crashes: II. Zonal generators. Accident; Analysis and Prevention, 27(5), 675-85.

Lovegrove, G. R., \& Sayed, T. (2006). Macro-level Collision Prediction Models for Evaluating Neighbourhood Traffic Safety. NRC Research Press, 621, 609-621. doi:10.1139/L06-013

Lovegrove, G. R., \& Sayed, T. (2007). Macrolevel Collision Prediction Models to Enhance Traditional Reactive Road Safety Improvement Programs. Transportation Research Record, 2019(-1), 65-73. doi:10.3141/2019-09

Lovegrove, G., \& Sayed, T. (2006). Using Macrolevel Collision Prediction Models in Road Safety Planning Applications. Transportation Research Record, 1950(1), 73-82. doi:10.3141/1950-09

Ma, J., Kockelman, K. M., \& Damien, P. (2008). A multivariate Poisson-lognormal regression model for prediction of crash counts by severity, using Bayesian methods. Accident; Analysis and Prevention, 40(3), 964-75. doi:10.1016/j.aap.2007.11.002

MacNab, Y. C. (2004). Bayesian spatial and ecological models for small-area accident and injury analysis. Accident; Analysis and Prevention, 36(6), 1019-1028. doi:10.1016/j.aap.2002.05.001

Management, R. S., Lee, J., Mannering, F., Monitor, T., Milton, J., State, W., \& Commission, T. (n.d.). Analysis of Roadside Accident Frequency and Severity and Roadside Safety Management .Final Research Report Research Project T9903, Task 97 Analysis of Roadside Accident Severity \& Roadside Safety Management. 
Miaou, S., \& Lord, D. (2003). Modeling Traffic Crash-Flow Relationship for Intersections: Dispersion Parameter, Functional Form, and Bayes Versus Empirical Bayes. Prepared for Transportation Research Board 82 nd Annual Meeting, January 2003, 250(January).

Miaou, S.-P. (1993). The Relationship Between Truck Accidents and Geometric Design of Road Sections: Poisson Versus Negative Binomial Regressions. Accident Analysis \& Prevention ., 4(26), 471-482.

Mohamed E.A. Sarhan, B. S. (2004). Safety Performance of Freeway Merge and Diverge Areas. A thesis Submitted to the Faculity of Graduate Studies and Reserche in Partial Fulfillment of the Requirments for the Degree of Master of Applied Scence.

Noland, R. B., \& Oh, L. (2004). The effect of infrastructure and demographic change on trafficrelated fatalities and crashes: a case study of Illinois county-level data. Accident; Analysis and Prevention, 36(4), 525-532. doi:10.1016/S0001-4575(03)00058-7

Noland, R. B., \& Quddus, M. a. (2004). A spatially disaggregate analysis of road casualties in England. Accident; Analysis and Prevention, 36(6), 973-984. doi:10.1016/j.aap.2003.11.001

Obeng, D. A. (2013). Characteristics of Pedestrian Accidents on Trunk Roads in Ghana, 2(5), $46-54$.

Quddus, M. a. (2008). Modelling area-wide count outcomes with spatial correlation and heterogeneity: An analysis of London crash data. Accident Analysis \& Prevention, 40(4), 1486-1497. doi:10.1016/j.aap.2008.03.009 
Sayed, Z. S. and T. (2001). Evaluating Safety of Urban Arterial Roadways.Journal of Transportation Engineering., 2(April), 151-158.

Siddiqui, C., \& Abdel-Aty, M. (2012). Nature of Modeling Boundary Pedestrian Crashes at Zones. Transportation Research Record: Journal of the Transportation Research Board, 2299(-1), 31-40. doi:10.3141/2299-04

Song, J. J., Ghosh, M., Miaou, S., \& Mallick, B. (2006). Bayesian multivariate spatial models for roadway traffic crash mapping. Journal of Multivariate Analysis, 97(1), 246-273. doi:10.1016/j.jmva.2005.03.007

Sun, J., \& Lovegrove, G. (2013). Comparing the road safety of neighbourhood development patterns: traditional versus sustainable communities. NRC Research Press, 45(October 2012), 35-45.

Trentacoste, M. F. (2000). Prediction of the Expected Safety Performance of Rural Two-Lane Highways. US Department of Transportation, (December), 1-200.

Turner, S. A., Roozenburg, A. P., \& Francis, T. (2006). Predicting Accident Rates for Cyclists and Pedestrians. Research Report 289. Land Transport NewZealand.

Wang, X., Jin, Y., Abdel-Aty, M., Tremont, P. J., \& Chen, X. (2012). Macrolevel Model Development for Safety Assessment of Road Network Structures. Transportation Research Record: Journal of the Transportation Research Board, 2280(-1), 100-109. doi:10.3141/2280-11 
Wei, F. (2010). Boundary Effects in Developing Macro-level CPMs: A Case Study of City of Ottawa. In PhD student.

WHO. (2013). GLOBAL STATUS REPORT ON ROAD (pp. 1-12).

Wier, M., Weintraub, J., Humphreys, E. H., Seto, E., \& Bhatia, R. (2009). An area-level Model of Vehicle-Pedestrian Injury Collisions with Implications for Land Use and Transportation Planning. Accident; Analysis and Prevention, 4l(1), 137-145. doi:10.1016/j.aap.2008.10.001

Zajac, S. S., \& Ivan, J. N. (2003). Factors Influencing Injury Severity of Motor Vehicle-crossing Pedestrian Crashes in Rural Connecticut. Accident; Analysis and Prevention, 35(3), 369379. 


\section{Appendix A: Spatial Analysis and Descriptive Statistics of Data}




\section{A.1 Motor Vehicle Collisions}

Table A.6 Descriptive Statistics of the Different Types of Collsions Within Ottawa's TAZs (Boundary Data Assigned Using Equal-proportion-based assignment Method)

\begin{tabular}{|c|c|c|c|c|c|c|c|}
\hline Collisions & Observations & Total & Min. & Max. & Median & Mean & $\begin{array}{c}\text { Standard } \\
\text { deviation }\end{array}$ \\
\hline Total & 422 & 63978 & 0 & 759 & 130.50 & 151.61 & 113.26 \\
\hline Total PDO & 422 & 50776 & 0 & 598 & 102.50 & 120.32 & 89.59 \\
\hline $\begin{array}{c}\text { Total Non-Fatal } \\
\text { Injury }\end{array}$ & 422 & 12443 & 0 & 151 & 25.00 & 29.49 & 23.22 \\
\hline $\begin{array}{c}\text { Total Fatal } \\
\text { Total Bike-involved }\end{array}$ & 422 & 117 & 0 & 5 & 0.00 & 0.34 & 0.71 \\
\hline $\begin{array}{c}\text { Total Pedestrian- } \\
\text { involved }\end{array}$ & 422 & 1760 & 0 & 35 & 2.50 & 4.17 & 4.82 \\
\hline
\end{tabular}

Table A.7 Descriptive Statistics of the Different Types of Collsions Within Ottawa's TAZs (Boundary Data Assigned Using Population-based-assignment Method)

\begin{tabular}{|c|c|c|c|c|c|c|c|}
\hline Collisions & Observations & Total & Min. & Max. & Median & Mean & $\begin{array}{c}\text { Standard } \\
\text { deviation }\end{array}$ \\
\hline Total & 422 & 61826 & 0 & 803 & 110.00 & 146.51 & 137.36 \\
\hline Total PDO & 422 & 49101 & 0 & 631 & 89.12 & 116.35 & 108.68 \\
\hline $\begin{array}{c}\text { Total Non-Fatal } \\
\text { Injury }\end{array}$ & 422 & 11949 & 0 & 161 & 21.17 & 28.32 & 27.83 \\
\hline $\begin{array}{c}\text { Total Fatal } \\
\text { Total Bike-involved }\end{array}$ & 422 & 114 & 0 & 5 & 0.00 & 0.27 & 0.64 \\
\hline $\begin{array}{c}\text { Total Pedestrian- } \\
\text { involved }\end{array}$ & 422 & 1685 & 0 & 43 & 1.58 & 3.99 & 5.63 \\
\hline
\end{tabular}


Table A.8 Descriptive Statistics of the Different Types of Collsions Within Ottawa's TAZs (Boundary Data Assigned Using Population-Employment-based assignment Method)

\begin{tabular}{|c|c|c|c|c|c|c|c|}
\hline Collisions & Observations & Total & Min. & Max. & Median & Mean & $\begin{array}{c}\text { Standard } \\
\text { deviation }\end{array}$ \\
\hline Total & 422 & 63937 & 0 & 793 & 123.5 & 151.51 & 125.25 \\
\hline Total PDO & 422 & 50744 & 0 & 624 & 100 & 120.25 & 99.16 \\
\hline $\begin{array}{c}\text { Total Non-Fatal } \\
\text { Injury }\end{array}$ & 422 & 12393 & 0 & 159 & 24 & 29.37 & 25.45 \\
\hline Total Fatal & 422 & 114 & 0 & 5 & 0 & 0.27 & 0.67 \\
\hline Total Bike-involved & 422 & 850 & 0 & 12 & 1 & 2.01 & 2.27 \\
\hline $\begin{array}{c}\text { Total Pedestrian- } \\
\text { involved }\end{array}$ & 422 & 1713 & 0 & 35 & 2 & 4.06 & 5.12 \\
\hline
\end{tabular}

Table A.9 Descriptive Statistics of the Different Types of Collsions Within Ottawa's TAZs (Boundary Data Assigned Using TLKM1-based assignment Method)

\begin{tabular}{|c|c|c|c|c|c|c|c|}
\hline Collisions & Observations & Total & Min. & Max. & Median & Mean & $\begin{array}{c}\text { Standard } \\
\text { deviation }\end{array}$ \\
\hline Total & 422 & 63933 & 0 & 824 & 114.5 & 151.50 & 137.50 \\
\hline Total PDO & 422 & 50749 & 0 & 643 & 92 & 120.26 & 108.42 \\
\hline $\begin{array}{c}\text { Total Non-Fatal } \\
\text { Injury }\end{array}$ & 422 & 12404 & 0 & 184 & 22 & 29.39 & 28.29 \\
\hline $\begin{array}{c}\text { Total Fatal } \\
\text { Total Bike-involved }\end{array}$ & 422 & 117 & 0 & 5 & 0 & 0.28 & 0.71 \\
\hline $\begin{array}{c}\text { Total Pedestrian- } \\
\text { involved }\end{array}$ & 422 & 1714 & 0 & 35 & 2 & 4.06 & 5.30 \\
\hline
\end{tabular}


Table A.10 Descriptive Statistics of the Different Types of Collsions Within Ottawa's TAZs (Boundary Data Assigned Using TLKM2-based assignment Method)

\begin{tabular}{|c|c|c|c|c|c|c|c|}
\hline Collisions & Observations & Total & Min. & Max. & Median & Mean & $\begin{array}{c}\text { Standard } \\
\text { deviation }\end{array}$ \\
\hline Total & 422 & 63928 & 0 & 827 & 116 & 151.49 & 137.87 \\
\hline Total PDO & 422 & 50727 & 0 & 651 & 93.5 & 120.21 & 108.96 \\
\hline $\begin{array}{c}\text { Total Non-Fatal } \\
\text { Injury }\end{array}$ & 422 & 12391 & 0 & 169 & 21.5 & 29.36 & 28.07 \\
\hline $\begin{array}{c}\text { Total Fatal } \\
\text { Total Bike-involved }\end{array}$ & 422 & 117 & 0 & 5 & 0 & 0.28 & 0.70 \\
\hline $\begin{array}{c}\text { Total Pedestrian- } \\
\text { involved }\end{array}$ & 422 & 1710 & 0 & 39 & 2 & 4.05 & 5.43 \\
\hline
\end{tabular}

Table A.11 Descriptive Statistics of the Different Types of Collsions Within Ottawa's TAZs (Boundary Data Assigned Using TLKM3-based assignment Method)

\begin{tabular}{|c|c|c|c|c|c|c|c|}
\hline Collisions & Observations & Total & Min. & Max. & Median & Mean & $\begin{array}{c}\text { Standard } \\
\text { deviation }\end{array}$ \\
\hline Total & 422 & 63945 & 0 & 825 & 116 & 151.53 & 136.33 \\
\hline Total PDO & 422 & 50741 & 0 & 646 & 92.5 & 120.24 & 107.67 \\
\hline $\begin{array}{c}\text { Total Non-Fatal } \\
\text { Injury }\end{array}$ & 422 & 12387 & 0 & 169 & 22 & 29.35 & 27.78 \\
\hline $\begin{array}{c}\text { Total Fatal } \\
\text { Total Bike-involved }\end{array}$ & 422 & 117 & 0 & 5 & 0 & 0.28 & 0.70 \\
\hline $\begin{array}{c}\text { Total Pedestrian- } \\
\text { involved }\end{array}$ & 422 & 1720 & 0 & 35 & 2 & 4.08 & 2.36 \\
\hline
\end{tabular}


Table A.12 Descriptive Statistics of the Different Types of Collsions Within Ottawa's TAZs (Boundary Data Assigned Using VKT1-based assignment Method)

\begin{tabular}{|c|c|c|c|c|c|c|c|}
\hline Collisions & Observations & Total & Min. & Max. & Median & Mean & $\begin{array}{c}\text { Standard } \\
\text { deviation }\end{array}$ \\
\hline Total & 422 & 63933 & 0 & 843 & 116.5 & 151.50 & 136.23 \\
\hline Total PDO & 422 & 50743 & 0 & 654 & 95 & 120.24 & 107.10 \\
\hline $\begin{array}{c}\text { Total Non-Fatal } \\
\text { Injury }\end{array}$ & 422 & 12394 & 0 & 192 & 23 & 29.37 & 28.42 \\
\hline $\begin{array}{c}\text { Total Fatal } \\
\text { Total Bike-involved }\end{array}$ & 422 & 114 & 0 & 5 & 0 & 0.27 & 0.70 \\
\hline $\begin{array}{c}\text { Total Pedestrian- } \\
\text { involved }\end{array}$ & 422 & 1712 & 0 & 35 & 2 & 4.06 & 5.17 \\
\hline
\end{tabular}

Table A.13 Descriptive Statistics of the Different Types of Collsions Within Ottawa's TAZs (Boundary Data Assigned Using VKT2-based assignment Method)

\begin{tabular}{|c|c|c|c|c|c|c|c|}
\hline Collisions & Observations & Total & Min. & Max. & Median & Mean & $\begin{array}{c}\text { Standard } \\
\text { deviation }\end{array}$ \\
\hline Total & 422 & 63905 & 0 & 844 & 112 & 151.43 & 140.79 \\
\hline Total PDO & 422 & 50716 & 0 & 655 & 90 & 120.18 & 110.87 \\
\hline $\begin{array}{c}\text { Total Non-Fatal } \\
\text { Injury }\end{array}$ & 422 & 12400 & 0 & 175 & 22 & 29.38 & 29.09 \\
\hline $\begin{array}{c}\text { Total Fatal } \\
\text { Total Bike-involved }\end{array}$ & 422 & 112 & 0 & 5 & 0 & 0.27 & 0.70 \\
\hline $\begin{array}{c}\text { Total Pedestrian- } \\
\text { involved }\end{array}$ & 422 & 1718 & 0 & 38 & 2 & 4.07 & 5.37 \\
\hline
\end{tabular}


Table A.14 Descriptive Statistics of the Different Types of Collsions Within Ottawa's TAZs (Boundary Data Assigned Using VKT3-based assignment Method)

\begin{tabular}{|c|c|c|c|c|c|c|c|}
\hline Collisions & Observations & Total & Min. & Max. & Median* & Mean & $\begin{array}{c}\text { Standard } \\
\text { deviation }\end{array}$ \\
\hline Total & 422 & 63934 & 0 & 844 & 116.5 & 151.50 & 138.02 \\
\hline Total PDO & 422 & 50736 & 0 & 655 & 94.5 & 120.23 & 108.65 \\
\hline $\begin{array}{c}\text { Total Non-Fatal } \\
\text { Injury }\end{array}$ & 422 & 12406 & 0 & 177 & 23 & 29.40 & 28.60 \\
\hline Total Fatal & 422 & 112 & 0 & 5 & 0 & 0.27 & 0.69 \\
\hline $\begin{array}{c}\text { Total Bike-involved } \\
\text { Total Pedestrian- } \\
\text { involved }\end{array}$ & 422 & 838 & 0 & 13 & 1 & 1.99 & 2.36 \\
\hline
\end{tabular}




\section{A.2 Roadways and Intersections}

Table A.15 Descriptive Statistics of the Roadways and Intersections Variables Within Ottawa's TAZs (Boundary Data Assigned Using Equal-proportion-based assignment Method)

\begin{tabular}{|c|c|c|c|c|c|}
\hline Variables & Observation & Minimum & Maximum & Mean & $\begin{array}{l}\text { Standard } \\
\text { deviation }\end{array}$ \\
\hline $\begin{array}{l}\text { Total Arterial Road } \\
\text { Kilometres }(\mathrm{km})\end{array}$ & 422 & 0.00 & 75.23 & 3.01 & 8.13 \\
\hline $\begin{array}{l}\text { Total Collector Road } \\
\text { Kilometres }(\mathrm{km})\end{array}$ & 422 & 0.00 & 137.73 & 3.88 & 14.70 \\
\hline $\begin{array}{l}\text { Total local Road } \\
\text { Kilometres }\end{array}$ & 422 & 0.00 & 36.33 & 5.39 & 6.16 \\
\hline $\begin{array}{l}\text { Total Major Collector } \\
\text { Road Kilometres }(\mathrm{km})\end{array}$ & 422 & 0.00 & 4.98 & 0.18 & 0.45 \\
\hline $\begin{array}{l}\text { Total Roadways } \\
\text { Kilometres }(\mathrm{km})\end{array}$ & 422 & 0.00 & 229.20 & 12.46 & 25.88 \\
\hline $\begin{array}{l}\text { Length of Roadways with } \\
40 \mathrm{~km} / \mathrm{h} \text { Speed Limit }(\mathrm{km})\end{array}$ & 422 & 0.00 & 30.46 & 2.05 & 4.48 \\
\hline $\begin{array}{l}\text { Length of Roadways with } \\
50 \mathrm{~km} / \mathrm{h} \text { Speed Limit }(\mathrm{km})\end{array}$ & 422 & 0.00 & 40.34 & 4.68 & 5.73 \\
\hline $\begin{array}{l}\text { Length of Roadways with } \\
60 \mathrm{~km} / \mathrm{h} \text { Speed Limit }(\mathrm{km})\end{array}$ & 422 & 0.00 & 34.58 & 0.95 & 2.56 \\
\hline $\begin{array}{l}\text { Length of Roadways with } \\
70 \mathrm{~km} / \mathrm{h} \text { Speed Limit }(\mathrm{km})\end{array}$ & 422 & 0.00 & 19.34 & 0.21 & 1.13 \\
\hline $\begin{array}{l}\text { Length of Roadways with } \\
80 \mathrm{~km} / \mathrm{h} \text { Speed Limit }(\mathrm{km})\end{array}$ & 422 & 0.00 & 203.28 & 4.53 & 20.87 \\
\hline $\begin{array}{l}\text { Length of Roadways with } \\
90 \mathrm{~km} / \mathrm{h} \text { Speed Limit }(\mathrm{km})\end{array}$ & 422 & 0.00 & 10.23 & 0.03 & 0.50 \\
\hline $\begin{array}{l}\text { Total Number } \\
\text { Intersections }\end{array}$ & 422 & 0.00 & 182.30 & 33.41 & 30.60 \\
\hline $\begin{array}{l}\text { Total Number Signalized } \\
\text { Intersections }\end{array}$ & 422 & 0.00 & 17.50 & 2.63 & 2.01 \\
\hline $\begin{array}{c}\text { Total Number Non- } \\
\text { signalized Intersections }\end{array}$ & 422 & 0.00 & 181.80 & 30.78 & 30.43 \\
\hline $\begin{array}{c}\text { Total Number of Bus } \\
\text { Stops }\end{array}$ & 422 & 0.00 & 111.30 & 14.95 & 12.59 \\
\hline
\end{tabular}


Table A.16 Descriptive Statistics of the Roadways and Intersections Variables Within Ottawa's TAZs (Boundary Data Assigned Using Population-based assignment Method)

\begin{tabular}{|c|c|c|c|c|c|}
\hline Variables & Observation & Minimum & Maximum & Mean & $\begin{array}{c}\text { Standard } \\
\text { deviation }\end{array}$ \\
\hline $\begin{array}{c}\text { Total Arterial Road } \\
\text { Kilometres (km) }\end{array}$ & 422 & 0 & 77.20 & 2.96 & 8.24 \\
\hline $\begin{array}{c}\text { Total Collector Road } \\
\text { Kilometres (km) }\end{array}$ & 422 & 0 & 139.03 & 3.87 & 14.86 \\
\hline $\begin{array}{c}\text { Total local Road } \\
\text { Kilometres (km) }\end{array}$ & 422 & 0 & 36.16 & 5.38 & 6.23 \\
\hline $\begin{array}{c}\text { Total Major Collector } \\
\text { Road Kilometres (km) }\end{array}$ & 422 & 0 & 4.98 & 0.18 & 0.46 \\
\hline $\begin{array}{c}\text { Length of Roadways with } \\
40 \text { km/h Speed Limit (km) }\end{array}$ & 422 & 0 & 30.46 & 2.04 & 4.53 \\
\hline $\begin{array}{c}\text { Length of Roadways with } \\
50 \text { km/h Speed Limit (km) }\end{array}$ & 422 & 0 & 40.87 & 4.67 & 5.90 \\
\hline $\begin{array}{c}\text { Length of Roadways with } \\
60 \text { km/h Speed Limit (km) }\end{array}$ & 422 & 0 & 34.26 & 0.94 & 2.58 \\
\hline $\begin{array}{c}\text { Length of Roadways with } \\
70 \text { km/h Speed Limit (km) }\end{array}$ & 422 & 0 & 20.83 & 0.21 & 1.20 \\
\hline $\begin{array}{c}\text { Length of Roadways with } \\
80 \text { km/h Speed Limit (km) }\end{array}$ & 422 & 0 & 206.58 & 4.51 & 21.09 \\
\hline $\begin{array}{c}\text { Length of Roadways with } \\
90 \text { km/h Speed Limit (km) }\end{array}$ & 422 & 0 & 10.24 & 0.03 & 0.51 \\
\hline $\begin{array}{c}\text { Total Roadways } \\
\text { Kilometres (km) }\end{array}$ & 422 & 0 & 232.92 & 12.40 & 26.29 \\
\hline $\begin{array}{c}\text { Total Number } \\
\text { Intersections }\end{array}$ & 422 & 0 & 189 & 33.20 & 32.56 \\
\hline $\begin{array}{c}\text { Total Number Signalized } \\
\text { Intersections }\end{array}$ & 422 & 0 & 19 & 2.52 & 2.53 \\
\hline $\begin{array}{c}\text { Total Number Non- } \\
\text { signalized Intersections }\end{array}$ & 422 & 0 & 188 & 30.68 & 31.69 \\
\hline $\begin{array}{c}\text { Total Number of Bus } \\
\text { Stops }\end{array}$ & 422 & 0 & 115 & 14.70 & 14.27 \\
\hline
\end{tabular}


Table A.17 Descriptive Statistics of the Roadways and Intersections Variables Within Ottawa's TAZs (Boundary Data Assigned Using Population-Employment-based assignment Method)

\begin{tabular}{|c|c|c|c|c|c|}
\hline Variables & Observation & Minimum & Maximum & Mean & $\begin{array}{c}\text { Standard } \\
\text { deviation }\end{array}$ \\
\hline $\begin{array}{c}\text { Total Arterial Road } \\
\text { Kilometres (km) }\end{array}$ & 422 & 0 & 77.5 & 3.01 & 8.20 \\
\hline $\begin{array}{c}\text { Total Collector Road } \\
\text { Kilometres (km) }\end{array}$ & 422 & 0 & 139.159 & 3.88 & 14.85 \\
\hline $\begin{array}{c}\text { Total local Road } \\
\text { Kilometres (km) }\end{array}$ & 422 & 0 & 36.178 & 5.39 & 6.19 \\
\hline $\begin{array}{c}\text { Total Major Collector } \\
\text { Road Kilometres (km) }\end{array}$ & 422 & 0 & 4.977 & 0.18 & 0.46 \\
\hline $\begin{array}{c}\text { Length of Roadways with } \\
40 \text { km/h Speed Limit (km) }\end{array}$ & 422 & 0 & 30.464 & 2.05 & 4.51 \\
\hline $\begin{array}{c}\text { Length of Roadways with } \\
50 \mathrm{~km} / \mathrm{h} \mathrm{Speed} \mathrm{Limit} \mathrm{(km)}\end{array}$ & 422 & 0 & 40.867 & 4.68 & 5.80 \\
\hline $\begin{array}{c}\text { Length of Roadways with } \\
60 \mathrm{~km} / \mathrm{h} \text { Speed Limit (km) }\end{array}$ & 422 & 0 & 34.228 & 0.95 & 2.56 \\
\hline $\begin{array}{c}\text { Length of Roadways with } \\
70 \mathrm{~km} / \mathrm{h} \mathrm{Speed} \mathrm{Limit} \mathrm{(km)}\end{array}$ & 422 & 0 & 20.305 & 0.21 & 1.18 \\
\hline $\begin{array}{c}\text { Length of Roadways with } \\
80 \mathrm{~km} / \mathrm{h} \text { Speed Limit (km) }\end{array}$ & 422 & 0 & 207.012 & 4.53 & 21.08 \\
\hline $\begin{array}{c}\text { Length of Roadways with } \\
90 \mathrm{~km} / \mathrm{h} \text { Speed Limit (km) }\end{array}$ & 422 & 0 & 10.24 & 0.03 & 0.51 \\
\hline $\begin{array}{c}\text { Total Roadways } \\
\text { Kilometres (km) }\end{array}$ & 422 & 0 & 233.379 & 12.46 & 26.19 \\
\hline $\begin{array}{c}\text { Total Number } \\
\text { Intersections }\end{array}$ & 422 & 0 & 188 & 33.41 & 31.61 \\
\hline $\begin{array}{c}\text { Total Number Signalized } \\
\text { Intersections }\end{array}$ & 422 & 0 & 19 & 2.63 & 2.26 \\
\hline $\begin{array}{c}\text { Total Number Non- } \\
\text { signalized Intersections }\end{array}$ & 422 & 0 & 188 & 30.77 & 31.12 \\
\hline $\begin{array}{c}\text { Total Number of Bus } \\
\text { Stops }\end{array}$ & 422 & 0 & 115 & 14.95 & 13.58 \\
\hline
\end{tabular}


Table A.18 Descriptive Statistics of the Roadways and Intersections Variables Within Ottawa's TAZs (Boundary Data Assigned Using VKT1-based assignment Method)

\begin{tabular}{|c|c|c|c|c|c|}
\hline Variables & Observation & Maximum & Minimum & Mean & $\begin{array}{l}\text { Standard } \\
\text { deviation }\end{array}$ \\
\hline $\begin{array}{c}\text { Total Arterial Road } \\
\text { Kilometres }(\mathrm{km})\end{array}$ & 422 & 0 & 81 & 3.01 & 8.50 \\
\hline $\begin{array}{c}\text { Total Collector Road } \\
\text { Kilometres }(\mathrm{km}) \\
\end{array}$ & 422 & 0 & 140 & 3.87 & 15.02 \\
\hline $\begin{array}{l}\text { Total local Road } \\
\text { Kilometres }(\mathrm{km})\end{array}$ & 422 & 0 & 37 & 5.39 & 6.20 \\
\hline $\begin{array}{l}\text { Total Major Collector } \\
\text { Road Kilometres }(\mathrm{km})\end{array}$ & 422 & 0 & 5 & 0.18 & 0.46 \\
\hline $\begin{array}{l}\text { Length of Roadways with } \\
40 \mathrm{~km} / \mathrm{h} \text { Speed Limit }(\mathrm{km})\end{array}$ & 422 & 0 & 30 & 2.05 & 4.49 \\
\hline $\begin{array}{l}\text { Length of Roadways with } \\
50 \mathrm{~km} / \mathrm{h} \text { Speed Limit }(\mathrm{km})\end{array}$ & 422 & 0 & 41 & 4.68 & 5.84 \\
\hline $\begin{array}{l}\text { Length of Roadways with } \\
60 \mathrm{~km} / \mathrm{h} \text { Speed Limit }(\mathrm{km})\end{array}$ & 422 & 0 & 35 & 0.95 & 2.63 \\
\hline $\begin{array}{l}\text { Length of Roadways with } \\
70 \mathrm{~km} / \mathrm{h} \text { Speed Limit }(\mathrm{km})\end{array}$ & 422 & 0 & 21 & 0.21 & 1.22 \\
\hline $\begin{array}{l}\text { Length of Roadways with } \\
80 \mathrm{~km} / \mathrm{h} \text { Speed Limit }(\mathrm{km})\end{array}$ & 422 & 0 & 212 & 4.53 & 21.42 \\
\hline $\begin{array}{l}\text { Length of Roadways with } \\
90 \mathrm{~km} / \mathrm{h} \text { Speed Limit }(\mathrm{km})\end{array}$ & 422 & 0 & 10 & 0.03 & 0.51 \\
\hline $\begin{array}{l}\text { Total Roadways } \\
\text { Kilometres }(\mathrm{km})\end{array}$ & 422 & 0 & 238 & 12.45 & 26.52 \\
\hline $\begin{array}{l}\text { Total Number } \\
\text { Intersections }\end{array}$ & 422 & 0 & 195 & 33.41 & 31.64 \\
\hline $\begin{array}{c}\text { Total Number Signalized } \\
\text { Intersections } \\
\end{array}$ & 422 & 0 & 20 & 2.63 & 2.44 \\
\hline $\begin{array}{c}\text { Total Number Non- } \\
\text { signalized Intersections }\end{array}$ & 422 & 0 & 194 & 30.77 & 31.17 \\
\hline $\begin{array}{l}\text { Total Number of Bus } \\
\text { Stops }\end{array}$ & 422 & 0 & 118 & 14.95 & 13.64 \\
\hline
\end{tabular}


Table A.19 Descriptive Statistics of the Roadways and Intersections Variables Within Ottawa's TAZs (Boundary Data Assigned Using VKT2-based assignment Method)

\begin{tabular}{|c|c|c|c|c|c|}
\hline Variables & Observation & Maximum & Minimum & Mean & $\begin{array}{l}\text { Standard } \\
\text { deviation }\end{array}$ \\
\hline $\begin{array}{c}\text { Total Arterial Road } \\
\text { Kilometres }(\mathrm{km})\end{array}$ & 422 & 0 & 81 & 3.01 & 8.50 \\
\hline $\begin{array}{l}\text { Total Collector Road } \\
\text { Kilometres }(\mathrm{km})\end{array}$ & 422 & 0 & 140 & 3.87 & 15.03 \\
\hline $\begin{array}{l}\text { Total local Road } \\
\text { Kilometres }(\mathrm{km})\end{array}$ & 422 & 0 & 37 & 5.39 & 6.22 \\
\hline $\begin{array}{l}\text { Total Major Collector } \\
\text { Road Kilometres (km) }\end{array}$ & 422 & 0 & 5 & 0.18 & 0.46 \\
\hline $\begin{array}{l}\text { Length of Roadways with } \\
40 \mathrm{~km} / \mathrm{h} \text { Speed Limit }(\mathrm{km})\end{array}$ & 422 & 0 & 30 & 2.05 & 4.51 \\
\hline $\begin{array}{l}\text { Length of Roadways with } \\
50 \mathrm{~km} / \mathrm{h} \text { Speed Limit }(\mathrm{km})\end{array}$ & 422 & 0 & 41 & 4.68 & 5.90 \\
\hline $\begin{array}{l}\text { Length of Roadways with } \\
60 \mathrm{~km} / \mathrm{h} \text { Speed Limit }(\mathrm{km})\end{array}$ & 422 & 0 & 35 & 0.95 & 2.63 \\
\hline $\begin{array}{l}\text { Length of Roadways with } \\
70 \mathrm{~km} / \mathrm{h} \text { Speed Limit }(\mathrm{km})\end{array}$ & 422 & 0 & 21 & 0.21 & 1.22 \\
\hline $\begin{array}{l}\text { Length of Roadways with } \\
80 \mathrm{~km} / \mathrm{h} \text { Speed Limit }(\mathrm{km})\end{array}$ & 422 & 0 & 212 & 4.53 & 21.43 \\
\hline $\begin{array}{l}\text { Length of Roadways with } \\
90 \mathrm{~km} / \mathrm{h} \text { Speed Limit }(\mathrm{km})\end{array}$ & 422 & 0 & 10 & 0.03 & 0.50 \\
\hline $\begin{array}{l}\text { Total Roadways } \\
\text { Kilometres }(\mathrm{km}) \\
\end{array}$ & 422 & 0 & 238 & 12.45 & 26.56 \\
\hline $\begin{array}{l}\text { Total Number } \\
\text { Intersections }\end{array}$ & 422 & 0 & 195 & 33.40 & 32.14 \\
\hline $\begin{array}{c}\text { Total Number Signalized } \\
\text { Intersections } \\
\end{array}$ & 422 & 0 & 20 & 2.63 & 2.54 \\
\hline $\begin{array}{c}\text { Total Number Non- } \\
\text { signalized Intersections }\end{array}$ & 422 & 0 & 194 & 30.77 & 31.48 \\
\hline $\begin{array}{l}\text { Total Number of Bus } \\
\text { Stops }\end{array}$ & 422 & 0 & 118 & 14.94 & 14.05 \\
\hline
\end{tabular}


Table A.20 Descriptive Statistics of the Roadways and Intersections Variables Within Ottawa's TAZs (Boundary Data Assigned Using VKT3-based assignment Method)

\begin{tabular}{|c|c|c|c|c|c|}
\hline Variables & Observation & Maximum & Minimum & Mean & $\begin{array}{c}\text { Standard } \\
\text { deviation }\end{array}$ \\
\hline $\begin{array}{c}\text { Total Arterial Road } \\
\text { Kilometres (km) }\end{array}$ & 422 & 0 & 81 & 3.01 & 8.50 \\
\hline $\begin{array}{c}\text { Total Collector Road } \\
\text { Kilometres (km) }\end{array}$ & 422 & 0 & 140 & 3.87 & 15.03 \\
\hline $\begin{array}{c}\text { Total local Road } \\
\text { Kilometres (km) }\end{array}$ & 422 & 0 & 37 & 5.39 & 6.21 \\
\hline $\begin{array}{c}\text { Total Major Collector } \\
\text { Road Kilometres (km) }\end{array}$ & 422 & 0 & 5 & 0.18 & 0.46 \\
\hline $\begin{array}{c}\text { Length of Roadways with } \\
40 \text { km/h Speed Limit (km) }\end{array}$ & 422 & 0 & 30 & 2.05 & 4.50 \\
\hline $\begin{array}{c}\text { Length of Roadways with } \\
50 \text { km/h Speed Limit (km) }\end{array}$ & 422 & 0 & 41 & 4.68 & 5.87 \\
\hline $\begin{array}{c}\text { Length of Roadways with } \\
60 \text { km/h Speed Limit (km) }\end{array}$ & 422 & 0 & 35 & 0.95 & 2.63 \\
\hline $\begin{array}{c}\text { Length of Roadways with } \\
70 \text { km/h Speed Limit (km) }\end{array}$ & 422 & 0 & 21 & 0.21 & 1.22 \\
\hline $\begin{array}{c}\text { Length of Roadways with } \\
80 \mathrm{~km} / \mathrm{h} \text { Speed Limit (km) }\end{array}$ & 422 & 0 & 212 & 4.53 & 21.43 \\
\hline $\begin{array}{c}\text { Length of Roadways with } \\
90 \mathrm{~km} / \mathrm{h} \mathrm{Speed} \mathrm{Limit} \mathrm{(km)}\end{array}$ & 422 & 0 & 10 & 0.03 & 0.50 \\
\hline $\begin{array}{c}\text { Total Roadways } \\
\text { Kilometres (km) }\end{array}$ & 422 & 0 & 238 & 12.45 & 26.54 \\
\hline $\begin{array}{c}\text { Total Number } \\
\text { Intersections }\end{array}$ & 422 & 0 & 195 & 33.40 & 31.86 \\
\hline $\begin{array}{c}\text { Total Number Signalized } \\
\text { Intersections }\end{array}$ & 422 & 0 & 20 & 2.63 & 2.48 \\
\hline $\begin{array}{c}\text { Total Number Non- } \\
\text { signalized Intersections }\end{array}$ & 422 & 0 & 194 & 30.77 & 31.31 \\
\hline $\begin{array}{c}\text { Total Number of Bus } \\
\text { Stops }\end{array}$ & 422 & 0 & 118 & 14.95 & 13.84 \\
\hline
\end{tabular}




\section{A.3 Traffic Exposure Variables}

Table A.21 Descriptive Statistics of Exposure Variables Within Ottawa's TAZs (Boundary Data Assigned Using Equal proportion based assignment Method)

\begin{tabular}{|c|c|c|c|c|c|}
\hline Variables & Observation & Minimum & Maximum & Mean & $\begin{array}{c}\text { Standard } \\
\text { Deviation }\end{array}$ \\
\hline $\begin{array}{c}\text { Vehicle Kilometre } \\
\text { Traveled (veh/km/day) }\end{array}$ & 422 & 0.00 & 508369.00 & 17269.81 & 46958.76 \\
\hline
\end{tabular}

Table A.22 Descriptive Statistics of Exposure Variables Within Ottawa's TAZs (Boundary Data Assigned Using Population based assignment Method)

\begin{tabular}{|c|c|c|c|c|c|}
\hline Variables & Observation & Minimum & Maximum & Mean & $\begin{array}{c}\text { Standard } \\
\text { Deviation }\end{array}$ \\
\hline $\begin{array}{c}\text { Vehicle Kilometre } \\
\text { Traveled (veh/km/day) }\end{array}$ & 422 & 0 & 896429 & 17266.72 & 61804.3 \\
\hline
\end{tabular}

Table A.23 Descriptive Statistics of Exposure Variables Within Ottawa's TAZs (Boundary Data Assigned Using Population-Employment based assignment Method)

\begin{tabular}{|c|c|c|c|c|c|}
\hline Variables & Observation & Minimum & Maximum & Mean & $\begin{array}{c}\text { Standard } \\
\text { Deviation }\end{array}$ \\
\hline $\begin{array}{c}\text { Vehicle Kilometre } \\
\text { Traveled (veh/km/day) }\end{array}$ & 422 & 0 & 680260 & 17269.78 & 52428.91 \\
\hline
\end{tabular}




\section{A.4 SPATIAL ANALYSIS OF VARIABLES}

\section{A.4.1 Equal-proportion-based assignment Method}

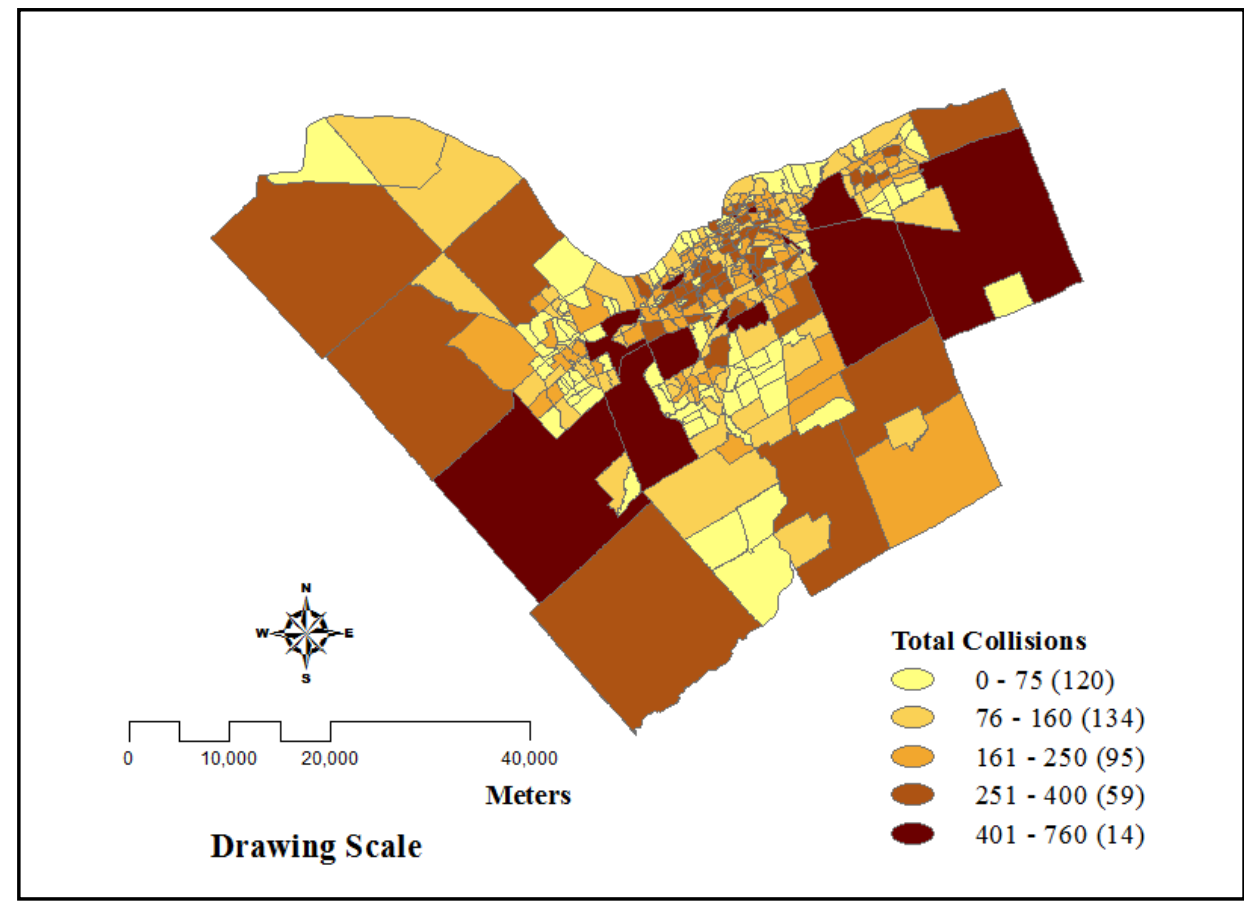

Figure A. 1 Spatial Distributions of Number of Total Collisions in the City of Ottawa.

Boundary Data Assigned Using Equal-proportion-based assignment Method.

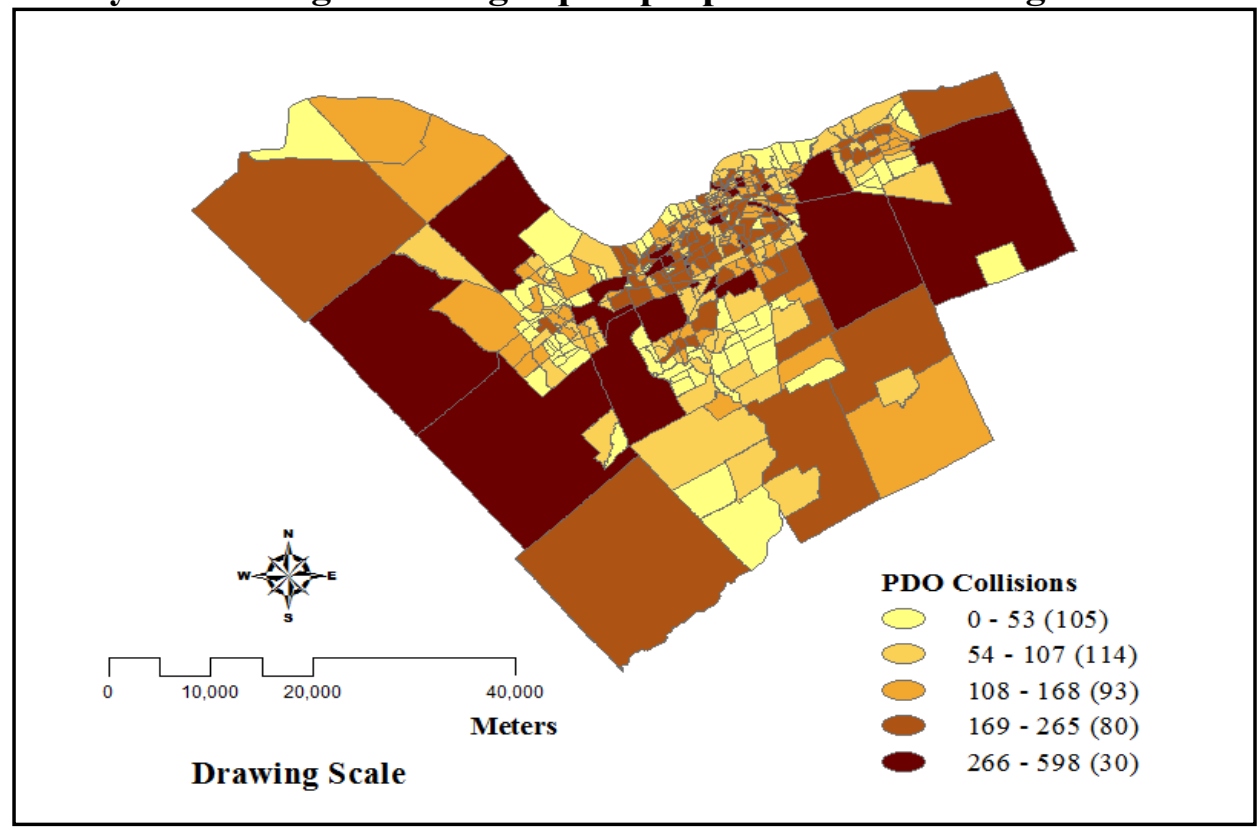

Figure A. 2 Spatial Distributions of Number of PDO Collisions in the City of Ottawa. Boundary Data Assigned Using Equal-proportion-based assignment Method. 


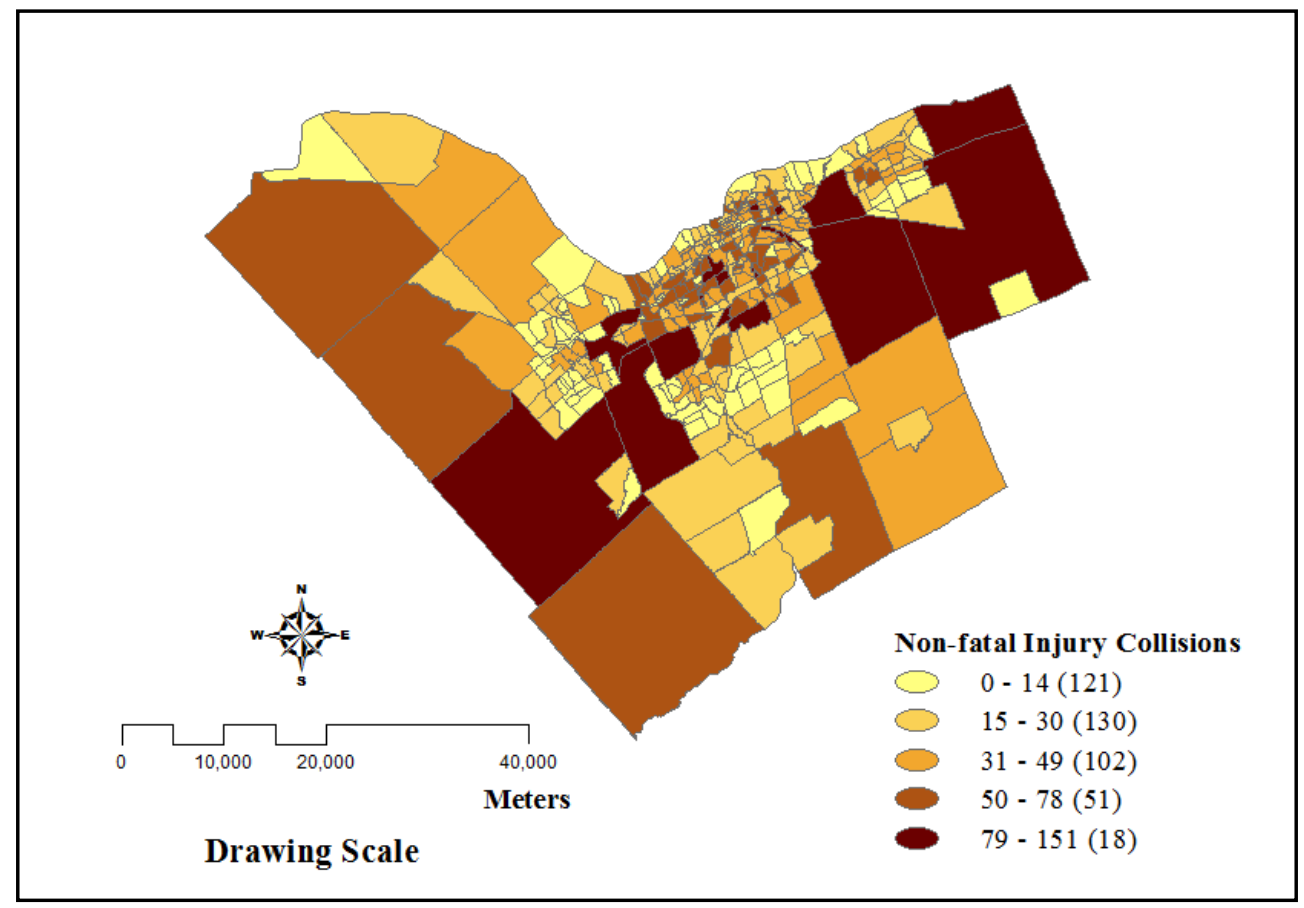

Figure A. 3 Spatial Distributions of Number of Non-fatal Injury Collisions in the City of Ottawa. Boundary Data Assigned Using Equal-proportion-based assignment Method.

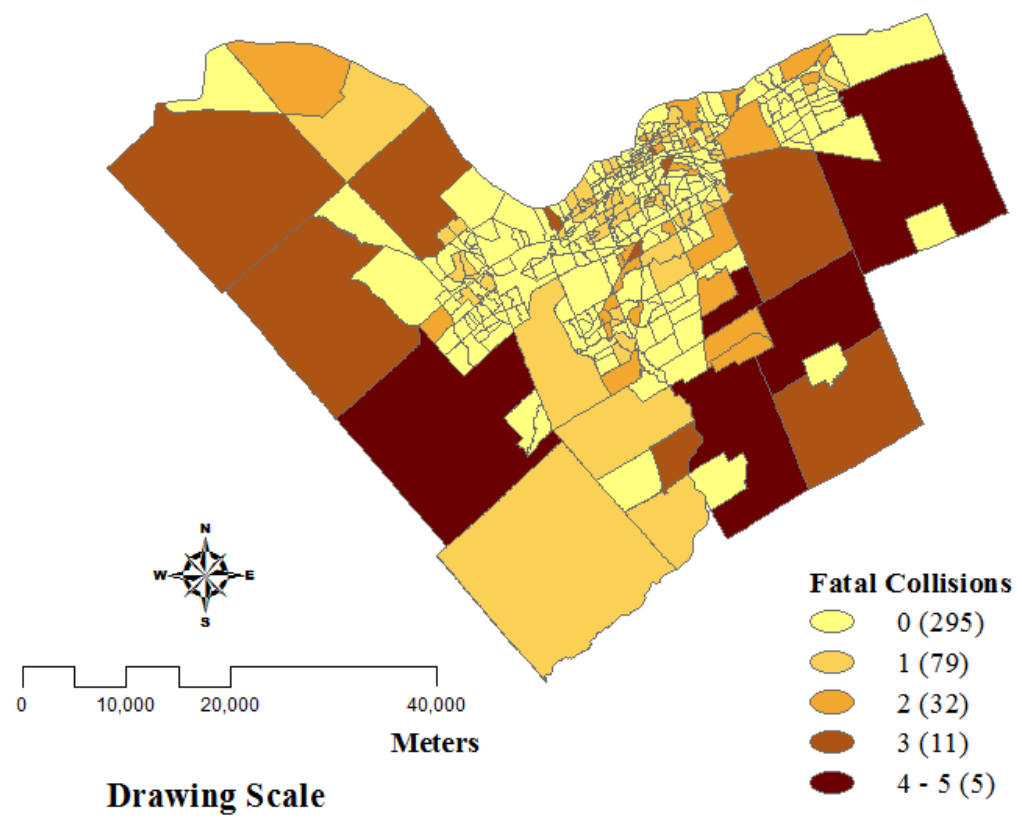

Figure A. 4 Spatial Distributions of Number of Fatal Collisions in the City of Ottawa. Boundary Data Assigned Using Equal-proportion-based assignment Method. 


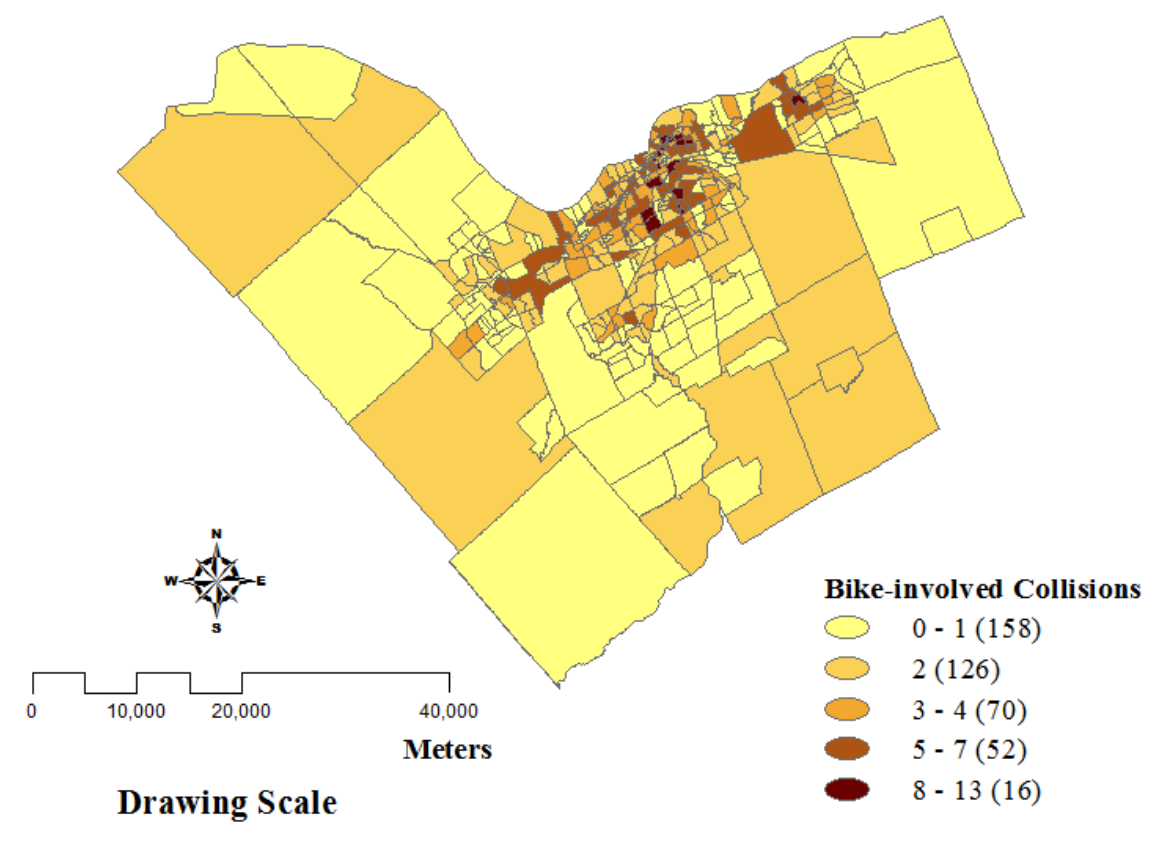

Figure A. 5 Spatial Distributions of Number of Bike-involved Collisions in the City of Ottawa. Boundary Data Assigned Using Equal-proportion-based assignment Method.

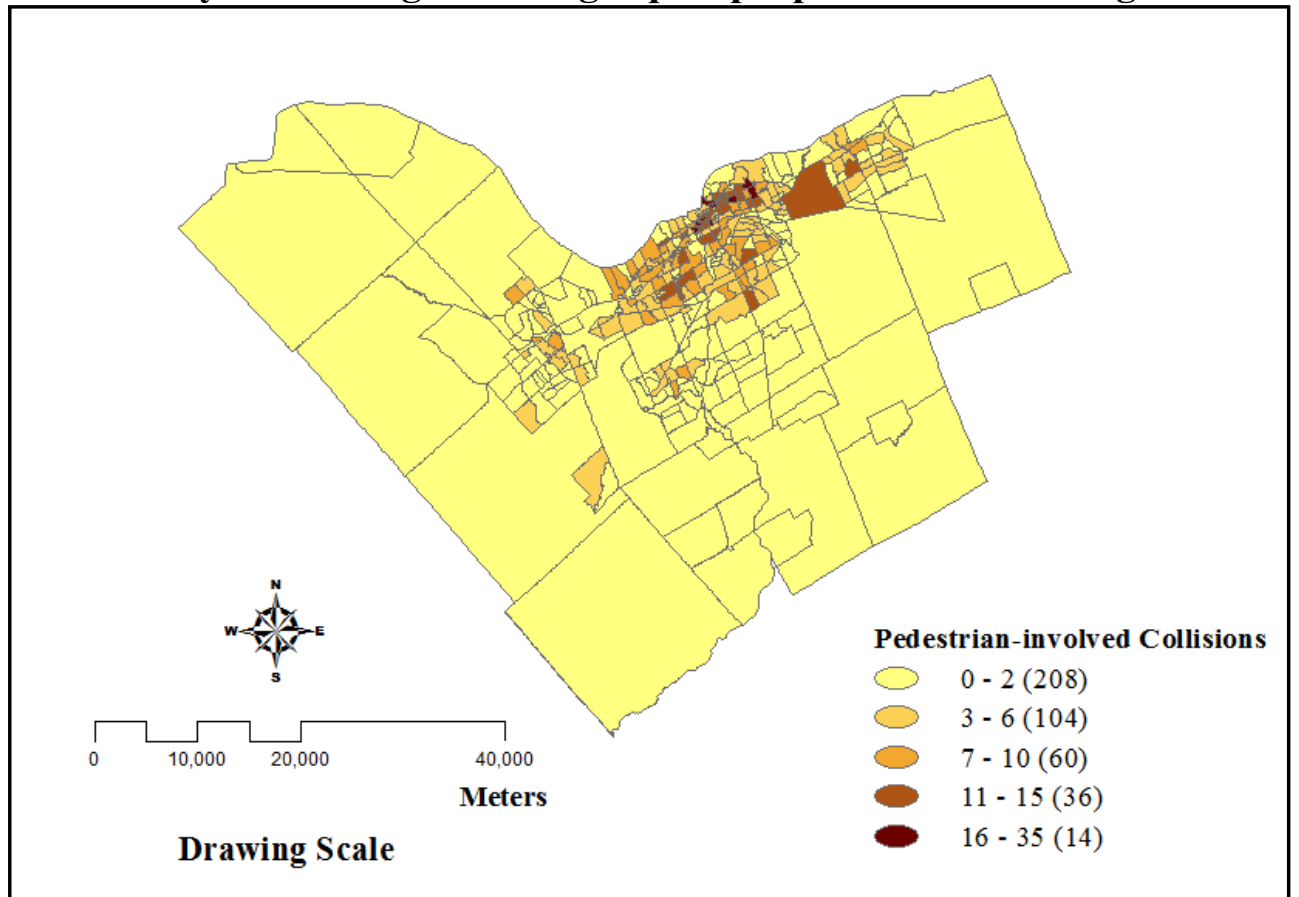

Figure A. 6 Spatial Distributions of Number of Pedestrian-involved Collisions in the City of Ottawa. Boundary Data Assigned Using Equal-proportion-based assignment Method. 


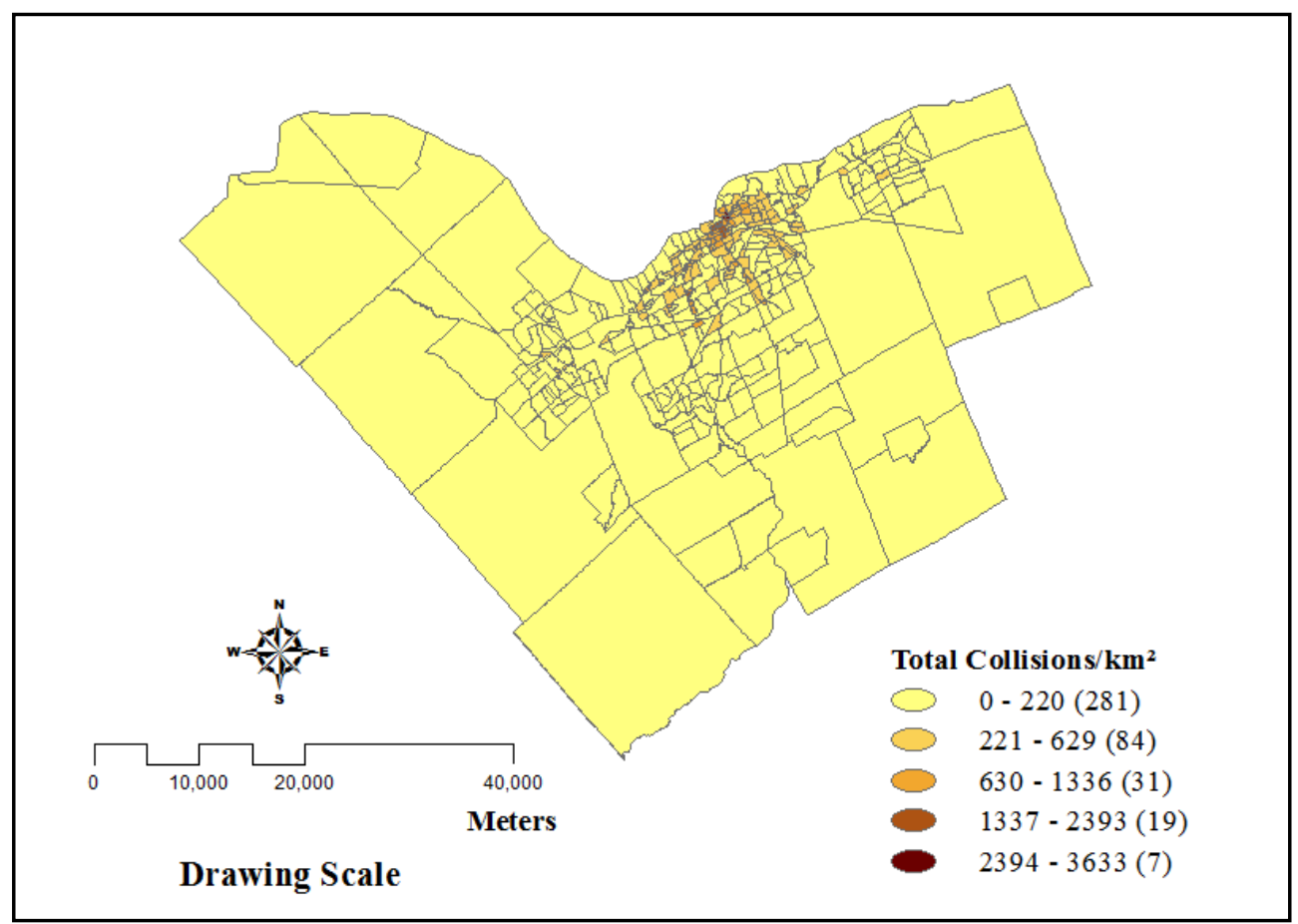

Figure A. 7 Spatial Distributions of Total Collisions Density in the City of Ottawa. Boundary Data Assigned Using Equal-proportion-based assignment Method. (Equal-

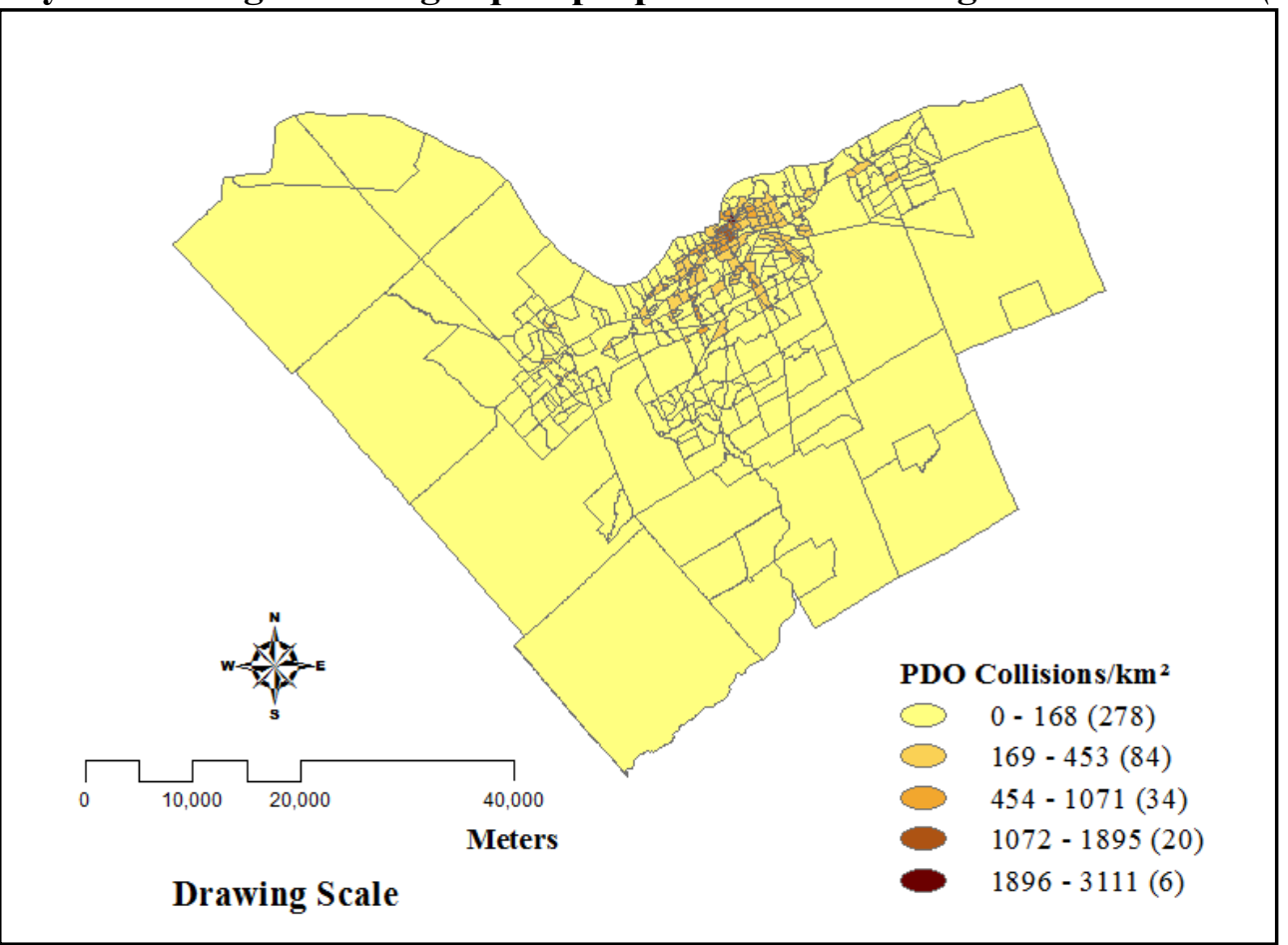

Figure A. 8 Spatial Distributions of PDO Collisions Density in the City of Ottawa. Boundary Data Assigned Using Equal-proportion-based assignment Method. (Equal- 


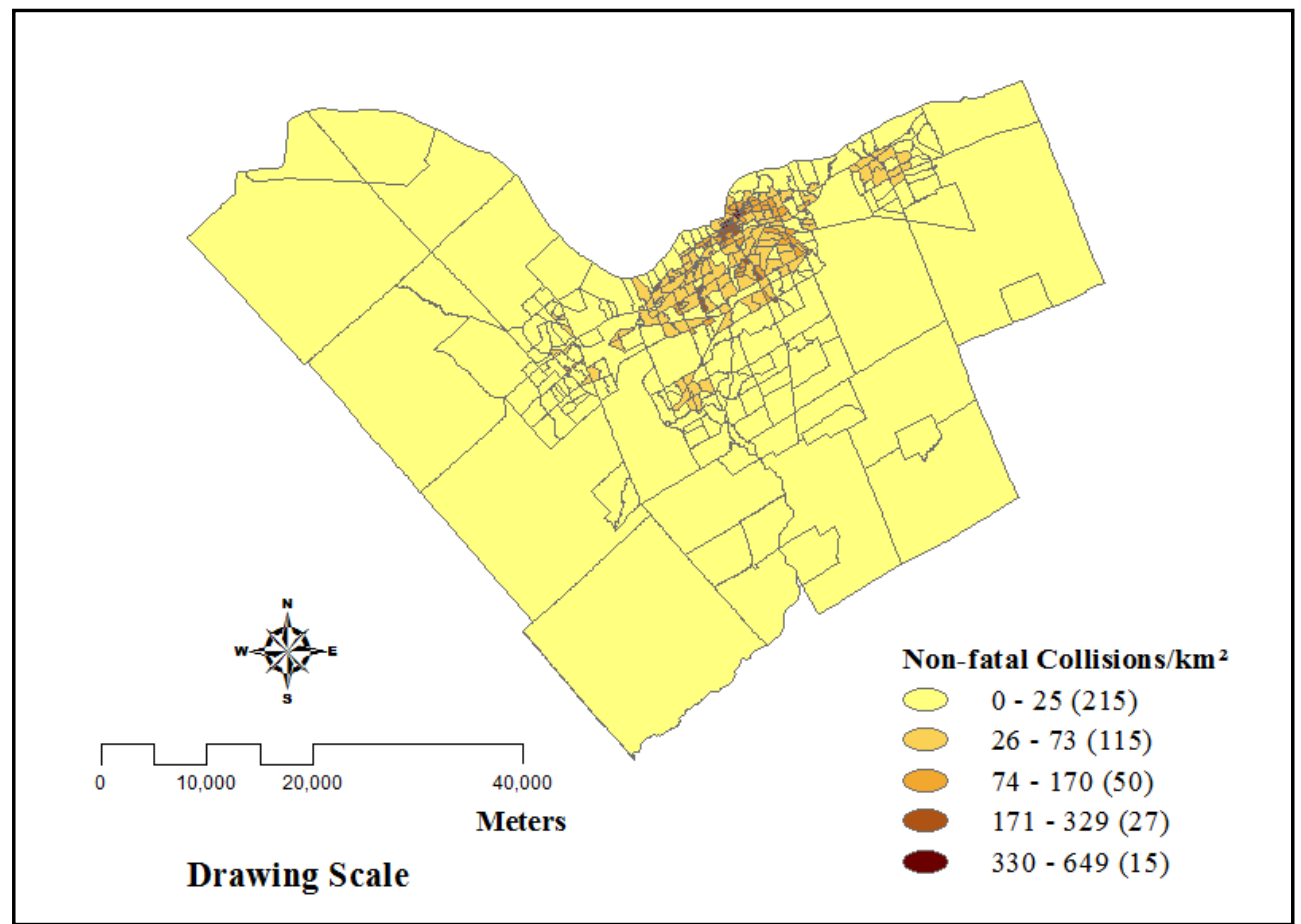

Figure A. 9 Spatial Distributions of Non-fatal Injury Collisions Density in the City of Ottawa. Boundary Data Assigned Using Equal-proportion-based assignment Method.

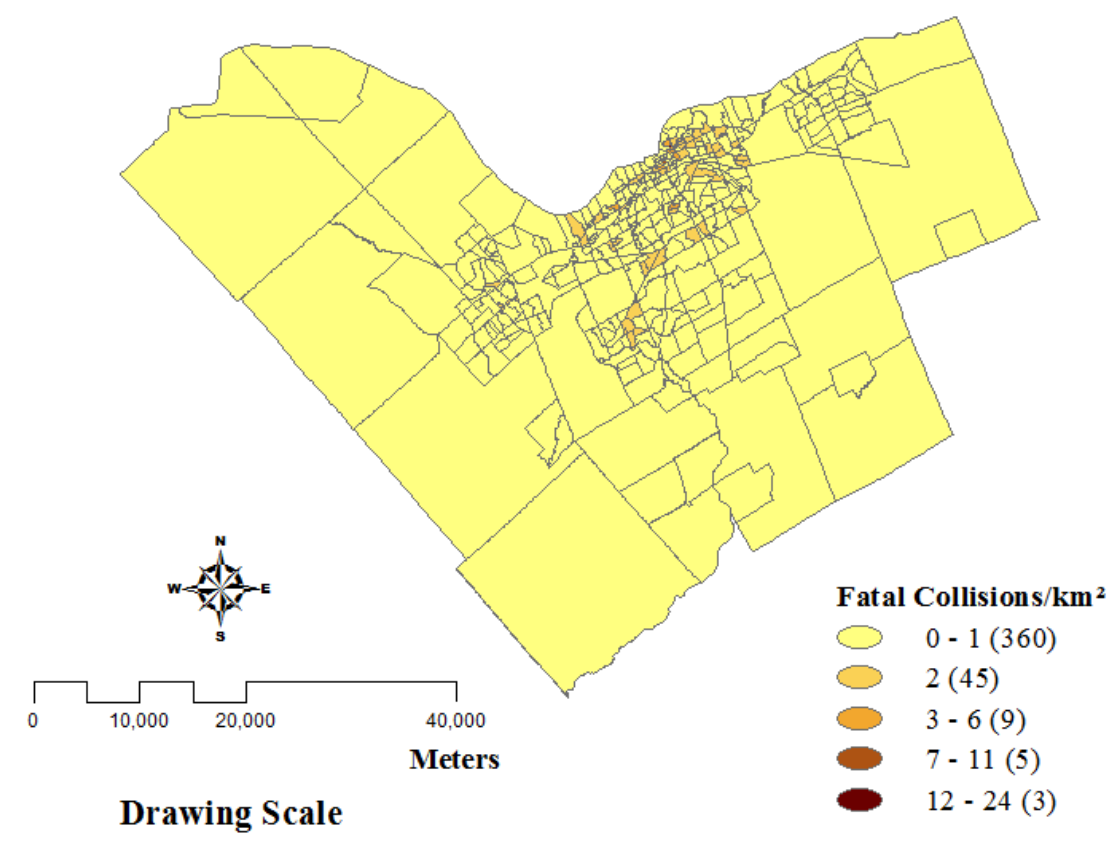

Figure A. 10 Spatial Distributions of Fatal Collisions Density in the City of Ottawa. Boundary Data Assigned Using Equal-proportion-based assignment Method. (Equal- 


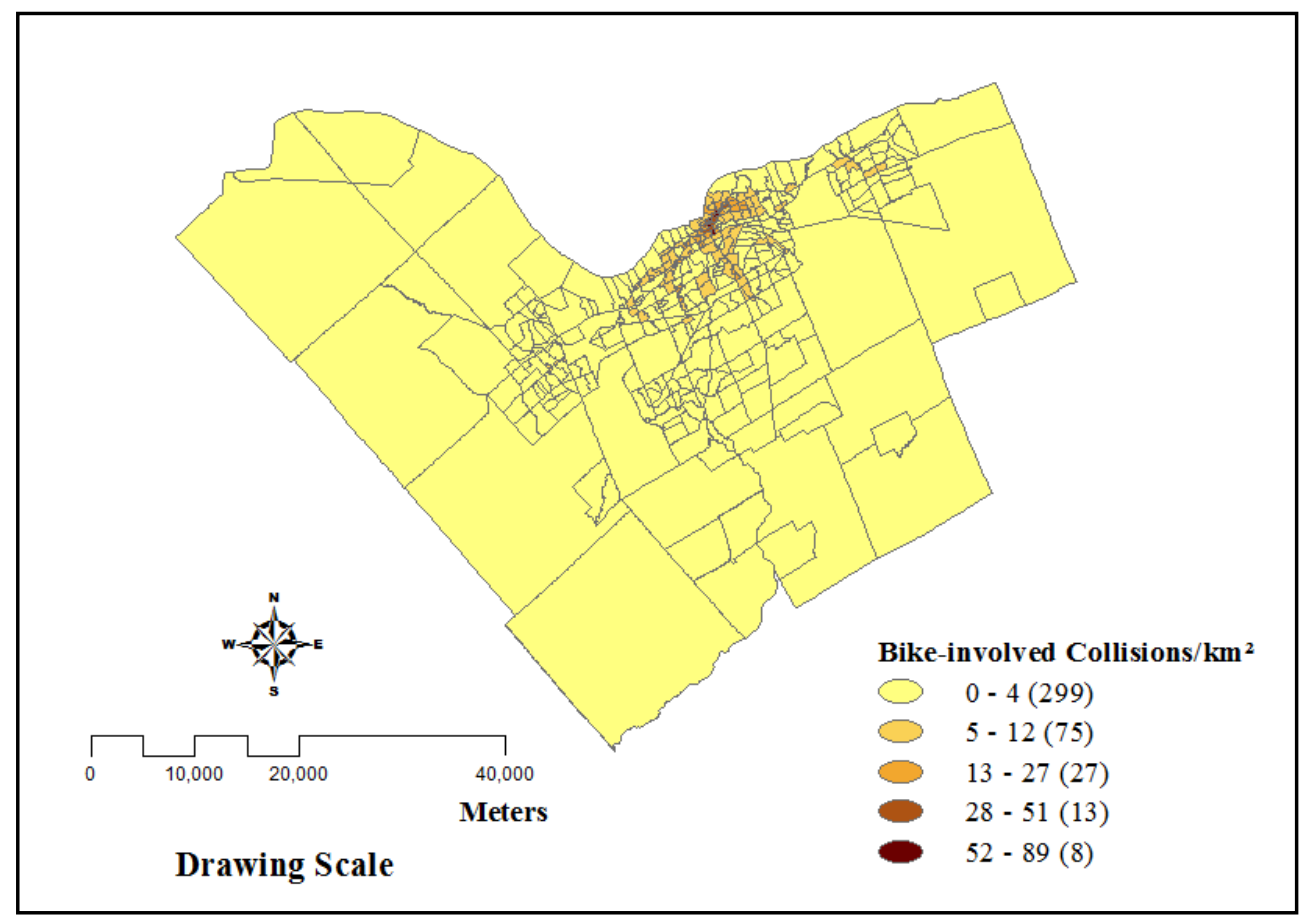

Figure A. 11 Spatial Distributions of Bike-involved Collisions Density in the City of Ottawa. Boundary Data Assigned Using Equal-proportion-based assignment Method.

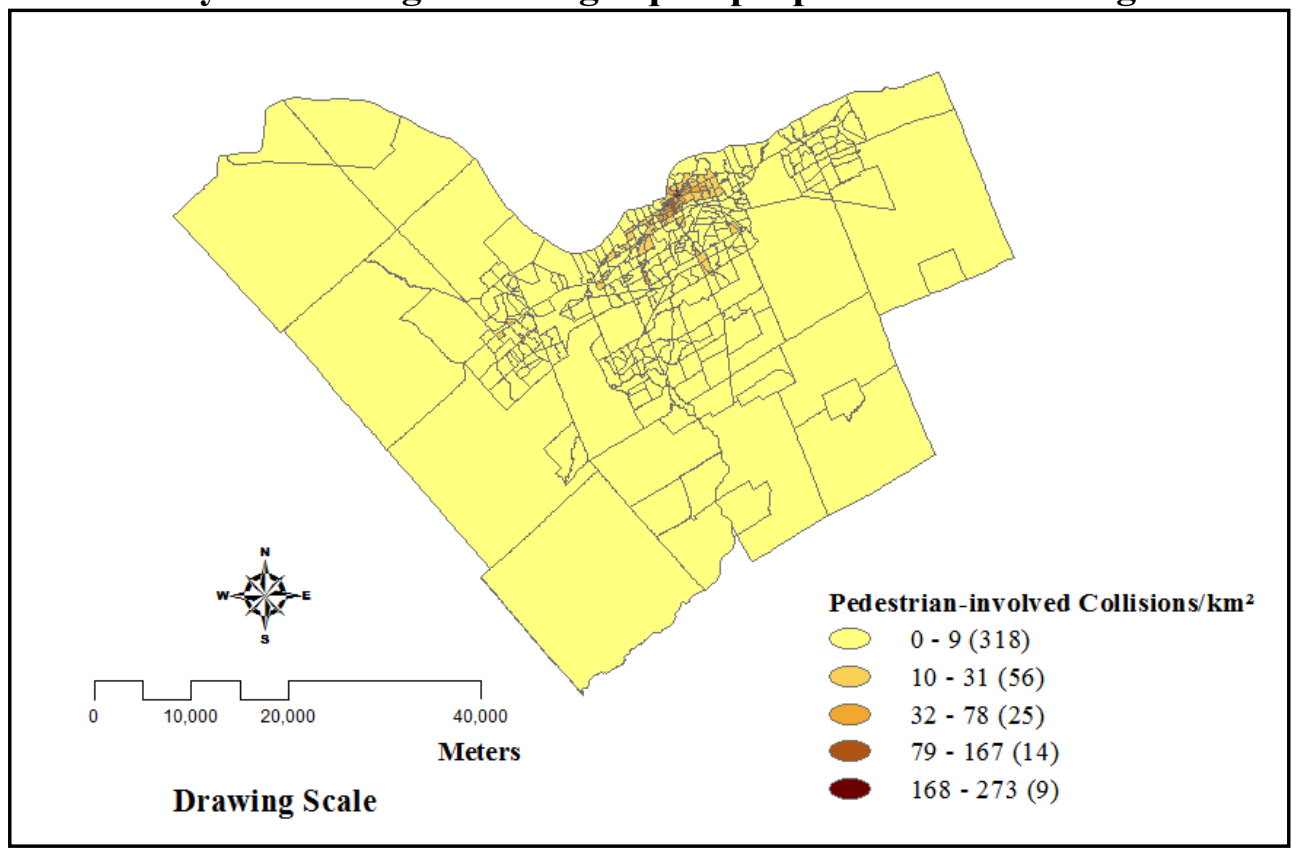

Figure A. 12 Spatial Distributions of Pedestrian-involved Collisions Density in the City of Ottawa. Boundary Data Assigned Using Equal-proportion-based assignment Method. 


\section{A.4.2 Population-based Assignment Method}

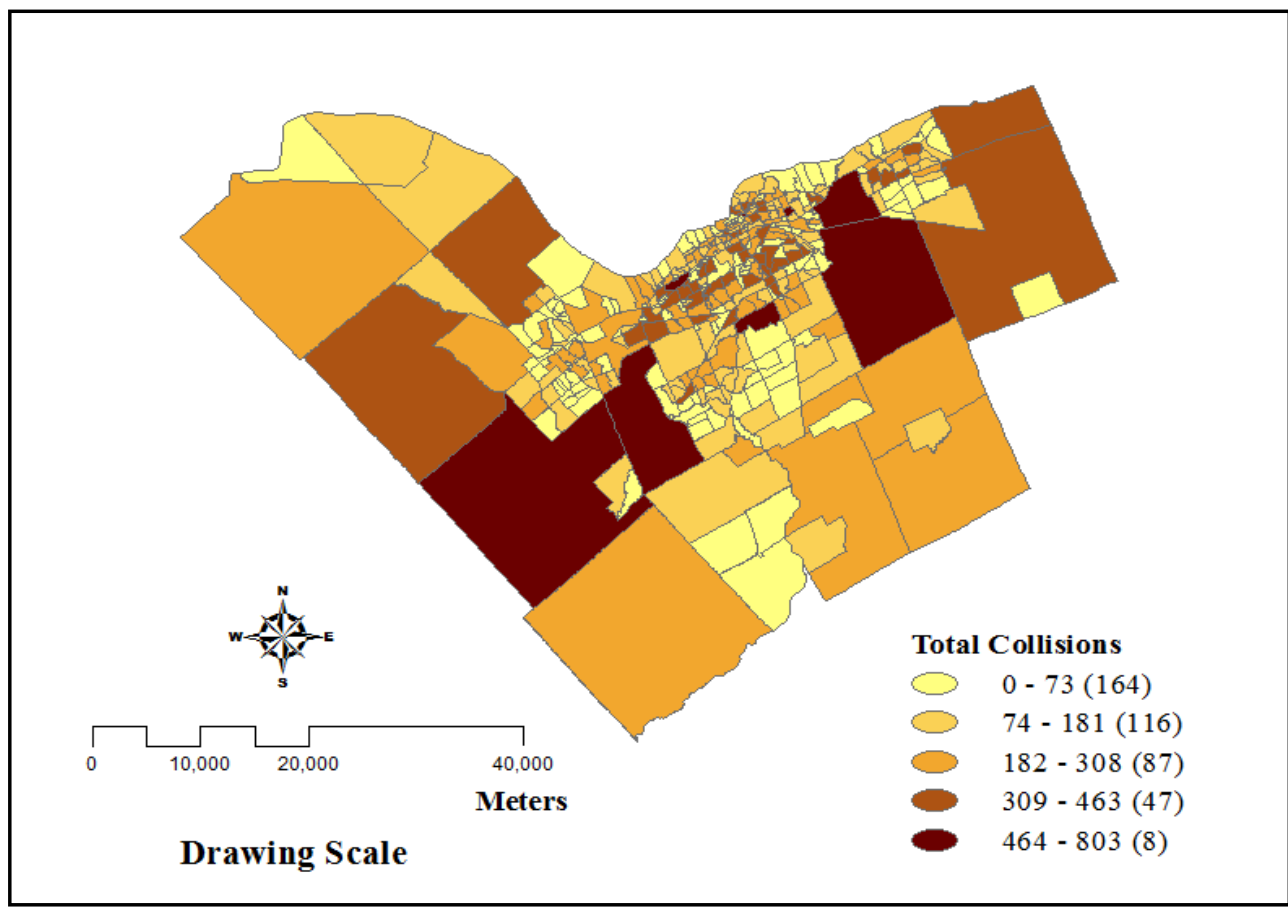

Figure A. 13 Spatial Distributions of Number of Total Collisions in the City of Ottawa. Boundary Data Assigned Using Population-based assignment Method.(Population-based Assignment Method)

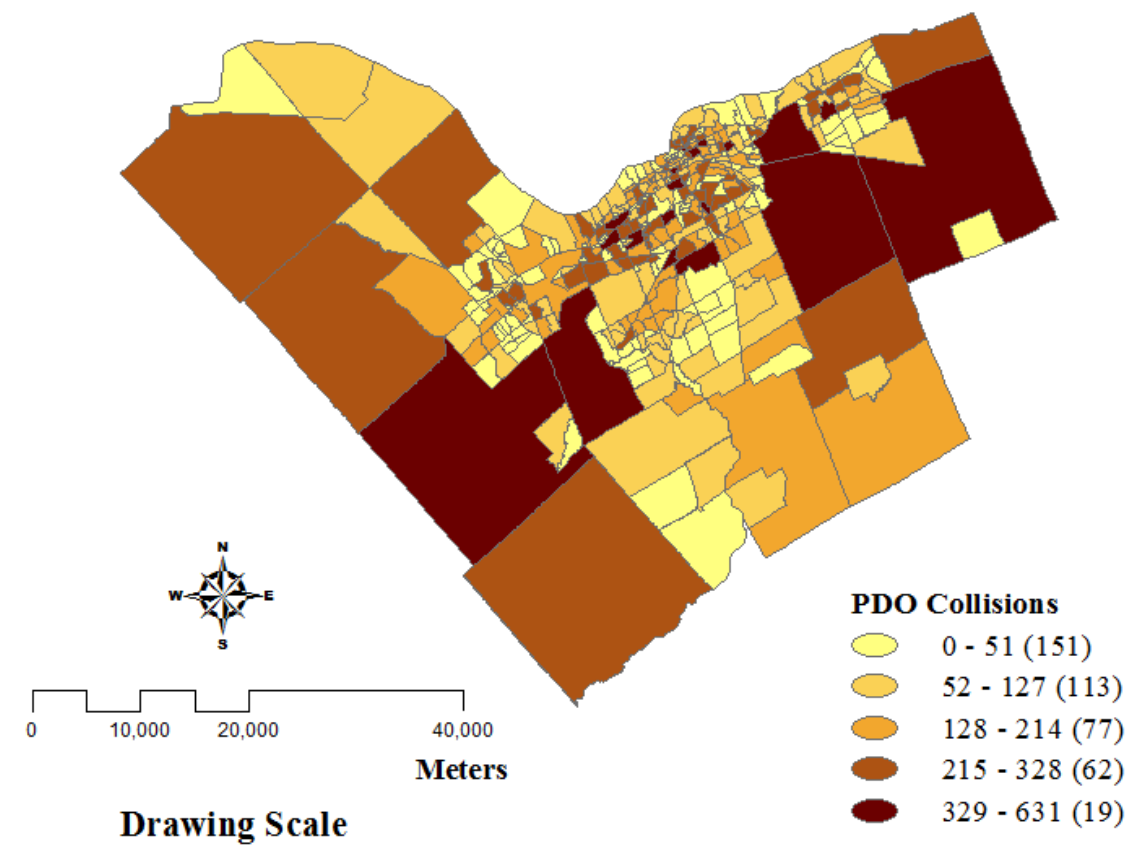

Figure A. 14 Spatial Distributions of Number of PDO Collisions in the City of Ottawa. Boundary Data Assigned Using Population-based assignment Method. 


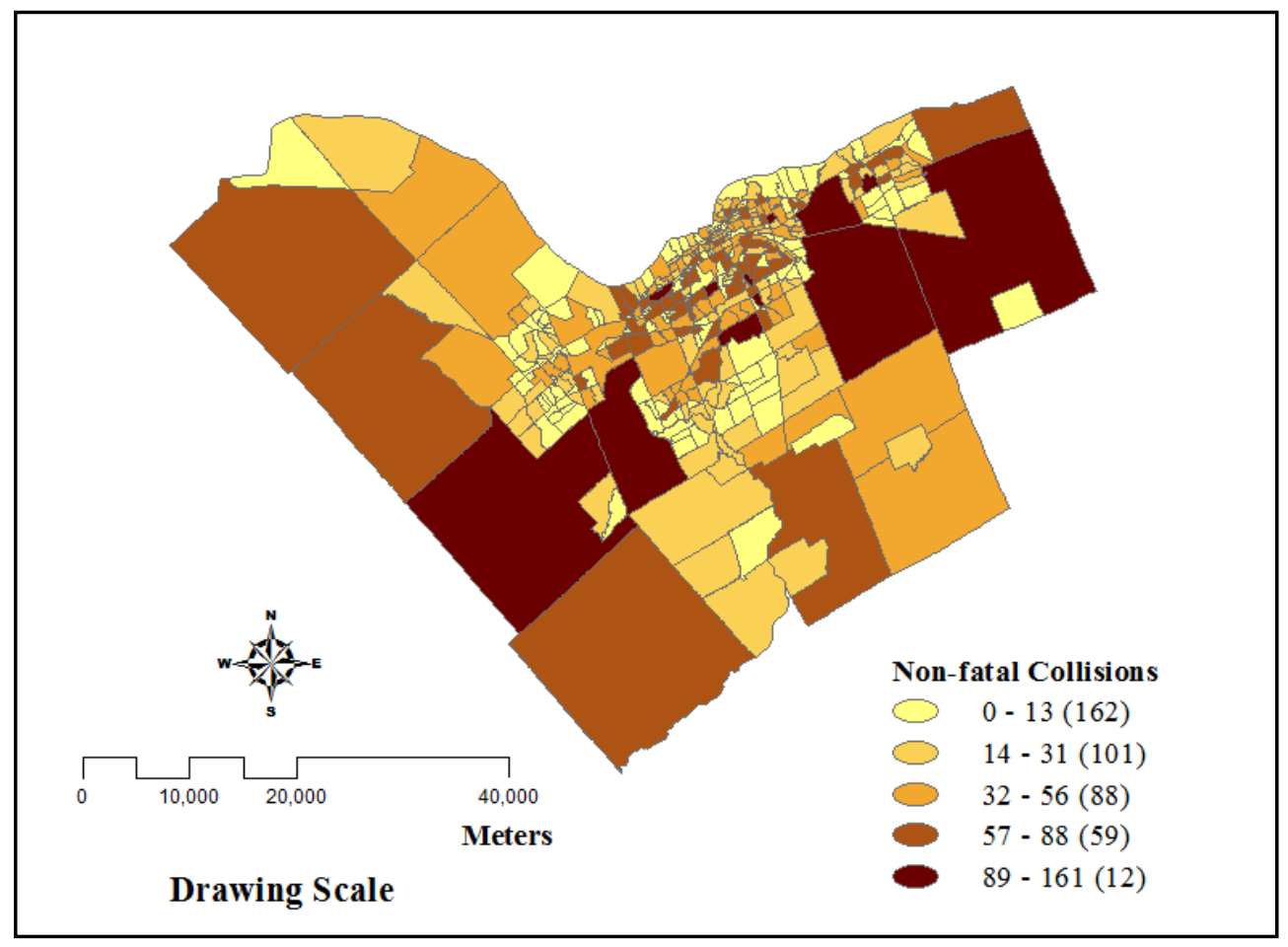

Figure A. 15 Spatial Distributions of Number of Non-fatal Injury Collisions in the City of Ottawa. Boundary Data Assigned Using Population-based assignment Method.

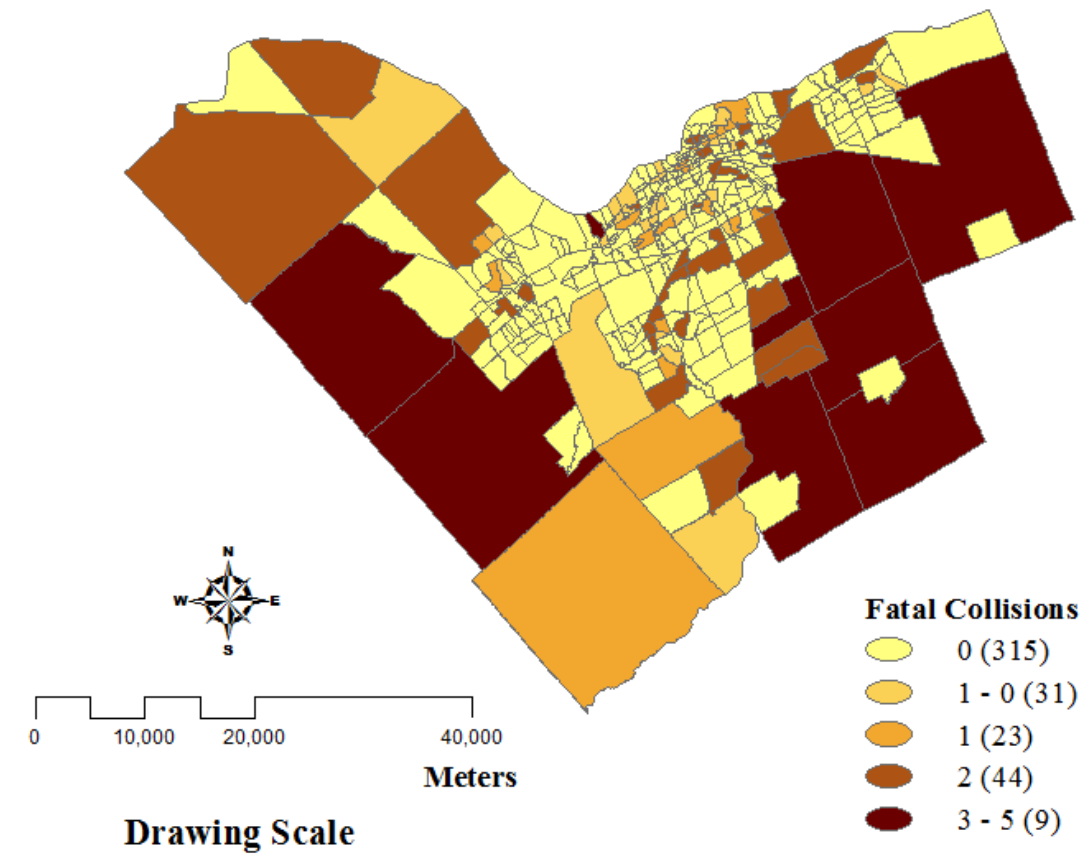

Figure A. 16 Spatial Distributions of Number of Fatal Collisions in the City of Ottawa. Boundary Data Assigned Using Population-based assignment Method. 


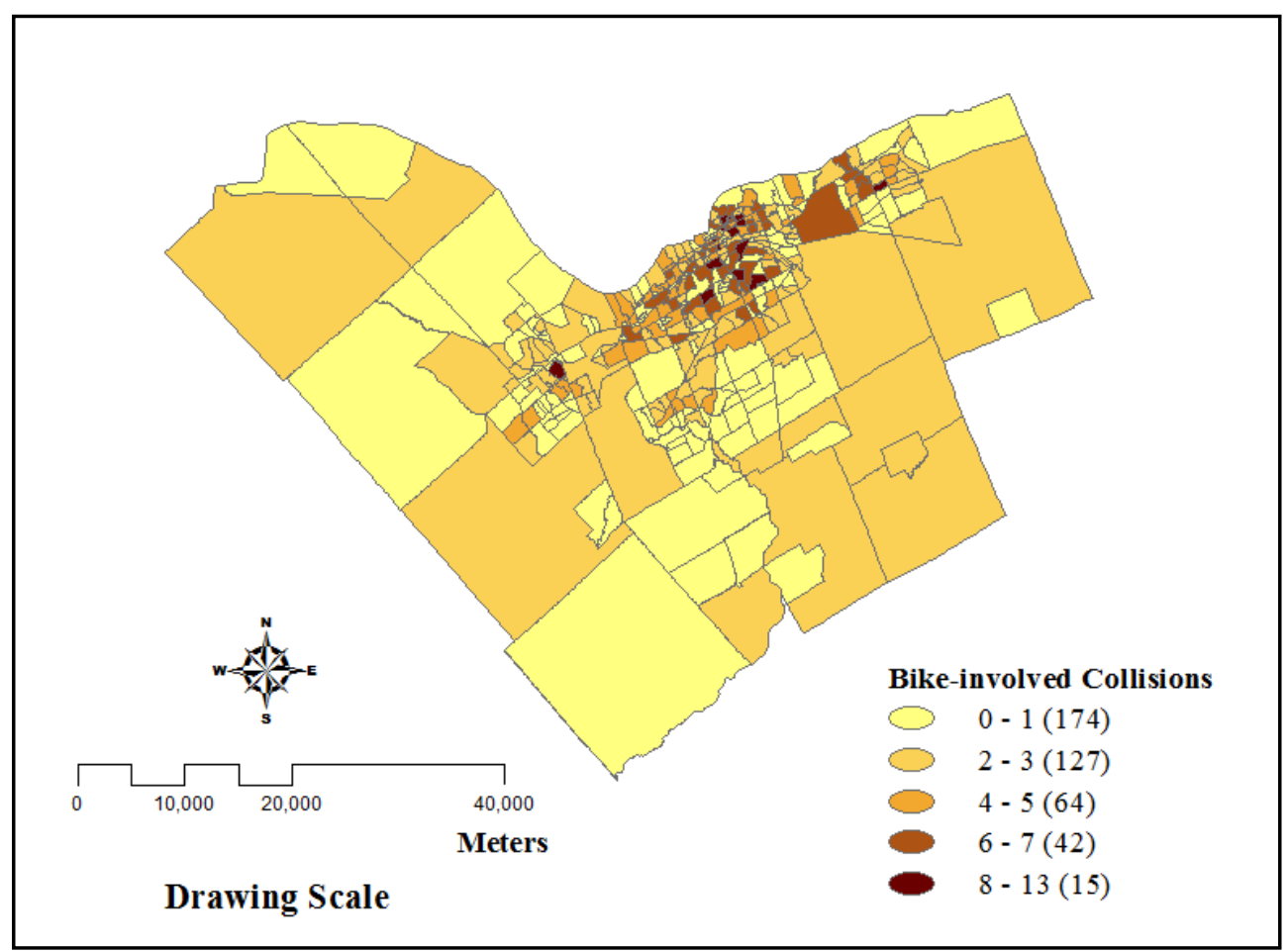

Figure A. 17 Spatial Distributions of Number of Bike-involved Collisions in the City of Ottawa. Boundary Data Assigned Using Population-based assignment Method.

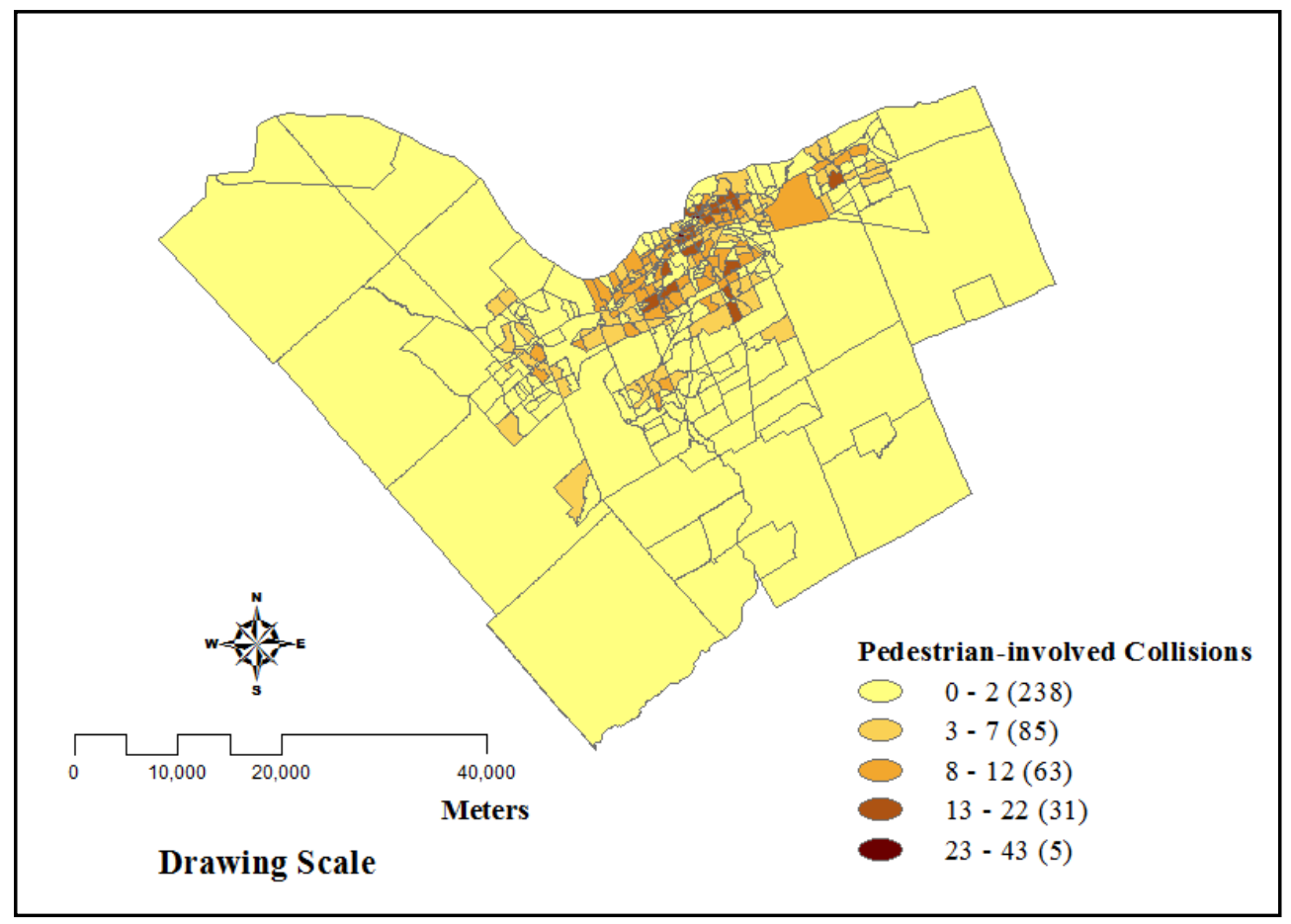

Figure A. 18 Spatial Distributions of Number of Pedestrian-involved Collisions in the City of Ottawa. Boundary Data Assigned Using Population-based assignment Method. 


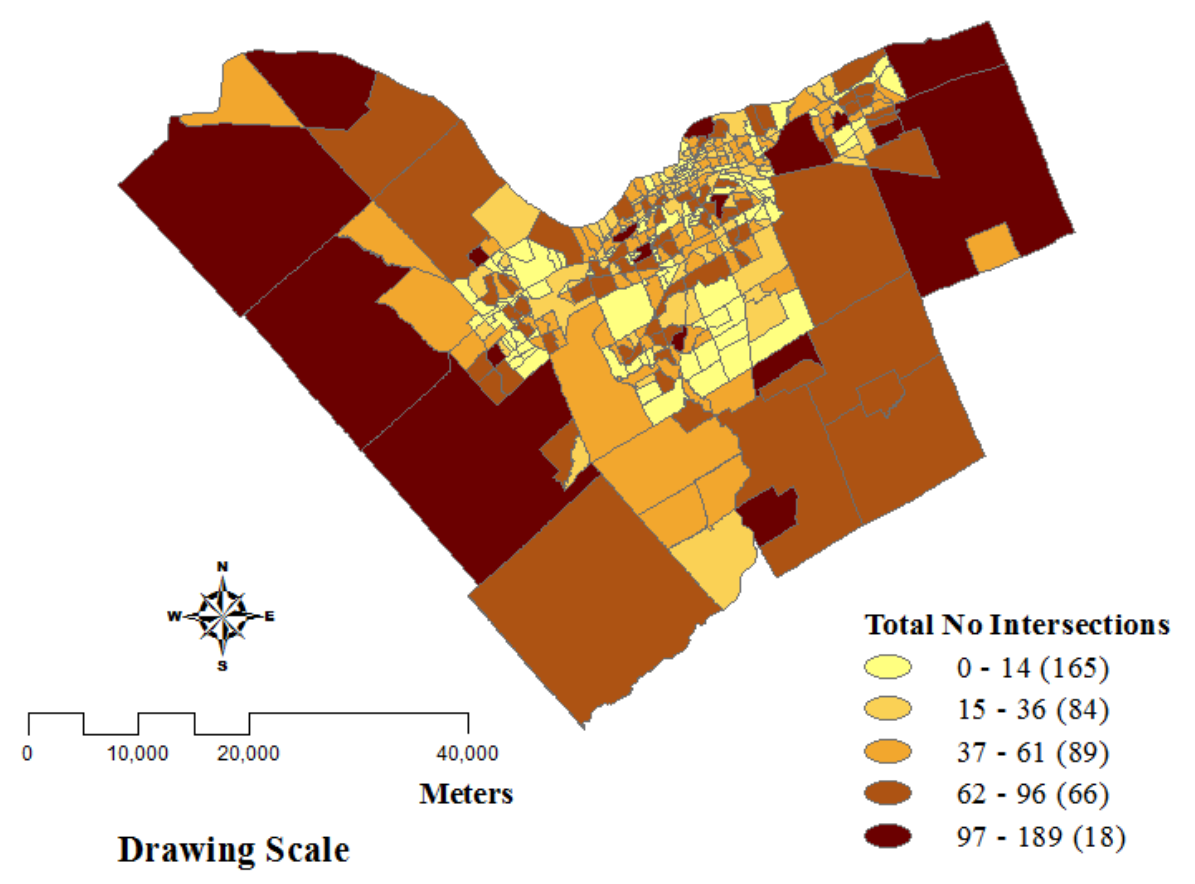

Figure A. 19 Spatial Distributions of Number of Intersections in the City of Ottawa. Boundary Data Assigned Using Population-based assignment Method.

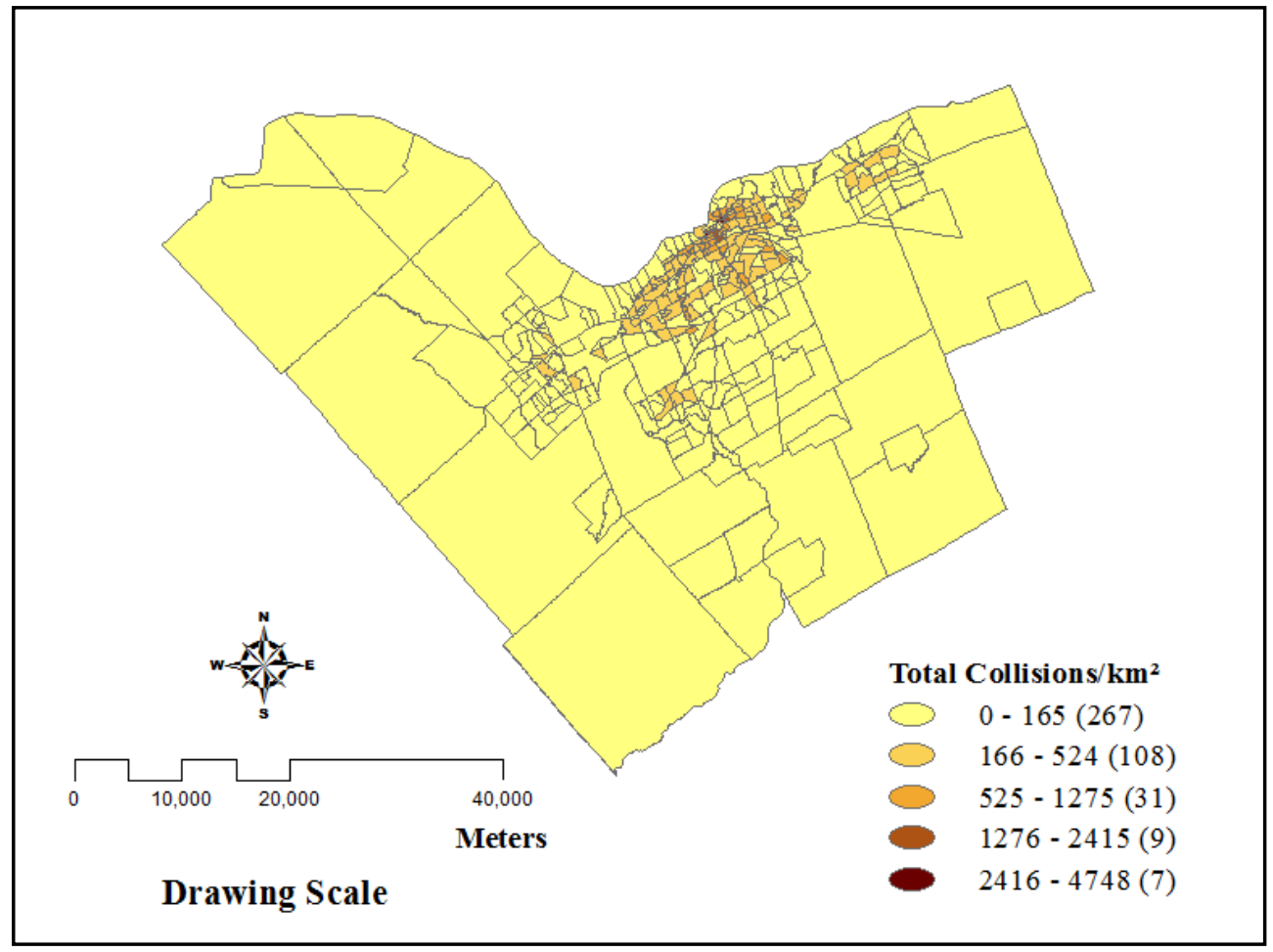

Figure A. 20 Spatial Distributions of Total Collisions Density in the City of Ottawa Boundary Data Assigned Using Population-based Assignment Method. 


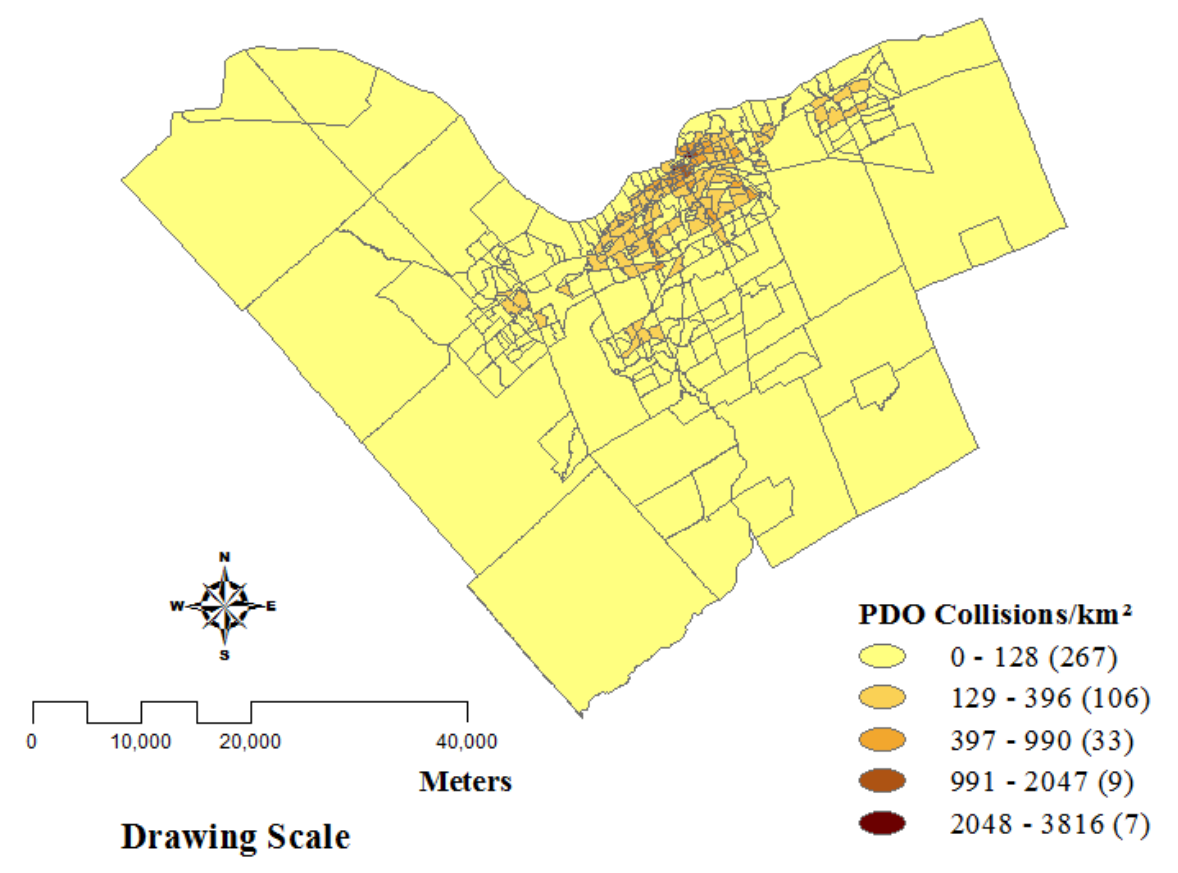

Figure A. 21 Spatial Distributions of PDO Collisions Density in the City of Ottawa. Boundary Data Assigned Using Population-based Assignment Method.

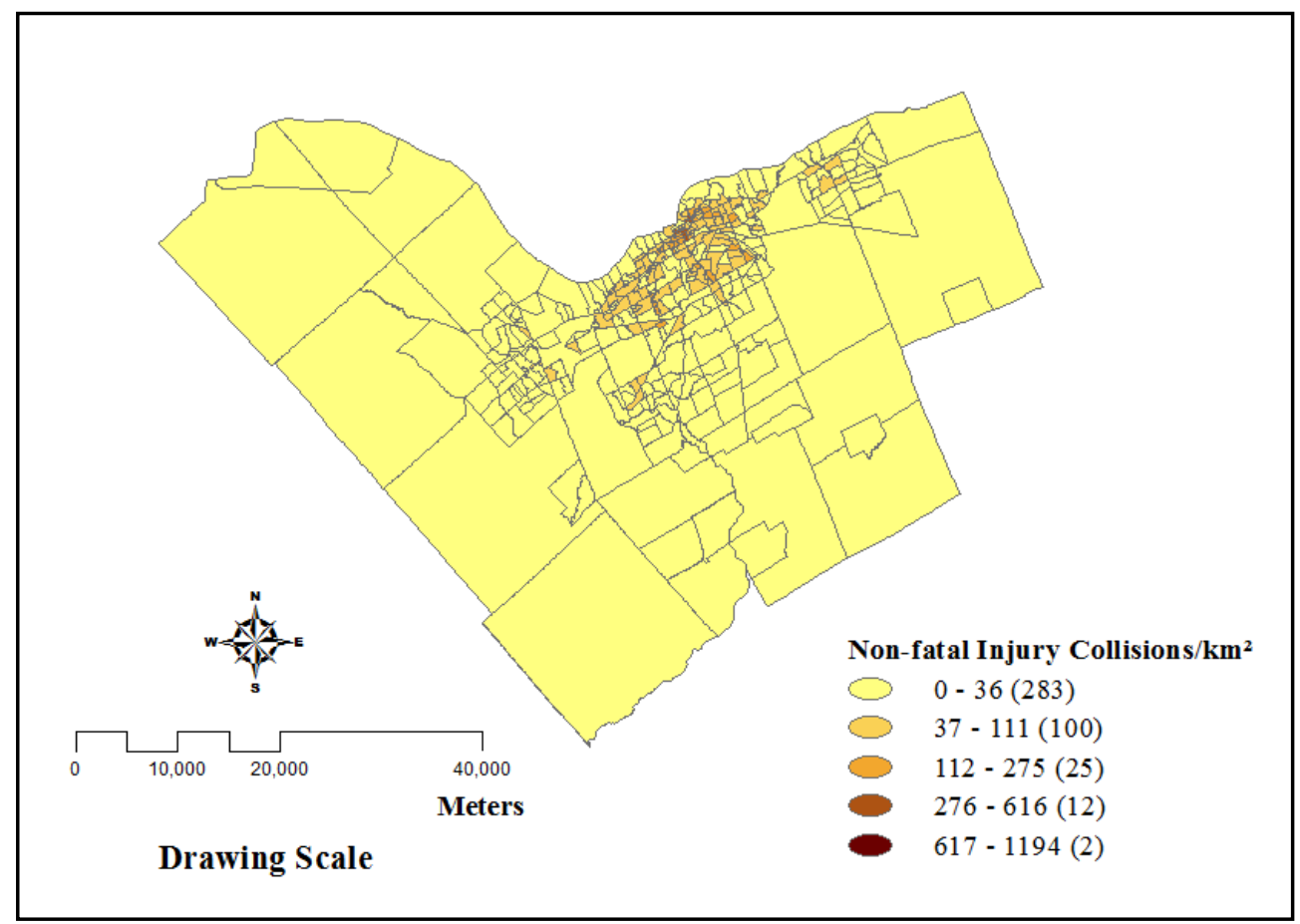

Figure A. 22 Spatial Distributions of Non-fatal Injury Collisions Density in the City of Ottawa. Boundary Data Assigned Using Population-based Assignment Method. 


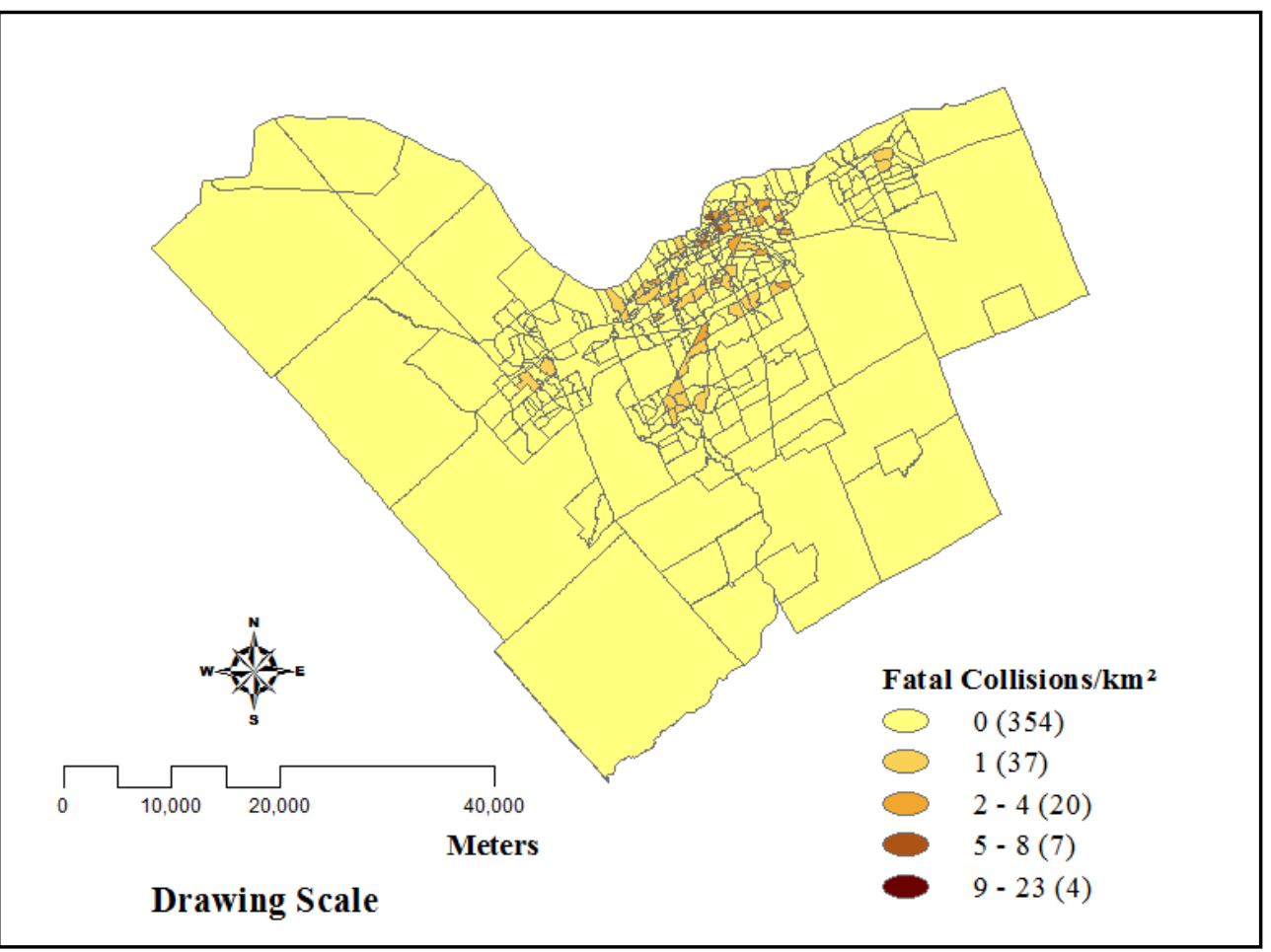

Figure A. 23 Spatial Distributions of Fatal Collisions Density in the City of Ottawa. Boundary Data Assigned Using Population-based Assignment Method.

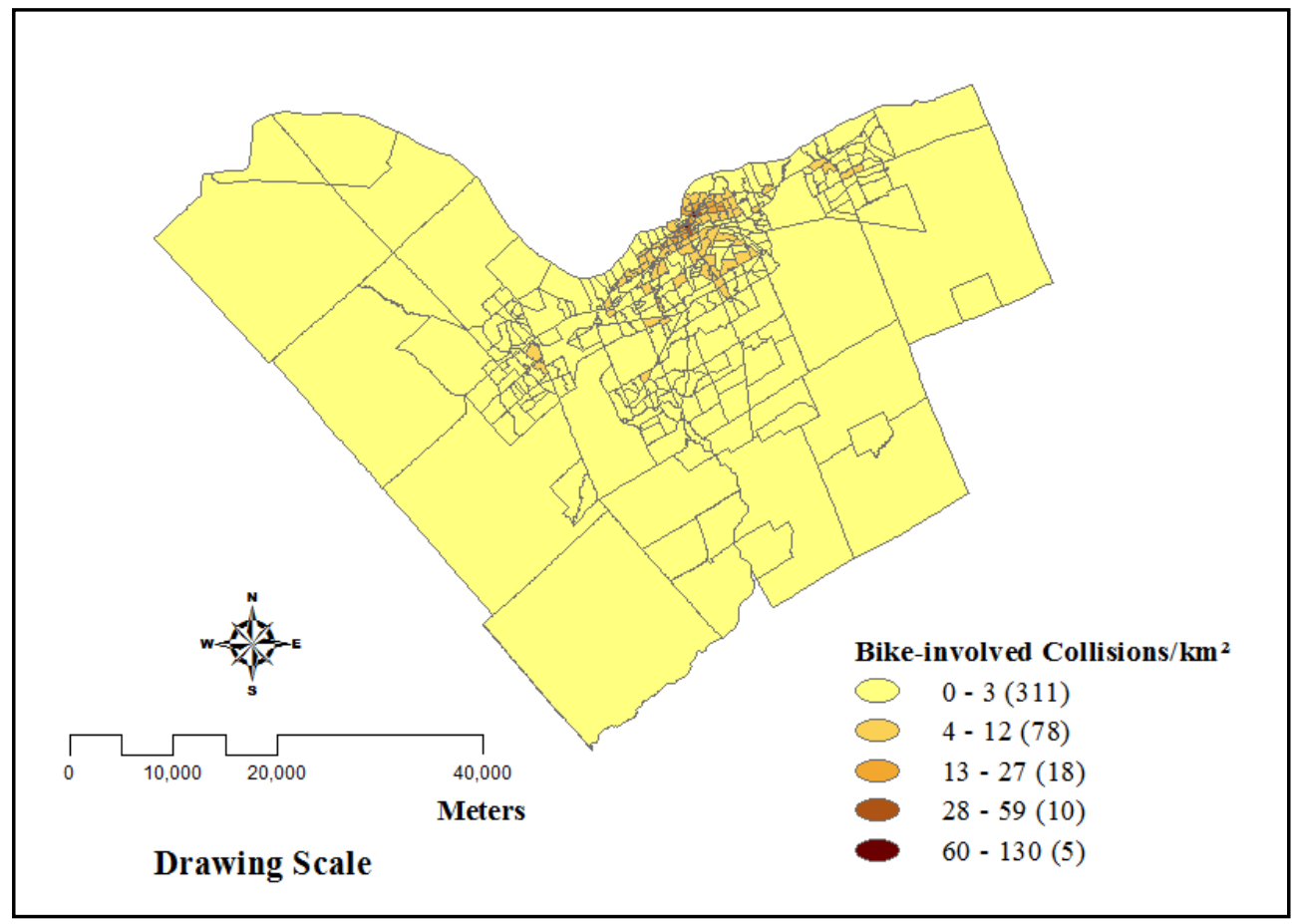

Figure A. 24 Spatial Distributions of Bike-involved Collisions Density in the City of Ottawa. Boundary Data Assigned Using Population-based Assignment Method. 


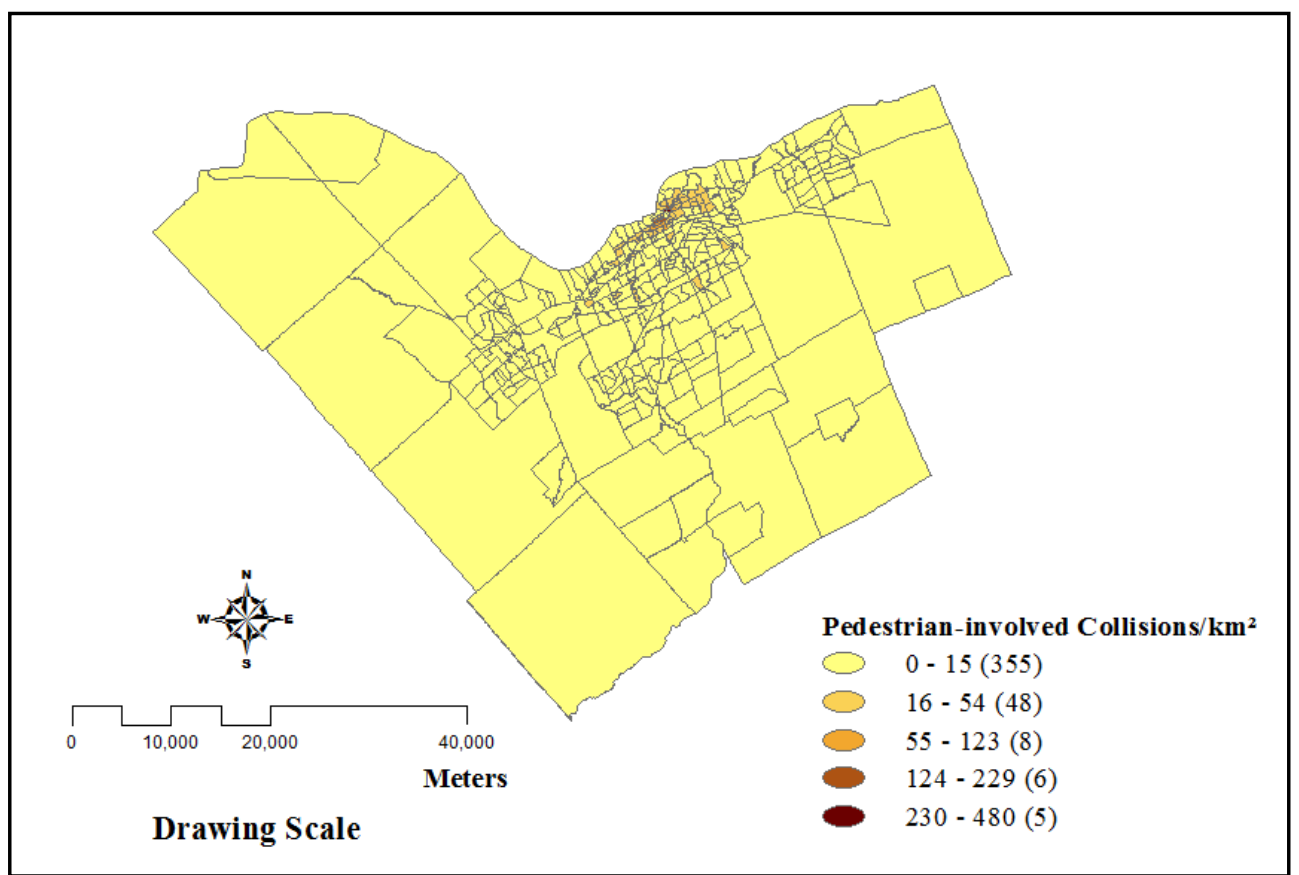

Figure A. 25 Spatial Distributions of Pedestrian-involved Collisions Density in the City of Ottawa. Boundary Data Assigned Using Population-based Assignment Method.

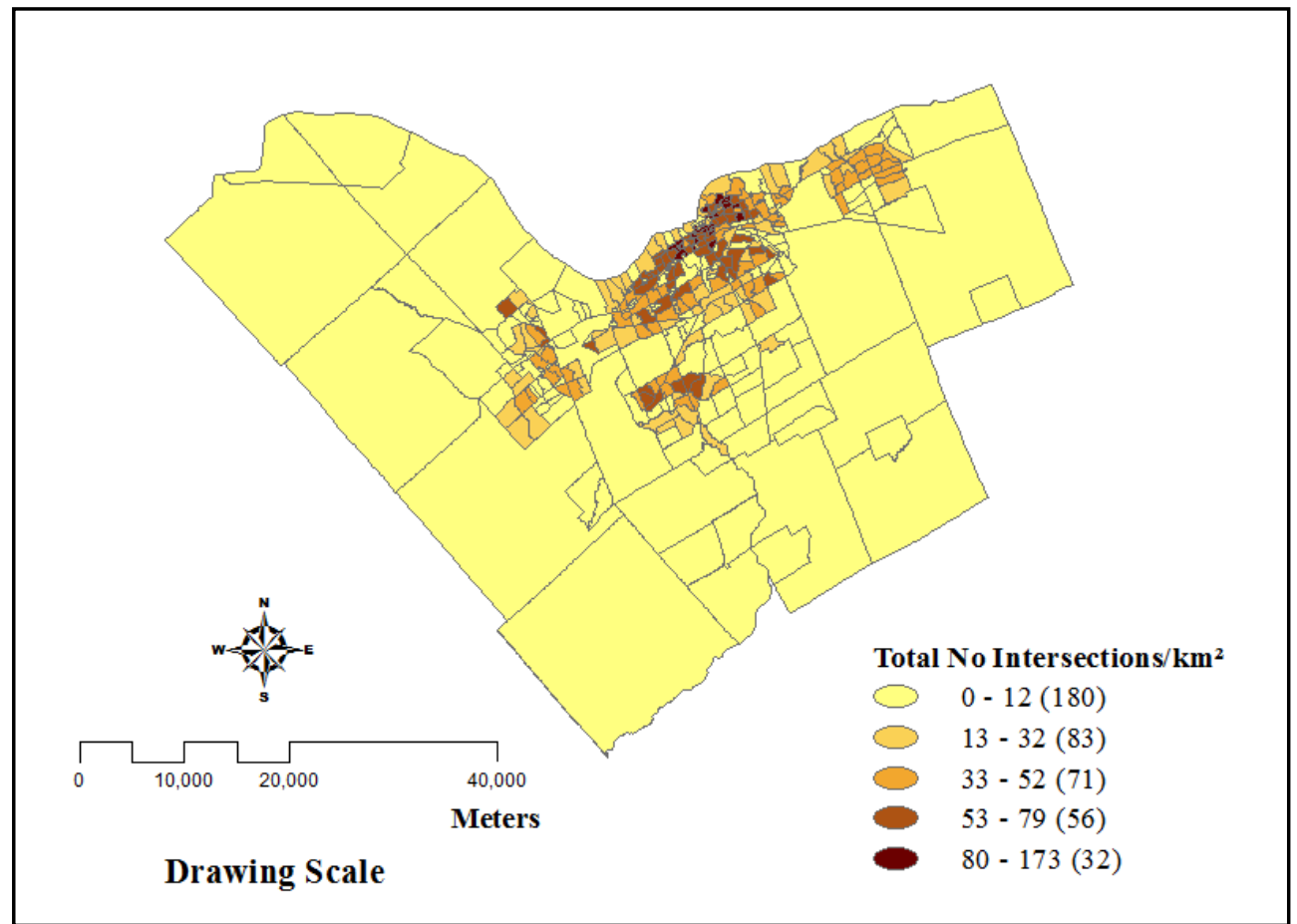

Figure A. 26 Spatial Distributions of Total Intersection Density in the City of Ottawa Boundary Data Assigned Using Population-based Assignment Method. 


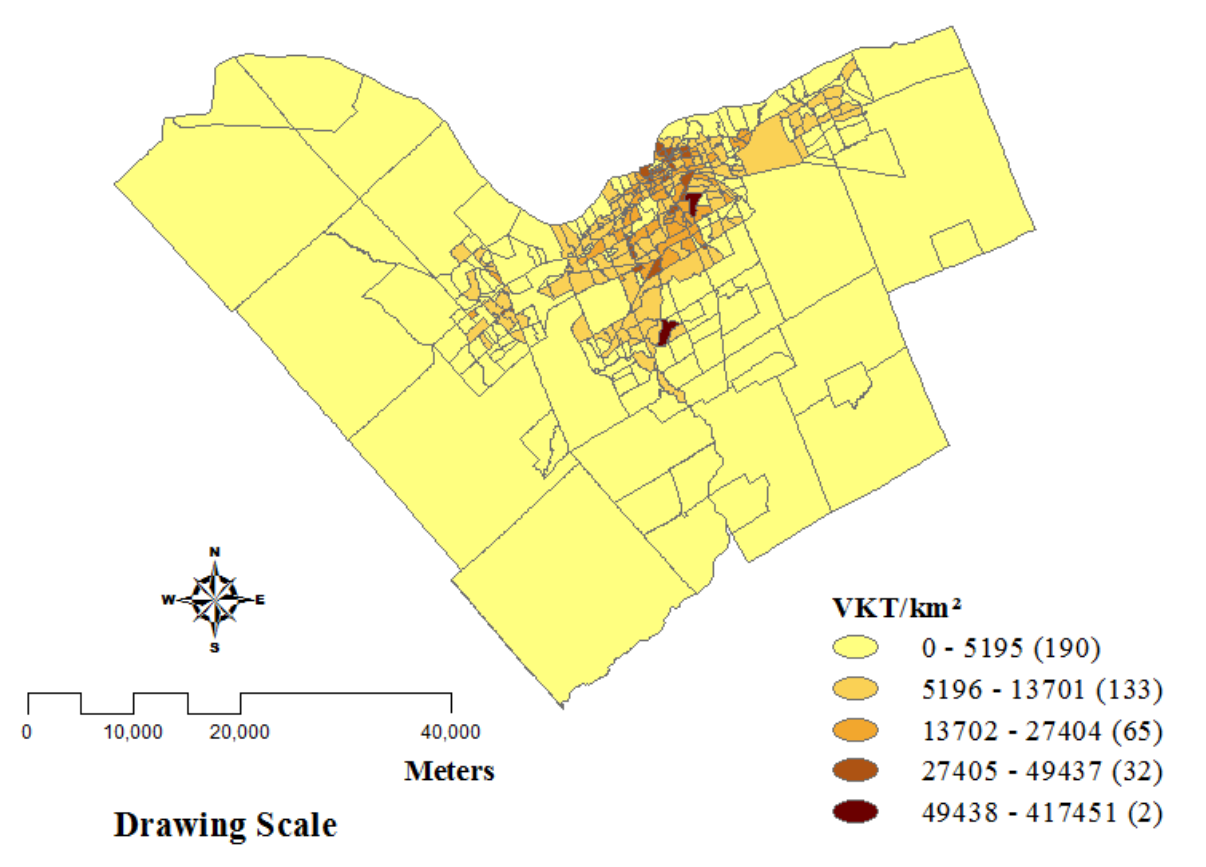

Figure A. 27 Spatial Distributions of VKT Density in the City of Ottawa. Boundary Data Assigned Using Population-based Assignment Method.

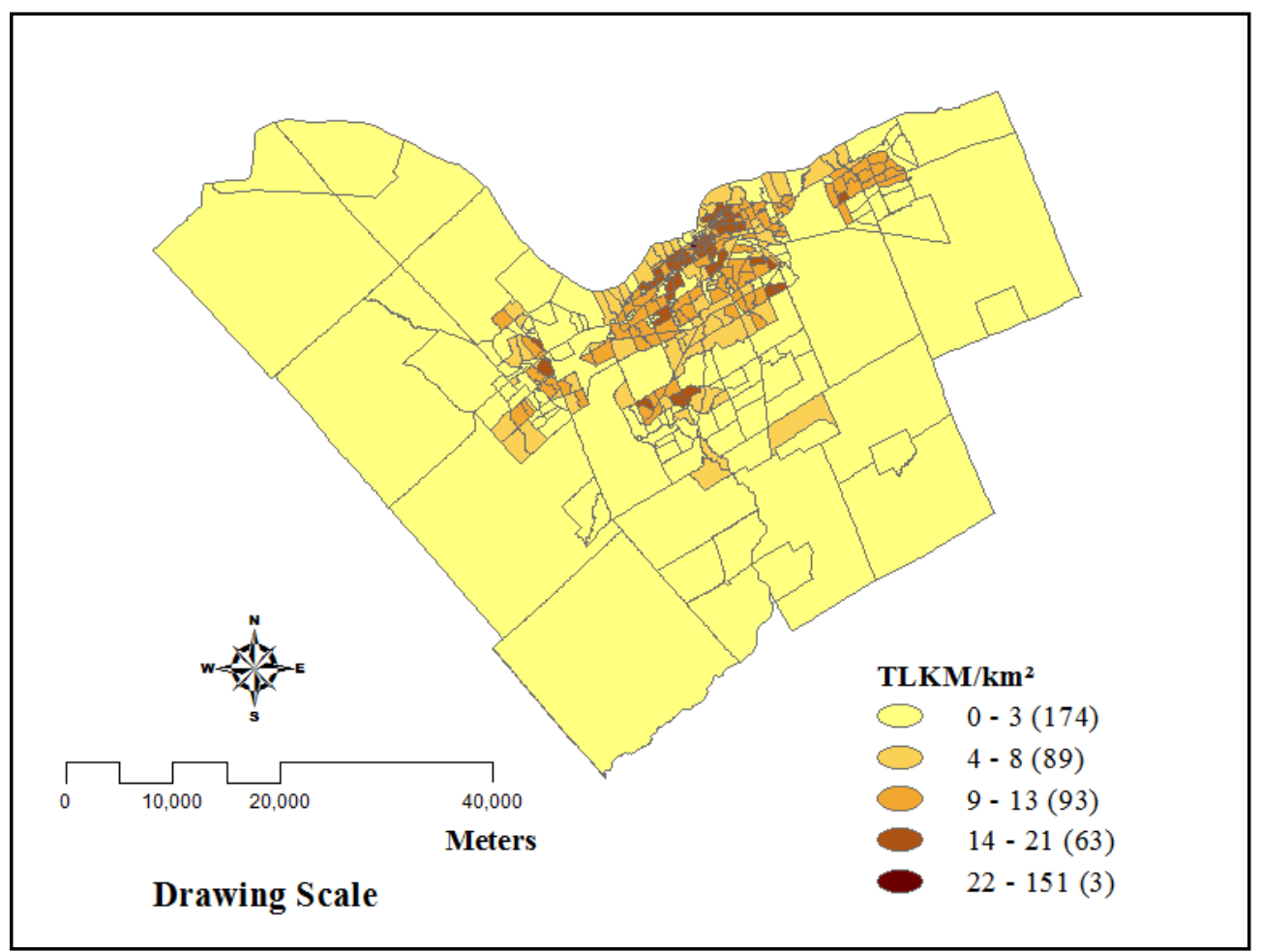

Figure A. 28 Spatial Distributions of TLKM Density in the City of Ottawa. Boundary Data Assigned Using Population-based Assignment Method. 


\section{A.4.3 Population-Employment-Based Assignment Method}

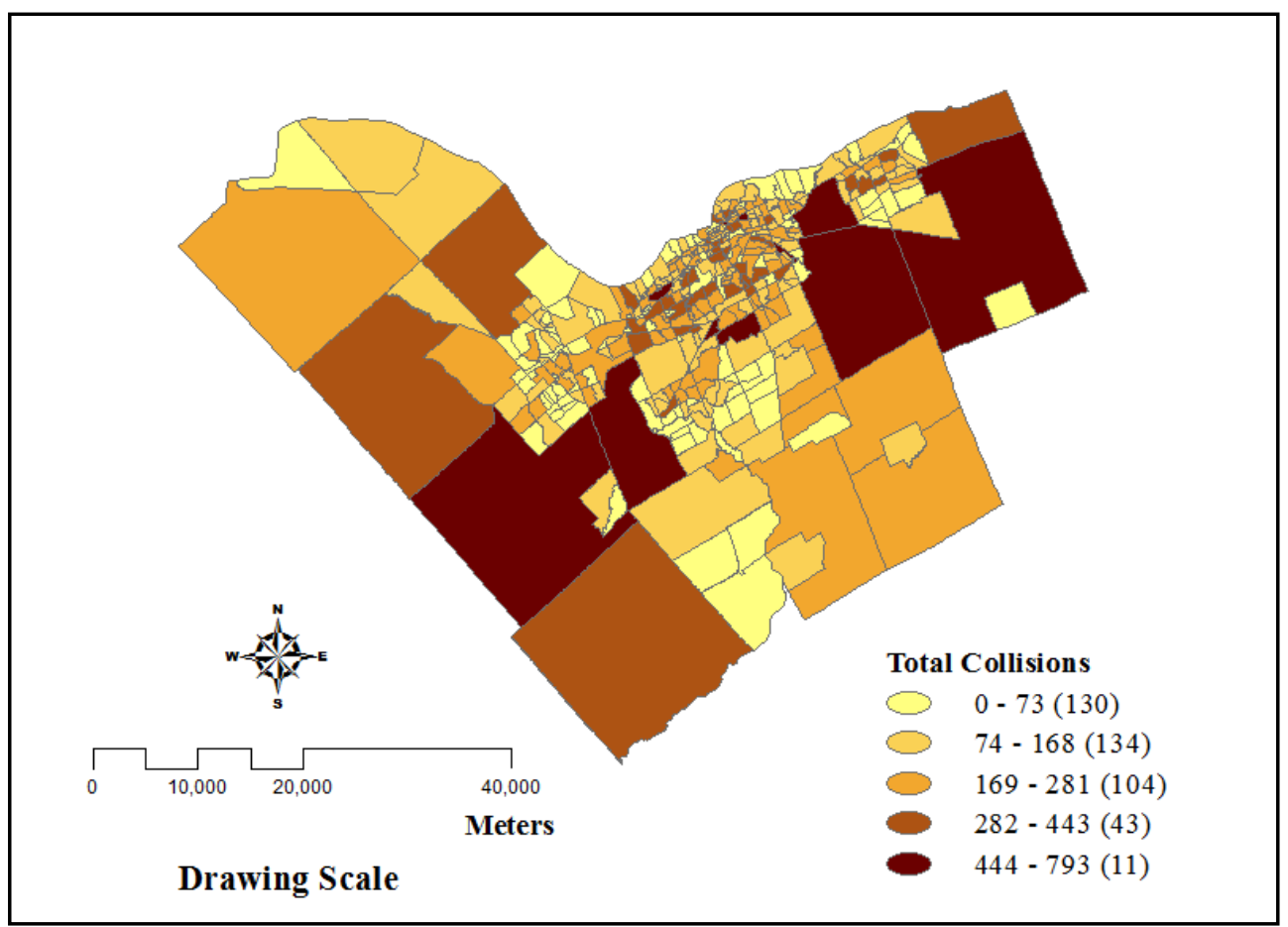

Figure A. 29 Spatial Distributions of Number of Total Collisions in the City of Ottawa. Boundary Data Assigned Using Population-Employment Based Assignment Method.

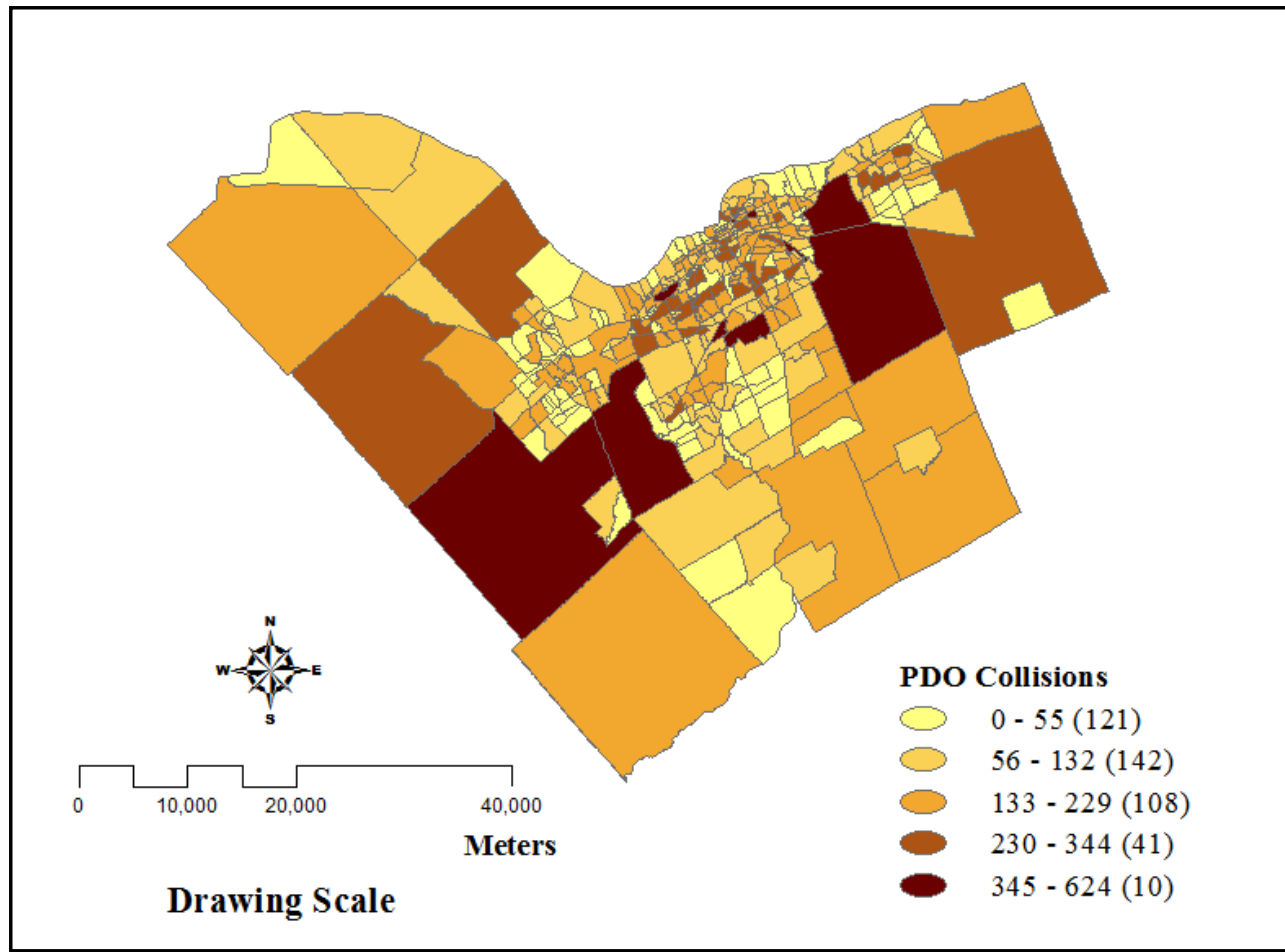

Figure A. 30 Spatial Distributions of Number of PDO Collisions in the City of Ottawa. Boundary Data Assigned Using Population-Employment Based Assignment Method. 


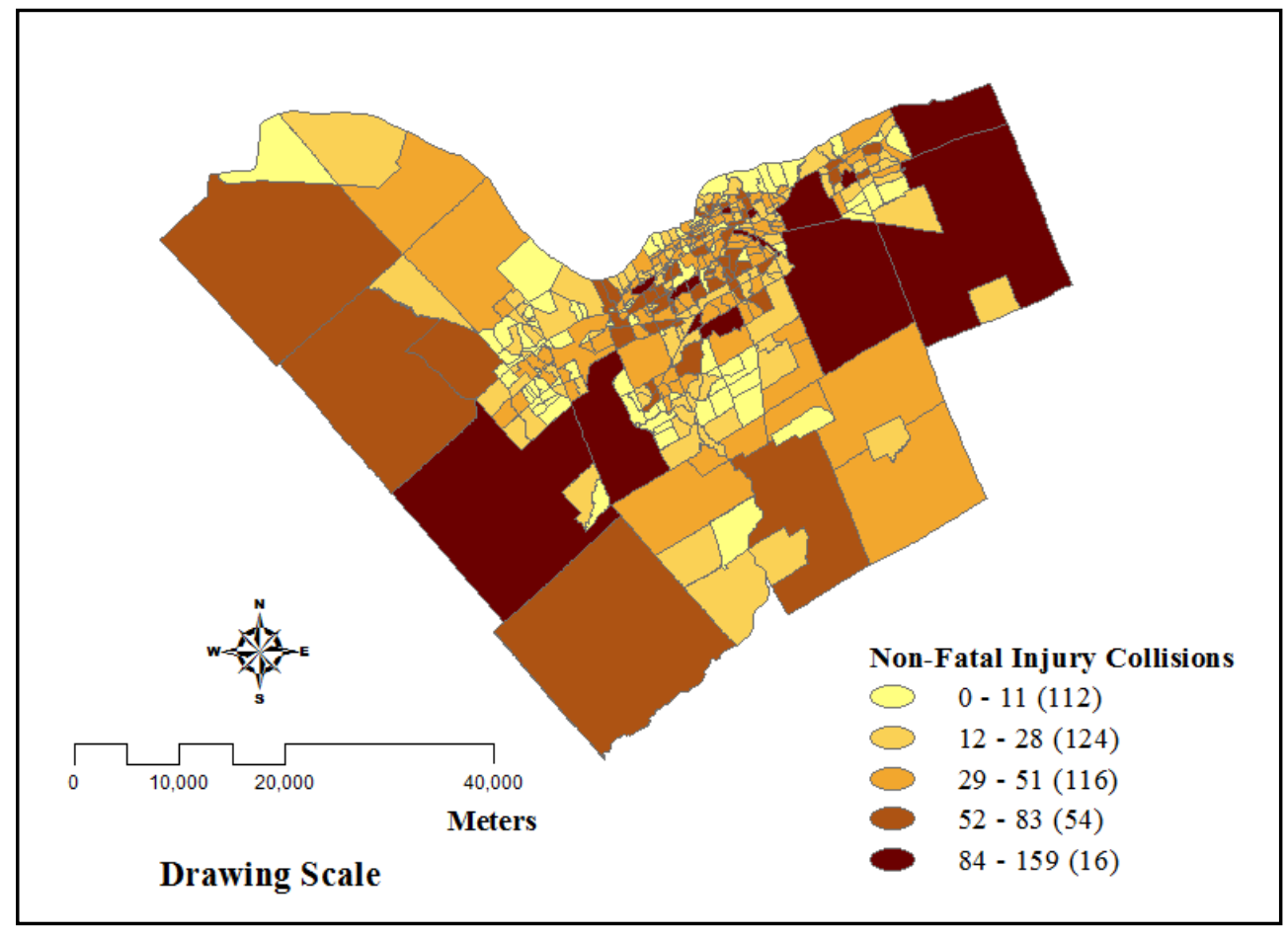

Figure A. 31 Spatial Distributions of Number of Non-fatal Injury Collisions in the City of Ottawa. Boundary Data Assigned Using Population-Employment Based Assignment Method.

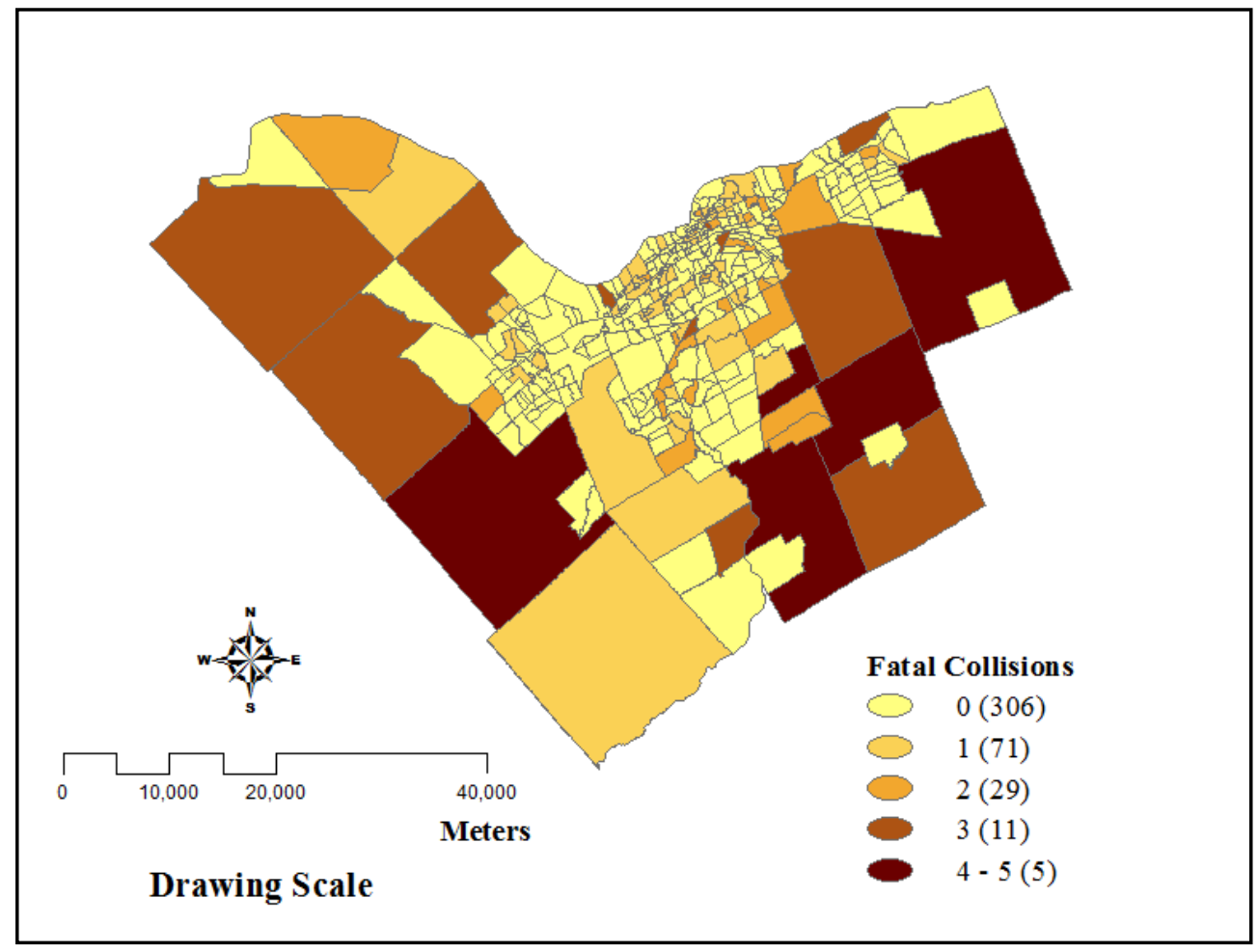

Figure A. 32 Spatial Distributions of Number of Fatal Collisions in the City of Ottawa Boundary Data Assigned Using Population-Employment Based Assignment Method. 


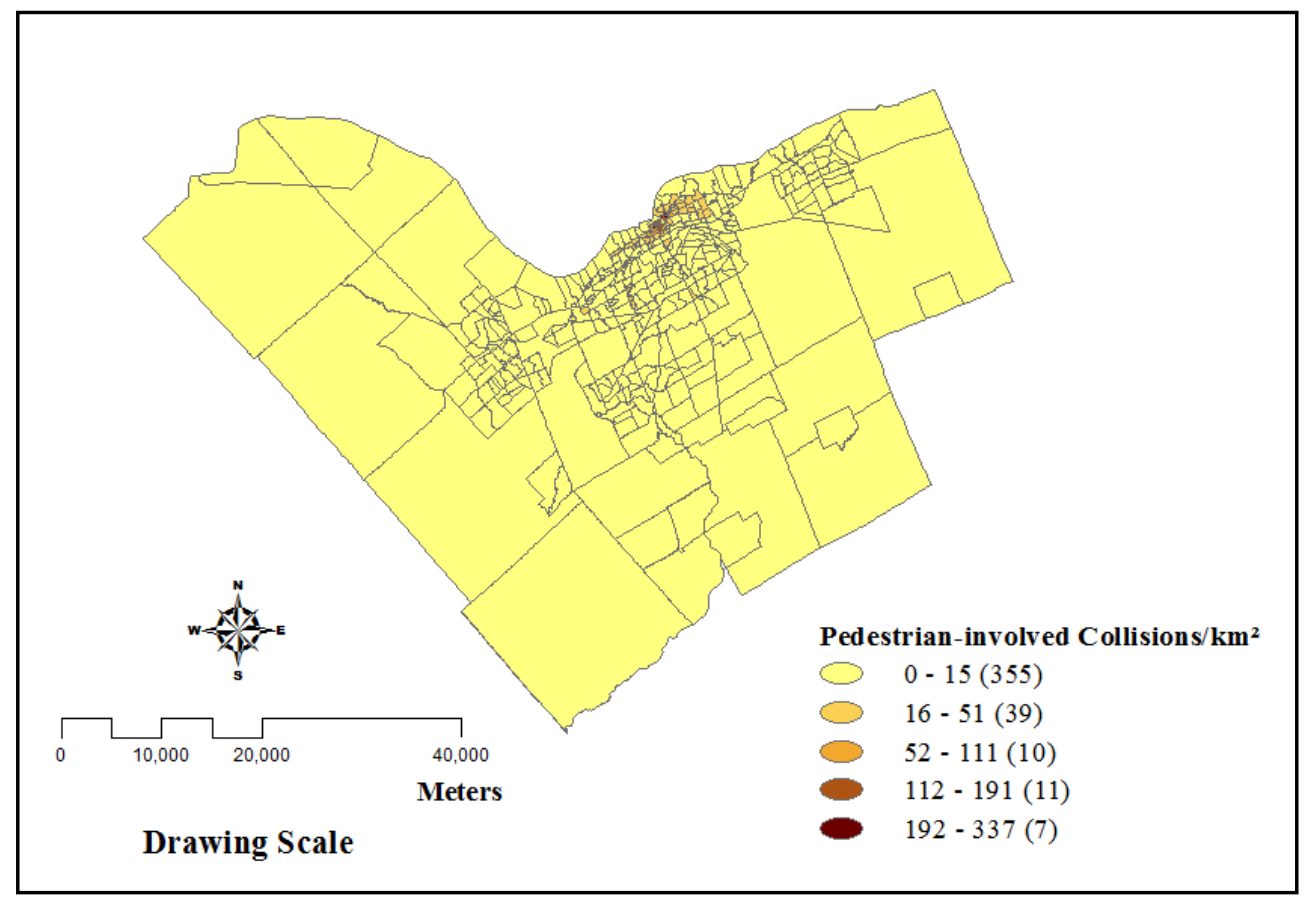

Figure A. 33 Spatial Distributions of Pedestrian-involved Collisions Density in the City of Ottawa. Boundary Data Assigned Using Population-Employment Based Assignment Method.

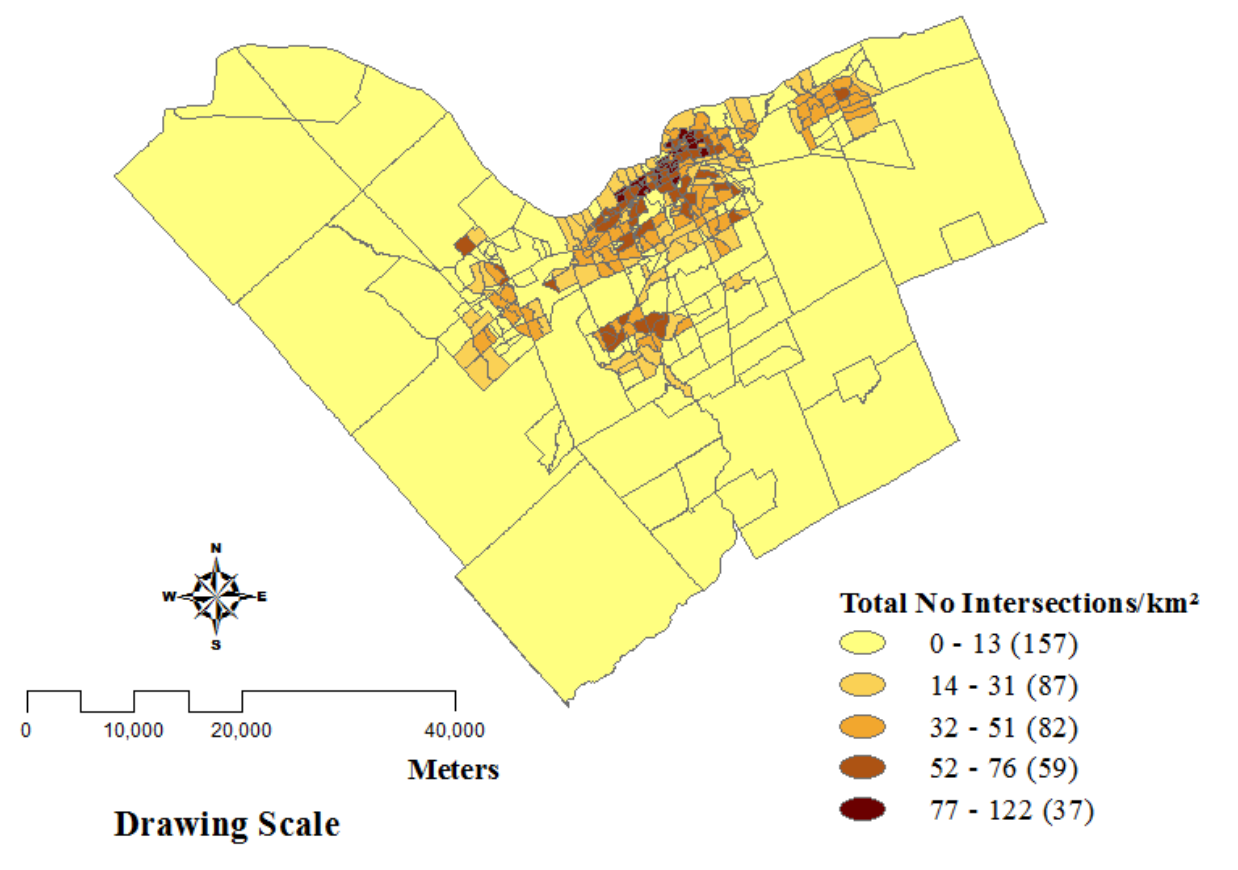

Figure A. 34 Spatial Distributions of Intersections Density in the City of Ottawa. Boundary Data Assigned Using Population-Employment Based Assignment Method. 


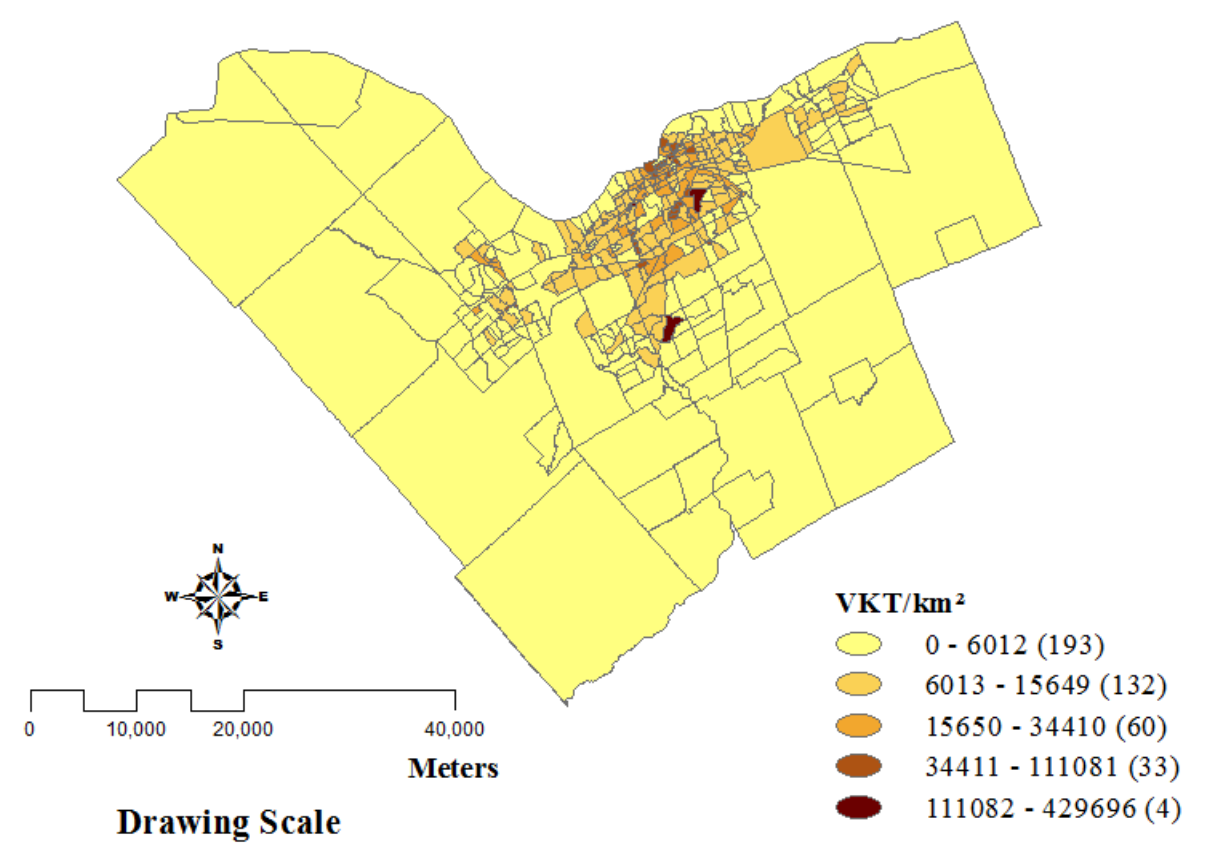

Figure A. 35 Spatial Distributions of VKT Density in the City of Ottawa. Boundary Data Assigned Using Population-Employment Based Assignment Method.

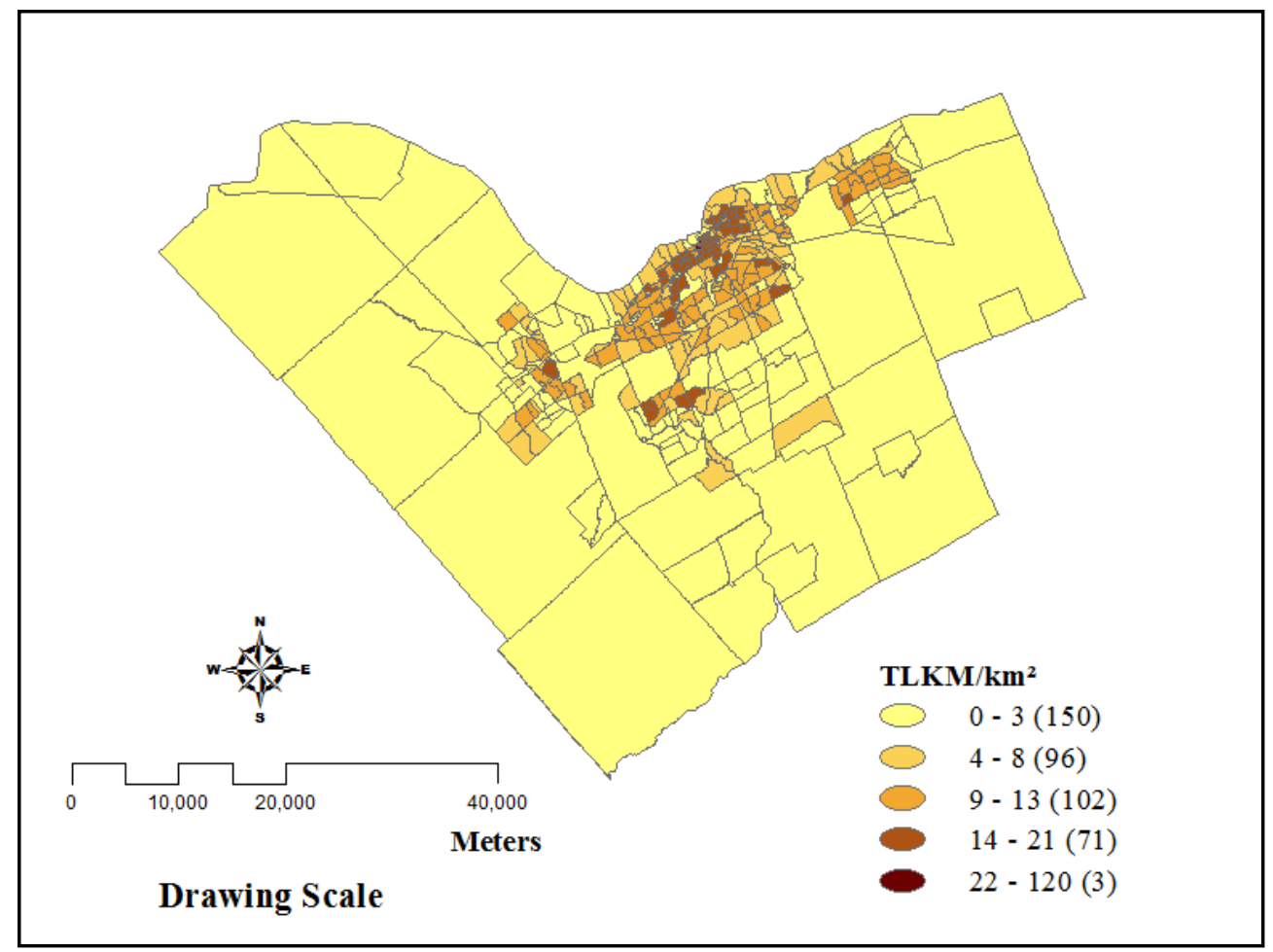

Figure A. 36 Spatial Distributions of TLKM Density in the City of Ottawa. Boundary Data Assigned Using Population-Employment Based Assignment Method. 


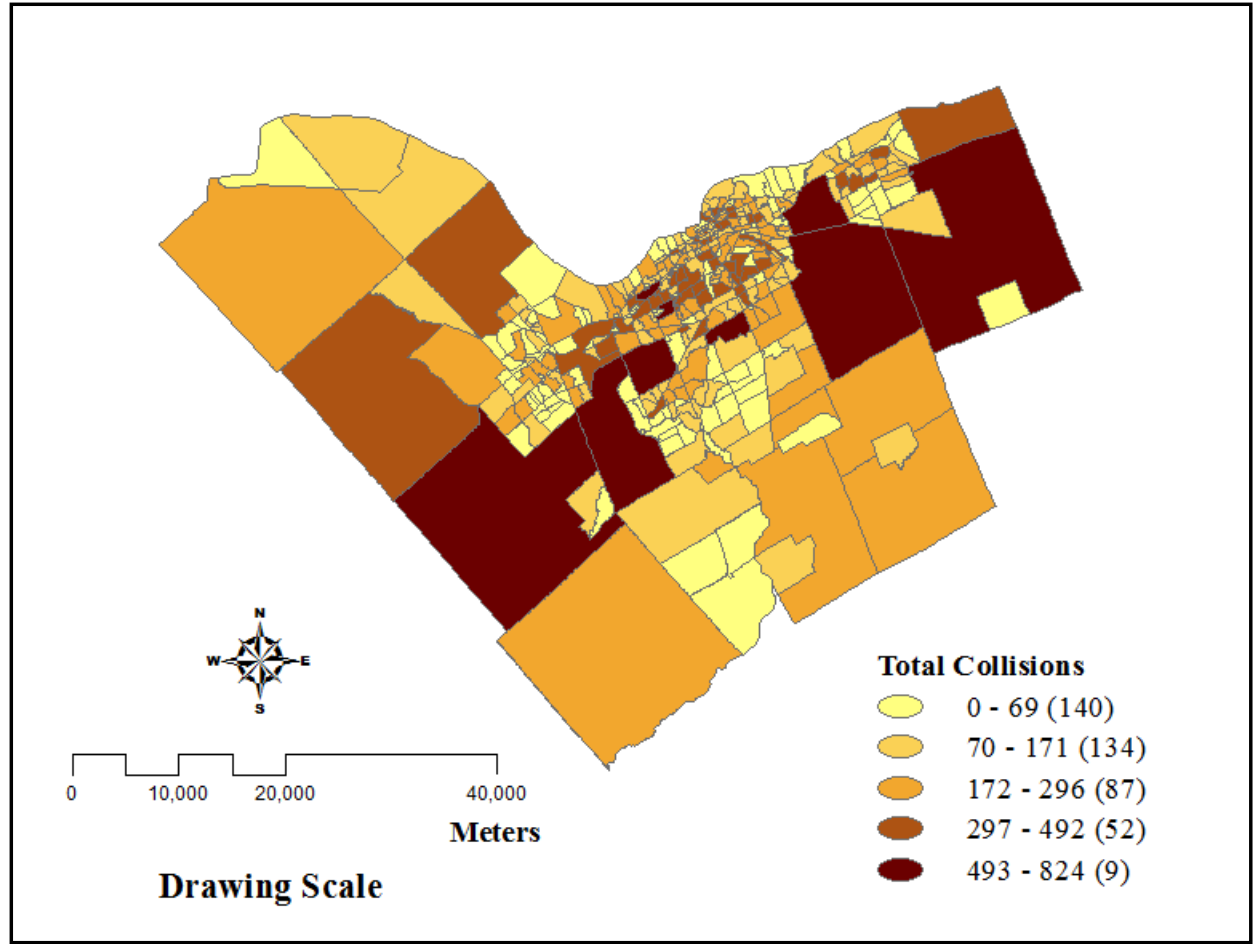

Figure A. 37 Spatial Distributions of Number of Total Collisions in the City of Ottawa. Boundary Data Assigned Using TLKM1-Based Assignment Method.

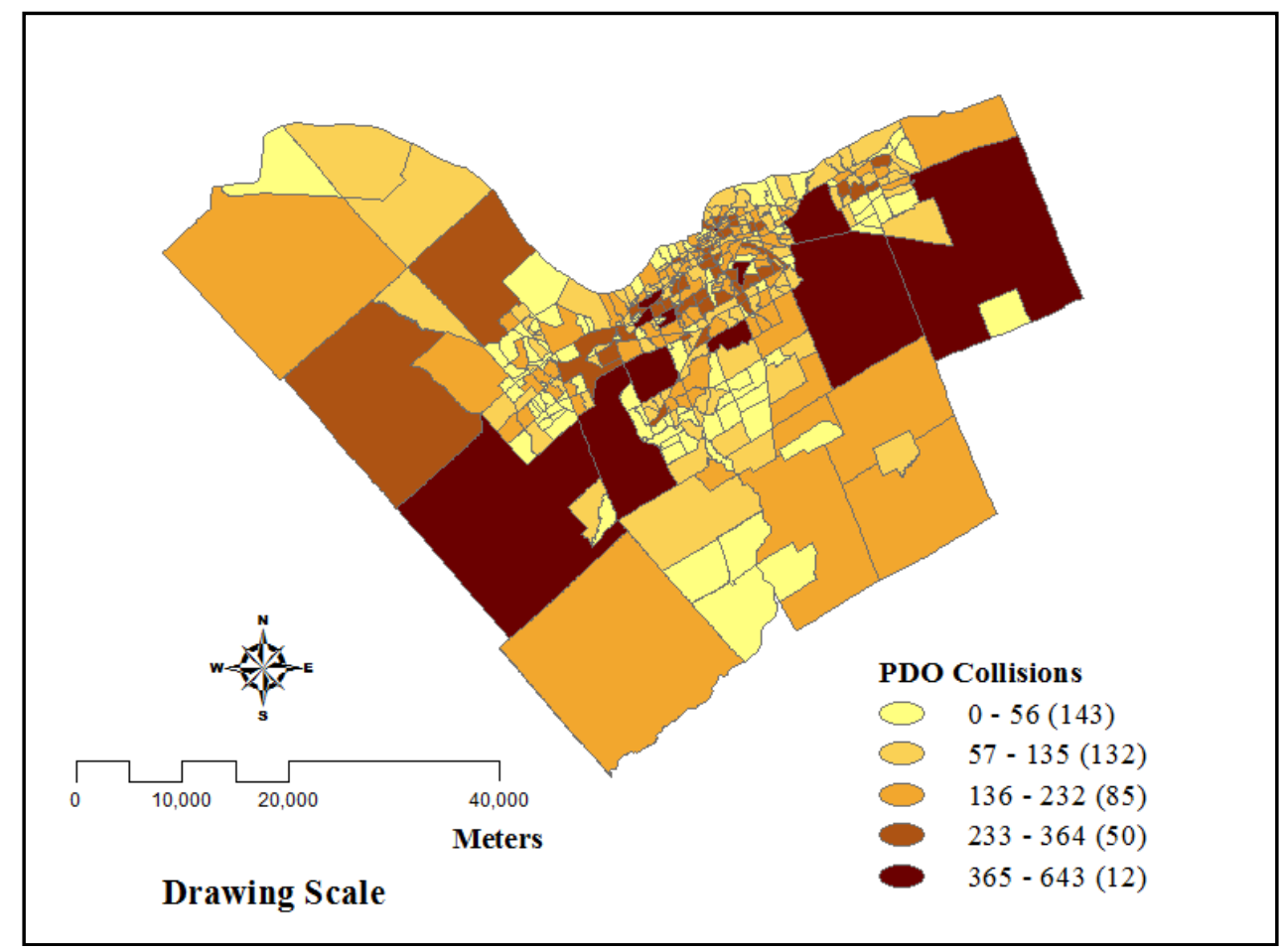

Figure A. 38 Spatial Distributions of Number of PDO Collisions in the City of Ottawa. Boundary Data Assigned Using TLKM1-Based Assignment Method. 


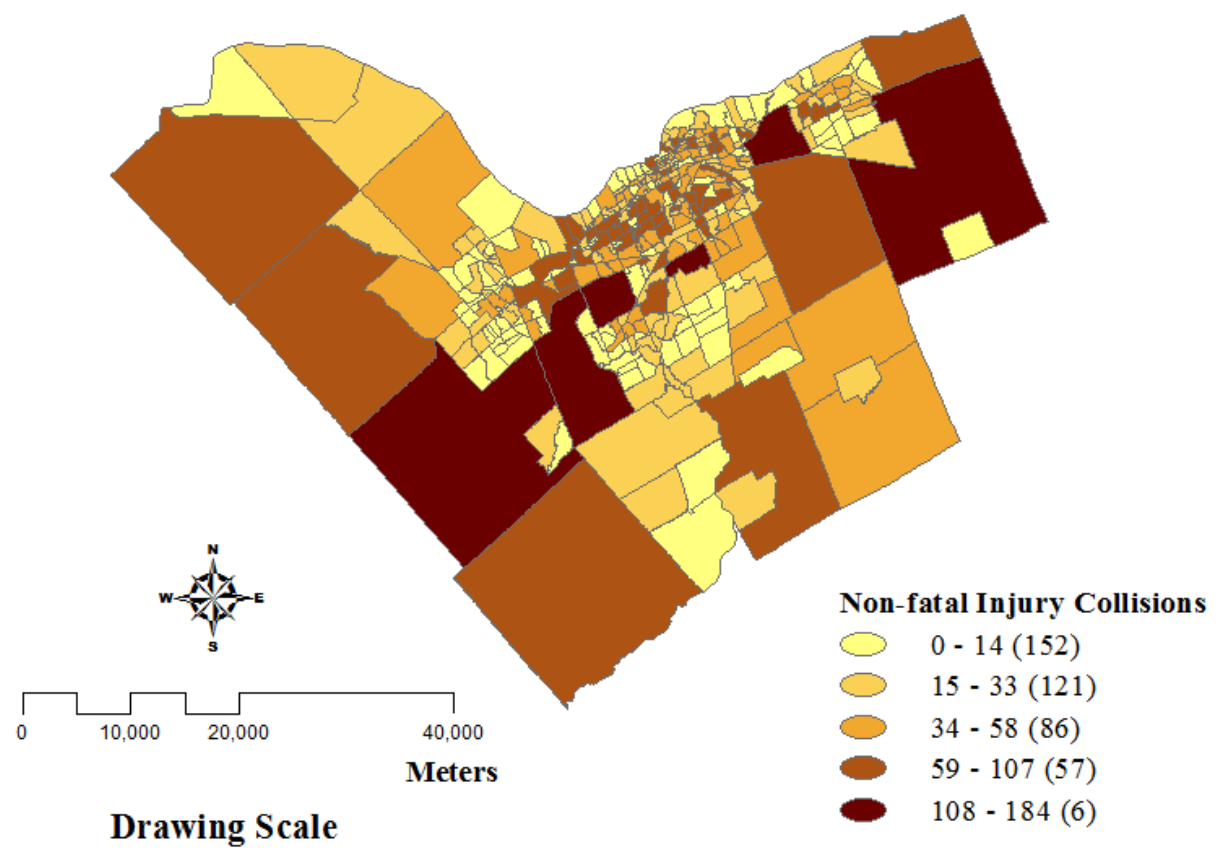

Figure A. 39 Spatial Distributions of Number of Non-fatal Injury Collisions in the City of Ottawa. Boundary Data Assigned Using TLKM1-Based Assignment Method.

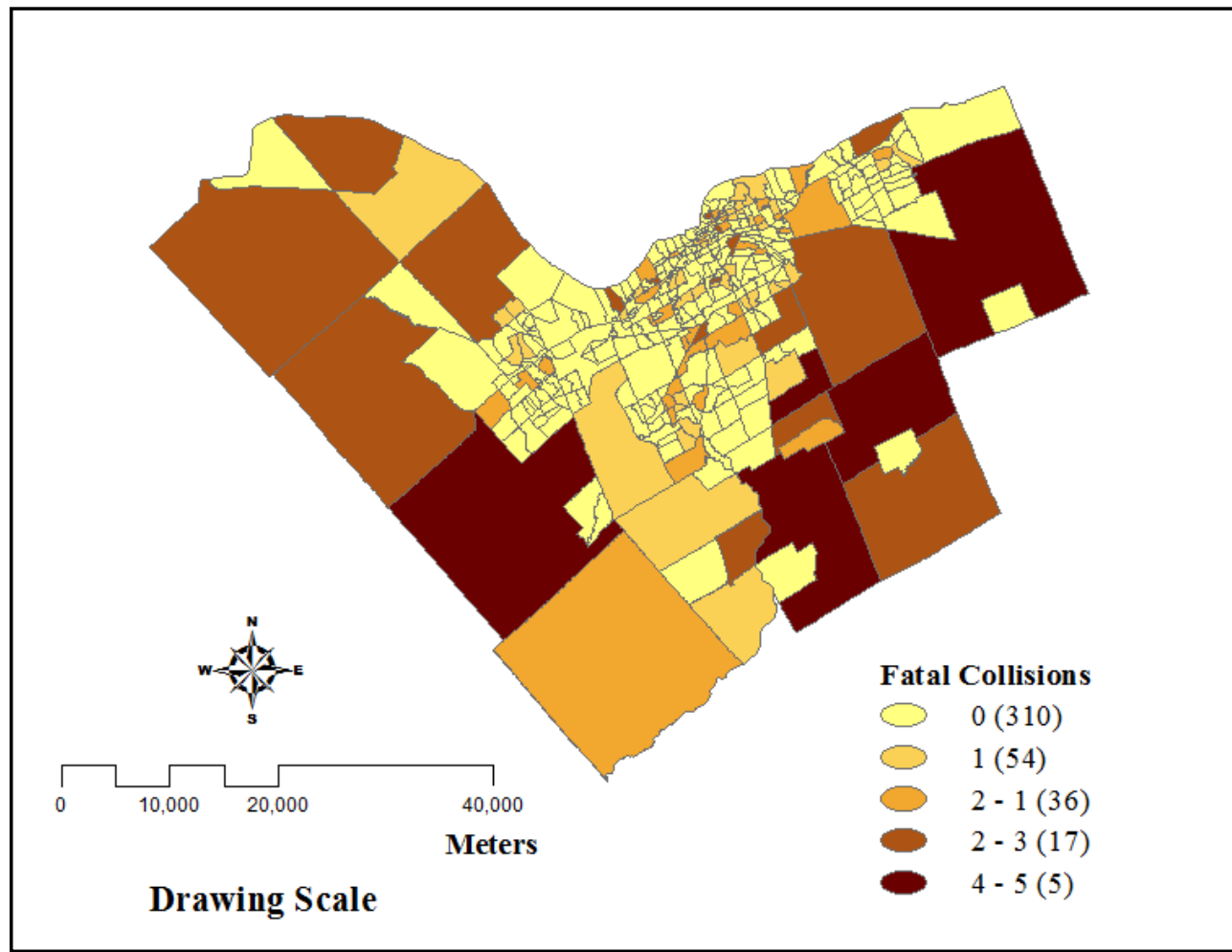

Figure A. 40 Spatial Distributions of Number of Fatal Collisions in the City of Ottawa Boundary Data Assigned Using TLKM1-Based Assignment Method. 


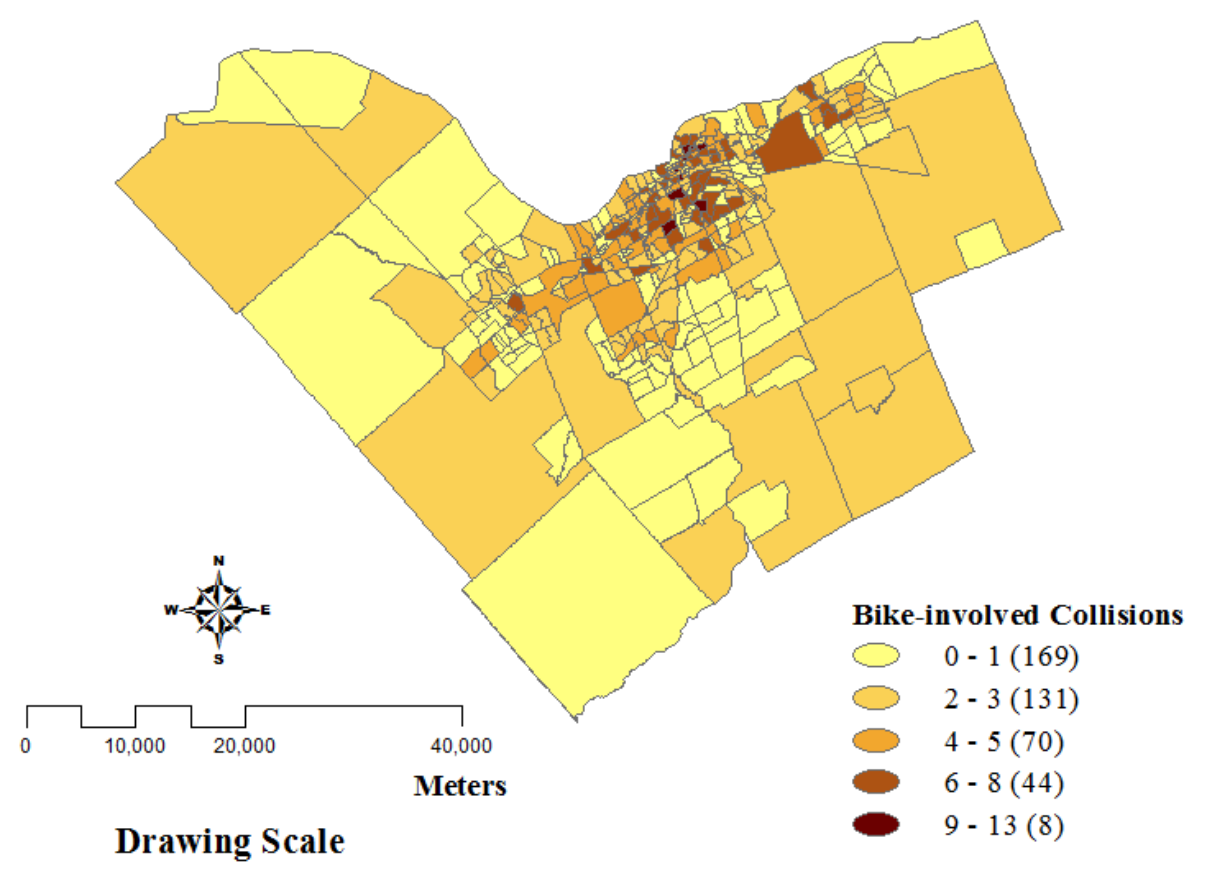

Figure A. 41 Spatial Distributions of Number of Bike-involved Collisions in the City of Ottawa. Boundary Data Assigned Using TLKM1-Based Assignment Method.

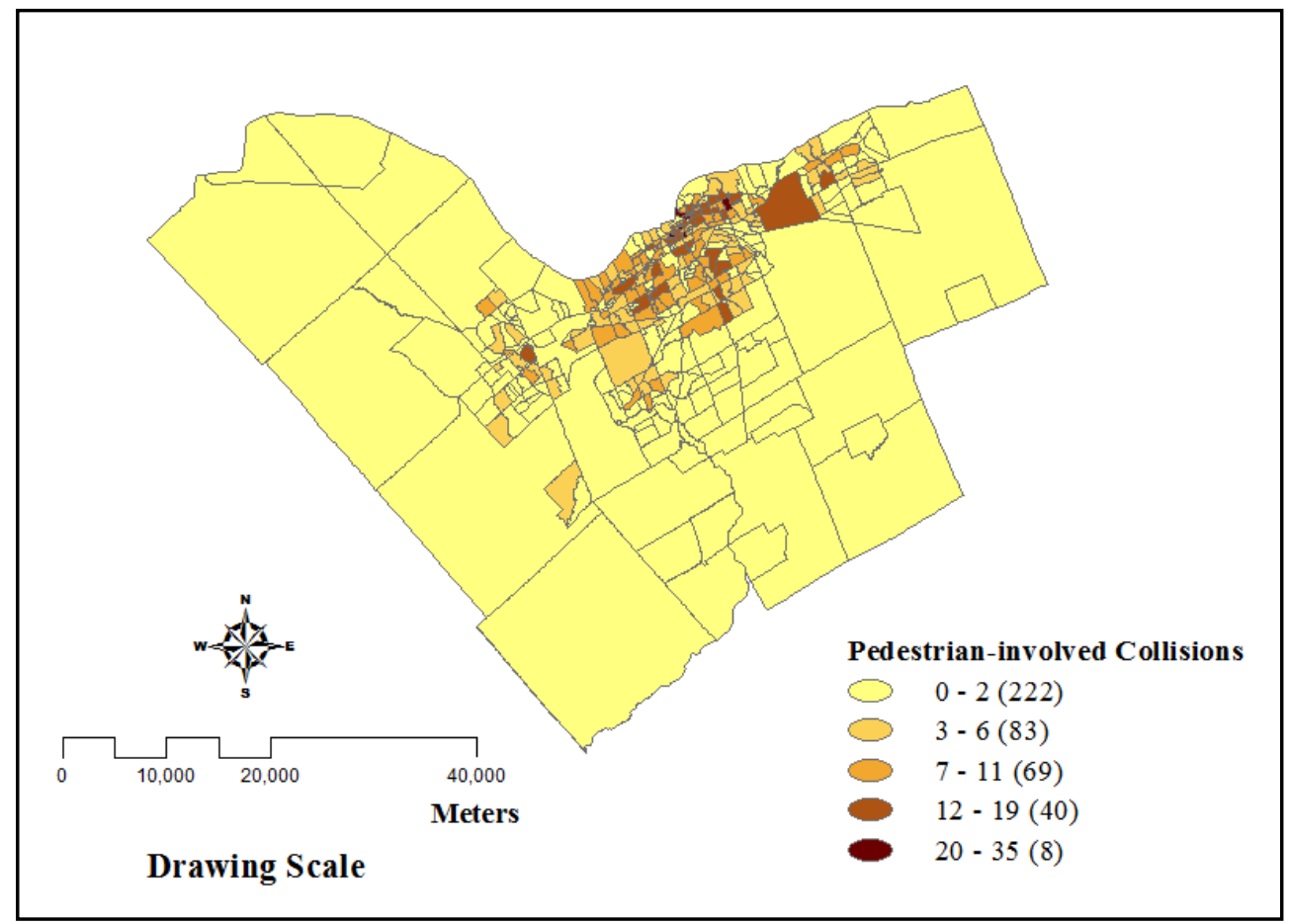

Figure A. 42 Spatial Distributions of Number of Pedestrian-involved Collisions in the City of Ottawa. Boundary Data Assigned Using TLKM1-Based Assignment Method. 


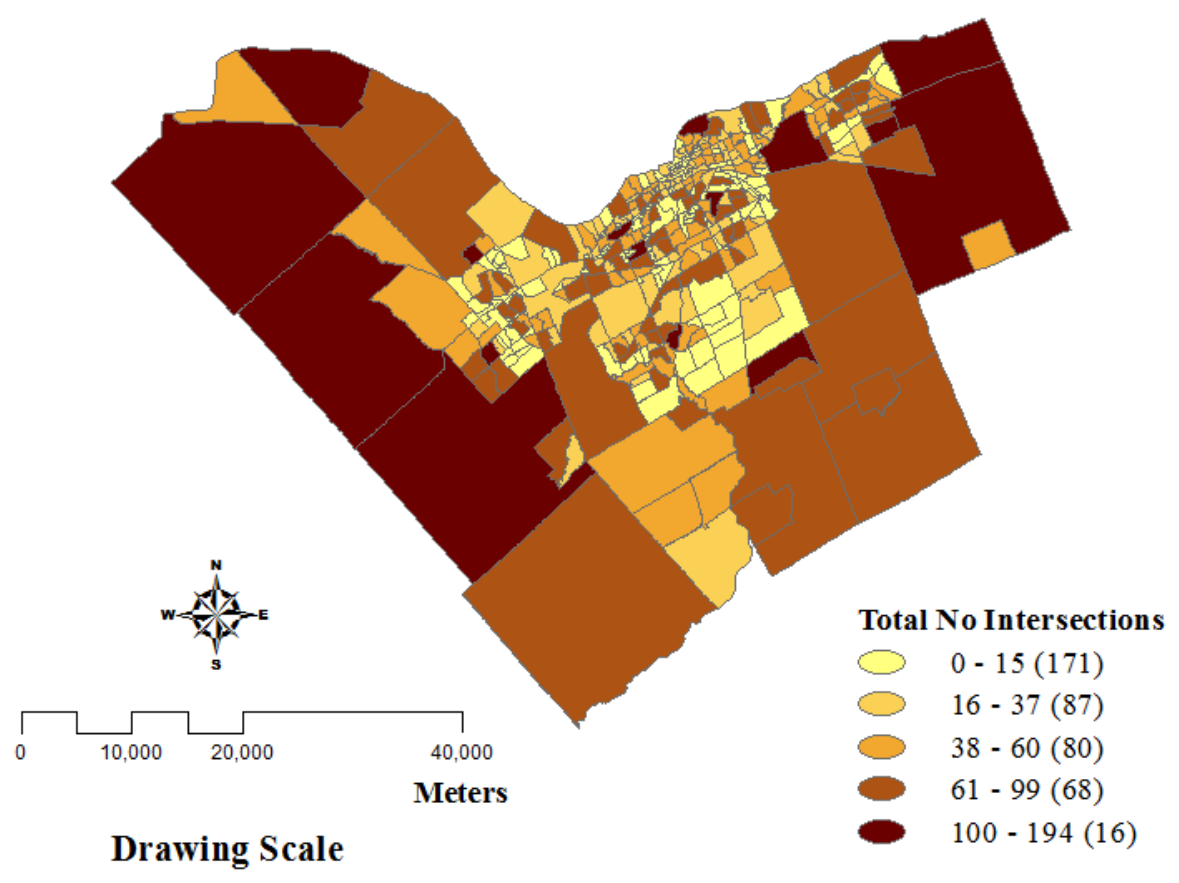

Figure A. 43 Spatial Distributions of Number of Intersections in the City of Ottawa. Boundary Data Assigned Using TLKM1-Based Assignment Method.

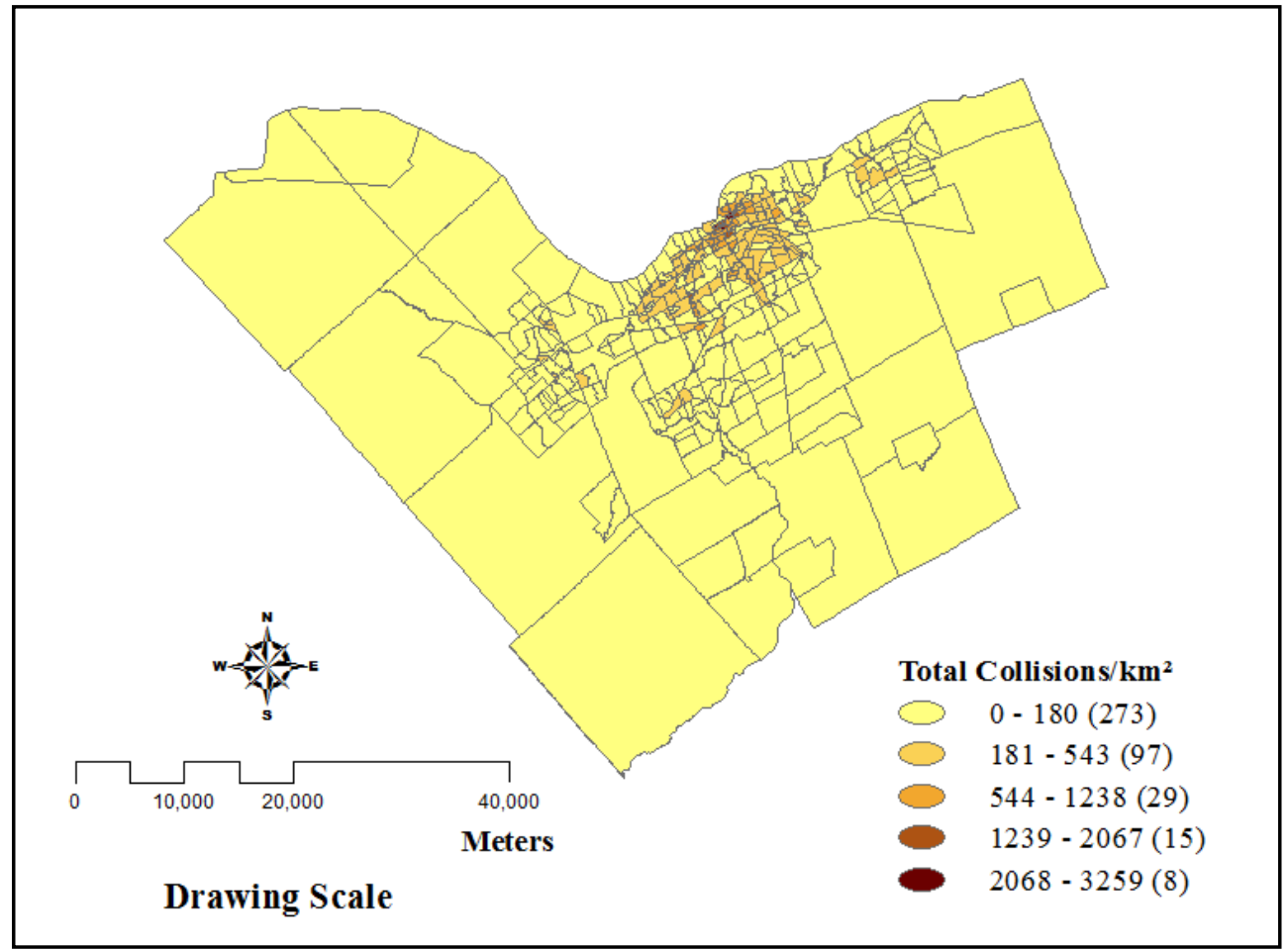

Figure A. 44 Spatial Distributions of Total Collisions Density in the City of Ottawa Boundary Data Assigned Using TLKM1-Based Assignment Method. 


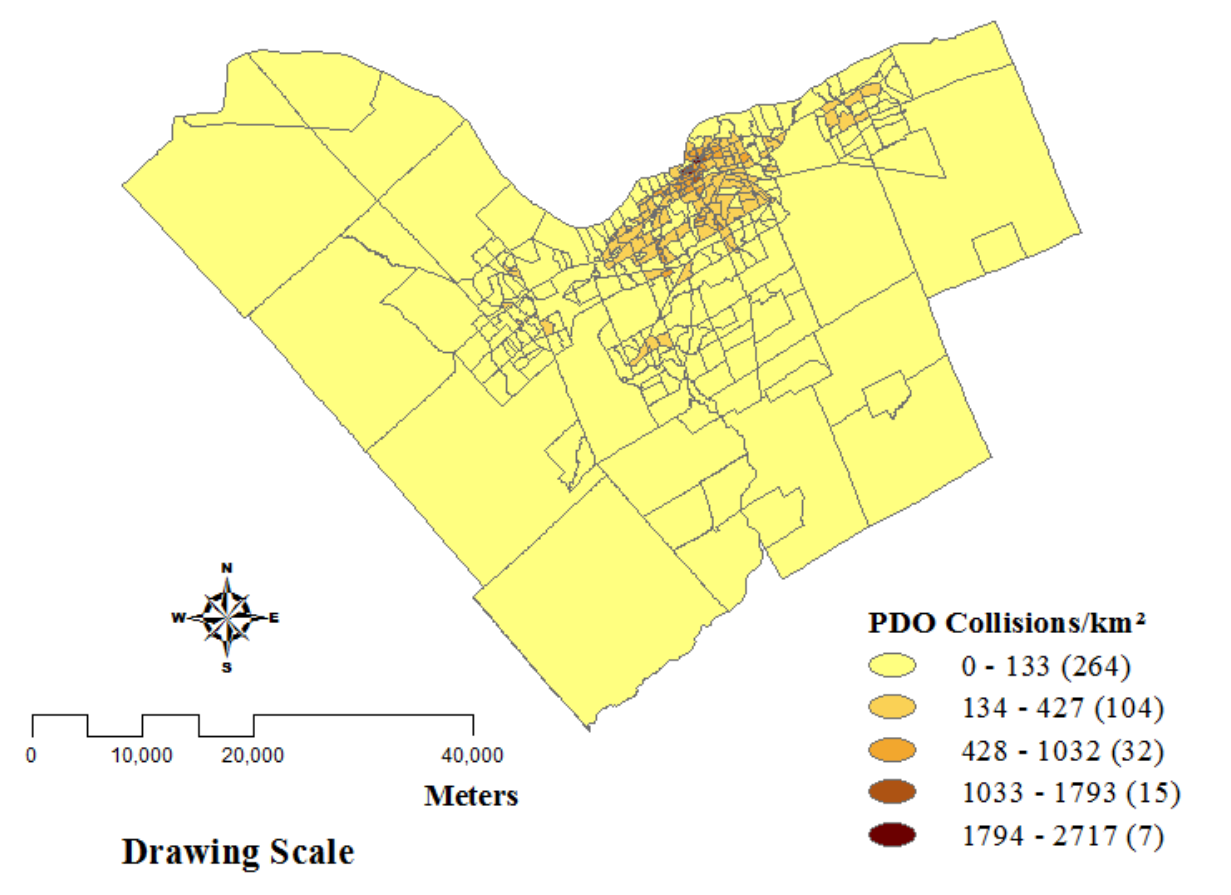

Figure A. 45 Spatial Distributions of PDO Collisions Density in the City of Ottawa. Boundary Data Assigned Using TLKM1-Based Assignment Method.

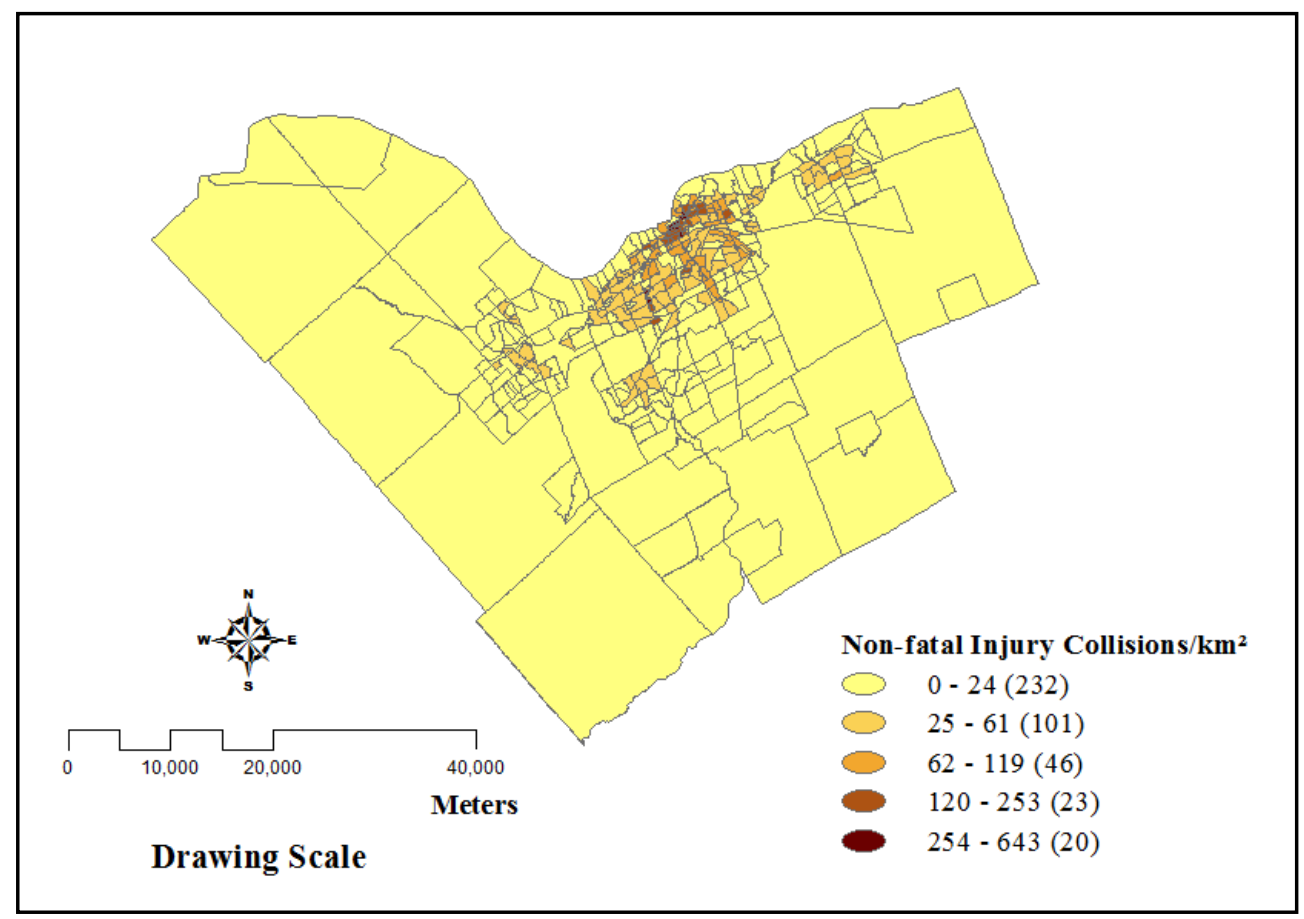

Figure A. 46 Spatial Distributions of Non-fatal Injury Collisions Density in the City of Ottawa. Boundary Data Assigned Using TLKM1-Based Assignment Method. 


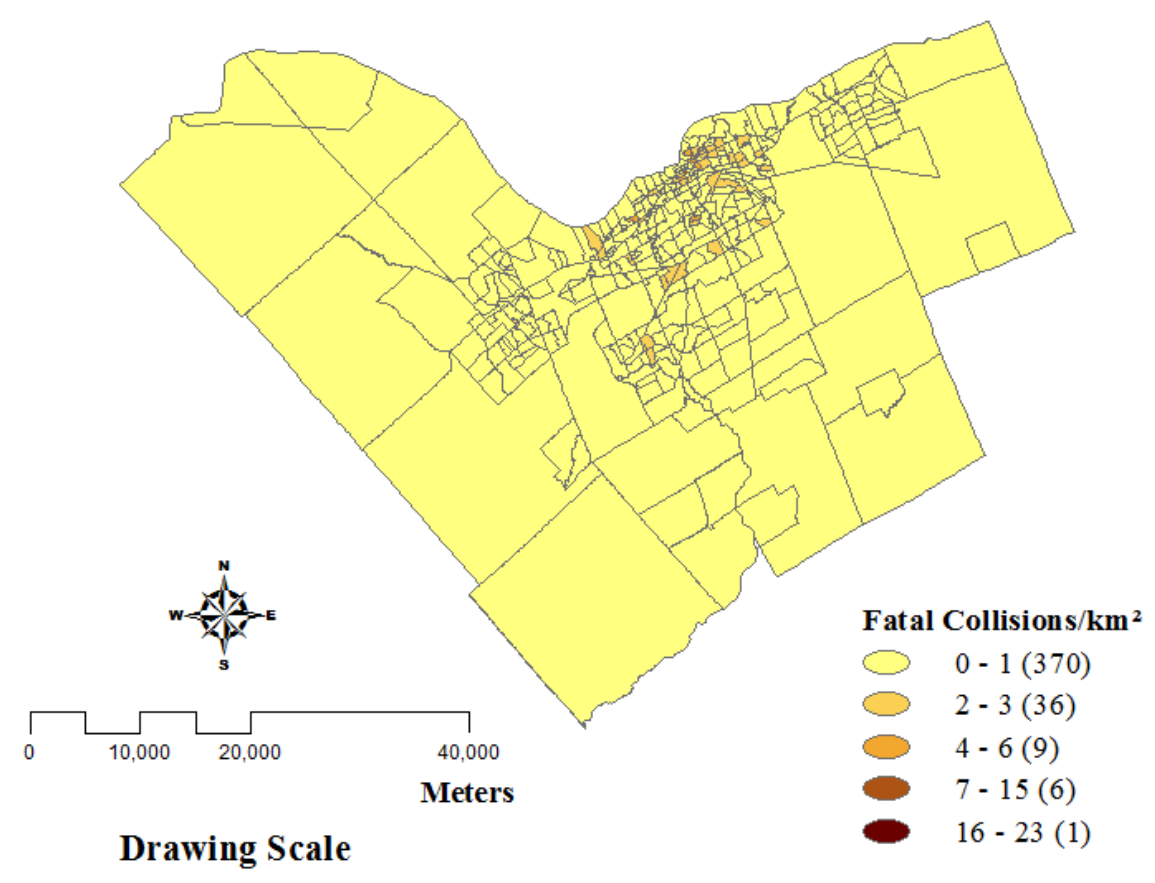

Figure A. 47 Spatial Distributions of Fatal Collisions Density in the City of Ottawa. Boundary Data Assigned Using TLKM1-Based Assignment Method.

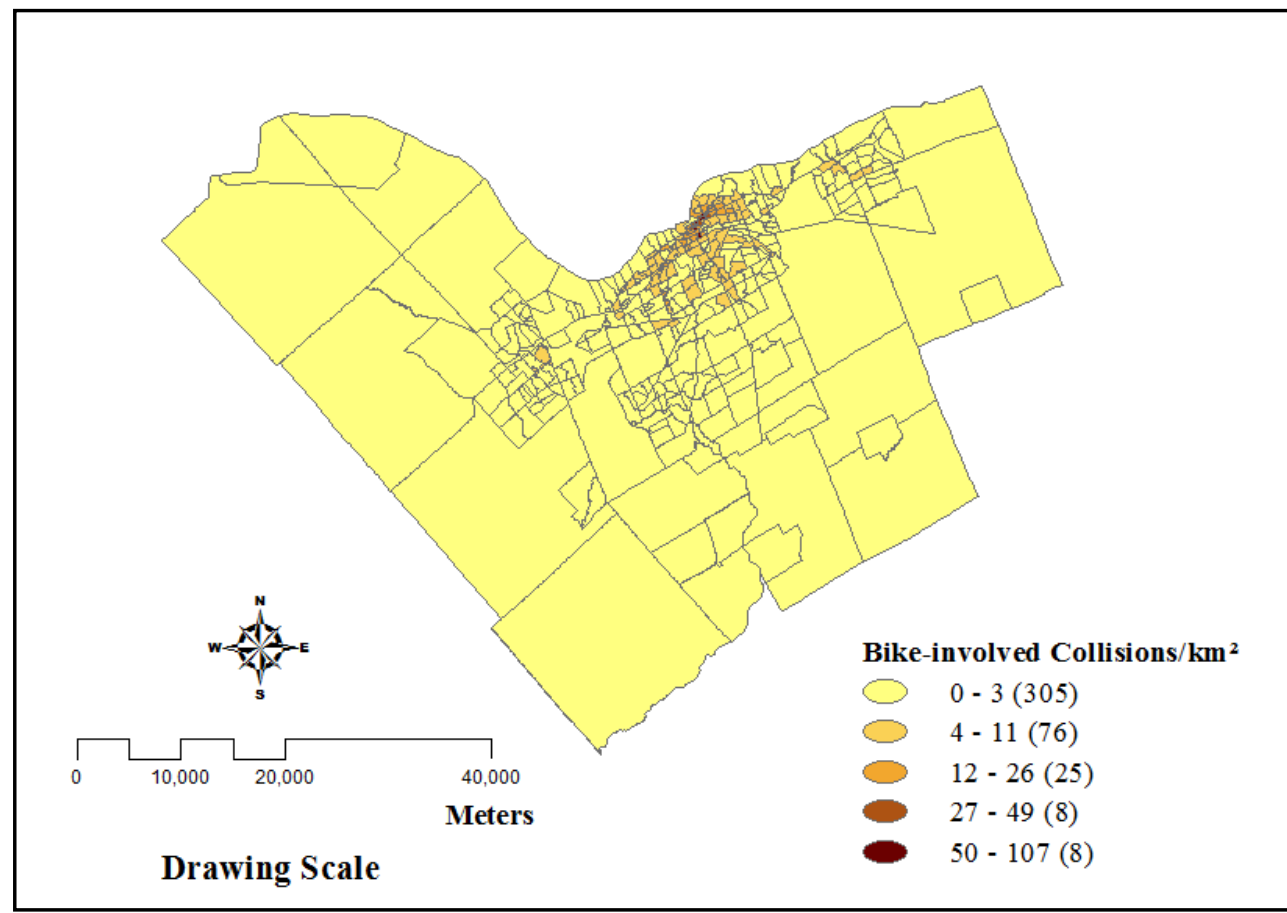

Figure A. 48 Spatial Distributions of Bike-involved Collisions Density in the City of Ottawa. Boundary Data Assigned Using TLKM1-Based Assignment Method. 


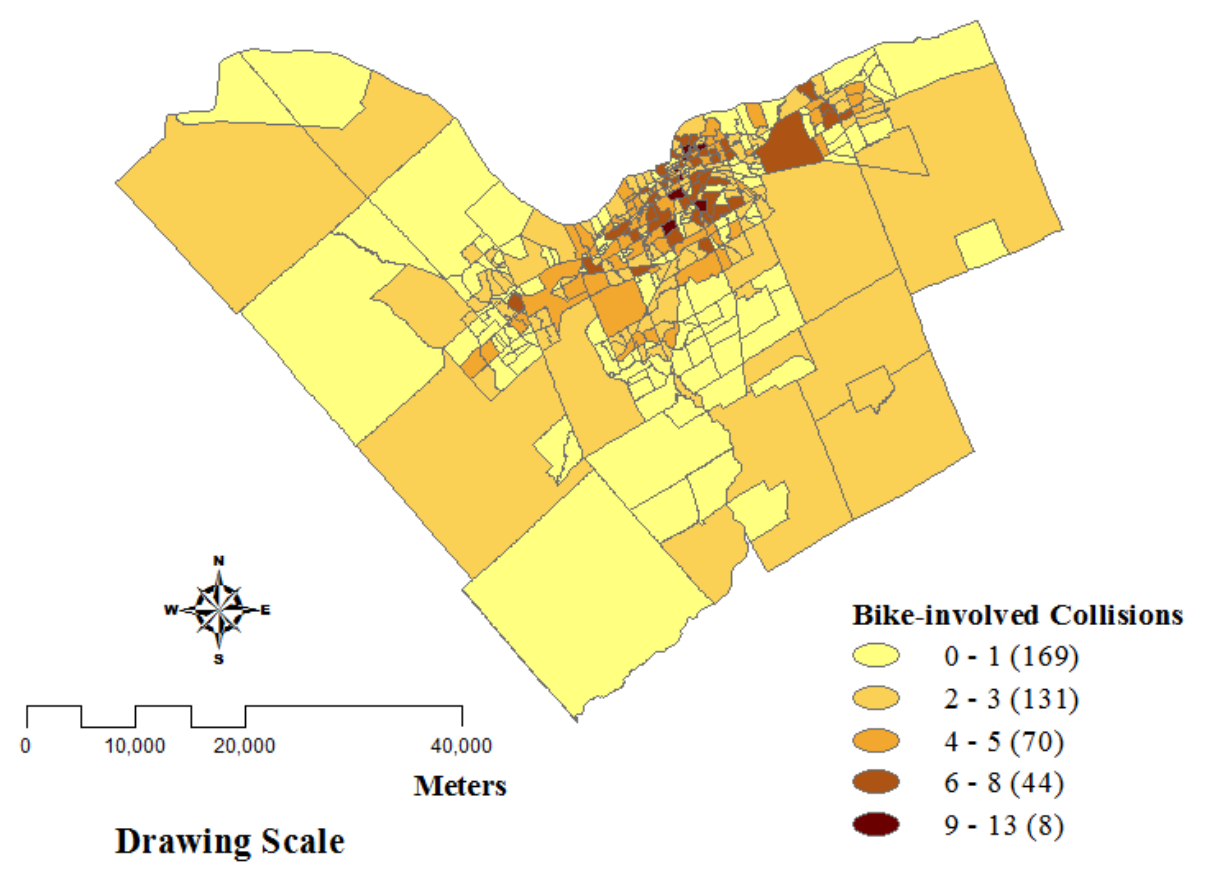

Figure A. 49 Spatial Distributions of Number of Bike-involved Collisions in the City of Ottawa. Boundary Data Assigned Using TLKM1-Based Assignment Method.

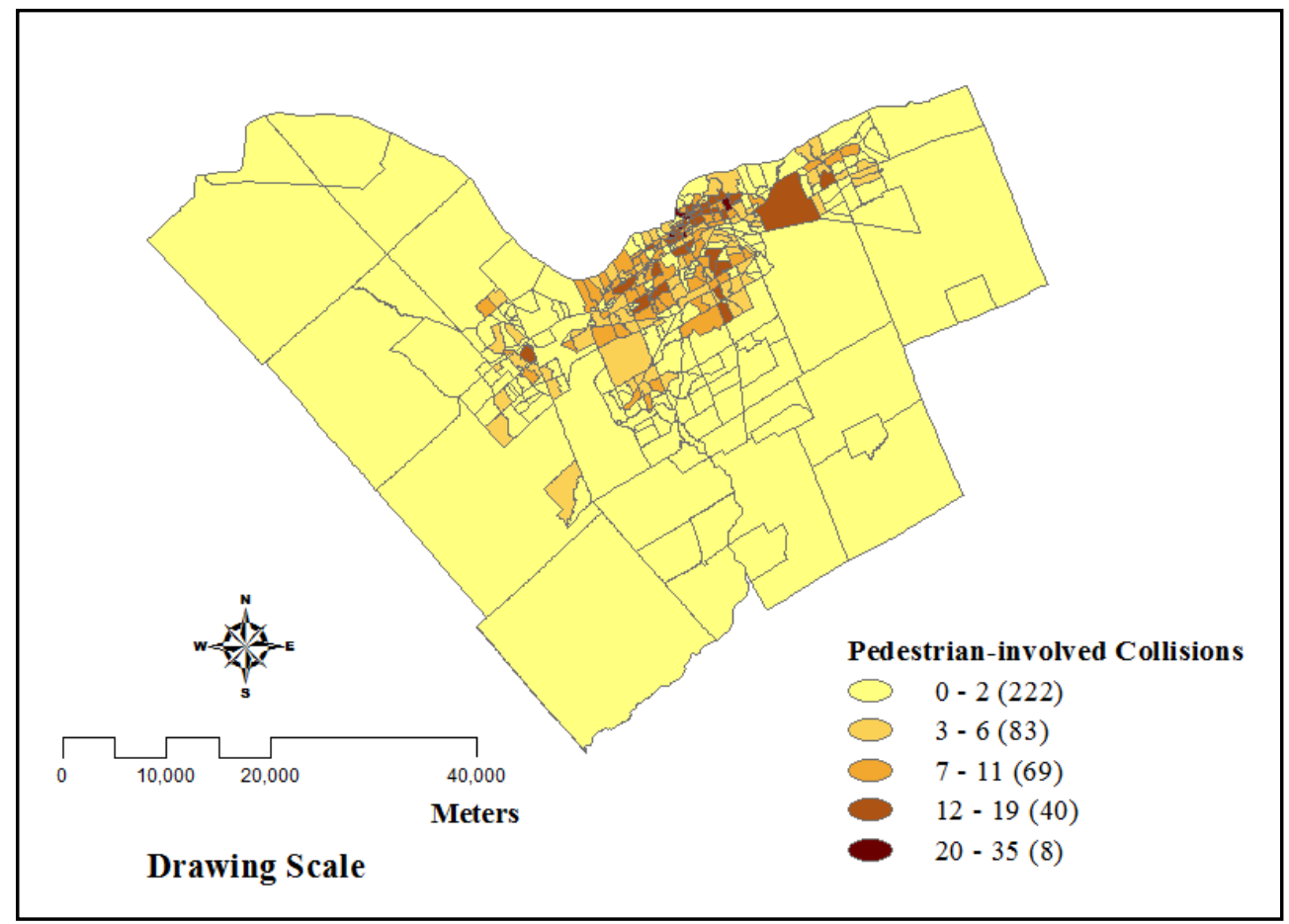

Figure A. 50 Spatial Distributions of Number of Pedestrian-involved Collisions in the City of Ottawa. Boundary Data Assigned Using TLKM1-Based Assignment Method. 


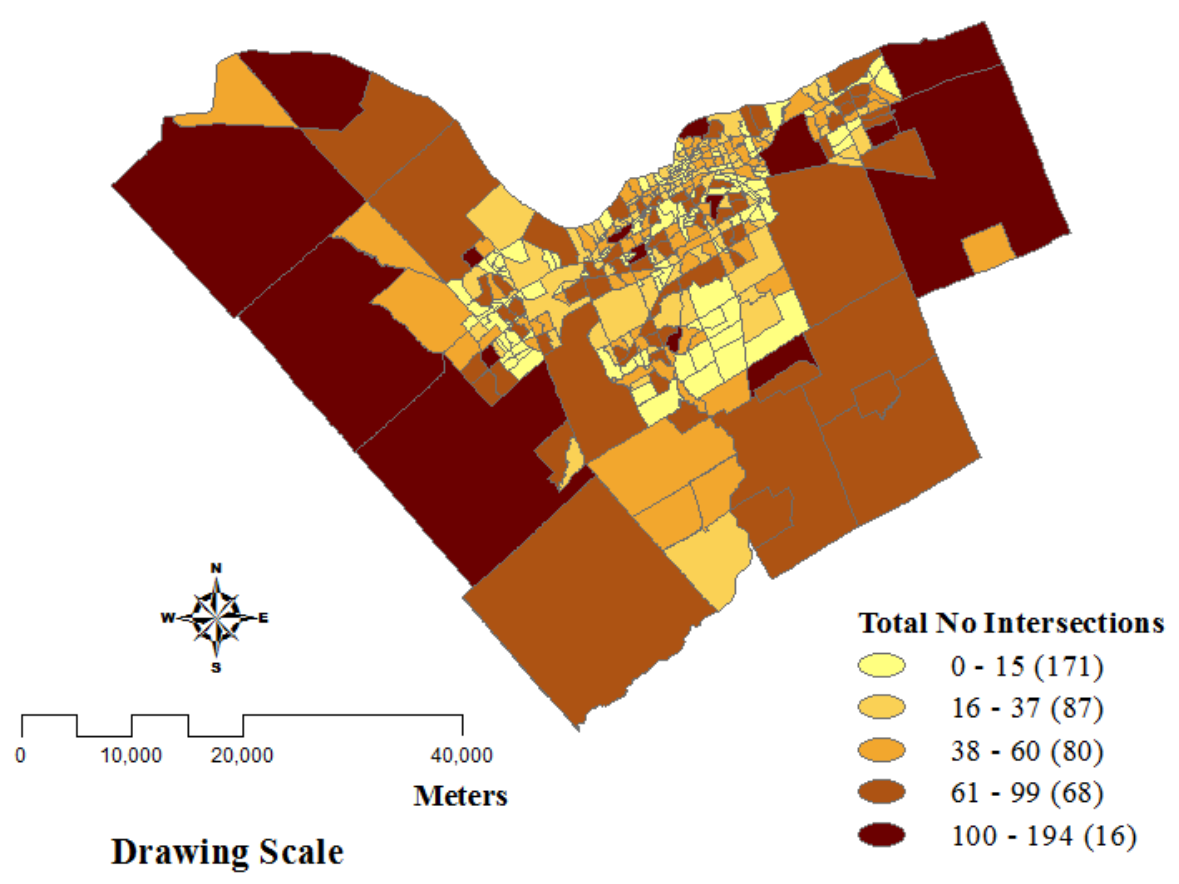

Figure A. 51 Spatial Distributions of Number of Intersections in the City of Ottawa. Boundary Data Assigned Using TLKM1-Based Assignment Method.

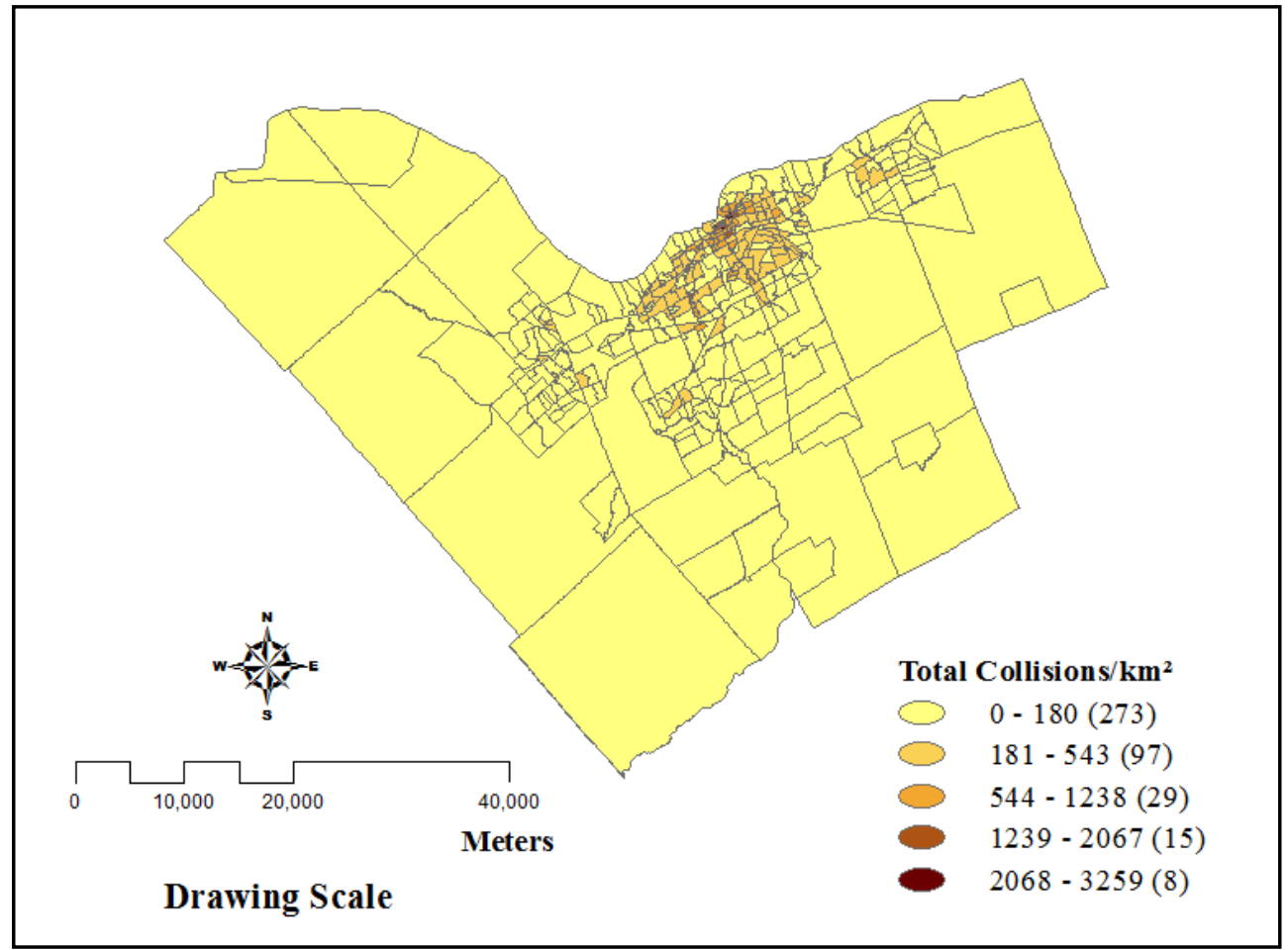

Figure A. 52 Spatial Distributions of Total Collisions Density in the City of Ottawa Boundary Data Assigned Using TLKM1-Based Assignment Method. 


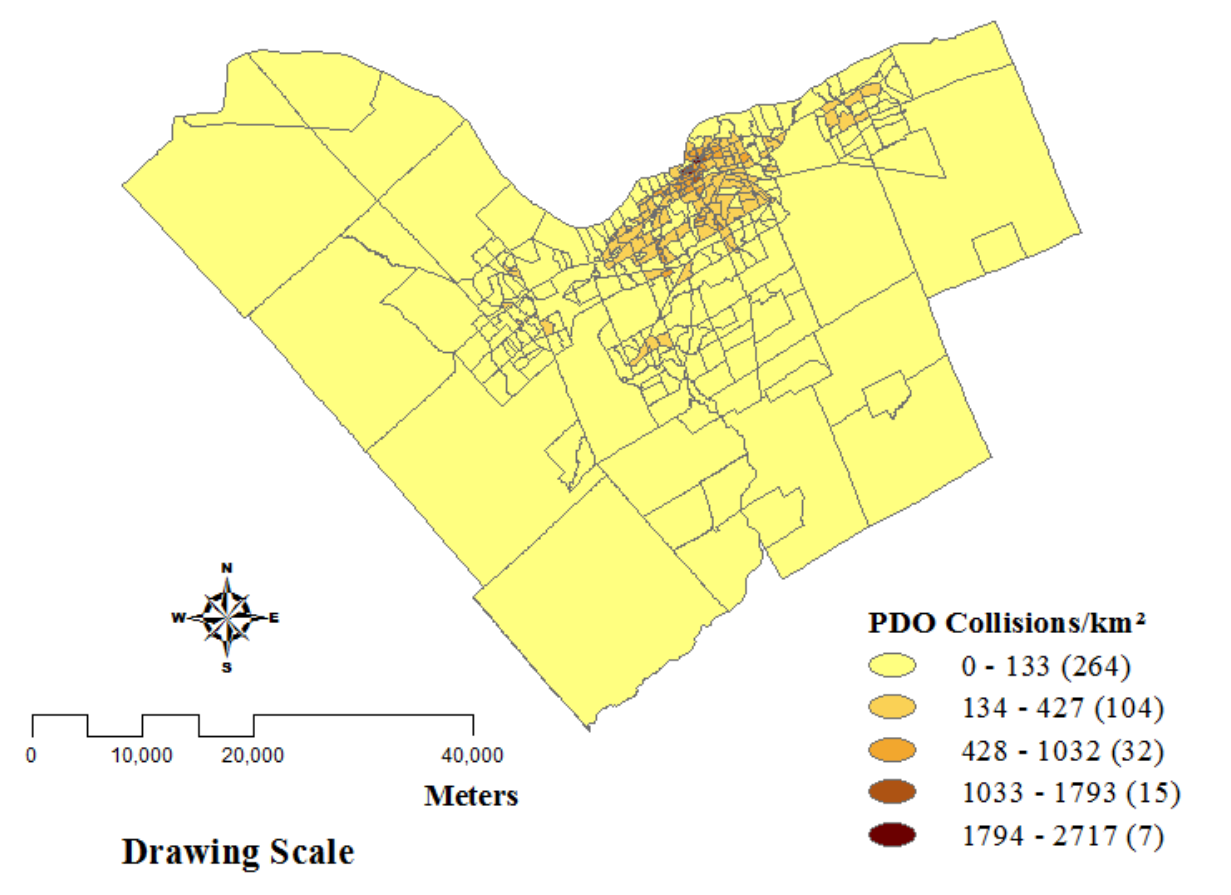

Figure A. 53 Spatial Distributions of PDO Collisions Density in the City of Ottawa. Boundary Data Assigned Using TLKM1-Based Assignment Method.

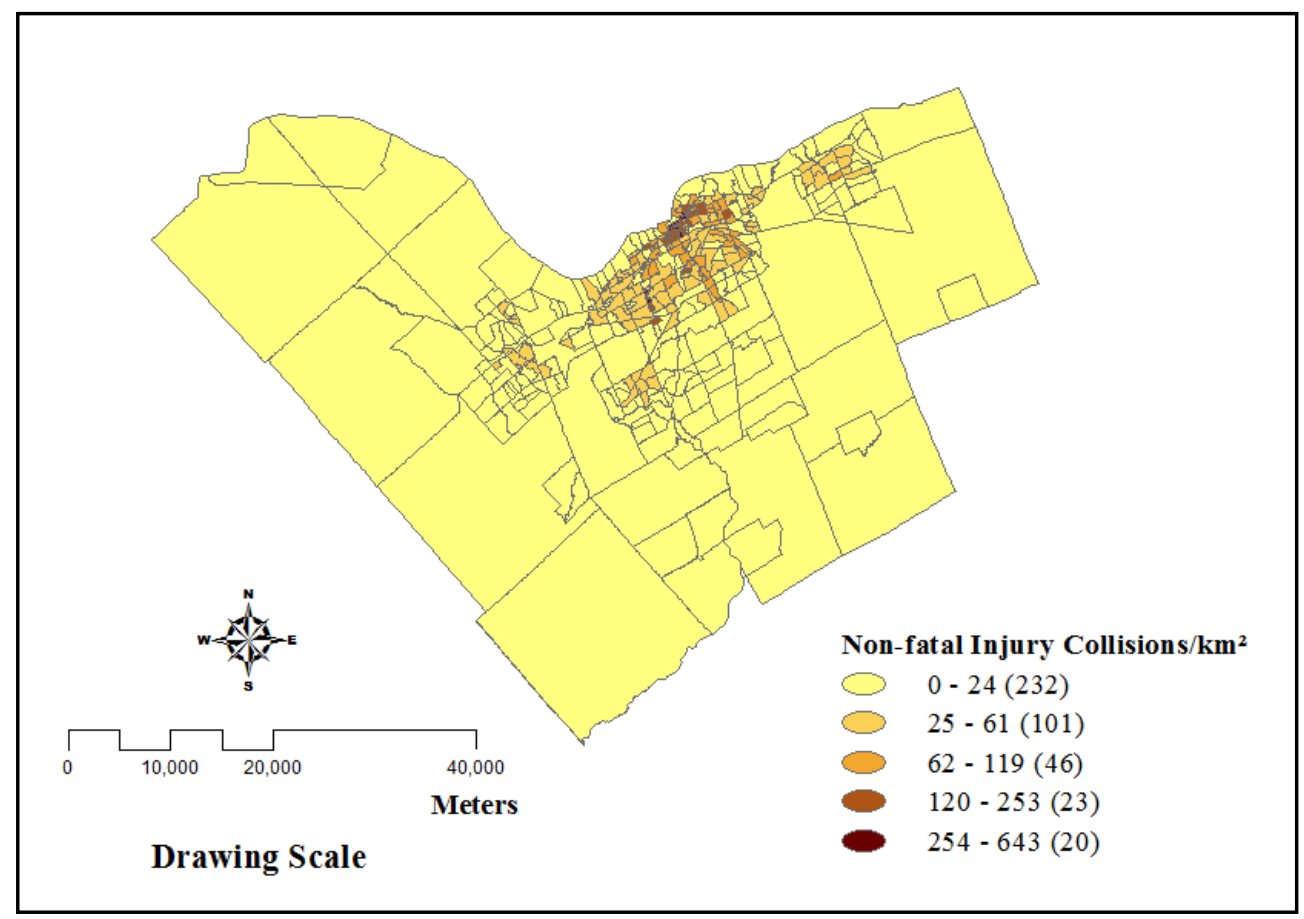

Figure A. 54 Spatial Distributions of Non-fatal Injury Collisions Density in the City of Ottawa. Boundary Data Assigned Using TLKM1-Based Assignment Method. 


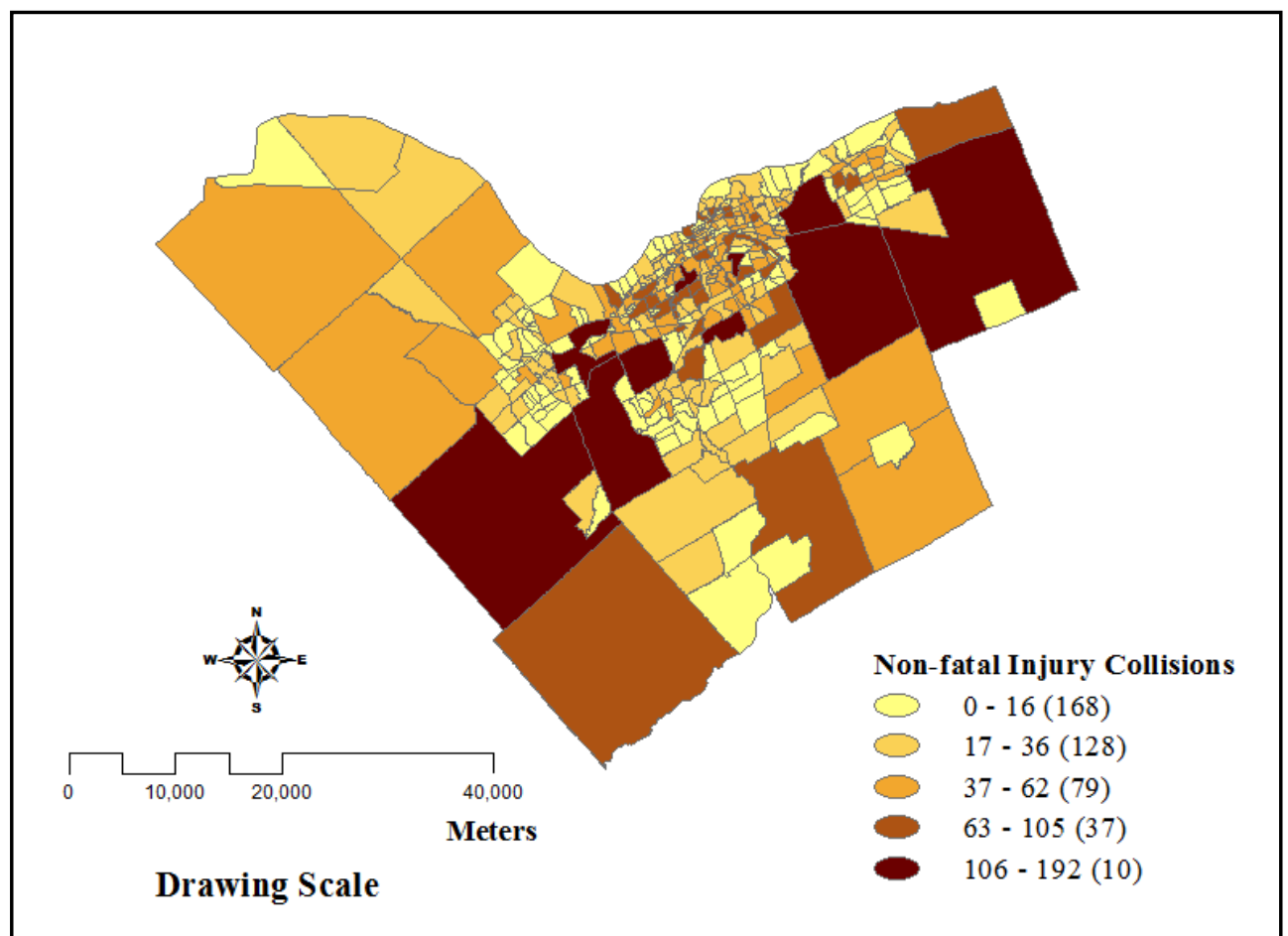

Figure A. 55 Spatial Distributions of Number of Non-fatal Injury Collisions in the City of Ottawa. Boundary Data Assigned Using VKT1-Based Assignment Method.

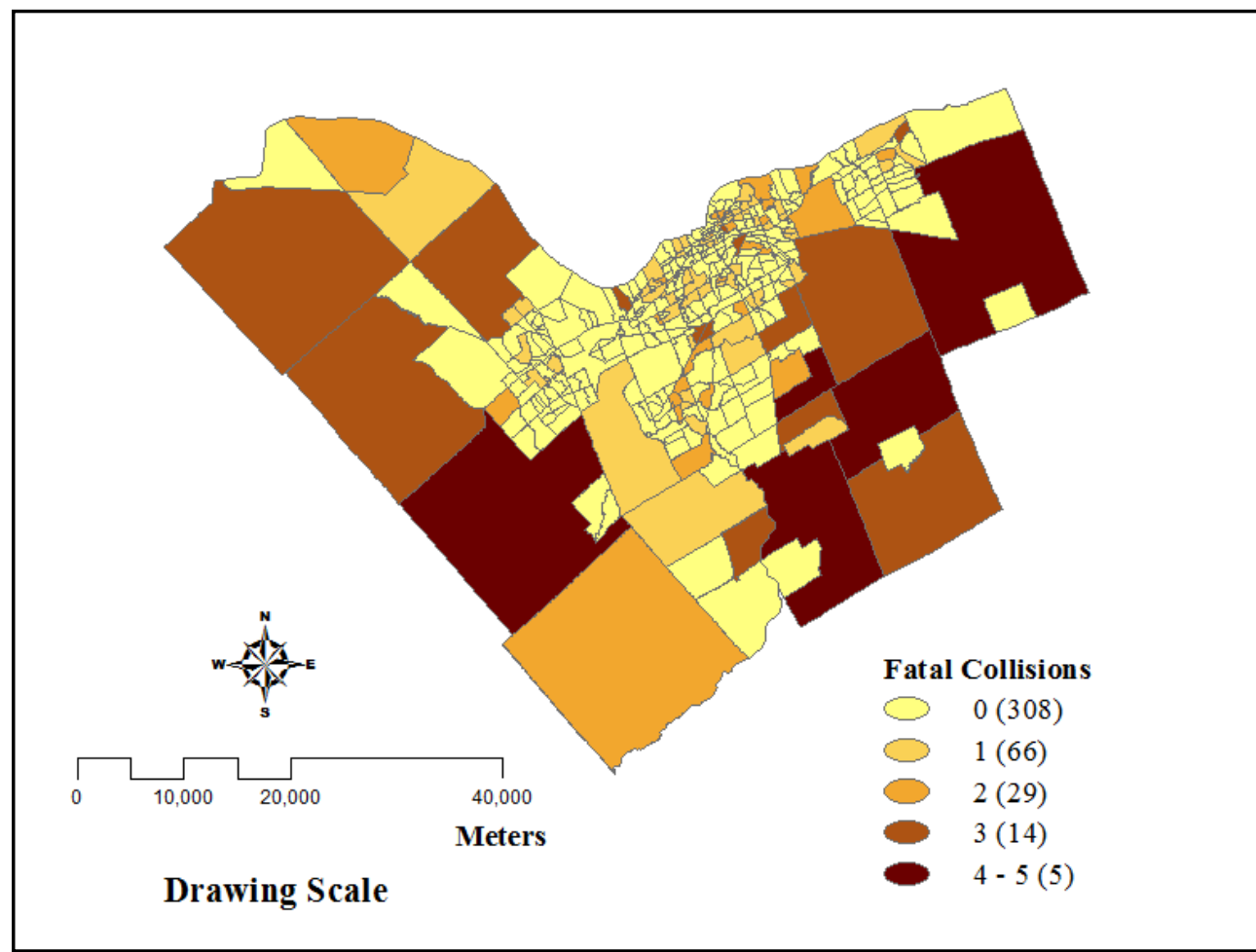

Figure A. 56 Spatial Distributions of Number of Fatal Collisions in the City of Ottawa. Boundary Data Assigned Using VKT1-Based Assignment Method. 


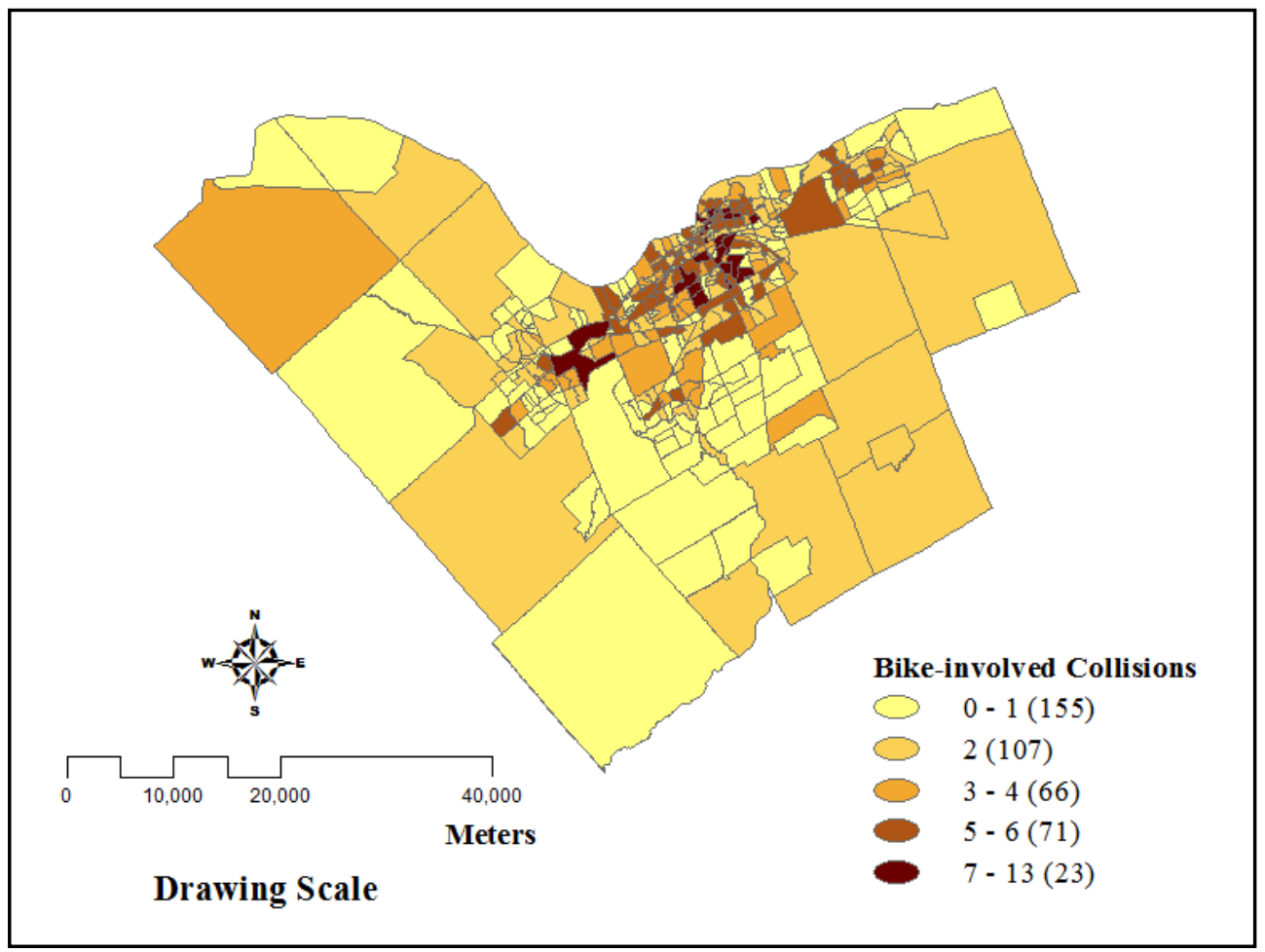

Figure A. 57 Spatial Distributions of Number of Bike-involved Collisions in the City of Ottawa. Boundary Data Assigned Using VKT1-Based Assignment Method.

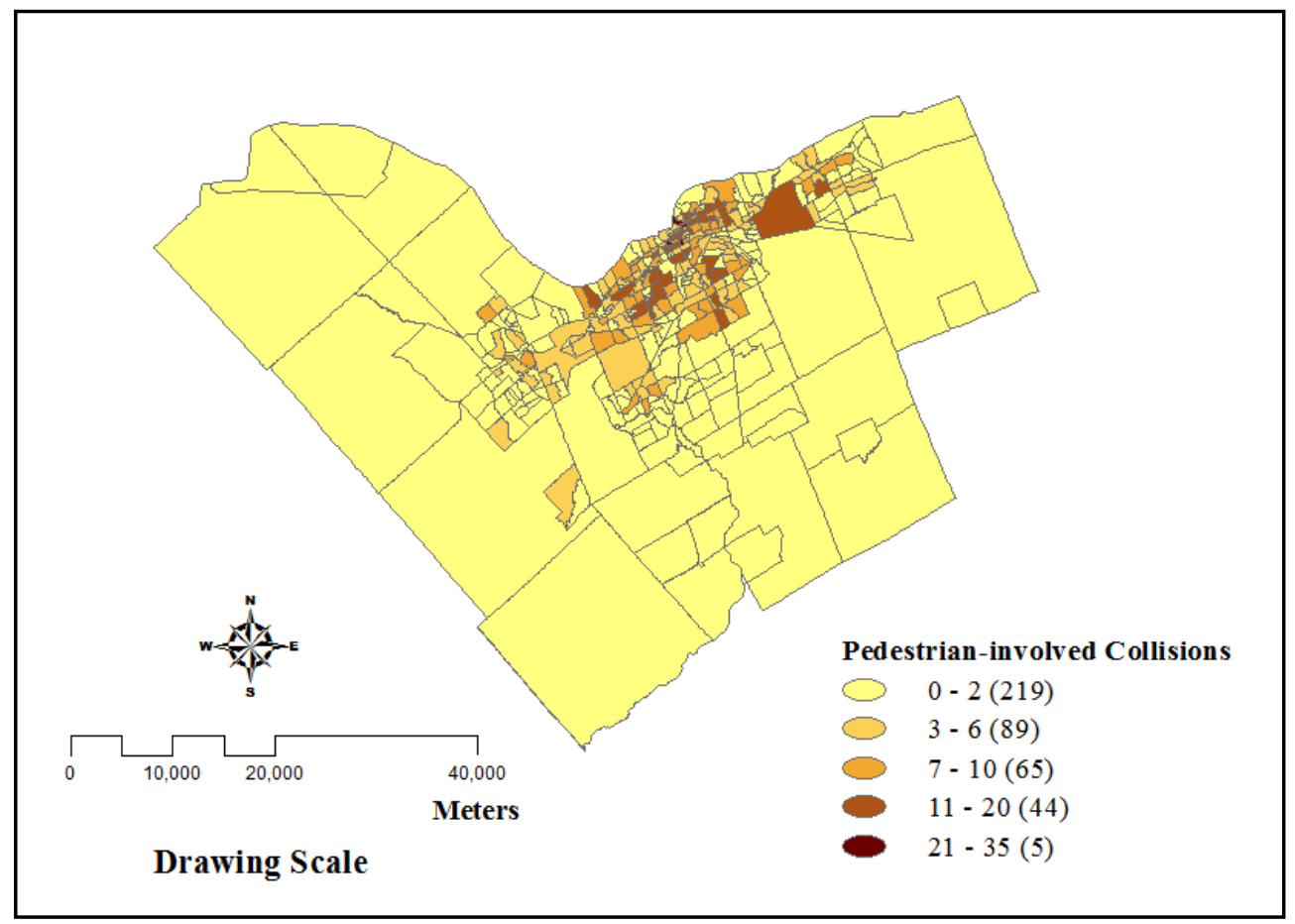

Figure A. 58 Spatial Distributions of Number of Pedestrian-involved Collisions in the City of Ottawa. Boundary Data Assigned Using VKT1-Based Assignment Method. 


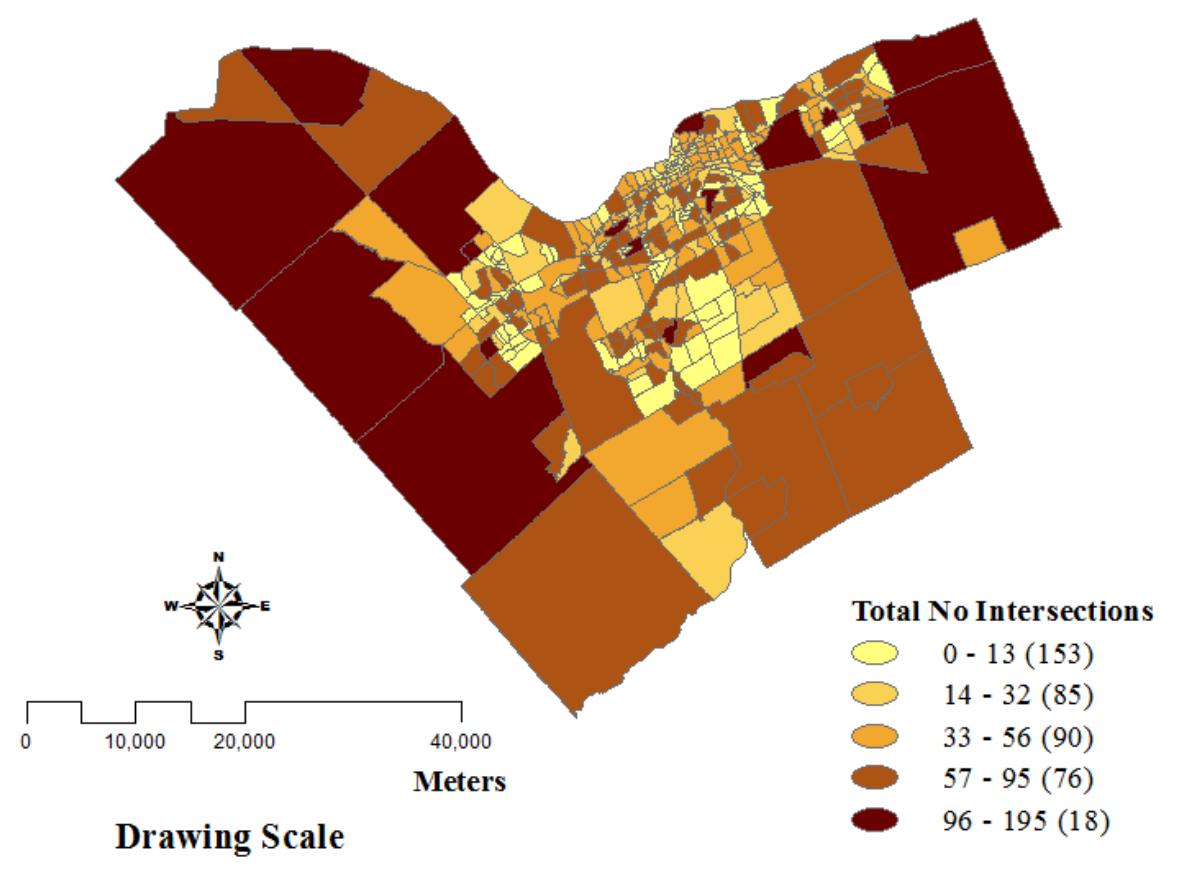

Figure A. 59 Spatial Distributions of Number of Intersections in the City of Ottawa. Boundary Data Assigned Using VKT1-Based Assignment Method.

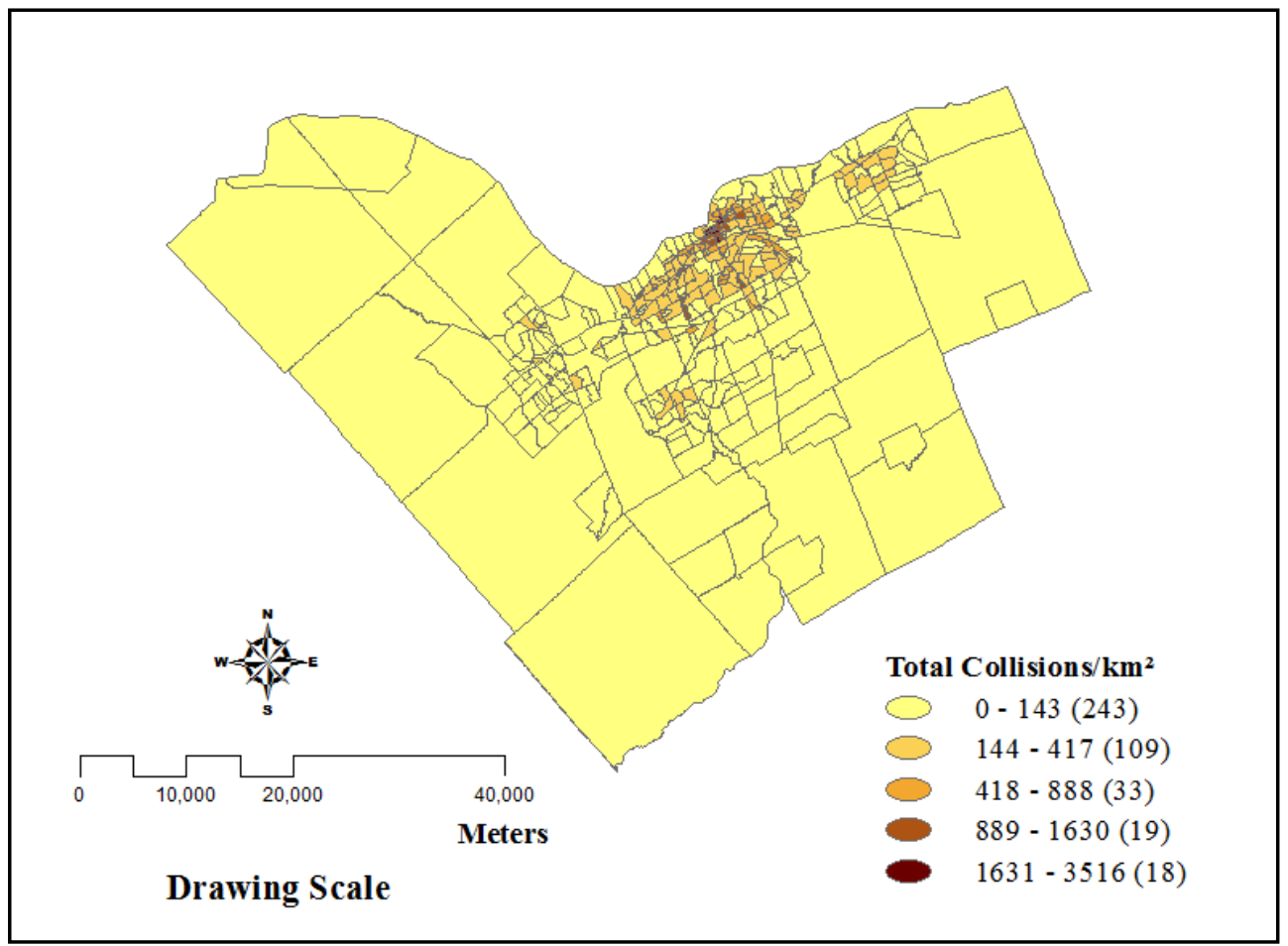

Figure A. 60 Spatial Distributions of Total Collisions Density in the City of Ottawa. Boundary Data Assigned Using VKT1-Based Assignment Method. 


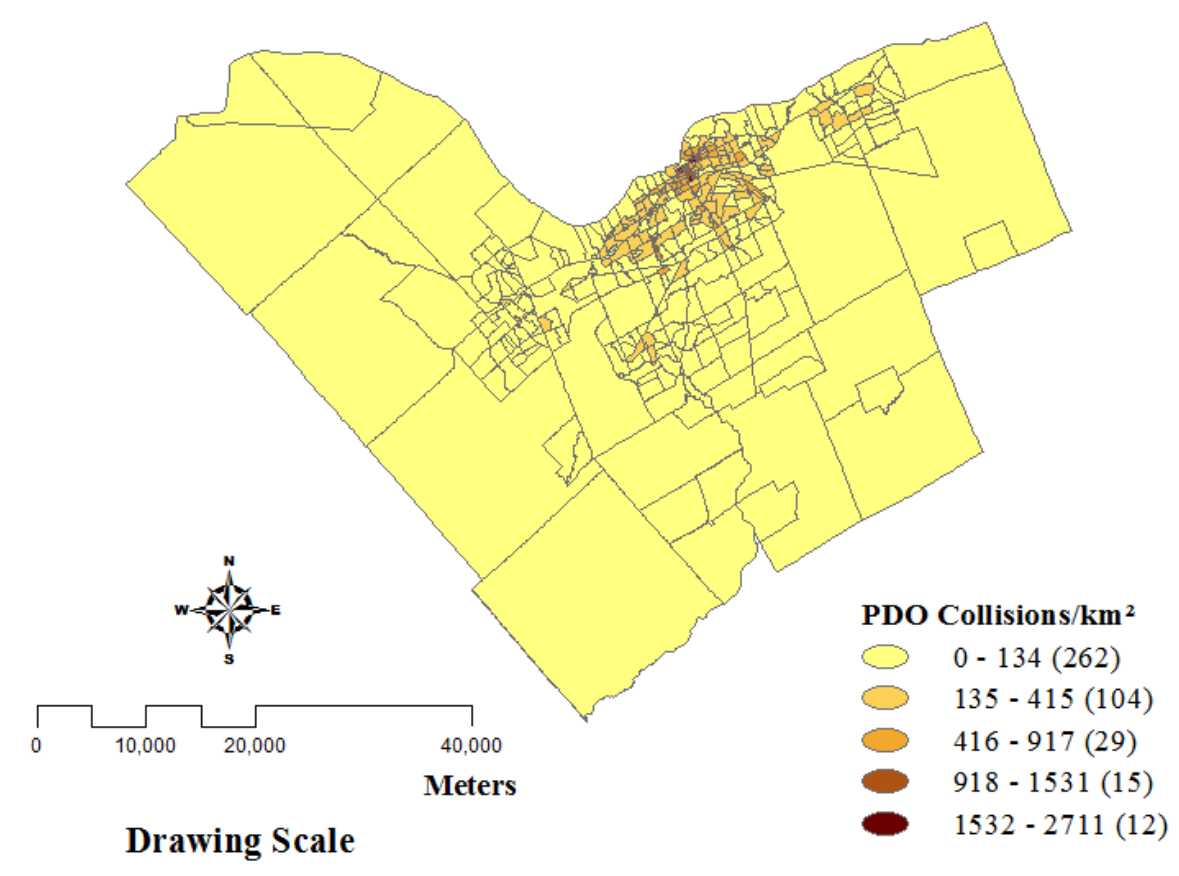

Figure A. 61 Spatial Distributions of PDO Collisions Density in the City of Ottawa. Boundary Data Assigned Using VKT1-Based Assignment Method.

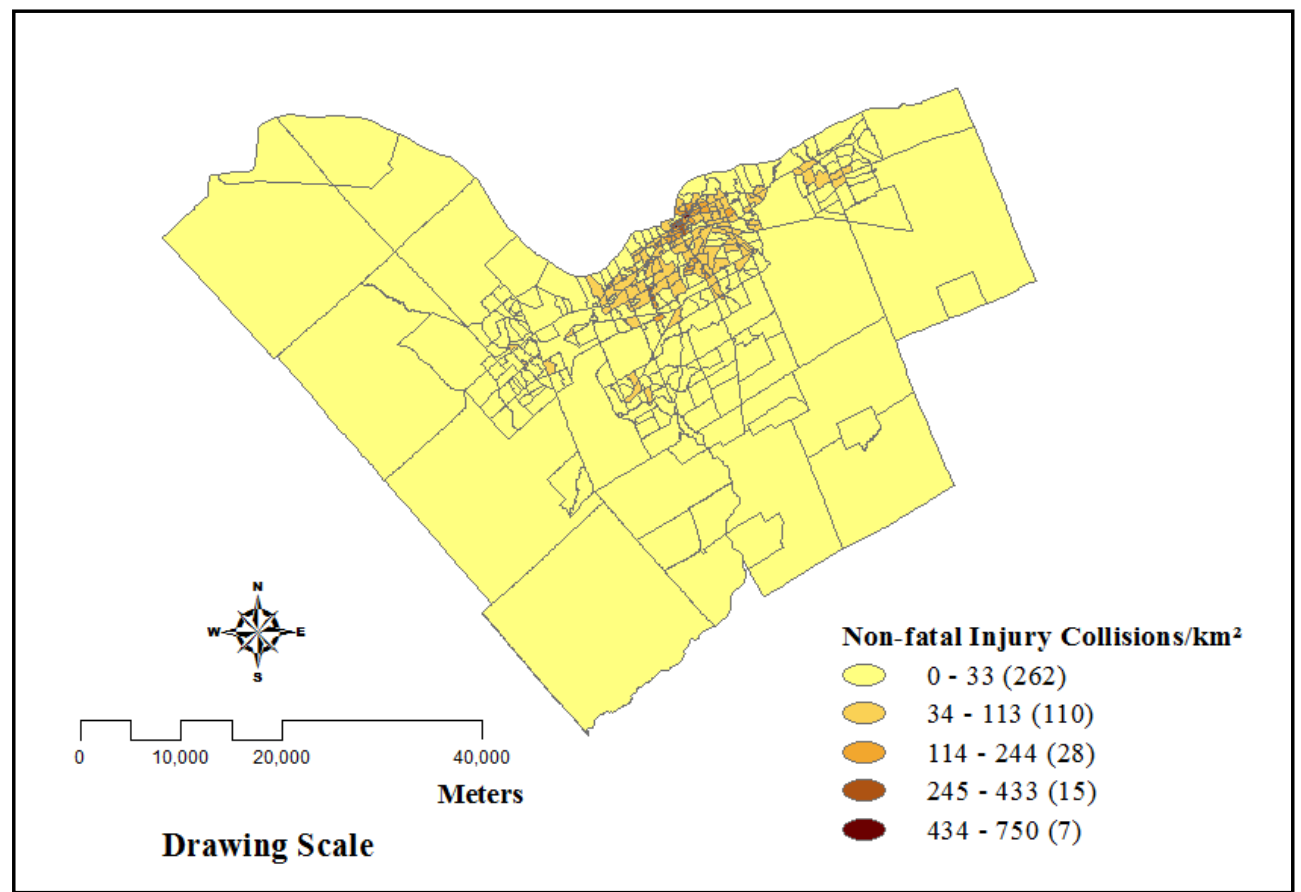

Figure A. 62 Spatial Distributions of Non-fatal Injury Collisions Density in the City of Ottawa. Boundary Data Assigned Using VKT1-Based Assignment Method. 


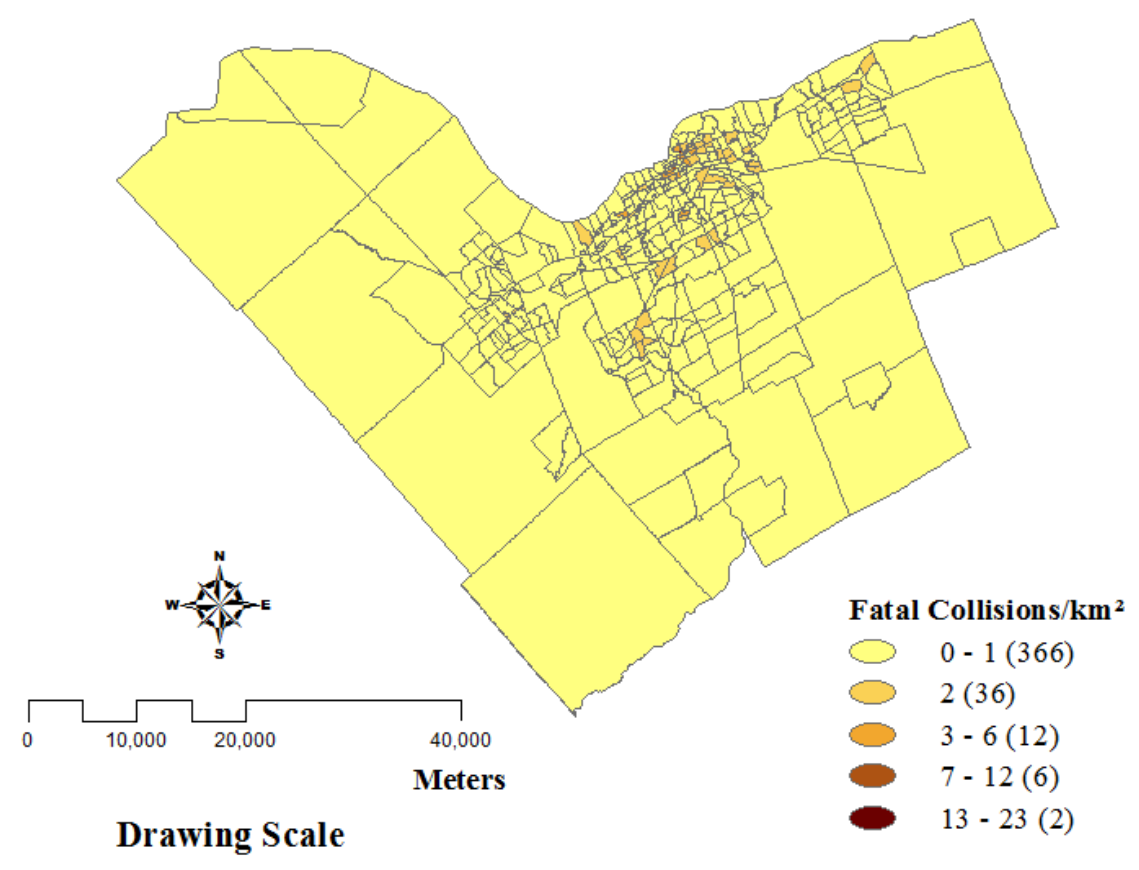

Figure A. 63 Spatial Distributions of Fatal Collisions Density in the City of Ottawa. Boundary Data Assigned Using VKT1-Based Assignment Method.

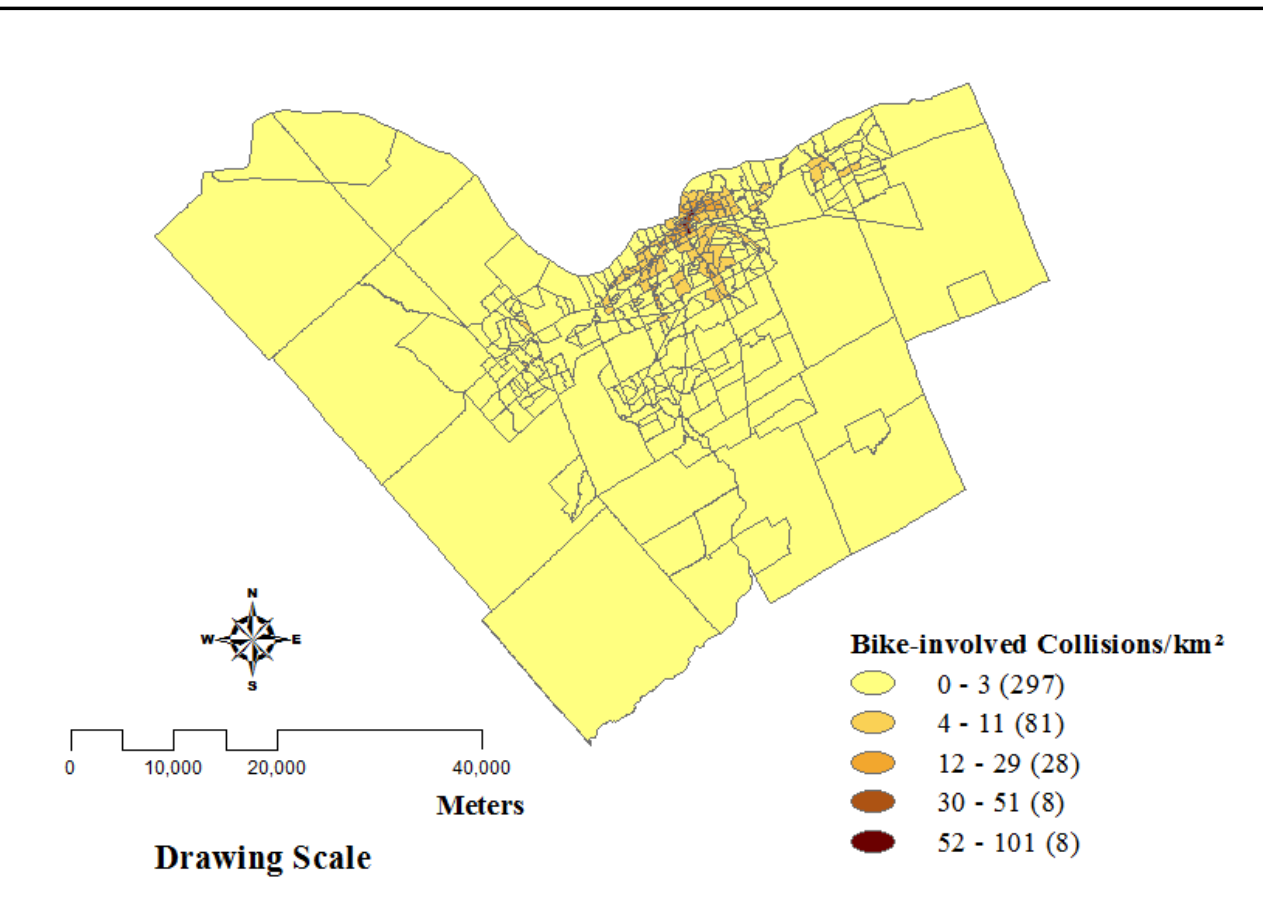

Figure A. 64 Spatial Distributions of Bike-involved Collisions Density in the City of Ottawa. Boundary Data Assigned Using VKT1-Based Assignment Method. 


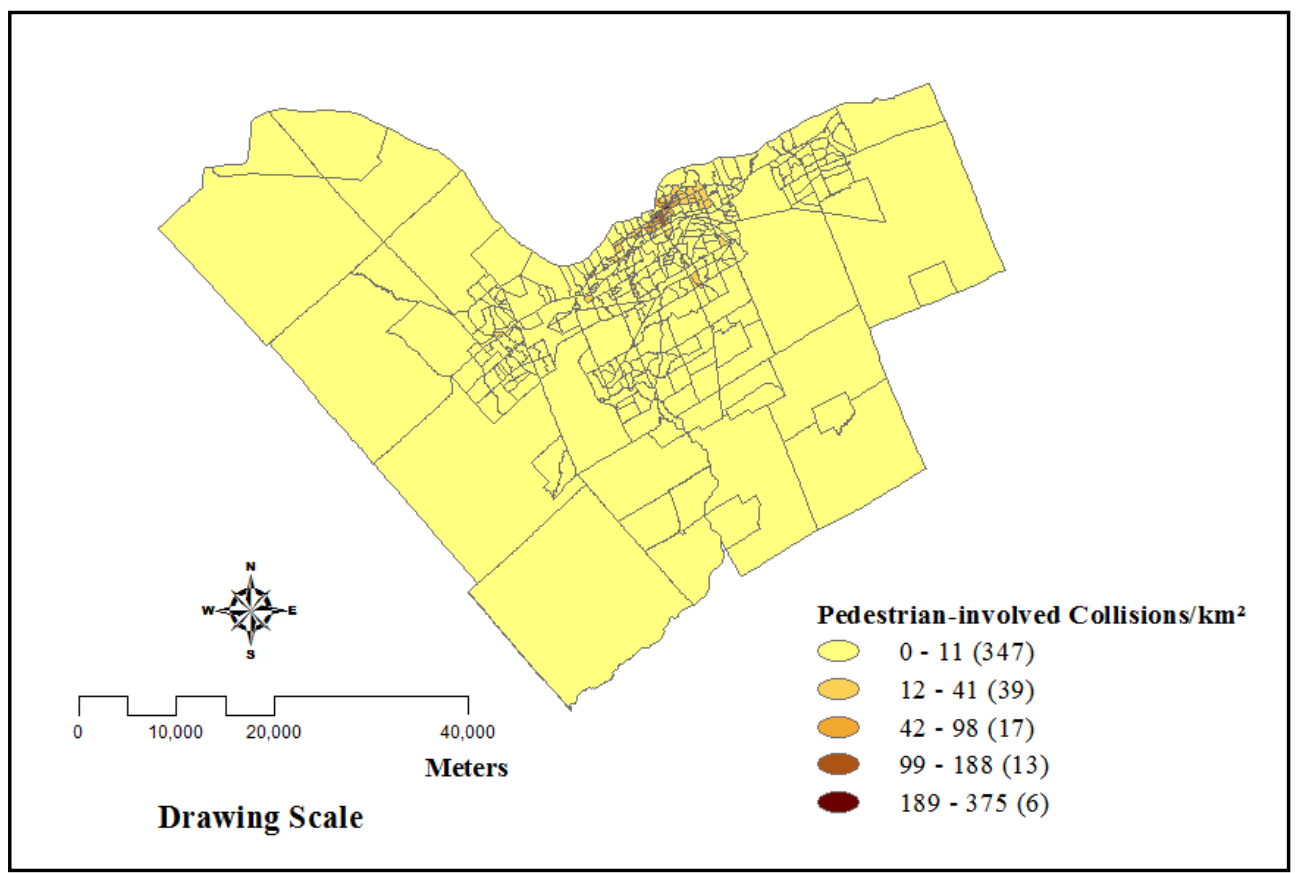

Figure A. 65 Spatial Distributions of Pedestrian-involved Collisions Density in the City of Ottawa. Boundary Data Assigned Using VKT1-Based Assignment Method.

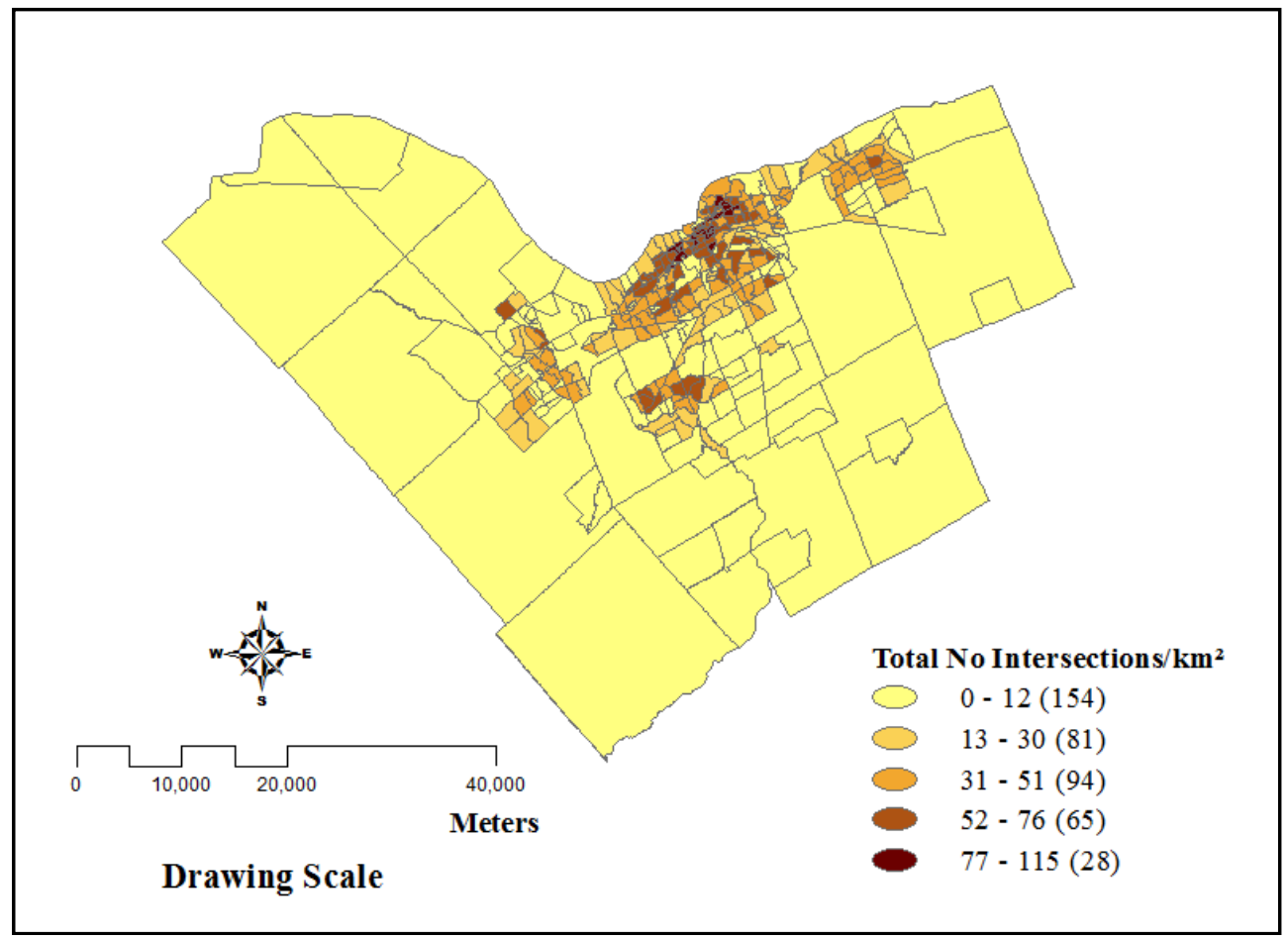

Figure A. 66 Spatial Distributions of Intersections Density in the City of Ottawa. Boundary Data Assigned Using VKT1-Based Assignment Method. 


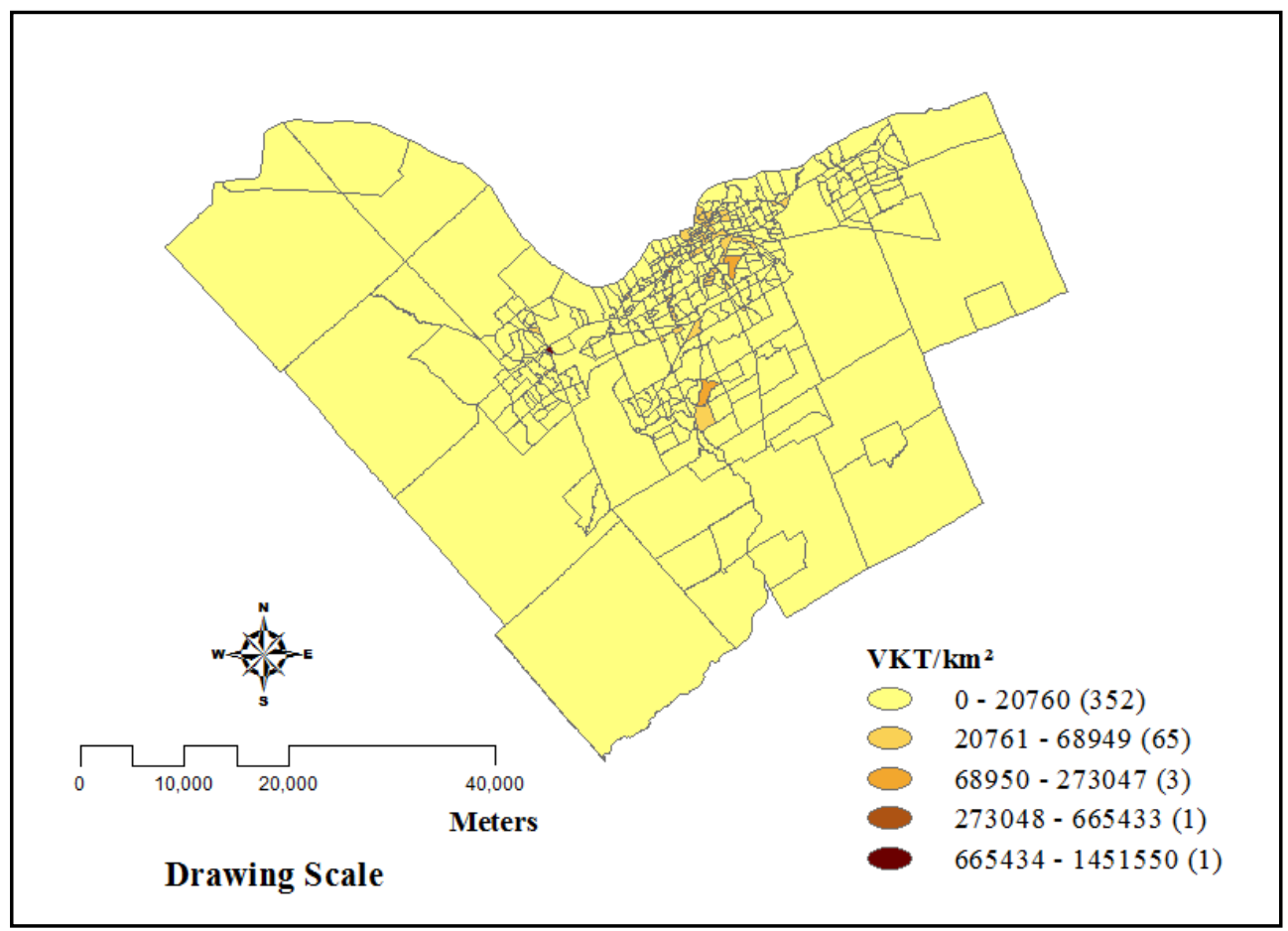

Figure A. 67 Spatial Distributions of VKT Density in the City of Ottawa. Boundary Data Assigned Using VKT1-Based Assignment Method.

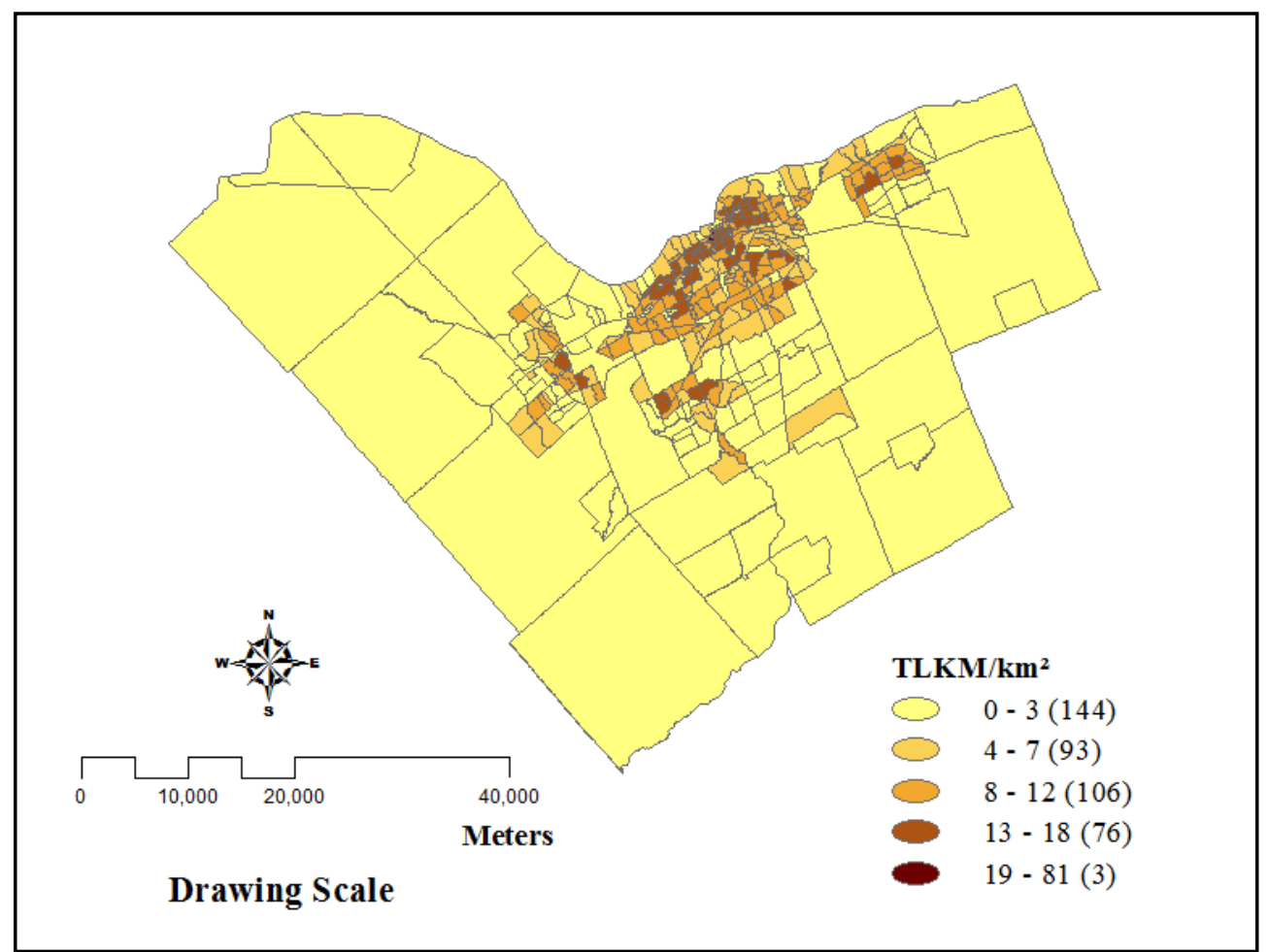

Figure A. 68 Spatial Distributions of TLKM Density in the City of Ottawa. Boundary Data Assigned Using VKT1-Based Assignment Method. 


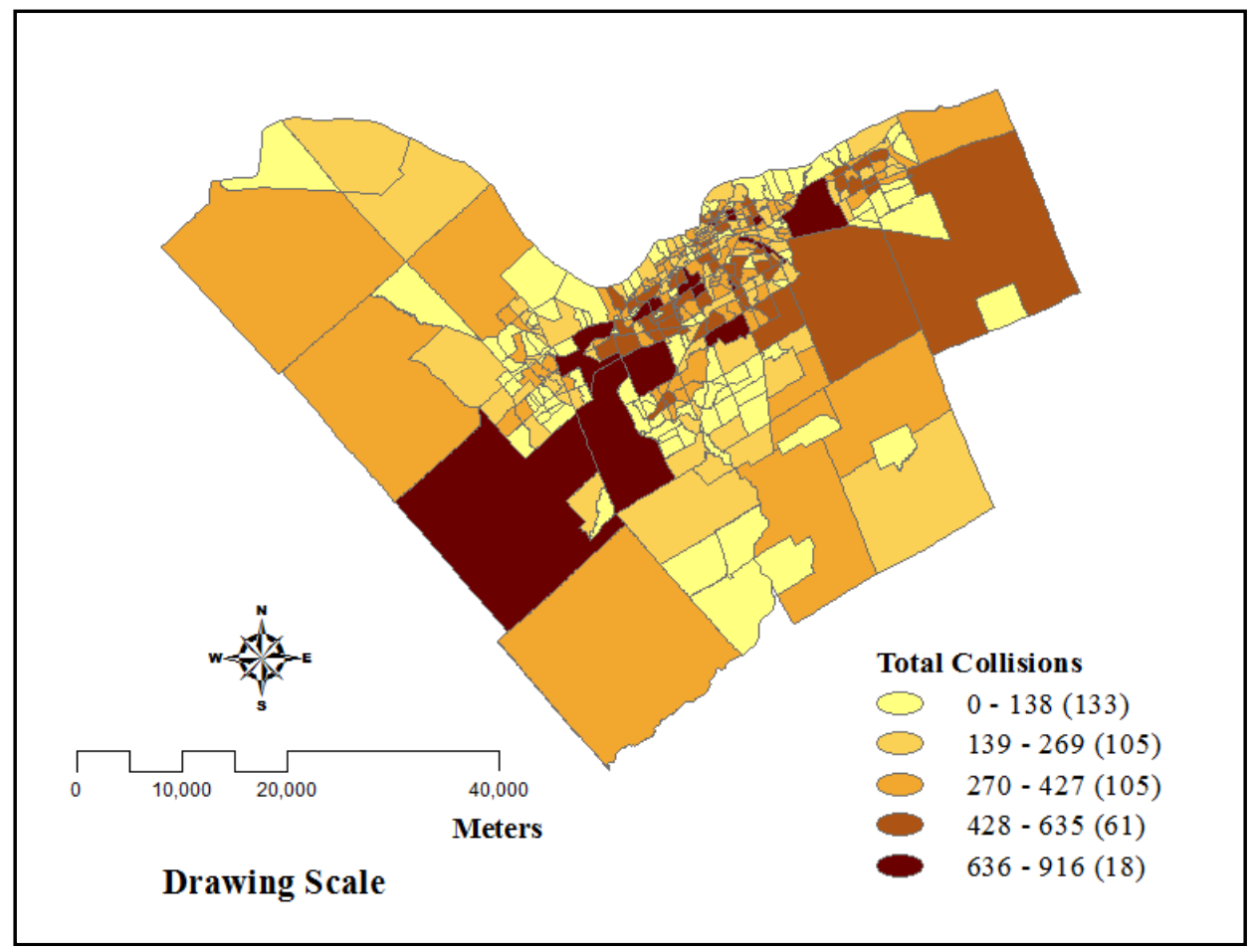

Figure A. 69 Spatial Distributions of Number of Total Collisions in the City of Ottawa. Boundary Data Assigned Using Multiple-Counted-Based Assignment Method.

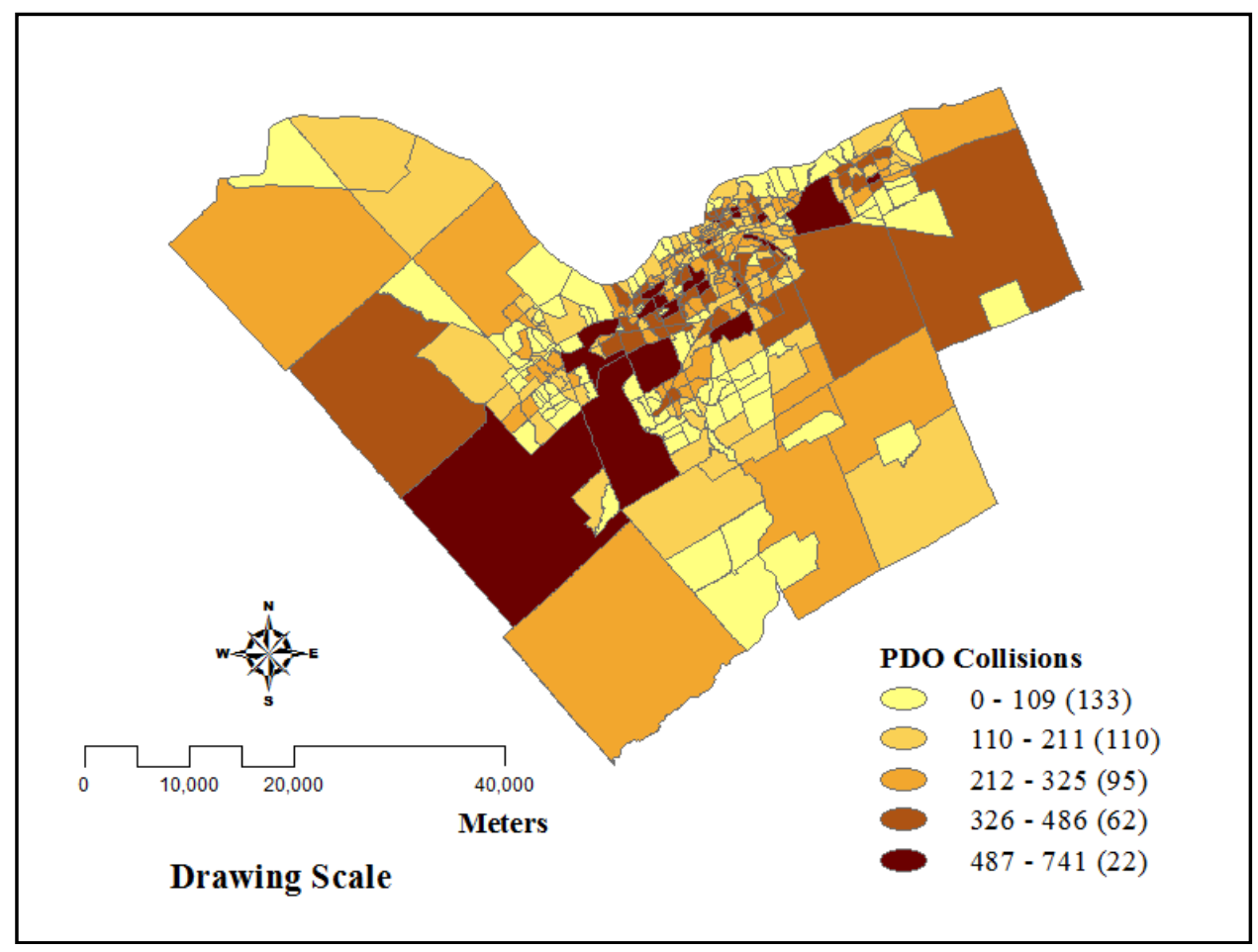

Figure A. 70 Spatial Distributions of Number of PDO Collisions in the City of Ottawa. Boundary Data Assigned Using Multiple-Counted-Based Assignment Method. 


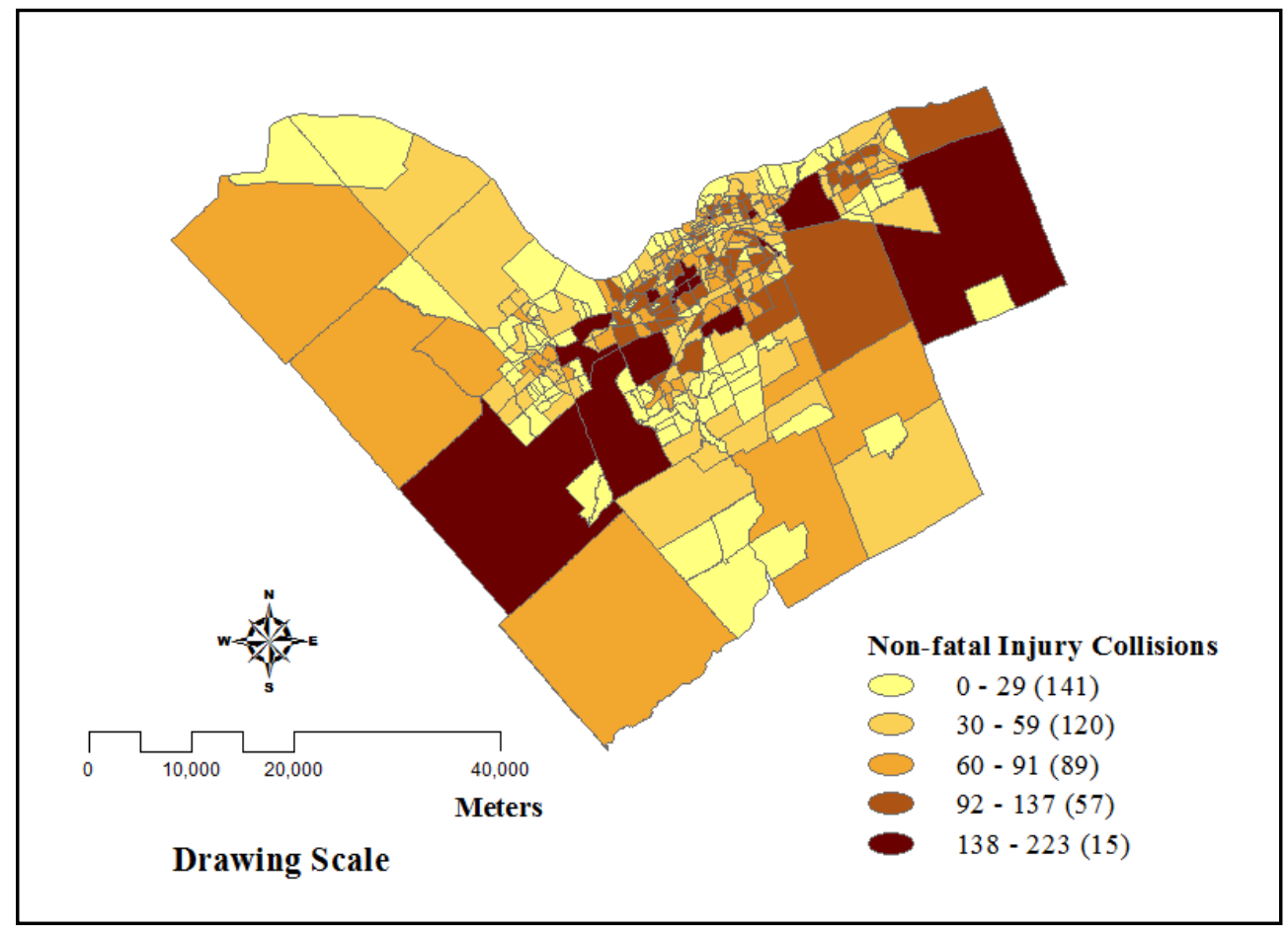

Figure A. 71 Spatial Distributions of Number of Non-fatal Injury Collisions in the City of Ottawa. Boundary Data Assigned Using Multiple-Counted-Based Assignment Method.

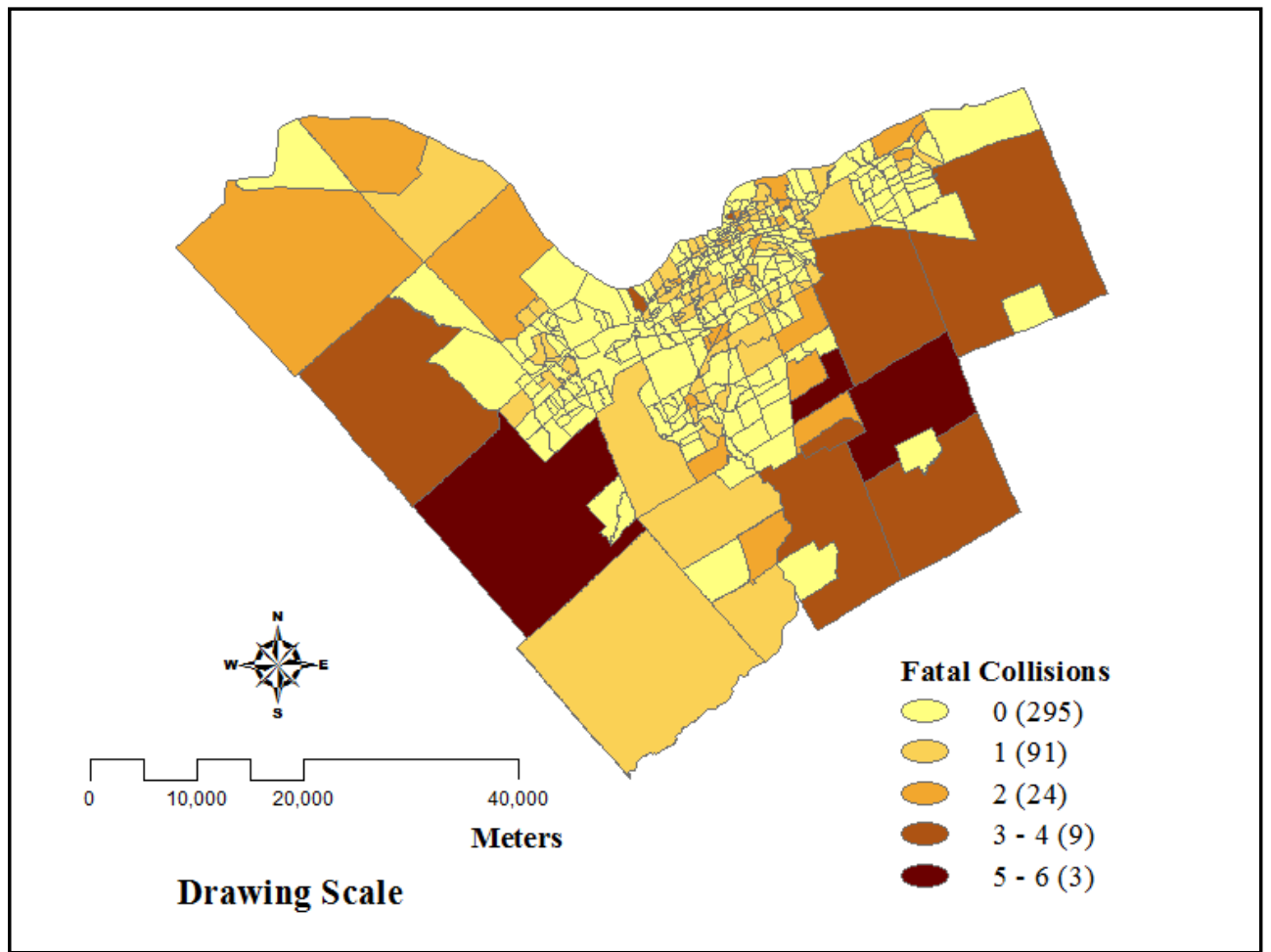

Figure A. 72 Spatial Distributions of Number of Fatal Collisions in the City of Ottawa. Boundary Data Assigned Using Multiple-Counted-Based Assignment Method. 


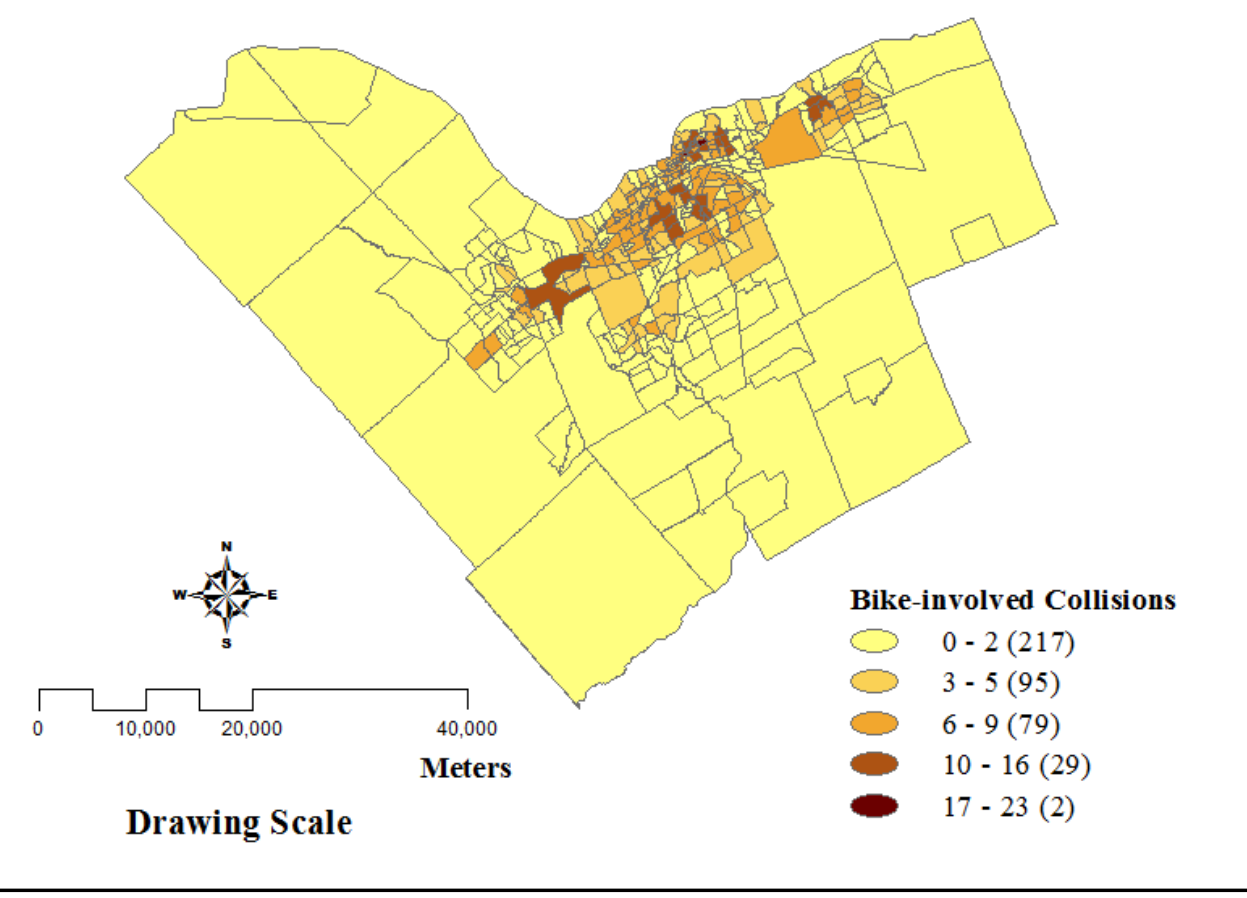

Figure A. 73 Spatial Distributions of Number of Bike-involved Collisions in the City of Ottawa. Boundary Data Assigned Using Multiple-Counted-Based Assignment Method.

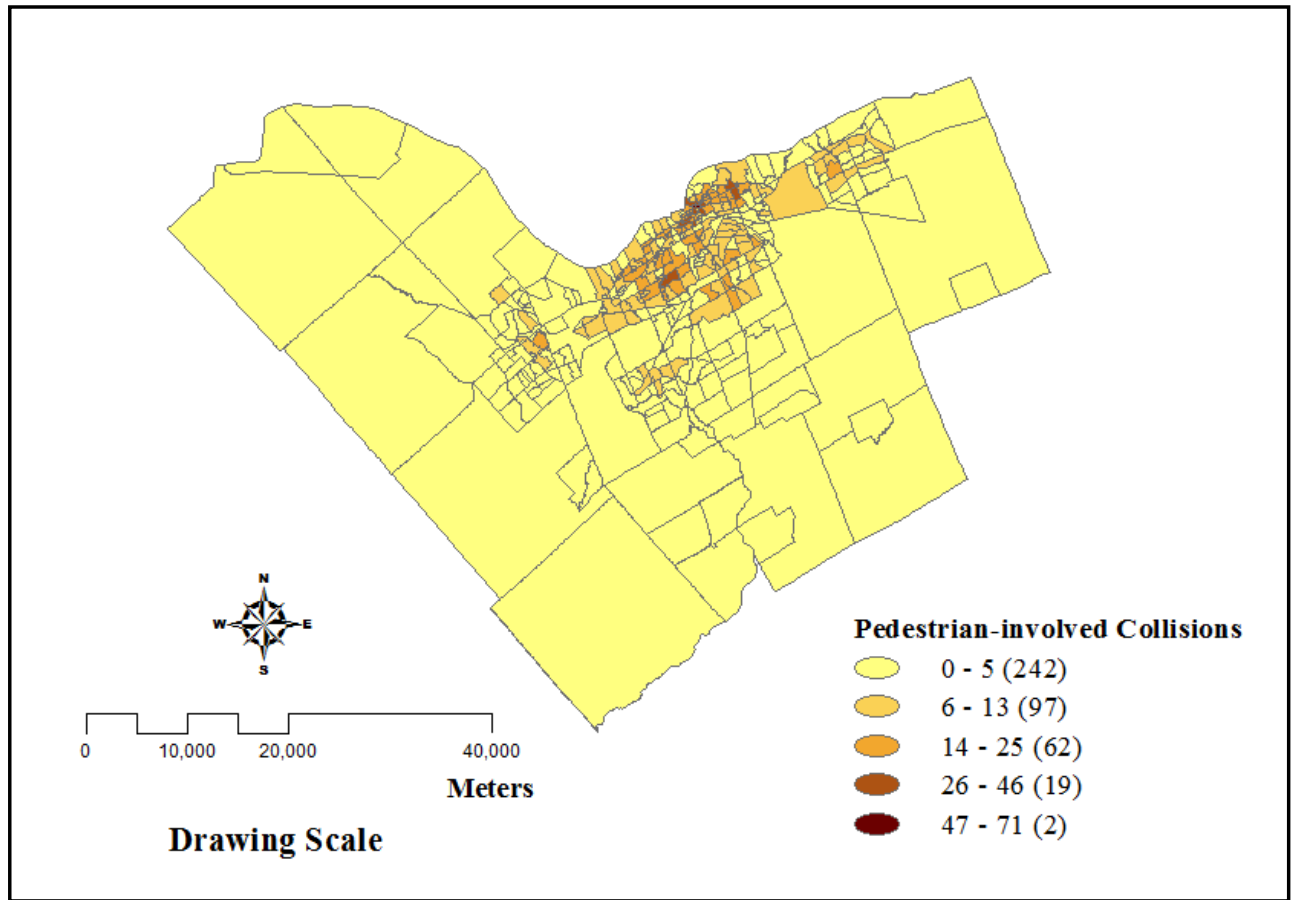

Figure A. 74 Spatial Distributions of Number of Pedestrian-involved Collisions in the City of Ottawa. Boundary Data Assigned Using Multiple-Counted-Based Assignment Method. 


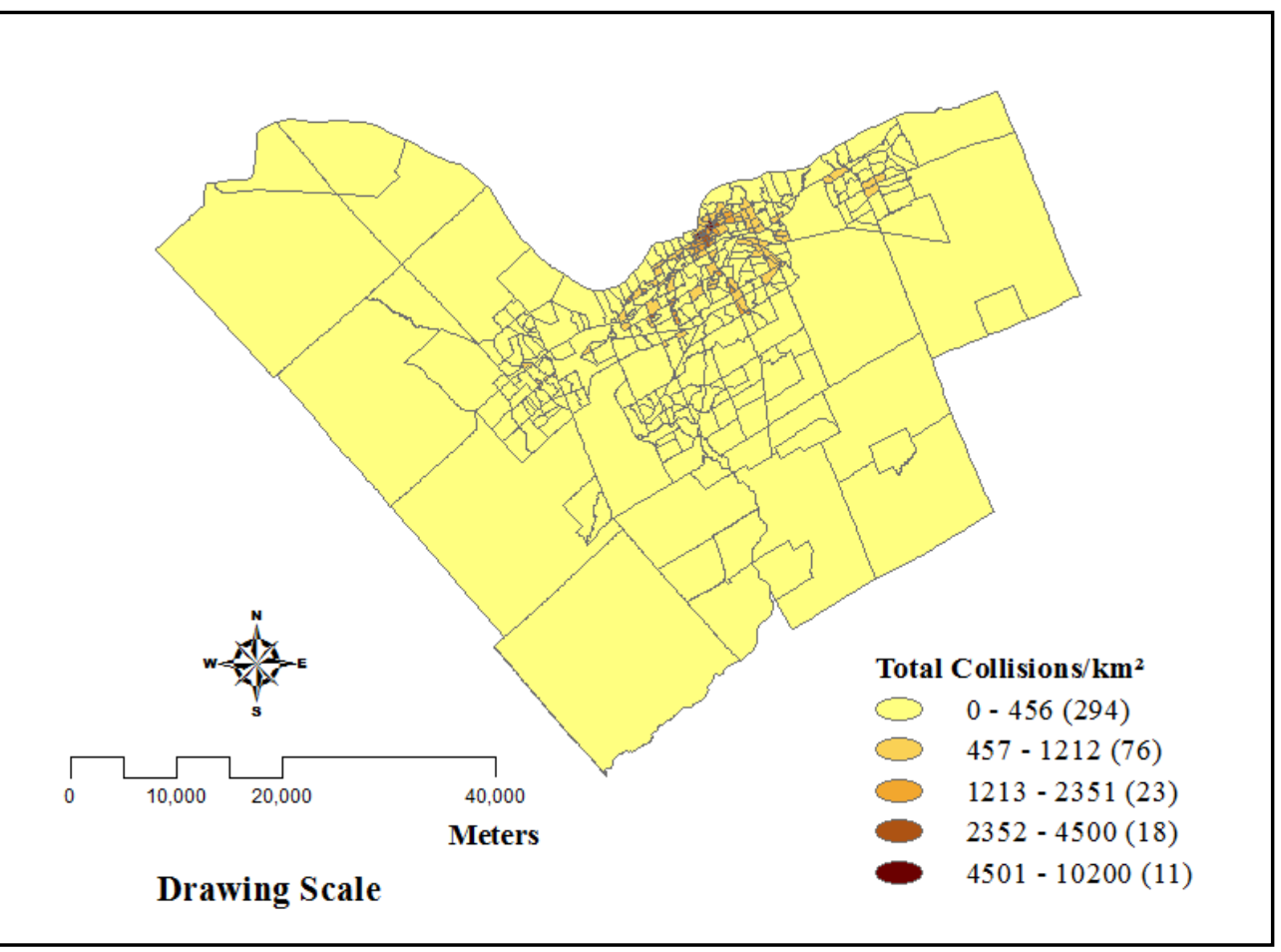

Figure A. 75 Spatial Distributions of Total Collisions Density in the City of Ottawa. Boundary Data Assigned Using Multiple-Counted-Based Assignment Method.

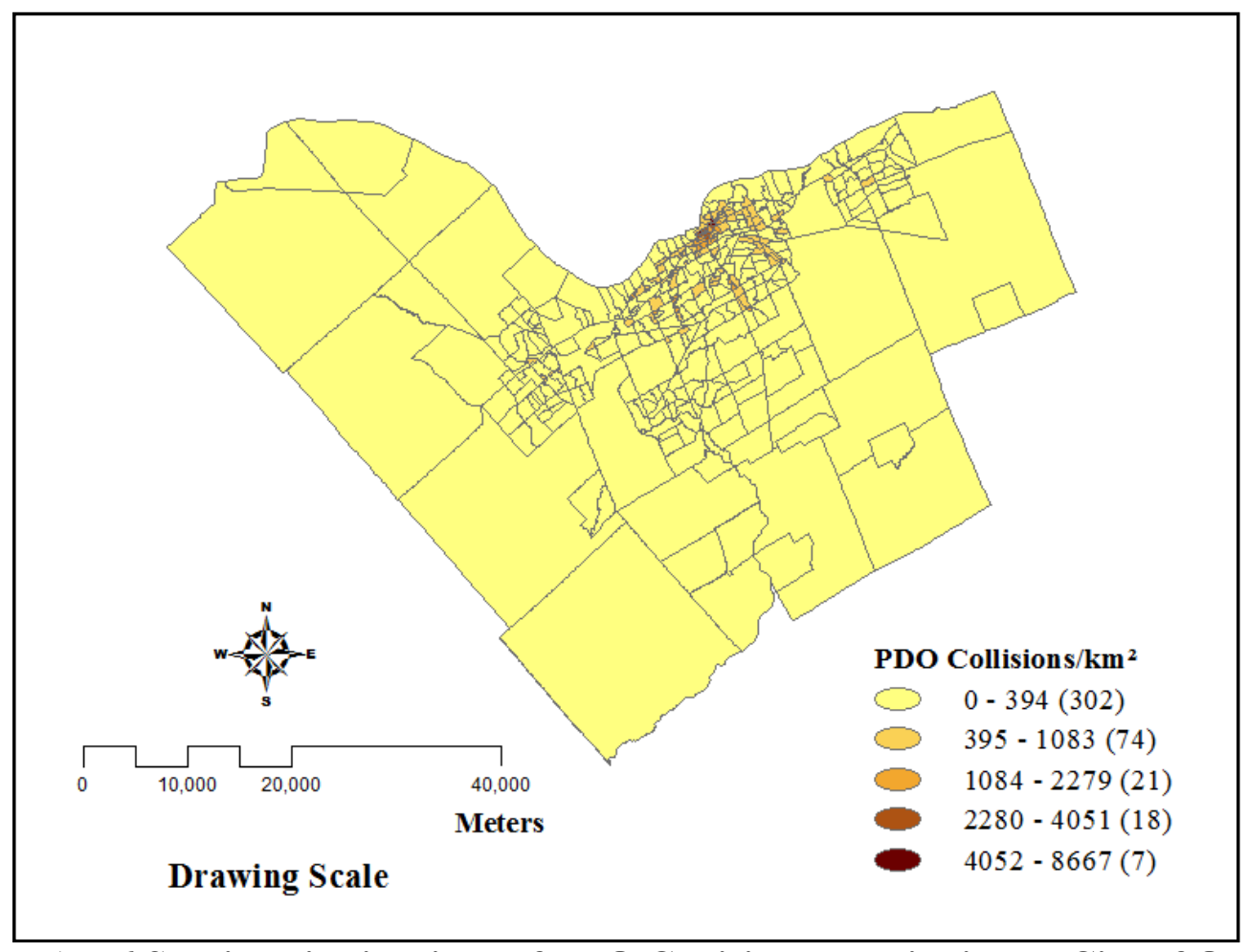

Figure A. 76 Spatial Distributions of PDO Collisions Density in the City of Ottawa. Boundary Data Assigned Using Multiple-Counted-Based Assignment Method. 


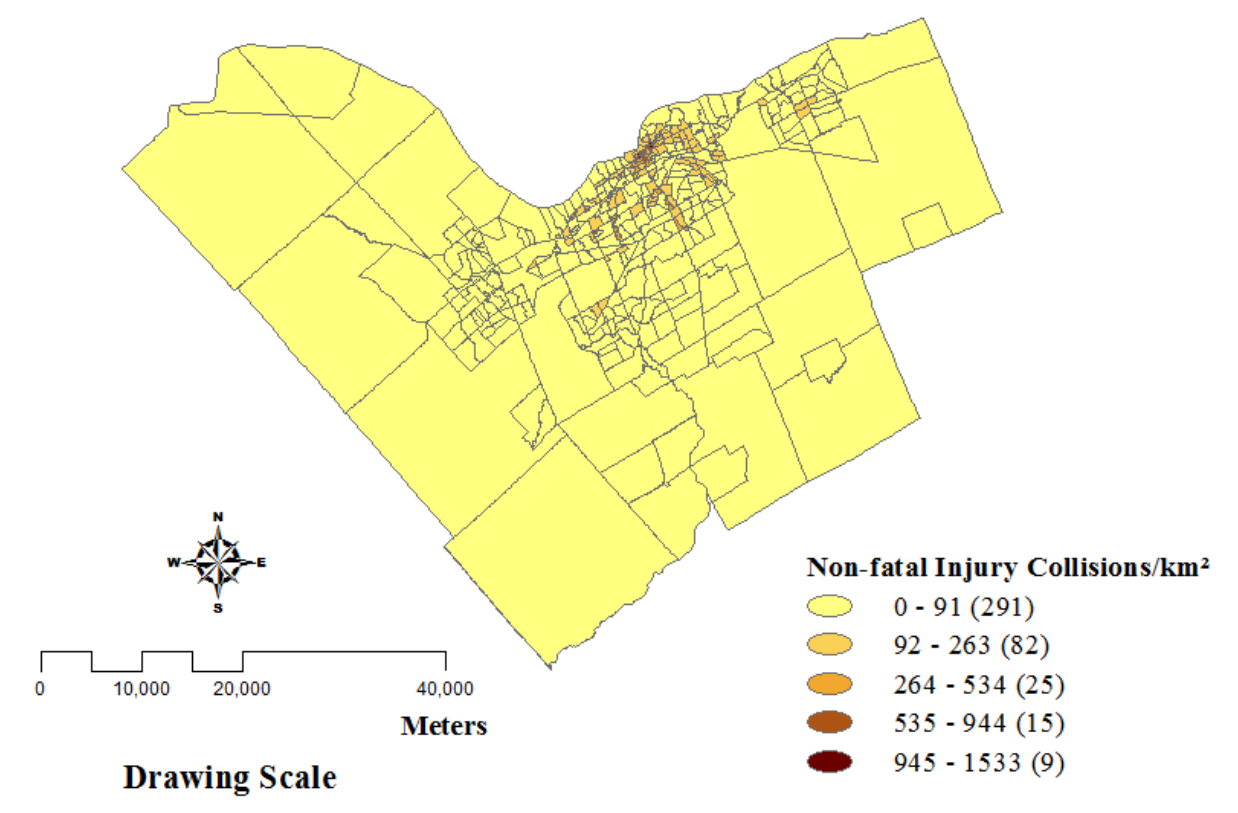

Figure A. 77 Spatial Distributions of Non-fatal Injury Collisions Density in the City of Ottawa. Boundary Data Assigned Using Multiple-Counted-Based Assignment Method.

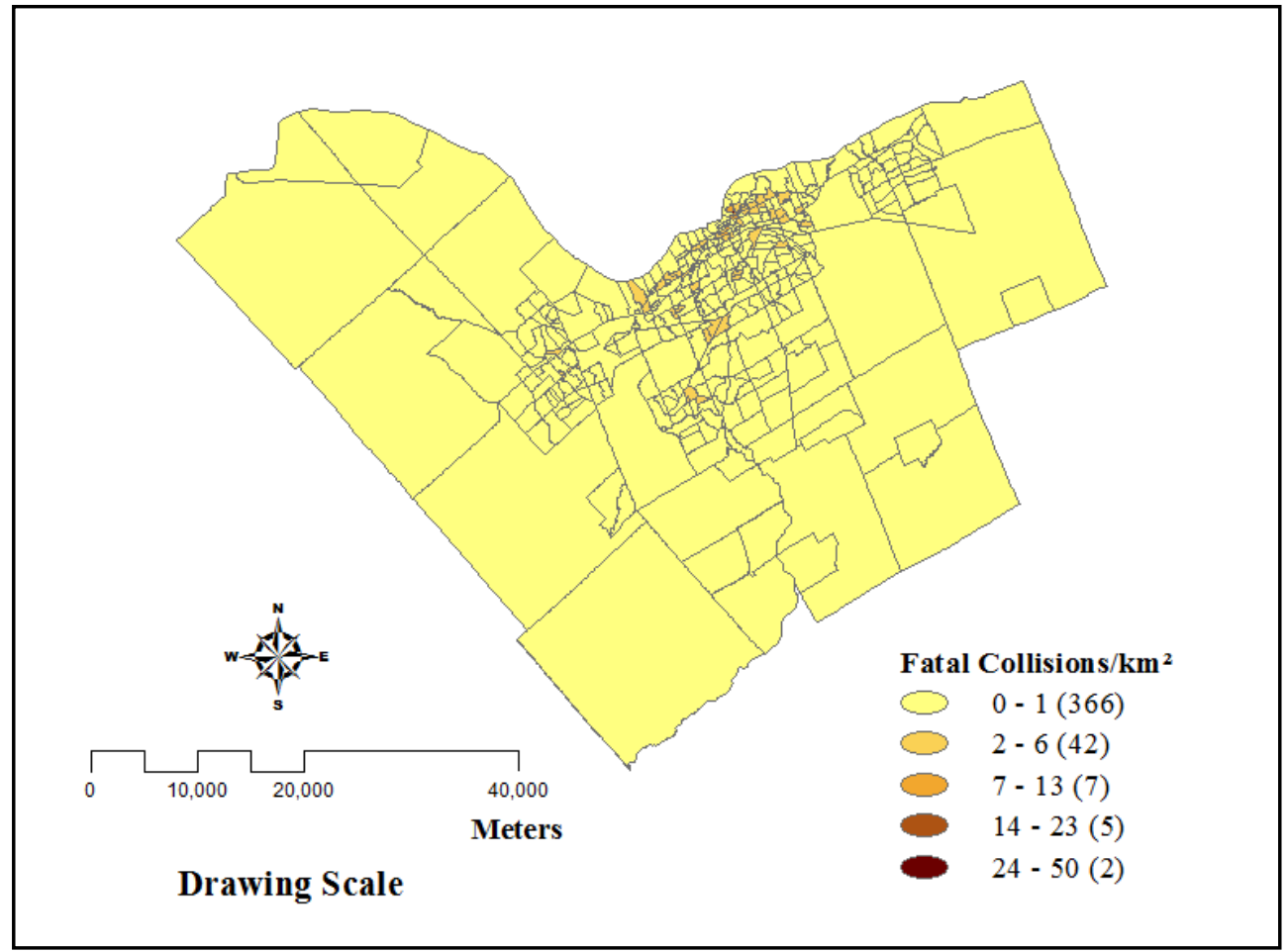

Figure A. 78 Spatial Distributions of Fatal Collisions Density in the City of Ottawa. Boundary Data Assigned Using Multiple-Counted-Based Assignment Method. 


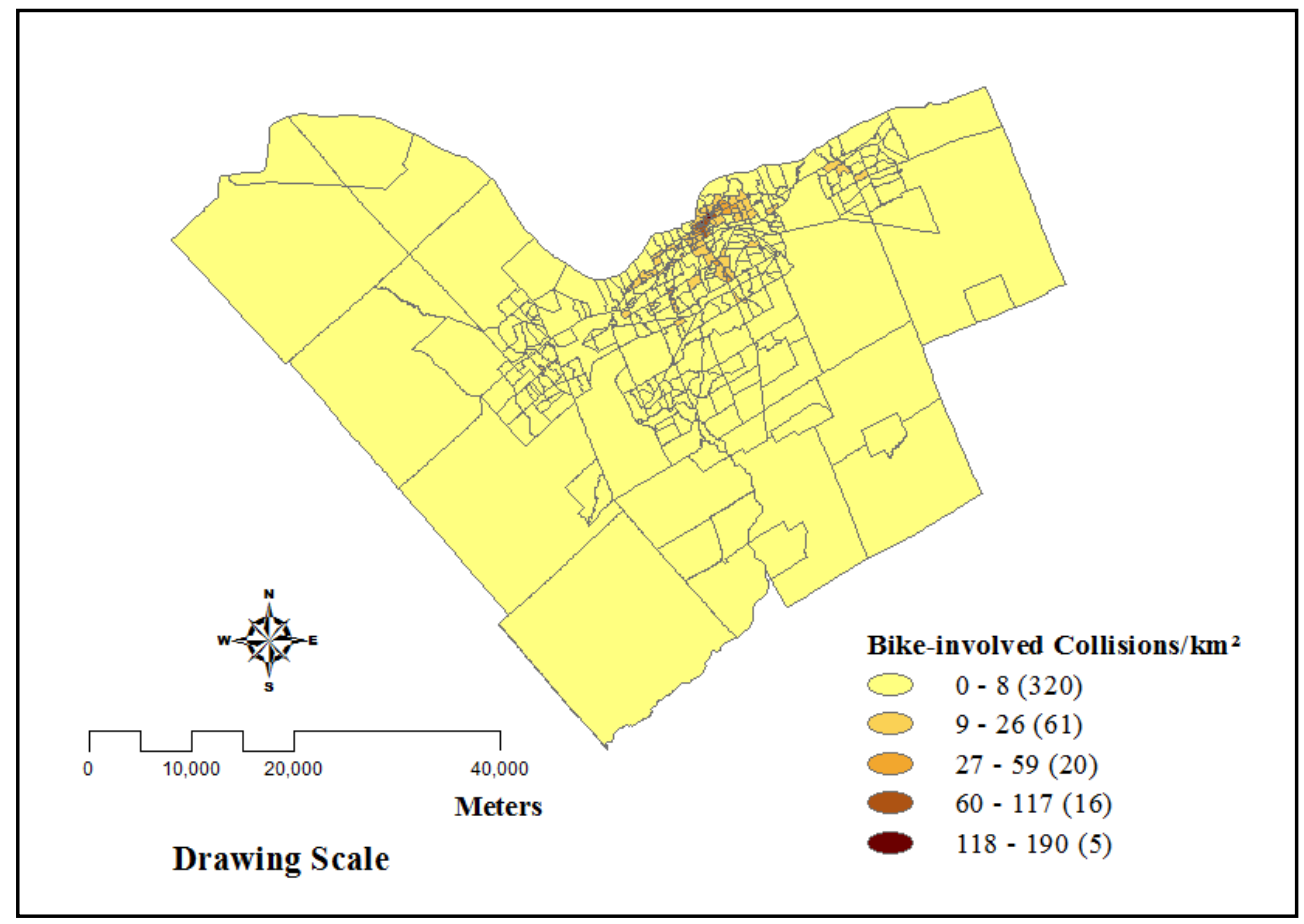

Figure A. 79 Spatial Distributions of Bike-involved Collisions Density in the City of Ottawa. Boundary Data Assigned Using Multiple-Counted-Based Assignment Method.

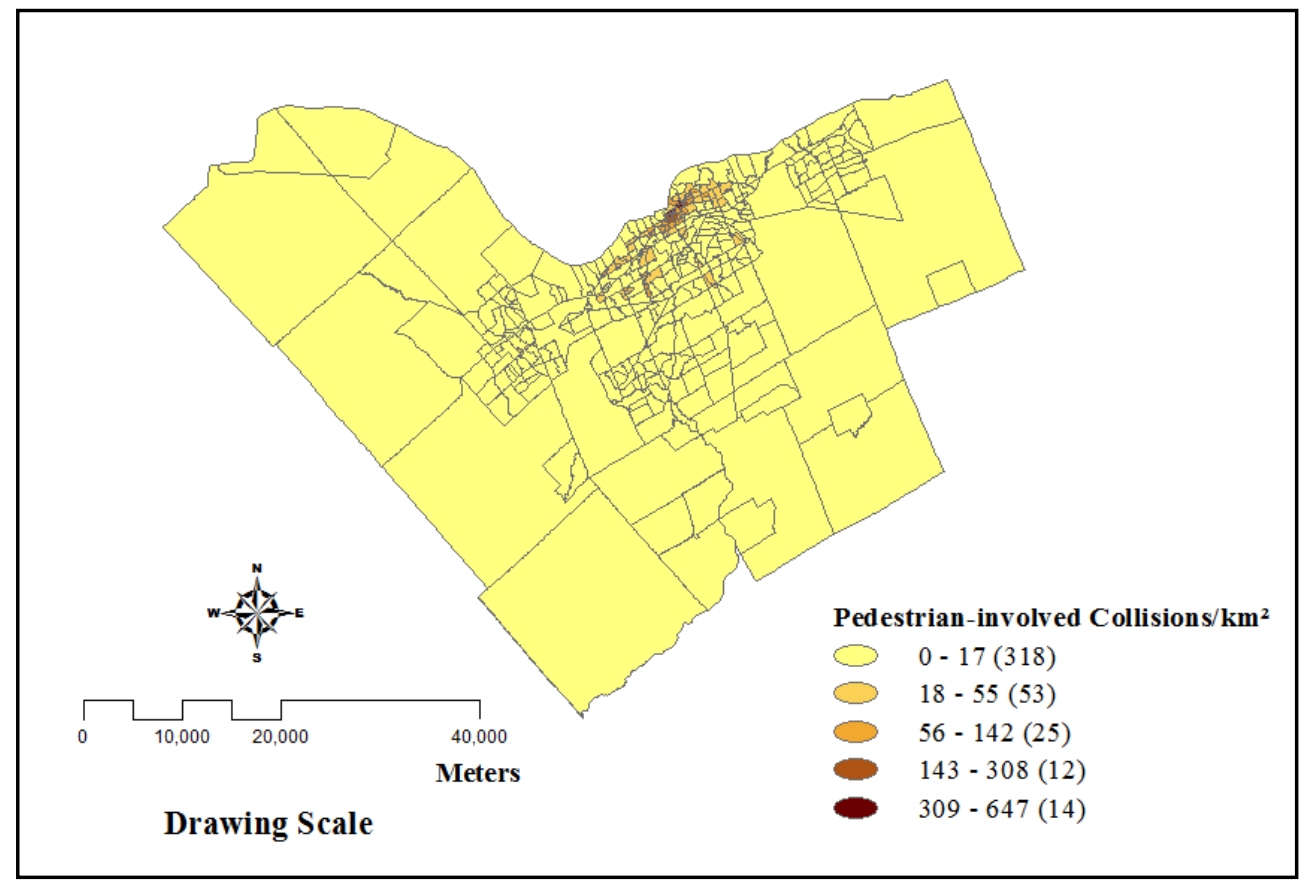

Figure A. 80 Spatial Distributions of Pedestrian-involved Collisions Density in the City of Ottawa. Boundary Data Assigned Using Multiple-Counted-Based Assignment Method. 


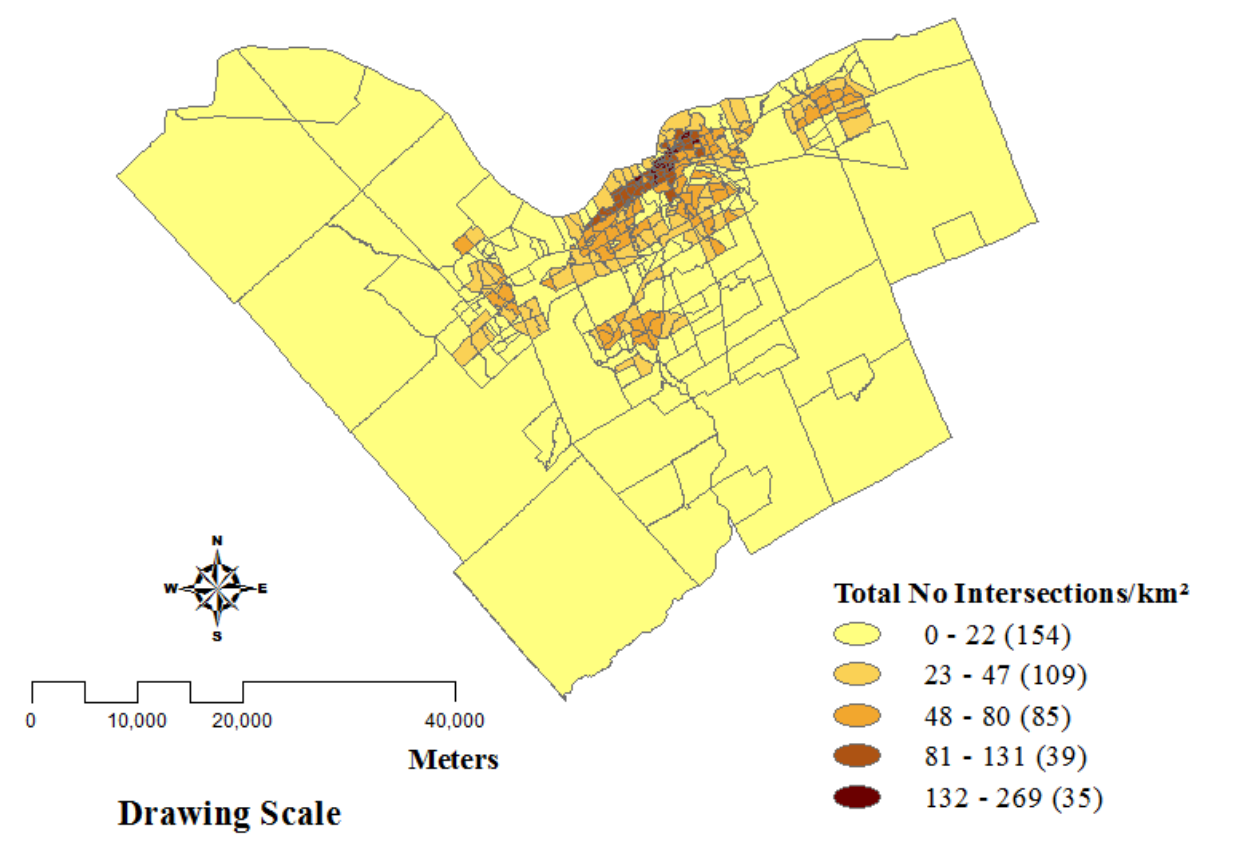

Figure A. 81 Spatial Distributions of Intersection Density in the City of Ottawa. Boundary Data Assigned Using Multiple-Counted-Based Assignment Method.

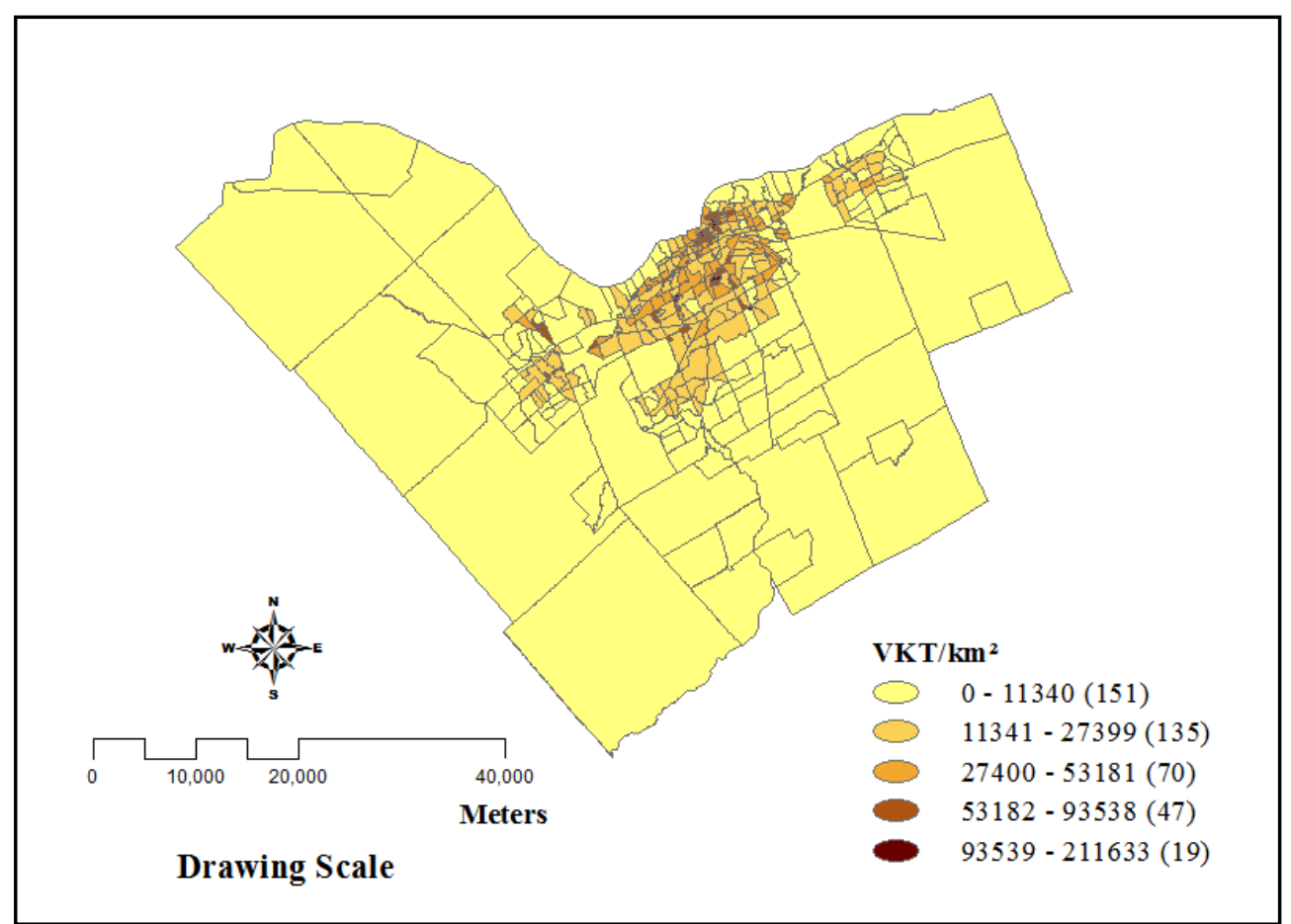

Figure A. 82 Spatial Distributions of VKT Density in the City of Ottawa. Boundary Data Assigned Using Multiple-Counted-Based Assignment Method. 


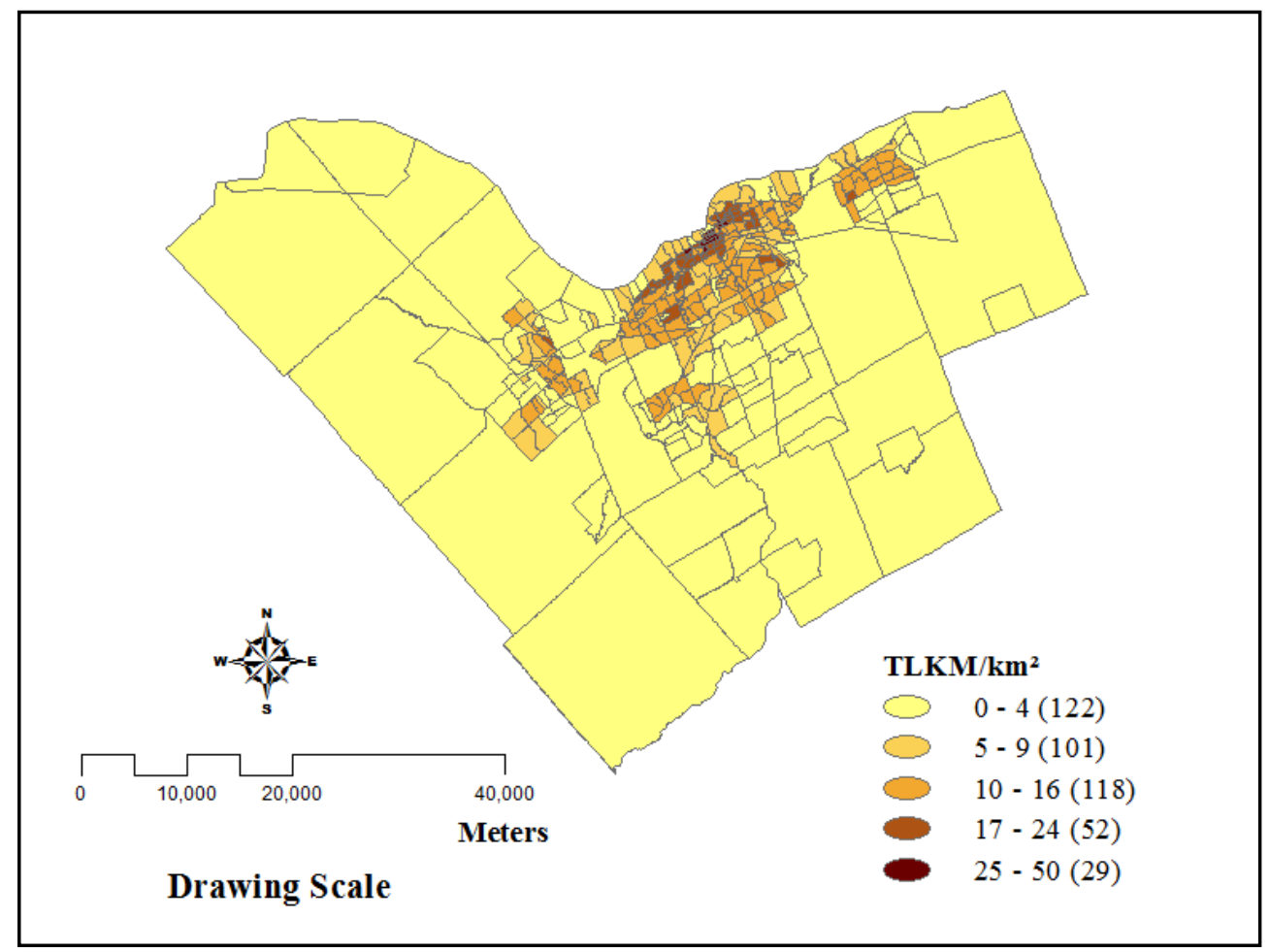

Figure A. 83 Spatial Distributions of TLKM Density in the City of Ottawa. Boundary Data Assigned Using Multiple-Counted-Based Assignment Method 


\section{Appendix B : Boundary Point Assignment Script and R Code.}




\section{B.1 Boundary Data Assignment Script}

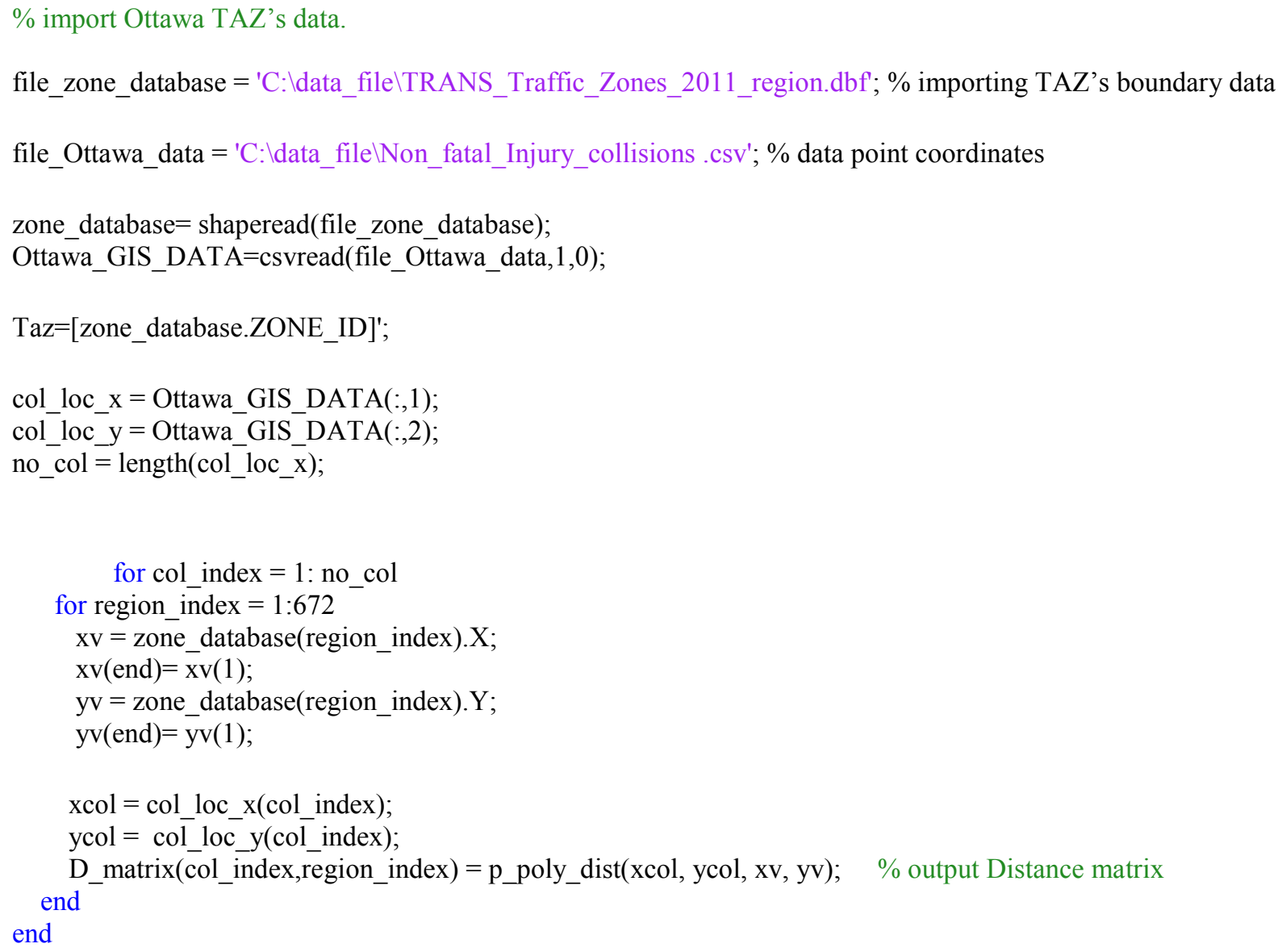




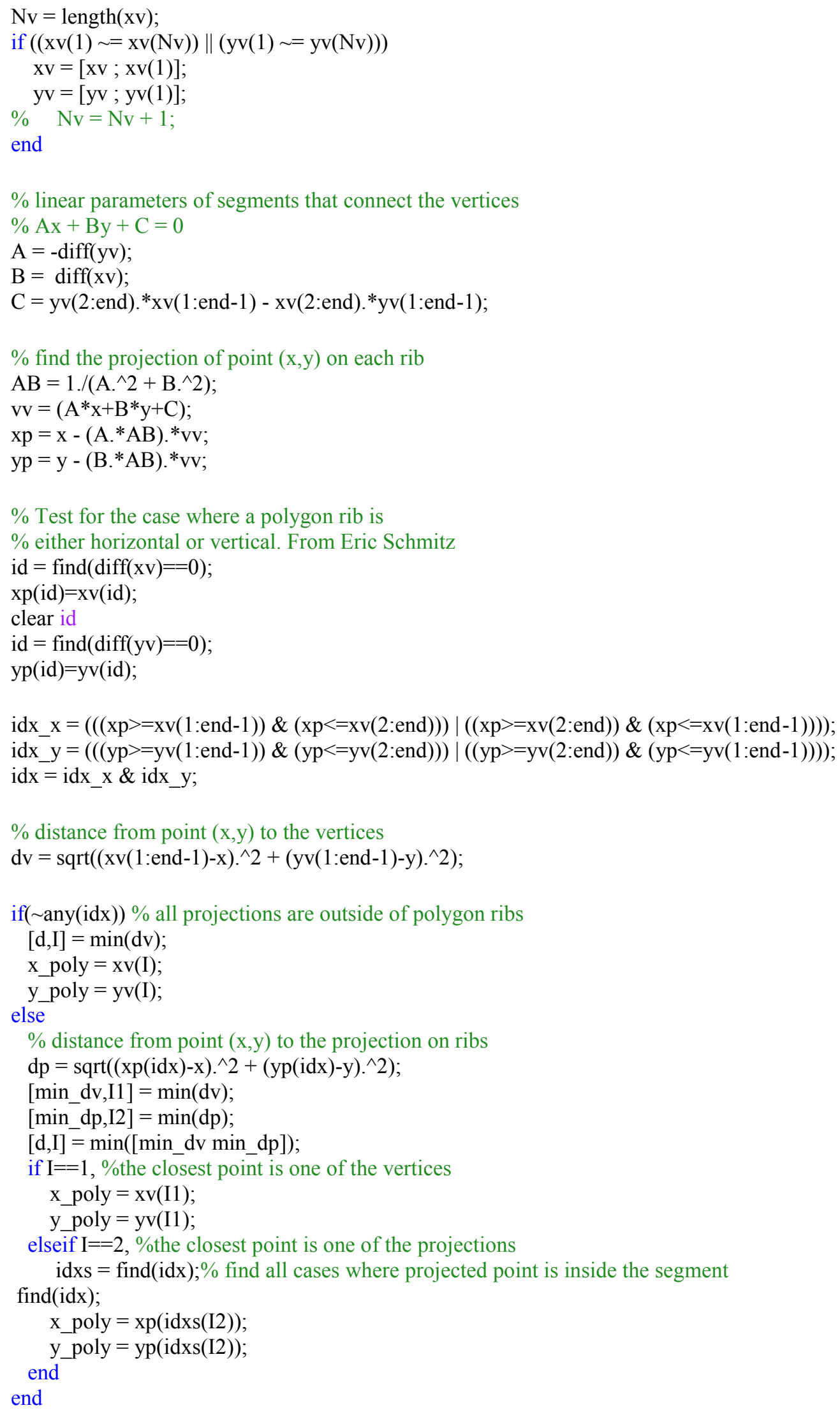


if(inpolygon(x, y, xv, yv))

$\mathrm{d}=-\mathrm{d}$;

end

$\%$ Creating boundary and non-boundary data matrix

boundary_Data_matrix $=$ abs(D_matrix);

boundary_Data_matrix $($ boundary_Data_matrix $<30)=1$;

boundary_Data_matrix(boundary_Data_matrix $\sim=1)=0$;

in_region_matrix $=$ D_matrix_schools;

in_region_matrix(in_region_matrix $<=0)=1$;

in_region_matrix(in_region_matrix $\sim=1)=0$;

non_boundary_Data_matrix $=$ D_matrix;

non_boundary_Data_matrix $($ non_boundary_Data_matrix $<-30)=1$;

non_boundary_Data_matrix(non_boundary_Data_matrix $=1)=0$;

Non_boundary_Data =sum(non_boundary_Data_matrix)'; 


\section{R Code For Developing Collisons models}

path_name1 <-("C: $\backslash \backslash U$ sers $\backslash \backslash$ aliihssian $\backslash \backslash$ Desktop $\backslash \backslash J u n e \backslash \backslash A-H A L F \backslash \backslash C o u n t e d \backslash \backslash A . c s v ")$

$\mathrm{Q}<-$ read.csv(path_name1,header=TRUE,sep = "," ) \# uploading data matrix

$\operatorname{dim}(\mathrm{Q})$

\# definition of Each Variables

Collisions $=(\mathrm{Q}[, 1])$

No.Schools $=(Q[, 2])$

No Bus stops $=(Q[, 3])$

Tot-Int $=(\mathrm{Q}[, 4])$

Sig-Int $=(Q[, 5])$

Non-Sig-Int $=(Q[, 6])$

$\operatorname{Artl}=(Q[, 7])$

Colle $=(\mathrm{Q}[, 8])$

$\mathrm{Loc}=(\mathrm{Q}[, 9])$

Maj-Colle $=(Q[, 10])$

$40 . \mathrm{Km} / \mathrm{h}=(\mathrm{Q}[, 11])$

$50 . \mathrm{Km} / \mathrm{h}=(\mathrm{Q}[, 12])$

$60 . \mathrm{Km} / \mathrm{h}=(\mathrm{Q}[, 13])$

$70 . \mathrm{Km} / \mathrm{h}=(\mathrm{Q}[, 14])$

$80 . \mathrm{Km} / \mathrm{h}=(\mathrm{Q}[, 15])$

$90 . \mathrm{Km} / \mathrm{h}=(\mathrm{Q}[, 16])$

$100 . \mathrm{Km} / \mathrm{h}=(\mathrm{Q}[, 17])$

Bike-Path $=(\mathrm{Q}[, 18])$

Area $=(Q[, 19])$

MS-Driver $=(\mathrm{Q}[, 20])$

MS-Passenger $=(\mathrm{Q}[, 21])$ 


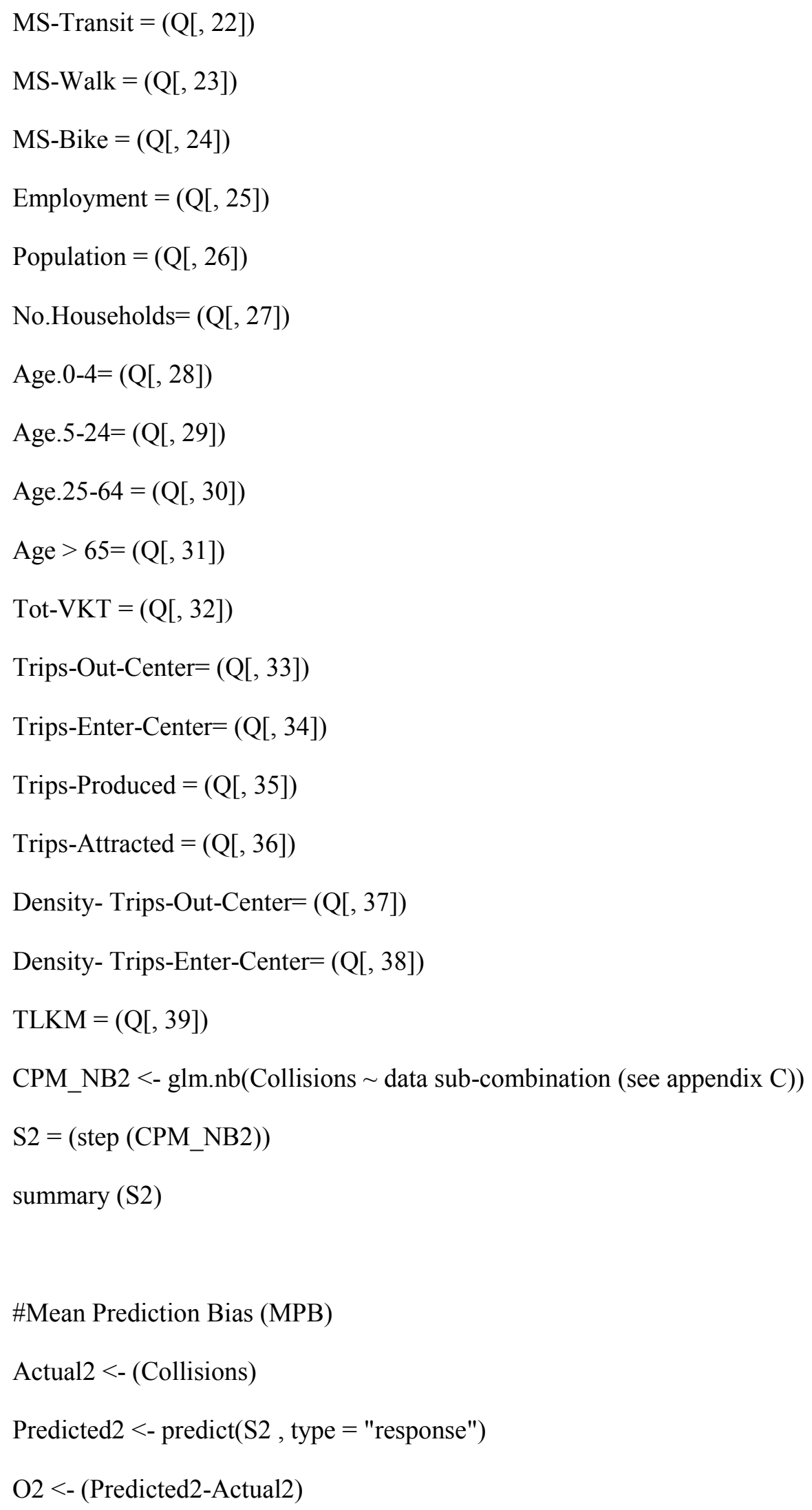




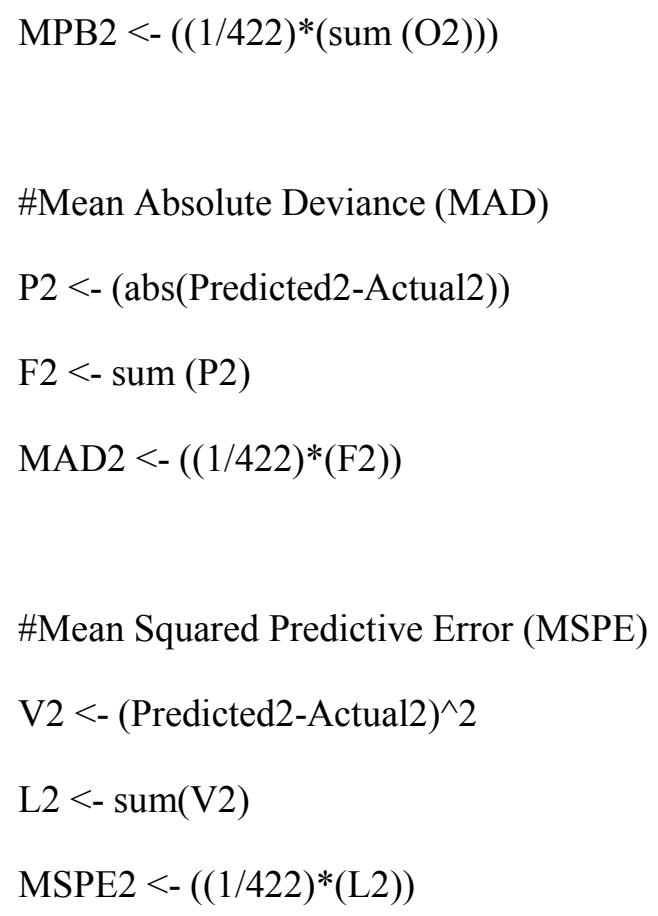


YY2 <- (Predicted2-Y2)

DD2 <- sum(XX2*YY2)

$\mathrm{ZZ2}<-\operatorname{sqrt}\left(\left(\operatorname{sum}\left(\mathrm{XX} 2^{\wedge} 2\right)\right) *\left(\operatorname{sum}\left(\mathrm{YY} 2^{\wedge} 2\right)\right)\right)$

$\mathrm{RP} 2<-\mathrm{DD} 2 / \mathrm{ZZ2}$

$\mathrm{RSQ} 2<-(\mathrm{RP} 2)^{\wedge} 2$

Coll2 <- (sum (Actual2))

Pred2 $<-($ sum $($ Predicted2) $)$

Theta $2<-$ S2\$theta

AIC2 <- S2\$aic

Deviance $2<$ - S2\$deviance

Fit1 <- cbind (MPB2 , MAD2 , MSPE2, RMSE2, E2 ,Theta2 ,AIC2, Deviance2 ,Coll2 ,Pred2,RSQ2) 


\section{Appendix C: Independent Variables' Correlation Matrix and Analysis Combination.}




\section{C.1 Count Data Correlation Matrix and Variables combinations}

\section{Table C.1 Correlation Matrix for Roadway-related and Exposure Variables}

\begin{tabular}{|c|c|c|c|c|c|c|c|c|c|c|c|c|c|c|c|c|c|}
\hline & 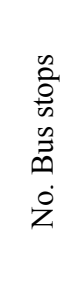 & 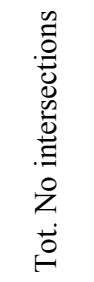 & 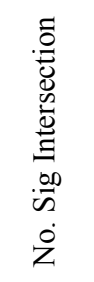 & 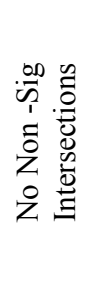 & 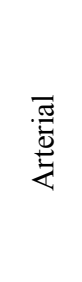 & $\begin{array}{l}\stackrel{0}{0} \\
\stackrel{0}{0} \\
0\end{array}$ & $\begin{array}{l}\bar{\Xi} \\
.0\end{array}$ & 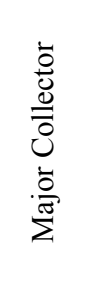 & 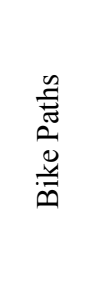 & $\stackrel{5}{>}$ & 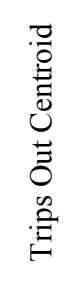 & 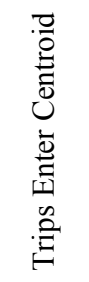 & 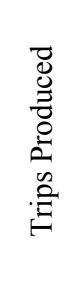 & 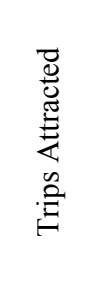 & 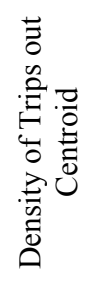 & 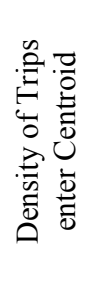 & $\underset{\exists}{\sum}$ \\
\hline No. Bus stops & 1.00 & & & & & & & & & & & & & & & & \\
\hline Tot. No intersections & 0.55 & 1.00 & & & & & & & & & & & & & & & \\
\hline No. Sig Intersection & 0.40 & 0.12 & 1.00 & & & & & & & & & & & & & & \\
\hline No Non -Sig Intersections & 0.52 & 1.00 & 0.06 & 1.00 & & & & & & & & & & & & & \\
\hline Arterial & 0.07 & 0.44 & -0.06 & 0.44 & 1.00 & & & & & & & & & & & & \\
\hline Collector & 0.01 & 0.48 & -0.18 & 0.50 & 0.90 & 1.00 & & & & & & & & & & & \\
\hline local & 0.52 & 0.89 & 0.08 & 0.89 & 0.44 & 0.49 & 1.00 & & & & & & & & & & \\
\hline Major Collector & 0.45 & 0.26 & 0.23 & 0.24 & -0.03 & -0.07 & 0.23 & 1.00 & & & & & & & & & \\
\hline Bike Paths & 0.38 & 0.23 & 0.21 & 0.22 & 0.06 & -0.04 & 0.24 & 0.34 & 1.00 & & & & & & & & \\
\hline VKT & 0.23 & 0.32 & 0.09 & 0.32 & 0.49 & 0.42 & 0.32 & 0.08 & 0.36 & 1.00 & & & & & & & \\
\hline Trips Out Centroid & 0.68 & 0.83 & 0.25 & 0.82 & 0.21 & 0.26 & 0.73 & 0.36 & 0.19 & 0.17 & 1.00 & & & & & & \\
\hline Trips Enter Centroid & 0.04 & -0.22 & 0.26 & -0.24 & -0.06 & -0.08 & -0.20 & 0.02 & -0.05 & -0.01 & -0.11 & 1.00 & & & & & \\
\hline Trips Produced & 0.65 & 0.72 & 0.26 & 0.71 & 0.05 & 0.09 & 0.65 & 0.38 & 0.21 & 0.12 & 0.90 & -0.20 & 1.00 & & & & \\
\hline Trips Attracted & 0.17 & 0.00 & 0.29 & -0.01 & -0.08 & -0.07 & 0.01 & 0.12 & 0.01 & -0.04 & 0.11 & 0.65 & 0.11 & 1.00 & & & \\
\hline Density of Trips out Centroid & -0.10 & -0.18 & 0.26 & -0.20 & -0.20 & -0.18 & -0.19 & -0.04 & -0.11 & -0.16 & 0.01 & 0.23 & 0.04 & 0.41 & 1.00 & & \\
\hline Density of Trips enter Centroid & -0.24 & -0.29 & 0.01 & -0.29 & -0.10 & -0.09 & -0.25 & -0.10 & -0.10 & -0.09 & -0.25 & 0.36 & -0.24 & 0.32 & 0.55 & 1.00 & \\
\hline TLKM & 0.16 & 0.63 & -0.10 & 0.64 & 0.93 & 0.97 & 0.66 & 0.02 & 0.06 & 0.47 & 0.39 & -0.11 & 0.23 & -0.06 & -0.21 & -0.14 & 1.00 \\
\hline
\end{tabular}

Note: The roadway were classified based on roadway classes 
Table C.2 Correlation Matrix for Roadway-related and Exposure Variables

\begin{tabular}{|c|c|c|c|c|c|c|c|c|c|c|c|c|c|c|c|c|c|c|c|c|}
\hline & $\begin{array}{l}0 \\
\hat{2} \\
0 \\
0 \\
0 \\
0 \\
0 \\
\dot{2}\end{array}$ & 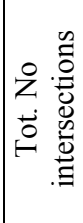 & 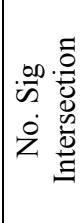 & 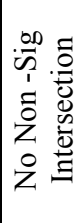 & $\begin{array}{l}\stackrel{f}{\xi} \\
\vdots \\
\dot{q}\end{array}$ & 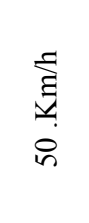 & 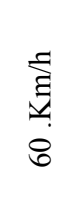 & 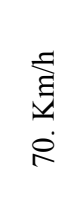 & $\frac{\sum_{\delta}^{E}}{\delta}$ & $\begin{array}{l}\dot{\Xi} \\
\dot{\Xi} \\
\dot{a}\end{array}$ & 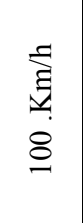 & 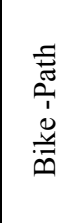 & $\stackrel{5}{5}$ & 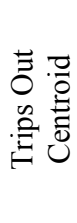 & 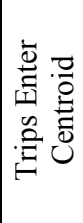 & 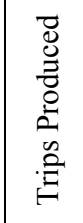 & 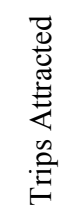 & 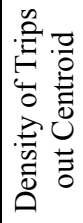 & 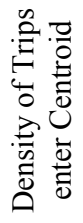 & $\underset{\boxminus}{\underline{\Xi}}$ \\
\hline No. Bus stops & 1.00 & & & & & & & & & & & & & & & & & & & \\
\hline Tot. No intersections & 0.55 & 1.00 & & & & & & & & & & & & & & & & & & \\
\hline No. Sig Intersection & 0.40 & 0.12 & 1.00 & & & & & & & & & & & & & & & & & \\
\hline No Non -Sig Intersections & 0.52 & 1.00 & 0.06 & 1.00 & & & & & & & & & & & & & & & & \\
\hline 40. $\mathrm{Km} / \mathrm{h}$ & 0.39 & 0.51 & 0.01 & 0.51 & 1.00 & & & & & & & & & & & & & & & \\
\hline $50 . \mathrm{Km} / \mathrm{h}$ & 0.40 & 0.69 & 0.14 & 0.68 & -0.02 & 1.00 & & & & & & & & & & & & & & \\
\hline 60. Km/h & 0.10 & 0.36 & -0.08 & 0.37 & 0.14 & 0.22 & 1.00 & & & & & & & & & & & & & \\
\hline 70. $\mathrm{Km} / \mathrm{h}$ & 0.03 & 0.17 & 0.06 & 0.17 & 0.06 & 0.23 & 0.20 & 1.00 & & & & & & & & & & & & \\
\hline $80 . \mathrm{Km} / \mathrm{h}$ & -0.01 & 0.42 & -0.15 & 0.44 & 0.09 & 0.28 & 0.61 & 0.17 & 1.00 & & & & & & & & & & & \\
\hline $90 . \mathrm{Km} / \mathrm{h}$ & 0.16 & 0.16 & -0.02 & 0.17 & 0.06 & 0.30 & 0.23 & -0.01 & 0.03 & 1.00 & & & & & & & & & & \\
\hline $100 . \mathrm{Km} / \mathrm{h}$ & 0.15 & 0.15 & -0.05 & 0.15 & -0.03 & 0.05 & 0.60 & -0.01 & 0.42 & 0.00 & 1.00 & & & & & & & & & \\
\hline Bike-Path & 0.38 & 0.23 & 0.21 & 0.22 & 0.20 & 0.11 & 0.09 & 0.02 & -0.02 & 0.03 & 0.10 & 1.00 & & & & & & & & \\
\hline VKT & 0.23 & 0.32 & 0.09 & 0.32 & 0.05 & 0.25 & 0.39 & 0.16 & 0.45 & 0.08 & 0.34 & 0.36 & 1.00 & & & & & & & \\
\hline Trips Out Centroid & 0.68 & 0.83 & 0.25 & 0.82 & 0.51 & 0.55 & 0.19 & 0.11 & 0.20 & 0.06 & 0.09 & 0.19 & 0.17 & 1.00 & & & & & & \\
\hline Trips Enter Centroid & 0.04 & -0.22 & 0.26 & -0.24 & -0.15 & -0.12 & -0.05 & -0.02 & -0.07 & -0.02 & -0.01 & -0.05 & -0.01 & -0.11 & 1.00 & & & & & \\
\hline Trips Produced & 0.65 & 0.72 & 0.26 & 0.71 & 0.47 & 0.50 & 0.07 & 0.08 & 0.04 & 0.02 & 0.01 & 0.21 & 0.12 & 0.90 & -0.20 & 1.00 & & & & \\
\hline Trips Attracted & 0.17 & 0.00 & 0.29 & -0.01 & 0.01 & 0.04 & -0.08 & -0.02 & -0.08 & -0.04 & -0.02 & 0.01 & -0.04 & 0.11 & 0.65 & 0.11 & 1.00 & & & \\
\hline Density of Trips out Centroid & -0.10 & -0.18 & 0.26 & -0.20 & -0.10 & -0.11 & -0.19 & -0.12 & -0.17 & -0.05 & -0.06 & -0.11 & -0.16 & 0.01 & 0.23 & 0.04 & 0.41 & 1.00 & & \\
\hline Density of Trips enter Centroid & -0.24 & -0.29 & 0.01 & -0.29 & -0.15 & -0.20 & -0.10 & -0.06 & -0.08 & -0.02 & -0.02 & -0.10 & -0.09 & -0.25 & 0.36 & -0.24 & 0.32 & 0.55 & 1.00 & \\
\hline TLKM & 0.16 & 0.63 & -0.10 & 0.64 & 0.26 & 0.48 & 0.68 & 0.26 & 0.95 & 0.14 & 0.40 & 0.06 & 0.47 & 0.39 & -0.11 & 0.23 & -0.06 & -0.21 & -0.14 & 1.00 \\
\hline
\end{tabular}

Note: The roadway were classified based on posted speed limits 
Table C.3 Correlation Matrix for Planning-related and Exposure Variables

\begin{tabular}{|c|c|c|c|c|c|c|c|c|c|c|c|c|c|c|c|c|c|c|c|c|c|c|}
\hline & $\begin{array}{l}\overline{0} \\
\overline{0} \\
\overline{0}\end{array}$ & 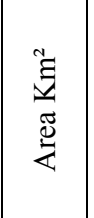 & 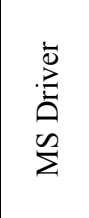 & 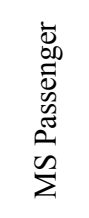 & 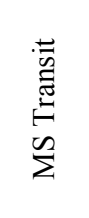 & $\begin{array}{l}\stackrel{y}{\sigma} \\
\sum^{\pi} \\
\sum_{\Sigma}^{\infty}\end{array}$ & 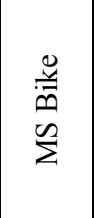 & 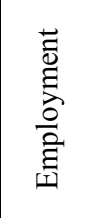 & 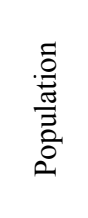 & $\begin{array}{l}0 \\
0 \\
0 \\
0 \\
0 \\
0 \\
0 \\
0 \\
0 \\
0 \\
z\end{array}$ & 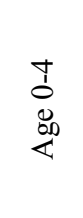 & 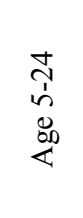 & 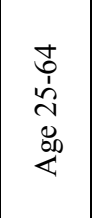 & 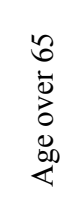 & $\stackrel{\vec{v}}{>}$ & 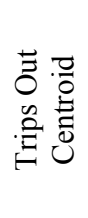 & 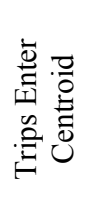 & 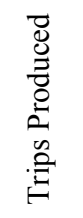 & 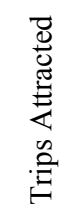 & 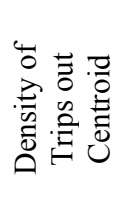 & 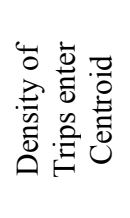 & $\underset{⿱ ⺌}{\underline{\exists}}$ \\
\hline School & 1.00 & & & & & & & & & & & & & & & & & & & & & \\
\hline Area $\mathrm{Km}^{2}$ & 0.05 & 1.00 & & & & & & & & & & & & & & & & & & & & \\
\hline MS Driver & -0.15 & 0.12 & 1.00 & & & & & & & & & & & & & & & & & & & \\
\hline MS Passenger & 0.15 & 0.05 & 0.17 & 1.00 & & & & & & & & & & & & & & & & & & \\
\hline MS Transit & 0.00 & -0.18 & -0.47 & -0.25 & 1.00 & & & & & & & & & & & & & & & & & \\
\hline MS Walk & 0.15 & -0.15 & -0.57 & -0.25 & 0.31 & 1.00 & & & & & & & & & & & & & & & & \\
\hline MS Bike & 0.00 & -0.09 & -0.32 & -0.19 & 0.31 & 0.40 & 1.00 & & & & & & & & & & & & & & & \\
\hline Employment & -0.21 & -0.09 & -0.01 & -0.19 & 0.36 & 0.09 & 0.15 & 1.00 & & & & & & & & & & & & & & \\
\hline Population & 0.59 & 0.11 & -0.19 & 0.17 & 0.10 & 0.16 & 0.03 & -0.34 & 1.00 & & & & & & & & & & & & & \\
\hline No Households & 0.58 & 0.04 & -0.26 & 0.12 & 0.15 & 0.28 & 0.11 & -0.32 & 0.93 & 1.00 & & & & & & & & & & & & \\
\hline Age 0-4 & 0.25 & 0.09 & -0.01 & 0.21 & -0.18 & 0.01 & -0.11 & -0.32 & 0.52 & 0.42 & 1.00 & & & & & & & & & & & \\
\hline Age 5-24 & 0.30 & 0.10 & -0.08 & 0.11 & 0.09 & 0.08 & -0.01 & -0.20 & 0.47 & 0.40 & 0.55 & 1.00 & & & & & & & & & & \\
\hline Age $25-64$ & 0.29 & 0.11 & -0.14 & 0.13 & -0.04 & 0.32 & 0.10 & -0.26 & 0.50 & 0.49 & 0.66 & 0.55 & 1.00 & & & & & & & & & \\
\hline Ageover65 & 0.37 & 0.05 & -0.10 & 0.07 & 0.01 & 0.26 & 0.12 & -0.18 & 0.40 & 0.45 & 0.31 & 0.36 & 0.63 & 1.00 & & & & & & & & \\
\hline VKT & 0.07 & 0.45 & 0.11 & 0.06 & -0.09 & -0.11 & -0.06 & -0.06 & 0.12 & 0.09 & 0.07 & 0.04 & 0.04 & 0.05 & 1.00 & & & & & & & \\
\hline $\begin{array}{l}\text { Trips Out } \\
\text { Centroid } \\
\end{array}$ & 0.55 & 0.21 & -0.06 & 0.24 & 0.00 & 0.05 & -0.05 & -0.19 & 0.93 & 0.83 & 0.51 & 0.45 & 0.45 & 0.31 & 0.17 & 1.00 & & & & & & \\
\hline $\begin{array}{l}\text { Trips Enter } \\
\text { Centroid }\end{array}$ & -0.14 & -0.08 & 0.10 & -0.08 & 0.24 & 0.00 & 0.10 & 0.90 & -0.28 & -0.26 & -0.29 & -0.14 & -0.28 & -0.17 & -0.01 & -0.11 & 1.00 & & & & & \\
\hline Trips Produced & 0.60 & 0.05 & -0.20 & 0.20 & 0.08 & 0.19 & 0.03 & -0.27 & 0.92 & 0.93 & 0.48 & 0.44 & 0.45 & 0.33 & 0.12 & 0.90 & -0.2 & 1.00 & & & & \\
\hline Trips Attracted & 0.19 & -0.08 & -0.21 & -0.11 & 0.49 & 0.17 & 0.15 & 0.71 & 0.02 & 0.02 & -0.14 & 0.20 & -0.12 & -0.03 & -0.0 & 0.11 & 0.65 & 0.1 & 1.00 & & & \\
\hline $\begin{array}{c}\text { Density of Trips } \\
\text { out Centroid }\end{array}$ & -0.05 & -0.18 & -0.43 & -0.18 & 0.53 & 0.51 & 0.27 & 0.45 & 0.02 & 0.07 & -0.16 & -0.09 & 0.07 & 0.02 & -0.1 & 0.01 & 0.23 & 0.04 & 0.4 & 1.00 & & \\
\hline $\begin{array}{c}\text { Density of Trips } \\
\text { enter Centroid }\end{array}$ & -0.19 & -0.08 & -0.19 & -0.01 & 0.37 & 0.15 & 0.07 & 0.43 & -0.27 & -0.25 & -0.31 & -0.25 & -0.24 & -0.20 & -0.0 & -0.25 & 0.36 & -0.2 & 0.3 & 0.5 & 1.00 & \\
\hline TLKM & 0.20 & 0.95 & 0.11 & 0.10 & -0.20 & -0.15 & -0.09 & -0.14 & 0.29 & 0.21 & 0.17 & 0.19 & 0.20 & 0.15 & 0.47 & 0.39 & -0.1 & 0.2 & -0.06 & -0.2 & -0.10 & 1.00 \\
\hline
\end{tabular}


Table C.4 Uncorrelated Independent Variables Combinations

\begin{tabular}{|c|c|}
\hline Models & Independent Variables Combination \\
\hline - Roa & characteristics, where the roadway classified based on roadway classes, and exposure variables. \\
\hline \multirow{12}{*}{ Model CI } & $\begin{array}{l}\text { - No Bus stops, Tot-Int, Sig-Int, Artl, Colle, Maj-Colle , Bike-Path, VKT, Trips-Attracted, Density- Trips-Out-Center, } \\
\text { Density- Trips-Enter-Center }\end{array}$ \\
\hline & $\begin{array}{l}\text { - No Bus stops, Tot-Int, Sig-Int, Maj-Colle , Bike-Path, VKT, Trips-Attracted, Density- Trips-Out-Center, Density- } \\
\text { Trips-Enter-Center, TLKM }\end{array}$ \\
\hline & $\begin{array}{l}\text { - No Bus stops, Non-Sig-Int, Sig-Int, Artl, Colle, Maj-Colle , Bike-Path, VKT, Trips-Attracted, Density- Trips-Out- } \\
\text { Center, Density- Trips-Enter-Center }\end{array}$ \\
\hline & $\begin{array}{l}\text { - No Bus stops, Non-Sig-Int, Sig-Int, Maj-Colle , Bike-Path, VKT, Trips-Attracted, Density- Trips-Out-Center, Density- } \\
\text { Trips-Enter-Center, TLKM }\end{array}$ \\
\hline & $\begin{array}{l}\text { - No Bus stops, Sig-Int, Artl, Colle, Loc , Maj-Colle , Bike-Path, VKT, Trips-Attracted, Trips-Produced, Density- } \\
\text { Trips-Out-Center, Density- Trips-Enter-Center }\end{array}$ \\
\hline & $\begin{array}{l}\text { - No Bus stops, Sig-Int, Loc, Maj-Colle, Bike-Path, VKT, Trips-Attracted, Trips-Produced, Density- Trips-Out-Center, } \\
\text { Density- Trips-Enter-Center, TLKM }\end{array}$ \\
\hline & $\begin{array}{l}\text { - No Bus stops, Tot-Int, Sig-Int, Artl, Colle, Maj-Colle , Bike-Path, VKT, Trips-Enter-Center, Density- Trips-Out- } \\
\text { Center, Density- Trips-Enter-Center }\end{array}$ \\
\hline & $\begin{array}{l}\text { - No Bus stops, Tot-Int, Sig-Int, Maj-Colle , Bike-Path, VKT, Trips-Enter-Center, Density- Trips-Out-Center, Density- } \\
\text { Trips-Enter-Center, TLKM }\end{array}$ \\
\hline & $\begin{array}{l}\text { - No Bus stops, Non-Sig-Int, Sig-Int, Artl, Colle, Maj-Colle , Bike-Path, VKT, Trips-Enter-Center, Density- Trips-Out- } \\
\text { Center, Density- Trips-Enter-Center }\end{array}$ \\
\hline & $\begin{array}{l}\text { - No Bus stops, Non-Sig-Int, Sig-Int, Maj-Colle , Bike-Path, VKT, Trips-Enter-Center, Density- Trips-Out-Center, } \\
\text { Density- Trips-Enter-Center, TLKM }\end{array}$ \\
\hline & $\begin{array}{l}\text { - Sig-Int, Artl, Colle, Loc, Maj-Colle , Bike-Path, VKT, Trips-Enter-Center, Density- Trips-Out-Center, Density- Trips- } \\
\text { Enter-Center }\end{array}$ \\
\hline & $\begin{array}{l}\text { - Sig-Int, Loc, Maj-Colle , Bike-Path, VKT, Trips-Enter-Center, Density- Trips-Out-Center, Density- Trips-Enter-Center } \\
\text {, TLKM }\end{array}$ \\
\hline
\end{tabular}


Table C.4 Uncorrelated Independent Variables Combinations (cont')

\begin{tabular}{|c|c|}
\hline Models & Independent Variables Combination \\
\hline \multicolumn{2}{|c|}{ - Roadway characteristics, where the roadway classified based on roadway classes, and exposure variables. } \\
\hline \multirow{2}{*}{ Model CI } & $\begin{array}{l}\text { - Sig-Int, Artl, Colle , Maj-Colle , Bike-Path , VKT, Trips-Enter-Center, Trips-Out-Center, Density- Trips-Out-Center, } \\
\text { Density- Trips-Enter-Center }\end{array}$ \\
\hline & $\begin{array}{l}\text { - Sig-Int, Maj-Colle , Bike-Path, VKT, Trips-Enter-Center, Trips-Out-Center , Density- Trips-Out-Center, Density- } \\
\text { Trips-Enter-Center, TLKM }\end{array}$ \\
\hline \multicolumn{2}{|c|}{ - Roadway characteristics, where the roadway classified based on posted speed limit, and exposure variables. } \\
\hline \multirow{10}{*}{ Model CII } & $\begin{array}{l}\text { - No Bus stops, Tot-Int, Sig-Int, } 40 . \mathrm{Km} / \mathrm{h}, 50 . \mathrm{Km} / \mathrm{h}, 60 . \mathrm{Km} / \mathrm{h}, 70 . \mathrm{Km} / \mathrm{h}, 80 . \mathrm{Km} / \mathrm{h}, 90 . \mathrm{Km} / \mathrm{h}, 100 . \mathrm{Km} / \mathrm{h}, \mathrm{Bike}-\mathrm{Path}, \mathrm{VKT} \text {, } \\
\text { Trips-Enter-Center, Density- Trips-Out-Center, Density- Trips-Enter-Center }\end{array}$ \\
\hline & $\begin{array}{l}\text { - No Bus stops, Tot-Int, Sig-Int, } 40 . \mathrm{Km} / \mathrm{h}, 50 . \mathrm{Km} / \mathrm{h}, 60 . \mathrm{Km} / \mathrm{h}, 70 . \mathrm{Km} / \mathrm{h}, 90 . \mathrm{Km} / \mathrm{h}, 100 . \mathrm{Km} / \mathrm{h} \text {, Bike-Path, VKT, Trips- } \\
\text { Enter-Center, Density- Trips-Out-Center, Density- Trips-Enter-Center, TLKM }\end{array}$ \\
\hline & $\begin{array}{l}\text { - No Bus stops, Non-Sig-Int, Sig-Int, } 40 . \mathrm{Km} / \mathrm{h}, 50 . \mathrm{Km} / \mathrm{h}, 60 . \mathrm{Km} / \mathrm{h}, 70 . \mathrm{Km} / \mathrm{h}, 80 . \mathrm{Km} / \mathrm{h}, 90 . \mathrm{Km} / \mathrm{h}, 100 . \mathrm{Km} / \mathrm{h}, \mathrm{Bike}-\mathrm{Path} \text {, } \\
\text { VKT, Trips-Enter-Center, Density- Trips-Out-Center, Density- Trips-Enter-Center }\end{array}$ \\
\hline & $\begin{array}{l}\text { - No Bus stops, Non-Sig-Int, Sig-Int, } 40 . \mathrm{Km} / \mathrm{h}, 50 . \mathrm{Km} / \mathrm{h}, 60 . \mathrm{Km} / \mathrm{h}, 70 . \mathrm{Km} / \mathrm{h}, 90 . \mathrm{Km} / \mathrm{h}, 100 . \mathrm{Km} / \mathrm{h}, \text { Bike-Path, VKT, } \\
\text { Trips-Enter-Center, Density- Trips-Out-Center, Density- Trips-Enter-Center, TLKM }\end{array}$ \\
\hline & $\begin{array}{l}\text { - Sig-Int, } 40 . \mathrm{Km} / \mathrm{h}, 50 . \mathrm{Km} / \mathrm{h}, 60 . \mathrm{Km} / \mathrm{h}, 70 . \mathrm{Km} / \mathrm{h}, 80 . \mathrm{Km} / \mathrm{h}, 90 . \mathrm{Km} / \mathrm{h}, 100 . \mathrm{Km} / \mathrm{h}, \text { Bike-Path , VKT, Trips-Enter-Center, } \\
\text { Trips-Out-Center, Density- Trips-Out-Center, Density- Trips-Enter-Center }\end{array}$ \\
\hline & $\begin{array}{l}\text { - Sig-Int, } 40 . \mathrm{Km} / \mathrm{h}, 50 . \mathrm{Km} / \mathrm{h}, 60 . \mathrm{Km} / \mathrm{h}, 70 . \mathrm{Km} / \mathrm{h}, 90 . \mathrm{Km} / \mathrm{h}, 100 . \mathrm{Km} / \mathrm{h}, \text { Bike-Path , VKT, Trips-Out-Center, Trips-Enter- } \\
\text { Center, Density- Trips-Out-Center, Density- Trips-Enter-Center, TLKM }\end{array}$ \\
\hline & $\begin{array}{l}\text { - No Bus stops, Tot-Int, Sig-Int, } 40 . \mathrm{Km} / \mathrm{h}, 50 . \mathrm{Km} / \mathrm{h}, 60 . \mathrm{Km} / \mathrm{h}, 70 . \mathrm{Km} / \mathrm{h}, 80 . \mathrm{Km} / \mathrm{h}, 90 . \mathrm{Km} / \mathrm{h}, 100 . \mathrm{Km} / \mathrm{h}, \text { Bike-Path, VKT, } \\
\text { Trips-Attracted, Density- Trips-Out-Center, Density- Trips-Enter-Center }\end{array}$ \\
\hline & $\begin{array}{l}\text { - No Bus stops, Tot-Int, Sig-Int, } 40 . \mathrm{Km} / \mathrm{h}, 50 . \mathrm{Km} / \mathrm{h}, 60 . \mathrm{Km} / \mathrm{h}, 70 . \mathrm{Km} / \mathrm{h}, 90 . \mathrm{Km} / \mathrm{h}, 100 . \mathrm{Km} / \mathrm{h} \text {, Bike-Path, VKT, Trips- } \\
\text { Attracted, Density- Trips-Out-Center, Density- Trips-Enter-Center, TLKM }\end{array}$ \\
\hline & $\begin{array}{l}\text { - No Bus stops, Non-Sig-Int, Sig-Int, } 40 . \mathrm{Km} / \mathrm{h}, 50 . \mathrm{Km} / \mathrm{h}, 60 . \mathrm{Km} / \mathrm{h}, 70 . \mathrm{Km} / \mathrm{h}, 80 . \mathrm{Km} / \mathrm{h}, 90 . \mathrm{Km} / \mathrm{h}, 100 . \mathrm{Km} / \mathrm{h}, \text { Bike-Path } \\
\text { VKT, Trips-Attracted, Density- Trips-Out-Center, Density- Trips-Enter-Center }\end{array}$ \\
\hline & $\begin{array}{l}\text { - No Bus stops, Non-Sig-Int, Sig-Int, } 40 . \mathrm{Km} / \mathrm{h}, 50 . \mathrm{Km} / \mathrm{h}, 60 . \mathrm{Km} / \mathrm{h}, 70 . \mathrm{Km} / \mathrm{h}, 90 . \mathrm{Km} / \mathrm{h}, 100 . \mathrm{Km} / \mathrm{h} \text {, Bike-Path, VKT, } \\
\text { Trips-Attracted , Density- Trips-Out-Center, Density- Trips-Enter-Center, TLKM }\end{array}$ \\
\hline
\end{tabular}


Table C.4 Uncorrelated Independent Variables Combinations (cont')

\begin{tabular}{|c|c|}
\hline Models & Independent Variables Combination \\
\hline \multicolumn{2}{|c|}{ - Roadway characteristics, where the roadway classified based on posted speed limit, and exposure variables. } \\
\hline \multirow{2}{*}{ Model CII } & $\begin{array}{l}\text { - Sig-Int, } 40 . \mathrm{Km} / \mathrm{h}, 50 . \mathrm{Km} / \mathrm{h}, 60 . \mathrm{Km} / \mathrm{h}, 70 . \mathrm{Km} / \mathrm{h}, 80 . \mathrm{Km} / \mathrm{h}, 90 . \mathrm{Km} / \mathrm{h}, 100 . \mathrm{Km} / \mathrm{h}, \text { Bike-Path, VKT, Trips-Produced, Trips- } \\
\text { Attracted , Density- Trips-Out-Center, Density- Trips-Enter-Center }\end{array}$ \\
\hline & $\begin{array}{l}\text { - Sig-Int, } 40 . \mathrm{Km} / \mathrm{h}, 50 . \mathrm{Km} / \mathrm{h}, 60 . \mathrm{Km} / \mathrm{h}, 70 . \mathrm{Km} / \mathrm{h}, 90 . \mathrm{Km} / \mathrm{h}, 100 . \mathrm{Km} / \mathrm{h}, \text { Bike-Path , VKT, Trips-Produced, Trips-Attracted } \\
\text {, Density- Trips-Out-Center, Density- Trips-Enter-Center, TLKM }\end{array}$ \\
\hline \multicolumn{2}{|c|}{ - Modal Split Variables and Exposure Variables. } \\
\hline \multirow{2}{*}{ Model CIII } & $\begin{array}{l}\text { - MS-Driver, MS-Passenger, MS-Transit, MS-Walk , MS-Bike, VKT, Trips-Produced, Trips-Attracted, Density- } \\
\text { Trips-Out-Center, Density- Trips-Enter-Center, TLKM }\end{array}$ \\
\hline & $\begin{array}{l}\text { - MS-Driver, MS-Passenger, MS-Transit, MS-Walk , MS-Bike, VKT, Trips-Out-Center, Trips-Enter-Center, } \\
\text { Density- Trips-Out-Center, Density- Trips-Enter-Center, TLKM }\end{array}$ \\
\hline \multicolumn{2}{|c|}{ - Modal Split Variables and Socio-demographic Characteristics Variables } \\
\hline \multirow{7}{*}{ Model CIV } & $\begin{array}{l}\text { - No.Schools, Area, MS-Driver, MS-Passenger, MS-Transit, MS-Walk, MS-Bike, Employment, Population, } \\
\text { Age.0-4, Age.5-24, Age.25-64, Age > 65, VKT, Density- Trips-Out-Center, Density- Trips-Enter-Center }\end{array}$ \\
\hline & $\begin{array}{l}\text { - No.Schools, MS-Driver, MS-Passenger, MS-Transit, MS-Walk, MS-Bike, Employment, Population, Age.0-4, } \\
\text { Age.5-24, Age.25-64, Age > 65, VKT, Density- Trips-Out-Center, Density- Trips-Enter-Center, TLKM }\end{array}$ \\
\hline & $\begin{array}{l}\text { - No.Schools, Area, MS-Driver, MS-Passenger, MS-Transit, MS-Walk, MS-Bike, Employment, No.Households, } \\
\text { Age.0-4, Age.5-24, Age.25-64, Age > 65, VKT, Density- Trips-Out-Center, Density- Trips-Enter-Center }\end{array}$ \\
\hline & $\begin{array}{l}\text { - No.Schools, MS-Driver, MS-Passenger, MS-Transit, MS-Walk, MS-Bike, Employment, No.Households, Age.0-4, } \\
\text { Age.5-24, Age.25-64, Age > 65, VKT, Density- Trips-Out-Center, Density- Trips-Enter-Center, TLKM }\end{array}$ \\
\hline & $\begin{array}{l}\text { - No.Schools, Area , MS-Driver, MS-Passenger, MS-Transit, MS-Walk , MS-Bike, Population, Age.0-4, Age.5-24, } \\
\text { Age.25-64, Age > 65, VKT, Trips-Enter-Center, Density- Trips-Out-Center, Density- Trips-Enter-Center }\end{array}$ \\
\hline & $\begin{array}{l}\text { - No.Schools, MS-Driver, MS-Passenger, MS-Transit, MS-Walk, MS-Bike, Population, Age.0-4, Age.5-24, } \\
\text { Age.25-64, Age > 65, VKT, Trips-Enter-Center, Density- Trips-Out-Center, Density- Trips-Enter-Center, TLKM }\end{array}$ \\
\hline & $\begin{array}{l}\text { - No.Schools, Area, MS-Driver, MS-Passenger, MS-Transit, MS-Walk , MS-Bike, No.Households, Age.0-4, Age.5- } \\
24 \text {, Age.25-64, Age > 65, VKT, Trips-Enter-Center, Density- Trips-Out-Center, Density- Trips-Enter-Center }\end{array}$ \\
\hline
\end{tabular}


Table C.4 Uncorrelated Independent Variables Combinations (cont')

\begin{tabular}{|c|c|}
\hline Models & Independent Variables Combination \\
\hline - $\mathrm{Mo}$ & olit Variables and Socio-demographic Characteristics Vari \\
\hline \multirow{13}{*}{ Model CIV } & $\begin{array}{l}\text { - No.Schools, MS-Driver, MS-Passenger, MS-Transit, MS-Walk, MS-Bike, No.Households, Age.0-4, Age.5-24, } \\
\text { Age.25-64, Age > 65, VKT, Trips-Enter-Center, Density- Trips-Out-Center, Density- Trips-Enter-Center, TLKM }\end{array}$ \\
\hline & $\begin{array}{l}\text { - No.Schools, Area , MS-Driver, MS-Passenger, MS-Transit, MS-Walk, MS-Bike, Employment, Age.0-4, Age.5- } \\
24, \text { Age.25-64, Age > 65, VKT, Trips-Out-Center, Density- Trips-Out-Center, Density- Trips-Enter-Center }\end{array}$ \\
\hline & $\begin{array}{l}\text { - No.Schools, MS-Driver, MS-Passenger, MS-Transit, MS-Walk, MS-Bike, Employment, Age.0-4, Age.5-24, } \\
\text { Age.25-64, Age > 65, VKT, Trips-Out-Center, Density- Trips-Out-Center, Density- Trips-Enter-Center, TLKM }\end{array}$ \\
\hline & $\begin{array}{l}\text { - No.Schools, Area , MS-Driver, MS-Passenger, MS-Transit, MS-Walk, MS-Bike, Age.0-4, Age.5-24, Age.25-64, } \\
\text { Age }>65, \text { VKT, Trips-Out-Center, Trips-Enter-Center, Density- Trips-Out-Center, Density- Trips-Enter-Center }\end{array}$ \\
\hline & $\begin{array}{l}\text { - No.Schools, MS-Driver, MS-Passenger, MS-Transit, MS-Walk , MS-Bike, Age.0-4, Age.5-24, Age.25-64, Age > } \\
\text { 65, VKT, Trips-Out-Center, Trips-Enter-Center, Density- Trips-Out-Center, Density- Trips-Enter-Center, TLKM }\end{array}$ \\
\hline & $\begin{array}{l}\text { - No.Schools, Area, MS-Driver, MS-Passenger, MS-Transit, MS-Walk, MS-Bike, Population, Age.0-4, Age.5-24, } \\
\text { Age.25-64, Age > 65, VKT, Trips-Attracted, Density- Trips-Out-Center, Density- Trips-Enter-Center }\end{array}$ \\
\hline & $\begin{array}{l}\text { - No.Schools, MS-Driver, MS-Passenger, MS-Transit, MS-Walk, MS-Bike, Population, Age.0-4, Age.5-24, } \\
\text { Age.25-64, Age > 65, VKT, Trips-Attracted, Density- Trips-Out-Center, Density- Trips-Enter-Center, TLKM }\end{array}$ \\
\hline & $\begin{array}{l}\text { - No.Schools, Area, MS-Driver, MS-Passenger, MS-Transit, MS-Walk, MS-Bike, No.Households, Age.0-4, Age.5- } \\
\text { 24, Age.25-64, Age > 65, VKT, Trips-Attracted, Density- Trips-Out-Center, Density- Trips-Enter-Center }\end{array}$ \\
\hline & $\begin{array}{l}\text { - No.Schools, MS-Driver, MS-Passenger, MS-Transit, MS-Walk, MS-Bike, No.Households, Age.0-4, Age.5-24, } \\
\text { Age.25-64, Age > 65, VKT, Trips-Attracted, Density- Trips-Out-Center, Density- Trips-Enter-Center, TLKM }\end{array}$ \\
\hline & $\begin{array}{l}\text { - No.Schools, Area , MS-Driver, MS-Passenger, MS-Transit, MS-Walk , MS-Bike, Employment, Age.0-4, Age.5- } \\
\text { 24, Age.25-64, Age > 65, VKT, Trips-Produced, Density- Trips-Out-Center, Density- Trips-Enter-Center }\end{array}$ \\
\hline & $\begin{array}{l}\text { - No.Schools, MS-Driver, MS-Passenger, MS-Transit, MS-Walk , MS-Bike, Employment, Age.0-4, Age.5-24, } \\
\text { Age.25-64, Age > 65, VKT, Trips-Produced, Density- Trips-Out-Center, Density- Trips-Enter-Center, TLKM }\end{array}$ \\
\hline & $\begin{array}{l}\text { - No.Schools, Area, MS-Driver, MS-Passenger, MS-Transit, MS-Walk, MS-Bike, Age.0-4, Age.5-24, Age.25-64, } \\
\text { Age }>65 \text {, VKT, Trips-Produced, Trips-Attracted, Density- Trips-Out-Center, Density- Trips-Enter-Center }\end{array}$ \\
\hline & $\begin{array}{l}\text { - No.Schools, MS-Driver, MS-Passenger, MS-Transit, MS-Walk , MS-Bike, Age.0-4, Age.5-24, Age.25-64, Age > } \\
\text { 65, VKT, Trips-Produced, Trips-Attracted, Density- Trips-Out-Center, Density- Trips-Enter-Center, TLKM }\end{array}$ \\
\hline
\end{tabular}


Table C.4 Uncorrelated Independent Variables Combinations (cont')

\begin{tabular}{|c|c|}
\hline Models & Independent Variables Combination \\
\hline \multicolumn{2}{|c|}{ - Modal split variables only } \\
\hline Model CV & - MS-Driver, MS-Passenger, MS-Transit, MS-Walk , MS-Bike \\
\hline \multicolumn{2}{|c|}{ - Exposure variables only } \\
\hline \multirow{2}{*}{ Model CVI } & - VKT, Trips-Produced, Trips-Attracted, Density- Trips-Out-Center, Density- Trips-Enter-Center, TLKM \\
\hline & - VKT, Trips-Out-Center, Trips-Enter-Center, Density- Trips-Out-Center, Density- Trips-Enter-Center, TLKM \\
\hline
\end{tabular}




\section{C.2 Density Data Correlation Matrix and Variables combinations}

Table C.5 Correlation Matrix for Roadway-related and Exposure Variables

\begin{tabular}{|c|c|c|c|c|c|c|c|c|c|c|c|c|c|c|c|}
\hline & 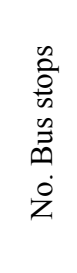 & 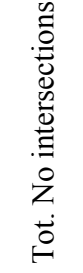 & 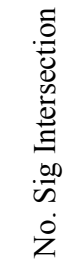 & 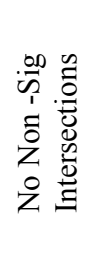 & 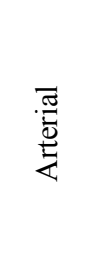 & $\begin{array}{l}\stackrel{\tilde{0}}{0} \\
\stackrel{0}{0} \\
\dot{0}\end{array}$ & تृ & 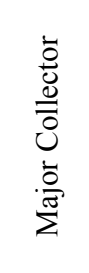 & 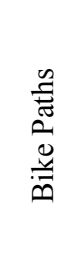 & $\stackrel{5}{>}$ & 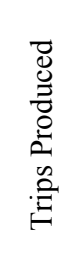 & 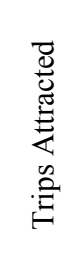 & 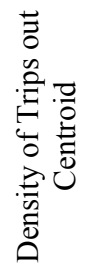 & 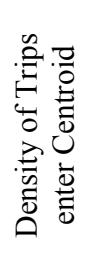 & $\underset{\exists}{\sum}$ \\
\hline No. Bus stops & 1.00 & & & & & & & & & & & & & & \\
\hline Tot. No intersections & 0.63 & 1.00 & & & & & & & & & & & & & \\
\hline No. Sig Intersection & 0.69 & 0.54 & 1.00 & & & & & & & & & & & & \\
\hline No Non-Sig Intersections & 0.34 & 0.87 & 0.06 & 1.00 & & & & & & & & & & & \\
\hline Arterial & 0.10 & 0.14 & 0.20 & 0.04 & 1.00 & & & & & & & & & & \\
\hline Collector & 0.03 & 0.16 & -0.19 & 0.30 & -0.09 & 1.00 & & & & & & & & & \\
\hline local & 0.41 & 0.84 & 0.19 & 0.88 & 0.06 & 0.31 & 1.00 & & & & & & & & \\
\hline Major Collector & 0.15 & 0.19 & 0.02 & 0.22 & -0.02 & -0.03 & 0.15 & 1.00 & & & & & & & \\
\hline Bike Paths & 0.16 & 0.18 & 0.22 & 0.09 & 0.06 & -0.01 & 0.13 & 0.05 & 1.00 & & & & & & \\
\hline VKT & 0.11 & 0.06 & 0.13 & -0.01 & 0.04 & -0.08 & -0.02 & -0.02 & 0.21 & 1.00 & & & & & \\
\hline Trips Produced & 0.49 & 0.64 & 0.58 & 0.42 & 0.19 & 0.07 & 0.53 & 0.06 & 0.13 & 0.06 & 1.00 & & & & \\
\hline Trips Attracted & 0.48 & 0.27 & 0.74 & -0.11 & 0.10 & -0.16 & -0.02 & -0.04 & 0.13 & 0.08 & 0.37 & 1.00 & & & \\
\hline Density of Trips out Centroid & 0.66 & 0.65 & 0.80 & 0.31 & 0.16 & 0.02 & 0.41 & 0.06 & 0.25 & 0.09 & 0.66 & 0.75 & 1.00 & & \\
\hline Density of Trips enter Centroid & 0.54 & 0.22 & 0.64 & -0.11 & 0.14 & -0.18 & -0.04 & -0.03 & 0.18 & 0.08 & 0.26 & 0.66 & 0.55 & 1.00 & \\
\hline TLKM & 0.19 & 0.33 & 0.23 & 0.26 & 0.97 & 0.03 & 0.30 & 0.04 & 0.09 & 0.03 & 0.31 & 0.09 & 0.25 & 0.12 & 1.00 \\
\hline
\end{tabular}

Note: The roadway were classified based on roadway classes 
Table C.6 Correlation Matrix for Roadway-related and Exposure Variables

\begin{tabular}{|c|c|c|c|c|c|c|c|c|c|c|c|c|c|c|c|c|c|c|}
\hline & $\begin{array}{l}n \\
\tilde{0} \\
\tilde{n} \\
0 \\
\tilde{n} \\
\dot{0} \\
\dot{z}\end{array}$ & 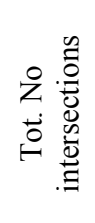 & 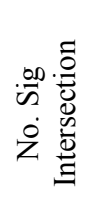 & 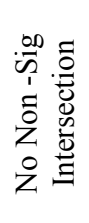 & $\begin{array}{l}\frac{\tilde{g}}{\mathfrak{g}} \\
\text { q }\end{array}$ & 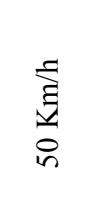 & $\frac{\tilde{\Xi}}{8}$ & 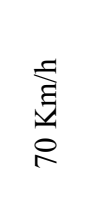 & $\begin{array}{l}\frac{1}{\delta} \\
\varnothing\end{array}$ & $\frac{\xi}{\frac{\xi}{2}}$ & 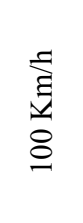 & 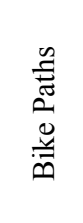 & $\stackrel{5}{5}$ & 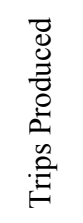 & 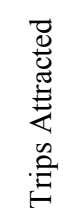 & 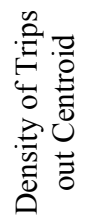 & 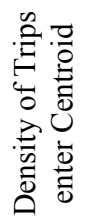 & $\underset{⿱ ⺌}{\Xi}$ \\
\hline No. Bus stops & 1.00 & & & & & & & & & & & & & & & & & \\
\hline Tot. No intersections & 0.63 & 1.00 & & & & & & & & & & & & & & & & \\
\hline No. Sig Intersection & 0.69 & 0.54 & 1.00 & & & & & & & & & & & & & & & \\
\hline $\begin{array}{l}\text { No Non-Sig } \\
\text { Intersections }\end{array}$ & 0.34 & 0.87 & 0.06 & 1.00 & & & & & & & & & & & & & & \\
\hline 40. $\mathrm{Km} / \mathrm{h}$ & -0.02 & 0.11 & -0.16 & 0.22 & 1.00 & & & & & & & & & & & & & \\
\hline $50 . \mathrm{Km} / \mathrm{h}$ & 0.19 & 0.33 & 0.25 & 0.24 & -0.10 & 1.00 & & & & & & & & & & & & \\
\hline 60. Km/h & 0.16 & -0.05 & -0.01 & -0.06 & 0.03 & -0.08 & 1.00 & & & & & & & & & & & \\
\hline 70. $\mathrm{Km} / \mathrm{h}$ & -0.10 & -0.14 & -0.07 & -0.12 & -0.04 & -0.06 & 0.01 & 1.00 & & & & & & & & & & \\
\hline $80 . \mathrm{Km} / \mathrm{h}$ & -0.34 & -0.37 & -0.20 & -0.32 & -0.08 & -0.13 & -0.12 & -0.04 & 1.00 & & & & & & & & & \\
\hline $90 . \mathrm{Km} / \mathrm{h}$ & -0.07 & -0.08 & -0.04 & -0.07 & -0.02 & -0.02 & -0.03 & -0.02 & 0.02 & 1.00 & & & & & & & & \\
\hline $100 . \mathrm{Km} / \mathrm{h}$ & -0.07 & -0.08 & -0.04 & -0.07 & -0.03 & -0.02 & -0.03 & -0.02 & 0.07 & -0.01 & 1.00 & & & & & & & \\
\hline Bike Paths & 0.16 & 0.18 & 0.22 & 0.09 & 0.05 & 0.08 & 0.07 & -0.03 & -0.11 & -0.02 & -0.03 & 1.00 & & & & & & \\
\hline VKT & 0.11 & 0.06 & 0.13 & -0.01 & -0.06 & 0.03 & 0.16 & -0.02 & -0.05 & -0.01 & -0.01 & 0.21 & 1.00 & & & & & \\
\hline Trips Produced & 0.49 & 0.64 & 0.58 & 0.42 & 0.04 & 0.31 & -0.08 & -0.12 & -0.25 & -0.05 & -0.05 & 0.13 & 0.06 & 1.00 & & & & \\
\hline Trips Attracted & 0.48 & 0.27 & 0.74 & -0.11 & -0.10 & 0.11 & -0.04 & -0.05 & -0.13 & -0.02 & -0.02 & 0.13 & 0.08 & 0.37 & 1.00 & & & \\
\hline $\begin{array}{c}\text { Density of Trips out } \\
\text { Centroid }\end{array}$ & 0.66 & 0.65 & 0.80 & 0.31 & 0.01 & 0.25 & 0.00 & -0.11 & -0.28 & -0.06 & -0.06 & 0.25 & 0.09 & 0.66 & 0.75 & 1.00 & & \\
\hline $\begin{array}{c}\text { Density of Trips enter } \\
\text { Centroid }\end{array}$ & 0.54 & 0.22 & 0.64 & -0.11 & -0.12 & 0.13 & 0.13 & 0.02 & -0.15 & -0.03 & -0.02 & 0.18 & 0.08 & 0.26 & 0.66 & 0.55 & 1.00 & \\
\hline TLKM & 0.19 & 0.33 & 0.23 & 0.26 & 0.04 & 0.99 & -0.02 & -0.05 & -0.13 & -0.03 & -0.03 & 0.09 & 0.03 & 0.31 & 0.09 & 0.25 & 0.12 & 1.00 \\
\hline
\end{tabular}

Note: The roadway were classified based posted speed limits 
Table C.7 Correlation Matrix for Planning-related and Exposure Variables

\begin{tabular}{|c|c|c|c|c|c|c|c|c|c|c|c|c|c|c|c|c|c|c|c|c|}
\hline & $\begin{array}{l}\overrightarrow{8} \\
\bar{\delta} \\
\bar{u} \\
\text { n. }\end{array}$ & 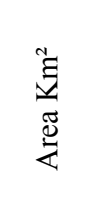 & 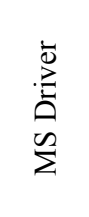 & 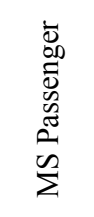 & 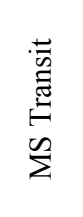 & $\begin{array}{l}\ddot{y} \\
\vec{J} \\
\sum^{\infty}\end{array}$ & 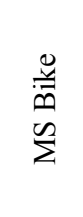 & 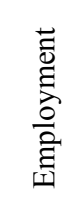 & 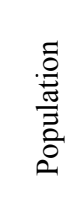 & $\begin{array}{l}n \\
0 \\
0 \\
0 \\
0 \\
0 \\
0 \\
0 \\
0 \\
0 \\
z\end{array}$ & \begin{tabular}{l}
+ \\
0 \\
0 \\
\multirow{8}{*}{} \\
$\ll$
\end{tabular} & 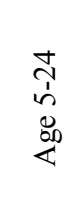 & 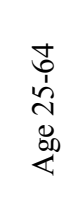 & $\begin{array}{l}n \\
\tilde{0} \\
\dot{0} \\
\dot{0} \\
\dot{8} \\
\dot{0}\end{array}$ & $\stackrel{5}{5}$ & 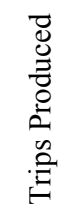 & 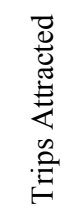 & 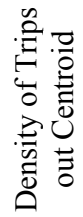 & 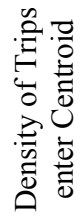 & $\underset{⿱ ⺌}{\sum}$ \\
\hline School & 1.00 & & & & & & & & & & & & & & & & & & & \\
\hline Area $\mathrm{Km}^{2}$ & -0.10 & 1.00 & & & & & & & & & & & & & & & & & & \\
\hline MS Driver & -0.23 & 0.12 & 1.00 & & & & & & & & & & & & & & & & & \\
\hline MS Passenger & -0.03 & 0.05 & 0.17 & 1.00 & & & & & & & & & & & & & & & & \\
\hline MS Transit & 0.10 & -0.18 & -0.47 & -0.25 & 1.00 & & & & & & & & & & & & & & & \\
\hline MS Walk & 0.37 & -0.15 & -0.57 & -0.25 & 0.31 & 1.00 & & & & & & & & & & & & & & \\
\hline MS Bike & 0.15 & -0.09 & -0.32 & -0.19 & 0.31 & 0.40 & 1.00 & & & & & & & & & & & & & \\
\hline Employment & -0.06 & -0.06 & -0.27 & -0.20 & 0.51 & 0.23 & 0.12 & 1.00 & & & & & & & & & & & & \\
\hline Population & 0.34 & -0.15 & -0.45 & -0.15 & 0.30 & 0.67 & 0.31 & 0.07 & 1.00 & & & & & & & & & & & \\
\hline No Households & 0.37 & -0.11 & -0.40 & -0.17 & 0.24 & 0.65 & 0.25 & 0.04 & 0.92 & 1.00 & & & & & & & & & & \\
\hline Age 0-4 & 0.06 & 0.09 & -0.01 & 0.21 & -0.18 & 0.01 & -0.11 & -0.24 & 0.10 & 0.01 & 1.00 & & & & & & & & & \\
\hline Age 5-24 & 0.14 & 0.10 & -0.08 & 0.11 & 0.09 & 0.08 & -0.01 & -0.20 & 0.19 & 0.07 & 0.55 & 1.00 & & & & & & & & \\
\hline Age 25-64 & 0.20 & 0.11 & -0.14 & 0.13 & -0.04 & 0.32 & 0.10 & -0.13 & 0.44 & 0.33 & 0.66 & 0.55 & 1.00 & & & & & & & \\
\hline Ageover65 & 0.19 & 0.05 & -0.10 & 0.07 & 0.01 & 0.26 & 0.12 & -0.12 & 0.29 & 0.23 & 0.31 & 0.36 & 0.63 & 1.00 & & & & & & \\
\hline VKT & -0.02 & -0.05 & 0.00 & -0.05 & 0.09 & 0.03 & 0.01 & 0.08 & -0.01 & 0.02 & -0.13 & -0.13 & -0.13 & -0.10 & 1.00 & & & & & \\
\hline Trips Produced & 0.31 & -0.15 & -0.44 & -0.17 & 0.38 & 0.64 & 0.26 & 0.36 & 0.83 & 0.88 & -0.04 & 0.04 & 0.25 & 0.14 & 0.06 & 1.00 & & & & \\
\hline Trips Attracted & -0.04 & -0.07 & -0.29 & -0.20 & 0.54 & 0.23 & 0.12 & 0.98 & 0.07 & 0.04 & -0.23 & -0.14 & -0.14 & -0.12 & 0.08 & 0.37 & 1.00 & & & \\
\hline $\begin{array}{c}\text { Density of Trips } \\
\text { out Centroid }\end{array}$ & 0.14 & -0.18 & -0.43 & -0.18 & 0.53 & 0.51 & 0.27 & 0.76 & 0.50 & 0.41 & -0.16 & -0.09 & 0.07 & 0.02 & 0.09 & 0.66 & 0.75 & 1.00 & & \\
\hline $\begin{array}{l}\text { Density of Trips } \\
\text { enter Centroid }\end{array}$ & -0.05 & -0.08 & -0.19 & -0.01 & 0.37 & 0.15 & 0.07 & 0.67 & 0.03 & 0.04 & -0.31 & -0.25 & -0.24 & -0.20 & 0.08 & 0.26 & 0.66 & 0.55 & 1.00 & \\
\hline TLKM & 0.13 & -0.08 & -0.10 & -0.10 & 0.12 & 0.20 & 0.19 & 0.10 & 0.41 & 0.35 & -0.01 & 0.02 & 0.14 & 0.10 & 0.03 & 0.31 & 0.09 & 0.25 & 0.12 & 1.00 \\
\hline
\end{tabular}


Table C.8 Uncorrelated Independent Variables Combinations

\begin{tabular}{|c|c|}
\hline Models & Independent Variables Combination \\
\hline - $\operatorname{Road}$ & haracteristics, where the roadway classified based on roadway classes, \\
\hline \multirow{12}{*}{ Model D-I } & $\begin{array}{l}\text { - No Bus stops, Tot-Int, Artl, Colle, Maj-Colle , Bike-Path , VKT, Trips-Produced, Trips-Attracted, Density- Trips- } \\
\text { Enter-Center }\end{array}$ \\
\hline & $\begin{array}{l}\text { - No Bus stops, Tot-Int, Colle, Maj-Colle , Bike-Path, VKT, Trips-Produced, Trips-Attracted, Density- Trips-Enter- } \\
\text { Center, TLKM }\end{array}$ \\
\hline & $\begin{array}{l}\text { - No Bus stops, Tot-Int, Artl, Colle, Maj-Colle , Bike-Path, VKT, Trips-Produced, Density- Trips-Out-Center, Density- } \\
\text { Trips-Enter-Center }\end{array}$ \\
\hline & $\begin{array}{l}\text { - No Bus stops, Tot-Int, Colle, Maj-Colle , Bike-Path, VKT, Trips-Produced, Density- Trips-Out-Center, Density- Trips- } \\
\text { Enter-Center, TLKM }\end{array}$ \\
\hline & $\begin{array}{l}\text { - No Bus stops, Artl, Colle, Loc, Maj-Colle , Bike-Path, VKT, Trips-Produced, Trips-Attracted, Density- Trips-Enter- } \\
\text { Center }\end{array}$ \\
\hline & $\begin{array}{l}\text { - No Bus stops, Colle, Loc ,Maj-Colle , Bike-Path, VKT, Trips-Produced, Trips-Attracted, Density- Trips-Enter-Center } \\
\text {,TLKM }\end{array}$ \\
\hline & $\begin{array}{l}\text { - No Bus stops, Artl, Colle, Loc, Maj-Colle , Bike-Path, VKT, Trips-Produced, Density- Trips-Out-Center, Density- } \\
\text { Trips-Enter-Center }\end{array}$ \\
\hline & $\begin{array}{l}\text { - No Bus stops, Colle , Loc ,Maj-Colle , Bike-Path, VKT, Trips-Produced, Density- Trips-Out-Center, Density- Trips- } \\
\text { Enter-Center, TLKM }\end{array}$ \\
\hline & - Sig-Int, Tot-Int, Artl, Colle, Maj-Colle , Bike-Path, VKT, Trips-Produced, Density- Trips-Enter-Center \\
\hline & - $\quad$ Sig-Int, Tot-Int, Colle, Maj-Colle , Bike-Path, VKT, Trips-Produced, Density- Trips-Enter-Center, TLKM \\
\hline & - Sig-Int, Artl, Colle, Loc, Maj-Colle , Bike-Path, VKT, Trips-Produced, Density- Trips-Enter-Center \\
\hline & - $\quad$ Sig-Int, Colle, Loc, Maj-Colle , Bike-Path, VKT, Trips-Produced, Density- Trips-Enter-Center, TLKM \\
\hline
\end{tabular}


Table C.8 Uncorrelated Independent Variables Combinations (cont')

\begin{tabular}{|c|c|}
\hline Models & Independent Variables Combination \\
\hline - Ro & haracteristics, where the roadway classifi \\
\hline \multirow{4}{*}{ Model DI } & $\begin{array}{l}\text { - No Bus stops, Non-Sig-Int, Artl, Colle, Maj-Colle , Bike-Path, VKT, Trips-Produced, Trips-Attracted, Density- Trips- } \\
\text { Enter-Center }\end{array}$ \\
\hline & $\begin{array}{l}\text { - No Bus stops, Non-Sig-Int, Colle, Maj-Colle , Bike-Path, VKT, Trips-Produced, Trips-Attracted, Density- Trips- } \\
\text { Enter-Center, TLKM }\end{array}$ \\
\hline & $\begin{array}{l}\text { - No Bus stops, Non-Sig-Int, Colle, Artl, Maj-Colle , Bike-Path, VKT, Trips-Produced, Density- Trips-Out-Center, } \\
\text { Density- Trips-Enter-Center }\end{array}$ \\
\hline & $\begin{array}{l}\text { - No Bus stops, Non-Sig-Int, Colle, Maj-Colle , Bike-Path, VKT, Trips-Produced, Density- Trips-Out-Center, Density- } \\
\text { Trips-Enter-Center, TLKM }\end{array}$ \\
\hline \multicolumn{2}{|c|}{ - Roadway characteristics, where the roadway classified based on posted speed limit, and exposure variables. } \\
\hline \multirow{6}{*}{ Model DII } & $\begin{array}{l}\text { - No Bus stops, Tot-Int, } 40 . \mathrm{Km} / \mathrm{h}, 50 . \mathrm{Km} / \mathrm{h}, 60 . \mathrm{Km} / \mathrm{h}, 70 . \mathrm{Km} / \mathrm{h}, 80 . \mathrm{Km} / \mathrm{h}, 90 . \mathrm{Km} / \mathrm{h}, 100 . \mathrm{Km} / \mathrm{h} \text {, Bike-Path, VKT, Trips- } \\
\text { Produced, Trips-Attracted, Density- Trips-Enter-Center, TLKM }\end{array}$ \\
\hline & $\begin{array}{l}\text { - No Bus stops, Tot-Int, } 40 . \mathrm{Km} / \mathrm{h}, 50 . \mathrm{Km} / \mathrm{h}, 60 . \mathrm{Km} / \mathrm{h}, 70 . \mathrm{Km} / \mathrm{h}, 80 . \mathrm{Km} / \mathrm{h}, 90 . \mathrm{Km} / \mathrm{h}, 100 . \mathrm{Km} / \mathrm{h} \text {, Bike-Path, VKT, Trips- } \\
\text { Produced, Density- Trips-Out-Center, Density- Trips-Enter-Center , TLKM }\end{array}$ \\
\hline & $\begin{array}{l}\text { - Tot-Int, Sig-Int, } 40 . \mathrm{Km} / \mathrm{h}, 50 . \mathrm{Km} / \mathrm{h}, 60 . \mathrm{Km} / \mathrm{h}, 70 . \mathrm{Km} / \mathrm{h}, 80 . \mathrm{Km} / \mathrm{h}, 90 . \mathrm{Km} / \mathrm{h}, 100 . \mathrm{Km} / \mathrm{h}, \text { Bike-Path, VKT, Trips- } \\
\text { Produced, Density- Trips-Enter-Center, TLKM }\end{array}$ \\
\hline & $\begin{array}{l}\text { - No Bus stops, Non-Sig-Int, } 40 . \mathrm{Km} / \mathrm{h}, 50 . \mathrm{Km} / \mathrm{h}, 60 . \mathrm{Km} / \mathrm{h}, 70 . \mathrm{Km} / \mathrm{h}, 80 . \mathrm{Km} / \mathrm{h}, 90 . \mathrm{Km} / \mathrm{h}, 100 . \mathrm{Km} / \mathrm{h}, \text { Bike-Path, VKT, } \\
\text { Trips-Produced, Trips-Attracted, Density- Trips-Enter-Center, TLKM }\end{array}$ \\
\hline & $\begin{array}{l}\text { - No Bus stops, Non-Sig-Int, } 40 . \mathrm{Km} / \mathrm{h}, 50 . \mathrm{Km} / \mathrm{h}, 60 . \mathrm{Km} / \mathrm{h}, 70 . \mathrm{Km} / \mathrm{h}, 80 . \mathrm{Km} / \mathrm{h}, 90 . \mathrm{Km} / \mathrm{h}, 100 . \mathrm{Km} / \mathrm{h} \text {, Bike-Path, VKT, } \\
\text { Trips-Produced, Density- Trips-Out-Center, Density- Trips-Enter-Center, TLKM }\end{array}$ \\
\hline & $\begin{array}{l}\text { - Sig-Int, Non-Sig-Int, } 40 . \mathrm{Km} / \mathrm{h}, 50 . \mathrm{Km} / \mathrm{h}, 60 . \mathrm{Km} / \mathrm{h}, 70 . \mathrm{Km} / \mathrm{h}, 80 . \mathrm{Km} / \mathrm{h}, 90 . \mathrm{Km} / \mathrm{h}, 100 . \mathrm{Km} / \mathrm{h} \text {, Bike-Path, VKT, Trips- } \\
\text { Produced, Density- Trips-Enter-Center, TLKM }\end{array}$ \\
\hline
\end{tabular}


Table C.8 Uncorrelated Independent Variables Combinations

\begin{tabular}{|c|c|}
\hline Models & Independent Variables Combination \\
\hline \multicolumn{2}{|c|}{ - Modal Split Variables and Exposure Variables. } \\
\hline \multirow{2}{*}{ Model DIII } & $\begin{array}{l}\text { - MS-Driver, MS-Passenger, MS-Transit, MS-Walk , MS-Bike, VKT, Trips-Produced, Trips-Attracted , Density- } \\
\text { Trips-Enter-Center, TLKM }\end{array}$ \\
\hline & $\begin{array}{l}\text { - MS-Driver, MS-Passenger, MS-Transit, MS-Walk , MS-Bike, VKT, Trips-Produced, Density- Trips-Out-Center, } \\
\text { Density- Trips-Enter-Center, TLKM }\end{array}$ \\
\hline \multicolumn{2}{|c|}{ - Modal Split Variables and Socio-demographic Characteristics Variables } \\
\hline \multirow{8}{*}{ Model DIV } & $\begin{array}{l}\text { - No.Schools, Area, MS-Driver, MS-Passenger, MS-Transit, MS-Walk , MS-Bike, Employment, Population, Age.0- } \\
\text { 4, Age.5-24, Age.25-64, Age > 65, VKT, TLKM }\end{array}$ \\
\hline & $\begin{array}{l}\text { - No.Schools, Area, MS-Driver, MS-Passenger, MS-Transit, MS-Walk , MS-Bike, Employment, No.Households, } \\
\text { Age.0-4, Age.5-24, Age.25-64, Age > 65, VKT, TLKM }\end{array}$ \\
\hline & $\begin{array}{l}\text { - No.Schools, Area, MS-Driver, MS-Passenger, MS-Transit, MS-Walk, MS-Bike, Population, Age.0-4, Age.5-24, } \\
\text { Age.25-64, Age > 65, VKT, Trips-Attracted, Density- Trips-Enter-Center, TLKM }\end{array}$ \\
\hline & $\begin{array}{l}\text { - No.Schools, Area, MS-Driver, MS-Passenger, MS-Transit, MS-Walk, MS-Bike, No.Households, Age.0-4, Age.5- } \\
\text { 24, Age.25-64, Age > 65, VKT, Trips-Attracted, Density- Trips-Enter-Center, TLKM }\end{array}$ \\
\hline & $\begin{array}{l}\text { - No.Schools, Area, MS-Driver, MS-Passenger, MS-Transit , MS-Walk , MS-Bike , Population , Age.0-4, Age.5-24, } \\
\text { Age.25-64, Age > 65, VKT, Density- Trips-Out-Center, Density- Trips-Enter-Center, TLKM }\end{array}$ \\
\hline & $\begin{array}{l}\text { - No.Schools, Area, MS-Driver, MS-Passenger, MS-Transit, MS-Walk, MS-Bike, No.Households, Age.0-4, Age.5- } \\
\text { 24, Age.25-64, Age > 65, VKT, Density- Trips-Out-Center, Density- Trips-Enter-Center, TLKM }\end{array}$ \\
\hline & $\begin{array}{l}\text { No.Schools, Area, MS-Driver, MS-Passenger, MS-Transit, MS-Walk , MS-Bike, Age.0-4, Age.5-24, Age.25-64, } \\
\text { Age > 65, VKT, Trips-Attracted, Trips-Produced, Density- Trips-Enter-Center, TLKM }\end{array}$ \\
\hline & $\begin{array}{l}\text { - No.Schools, Area, MS-Driver, MS-Passenger, MS-Transit, MS-Walk, MS-Bike, Age.0-4, Age.5-24, Age.25-64, } \\
\text { Age }>65, \text { VKT, Trips-Attracted, Trips-Produced, Density- Trips-Enter-Center ,TLKM }\end{array}$ \\
\hline \multicolumn{2}{|c|}{ - Exposure variables only } \\
\hline \multirow{2}{*}{ Model DVI } & - VKT, Trips-Produced, Trips-Attracted, Density- Trips-Enter-Center, TLKM \\
\hline & - VKT, Trips-Produced, Trips-Out-Center, Density- Trips-Enter-Center, TLKM \\
\hline
\end{tabular}




\section{Appendix D : The Best Ten CPMs for Each of exposure- related, Roadway-relate, and Planning-related for Each Type of Collisions.}




\section{D.1 Total Collisions Models}

\begin{tabular}{|c|c|c|c|c|c|}
\hline \multicolumn{6}{|c|}{$\begin{array}{l}\text { Call: } \quad \text { A-D-III } \\
\text { glm.nb(formula }=\text { Collisions } \sim \text { Sig-Int }+ \text { Non-Sig-Int }+40 . K m / h+50 . K m / h+70 . K m / h+100 . K m / h+\text { Bike- } \\
\text { Path + VKT + Density-Trips-Enter-Center + TLKM, init.theta }=1.670567423, \text { link=log) }\end{array}$} \\
\hline \multicolumn{6}{|l|}{ Deviance Residuals: } \\
\hline Min & 10 & Median & $3 Q$ & Max & \\
\hline \multirow[t]{2}{*}{-3.8967} & -0.8211 & -0.191 & 0.4097 & 3.4584 & \\
\hline & Estimate & Std.Error & $z$ value & $\operatorname{Pr}(>|z|)$ & \\
\hline (Intercept) & $4.68 \mathrm{E}+00$ & $7.55 \mathrm{E}-02$ & 62.009 & $<2 \mathrm{E}-16$ & $* * *$ \\
\hline Sig-Int & $1.69 \mathrm{E}-02$ & $3.97 E-03$ & 4.258 & $2.06 \mathrm{E}-05$ & $* * *$ \\
\hline Non-Sig-Int & $6.35 \mathrm{E}-03$ & $1.82 \mathrm{E}-03$ & 3.49 & 0.000483 & $* * *$ \\
\hline $40 . \mathrm{Km} / \mathrm{h}$ & $-9.40 \mathrm{E}-02$ & $3.89 \mathrm{E}-02$ & -2.418 & 0.015627 & $*$ \\
\hline $50 . \mathrm{Km} / \mathrm{h}$ & $-1.10 \mathrm{E}-01$ & $3.49 \mathrm{E}-02$ & -3.157 & 0.001594 & $* *$ \\
\hline $70 . \mathrm{Km} / \mathrm{h}$ & $-2.75 \mathrm{E}-01$ & $1.31 \mathrm{E}-01$ & -2.103 & 0.03545 & $*$ \\
\hline $100 . \mathrm{Km} / \mathrm{h}$ & $1.33 \mathrm{E}+03$ & $5.52 \mathrm{E}+02$ & 2.407 & 0.016064 & $*$ \\
\hline Bike-Path & $2.32 \mathrm{E}-02$ & $1.10 \mathrm{E}-02$ & 2.118 & 0.034158 & $*$ \\
\hline VKT & $-1.56 \mathrm{E}-06$ & $5.15 \mathrm{E}-07$ & -3.037 & 0.002389 & $* *$ \\
\hline Density-Trips-Enter-Center & $-1.41 \mathrm{E}-05$ & $2.89 \mathrm{E}-06$ & -4.894 & $9.90 \mathrm{E}-07$ & $* * *$ \\
\hline TLKM & $1.09 \mathrm{E}-01$ & $3.50 \mathrm{E}-02$ & 3.101 & 0.001929 & $* *$ \\
\hline $\begin{array}{l}\text { Signif.codes: }\left(0^{* * * *)}\right)(0 . \\
\text { (Dispersion parameter for } \\
\text { Null deviance: } 535.43 \text { on } 42 \\
\text { Residual deviance: } 473.160 \\
\text { AIC: } 5017.6 \\
\text { Number of Fisher Scoring it } \\
\text { Theta: } 1.671 \\
\text { Std.Error.: } 0.11 \\
\text { 2xlog-likelihood:-4S }\end{array}$ & $\begin{array}{l}\left.L^{\prime * * \prime}\right)\left(0.01^{\prime *}\right. \\
\text { ative Binom } \\
\text { legree of fre } \\
11 \text { degrees } \\
\text { tions: } 1 \\
589\end{array}$ & $\begin{array}{l}.05 \\
\text { m } \\
\text { eedom famil }\end{array}$ & $\begin{array}{l}\left(0.1^{\prime \prime}\right) \\
\text { n to be }\end{array}$ & & \\
\hline
\end{tabular}




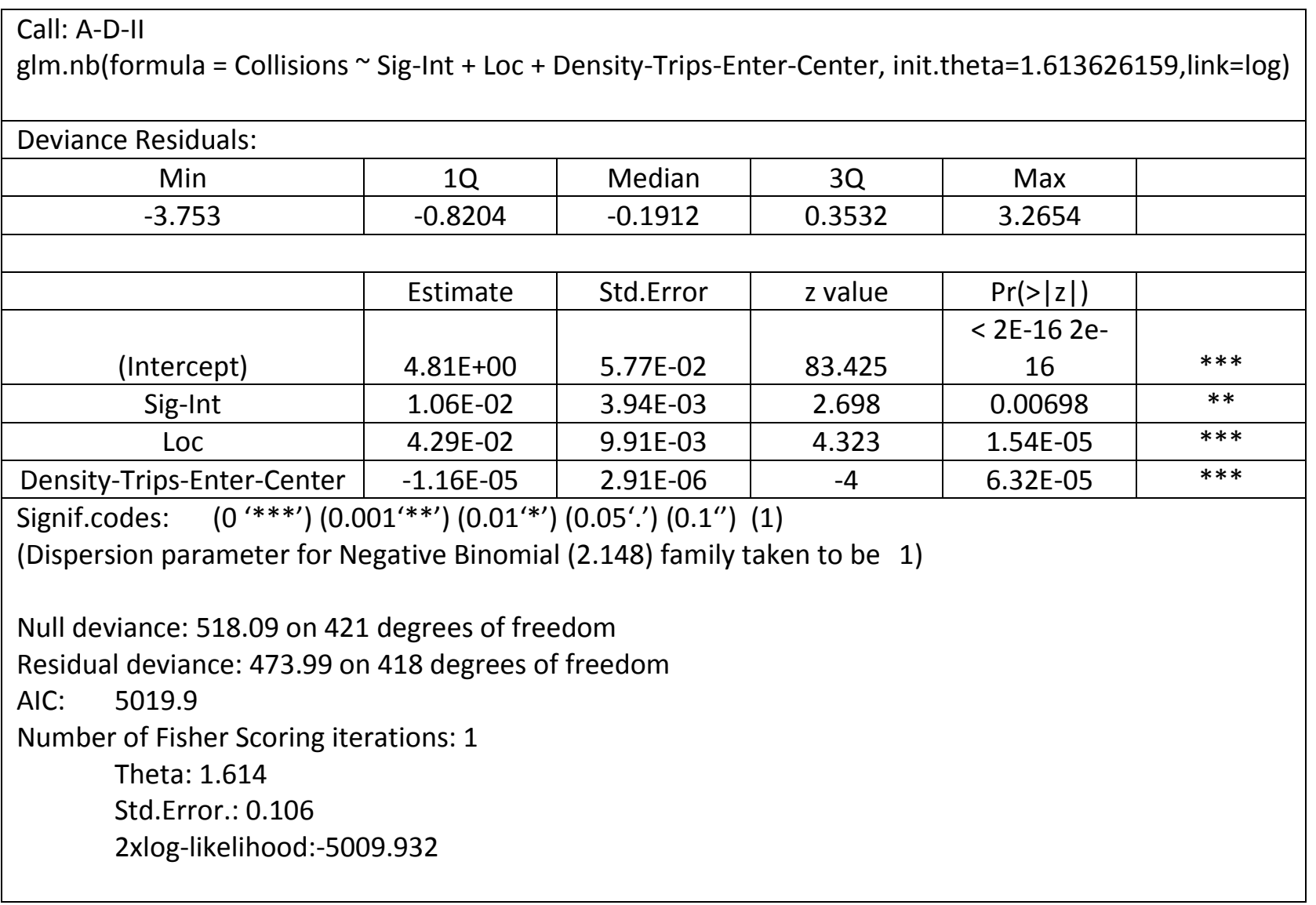




\begin{tabular}{|c|c|c|c|c|c|}
\hline \multicolumn{6}{|c|}{$\begin{array}{l}\text { A-D-IV } \\
\text { Call: } \\
\text { glm.nb(formula = Collisions MS-Driver + MS-Passenger + MS-Transit + MS-Walk + MS-Bike + Density- } \\
\text { Trips-Enter-Center, init.theta }=1.70689375 \text {,link=log) }\end{array}$} \\
\hline \multicolumn{6}{|l|}{ Deviance Residuals: } \\
\hline Min & 10 & Median & $3 Q$ & Max & \\
\hline \multirow[t]{2}{*}{-3.8795} & -0.8367 & -0.1502 & 0.3576 & 3.328 & \\
\hline & Estimate & Std.Error & $z$ value & $\operatorname{Pr}(>|z|)$ & \\
\hline (Intercept) & $3.01 \mathrm{E}+00$ & $2.46 \mathrm{E}-01$ & 12.225 & $<2 \mathrm{E}-16$ & $* * *$ \\
\hline MS-Driver & $1.38 \mathrm{E}-02$ & $2.92 \mathrm{E}-03$ & 4.722 & $2.34 \mathrm{E}-06$ & $* * *$ \\
\hline MS-Passenger & $4.12 \mathrm{E}-02$ & $6.10 \mathrm{E}-03$ & 6.765 & $1.33 \mathrm{E}-11$ & $* * *$ \\
\hline MS-Transit & $3.01 \mathrm{E}-02$ & $5.00 \mathrm{E}-03$ & 6.021 & $1.73 \mathrm{E}-09$ & $* * *$ \\
\hline MS-Walk & $2.61 \mathrm{E}-02$ & $4.88 \mathrm{E}-03$ & 5.348 & $8.88 \mathrm{E}-08$ & $* * *$ \\
\hline MS-Bike & $4.69 \mathrm{E}-02$ & $1.85 \mathrm{E}-02$ & 2.531 & 0.0114 & $*$ \\
\hline Density- Trips-Enter-Center & $-1.24 \mathrm{E}-05$ & $2.24 \mathrm{E}-06$ & -5.557 & $2.74 \mathrm{E}-08$ & $* * *$ \\
\hline \multicolumn{6}{|c|}{ 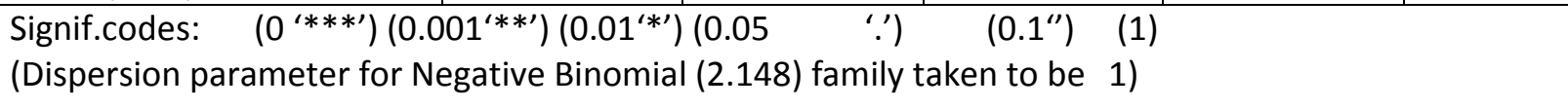 } \\
\hline \multicolumn{6}{|c|}{$\begin{array}{l}\text { Null deviance: } 546.46 \text { on } 421 \text { degrees of free } \\
\text { Residual deviance: } 472.06 \text { on } 415 \text { degrees of freedom } \\
\text { AIC: } 4998.9\end{array}$} \\
\hline \multicolumn{6}{|c|}{ Number of Fisher Scoring iterations: 1} \\
\hline \multicolumn{6}{|l|}{$\begin{array}{l}\text { Theta: } 1.707 \\
\text { Std. Err.: } 0.113\end{array}$} \\
\hline \multicolumn{6}{|l|}{2 x log-likelihood: -4982.891} \\
\hline
\end{tabular}




\begin{tabular}{|c|c|c|c|c|c|}
\hline \multicolumn{6}{|c|}{$\begin{array}{l}\text { A-D-V } \\
\text { Call: } \\
\text { glm.nb(formula =Collisions Area + MS-Driver + MS-Passenger + MS-Transit + MS-Walk + MS-Bike + } \\
\text { Employment + Age.25-64 + Age > 65, init.theta=1.910281991, link=log) }\end{array}$} \\
\hline \multicolumn{6}{|l|}{ Deviance Residuals: } \\
\hline Min & 10 & Median & $3 Q$ & Max & \\
\hline \multirow[t]{2}{*}{-3.9591} & -0.8805 & -0.1304 & 0.417 & 2.669 & \\
\hline & Estimate & Std Error & 7 value & $\operatorname{Pr}(>|z|)$ & \\
\hline (Intercept) & $2.65 \mathrm{E}+00$ & $2.41 \mathrm{E}-01$ & 11.008 & $<2 \mathrm{E}-16$ & $* * *$ \\
\hline Area & $7.23 \mathrm{E}-03$ & $1.31 \mathrm{E}-03$ & 5.514 & $3.51 \mathrm{E}-08$ & $* * *$ \\
\hline MS-Driver & $1.53 \mathrm{E}-02$ & $2.80 \mathrm{E}-03$ & 5.457 & $4.84 \mathrm{E}-08$ & $* * *$ \\
\hline MS-Passenger & $2.79 \mathrm{E}-02$ & $5.89 \mathrm{E}-03$ & 4.744 & $2.09 \mathrm{E}-06$ & $* * *$ \\
\hline MS-Transit & $3.51 \mathrm{E}-02$ & $5.02 \mathrm{E}-03$ & 6.983 & $2.90 \mathrm{E}-12$ & $* * *$ \\
\hline MS-Walk & $2.28 \mathrm{E}-02$ & 4.99E-03 & 4.57 & 4.87E-06 & $* * *$ \\
\hline MS-Bike & $4.42 \mathrm{E}-02$ & $1.76 \mathrm{E}-02$ & 2.515 & 0.01189 & $*$ \\
\hline Employment & $-4.70 \mathrm{E}-06$ & $1.73 \mathrm{E}-06$ & -2.72 & 0.00654 & $* *$ \\
\hline Age.25-64 & $4.68 \mathrm{E}-03$ & $1.90 \mathrm{E}-03$ & 2.462 & 0.0138 & $*$ \\
\hline Age $>65$ & $1.11 \mathrm{E}-02$ & $5.69 \mathrm{E}-03$ & 1.955 & 0.0506 & . \\
\hline \multicolumn{6}{|c|}{$\begin{array}{l}\text { Null deviance: } 607.94 \text { on } 421 \text { degrees of freedom } \\
\text { Residual deviance: } 470.49 \text { on } 412 \text { degrees of freedom } \\
\text { AIC: } 4953.5\end{array}$} \\
\hline \multicolumn{6}{|c|}{ Number of Fisher Scoring iterations: 1} \\
\hline \multicolumn{6}{|l|}{ Theta: 1.91} \\
\hline $2 \times$ log-likelihood: -4 & & & & & \\
\hline
\end{tabular}




\begin{tabular}{|c|c|c|c|c|c|}
\hline \multicolumn{6}{|c|}{$\begin{array}{l}\text { Call: A-C-IV } \\
\text { glm.nb(formula }=\text { Collisions } \sim \text { MS-Driver + MS-Passenger + MS-Transit + MS-Walk + MS-Bike + VKT + } \\
\text { Trips-Produced + Trips-Attracted + Density- Trips-Out-Center + Density- Trips-Enter-Center + TLKM, } \\
\text { init.theta }=2.147993471, \text { link=log) }\end{array}$} \\
\hline \multicolumn{6}{|l|}{ Deviance Residuals: } \\
\hline Min & 10 & Median & $3 Q$ & Max & \\
\hline \multirow[t]{2}{*}{-3.9507} & -0.8173 & -0.1287 & 0.3976 & 2.528 & \\
\hline & Estimate & Std.Error & $z$ value & $\operatorname{Pr}(>|z|)$ & \\
\hline (Intercept) & $2.70 \mathrm{E}+00$ & $2.27 \mathrm{E}-01$ & 11.89 & $<2 \mathrm{E}-16$ & $* * *$ \\
\hline MS-Driver & $1.34 \mathrm{E}-02$ & $2.68 \mathrm{E}-03$ & 4.98 & $6.36 \mathrm{E}-07$ & $* * *$ \\
\hline MS-Passenger & $3.20 \mathrm{E}-02$ & $5.76 \mathrm{E}-03$ & 5.564 & $2.63 \mathrm{E}-08$ & $* * *$ \\
\hline MS-Transit & $2.29 \mathrm{E}-02$ & $5.04 \mathrm{E}-03$ & 4.545 & $5.50 \mathrm{E}-06$ & $* * *$ \\
\hline MS-Walk & $2.35 \mathrm{E}-02$ & $4.80 \mathrm{E}-03$ & 4.889 & $1.01 \mathrm{E}-06$ & $* * *$ \\
\hline MS-Bike & $4.90 \mathrm{E}-02$ & $1.66 \mathrm{E}-02$ & 2.951 & 0.00317 & $* *$ \\
\hline VKT & $3.43 \mathrm{E}-06$ & $8.10 \mathrm{E}-07$ & 4.231 & $2.32 \mathrm{E}-05$ & $* * *$ \\
\hline Trips-Produced & $8.35 \mathrm{E}-05$ & $3.50 \mathrm{E}-05$ & 2.386 & 0.01703 & $*$ \\
\hline Trips-Attracted & $1.36 \mathrm{E}-04$ & $2.88 \mathrm{E}-05$ & 4.706 & $2.52 \mathrm{E}-06$ & $* * *$ \\
\hline Density- Trips-Out-Center & $4.72 \mathrm{E}-05$ & $2.05 \mathrm{E}-05$ & 2.304 & 0.02124 & $*$ \\
\hline Density- Trips-Enter-Center & $-1.25 \mathrm{E}-05$ & $2.62 \mathrm{E}-06$ & -4.762 & $1.92 \mathrm{E}-06$ & $* * *$ \\
\hline TLKM & $6.86 \mathrm{E}-03$ & $1.54 \mathrm{E}-03$ & 4.464 & 8.04E-06 & $* * *$ \\
\hline \multicolumn{6}{|c|}{$\begin{array}{l}\text { Signif.codes: }\left(0^{\prime * * * \prime}\right)\left(0.001^{\prime * * \prime}\right)\left(0.01^{\prime * \prime}\right)\left(0.05^{\prime .}\right)\left(0.1^{\prime \prime}\right)(1) \\
\text { (Dispersion parameter for Negative Binomial }(2.148) \text { family taken to be } 1)\end{array}$} \\
\hline \multicolumn{6}{|c|}{ Null deviance: 679.15 on 421 degrees of freedom } \\
\hline \multicolumn{6}{|c|}{ Residual deviance: 469.68 on 410 degrees of freedom } \\
\hline \multicolumn{6}{|l|}{ AIC: 4905.3} \\
\hline \multicolumn{6}{|c|}{ Number of Fisher Scoring iterations: 1} \\
\hline \multicolumn{6}{|l|}{ Theta: 2.148} \\
\hline \multicolumn{6}{|l|}{ Std. Err.: 0.146} \\
\hline \multicolumn{6}{|c|}{ Warning while fitting theta: alternation limit reached } \\
\hline \multicolumn{6}{|l|}{2 x log-likelihood: -4879.304} \\
\hline
\end{tabular}




\begin{tabular}{|c|c|c|c|c|c|}
\hline \multicolumn{6}{|c|}{$\begin{array}{l}\text { Call: } \\
\text { glm.nb (formula = Collisions } \sim \text { MS-Driver + MS-Passenger + MS-Transit + MS-Walk + MS Bike + } \\
\text { Population + Age.0-4 + Age.5-24 + Age.25-64 + VKT +Trips-Attracted + Density- Trips-Enter Center + } \\
\text { TLKM, init.theta }=2.245827836, \text { link }=\log \text { ) }\end{array}$} \\
\hline \multicolumn{6}{|l|}{ Deviance Residuals: } \\
\hline Min & 10 & Median & 30 & Max & \\
\hline \multirow[t]{2}{*}{-3.9526} & -0.8003 & -0.158 & 0.4198 & 2.8182 & \\
\hline & Estimate & Std.Error & $z$ value & $\operatorname{Pr}(>|z|)$ & \\
\hline (Intercept) & $2.73 \mathrm{E}+00$ & 2.27E-01 & 12.031 & $<2 \mathrm{E}-16$ & $* * *$ \\
\hline MS-Driver & $1.28 \mathrm{E}-02$ & $2.64 \mathrm{E}-03$ & 4.859 & $1.18 \mathrm{E}-06$ & $* * *$ \\
\hline MS-Passenger & $2.74 \mathrm{E}-02$ & $5.66 \mathrm{E}-03$ & 4.844 & $1.28 \mathrm{E}-06$ & $* * *$ \\
\hline MS-Transit & $2.24 \mathrm{E}-02$ & $5.02 \mathrm{E}-03$ & 4.469 & 7.85E-06 & $* * *$ \\
\hline MS-Walk & $1.93 \mathrm{E}-02$ & $4.78 \mathrm{E}-03$ & 4.039 & $5.36 \mathrm{E}-05$ & $* * *$ \\
\hline MS-Bike & $4.16 \mathrm{E}-02$ & $1.64 \mathrm{E}-02$ & 2.535 & 0.01126 & $*$ \\
\hline Population & $6.51 \mathrm{E}-05$ & $2.08 \mathrm{E}-05$ & 3.125 & 0.00178 & $* *$ \\
\hline Age.0-4 & $-3.01 \mathrm{E}-02$ & $1.65 \mathrm{E}-02$ & -1.825 & 0.06805 & . \\
\hline Age.5-24 & $-9.60 \mathrm{E}-03$ & 3.39E-03 & -2.835 & 0.00458 & $* *$ \\
\hline Age.25-64 & $9.66 \mathrm{E}-03$ & $2.12 \mathrm{E}-03$ & 4.561 & $5.08 \mathrm{E}-06$ & $* * *$ \\
\hline VKT & $3.28 \mathrm{E}-06$ & 7.94E-07 & 4.137 & $3.52 \mathrm{E}-05$ & $* * *$ \\
\hline Trips-Attracted & $1.89 \mathrm{E}-04$ & 2.97E-05 & 6.382 & $1.75 \mathrm{E}-10$ & $* * *$ \\
\hline Density- Trips-Enter-Center & $-1.03 E-05$ & $2.30 \mathrm{E}-06$ & -4.49 & 7.13E-06 & $* * *$ \\
\hline TLKM & $5.23 \mathrm{E}-03$ & 1.57E-03 & 3.333 & 0.00086 & $* * *$ \\
\hline \multicolumn{6}{|c|}{$\begin{array}{l}\text { Signif.codes: }\left(0^{\prime * * * \prime}\right)\left(0.001^{\prime * * \prime}\right)\left(0.01^{\prime * \prime}\right)\left(0.05^{\prime \prime}\right)\left(0.1^{\prime \prime}\right)(1) \\
\text { (Dispersion parameter for Negative Binomial }(2.148) \text { family taken to be } 1)\end{array}$} \\
\hline \multicolumn{6}{|c|}{$\begin{array}{l}\text { Null deviance: } 708.26 \text { on } 421 \text { degrees of freedom } \\
\text { Residual deviance: } 469.24 \text { on } 408 \text { degrees of freedom } \\
\text { AIC: } 4889.5\end{array}$} \\
\hline \multicolumn{6}{|c|}{ Number of Fisher Scoring iterations: 1} \\
\hline \multicolumn{6}{|l|}{ Theta: 2.246} \\
\hline \multicolumn{6}{|l|}{ Std. Err.: 0.153} \\
\hline $2 \times$ log-likelihood: -4859.52 & & & & & \\
\hline
\end{tabular}




\begin{tabular}{|c|c|c|c|c|c|}
\hline \multicolumn{6}{|c|}{$\begin{array}{l}\text { Call: } \\
\text { glm.nb (formula }=\text { Collisions } \sim \text { VKT }+ \text { Trips-Out-Center }+ \text { Trips-Enter-Center + Density- Trips-Out-Center + } \\
\text { Density- Trips-Enter-Center + TLKM, init.theta }=2.080503998, \text { link }=\log \text { ) }\end{array}$} \\
\hline \multicolumn{6}{|l|}{ Deviance Residuals: } \\
\hline Min & 10 & Median & $3 Q$ & Max & \\
\hline \multirow[t]{2}{*}{-4.015} & -0.8174 & -0.1868 & 0.4289 & 3.1936 & \\
\hline & Estimate & Std.Error & $z$ value & $\operatorname{Pr}(>|z|)$ & \\
\hline (Intercept) & $4.06 \mathrm{E}+00$ & 7.09E-02 & 57.268 & $<2 \mathrm{E}-16$ & $* * *$ \\
\hline VKT & 3.97E-06 & $8.21 \mathrm{E}-07$ & 4.837 & $1.32 \mathrm{E}-06$ & $* * *$ \\
\hline Trips-Out-Center & $1.85 \mathrm{E}-04$ & $3.03 \mathrm{E}-05$ & 6.092 & $1.11 \mathrm{E}-09$ & $* * *$ \\
\hline Trips-Enter-Center & $1.68 \mathrm{E}-04$ & $2.15 \mathrm{E}-05$ & 7.786 & $6.94 \mathrm{E}-15$ & $* * *$ \\
\hline Density- Trips-Out-Center & $1.10 \mathrm{E}-04$ & $1.75 \mathrm{E}-05$ & 6.27 & $3.62 \mathrm{E}-10$ & $* * *$ \\
\hline Density- Trips-Enter-Center & $-1.33 \mathrm{E}-05$ & $2.66 \mathrm{E}-06$ & -5.001 & $5.72 \mathrm{E}-07$ & $* * *$ \\
\hline TLKM & $3.85 \mathrm{E}-03$ & $1.63 \mathrm{E}-03$ & 2.359 & 0.0183 & $*$ \\
\hline \multicolumn{6}{|c|}{$\begin{array}{l}\text { Signif.codes: }\left(0^{* * * \prime}\right)\left(0.001^{\prime * * \prime}\right)\left(0.01^{(* \prime}\right)\left(0.05^{\prime \prime} .\right)\left(0.1^{\prime \prime}\right)(1) \\
\text { (Dispersion parameter for Negative Binomial }(2.148) \text { family taken to be } 1)\end{array}$} \\
\hline \multicolumn{6}{|c|}{ Null deviance: 659 on 421 degrees of freedom } \\
\hline \multicolumn{6}{|c|}{ Residual deviance: 470.7 on 415 degrees of freedom } \\
\hline \multicolumn{6}{|l|}{ AIC: 4910.2} \\
\hline \multicolumn{6}{|c|}{ Number of Fisher Scoring iterations: 1} \\
\hline \multicolumn{6}{|l|}{ Theta: 2.081} \\
\hline \multicolumn{6}{|l|}{ Std. Err.: 0.141} \\
\hline \multicolumn{6}{|c|}{ Warning while fitting theta: alternation limit reached } \\
\hline 2 x log-likelihood: -4894.24 & & & & & \\
\hline
\end{tabular}




\begin{tabular}{|c|c|c|c|c|c|}
\hline \multicolumn{6}{|c|}{$\begin{array}{l}\text { Call: } \\
\text { glm.nb (formula }=\text { Collisions } \sim \text { Density- Trips-Out-Center + Density- Trips-Enter-Center, init.theta }= \\
\text { 1.555701382, link }=\log \text { ) }\end{array}$} \\
\hline \multicolumn{6}{|l|}{ Deviance Residuals: } \\
\hline Min & 10 & Median & $3 Q$ & Max & \\
\hline-3.8598 & -0.8771 & -0.206 & 0.4163 & 2.9706 & \\
\hline & Estimate & Std.Error & $z$ value & $\operatorname{Pr}(>|z|)$ & \\
\hline (Intercept) & $4.91 \mathrm{E}+00$ & $5.15 \mathrm{E}-02$ & 95.449 & $<2 \mathrm{E}-16$ & $* * *$ \\
\hline Density- Trips-Out-Center & $8.14 \mathrm{E}-05$ & $1.89 \mathrm{E}-05$ & 4.314 & $1.60 \mathrm{E}-05$ & $* * *$ \\
\hline Density- Trips-Enter-Center & $-1.25 \mathrm{E}-05$ & 2.69E-06 & -4.669 & $3.02 \mathrm{E}-06$ & $* * *$ \\
\hline \multicolumn{6}{|c|}{$\begin{array}{l}\text { Signif.codes: }\left(0^{* * * \prime}\right)\left(0.001^{\prime * * \prime}\right)\left(0.01^{(* \prime}\right)\left(0.05^{\prime} . \prime\right)\left(0.1^{\prime \prime}\right)(1) \\
\text { (Dispersion parameter for Negative Binomial }(2.148) \text { family taken to be } 1)\end{array}$} \\
\hline \multicolumn{6}{|c|}{$\begin{array}{l}\text { Null deviance: } 500.41 \text { on } 421 \text { degrees of freedom } \\
\text { Residual deviance: } 474.9 \text { on } 419 \text { degrees of freedom } \\
\text { AIC: } 5035.3\end{array}$} \\
\hline \multicolumn{6}{|c|}{ Number of Fisher Scoring iterations: 1} \\
\hline \multicolumn{6}{|l|}{ Theta: 1.556} \\
\hline $2 \times$ log-likelihood: -5027.25 & & & & & \\
\hline
\end{tabular}




\begin{tabular}{|c|c|c|c|c|c|}
\hline \multicolumn{6}{|c|}{$\begin{array}{l}\text { Call: } \\
\text { glm.nb(formula }=\text { Collisions } \sim \text { No Bus stops }+ \text { Non-Sig-Int }+40 . \mathrm{Km} / \mathrm{h}+50 . \mathrm{Km} / \mathrm{h}+60 . \mathrm{Km} / \mathrm{h}+70 . \mathrm{Km} / \mathrm{h}+ \\
80 . \mathrm{Km} / \mathrm{h}+90 . \mathrm{Km} / \mathrm{h}+100 . \mathrm{Km} / \mathrm{h}+\text { VKT+Trips-Attracted }+ \text { Density- Trips-Enter-Center }+ \text { TLKM, init.theta }= \\
\text { 1.191452324, link }=\log )\end{array}$} \\
\hline \multicolumn{6}{|l|}{ Deviance Residuals: } \\
\hline Min & 10 & Median & $3 Q$ & Max & \\
\hline \multirow[t]{2}{*}{-3.3481} & -0.8374 & -0.2432 & 0.3583 & 3.4744 & \\
\hline & Estimate & Std.Error & $z$ value & $\operatorname{Pr}(>|z|)$ & \\
\hline (Intercept) & $4.21 \mathrm{E}+00$ & $9.18 \mathrm{E}-02$ & 45.907 & $<2 \mathrm{E}-16$ & $* * *$ \\
\hline No Bus stops & $1.96 \mathrm{E}-02$ & $3.78 \mathrm{E}-03$ & 5.178 & $2.24 \mathrm{E}-07$ & $* * *$ \\
\hline Non-Sig-Int & $5.41 \mathrm{E}-03$ & $2.64 \mathrm{E}-03$ & 2.047 & 0.0407 & $*$ \\
\hline $40 . \mathrm{Km} / \mathrm{h}$ & $-3.83 E+01$ & $1.76 \mathrm{E}+01$ & -2.17 & 0.03 & $*$ \\
\hline $50 . \mathrm{Km} / \mathrm{h}$ & $-3.83 E+01$ & $1.76 \mathrm{E}+01$ & -2.17 & 0.03 & $*$ \\
\hline $60 . \mathrm{Km} / \mathrm{h}$ & $-3.82 E+01$ & $1.76 \mathrm{E}+01$ & -2.163 & 0.0305 & $*$ \\
\hline $70 . \mathrm{Km} / \mathrm{h}$ & $-3.80 \mathrm{E}+01$ & $1.76 \mathrm{E}+01$ & -2.156 & 0.0311 & $*$ \\
\hline $80 . \mathrm{Km} / \mathrm{h}$ & $-3.78 \mathrm{E}+01$ & $1.76 \mathrm{E}+01$ & -2.144 & 0.032 & $*$ \\
\hline $90 . \mathrm{Km} / \mathrm{h}$ & $-3.48 \mathrm{E}+01$ & $1.79 \mathrm{E}+01$ & -1.941 & 0.0522 & . \\
\hline $100 . \mathrm{Km} / \mathrm{h}$ & $1.52 \mathrm{E}+03$ & $6.54 \mathrm{E}+02$ & 2.318 & 0.0205 & $*$ \\
\hline VKT & $3.40 \mathrm{E}-06$ & $1.47 \mathrm{E}-06$ & 2.318 & 0.0204 & $*$ \\
\hline Trips-Attracted & $3.48 \mathrm{E}-05$ & $6.30 \mathrm{E}-06$ & 5.523 & $3.34 \mathrm{E}-08$ & $* * *$ \\
\hline Density-Trips-Enter-Center & $-5.85 \mathrm{E}-05$ & $7.89 \mathrm{E}-06$ & -7.412 & $1.25 \mathrm{E}-13$ & $* * *$ \\
\hline TLKM & $3.83 \mathrm{E}+01$ & $1.76 \mathrm{E}+01$ & 2.171 & 0.0299 & $*$ \\
\hline \multicolumn{6}{|c|}{$\begin{array}{l}\text { Signif.codes: }\left(0^{\prime * * * \prime}\right)\left(0.001^{\prime * * \prime}\right)\left(0.01^{\prime * \prime}\right)\left(0.05^{\prime} .\right)\left(0.1^{\prime \prime}\right)(1) \\
\text { (Dispersion parameter for Negative Binomial }(2.148) \text { family taken to be } 1)\end{array}$} \\
\hline \multicolumn{6}{|c|}{ Null deviance: 616.5 on 421 degrees of freedom } \\
\hline \multicolumn{6}{|c|}{ Residual deviance: 494.9 on 408 degrees of freedom } \\
\hline \multicolumn{6}{|l|}{ AIC: 5004.8} \\
\hline \multicolumn{6}{|c|}{ Number of Fisher Scoring iterations: 1} \\
\hline \multicolumn{6}{|l|}{ Theta: 1.1915} \\
\hline \multicolumn{6}{|l|}{ Std. Err.: 0.0796} \\
\hline \multicolumn{6}{|l|}{$2 \times$ log-likelihood: -4974.788} \\
\hline
\end{tabular}




\begin{tabular}{|c|c|c|c|c|c|}
\hline $\begin{array}{l}\text { C-C-I } \\
\text { Call: } \\
\text { glm.nb(formula = Collisions } \\
\text { Density- Trips-Out-Center + } \\
\text { link = log) }\end{array}$ & $\begin{array}{l}\text { /KT + Trips-C } \\
\text { nsity- Trips }\end{array}$ & $\begin{array}{l}\text { Center + Tri } \\
\text { r-Center + }\end{array}$ & ter-Cent & 557445358 & \\
\hline Deviance Residuals: & & & & & \\
\hline Min & 10 & Median & $3 Q$ & Max & \\
\hline-3.57 & -0.78 & -0.1755 & 0.4301 & 2.4676 & \\
\hline & Estimate & Std.Error & $z$ value & $\operatorname{Pr}(>|z|)$ & \\
\hline (Intercept) & $3.44 \mathrm{E}+00$ & $8.19 \mathrm{E}-02$ & 41.995 & $<2 \mathrm{E}-16$ & $* * *$ \\
\hline VKT & $2.91 \mathrm{E}-06$ & $8.36 \mathrm{E}-07$ & 3.475 & 0.000512 & $* * *$ \\
\hline Trips-Out-Center & $2.70 \mathrm{E}-04$ & $3.76 \mathrm{E}-05$ & 7.185 & $6.72 \mathrm{E}-13$ & $* * *$ \\
\hline Trips-Enter-Center & $2.87 \mathrm{E}-04$ & $2.54 \mathrm{E}-05$ & 11.3 & $<2 \mathrm{E}-16$ & $* * *$ \\
\hline Density- Trips-Out-Center & $2.44 \mathrm{E}-04$ & $2.61 \mathrm{E}-05$ & 9.339 & $<2 \mathrm{E}-16$ & $* * *$ \\
\hline Density- Trips-Enter-Center & $-3.67 \mathrm{E}-05$ & 5.01E-06 & -7.322 & $2.45 \mathrm{E}-13$ & $* * *$ \\
\hline TLKM & 7.00E-03 & $1.84 \mathrm{E}-03$ & 3.795 & 0.000148 & $* * *$ \\
\hline $\begin{array}{l}\text { Signif.codes: }\left(0^{\prime * * * \prime}\right)(0.001 \\
\text { (Dispersion parameter for } \mathrm{N}\end{array}$ & $\begin{array}{l}\left.*^{\prime \prime}\right)\left(0.01^{(* \prime}\right) \\
\text { ative Binom }\end{array}$ & $\begin{array}{l}\left..^{\prime \prime}\right)\left(0.1^{\prime \prime}\right) \\
.148) \text { fami }\end{array}$ & n to be & & \\
\hline Null deviance: 786.39 on 42 & degrees of $f$ & & & & \\
\hline Residual deviance: 496.91 o & 415 degrees & eedom & & & \\
\hline AIC: 4875.4 & & & & & \\
\hline Number of Fisher Scoring ite & tions: 1 & & & & \\
\hline Theta: 1.557 & & & & & \\
\hline Std. Err.: 0.109 & & & & & \\
\hline $2 \times$ log-likelihood: -4859.38 & & & & & \\
\hline
\end{tabular}




\begin{tabular}{|c|c|c|c|c|c|}
\hline \multicolumn{6}{|c|}{$\begin{array}{l}\text { D-D-II } \\
\text { Call: } \\
\text { glm.nb(formula = Collisions No Bus stops + Loc + Bike-Path + } \\
\text { VKT + Density- Trips-Enter-Center, init.theta }=0.97668282, \text { link }=\text { log) }\end{array}$} \\
\hline \multicolumn{6}{|l|}{ Deviance Residuals: } \\
\hline Min & $1 Q$ & Median & $3 Q$ & Max & \\
\hline-3.1941 & -0.8881 & -0.2641 & 0.2841 & 3.0733 & \\
\hline & Estimate & Std.Error & $z$ value & $\operatorname{Pr}(>|z|)$ & \\
\hline (Intercept) & $4.63 \mathrm{E}+00$ & $7.80 \mathrm{E}-02$ & 59.396 & $<2 \mathrm{E}-16$ & $* * *$ \\
\hline No Bus stops & $1.24 \mathrm{E}-02$ & $3.72 \mathrm{E}-03$ & 3.326 & 0.00088 & $* * *$ \\
\hline Loc & $4.22 \mathrm{E}-02$ & $1.50 \mathrm{E}-02$ & 2.822 & 0.00477 & $* *$ \\
\hline Bike-Path & $2.67 \mathrm{E}-02$ & $1.43 \mathrm{E}-02$ & 1.869 & 0.06163 & . \\
\hline VKT & $-1.54 \mathrm{E}-06$ & $6.54 \mathrm{E}-07$ & -2.358 & 0.01836 & $*$ \\
\hline Density- Trips-Enter-Center & $-1.52 \mathrm{E}-05$ & $3.15 \mathrm{E}-06$ & -4.809 & $1.52 \mathrm{E}-06$ & $* * *$ \\
\hline \multicolumn{6}{|c|}{$\begin{array}{l}\text { Signif.codes: }\left(0^{\prime * * * \prime}\right)\left(0.001^{* * \prime}\right)\left(0.01^{* \prime}\right)\left(0.05^{\prime}{ }^{\prime}\right)\left(0.1^{\prime \prime}\right)(1) \\
\text { (Dispersion parameter for Negative Binomial }(2.148) \text { family taken to be } 1)\end{array}$} \\
\hline \multicolumn{6}{|c|}{$\begin{array}{l}\text { Null deviance: } 558.14 \text { on } 421 \text { degrees of freedom } \\
\text { Residual deviance: } 496.83 \text { on } 416 \text { degrees of freedom } \\
\text { AIC: } 5035.3\end{array}$} \\
\hline \multicolumn{6}{|c|}{ Number of Fisher Scoring iterations: 1} \\
\hline \multicolumn{6}{|l|}{ Theta: 0.9767} \\
\hline $2 \times$ log-likelihood: -5021.25 & & & & & \\
\hline
\end{tabular}




\begin{tabular}{|c|c|c|c|c|c|}
\hline $\begin{array}{l}\text { D-D-III } \\
\text { Call: } \\
\text { glm.nb(formula }=\text { Collisions } \\
80 . \mathrm{Km} / \mathrm{h}+100 . \mathrm{Km} / \mathrm{h}+\text { Bike } \\
\text { init.theta }=1.007662922 \text {, li }\end{array}$ & $\begin{array}{l}\text { lo Bus stop } \\
\text { th + VKT + } \\
=\log )\end{array}$ & $\begin{array}{l}\text { on-Sig-Int + } \\
\text { ity- Trips-E }\end{array}$ & Center, & & \\
\hline Deviance Residuals: & & & & & \\
\hline Min & 10 & Median & $3 Q$ & Max & \\
\hline-3.2669 & -0.8648 & -0.2833 & 0.3417 & 3.1621 & \\
\hline & Estimate & Std.Error & $z$ value & $\operatorname{Pr}(>|z|)$ & \\
\hline (Intercept) & $4.40 \mathrm{E}+00$ & $9.45 \mathrm{E}-02$ & 46.543 & $<2 \mathrm{E}-16$ & $* * *$ \\
\hline No Bus stops & $1.68 \mathrm{E}-02$ & $3.62 \mathrm{E}-03$ & 4.646 & $3.38 \mathrm{E}-06$ & $* * *$ \\
\hline Non-Sig-Int & $8.85 \mathrm{E}-03$ & $2.54 \mathrm{E}-03$ & 3.488 & 0.000487 & $* * *$ \\
\hline $80 . \mathrm{Km} / \mathrm{h}$ & $3.06 \mathrm{E}-01$ & $1.05 \mathrm{E}-01$ & 2.904 & 0.003683 & $* *$ \\
\hline $100 . \mathrm{Km} / \mathrm{h}$ & $1.64 \mathrm{E}+03$ & $7.10 \mathrm{E}+02$ & 2.309 & 0.020959 & $*$ \\
\hline Bike-Path & $3.13 \mathrm{E}-02$ & $1.40 \mathrm{E}-02$ & 2.23 & 0.025734 & $*$ \\
\hline VKT & $-1.61 \mathrm{E}-06$ & $6.46 \mathrm{E}-07$ & -2.487 & 0.012882 & $*$ \\
\hline Density- Trips-Enter-Center & $-1.40 \mathrm{E}-05$ & $3.21 \mathrm{E}-06$ & -4.366 & $1.27 \mathrm{E}-05$ & $* * *$ \\
\hline $\begin{array}{l}\text { Signif.codes: }\left(0^{* * * * \prime)}(0.001\right. \\
\text { (Dispersion parameter for } N\end{array}$ & $\begin{array}{l}\left.\varepsilon^{\prime}\right)\left(0.01^{\prime * \prime}\right) \\
\text { ative Binom }\end{array}$ & $\begin{array}{l}\left..^{\prime}\right)\left(0.1^{\prime \prime}\right) \\
.148) \text { famil }\end{array}$ & n to be 1 & & \\
\hline Null deviance: 574.64 on 42 & degrees of $t$ & & & & \\
\hline Residual deviance: 495.95 o & 14 degrees & eedom & & & \\
\hline AIC: 5024 & & & & & \\
\hline Number of Fisher Scoring it & tions: 1 & & & & \\
\hline Theta: 1.0077 & & & & & \\
\hline Std. Err.: 0.0652 & & & & & \\
\hline 2 x log-likelihood: -5006.04 & & & & & \\
\hline
\end{tabular}




\begin{tabular}{|c|c|c|c|c|c|}
\hline $\begin{array}{l}\text { D-C-V } \\
\text { Call: } \\
\text { glm.nb(formula = Collisions } \\
\text { MS-Walk + MS-Bike + Popul } \\
\text { Trips-Attracted + Density- T } \\
\text { link = log) }\end{array}$ & $\begin{array}{l}\text { AS-Driver + } \\
\text { on + Age.5- } \\
\text { s-Enter-Cen }\end{array}$ & $\begin{array}{l}\text { Passenger + } \\
\text { Age. } 25-64 \\
\text { TLKM, init }\end{array}$ & $\begin{array}{l}\text { ransit + } \\
+ \\
=1.3377\end{array}$ & & \\
\hline Deviance Residuals: & & & & & \\
\hline Min & 10 & Median & $3 Q$ & Max & \\
\hline-3.3787 & -0.7826 & -0.2444 & 0.3235 & 2.8536 & \\
\hline & Estimate & Std.Error & $z$ value & $\operatorname{Pr}(>|z|)$ & \\
\hline (Intercept) & $1.86 \mathrm{E}+00$ & $2.97 \mathrm{E}-01$ & 6.264 & $3.74 \mathrm{E}-10$ & $* * *$ \\
\hline MS-Driver & $1.76 \mathrm{E}-02$ & $3.45 \mathrm{E}-03$ & 5.089 & $3.60 \mathrm{E}-07$ & $* * *$ \\
\hline MS-Passenger & $2.99 \mathrm{E}-02$ & $7.36 \mathrm{E}-03$ & 4.071 & 4.69E-05 & $* * *$ \\
\hline MS-Transit & $3.29 \mathrm{E}-02$ & $6.48 \mathrm{E}-03$ & 5.069 & 4.00E-07 & $* * *$ \\
\hline MS-Walk & $2.68 \mathrm{E}-02$ & $6.10 \mathrm{E}-03$ & 4.395 & $1.11 \mathrm{E}-05$ & $* * *$ \\
\hline MS-Bike & $5.55 \mathrm{E}-02$ & $2.11 \mathrm{E}-02$ & 2.626 & 0.00864 & $* *$ \\
\hline Population & $1.33 \mathrm{E}-04$ & $2.61 \mathrm{E}-05$ & 5.094 & $3.51 \mathrm{E}-07$ & $* * *$ \\
\hline Age.5-24 & $-1.78 \mathrm{E}-02$ & $4.29 \mathrm{E}-03$ & -4.145 & $3.40 \mathrm{E}-05$ & $* * *$ \\
\hline Age.25-64 & $1.08 \mathrm{E}-02$ & $2.44 \mathrm{E}-03$ & 4.44 & 9.01E-06 & $* * *$ \\
\hline VKT & $5.18 \mathrm{E}-06$ & $1.03 \mathrm{E}-06$ & 5.051 & 4.39E-07 & $* * *$ \\
\hline Trips-Attracted & $2.45 \mathrm{E}-04$ & $3.86 \mathrm{E}-05$ & 6.352 & $2.12 \mathrm{E}-10$ & $* * *$ \\
\hline Density- Trips-Enter-Center & $-1.42 \mathrm{E}-05$ & $3.24 \mathrm{E}-06$ & -4.398 & $1.09 \mathrm{E}-05$ & $* * *$ \\
\hline TLKM & $6.40 \mathrm{E}-03$ & $2.01 \mathrm{E}-03$ & 3.182 & 0.00146 & $* *$ \\
\hline $\begin{array}{l}\text { Signif.codes: }\left(0^{\prime * * * \prime}\right)(0.001 \\
\text { (Dispersion parameter for } \mathrm{N}\end{array}$ & $\begin{array}{l}\left.\mathrm{k}^{\prime}\right)\left(0.01^{(* \prime}\right) \\
\text { ative Binom }\end{array}$ & $\begin{array}{l}5 . ')\left(0.1^{\prime \prime}\right) \\
2.148) \text { famil }\end{array}$ & to be 1 & & \\
\hline Null deviance: 747.82 on 42 & degrees of $\mathrm{fr}$ & om & & & \\
\hline Residual deviance: 491.63 o & 09 degrees & eedom & & & \\
\hline AIC: 4902.4 & & & & & \\
\hline Number of Fisher Scoring ite & tions: 1 & & & & \\
\hline $\begin{array}{l}\text { Theta: } 1.3378 \\
\text { Std. Err.: } 0.0905\end{array}$ & & & & & \\
\hline 2 x log-likelihood: -4874.44 & & & & & \\
\hline
\end{tabular}


D-C-I

Call:

glm.nb(formula $=$ Collisions $\sim$ VKT + Trips-Out-Center + Trips-Enter-Center +

Density- Trips-Out-Center + Density- Trips-Enter-Center, init.theta $=1.181866748$,

link $=\log$ )

Deviance Residuals:

\begin{tabular}{|c|c|c|c|c|c|}
\hline Min & $1 \mathrm{Q}$ & Median & $3 \mathrm{Q}$ & Max & \\
\hline-3.7257 & -0.8354 & -0.2233 & 0.365 & 3.3259 & \\
\hline \multicolumn{7}{|l|}{} \\
\hline \multicolumn{7}{|c|}{} & Estimate & Std.Error & z value & $\operatorname{Pr}(>|\mathrm{z}|)$ & \\
\hline (Intercept) & $3.73 \mathrm{E}+00$ & $9.36 \mathrm{E}-02$ & 39.851 & $<2 \mathrm{E}-16$ & $* * *$ \\
\hline VKT & $8.23 \mathrm{E}-06$ & $9.85 \mathrm{E}-07$ & 8.354 & $<2 \mathrm{E}-16$ & $* * *$ \\
\hline Trips-Out-Center & $3.21 \mathrm{E}-04$ & $3.73 \mathrm{E}-05$ & 8.593 & $<2 \mathrm{E}-16$ & $* * *$ \\
\hline Trips-Enter-Center & $1.58 \mathrm{E}-04$ & $2.85 \mathrm{E}-05$ & 5.537 & $3.07 \mathrm{E}-08$ & $* * *$ \\
\hline Density- Trips-Out-Center & $1.35 \mathrm{E}-04$ & $2.33 \mathrm{E}-05$ & 5.788 & $7.12 \mathrm{E}-09$ & $* * *$ \\
\hline Density- Trips-Enter-Center & $-1.64 \mathrm{E}-05$ & $3.74 \mathrm{E}-06$ & -4.376 & $1.21 \mathrm{E}-05$ & $* * *$ \\
\hline Signif.codes: (0**)
\end{tabular}

Signif.codes: $\left(0^{\prime * * * \prime}\right)\left(0.001^{(* * \prime}\right)\left(0.01^{\prime * \prime}\right)\left(0.05^{\prime \prime}\right)\left(0.1^{\prime \prime}\right)$ (1)

(Dispersion parameter for Negative Binomial (2.148) family taken to be 1 )

Null deviance: 666.6 on 421 degrees of freedom

Residual deviance: 493.54 on 416 degrees of freedom

AIC: 4945.4

Number of Fisher Scoring iterations: 1

Theta: 1.1819

Std. Err.: 0.0785

Warning while fitting theta: alternation limit reached

2 x log-likelihood: -4931.416 


\begin{tabular}{|c|c|c|c|c|c|}
\hline $\begin{array}{l}\text { D-D-I } \\
\text { Call: } \\
\text { glm.nb(formula = Collisions } \\
\text { init.theta }=0.9150710852 \text {, I }\end{array}$ & $\begin{array}{l}\text { ensity- Trip } \\
=\log )\end{array}$ & t-Center + & ty- Trips-E & enter, & \\
\hline Deviance Residuals: & & & & & \\
\hline Min & 10 & Median & $3 Q$ & Max & \\
\hline-3.1292 & -0.9248 & -0.25 & 0.3776 & 2.4553 & \\
\hline & Estimate & Std.Error & $z$ value & $\operatorname{Pr}(>|z|)$ & \\
\hline (Intercept) & $4.89 E+00$ & $6.71 \mathrm{E}-02$ & 72.921 & $<2 \mathrm{E}-16$ & $* * *$ \\
\hline Density- Trips-Out-Center & 9.85E-05 & $2.52 \mathrm{E}-05$ & 3.911 & $9.18 \mathrm{E}-05$ & $* * *$ \\
\hline Density- Trips-Enter-Center & $-1.76 \mathrm{E}-05$ & $3.74 \mathrm{E}-06$ & -4.712 & $2.45 \mathrm{E}-06$ & $* * *$ \\
\hline $\begin{array}{l}\text { Signif.codes: }\left(0^{\prime * * * \prime}\right)(0.001 \\
\text { (Dispersion parameter for } \mathrm{N}\end{array}$ & $\begin{array}{l}\text { ") }\left(0.01^{* \prime}\right) \\
\text { ative Binom }\end{array}$ & $\begin{array}{l}\left.{ }^{\prime}\right)\left(0.1^{\prime \prime}\right) \\
.148) \text { famil }\end{array}$ & $n$ to be 1 & & \\
\hline Null deviance: 525.19 on 42 & degrees of $f$ & & & & \\
\hline Residual deviance: 499.15 or & 19 degrees & eedom & & & \\
\hline AIC: 5061.7 & & & & & \\
\hline Number of Fisher Scoring ite & tions: 1 & & & & \\
\hline Theta: 0.9151 & & & & & \\
\hline Std. Err.: 0.0584 & & & & & \\
\hline Warning while fitting theta: & ernation lin & ached & & & \\
\hline $2 \times$ log-likelihood: -5053.695 & & & & & \\
\hline
\end{tabular}


E-C-I

Call:

glm.nb(formula $=$ Collisions $\sim$ VKT + Trips-Out-Center + Trips-Enter-Center + Density- Trips-Out-Center + Density- Trips-Enter-Center + TLKM, init.theta $=0.9575477213$, link $=\log$ )

Deviance Residuals:

\begin{tabular}{|c|c|c|c|c|c|}
\hline Min & $1 \mathrm{Q}$ & Median & $3 \mathrm{Q}$ & Max & \\
\hline-3.3091 & -0.7844 & -0.1954 & 0.2968 & 2.6835 & \\
\hline \multicolumn{7}{|l|}{} \\
\hline & Estimate & Std.Error & z value & $\operatorname{Pr}(>|\mathrm{z}|)$ & \\
\hline (Intercept) & $3.66 \mathrm{E}+00$ & $1.04 \mathrm{E}-01$ & 35.258 & $<2 \mathrm{E}-16$ & $* * *$ \\
\hline VKT & $3.59 \mathrm{E}-06$ & $8.78 \mathrm{E}-07$ & 4.094 & $4.25 \mathrm{E}-05$ & $* * *$ \\
\hline Trips-Out-Center & $2.40 \mathrm{E}-04$ & $4.79 \mathrm{E}-05$ & 5.013 & $5.36 \mathrm{E}-07$ & $* * *$ \\
\hline Trips-Enter-Center & $2.01 \mathrm{E}-04$ & $3.24 \mathrm{E}-05$ & 6.212 & $5.22 \mathrm{E}-10$ & $* * *$ \\
\hline Density- Trips-Out-Center & $2.43 \mathrm{E}-04$ & $3.32 \mathrm{E}-05$ & 7.316 & $2.56 \mathrm{E}-13$ & $* * *$ \\
\hline Density- Trips-Enter-Center & $-3.93 \mathrm{E}-05$ & $6.38 \mathrm{E}-06$ & -6.155 & $7.49 \mathrm{E}-10$ & $* * *$ \\
\hline TLKM & $7.17 \mathrm{E}-03$ & $2.29 \mathrm{E}-03$ & 3.128 & 0.00176 & $* *$ \\
\hline
\end{tabular}

Signif.codes: $\left(0^{\prime * * * \prime}\right)\left(0.001^{\prime * * \prime}\right)\left(0.01^{\prime * \prime}\right)\left(0.05^{\prime \prime}.\right)\left(0.1^{\prime \prime}\right)$ (1)

(Dispersion parameter for Negative Binomial (2.148) family taken to be 1 )

Null deviance: 675.09 on 421 degrees of freedom

Residual deviance: 508.37 on 415 degrees of freedom

AIC: 4925.7

Number of Fisher Scoring iterations: 1

Theta: 0.9575

Std. Err.: 0.0652

2 x log-likelihood: -4909.735 


\begin{tabular}{|c|c|c|c|c|c|}
\hline \multicolumn{6}{|c|}{$\begin{array}{l}\text { Call: } \\
\text { glm.nb(formula }=\text { Collisions } \sim \text { VKT }+ \text { Density- Trips-Enter-Center }+ \text { TLKM, init.theta }=1.70654907, \text { link }= \\
\text { log) }\end{array}$} \\
\hline \multicolumn{6}{|l|}{ Deviance Residuals: } \\
\hline Min & 10 & Median & $3 Q$ & Max & \\
\hline-4.0433 & -0.8829 & -0.1659 & 0.3843 & 2.597 & \\
\hline & Estimate & Std.Error & $z$ value & $\operatorname{Pr}(>|z|)$ & \\
\hline (Intercept) & $5.21 \mathrm{E}+00$ & $6.02 \mathrm{E}-02$ & 86.584 & $<2 \mathrm{E}-16$ & $* * *$ \\
\hline VKT & $9.37 \mathrm{E}-06$ & $2.07 \mathrm{E}-06$ & 4.531 & 5.87E-06 & $* * *$ \\
\hline Density- Trips-Enter-Center & $-1.51 \mathrm{E}-05$ & $2.53 \mathrm{E}-06$ & -5.955 & $2.61 \mathrm{E}-09$ & $* * *$ \\
\hline TLKM & $1.72 \mathrm{E}-02$ & $6.75 \mathrm{E}-03$ & 2.554 & 0.0106 & $*$ \\
\hline \multicolumn{6}{|c|}{$\begin{array}{l}\text { Signif.codes: }\left(0^{(* * * \prime}\right)\left(0.001^{\prime * *^{\prime}}\right)\left(0.01^{\prime * \prime}\right)\left(0.05^{\prime \prime}\right)\left(0.1^{\prime \prime}\right)(1) \\
\text { (Dispersion parameter for Negative Binomial }(2.148) \text { family taken to be } 1)\end{array}$} \\
\hline \multicolumn{6}{|c|}{ Null deviance: 538.03 on 421 degrees of freedom } \\
\hline \multicolumn{6}{|c|}{ Residual deviance: 473.09 on 418 degrees of freedom } \\
\hline \multicolumn{6}{|c|}{ AIC: 5470.3} \\
\hline \multicolumn{6}{|c|}{ Number of Fisher Scoring iterations: 1} \\
\hline \multicolumn{6}{|l|}{ Theta: 1.707} \\
\hline \multicolumn{6}{|l|}{ Std. Err.: 0.112} \\
\hline 2 x log-likelihood: -5460.25 & & & & & \\
\hline
\end{tabular}




\begin{tabular}{|c|c|c|c|c|c|}
\hline $\begin{array}{l}\text { K-D-II } \\
\text { Call: } \\
\text { glm.nb(formula = Collisions } \\
\text { TLKM, init.theta }=1.669845\end{array}$ & $\begin{array}{l}\text { oc + Bike-Pa } \\
\text { 8, link = log) }\end{array}$ & VKT + Trip & cted $+\mathrm{D}$ & Trips-Ent & er + \\
\hline Deviance Residuals: & & & & & \\
\hline Min & 10 & Median & $3 Q$ & Max & \\
\hline-3.7603 & -0.8317 & -0.1816 & 0.3567 & 3.5245 & \\
\hline & Estimate & Std.Error & z value & $\operatorname{Pr}(>|z|)$ & \\
\hline (Intercept) & $4.76 \mathrm{E}+00$ & $6.34 \mathrm{E}-02$ & 75.025 & $<2 \mathrm{E}-16$ & $* * *$ \\
\hline LoC & $9.03 \mathrm{E}-02$ & $2.26 \mathrm{E}-02$ & 3.996 & $6.43 \mathrm{E}-05$ & $* * *$ \\
\hline Bike-Path & $1.97 \mathrm{E}-02$ & $1.07 \mathrm{E}-02$ & 1.833 & 0.06686 & . \\
\hline VKT & $1.25 \mathrm{E}-05$ & $2.66 \mathrm{E}-06$ & 4.705 & $2.54 \mathrm{E}-06$ & $* * *$ \\
\hline Trips-Attracted & $9.02 \mathrm{E}-06$ & $3.89 \mathrm{E}-06$ & 2.32 & 0.02033 & $*$ \\
\hline Density- Trips-Enter-Center & $-1.52 \mathrm{E}-05$ & 3.19E-06 & -4.758 & $1.95 \mathrm{E}-06$ & $* * *$ \\
\hline TLKM & $-5.36 \mathrm{E}-02$ & $1.89 \mathrm{E}-02$ & -2.839 & 0.00453 & $* *$ \\
\hline $\begin{array}{l}\text { Signif.codes: }\left(0^{* * * \prime)}(0.001\right. \\
\text { (Dispersion parameter for } \mathrm{N}\end{array}$ & $\begin{array}{l}\left.\mathrm{k}^{\prime}\right)\left(0.01^{(* \prime}\right) \\
\text { ative Binom }\end{array}$ & $\begin{array}{l}\left.\because^{\prime}\right)\left(0.1^{\prime \prime}\right) \\
.148) \text { fami }\end{array}$ & n to be 1 & & \\
\hline $\begin{array}{l}\text { Null deviance: } 535.21 \text { on } 42 \\
\text { Residual deviance: } 473.28 \text { o } \\
\text { AIC: } 5009.9\end{array}$ & $\begin{array}{l}\text { degrees of } \mathrm{fi} \\
15 \text { degrees }\end{array}$ & eedom & & & \\
\hline Number of Fisher Scoring ite & tions: 1 & & & & \\
\hline $\begin{array}{l}\text { Theta: } 1.67 \\
\text { Std. Err.: } 0.11\end{array}$ & & & & & \\
\hline 2 x log-likelihood: -4993.89 & & & & & \\
\hline
\end{tabular}




\begin{tabular}{|c|c|c|c|c|c|}
\hline $\begin{array}{l}\text { K-D-III } \\
\text { Call: } \\
\text { glm.nb(formula = Collisions } \\
\text { Density- Trips-Enter-Center }\end{array}$ & $\begin{array}{l}0 . \mathrm{Km} / \mathrm{h}+5 \mathrm{C} \\
\mathrm{LKM} \text {, init.th }\end{array}$ & $\begin{array}{l}/ \mathrm{h}+60 . \mathrm{Km} \\
=1.705938\end{array}$ & $\begin{array}{l}0 . \mathrm{Km} / \mathrm{h} \\
\mathrm{nk}=\log )\end{array}$ & $\mathrm{m} / \mathrm{h}+\mathrm{Bike}$ & VKT + \\
\hline Deviance Residuals: & & & & & \\
\hline Min & 10 & Median & $3 Q$ & Max & \\
\hline-3.8568 & -0.8608 & -0.1495 & 0.3495 & 3.4614 & \\
\hline & Estimate & Std.Error & $z$ value & $\operatorname{Pr}(>|z|)$ & \\
\hline (Intercept) & $4.85 \mathrm{E}+00$ & $7.88 \mathrm{E}-02$ & 61.508 & $<2 \mathrm{E}-16$ & $* * *$ \\
\hline $40 . \mathrm{Km} / \mathrm{h}$ & $2.99 \mathrm{E}-01$ & $6.99 \mathrm{E}-02$ & 4.279 & $1.88 \mathrm{E}-05$ & $* * *$ \\
\hline $50 . \mathrm{Km} / \mathrm{h}$ & $2.70 \mathrm{E}-01$ & $6.81 \mathrm{E}-02$ & 3.971 & $7.15 \mathrm{E}-05$ & $* * *$ \\
\hline $60 . \mathrm{Km} / \mathrm{h}$ & $2.27 \mathrm{E}-01$ & $7.05 E-02$ & 3.215 & 0.001302 & $* *$ \\
\hline $80 . \mathrm{Km} / \mathrm{h}$ & $1.57 \mathrm{E}-01$ & 8.07E-02 & 1.948 & 0.051382 & . \\
\hline $100 . \mathrm{Km} / \mathrm{h}$ & $8.59 E+02$ & $3.45 \mathrm{E}+02$ & 2.489 & 0.012809 & $*$ \\
\hline Bike-Path & $1.87 \mathrm{E}-02$ & $1.06 \mathrm{E}-02$ & 1.761 & 0.078299 & . \\
\hline VKT & $1.12 \mathrm{E}-05$ & $2.53 \mathrm{E}-06$ & 4.433 & $9.31 \mathrm{E}-06$ & $* * *$ \\
\hline Density- Trips-Enter-Center & $-1.66 \mathrm{E}-05$ & $2.67 \mathrm{E}-06$ & -6.205 & $5.46 \mathrm{E}-10$ & $* * *$ \\
\hline TLKM & $-2.71 \mathrm{E}-01$ & $6.98 \mathrm{E}-02$ & -3.877 & 0.000106 & $* * *$ \\
\hline $\begin{array}{l}\text { Signif.codes: }\left(0^{` * * *}\right)(0.001 \\
\text { (Dispersion parameter for } \mathrm{N}\end{array}$ & $\begin{array}{l}\left.{ }^{\prime \prime}\right)\left(0.01^{\prime * \prime}\right)( \\
\text { ative Binom }\end{array}$ & $\begin{array}{l}\text { ') }\left(0.1^{\prime \prime}\right) \\
\text { 148) famil }\end{array}$ & $\mathrm{n}$ to be 1 & & \\
\hline Null deviance: 546.17 on 42 & degrees of $\mathrm{fr}$ & & & & \\
\hline Residual deviance: 472.72 o & 12 degrees & eedom & & & \\
\hline AIC: 5005.8 & & & & & \\
\hline Number of Fisher Scoring ite & tions: 1 & & & & \\
\hline Theta: 1.706 & & & & & \\
\hline Std. Err.: 0.113 & & & & & \\
\hline $2 \times$ log-likelihood: -4983.79 & & & & & \\
\hline
\end{tabular}




\begin{tabular}{|c|c|c|c|c|c|}
\hline $\begin{array}{l}\text { K-D-IV } \\
\text { Call: } \\
\text { glm.nb(formula = Collisions } \\
\text { Density- Trips-Enter-Center, }\end{array}$ & $\begin{array}{l}\text { AS-Driver }+1 \\
\text { t.theta }=1.7\end{array}$ & $\begin{array}{l}\text { Passenger + } \\
\text { 43984, link }\end{array}$ & ransit + 1 & alk + MS-Bi & $\langle T+$ \\
\hline Deviance Residuals: & & & & & \\
\hline Min & 10 & Median & $3 Q$ & Max & \\
\hline-3.8504 & -0.8929 & -0.1704 & 0.3749 & 3.5881 & \\
\hline & Estimate & Std.Error & $z$ value & $\operatorname{Pr}(>|z|)$ & \\
\hline (Intercept) & $3.03 E+00$ & $2.42 \mathrm{E}-01$ & 12.515 & $<2 \mathrm{E}-16$ & $* * *$ \\
\hline MS-Driver & $1.15 \mathrm{E}-02$ & $2.89 \mathrm{E}-03$ & 3.969 & $7.22 \mathrm{E}-05$ & $* * *$ \\
\hline MS-Passenger & $4.58 \mathrm{E}-02$ & $6.04 \mathrm{E}-03$ & 7.58 & $3.46 \mathrm{E}-14$ & $* * *$ \\
\hline MS-Transit & $2.50 \mathrm{E}-02$ & $5.02 \mathrm{E}-03$ & 4.981 & $6.32 \mathrm{E}-07$ & $* * *$ \\
\hline MS-Walk & $1.91 \mathrm{E}-02$ & $4.92 \mathrm{E}-03$ & 3.878 & 0.000105 & $* * *$ \\
\hline MS-Bike & 4.66E-02 & $1.83 \mathrm{E}-02$ & 2.549 & 0.010789 & $*$ \\
\hline VKT & $7.26 \mathrm{E}-06$ & $1.70 \mathrm{E}-06$ & 4.273 & $1.93 \mathrm{E}-05$ & $* * *$ \\
\hline Density- Trips-Enter-Center & $-1.78 \mathrm{E}-05$ & $2.64 \mathrm{E}-06$ & -6.738 & $1.61 \mathrm{E}-11$ & $* * *$ \\
\hline $\begin{array}{l}\text { Signif.codes: }\left(0^{* * * *}\right)(0.001 \\
\text { (Dispersion parameter for } \mathrm{N}\end{array}$ & $\begin{array}{l}\left.k^{\prime}\right)\left(0.01^{\prime * \prime}\right) \\
\text { ative Binom }\end{array}$ & $\begin{array}{l}\because \prime)\left(0.1^{\prime \prime}\right) \\
.148) \text { famil }\end{array}$ & $\mathrm{n}$ to be 1 & & \\
\hline Null deviance: 563.91 on 42 & degrees of $\mathrm{fr}$ & & & & \\
\hline Residual deviance: 471.61 or & 14 degrees & eedom & & & \\
\hline AIC: 4985.7 & & & & & \\
\hline Number of Fisher Scoring ite & tions: 1 & & & & \\
\hline Theta: 1.764 & & & & & \\
\hline Std. Err.: 0.117 & & & & & \\
\hline 2 x log-likelihood: -4967.695 & & & & & \\
\hline
\end{tabular}




\begin{tabular}{|c|c|c|c|c|c|}
\hline $\begin{array}{l}\text { K-D-V } \\
\text { Call: } \\
\text { glm.nb(formula = Collisions } \\
\text { Age. } 25-64+\text { Age }>65+\text { VKT }\end{array}$ & $\begin{array}{l}\text { Area + MS-D } \\
\text { Density- Trip }\end{array}$ & $\begin{array}{l}\text { + MS-Pass } \\
\text { ter-Center, }\end{array}$ & $\begin{array}{l}+ \text { MS-Tra } \\
\text { leta }=2.1\end{array}$ & $\begin{array}{l}\text { MS-Walk } \\
344 \text {, link = }\end{array}$ & ike + \\
\hline Deviance Residuals: & & & & & \\
\hline Min & 10 & Median & $3 Q$ & Max & \\
\hline-3.8999 & -0.8483 & -0.1527 & 0.4308 & 2.7221 & \\
\hline & Estimate & Std.Error & z value & $\operatorname{Pr}(>|z|)$ & \\
\hline (Intercept) & $2.56 \mathrm{E}+00$ & $2.28 \mathrm{E}-01$ & 11.224 & $<2 \mathrm{E}-16$ & $* * *$ \\
\hline Area & $8.50 \mathrm{E}-03$ & $1.24 \mathrm{E}-03$ & 6.857 & $7.05 \mathrm{E}-12$ & $* * *$ \\
\hline MS-Driver & 1.19E-02 & 2.67E-03 & 4.468 & $7.90 \mathrm{E}-06$ & $* * *$ \\
\hline MS-Passenger & $3.49 \mathrm{E}-02$ & $5.66 \mathrm{E}-03$ & 6.157 & $7.41 \mathrm{E}-10$ & $* * *$ \\
\hline MS-Transit & $2.93 \mathrm{E}-02$ & $4.63 \mathrm{E}-03$ & 6.337 & $2.34 \mathrm{E}-10$ & $* * *$ \\
\hline MS-Walk & $1.25 \mathrm{E}-02$ & 4.89E-03 & 2.549 & 0.01081 & $*$ \\
\hline MS-Bike & $4.36 \mathrm{E}-02$ & $1.66 \mathrm{E}-02$ & 2.63 & 0.00853 & $* *$ \\
\hline Age.25-64 & $5.83 \mathrm{E}-03$ & $1.83 \mathrm{E}-03$ & 3.182 & 0.00146 & $* *$ \\
\hline Age $>65$ & $1.24 \mathrm{E}-02$ & $5.37 \mathrm{E}-03$ & 2.314 & 0.02069 & $*$ \\
\hline VKT & $1.18 \mathrm{E}-05$ & $1.57 \mathrm{E}-06$ & 7.531 & $5.03 E-14$ & $* * *$ \\
\hline Density-Trips-Enter-Center & $-1.73 \mathrm{E}-05$ & $2.51 \mathrm{E}-06$ & -6.91 & $4.86 \mathrm{E}-12$ & $* * *$ \\
\hline $\begin{array}{l}\text { Signif.codes: }\left(0^{* * * \prime}\right)(0.001 \\
\text { (Dispersion parameter for } \mathrm{N}\end{array}$ & $\begin{array}{l}\left.*^{\prime}\right)\left(0.01^{* \prime}\right) \\
\text { ative Binom }\end{array}$ & $\begin{array}{l}5 . \prime)\left(0.1^{\prime \prime}\right) \\
2.148) \text { famil }\end{array}$ & $n$ to be 1 & & \\
\hline $\begin{array}{l}\text { Null deviance: } 681.6 \text { on } 421 \\
\text { Residual deviance: } 469.36 \text { o } \\
\text { AIC: } 4901.3\end{array}$ & $\begin{array}{l}\text { egrees of fre } \\
11 \text { degrees }\end{array}$ & $\begin{array}{l}\mathrm{m} \\
\text { eedom }\end{array}$ & & & \\
\hline Number of Fisher Scoring ite & tions: 1 & & & & \\
\hline $\begin{array}{l}\text { Theta: } 2.156 \\
\text { Std. Err.: } 0.147\end{array}$ & & & & & \\
\hline Std. Err.: 0.147 & & & & & \\
\hline 2 x log-likelihood: -4877.33 & & & & & \\
\hline
\end{tabular}




\begin{tabular}{|c|c|c|c|c|c|}
\hline \multicolumn{6}{|c|}{$\begin{array}{l}\text { Call: } \\
\text { glm.nb(formula }=\text { Collisions } \sim \text { VKT }+ \text { Density- Trips-Enter-Center }+ \text { TLKM, init.theta }=1.616010018, \text { link }= \\
\text { log) }\end{array}$} \\
\hline \multicolumn{6}{|l|}{ Deviance Residuals: } \\
\hline Min & 10 & Median & $3 Q$ & Max & \\
\hline \multirow[t]{2}{*}{-3.7512} & -0.8139 & -0.2079 & 0.3782 & 3.3583 & \\
\hline & Estimate & Std.Error & $z$ value & $\operatorname{Pr}(>|z|)$ & \\
\hline (Intercept) & $4.76 \mathrm{E}+00$ & $6.21 \mathrm{E}-02$ & 76.622 & $<2 \mathrm{E}-16$ & $* * *$ \\
\hline VKT & $6.25 \mathrm{E}-06$ & $2.13 \mathrm{E}-06$ & 2.934 & 0.00334 & $* *$ \\
\hline Density- Trips-Enter-Center & $-1.55 \mathrm{E}-05$ & $2.69 \mathrm{E}-06$ & -5.76 & 8.39E-09 & $* * *$ \\
\hline TLKM & $1.67 \mathrm{E}-02$ & $6.96 \mathrm{E}-03$ & 2.392 & 0.01677 & $*$ \\
\hline \multicolumn{6}{|c|}{$\begin{array}{l}\text { Signif.codes: }\left(0^{\prime * * * \prime}\right)\left(0.001^{\prime * * \prime}\right)\left(0.01^{\prime * \prime}\right)\left(0.05^{\prime \prime}\right)\left(0.1^{\prime \prime}\right)(1) \\
\text { (Dispersion parameter for Negative Binomial }(2.148) \text { family taken to be } 1)\end{array}$} \\
\hline \multicolumn{6}{|c|}{ Null deviance: 518.82 on 421 degrees of freedom } \\
\hline \multicolumn{6}{|c|}{ Residual deviance: 474.15 on 418 degrees of freedom } \\
\hline \multicolumn{6}{|l|}{ AIC: 5019.4} \\
\hline \multicolumn{6}{|c|}{ Number of Fisher Scoring iterations: 1} \\
\hline \multicolumn{6}{|l|}{ Theta: 1.616} \\
\hline \multicolumn{6}{|l|}{ Std. Err.: 0.106} \\
\hline $2 \times$ log-likelihood: -5009.42 & & & & & \\
\hline
\end{tabular}


L-D-III

Call:

glm.nb(formula $=$ Collisions $\sim$ No Bus stops + Tot-Int $+40 . \mathrm{Km} / \mathrm{h}+50 . \mathrm{Km} / \mathrm{h}+60 . \mathrm{Km} / \mathrm{h}+80 . \mathrm{Km} / \mathrm{h}+$ 100. Km/h + Bike-Path + VKT + Density- Trips-Out-Center + Density- Trips-Enter-Center + TLKM, init.theta $=0.8001580795$, link $=\log$ )

Deviance Residuals:

\begin{tabular}{|c|c|c|c|c|c|}
\hline Min & 10 & Median & $3 Q$ & Max & \\
\hline-2.8338 & -0.8266 & -0.2502 & 0.2615 & 2.9876 & \\
\hline & Estimate & Std.Error & $\mathrm{z}$ value & $\operatorname{Pr}(>|z|)$ & \\
\hline (Intercept) & $4.57 E+00$ & $1.18 \mathrm{E}-01$ & 38.728 & $<2 \mathrm{E}-16$ & $* * *$ \\
\hline No Bus stops & $9.62 \mathrm{E}-03$ & 5.55E-03 & 1.734 & 0.082959 & . \\
\hline Tot-Int & 1.59E-02 & $3.82 \mathrm{E}-03$ & 4.162 & $3.15 \mathrm{E}-05$ & $* * *$ \\
\hline $40 . \mathrm{Km} / \mathrm{h}$ & $4.03 E-01$ & 1.07E-01 & 3.75 & 0.000177 & $* * *$ \\
\hline $50 . \mathrm{Km} / \mathrm{h}$ & $3.51 \mathrm{E}-01$ & $1.05 \mathrm{E}-01$ & 3.354 & 0.000795 & $* * *$ \\
\hline $60 . \mathrm{Km} / \mathrm{h}$ & $2.45 \mathrm{E}-01$ & $1.08 \mathrm{E}-01$ & 2.258 & 0.023934 & $*$ \\
\hline $80 . \mathrm{Km} / \mathrm{h}$ & $3.18 \mathrm{E}-01$ & $1.22 \mathrm{E}-01$ & 2.599 & 0.009355 & $* *$ \\
\hline $100 . \mathrm{Km} / \mathrm{h}$ & $1.09 E+03$ & $5.04 \mathrm{E}+02$ & 2.154 & 0.031242 & $*$ \\
\hline Bike-Path & $4.06 \mathrm{E}-02$ & $2.15 \mathrm{E}-02$ & 1.89 & 0.058709 & . \\
\hline VKT & $7.81 \mathrm{E}-06$ & 4.04E-06 & 1.932 & 0.053383 & . \\
\hline Density- Trips-Out-Center & 7.71E-05 & $4.60 \mathrm{E}-05$ & 1.675 & 0.093893 & . \\
\hline Density- Trips-Enter-Center & $-2.83 \mathrm{E}-05$ & 7.00E-06 & -4.039 & $5.37 \mathrm{E}-05$ & $* * *$ \\
\hline TLKM & $-4.05 E-01$ & $1.07 \mathrm{E}-01$ & -3.781 & 0.000156 & $* * *$ \\
\hline \multicolumn{6}{|c|}{$\begin{array}{l}\text { Signif.codes: }\left(0^{* * * \prime}\right)\left(0.001^{\prime * * \prime}\right)\left(0.01^{\prime * \prime}\right)\left(0.05^{\prime \prime}\right)\left(0.1^{\prime \prime}\right)(1) \\
\text { (Dispersion parameter for Negative Binomial }(2.148) \text { family taken to be } 1)\end{array}$} \\
\hline $\begin{array}{l}\text { Null deviance: } 644.14 \text { on } 42 \\
\text { Residual deviance: } 510.94 \text { o } \\
\text { AIC: } 4906.9\end{array}$ & $\begin{array}{l}\text { degrees of } f \\
09 \text { degrees }\end{array}$ & om & & & \\
\hline \multicolumn{6}{|c|}{ Number of Fisher Scoring iterations: 1} \\
\hline \multicolumn{6}{|l|}{ Theta: 0.8002} \\
\hline \multicolumn{6}{|l|}{2 x log-likelihood: -4878.874} \\
\hline
\end{tabular}


L-D-V

Call:

glm.nb(formula $=$ Collisions $\sim$ Area + MS-Driver + MS-Passenger + MS-Transit + MS-Walk + Employment +

Age.5-24 + Age. $25-64+$ Age $>65+$ VKT + TLKM, init.theta $=1.011514045$, link = log)

Deviance Residuals:

\begin{tabular}{|c|c|c|c|c|c|}
\hline Min & $1 \mathrm{Q}$ & Median & $3 \mathrm{Q}$ & Max & \\
\hline-3.2251 & -0.7977 & -0.1796 & 0.3037 & 3.5721 & \\
\hline \multicolumn{5}{|l|}{} \\
\hline \multicolumn{7}{|l|}{} & Estimate & Std.Error & z value & $\operatorname{Pr}(>|\mathrm{z}|)$ & \\
\hline (Intercept) & $2.43 \mathrm{E}-01$ & $3.79 \mathrm{E}-01$ & 0.64 & 0.52209 & \\
\hline Area & $9.24 \mathrm{E}-03$ & $1.83 \mathrm{E}-03$ & 5.04 & $4.65 \mathrm{E}-07$ & $* * *$ \\
\hline MS-Driver & $2.18 \mathrm{E}-02$ & $4.30 \mathrm{E}-03$ & 5.082 & $3.74 \mathrm{E}-07$ & $* * *$ \\
\hline MS-Passenger & $4.87 \mathrm{E}-02$ & $8.34 \mathrm{E}-03$ & 5.839 & $5.24 \mathrm{E}-09$ & $* * *$ \\
\hline MS-Transit & $4.47 \mathrm{E}-02$ & $7.55 \mathrm{E}-03$ & 5.912 & $3.38 \mathrm{E}-09$ & $* * *$ \\
\hline MS-Walk & $2.16 \mathrm{E}-02$ & $7.87 \mathrm{E}-03$ & 2.743 & 0.006096 & $* *$ \\
\hline Employment & $-1.14 \mathrm{E}-05$ & $2.64 \mathrm{E}-06$ & -4.326 & $1.52 \mathrm{E}-05$ & $* * *$ \\
\hline Age.5-24 & $1.66 \mathrm{E}-02$ & $4.59 \mathrm{E}-03$ & 3.612 & 0.000304 & $* * *$ \\
\hline Age.25-64 & $1.82 \mathrm{E}-02$ & $2.99 \mathrm{E}-03$ & 6.074 & $1.24 \mathrm{E}-09$ & $* * *$ \\
\hline Age $>65$ & $2.67 \mathrm{E}-02$ & $7.86 \mathrm{E}-03$ & 3.398 & 0.000679 & $* * *$ \\
\hline VKT & $5.55 \mathrm{E}-06$ & $2.84 \mathrm{E}-06$ & 1.958 & 0.050196 &. \\
\hline TLKM & $2.54 \mathrm{E}-02$ & $1.20 \mathrm{E}-02$ & 2.118 & 0.034198 & $*$ \\
\hline
\end{tabular}

Signif.codes: $\left(0^{\prime * * * \prime}\right)\left(0.001^{* * \prime}\right)\left(0.01^{\prime * \prime}\right)\left(0.05^{\prime \prime}{ }^{\prime}\right)\left(0.1^{\prime \prime}\right)$ (1)

(Dispersion parameter for Negative Binomial (2.148) family taken to be 1 )

Null deviance: 793.7 on 421 degrees of freedom

Residual deviance: 511.16 on 410 degrees of freedom

AIC: 4802.5

Number of Fisher Scoring iterations: 1

Theta: 1.0115

Std. Err.: 0.0708

2 x log-likelihood: $-\mathbf{4 7 7 6 . 5 2 5}$ 


\begin{tabular}{|c|c|c|c|c|c|}
\hline $\begin{array}{l}\text { L-D-I } \\
\text { Call: } \\
\text { glm.nb(formula = Collisions } \\
0.7299224942 \text {, link = log) }\end{array}$ & rips-Attrac & Density- Tr & ter-Cent & KM, init.tt & \\
\hline Deviance Residuals: & & & & & \\
\hline Min & 10 & Median & $3 Q$ & Max & \\
\hline-2.7765 & -0.8527 & -0.2535 & 0.2677 & 2.875 & \\
\hline & Estimate & Std.Error & $z$ value & $\operatorname{Pr}(>|z|)$ & \\
\hline (Intercept) & $4.49 \mathrm{E}+00$ & $9.37 \mathrm{E}-02$ & 47.914 & $<2 \mathrm{E}-16$ & $* * *$ \\
\hline Trips-Attracted & $4.62 \mathrm{E}-05$ & $8.00 \mathrm{E}-06$ & 5.768 & $8.01 \mathrm{E}-09$ & $* * *$ \\
\hline Density- Trips-Enter-Center & $-8.69 \mathrm{E}-05$ & $1.02 \mathrm{E}-05$ & -8.53 & $<2 \mathrm{E}-16$ & $* * *$ \\
\hline TLKM & $6.91 \mathrm{E}-02$ & $8.76 \mathrm{E}-03$ & 7.892 & $2.97 \mathrm{E}-15$ & $* * *$ \\
\hline $\begin{array}{l}\text { Signif.codes: }\left(0^{* * * * \prime)}(0.001\right. \\
\text { (Dispersion parameter for } N\end{array}$ & $\begin{array}{l}\left.\varepsilon^{\prime}\right)\left(0.01^{\prime * \prime}\right) \\
\text { ative Binom }\end{array}$ & $\begin{array}{l}\text { ') }\left(0.1^{\prime \prime}\right) \\
.148) \text { famil }\end{array}$ & $\mathrm{n}$ to be 1 & & \\
\hline $\begin{array}{l}\text { Null deviance: } 593.36 \text { on } 42 \\
\text { Residual deviance: } 510.89 \text { o } \\
\text { AIC: } 4930\end{array}$ & 18 degrees & $\begin{array}{l}\text { om } \\
\text { eedom }\end{array}$ & & & \\
\hline Number of Fisher Scoring it & tions: 1 & & & & \\
\hline $\begin{array}{l}\text { Theta: } 0.7299 \\
\text { Std. Err.: } 0.0482\end{array}$ & & & & & \\
\hline 2 x log-likelihood: -4920.01 & & & & & \\
\hline
\end{tabular}




\begin{tabular}{|c|c|c|c|c|c|}
\hline $\begin{array}{l}\text { M-D-II } \\
\text { Call: } \\
\text { glm.nb(formula = Collisions } \\
\text { Trips-Out-Center + Density- }\end{array}$ & $\begin{array}{l}\text { Jo Bus stops } \\
\text { ps-Enter-Ce }\end{array}$ & $\begin{array}{l}\text { t-Int + Maj } \\
\text { init.theta }\end{array}$ & $\begin{array}{l}\text { le }+V K T \\
467772\end{array}$ & $\begin{array}{l}\text {-Produced } \\
=\log )\end{array}$ & sity- \\
\hline Deviance Residuals: & & & & & \\
\hline Min & 10 & Median & $3 Q$ & Max & \\
\hline-3.2848 & -0.808 & -0.2139 & 0.3267 & 3.0812 & \\
\hline & Estimate & Std.Error & $z$ value & $\operatorname{Pr}(>|z|)$ & \\
\hline (Intercept) & $4.65 \mathrm{E}+00$ & $7.65 \mathrm{E}-02$ & 60.772 & $<2 \mathrm{E}-16$ & $* * *$ \\
\hline No Bus stops & $8.14 \mathrm{E}-03$ & 4.79E-03 & 1.701 & 0.08901 & . \\
\hline Tot-Int & $4.44 \mathrm{E}-03$ & $2.68 \mathrm{E}-03$ & 1.659 & 0.09713 & - \\
\hline Major-Colle & $-1.16 \mathrm{E}-01$ & $5.48 \mathrm{E}-02$ & -2.108 & 0.03505 & $*$ \\
\hline VKT & 4.70E-06 & $2.31 \mathrm{E}-06$ & 2.039 & 0.04145 & $*$ \\
\hline Trips-Produced & $-4.97 E-05$ & $3.37 \mathrm{E}-05$ & -1.476 & 0.13988 & \\
\hline Density- Trips-Out-Center & $1.00 \mathrm{E}-04$ & $3.69 \mathrm{E}-05$ & 2.708 & 0.00677 & $* *$ \\
\hline Density- Trips-Enter-Center & $-2.59 \mathrm{E}-05$ & $5.15 E-06$ & -5.035 & 4.77E-07 & $* * *$ \\
\hline $\begin{array}{l}\text { Signif.codes: }\left(0^{* * * \prime}\right)(0.001 \\
\text { (Dispersion parameter for } \mathrm{N}\end{array}$ & $\begin{array}{l}\text { (') }\left(0.01^{\prime * \prime}\right) \\
\text { ative Binom }\end{array}$ & $\begin{array}{l}\text {.') }\left(0.1^{\prime \prime}\right) \\
.148) \text { famil }\end{array}$ & $\mathrm{n}$ to be 1 & & \\
\hline Null deviance: 556.35 on 42 & degrees of $\mathrm{fr}$ & & & & \\
\hline Residual deviance: 498.02 or & 14 degrees & eedom & & & \\
\hline AIC: 5046.4 & & & & & \\
\hline Number of Fisher Scoring ite & tions: 1 & & & & \\
\hline Theta: 1.0647 & & & & & \\
\hline Std. Err.: 0.0701 & & & & & \\
\hline $2 \times$ log-likelihood: -5028.40 & & & & & \\
\hline
\end{tabular}


M-D-III

Call:

glm.nb(formula $=$ Collisions $\sim$ Tot-Int $+40 . \mathrm{Km} / \mathrm{h}+50 . \mathrm{Km} / \mathrm{h}+100 . \mathrm{Km} / \mathrm{h}+$ VKT + Density- Trips-Out-

Center + Density- Trips-Enter-Center + TLKM, init.theta $=1.090452994$, link $=\log$ )

Deviance Residuals:

\begin{tabular}{|c|c|c|c|c|c|}
\hline Min & $1 \mathrm{Q}$ & Median & $3 \mathrm{Q}$ & Max & \\
\hline-3.2861 & -0.7739 & -0.1989 & 0.3235 & 3.1262 & \\
\hline \multicolumn{5}{|l|}{} \\
\hline & Estimate & Std.Error & z value & $\operatorname{Pr}(>|\mathrm{z}|)$ & \\
\hline \multicolumn{7}{|c|}{} & $4.72 \mathrm{E}+00$ & $9.22 \mathrm{E}-02$ & 51.212 & $<2 \mathrm{E}-16$ & $* * *$ \\
\hline (Intercept) & $8.23 \mathrm{E}-03$ & $2.69 \mathrm{E}-03$ & 3.064 & 0.002186 & $* *$ \\
\hline Tot-Int & $1.14 \mathrm{E}-01$ & $3.76 \mathrm{E}-02$ & 3.037 & 0.002389 & $* *$ \\
\hline $40 . \mathrm{Km} / \mathrm{h}$ & $7.15 \mathrm{E}-02$ & $2.88 \mathrm{E}-02$ & 2.487 & 0.012871 & $*$ \\
\hline $50 . \mathrm{Km} / \mathrm{h}$ & $9.83 \mathrm{E}+02$ & $4.31 \mathrm{E}+02$ & 2.279 & 0.022663 & $*$ \\
\hline $100 . \mathrm{Km} / \mathrm{h}$ & $1.30 \mathrm{E}-05$ & $3.35 \mathrm{E}-06$ & 3.871 & 0.000108 & $* * *$ \\
\hline VKT & $9.41 \mathrm{E}-05$ & $3.63 \mathrm{E}-05$ & 2.59 & 0.009585 & $* *$ \\
\hline Density- Trips-Out-Center & $-2.43 \mathrm{E}-05$ & $4.87 \mathrm{E}-06$ & -4.98 & $6.35 \mathrm{E}-07$ & $* * *$ \\
\hline Density- Trips-Enter-Center & $-1.06 \mathrm{E}-01$ & $3.57 \mathrm{E}-02$ & -2.958 & 0.003101 & $* *$ \\
\hline TLKM &
\end{tabular}

Signif.codes: $\left(0^{\prime * * * \prime}\right)\left(0.001^{\prime * * \prime}\right)\left(0.01^{\prime * \prime}\right)\left(0.05^{\prime \prime}.\right)\left(0.1^{\prime \prime}\right)(1)$

(Dispersion parameter for Negative Binomial (2.148) family taken to be 1 )

Null deviance: 568.64 on 421 degrees of freedom

Residual deviance: 497.85 on 413 degrees of freedom

AIC: 5037.4

Number of Fisher Scoring iterations: 1

Theta: 1.0905

Std. Err.: 0.0721

2 x log-likelihood: -5017.437 


\begin{tabular}{|c|c|c|c|c|c|}
\hline \multicolumn{6}{|c|}{$\begin{array}{l}\text { M-D-IV } \\
\text { Call: } \\
\text { glm.nb(formula = Collisions MS-Driver + MS-Passenger + MS-Transit + MS-Walk + MS-Bike + VKT + } \\
\text { Density- Trips-Out-Center + Density- Trips-Enter-Center, init.theta }=1.128389284, \text { link }=\text { log) }\end{array}$} \\
\hline \multicolumn{6}{|l|}{ Deviance Residuals: } \\
\hline Min & 10 & Median & $3 Q$ & Max & \\
\hline \multirow{2}{*}{-3.3727} & -0.8127 & -0.2206 & 0.3671 & 3.2781 & \\
\hline & Estimate & Std.Error & $z$ value & $\operatorname{Pr}(>|z|)$ & \\
\hline (Intercept) & $1.88 \mathrm{E}+00$ & $3.13 E-01$ & 6.005 & $1.91 \mathrm{E}-09$ & $* * *$ \\
\hline MS-Driver & $2.39 \mathrm{E}-02$ & $3.72 \mathrm{E}-03$ & 6.438 & $1.21 \mathrm{E}-10$ & $* * *$ \\
\hline MS-Passenger & $5.45 \mathrm{E}-02$ & $7.72 \mathrm{E}-03$ & 7.056 & $1.71 \mathrm{E}-12$ & $* * *$ \\
\hline MS-Transit & $4.22 \mathrm{E}-02$ & $6.60 \mathrm{E}-03$ & 6.393 & $1.63 \mathrm{E}-10$ & $* * *$ \\
\hline MS-Walk & $2.76 \mathrm{E}-02$ & $6.61 \mathrm{E}-03$ & 4.177 & $2.95 \mathrm{E}-05$ & $* * *$ \\
\hline MS-Bike & $4.08 \mathrm{E}-02$ & $2.29 \mathrm{E}-02$ & 1.782 & 0.0747 & . \\
\hline VKT & $3.89 \mathrm{E}-06$ & $2.26 \mathrm{E}-06$ & 1.722 & 0.08501 & . \\
\hline Density- Trips-Out-Center & $9.60 \mathrm{E}-05$ & $3.22 \mathrm{E}-05$ & 2.984 & 0.00285 & $* *$ \\
\hline Density- Trips-Enter-Center & $-3.08 \mathrm{E}-05$ & 5.01E-06 & -6.155 & $7.49 \mathrm{E}-10$ & $* * *$ \\
\hline \multicolumn{6}{|c|}{$\begin{array}{l}\text { Null deviance: } 586.67 \text { on } 421 \text { degrees of freedom } \\
\text { Residual deviance: } 495.81 \text { on } 413 \text { degrees of freedom } \\
\text { AIC: } 5020\end{array}$} \\
\hline \multicolumn{6}{|c|}{ Number of Fisher Scoring iterations: 1} \\
\hline \multicolumn{6}{|l|}{$\begin{array}{l}\text { Theta: } 1.1284 \\
\text { Std. Err.: } 0.0748\end{array}$} \\
\hline \multicolumn{6}{|l|}{$2 \times$ log-likelihood: -5000.017} \\
\hline
\end{tabular}




\begin{tabular}{|c|c|c|c|c|c|}
\hline $\begin{array}{l}\text { M-D-V } \\
\text { Call: } \\
\text { glm.nb(formula = Col } \\
\text { Employment + Age. } 5\end{array}$ & $\begin{array}{l}\text { rea + MS-D } \\
.25-64+A g\end{array}$ & $\begin{array}{l}\text { + MS-Pass } \\
5+\text { VKT, ini }\end{array}$ & $\begin{array}{r} \\
+\mathrm{MS}-\mathrm{Tr} \\
\mathrm{a}=1.259 \\
\end{array}$ & $\begin{array}{l}\text { MS-Walk } \\
7 \text {, link = lo }\end{array}$ & ike + \\
\hline Deviance Residuals: & & & & & \\
\hline Min & 10 & Median & $3 Q$ & Max & \\
\hline-3.5818 & -0.7629 & -0.1404 & 0.3951 & 2.833 & \\
\hline & Estimate & Std.Error & $z$ value & $\operatorname{Pr}(>|z|)$ & \\
\hline (Intercept) & $1.29 \mathrm{E}+00$ & $3.15 \mathrm{E}-01$ & 4.093 & $4.26 \mathrm{E}-05$ & $* * *$ \\
\hline Area & $8.62 \mathrm{E}-03$ & $1.63 \mathrm{E}-03$ & 5.303 & $1.14 \mathrm{E}-07$ & $* * *$ \\
\hline MS-Driver & $2.50 \mathrm{E}-02$ & $3.62 \mathrm{E}-03$ & 6.903 & $5.10 \mathrm{E}-12$ & $* * *$ \\
\hline MS-Passenger & 3.67E-02 & $7.33 \mathrm{E}-03$ & 5.009 & $5.46 \mathrm{E}-07$ & $* * *$ \\
\hline MS-Transit & $4.27 \mathrm{E}-02$ & $6.67 \mathrm{E}-03$ & 6.397 & $1.58 \mathrm{E}-10$ & $* * *$ \\
\hline MS-Walk & $2.60 \mathrm{E}-02$ & $6.46 \mathrm{E}-03$ & 4.033 & $5.50 \mathrm{E}-05$ & $* * *$ \\
\hline MS-Bike & $4.38 \mathrm{E}-02$ & $2.18 \mathrm{E}-02$ & 2.009 & 0.04457 & $*$ \\
\hline Employment & $-6.59 \mathrm{E}-06$ & $2.33 \mathrm{E}-06$ & -2.829 & 0.00467 & $* *$ \\
\hline Age.5-24 & $8.05 \mathrm{E}-03$ & $4.13 \mathrm{E}-03$ & 1.952 & 0.05098 & . \\
\hline Age.25-64 & $7.10 \mathrm{E}-03$ & $2.65 \mathrm{E}-03$ & 2.681 & 0.00735 & $* *$ \\
\hline Age $>65$ & $1.48 \mathrm{E}-02$ & $7.00 \mathrm{E}-03$ & 2.112 & 0.03465 & $*$ \\
\hline VKT & $8.40 \mathrm{E}-06$ & $1.96 \mathrm{E}-06$ & 4.283 & $1.84 \mathrm{E}-05$ & $* * *$ \\
\hline $\begin{array}{l}\text { Signif.codes: }\left(0^{\prime * * * \prime}\right) \\
\text { (Dispersion paramet }\end{array}$ & $\begin{array}{l}\left.k^{\prime}\right)\left(0.01^{\prime * \prime}\right) \\
\text { ative Binom }\end{array}$ & $\begin{array}{l}\left.y^{\prime}\right)\left(0.1^{\prime \prime}\right) \\
.148) \text { famil }\end{array}$ & to be 1 & & \\
\hline Null deviance: 648.6 & degrees of $t$ & & & & \\
\hline Residual deviance: 49 & 10 degrees & eedom & & & \\
\hline AIC: 4978.1 & & & & & \\
\hline Number of Fisher Sco & tions: 1 & & & & \\
\hline Theta: 1.2597 & & & & & \\
\hline Std. Err.: 0.0851 & & & & & \\
\hline $2 \times$ log-likelihood: -4 & & & & & \\
\hline
\end{tabular}




\begin{tabular}{|c|c|c|c|c|c|}
\hline \multicolumn{6}{|c|}{$\begin{array}{l}\text { Call: } \\
\text { glm.nb(formula }=\text { Collisions } \sim \text { VKT + Trips-Attracted + Density- Trips-Enter-Center TLKM, init.theta }= \\
\text { 1.060347495, link }=\text { log) }\end{array}$} \\
\hline \multicolumn{6}{|l|}{ Deviance Residuals: } \\
\hline Min & 10 & Median & $3 Q$ & Max & \\
\hline \multirow[t]{2}{*}{-3.412} & -0.8437 & -0.2532 & 0.3186 & 2.949 & \\
\hline & Estimate & Std.Error & $z$ value & $\operatorname{Pr}(>|z|)$ & \\
\hline (Intercept) & $4.68 \mathrm{E}+00$ & 7.81E-02 & 59.962 & $<2 \mathrm{E}-16$ & $* * *$ \\
\hline VKT & $6.40 \mathrm{E}-06$ & $2.70 \mathrm{E}-06$ & 2.372 & 0.017704 & $*$ \\
\hline Trips-Attracted & $3.24 \mathrm{E}-05$ & $6.42 \mathrm{E}-06$ & 5.044 & $4.55 E-07$ & $* * *$ \\
\hline Density- Trips-Enter-Center & $-6.03 E-05$ & $8.50 \mathrm{E}-06$ & -7.1 & $1.25 \mathrm{E}-12$ & $* * *$ \\
\hline TLKM & $3.37 \mathrm{E}-02$ & $8.84 \mathrm{E}-03$ & 3.815 & 0.000136 & $* * *$ \\
\hline \multicolumn{6}{|c|}{$\begin{array}{l}\text { Signif.codes: }\left(0^{\prime * * * \prime}\right)\left(0.001^{\prime * * \prime}\right)\left(0.01^{\prime * \prime}\right)\left(0.05^{\prime \prime}\right)\left(0.1^{\prime \prime}\right)(1) \\
\text { (Dispersion parameter for Negative Binomial }(2.148) \text { family taken to be } 1)\end{array}$} \\
\hline \multicolumn{6}{|c|}{$\begin{array}{l}\text { Null deviance: } 554.28 \text { on } 421 \text { degrees of freedom } \\
\text { Residual deviance: } 497.2 \text { on } 417 \text { degrees of freedom } \\
\text { AIC: } 5041.4\end{array}$} \\
\hline \multicolumn{6}{|c|}{ Number of Fisher Scoring iterations: 1} \\
\hline \multicolumn{6}{|l|}{$\begin{array}{l}\text { Theta: } 1.0603 \\
\text { Std. Err.: } 0.0697\end{array}$} \\
\hline \multicolumn{6}{|l|}{2 x log-likelihood: -5029.424} \\
\hline
\end{tabular}




\section{D.2 PDO Collisions Models}

A-D-II

Call:

glm.nb(formula $=$ Collisions $~ S i g-I n t ~+$ Loc + Density- Trips-Enter-Center, init.theta $=1.656885149$, link $=$ $\log )$

Deviance Residuals:

\begin{tabular}{|c|c|c|c|c|c|}
\hline Min & $1 \mathrm{Q}$ & Median & $3 \mathrm{Q}$ & Max & \\
\hline-3.6837 & -0.8011 & -0.2018 & 0.3418 & 3.3257 & \\
\hline \multicolumn{5}{|l|}{} \\
\hline & Estimate & Std.Error & z value & $\operatorname{Pr}(>|\mathrm{z}|)$ & \\
\hline (Intercept) & $4.56 \mathrm{E}+00$ & $5.70 \mathrm{E}-02$ & 80.028 & $<2 \mathrm{E}-16$ & $* * *$ \\
\hline Sig-Int & $1.06 \mathrm{E}-02$ & $3.93 \mathrm{E}-03$ & 2.705 & 0.00683 & $* *$ \\
\hline Loc & $4.61 \mathrm{E}-02$ & $9.80 \mathrm{E}-03$ & 4.702 & $2.57 \mathrm{E}-06$ & $* * *$ \\
\hline Density- Trips-Enter-Center & $-1.18 \mathrm{E}-05$ & $2.93 \mathrm{E}-06$ & -4.003 & $6.24 \mathrm{E}-05$ & $* * *$ \\
\hline
\end{tabular}

Signif.codes: $\left(0^{\prime * * * \prime}\right)\left(0.001^{\left(* *^{\prime}\right.}\right)\left(0.01^{\prime * \prime}\right)\left(0.05^{\prime \prime}.\right)\left(0.1^{\prime \prime}\right)(1)$

(Dispersion parameter for Negative Binomial (2.148) family taken to be 1 )

Null deviance: 522.78 on 421 degrees of freedom

Residual deviance: 473.51 on 418 degrees of freedom

AIC: 4818.8

Number of Fisher Scoring iterations: 1

Theta: 1.657

Std. Err.: 0.11

2 x log-likelihood: -4808.788 


\begin{tabular}{|c|c|c|c|c|c|}
\hline \multicolumn{6}{|c|}{$\begin{array}{l}\text { Call: } \\
\text { glm.nb(formula }=\text { Collisions } \sim \text { Sig-Int }+ \text { Non-Sig-Int }+40 . \mathrm{Km} / \mathrm{h}+50 . \mathrm{Km} / \mathrm{h}+70 . \mathrm{Km} / \mathrm{h}+100 . \mathrm{Km} / \mathrm{h}+\text { Bike- } \\
\text { Path + VKT + Density- Trips-Enter-Center + TLKM, init.theta }=1.708717348, \text { link }=\log )\end{array}$} \\
\hline \multicolumn{6}{|l|}{ Deviance Residuals: } \\
\hline Min & 10 & Median & $3 Q$ & Max & \\
\hline \multirow[t]{2}{*}{-3.8179} & -0.8206 & -0.203 & 0.3904 & 3.4981 & \\
\hline & Estimate & Std.Error & $z$ value & $\operatorname{Pr}(>|z|)$ & \\
\hline (Intercept) & $4.45 \mathrm{E}+00$ & $7.48 \mathrm{E}-02$ & 59.451 & $<2 \mathrm{E}-16$ & $* * *$ \\
\hline Sig-Int & $1.68 \mathrm{E}-02$ & $3.96 \mathrm{E}-03$ & 4.237 & $2.26 \mathrm{E}-05$ & $* * *$ \\
\hline Non-Sig-Int & $6.79 \mathrm{E}-03$ & $1.80 \mathrm{E}-03$ & 3.767 & 0.000165 & $* * *$ \\
\hline $40 . \mathrm{Km} / \mathrm{h}$ & $-8.51 \mathrm{E}-02$ & $3.85 \mathrm{E}-02$ & -2.212 & 0.026991 & $*$ \\
\hline $50 . \mathrm{Km} / \mathrm{h}$ & $-1.02 \mathrm{E}-01$ & $3.46 \mathrm{E}-02$ & -2.941 & 0.003267 & $* *$ \\
\hline $70 . \mathrm{Km} / \mathrm{h}$ & $-2.78 \mathrm{E}-01$ & $1.30 \mathrm{E}-01$ & -2.146 & 0.031896 & $*$ \\
\hline $100 . \mathrm{Km} / \mathrm{h}$ & $1.30 \mathrm{E}+03$ & $5.46 \mathrm{E}+02$ & 2.387 & 0.01697 & $*$ \\
\hline Bike-Path & $2.26 \mathrm{E}-02$ & $1.09 \mathrm{E}-02$ & 2.077 & 0.037758 & * \\
\hline VKT & $-1.46 \mathrm{E}-06$ & $5.12 \mathrm{E}-07$ & -2.849 & 0.004391 & $* *$ \\
\hline Density- Trips-Enter-Center & $-1.42 \mathrm{E}-05$ & $2.92 \mathrm{E}-06$ & -4.849 & $1.24 \mathrm{E}-06$ & $* * *$ \\
\hline TLKM & $1.00 \mathrm{E}-01$ & $3.47 \mathrm{E}-02$ & 2.884 & 0.003923 & $* *$ \\
\hline \multicolumn{6}{|c|}{$\begin{array}{l}\text { Signif.codes: }\left(0^{\prime * * * \prime}\right)\left(0.001^{\prime * * \prime}\right)\left(0.01^{\prime * \prime}\right)\left(0.05^{\prime \prime}\right)\left(0.1^{\prime \prime}\right)(1) \\
\text { (Dispersion parameter for Negative Binomial }(2.148) \text { family taken to be } 1)\end{array}$} \\
\hline \multicolumn{6}{|c|}{$\begin{array}{l}\text { Null deviance: } 538.18 \text { on } 421 \text { degrees of freedom } \\
\text { Residual deviance: } 472.82 \text { on } 411 \text { degrees of freedom } \\
\text { AIC: } 4818.4\end{array}$} \\
\hline \multicolumn{6}{|c|}{ Number of Fisher Scoring iterations: 1} \\
\hline \multicolumn{6}{|l|}{ Theta: 1.709} \\
\hline $2 \times$ log-likelihood: -4794.43 & & & & & \\
\hline
\end{tabular}




\begin{tabular}{|c|c|c|c|c|c|}
\hline \multicolumn{6}{|c|}{$\begin{array}{l}\text { Call: } \\
\text { glm.nb(formula = Collisions } \sim \text { MS-Driver + MS-Passenger + MS-Transit + MS-Walk + MS-Bike + VKT + } \\
\text { Trips-Produced + Trips-Attracted + Density- Trips-Out-Center + Density- Trips-Enter-Center + TLKM, } \\
\text { init.theta }=2.21358958, \text { link }=\log \text { ) }\end{array}$} \\
\hline \multicolumn{6}{|l|}{ Deviance Residuals: } \\
\hline Min & 10 & Median & $3 Q$ & Max & \\
\hline \multirow[t]{2}{*}{-3.8654} & -0.7972 & -0.1289 & 0.404 & 2.593 & \\
\hline & Estimate & Std.Error & $z$ value & $\operatorname{Pr}(>|z|)$ & \\
\hline (Intercept) & $2.51 \mathrm{E}+00$ & 2.27E-01 & 11.057 & $<2 \mathrm{E}-16$ & $* * *$ \\
\hline MS-Driver & $1.29 \mathrm{E}-02$ & 2.67E-03 & 4.823 & $1.41 \mathrm{E}-06$ & $* * *$ \\
\hline MS-Passenger & $3.14 \mathrm{E}-02$ & 5.70E-03 & 5.504 & $3.72 \mathrm{E}-08$ & $* * *$ \\
\hline MS-Transit & $2.09 \mathrm{E}-02$ & 4.99E-03 & 4.188 & $2.81 \mathrm{E}-05$ & $* * *$ \\
\hline MS-Walk & $2.38 \mathrm{E}-02$ & 4.75E-03 & 5.015 & $5.29 \mathrm{E}-07$ & $* * *$ \\
\hline MS-Bike & $5.15 \mathrm{E}-02$ & $1.64 \mathrm{E}-02$ & 3.136 & 0.00171 & $* *$ \\
\hline VKT & $3.27 \mathrm{E}-06$ & $7.98 \mathrm{E}-07$ & 4.095 & $4.22 \mathrm{E}-05$ & $* * *$ \\
\hline Trips-Produced & $8.97 \mathrm{E}-05$ & $3.46 \mathrm{E}-05$ & 2.591 & 0.00956 & $* *$ \\
\hline Trips-Attracted & $1.36 \mathrm{E}-04$ & $2.84 \mathrm{E}-05$ & 4.782 & $1.73 \mathrm{E}-06$ & $* * *$ \\
\hline Density- Trips-Out-Center & $5.03 \mathrm{E}-05$ & $2.04 \mathrm{E}-05$ & 2.47 & 0.01351 & $*$ \\
\hline Density- Trips-Enter-Center & $-1.26 \mathrm{E}-05$ & 2.67E-06 & -4.724 & $2.31 \mathrm{E}-06$ & $* * *$ \\
\hline TLKM & $6.90 \mathrm{E}-03$ & $1.52 \mathrm{E}-03$ & 4.558 & $5.17 \mathrm{E}-06$ & $* * *$ \\
\hline \multicolumn{6}{|c|}{$\begin{array}{l}\text { Signif.codes: }\left(0^{\left.* * * *^{\prime}\right)}\left(0.001^{\prime * * \prime}\right)\left(0.01^{\prime * \prime}\right)\left(0.05^{\prime \prime}\right)\left(0.1^{\prime \prime}\right)(1)\right. \\
\text { (Dispersion parameter for Negative Binomial }(2.148) \text { family taken to be } 1)\end{array}$} \\
\hline \multicolumn{6}{|c|}{ Null deviance: 686.26 on 421 degrees of freedom } \\
\hline \multicolumn{6}{|c|}{ Residual deviance: 469.72 on 410 degrees of freedom } \\
\hline \multicolumn{6}{|l|}{ AIC: 4704.3} \\
\hline \multicolumn{6}{|c|}{ Number of Fisher Scoring iterations: 1} \\
\hline \multicolumn{6}{|l|}{ Theta: 2.214} \\
\hline \multicolumn{6}{|l|}{ Std. Err.: 0.152} \\
\hline \multicolumn{6}{|c|}{ Warning while fitting theta: alternation limit reached } \\
\hline \multicolumn{6}{|l|}{2 x log-likelihood: -4678.277} \\
\hline
\end{tabular}




\begin{tabular}{|c|c|c|c|c|c|}
\hline $\begin{array}{l}\text { A-C-V } \\
\text { Call: } \\
\text { glm.nb(formula = Collisions } \\
\text { Bike + Employment + Age.0- } \\
\text { TLKM, init.theta }=2.4898398\end{array}$ & $\begin{array}{l}\text { o.Schools } \\
\text { Age.25-64 } \\
\text {, link = log }\end{array}$ & $\begin{array}{l}\text { Driver + M } \\
T+\text { Trips-O }\end{array}$ & $\begin{array}{l}\text { enger + } \\
\text { ter + De }\end{array}$ & $\begin{array}{l}\text { ransit + MS } \\
\text { Trips-Enter }\end{array}$ & $\begin{array}{l}+ \text { MS- } \\
r+\end{array}$ \\
\hline Deviance Residuals: & & & & & \\
\hline Min & 10 & Median & $3 Q$ & Max & \\
\hline-3.7944 & -0.7647 & -0.1501 & 0.3692 & 3.54 & \\
\hline & Estimate & Std.Error & $z$ value & $\operatorname{Pr}(>|z|)$ & \\
\hline (Intercept) & $2.58 \mathrm{E}+00$ & $2.19 \mathrm{E}-01$ & 11.783 & $<2 \mathrm{E}-16$ & $* * *$ \\
\hline No.Schools & $5.72 \mathrm{E}-02$ & $3.23 \mathrm{E}-02$ & 1.771 & 0.07659 & . \\
\hline MS-Driver & $7.84 \mathrm{E}-03$ & $2.59 \mathrm{E}-03$ & 3.026 & 0.00248 & $* *$ \\
\hline MS-Passenger & $2.80 \mathrm{E}-02$ & 5.50E-03 & 5.099 & $3.41 \mathrm{E}-07$ & $* * *$ \\
\hline MS-Transit & $2.10 \mathrm{E}-02$ & $4.51 \mathrm{E}-03$ & 4.66 & $3.15 \mathrm{E}-06$ & $* * *$ \\
\hline MS-Walk & $1.88 \mathrm{E}-02$ & 4.57E-03 & 4.11 & $3.96 \mathrm{E}-05$ & $* * *$ \\
\hline MS-Bike & $4.86 \mathrm{E}-02$ & $1.57 \mathrm{E}-02$ & 3.097 & 0.00196 & $* *$ \\
\hline Employment & $1.51 \mathrm{E}-04$ & $2.02 \mathrm{E}-05$ & 7.49 & $6.86 \mathrm{E}-14$ & $* * *$ \\
\hline Age.0-4 & $-3.78 \mathrm{E}-02$ & $1.54 \mathrm{E}-02$ & -2.453 & 0.01416 & $*$ \\
\hline Age.25-64 & $7.55 \mathrm{E}-03$ & 1.91E-03 & 3.955 & $7.66 \mathrm{E}-05$ & $* * *$ \\
\hline VKT & $3.13 \mathrm{E}-06$ & $7.54 \mathrm{E}-07$ & 4.152 & $3.30 \mathrm{E}-05$ & $* * *$ \\
\hline Trips-Out-Center & 1.61E-04 & $3.55 \mathrm{E}-05$ & 4.524 & $6.05 \mathrm{E}-06$ & $* * *$ \\
\hline Density- Trips-Enter-Center & $-1.09 \mathrm{E}-05$ & $2.30 \mathrm{E}-06$ & -4.715 & $2.42 \mathrm{E}-06$ & $* * *$ \\
\hline TLKM & 4.09E-03 & $1.53 \mathrm{E}-03$ & 2.671 & 0.00756 & $* *$ \\
\hline (Intercept) & $2.58 \mathrm{E}+00$ & $2.19 \mathrm{E}-01$ & 11.783 & $<2 \mathrm{E}-16$ & $* * *$ \\
\hline No.Schools & $5.72 \mathrm{E}-02$ & $3.23 \mathrm{E}-02$ & 1.771 & 0.07659 & . \\
\hline MS-Driver & $7.84 \mathrm{E}-03$ & $2.59 \mathrm{E}-03$ & 3.026 & 0.00248 & $* *$ \\
\hline MS-Passenger & $2.80 \mathrm{E}-02$ & $5.50 \mathrm{E}-03$ & 5.099 & $3.41 \mathrm{E}-07$ & $* * *$ \\
\hline $\begin{array}{l}\text { Signif.codes: }\left(0^{* * * * \prime}\right)(0.001 \\
\text { (Dispersion parameter for } N\end{array}$ & $\begin{array}{l}\left.k^{\prime}\right)\left(0.01^{\prime * \prime}\right) \\
\text { ative Binom }\end{array}$ & $\begin{array}{l}\text { '.) }\left(0.1^{\prime \prime}\right) \\
.148) \text { fami }\end{array}$ & n to be 1 & & \\
\hline $\begin{array}{l}\text { Null deviance: } 765.87 \text { on } 42 \\
\text { Residual deviance: } 469.36 \text { ol } \\
\text { AIC: } 4657.6\end{array}$ & $\begin{array}{l}\text { degrees of } t \\
08 \text { degrees }\end{array}$ & $\begin{array}{l}\text { om } \\
\text { eedom }\end{array}$ & & & \\
\hline Number of Fisher Scoring ite & tions: 1 & & & & \\
\hline $\begin{array}{l}\text { Theta: } 2.49 \\
\text { Std. Err.: } 0.174\end{array}$ & & & & & \\
\hline $2 \times$ log-likelihood: -4627.553 & & & & & \\
\hline
\end{tabular}




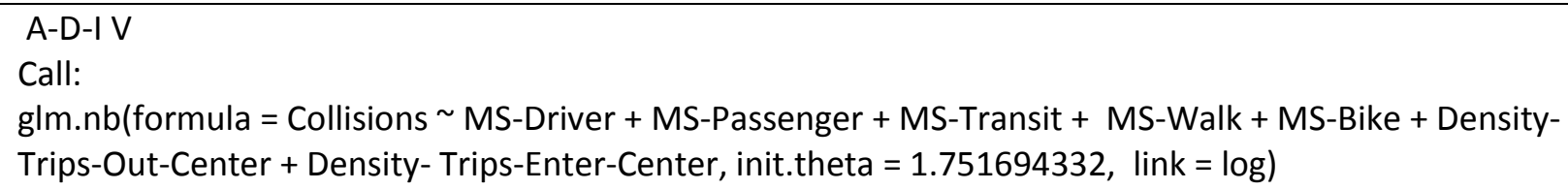

Deviance Residuals:

\begin{tabular}{|c|c|c|c|c|c|}
\hline Min & $1 \mathrm{Q}$ & Median & $3 Q$ & Max & \\
\hline-3.8078 & -0.824 & -0.1986 & 0.3804 & 3.3836 & \\
\hline \multicolumn{7}{|l|}{} \\
\hline & Estimate & Std.Error & z value & $\operatorname{Pr}(>|\mathrm{z}|)$ & \\
\hline & $2.83 \mathrm{E}+00$ & $2.45 \mathrm{E}-01$ & 11.523 & $<2 \mathrm{E}-16$ & $* * *$ \\
\hline (Intercept) & $1.30 \mathrm{E}-02$ & $2.90 \mathrm{E}-03$ & 4.478 & $7.55 \mathrm{E}-06$ & $* * *$ \\
\hline MS-Driver & $4.21 \mathrm{E}-02$ & $6.04 \mathrm{E}-03$ & 6.957 & $3.48 \mathrm{E}-12$ & $* * *$ \\
\hline MS-Passenger & $2.55 \mathrm{E}-02$ & $5.17 \mathrm{E}-03$ & 4.929 & $8.27 \mathrm{E}-07$ & $* * *$ \\
\hline MS-Transit & $2.29 \mathrm{E}-02$ & $5.22 \mathrm{E}-03$ & 4.39 & $1.13 \mathrm{E}-05$ & $* * *$ \\
\hline MS-Walk & $4.93 \mathrm{E}-02$ & $1.83 \mathrm{E}-02$ & 2.69 & 0.00714 & $* *$ \\
\hline MS-Bike & $3.54 \mathrm{E}-05$ & $2.21 \mathrm{E}-05$ & 1.598 & 0.10997 & $*$ \\
\hline Density- Trips-Out-Center & $-1.41 \mathrm{E}-05$ & $2.63 \mathrm{E}-06$ & -5.371 & $7.83 \mathrm{E}-08$ & $* * *$ \\
\hline Density- Trips-Enter-Center &
\end{tabular}

Signif.codes: $\left(0^{(* * * \prime}\right)\left(0.001^{(* * \prime}\right)\left(0.01^{\prime * \prime}\right)\left(0.05^{\prime \prime}.\right)\left(0.1^{\prime \prime}\right)$ (1)

(Dispersion parameter for Negative Binomial (2.148) family taken to be 1 )

Null deviance: 550.93 on 421 degrees of freedom

Residual deviance: 471.64 on 414 degrees of freedom

AIC: 4800.3

Number of Fisher Scoring iterations: 1

Theta: 1.752

Std. Err.: 0.117

2 x log-likelihood: -4782.27 


\begin{tabular}{|c|c|c|c|c|c|}
\hline $\begin{array}{l}\text { A-D-V } \\
\text { Call: } \\
\text { glm.nb(formula = Collisions } \\
\text { Age. } 25-64+\text { Age }>65+\text { Dens }\end{array}$ & $\begin{array}{l}\text { rea + MS-D } \\
\text { - Trips-Ente }\end{array}$ & $\begin{array}{l}\text { + MS-Passe } \\
\text { hter, init.th }\end{array}$ & $\begin{array}{l}+\mathrm{MS}-\mathrm{Tr} \\
1.992516\end{array}$ & $\begin{array}{l}\text { MS-Walk } \\
\text { ink = log) }\end{array}$ & ike + \\
\hline Deviance Residuals: & & & & & \\
\hline Min & 10 & Median & $3 Q$ & Max & \\
\hline-3.8013 & -0.8938 & -0.1329 & 0.4106 & 2.6803 & \\
\hline & Estimate & Std.Error & z value & $\operatorname{Pr}(>|z|)$ & \\
\hline (Intercept) & $2.46 \mathrm{E}+00$ & 2.39E-01 & 10.303 & $<2 \mathrm{E}-16$ & $* * *$ \\
\hline Area & 7.09E-03 & $1.28 \mathrm{E}-03$ & 5.522 & $3.35 \mathrm{E}-08$ & $* * *$ \\
\hline MS-Driver & $1.46 \mathrm{E}-02$ & $2.77 \mathrm{E}-03$ & 5.256 & $1.47 \mathrm{E}-07$ & $* * *$ \\
\hline MS-Passenger & $3.17 E-02$ & $5.88 \mathrm{E}-03$ & 5.399 & $6.71 \mathrm{E}-08$ & $* * *$ \\
\hline MS-Transit & $3.37 \mathrm{E}-02$ & 4.73E-03 & 7.126 & $1.03 \mathrm{E}-12$ & $* * *$ \\
\hline MS-Walk & $2.54 \mathrm{E}-02$ & $4.94 \mathrm{E}-03$ & 5.151 & $2.59 \mathrm{E}-07$ & $* * *$ \\
\hline MS-Bike & $4.67 E-02$ & $1.72 \mathrm{E}-02$ & 2.71 & 0.00673 & $* *$ \\
\hline Age.25-64 & $3.90 \mathrm{E}-03$ & $1.90 \mathrm{E}-03$ & 2.054 & 0.03996 & $*$ \\
\hline Age $>65$ & $1.01 \mathrm{E}-02$ & $5.58 \mathrm{E}-03$ & 1.817 & 0.06925 & . \\
\hline Density- Trips-Enter-Center & $-9.66 \mathrm{E}-06$ & $2.22 \mathrm{E}-06$ & -4.361 & $1.29 \mathrm{E}-05$ & $* * *$ \\
\hline $\begin{array}{l}\text { Signif.codes: }\left(0^{\prime * * * \prime}\right)(0.001 \\
\text { (Dispersion parameter for } \mathrm{N}\end{array}$ & $\begin{array}{l}\text { ") }\left(0.01^{\prime * \prime}\right) \\
\text { ative Binom }\end{array}$ & $\begin{array}{l}\left..^{\prime}\right)\left(0.1^{\prime \prime}\right) \\
.148) \text { famil }\end{array}$ & $n$ to be 1 & & \\
\hline $\begin{array}{l}\text { Null deviance: } 621.85 \text { on } 42 \\
\text { Residual deviance: } 469.89 \text { o } \\
\text { AIC: } 4746\end{array}$ & $\begin{array}{l}\text { legrees of } f \\
12 \text { degrees }\end{array}$ & eedom & & & \\
\hline Number of Fisher Scoring ite & ions: 1 & & & & \\
\hline $\begin{array}{l}\text { Theta: } 1.993 \\
\text { Std. Err.: } 0.135\end{array}$ & & & & & \\
\hline $2 \times$ log-likelihood: -4724.02 & & & & & \\
\hline
\end{tabular}




\begin{tabular}{|c|c|c|c|c|c|}
\hline \multicolumn{6}{|c|}{$\begin{array}{l}\text { Call: } \\
\text { glm.nb(formula }=\text { Collisions } \sim \text { VKT + Trips-Out-Center + Trips-Enter-Center + Density- Trips-Out-Center + } \\
\text { Density- Trips-Enter-Center + TLKM, init.theta }=2.152975344, \text { link }=\text { log) }\end{array}$} \\
\hline \multicolumn{6}{|l|}{ Deviance Residuals: } \\
\hline Min & 10 & Median & $3 Q$ & Max & \\
\hline \multirow[t]{2}{*}{-3.9473} & -0.8287 & -0.1798 & 0.4324 & 3.3339 & \\
\hline & Estimate & Std.Error & z value & $\operatorname{Pr}(>|z|)$ & \\
\hline (Intercept) & $3.81 \mathrm{E}+00$ & 6.99E-02 & 54.52 & $<2 \mathrm{E}-16$ & $* * *$ \\
\hline VKT & $3.76 \mathrm{E}-06$ & $8.08 \mathrm{E}-07$ & 4.657 & $3.20 \mathrm{E}-06$ & $* * *$ \\
\hline Trips-Out-Center & $1.91 \mathrm{E}-04$ & $3.00 \mathrm{E}-05$ & 6.368 & $1.92 \mathrm{E}-10$ & $* * *$ \\
\hline Trips-Enter-Center & 1.67E-04 & $2.12 \mathrm{E}-05$ & 7.858 & $3.90 \mathrm{E}-15$ & $* * *$ \\
\hline Density- Trips-Out-Center & $1.14 \mathrm{E}-04$ & $1.75 \mathrm{E}-05$ & 6.505 & $7.75 \mathrm{E}-11$ & $* * *$ \\
\hline Density- Trips-Enter-Center & $-1.36 \mathrm{E}-05$ & $2.72 \mathrm{E}-06$ & -4.995 & $5.87 \mathrm{E}-07$ & $* * *$ \\
\hline TLKM & $3.93 \mathrm{E}-03$ & $1.61 \mathrm{E}-03$ & 2.45 & 0.0143 & $*$ \\
\hline \multicolumn{6}{|c|}{$\begin{array}{l}\text { Signif.codes: }\left(0^{\prime * * * \prime}\right)\left(0.001^{\prime * * \prime}\right)\left(0.01^{\prime * \prime}\right)\left(0.05^{\prime \prime} .\right)\left(0.1^{\prime \prime}\right)(1) \\
\text { (Dispersion parameter for Negative Binomial }(2.148) \text { family taken to be } 1)\end{array}$} \\
\hline \multicolumn{6}{|c|}{ Null deviance: 668.66 on 421 degrees of freedom } \\
\hline \multicolumn{6}{|c|}{ Residual deviance: 470.88 on 415 degrees of freedom } \\
\hline \multicolumn{6}{|l|}{ AIC: 4707.4} \\
\hline \multicolumn{6}{|c|}{ Number of Fisher Scoring iterations: 1} \\
\hline \multicolumn{6}{|l|}{ Theta: 2.153} \\
\hline \multicolumn{6}{|l|}{ Std. Err.: 0.148} \\
\hline \multicolumn{6}{|c|}{ Warning while fitting theta: alternation limit reached } \\
\hline \multicolumn{6}{|l|}{2 x log-likelihood: -4691.418} \\
\hline
\end{tabular}




\begin{tabular}{|c|c|c|c|c|c|}
\hline \multicolumn{6}{|c|}{$\begin{array}{l}\text { A-D-I } \\
\text { Call: } \\
\text { glm.nb(formula = Collisions Density- Trips-Out-Center + Density- Trips-Enter-Center, init.theta = } \\
1.587973135, \text { link }=\log )\end{array}$} \\
\hline \multicolumn{6}{|l|}{ Deviance Residuals: } \\
\hline Min & 10 & Median & $3 Q$ & Max & \\
\hline \multirow[t]{2}{*}{-3.8004} & -0.8658 & -0.2162 & 0.4371 & 3 & \\
\hline & Estimate & Std.Error & $z$ value & $\operatorname{Pr}(>|z|)$ & \\
\hline (Intercept) & $4.67 \mathrm{E}+00$ & $5.11 \mathrm{E}-02$ & 91.53 & $<2 \mathrm{E}-16$ & $* * *$ \\
\hline Density- Trips-Out-Center & $8.55 \mathrm{E}-05$ & $1.89 \mathrm{E}-05$ & 4.532 & $5.85 \mathrm{E}-06$ & $* * *$ \\
\hline Density- Trips-Enter-Center & $-1.28 \mathrm{E}-05$ & $2.73 E-06$ & -4.71 & $2.48 \mathrm{E}-06$ & $* * *$ \\
\hline \multicolumn{6}{|c|}{$\begin{array}{l}\text { Signif.codes: }\left(0^{\left.* * * *^{\prime}\right)}\left(0.001^{\prime * *^{\prime}}\right)\left(0.01^{\prime * \prime}\right)\left(0.05^{\prime} .^{\prime}\right)\left(0.1^{\prime \prime}\right)(1)\right. \\
\text { (Dispersion parameter for Negative Binomial }(2.148) \text { family taken to be } 1)\end{array}$} \\
\hline \multicolumn{6}{|c|}{$\begin{array}{l}\text { Null deviance: } 502.24 \text { on } 421 \text { degrees of freedom } \\
\text { Residual deviance: } 474.52 \text { on } 419 \text { degrees of freedom } \\
\text { AIC: } 4836.7\end{array}$} \\
\hline \multicolumn{6}{|c|}{ Number of Fisher Scoring iterations: 1} \\
\hline \multicolumn{6}{|l|}{$\begin{array}{l}\text { Theta: } 1.588 \\
\text { Std. Err.: } 0.105\end{array}$} \\
\hline \multicolumn{6}{|l|}{2 x log-likelihood: -4828.728} \\
\hline
\end{tabular}




\begin{tabular}{|c|c|c|c|c|c|}
\hline \multicolumn{6}{|c|}{$\begin{array}{l}\text { Call: } \\
\text { glm.nb(formula }=\text { Collisions } \sim \text { No Bus stops }+ \text { Tot-Int }+40 . \mathrm{Km} / \mathrm{h}+50 . \mathrm{Km} / \mathrm{h}+60 . \mathrm{Km} / \mathrm{h}+70 . \mathrm{Km} / \mathrm{h}+ \\
80 . \mathrm{Km} / \mathrm{h}+90 . \mathrm{Km} / \mathrm{h}+100 . \mathrm{Km} / \mathrm{h}+\text { Trips-Attracted }+ \text { Density- Trips-Enter-Center }+ \text { TLKM, init.theta }= \\
\text { 1.241661687, link }=\log )\end{array}$} \\
\hline \multicolumn{6}{|l|}{ Deviance Residuals: } \\
\hline Min & $1 Q$ & Median & $3 Q$ & Max & \\
\hline \multirow[t]{2}{*}{-3.2462} & -0.833 & -0.2552 & 0.3851 & 3.5638 & \\
\hline & Estimate & Std.Error & $z$ value & $\operatorname{Pr}(>|z|)$ & \\
\hline (Intercept) & $3.95 \mathrm{E}+00$ & $8.84 \mathrm{E}-02$ & 44.668 & $<2 \mathrm{E}-16$ & $* * *$ \\
\hline No Bus stops & $1.41 \mathrm{E}-02$ & $4.01 \mathrm{E}-03$ & 3.516 & 0.000438 & $* * *$ \\
\hline Tot-Int & $1.15 \mathrm{E}-02$ & $2.53 \mathrm{E}-03$ & 4.549 & $5.39 \mathrm{E}-06$ & $* * *$ \\
\hline $40 . \mathrm{Km} / \mathrm{h}$ & $-3.80 \mathrm{E}+01$ & $1.74 \mathrm{E}+01$ & -2.184 & 0.028926 & $*$ \\
\hline $50 . \mathrm{Km} / \mathrm{h}$ & $-3.80 \mathrm{E}+01$ & $1.74 \mathrm{E}+01$ & -2.185 & 0.028899 & $*$ \\
\hline $60 . \mathrm{Km} / \mathrm{h}$ & $-3.78 \mathrm{E}+01$ & $1.74 \mathrm{E}+01$ & -2.175 & 0.029597 & $*$ \\
\hline $70 . \mathrm{Km} / \mathrm{h}$ & $-3.76 \mathrm{E}+01$ & $1.74 \mathrm{E}+01$ & -2.165 & 0.030387 & $*$ \\
\hline $80 . \mathrm{Km} / \mathrm{h}$ & $-3.75 \mathrm{E}+01$ & $1.74 \mathrm{E}+01$ & -2.156 & 0.031071 & $*$ \\
\hline $90 . \mathrm{km} / \mathrm{h}$ & $-3.47 \mathrm{E}+01$ & $1.77 \mathrm{E}+01$ & -1.967 & 0.049215 & $*$ \\
\hline $100 . \mathrm{Km} / \mathrm{h}$ & $1.50 \mathrm{E}+03$ & $6.41 \mathrm{E}+02$ & 2.339 & 0.019356 & $*$ \\
\hline Trips-Attracted & 3.39E-05 & $6.26 \mathrm{E}-06$ & 5.415 & $6.14 \mathrm{E}-08$ & $* * *$ \\
\hline Density- Trips-Enter-Center & $-5.94 \mathrm{E}-05$ & 7.79E-06 & -7.625 & $2.44 \mathrm{E}-14$ & $* * *$ \\
\hline TLKM & $3.80 \mathrm{E}+01$ & $1.74 \mathrm{E}+01$ & 2.185 & 0.028872 & * \\
\hline \multicolumn{6}{|c|}{$\begin{array}{l}\text { Signif.codes: }\left(0^{\prime * * * \prime}\right)\left(0.001^{\prime * * \prime}\right)\left(0.01^{\prime * \prime}\right)\left(0.05^{\prime \prime}{ }^{\prime}\right)\left(0.1^{\prime \prime}\right)(1) \\
\text { (Dispersion parameter for Negative Binomial }(2.148) \text { family taken to be } 1)\end{array}$} \\
\hline \multicolumn{6}{|c|}{ Null deviance: 626.75 on 421 degrees of freedom } \\
\hline \multicolumn{6}{|c|}{ Residual deviance: 493.7 on 409 degrees of freedom } \\
\hline \multicolumn{6}{|l|}{ AIC: 4799.8} \\
\hline \multicolumn{6}{|c|}{ Number of Fisher Scoring iterations: 1} \\
\hline \multicolumn{6}{|l|}{ Theta: 1.2417} \\
\hline \multicolumn{6}{|l|}{ Std. Err.: 0.0838} \\
\hline 2 x log-likelihood: -4771.817 & & & & & \\
\hline
\end{tabular}




\begin{tabular}{|c|c|c|c|c|c|}
\hline \multicolumn{6}{|c|}{$\begin{array}{l}\text { Call: } \\
\text { glm.nb(formula = Collisions } \sim \text { VKT + Trips-Out-Center + Trips-Enter-Center + Density- Trips-Out-Center + } \\
\text { Density- Trips-Enter-Center + TLKM, init.theta }=1.631305154, \text { link }=\log )\end{array}$} \\
\hline \multicolumn{6}{|l|}{ Deviance Residuals: } \\
\hline Min & 10 & Median & $3 Q$ & Max & \\
\hline \multirow[t]{2}{*}{-3.5337} & -0.8029 & -0.1719 & 0.4288 & 2.6469 & \\
\hline & Estimate & Std.Error & $z$ value & $\operatorname{Pr}(>|z|)$ & \\
\hline (Intercept) & $3.20 \mathrm{E}+00$ & $8.04 \mathrm{E}-02$ & 39.786 & $<2 \mathrm{E}-16$ & $* * *$ \\
\hline VKT & $2.72 \mathrm{E}-06$ & $8.18 \mathrm{E}-07$ & 3.329 & 0.000871 & $* * *$ \\
\hline Trips-Out-Center & $2.73 \mathrm{E}-04$ & $3.68 \mathrm{E}-05$ & 7.428 & $1.10 \mathrm{E}-13$ & $* * *$ \\
\hline Trips-Enter-Center & $2.85 \mathrm{E}-04$ & $2.49 \mathrm{E}-05$ & 11.463 & $<2 \mathrm{E}-16$ & $* * *$ \\
\hline Density- Trips-Out-Center & $2.48 \mathrm{E}-04$ & 2.57E-05 & 9.653 & $<2 \mathrm{E}-16$ & $* * *$ \\
\hline Density- Trips-Enter-Center & $-3.71 \mathrm{E}-05$ & $4.95 \mathrm{E}-06$ & -7.499 & $6.41 \mathrm{E}-14$ & $* * *$ \\
\hline TLKM & $7.08 \mathrm{E}-03$ & $1.80 \mathrm{E}-03$ & 3.927 & $8.60 \mathrm{E}-05$ & $* * *$ \\
\hline \multicolumn{6}{|c|}{$\begin{array}{l}\text { Signif.codes: }\left(0^{* * * \prime}\right)\left(0.001^{\prime * * \prime}\right)\left(0.01^{\prime * \prime}\right)\left(0.05^{\prime \prime}\right)\left(0.1^{\prime \prime}\right)(1) \\
\text { (Dispersion parameter for Negative Binomial }(2.148) \text { family taken to be } 1)\end{array}$} \\
\hline $\begin{array}{l}\text { Null deviance: } 801.59 \text { on } 42 \\
\text { Residual deviance: } 497.06 \text { o } \\
\text { AIC: } 4672.9\end{array}$ & $\begin{array}{l}\text { degrees of } f \\
+15 \text { degrees }\end{array}$ & $\begin{array}{l}\text { om } \\
\text { eedom }\end{array}$ & & & \\
\hline \multicolumn{6}{|c|}{ Number of Fisher Scoring iterations: 1} \\
\hline \multicolumn{6}{|l|}{ Theta: 1.631} \\
\hline 2 x log-likelihood: -4656.93 & & & & & \\
\hline
\end{tabular}




\begin{tabular}{|c|c|c|c|c|c|}
\hline \multicolumn{6}{|c|}{$\begin{array}{l}\text { Call: } \\
\text { glm.nb(formula }=\text { Collisions } \sim \text { No Bus stops + Loc + Bike-Path + VKT + Density- Trips-Enter-Center, } \\
\text { init.theta }=1.002348038, \text { link }=\log )\end{array}$} \\
\hline \multicolumn{6}{|l|}{ Deviance Residuals: } \\
\hline Min & 10 & Median & $3 Q$ & Max & \\
\hline \multirow[t]{2}{*}{-3.145} & -0.888 & -0.2741 & 0.3066 & 3.1159 & \\
\hline & Estimate & Std.Error & z value & $\operatorname{Pr}(>|z|)$ & \\
\hline (Intercept) & $4.39 \mathrm{E}+00$ & 7.71E-02 & 56.932 & $<2 \mathrm{E}-16$ & $* * *$ \\
\hline No Bus stops & $1.20 \mathrm{E}-02$ & 3.69E-03 & 3.252 & 0.00115 & $* *$ \\
\hline Loc & $4.61 \mathrm{E}-02$ & $1.48 \mathrm{E}-02$ & 3.116 & 0.00183 & $* *$ \\
\hline Bike-Path & $2.61 \mathrm{E}-02$ & $1.41 \mathrm{E}-02$ & 1.851 & 0.0642 & . \\
\hline VKT & $-1.52 \mathrm{E}-06$ & $6.51 \mathrm{E}-07$ & -2.329 & 0.01987 & $*$ \\
\hline Density- Trips-Enter-Center & $-1.51 \mathrm{E}-05$ & 3.17E-06 & -4.769 & $1.85 \mathrm{E}-06$ & $* * *$ \\
\hline \multicolumn{6}{|c|}{$\begin{array}{l}\text { Signif.codes: }\left(0^{\prime * * * \prime}\right)\left(0.001^{\prime * * \prime}\right)\left(0.01^{\prime * \prime}\right)\left(0.05^{\prime}{ }^{\prime}\right)\left(0.1^{\prime \prime}\right)(1) \\
\text { (Dispersion parameter for Negative Binomial }(2.148) \text { family taken to be } 1)\end{array}$} \\
\hline \multicolumn{6}{|c|}{ Null deviance: 561.69 on 421 degrees of freedom } \\
\hline \multicolumn{6}{|c|}{ Residual deviance: 496.36 on 416 degrees of freedom } \\
\hline \multicolumn{6}{|l|}{ AIC: 4838.8} \\
\hline \multicolumn{6}{|c|}{ Number of Fisher Scoring iterations: 1} \\
\hline \multicolumn{6}{|l|}{ Theta: 1.0023} \\
\hline \multicolumn{6}{|l|}{ Std. Err.: 0.0652} \\
\hline \multicolumn{6}{|l|}{2 x log-likelihood: -4824.784} \\
\hline
\end{tabular}


D-D-III

Call:

glm.nb(formula $=$ Collisions $~$ No Bus stops + Non-Sig-Int $+80 . \mathrm{Km} / \mathrm{h}+100 . \mathrm{Km} / \mathrm{h}+$ Bike-Path + VKT + Density- Trips-Enter-Center, init.theta $=1.033518562$, link $=\log$ )

Deviance Residuals:

\begin{tabular}{|c|c|c|c|c|c|}
\hline Min & 10 & Median & 30 & Max & \\
\hline-3.224 & -0.8406 & -0.2814 & 0.3478 & 3.2001 & \\
\hline & Estimate & Std.Error & $z$ value & $\operatorname{Pr}(>|z|)$ & \\
\hline (Intercept) & $4.16 \mathrm{E}+00$ & $9.35 \mathrm{E}-02$ & 44.493 & $<2 \mathrm{E}-16$ & $* * *$ \\
\hline No Bus stops & $1.64 \mathrm{E}-02$ & $3.59 \mathrm{E}-03$ & 4.58 & $4.64 \mathrm{E}-06$ & $* * *$ \\
\hline Non-Sig-Int & $9.45 \mathrm{E}-03$ & $2.51 E-03$ & 3.763 & 0.000168 & $* * *$ \\
\hline $80 . \mathrm{Km} / \mathrm{h}$ & $3.04 \mathrm{E}-01$ & $1.04 \mathrm{E}-01$ & 2.923 & 0.003466 & $* *$ \\
\hline $100 . \mathrm{Km} / \mathrm{h}$ & $1.61 \mathrm{E}+03$ & $7.01 \mathrm{E}+02$ & 2.294 & 0.021784 & $*$ \\
\hline Bike-Path & 3.07E-02 & $1.39 \mathrm{E}-02$ & 2.211 & 0.027021 & $*$ \\
\hline VKT & $-1.58 \mathrm{E}-06$ & $6.44 \mathrm{E}-07$ & -2.454 & 0.014109 & $*$ \\
\hline Density- Trips-Enter-Center & $-1.39 \mathrm{E}-05$ & $3.22 \mathrm{E}-06$ & -4.308 & $1.65 \mathrm{E}-05$ & $* * *$ \\
\hline
\end{tabular}

Signif.codes: $\left(0^{(* * * \prime}\right)\left(0.001^{\prime * * \prime}\right)\left(0.01^{\prime * \prime}\right)\left(0.05^{\prime \prime}\right)\left(0.1^{\prime \prime}\right)$ (1)

(Dispersion parameter for Negative Binomial (2.148) family taken to be 1 )

Null deviance: 577.84 on 421 degrees of freedom

Residual deviance: 495.57 on 414 degrees of freedom

AIC: 4828.1

Number of Fisher Scoring iterations: 1

Theta: 1.0335

Std. Err.: 0.0676

2 x log-likelihood: -4810.072 


\begin{tabular}{|c|c|c|c|c|c|}
\hline $\begin{array}{l}\text { D-D-V } \\
\text { Call: } \\
\text { glm.nb(formula = Collisions } \\
\text { Age. } 25-64+\text { Age }>65+\text { Dens }\end{array}$ & $\begin{array}{l}\text { Irea + MS-D } \\
\text { - Trips-Ente }\end{array}$ & $\begin{array}{l}\text { + MS-Passe } \\
\text { ter, init.th }\end{array}$ & $\begin{array}{r}\text { MS-Tra } \\
1.159811 \\
\end{array}$ & $\begin{array}{l}\text { MS-Walk + } \\
\text { ink = log) }\end{array}$ & ke + \\
\hline Deviance Residuals: & & & & & \\
\hline Min & 10 & Median & $3 Q$ & Max & \\
\hline-3.0622 & -0.829 & -0.2121 & 0.3997 & 2.6054 & \\
\hline & Estimate & Std.Error & z value & $\operatorname{Pr}(>|z|)$ & \\
\hline (Intercept) & $1.66 \mathrm{E}+00$ & $3.18 \mathrm{E}-01$ & 5.207 & $1.92 \mathrm{E}-07$ & $* * *$ \\
\hline Area & $8.66 \mathrm{E}-03$ & $1.68 \mathrm{E}-03$ & 5.158 & $2.50 \mathrm{E}-07$ & $* * *$ \\
\hline MS-Driver & $1.88 \mathrm{E}-02$ & 3.67E-03 & 5.122 & 3.03E-07 & $* * *$ \\
\hline MS-Passenger & $4.17 \mathrm{E}-02$ & $7.72 \mathrm{E}-03$ & 5.403 & $6.56 \mathrm{E}-08$ & $* * *$ \\
\hline MS-Transit & $4.82 \mathrm{E}-02$ & $6.26 \mathrm{E}-03$ & 7.698 & $1.38 \mathrm{E}-14$ & $* * *$ \\
\hline MS-Walk & $3.25 \mathrm{E}-02$ & $6.49 \mathrm{E}-03$ & 5.005 & $5.59 \mathrm{E}-07$ & $* * *$ \\
\hline MS-Bike & $5.25 \mathrm{E}-02$ & $2.25 \mathrm{E}-02$ & 2.333 & 0.0196 & $*$ \\
\hline Age.25-64 & $6.02 \mathrm{E}-03$ & $2.48 \mathrm{E}-03$ & 2.424 & 0.0154 & $*$ \\
\hline Age $>65$ & $1.48 \mathrm{E}-02$ & 7.30E-03 & 2.034 & 0.042 & $*$ \\
\hline Density- Trips-Enter-Center & $-1.47 \mathrm{E}-05$ & $3.06 \mathrm{E}-06$ & -4.822 & $1.42 \mathrm{E}-06$ & $* * *$ \\
\hline $\begin{array}{l}\text { Signif.codes: }\left(0^{* * * \prime}\right)(0.001 \\
\text { (Dispersion parameter for } \mathrm{N}\end{array}$ & $\begin{array}{l}\left.k^{\prime}\right)\left(0.01^{\prime * \prime}\right) \\
\text { ative Binom }\end{array}$ & $\begin{array}{l}\left..^{\prime}\right)\left(0.1^{\prime \prime}\right) \\
.148) \text { famil }\end{array}$ & $\mathrm{n}$ to be 1 & & \\
\hline $\begin{array}{l}\text { Null deviance: } 642.8 \text { on } 421 \\
\text { Residual deviance: } 492.77 \text { o } \\
\text { AIC: } 4777.4\end{array}$ & $\begin{array}{l}\text { egrees of fre } \\
12 \text { degrees }\end{array}$ & eedom & & & \\
\hline Number of Fisher Scoring ite & tions: 1 & & & & \\
\hline $\begin{array}{l}\text { Theta: } 1.1598 \\
\text { Std. Err.: } 0.0771\end{array}$ & & & & & \\
\hline 2 x log-likelihood: -4755.42 & & & & & \\
\hline
\end{tabular}


D-C-I

Call:

glm.nb(formula $=$ Collisions $\sim$ VKT + Trips-Produced + Trips-Attracted + Density- Trips-Out-Center + Density- Trips-Enter-Center + TLKM, init.theta $=1.174702883$, link $=\log$ )

Deviance Residuals:

\begin{tabular}{|c|c|c|c|c|c|}
\hline Min & $1 \mathrm{Q}$ & Median & $3 \mathrm{Q}$ & Max & \\
\hline-3.3782 & -0.8848 & -0.202 & 0.3318 & 2.8768 & \\
\hline \multicolumn{5}{|l|}{} \\
\hline & Estimate & Std.Error & z value & $\operatorname{Pr}(>|\mathrm{z}|)$ & \\
\hline (Intercept) & $3.78 \mathrm{E}+00$ & $8.04 \mathrm{E}-02$ & 46.973 & $<2 \mathrm{E}-16$ & $* * *$ \\
\hline VKT & $6.63 \mathrm{E}-06$ & $1.09 \mathrm{E}-06$ & 6.086 & $1.16 \mathrm{E}-09$ & $* * *$ \\
\hline Trips-Produced & $2.59 \mathrm{E}-04$ & $4.45 \mathrm{E}-05$ & 5.82 & $5.89 \mathrm{E}-09$ & $* * *$ \\
\hline Trips-Attracted & $1.75 \mathrm{E}-04$ & $3.68 \mathrm{E}-05$ & 4.753 & $2.00 \mathrm{E}-06$ & $* * *$ \\
\hline Density- Trips-Out-Center & $1.23 \mathrm{E}-04$ & $2.50 \mathrm{E}-05$ & 4.913 & $8.97 \mathrm{E}-07$ & $* * *$ \\
\hline Density- Trips-Enter-Center & $-1.53 \mathrm{E}-05$ & $3.99 \mathrm{E}-06$ & -3.848 & 0.000119 & $* * *$ \\
\hline TLKM & $4.98 \mathrm{E}-03$ & $2.05 \mathrm{E}-03$ & 2.433 & 0.014959 & $*$ \\
\hline
\end{tabular}

Signif.codes: $\left(0^{\prime * * * \prime}\right)\left(0.001^{\prime * * \prime}\right)\left(0.01^{\prime * \prime}\right)\left(0.05^{\prime \prime}.\right)\left(0.1^{\prime \prime}\right)$ (1)

(Dispersion parameter for Negative Binomial (2.148) family taken to be 1 )

Null deviance: 650.41 on 421 degrees of freedom

Residual deviance: 494.8 on 415 degrees of freedom

AIC: 4767.8

Number of Fisher Scoring iterations: 1

Theta: 1.1747

Std. Err.: 0.0785

Warning while fitting theta: alternation limit reached

2 x log-likelihood: -4751.765 


\section{D-D-I}

Call:

glm.nb(formula $=$ Collisions $\sim$ Density- Trips-Out-Center + Density- Trips-Enter-Center, init.theta $=$ 0.9344494795, link = log)

Deviance Residuals:

\begin{tabular}{|c|c|c|c|c|c|}
\hline Min & $1 \mathrm{Q}$ & Median & $3 \mathrm{Q}$ & Max & \\
\hline-3.0898 & -0.902 & -0.2335 & 0.3695 & 2.4762 & \\
\hline \multicolumn{5}{|c|}{} \\
\hline & Estimate & Std.Error & z value & $\operatorname{Pr}(>|\mathrm{z}|)$ & \\
\hline (Intercept) & $4.65 \mathrm{E}+00$ & $6.66 \mathrm{E}-02$ & 69.934 & $<2 \mathrm{E}-16$ & $* * *$ \\
\hline Density- Trips-Out-Center & $1.03 \mathrm{E}-04$ & $2.53 \mathrm{E}-05$ & 4.048 & $5.17 \mathrm{E}-05$ & $* * *$ \\
\hline Density- Trips-Enter-Center & $-1.79 \mathrm{E}-05$ & $3.84 \mathrm{E}-06$ & -4.671 & $3.00 \mathrm{E}-06$ & $* * *$ \\
\hline
\end{tabular}

Signif.codes: $\left(0^{(* * * \prime}\right)\left(0.001^{\left(* *^{\prime}\right.}\right)\left(0.01^{\prime * \prime}\right)\left(0.05^{\prime \prime}.\right)\left(0.1^{\prime \prime}\right)$ (1)

(Dispersion parameter for Negative Binomial (2.148) family taken to be 1 )

Null deviance: 526.33 on 421 degrees of freedom

Residual deviance: 498.83 on 419 degrees of freedom

AIC: 4867.4

Number of Fisher Scoring iterations: 1

Theta: 0.9344

Std. Err.: 0.0602

2 x log-likelihood: -4859.357 


\begin{tabular}{|c|c|c|c|c|c|}
\hline \multicolumn{6}{|c|}{$\begin{array}{l}\text { Call: } \\
\text { glm.nb(formula = Collisions } \sim \text { VKT + Trips-Out-Center + Trips-Enter-Center + Density- Trips-Out-Center + } \\
\text { Density- Trips-Enter-Center + TLKM, init.theta }=1.007607714 \text {, link }=\text { log) }\end{array}$} \\
\hline \multicolumn{6}{|l|}{ Deviance Residuals: } \\
\hline Min & 10 & Median & $3 Q$ & Max & \\
\hline \multirow[t]{2}{*}{-3.3145} & -0.7594 & -0.1991 & 0.318 & 2.7342 & \\
\hline & Estimate & Std.Error & $z$ value & $\operatorname{Pr}(>|z|)$ & \\
\hline (Intercept) & $3.41 \mathrm{E}+00$ & 1.01E-01 & 33.656 & $<2 \mathrm{E}-16$ & $* * *$ \\
\hline VKT & $3.41 \mathrm{E}-06$ & $8.56 \mathrm{E}-07$ & 3.983 & $6.79 \mathrm{E}-05$ & $* * *$ \\
\hline Trips-Out-Center & $2.45 \mathrm{E}-04$ & $4.68 \mathrm{E}-05$ & 5.223 & $1.76 \mathrm{E}-07$ & $* * *$ \\
\hline Trips-Enter-Center & $2.01 \mathrm{E}-04$ & $3.16 \mathrm{E}-05$ & 6.364 & $1.96 \mathrm{E}-10$ & $* * *$ \\
\hline Density- Trips-Out-Center & $2.47 \mathrm{E}-04$ & $3.25 \mathrm{E}-05$ & 7.586 & $3.30 \mathrm{E}-14$ & $* * *$ \\
\hline Density- Trips-Enter-Center & $-3.97 \mathrm{E}-05$ & $6.27 \mathrm{E}-06$ & -6.329 & $2.48 \mathrm{E}-10$ & $* * *$ \\
\hline TLKM & $7.24 \mathrm{E}-03$ & $2.24 \mathrm{E}-03$ & 3.238 & 0.00121 & $* *$ \\
\hline \multicolumn{6}{|c|}{$\begin{array}{l}\text { Signif.codes: }\left(0^{\prime * * * \prime}\right)\left(0.001^{\prime * * \prime}\right)\left(0.01^{\prime * \prime}\right)\left(0.05^{\prime \prime} .\right)\left(0.1^{\prime \prime}\right)(1) \\
\text { (Dispersion parameter for Negative Binomial }(2.148) \text { family taken to be } 1)\end{array}$} \\
\hline \multicolumn{6}{|c|}{ Null deviance: 684.68 on 421 degrees of freedom } \\
\hline \multicolumn{6}{|c|}{ Residual deviance: 507.8 on 415 degrees of freedom } \\
\hline \multicolumn{6}{|l|}{ AIC: 4730} \\
\hline \multicolumn{6}{|c|}{ Number of Fisher Scoring iterations: 1} \\
\hline \multicolumn{6}{|l|}{ Theta: 1.0076} \\
\hline \multicolumn{6}{|l|}{ Std. Err.: 0.0694} \\
\hline \multicolumn{6}{|l|}{2 x log-likelihood: -4714.001} \\
\hline
\end{tabular}




\begin{tabular}{|c|c|c|c|c|c|}
\hline \multicolumn{6}{|c|}{$\begin{array}{l}\text { Call: } \\
\text { glm.nb(formula }=\text { Collisions } \sim \text { VKT }+ \text { Density- Trips-Enter-Center }+ \text { TLKM, init.theta }=1.742392744 \text {, link }= \\
\text { log) }\end{array}$} \\
\hline \multicolumn{6}{|l|}{ Deviance Residuals: } \\
\hline Min & 10 & Median & $3 Q$ & Max & \\
\hline \multirow[t]{2}{*}{-3.9708} & -0.8724 & -0.1814 & 0.383 & 2.6377 & \\
\hline & Estimate & Std.Error & $z$ value & $\operatorname{Pr}(>|z|)$ & \\
\hline (Intercept) & $4.97 \mathrm{E}+00$ & 5.97E-02 & 83.21 & $<2 \mathrm{E}-16$ & $* * *$ \\
\hline VKT & $9.10 \mathrm{E}-06$ & $2.05 \mathrm{E}-06$ & 4.442 & $8.92 \mathrm{E}-06$ & *** \\
\hline Density- Trips-Enter-Center & $-1.52 \mathrm{E}-05$ & $2.53 \mathrm{E}-06$ & -6.002 & $1.95 \mathrm{E}-09$ & $* * *$ \\
\hline TLKM & $1.84 \mathrm{E}-02$ & $6.69 \mathrm{E}-03$ & 2.75 & 0.00596 & $* *$ \\
\hline \multicolumn{6}{|c|}{$\begin{array}{l}\text { Signif.codes: }\left(0^{\prime * * * \prime}\right)\left(0.001^{\prime * * \prime}\right)\left(0.01^{\prime * \prime}\right)\left(0.05^{\prime \prime}\right)\left(0.1^{\prime \prime}\right)(1) \\
\text { (Dispersion parameter for Negative Binomial }(2.148) \text { family taken to be } 1)\end{array}$} \\
\hline \multicolumn{6}{|c|}{ Null deviance: 540.43 on 421 degrees of freedom } \\
\hline \multicolumn{6}{|c|}{ Residual deviance: 472.78 on 418 degrees of freedom } \\
\hline \multicolumn{6}{|l|}{ AIC: 5261} \\
\hline \multicolumn{6}{|c|}{ Number of Fisher Scoring iterations: 1} \\
\hline \multicolumn{6}{|l|}{ Theta: 1.742} \\
\hline \multicolumn{6}{|l|}{ Std. Err.: 0.115} \\
\hline 2 x log-likelihood: -5250.99 & & & & & \\
\hline
\end{tabular}




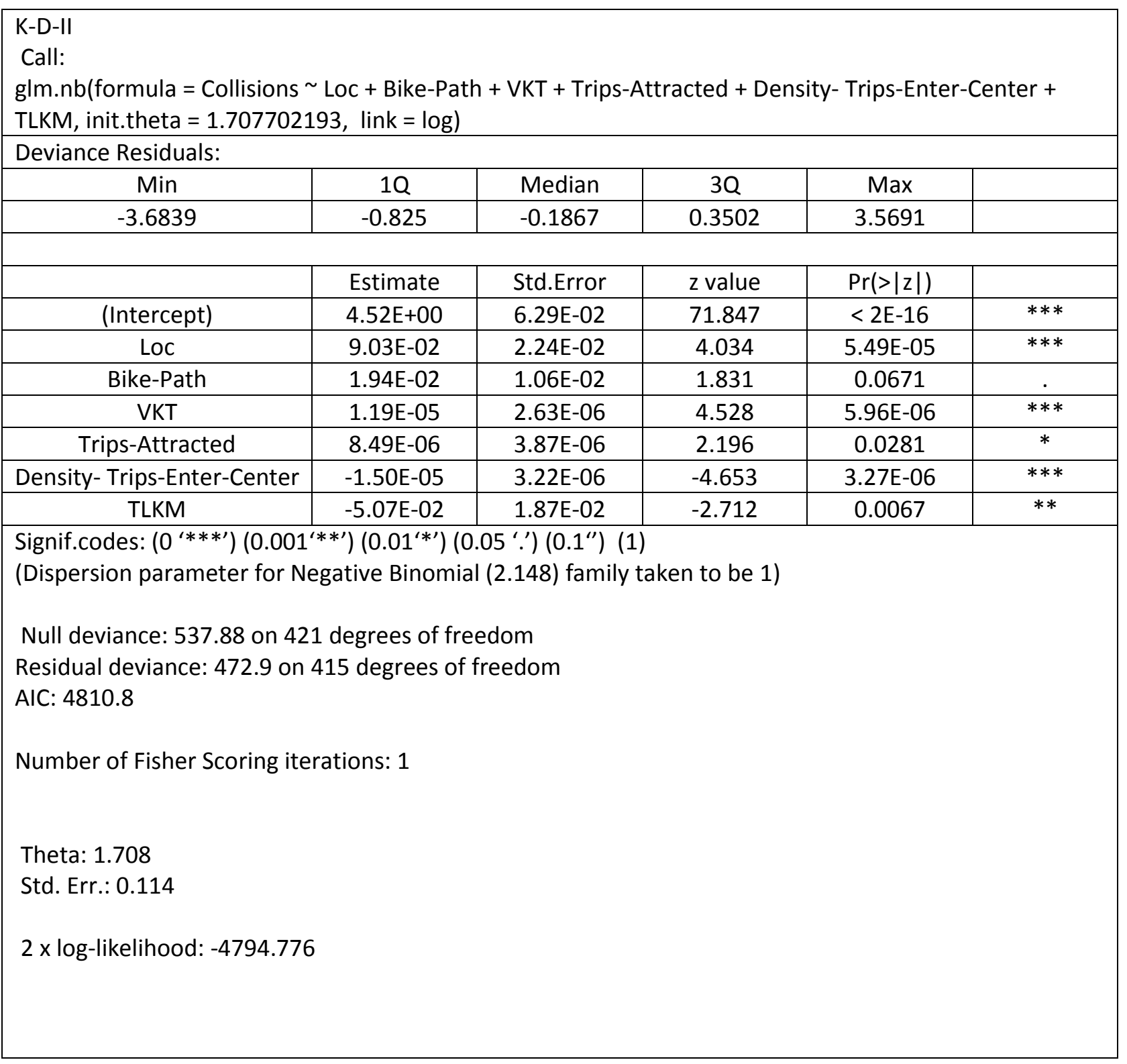




\begin{tabular}{|c|c|c|c|c|c|}
\hline $\begin{array}{l}\text { K-D-III } \\
\text { Call: } \\
\text { glm.nb(formula = Collisions } \\
\text { Bike-Path + VKT + Density- T }\end{array}$ & $\begin{array}{l}\text { Jon-Sig-Int + } \\
\text { S-Enter-Cen }\end{array}$ & $\begin{array}{l}\mathrm{Km} / \mathrm{h}+50 \\
\mathrm{TLKM}, \text { init }\end{array}$ & $\begin{array}{r}+60 . \mathrm{Km} \\
=1.7524 \\
\end{array}$ & $\begin{array}{l}\mathrm{Km} / \mathrm{h}+1( \\
\text { link = log) }\end{array}$ & \\
\hline Deviance Residuals: & & & & & \\
\hline Min & $1 Q$ & Median & $3 Q$ & Max & \\
\hline-3.774 & -0.8324 & -0.1304 & 0.3364 & 3.5698 & \\
\hline & Estimate & Std.Error & $z$ value & $\operatorname{Pr}(>|z|)$ & \\
\hline (Intercept) & $4.58 \mathrm{E}+00$ & 8.06E-02 & 56.843 & $<2 \mathrm{E}-16$ & $* * *$ \\
\hline Non-Sig-Int & $4.31 \mathrm{E}-03$ & $2.75 \mathrm{E}-03$ & 1.569 & 0.11667 & \\
\hline $40 . \mathrm{Km} / \mathrm{h}$ & $3.02 \mathrm{E}-01$ & $6.96 \mathrm{E}-02$ & 4.342 & $1.41 \mathrm{E}-05$ & $* * *$ \\
\hline $50 . \mathrm{Km} / \mathrm{h}$ & $2.73 \mathrm{E}-01$ & $6.78 \mathrm{E}-02$ & 4.028 & $5.62 \mathrm{E}-05$ & $* * *$ \\
\hline $60 . \mathrm{Km} / \mathrm{h}$ & $2.15 \mathrm{E}-01$ & 7.04E-02 & 3.046 & 0.00232 & $* *$ \\
\hline $80 . \mathrm{Km} / \mathrm{h}$ & $1.71 \mathrm{E}-01$ & $8.05 \mathrm{E}-02$ & 2.126 & 0.03348 & $*$ \\
\hline $100 . \mathrm{Km} / \mathrm{h}$ & $8.56 \mathrm{E}+02$ & $3.41 E+02$ & 2.511 & 0.01205 & $*$ \\
\hline Bike-Path & $1.80 \mathrm{E}-02$ & $1.05 \mathrm{E}-02$ & 1.715 & 0.08643 & . \\
\hline VKT & $1.28 \mathrm{E}-05$ & $2.83 \mathrm{E}-06$ & 4.534 & $5.79 \mathrm{E}-06$ & $* * *$ \\
\hline Density-Trips-Enter-Center & $-1.53 \mathrm{E}-05$ & 2.87E-06 & -5.343 & $9.13 \mathrm{E}-08$ & $* * *$ \\
\hline TLKM & $-2.85 \mathrm{E}-01$ & 7.01E-02 & -4.066 & $4.78 \mathrm{E}-05$ & $* * *$ \\
\hline $\begin{array}{l}\text { Signif.codes: }\left(0^{* * * \prime)}(0.001\right. \\
\text { (Dispersion parameter for } N\end{array}$ & $\begin{array}{l}\left.\mathrm{k}^{\prime}\right)\left(0.01^{(* \prime}\right) \\
\text { ative Binom }\end{array}$ & $\begin{array}{l}y^{\prime \prime}\left(0.1^{\prime \prime}\right) \\
.148) \text { fami }\end{array}$ & n to be 1 & & \\
\hline $\begin{array}{l}\text { Null deviance: } 551.15 \text { on } 42 \\
\text { Residual deviance: } 472.03 \text { or } \\
\text { AIC: } 4806.5\end{array}$ & $\begin{array}{l}\text { degrees of } \mathrm{ft} \\
11 \text { degrees }\end{array}$ & $\begin{array}{l}\text { om } \\
\text { eedom }\end{array}$ & & & \\
\hline Number of Fisher Scoring ite & tions: 1 & & & & \\
\hline $\begin{array}{l}\text { Theta: } 1.752 \\
\text { Std. Err.: } 0.117\end{array}$ & & & & & \\
\hline $2 \times$ log-likelihood: -4782.466 & & & & & \\
\hline
\end{tabular}




\begin{tabular}{|c|c|c|c|c|c|}
\hline \multicolumn{6}{|c|}{$\begin{array}{l}\text { Call: } \\
\text { glm.nb(formula }=\text { Collisions } \sim \text { MS-Driver + MS-Passenger + MS-Transit + MS-Walk + MS-Bike + VKT + } \\
\text { Density- Trips-Enter-Center, init.theta }=1.800884235, \text { link }=\text { log) }\end{array}$} \\
\hline \multicolumn{6}{|l|}{ Deviance Residuals: } \\
\hline Min & 10 & Median & $3 Q$ & Max & \\
\hline \multirow[t]{2}{*}{-3.7755} & -0.8797 & -0.173 & 0.3905 & 3.5898 & \\
\hline & Estimate & Std.Error & $z$ value & $\operatorname{Pr}(>|z|)$ & \\
\hline (Intercept) & $2.85 \mathrm{E}+00$ & $2.42 \mathrm{E}-01$ & 11.777 & $<2 \mathrm{E}-16$ & $* * *$ \\
\hline MS-Driver & $1.09 \mathrm{E}-02$ & $2.88 \mathrm{E}-03$ & 3.768 & 0.000164 & $* * *$ \\
\hline MS-Passenger & $4.56 \mathrm{E}-02$ & $6.00 \mathrm{E}-03$ & 7.6 & $2.97 \mathrm{E}-14$ & $* * *$ \\
\hline MS-Transit & $2.35 \mathrm{E}-02$ & 4.99E-03 & 4.7 & $2.61 \mathrm{E}-06$ & $* * *$ \\
\hline MS-Walk & $2.01 \mathrm{E}-02$ & 4.89E-03 & 4.121 & $3.78 \mathrm{E}-05$ & $* * *$ \\
\hline MS-Bike & $4.87 \mathrm{E}-02$ & $1.81 \mathrm{E}-02$ & 2.69 & 0.007142 & $* *$ \\
\hline VKT & $7.09 \mathrm{E}-06$ & $1.69 \mathrm{E}-06$ & 4.207 & $2.59 \mathrm{E}-05$ & $* * *$ \\
\hline Density- Trips-Enter-Center & $-1.78 \mathrm{E}-05$ & $2.65 \mathrm{E}-06$ & -6.701 & $2.07 \mathrm{E}-11$ & $* * *$ \\
\hline \multicolumn{6}{|c|}{$\begin{array}{l}\text { Signif.codes: }\left(0^{* * * \prime}\right)\left(0.001^{\prime * * \prime}\right)\left(0.01^{\prime * \prime}\right)\left(0.05^{\prime \prime}\right)\left(0.1^{\prime \prime}\right)(1) \\
\text { (Dispersion parameter for Negative Binomial }(2.148) \text { family taken to be } 1)\end{array}$} \\
\hline \multicolumn{6}{|c|}{ Null deviance: 565.48 on 421 degrees of freedom } \\
\hline \multicolumn{6}{|c|}{ Residual deviance: 471.21 on 414 degrees of freedom } \\
\hline \multicolumn{6}{|l|}{ AIC: 4787.6} \\
\hline \multicolumn{6}{|c|}{ Number of Fisher Scoring iterations: 1} \\
\hline \multicolumn{6}{|l|}{ Theta: 1.801} \\
\hline \multicolumn{6}{|l|}{ Std. Err.: 0.12} \\
\hline $2 \times$ log-likelihood: -4769.63 & & & & & \\
\hline
\end{tabular}




\begin{tabular}{|c|c|c|c|c|c|}
\hline \multicolumn{6}{|c|}{$\begin{array}{l}\text { Call: } \\
\text { glm.nb(formula }=\text { Collisions } \sim \text { Area + MS-Driver + MS-Passenger + MS-Transit + MS-Walk + MS-Bike + } \\
\text { Age. } 25-64+\text { Age }>65+\text { VKT + Density- Trips-Enter-Center, init.theta }=2.205503251 \text {, link }=\text { log) }\end{array}$} \\
\hline \multicolumn{6}{|l|}{ Deviance Residuals: } \\
\hline Min & 10 & Median & $3 Q$ & Max & \\
\hline \multirow[t]{2}{*}{-3.7985} & -0.8494 & -0.1325 & 0.4094 & 2.7569 & \\
\hline & Estimate & Std.Error & $z$ value & $\operatorname{Pr}(>|z|)$ & \\
\hline (Intercept) & $2.38 \mathrm{E}+00$ & $2.29 \mathrm{E}-01$ & 10.393 & $<2 \mathrm{E}-16$ & $* * *$ \\
\hline Area & $8.36 \mathrm{E}-03$ & $1.23 \mathrm{E}-03$ & 6.812 & $9.65 \mathrm{E}-12$ & $* * *$ \\
\hline MS-Driver & $1.14 \mathrm{E}-02$ & $2.67 \mathrm{E}-03$ & 4.262 & 2.03E-05 & $* * *$ \\
\hline MS-Passenger & $3.47 \mathrm{E}-02$ & $5.63 \mathrm{E}-03$ & 6.159 & $7.32 \mathrm{E}-10$ & $* * *$ \\
\hline MS-Transit & $2.78 \mathrm{E}-02$ & $4.61 \mathrm{E}-03$ & 6.035 & 1.59E-09 & $* * *$ \\
\hline MS-Walk & $1.35 \mathrm{E}-02$ & $4.86 \mathrm{E}-03$ & 2.775 & 0.005522 & $* *$ \\
\hline MS-Bike & $4.56 \mathrm{E}-02$ & $1.64 \mathrm{E}-02$ & 2.775 & 0.005517 & $* *$ \\
\hline Age.25-64 & $5.98 \mathrm{E}-03$ & $1.82 \mathrm{E}-03$ & 3.293 & 0.000989 & $* * *$ \\
\hline Age $>65$ & $1.20 \mathrm{E}-02$ & $5.32 \mathrm{E}-03$ & 2.255 & 0.024154 & $*$ \\
\hline VKT & $1.16 \mathrm{E}-05$ & $1.56 \mathrm{E}-06$ & 7.424 & $1.14 \mathrm{E}-13$ & $* * *$ \\
\hline Density- Trips-Enter-Center & $-1.73 \mathrm{E}-05$ & $2.53 \mathrm{E}-06$ & -6.841 & $7.84 \mathrm{E}-12$ & $* * *$ \\
\hline \multicolumn{6}{|c|}{$\begin{array}{l}\text { Signif.codes: }\left(0^{\prime * * * \prime}\right)\left(0.001^{\prime * * \prime}\right)\left(0.01^{\prime * \prime}\right)\left(0.05^{\prime} .^{\prime}\right)\left(0.1^{\prime \prime}\right)(1) \\
\text { (Dispersion parameter for Negative Binomial }(2.148) \text { family taken to be } 1)\end{array}$} \\
\hline \multicolumn{6}{|c|}{ Null deviance: 683.91 on 421 degrees of freedom } \\
\hline \multicolumn{6}{|c|}{ Residual deviance: 469.36 on 411 degrees of freedom } \\
\hline \multicolumn{6}{|l|}{ AIC: 4703.5} \\
\hline \multicolumn{6}{|c|}{ Number of Fisher Scoring iterations: 1} \\
\hline \multicolumn{6}{|l|}{ Theta: 2.206} \\
\hline \multicolumn{6}{|l|}{ Std. Err.: 0.151} \\
\hline 2 x log-likelihood: -4679.49 & & & & & \\
\hline
\end{tabular}




\begin{tabular}{|c|c|c|c|c|c|}
\hline $\begin{array}{l}\text { K-D-I } \\
\text { Call: } \\
\text { glm.nb(formula = Collisions } \\
\text { log) }\end{array}$ & KT + Densit & ips-Enter-C & TLKM, i & $\mathrm{ta}=1.65 \mathrm{C}$ & link = \\
\hline Deviance Residuals: & & & & & \\
\hline Min & 10 & Median & $3 Q$ & Max & \\
\hline-3.678 & -0.7927 & -0.1743 & 0.3633 & 3.3929 & \\
\hline & Estimate & Std.Error & $z$ value & $\operatorname{Pr}(>|z|)$ & \\
\hline (Intercept) & $4.52 \mathrm{E}+00$ & $6.16 \mathrm{E}-02$ & 73.343 & $<2 \mathrm{E}-16$ & $* * *$ \\
\hline VKT & $5.65 \mathrm{E}-06$ & $2.11 \mathrm{E}-06$ & 2.674 & 0.0075 & $* *$ \\
\hline Density- Trips-Enter-Center & $-1.56 \mathrm{E}-05$ & $2.72 \mathrm{E}-06$ & -5.731 & $9.96 \mathrm{E}-09$ & $* * *$ \\
\hline TLKM & $1.92 \mathrm{E}-02$ & $6.91 \mathrm{E}-03$ & 2.775 & 0.00551 & $* *$ \\
\hline $\begin{array}{l}\text { Signif.codes: }\left(0^{* * * \prime}\right)(0.001 \\
\text { (Dispersion parameter for } \mathrm{N}\end{array}$ & $\begin{array}{l}\left.k^{\prime}\right)\left(0.01^{* \prime}\right) \\
\text { ative Binom }\end{array}$ & $\begin{array}{l}\because \prime)\left(0.1^{\prime \prime}\right) \\
.148) \text { famil }\end{array}$ & $n$ to be 1 & & \\
\hline Null deviance: 520.99 on 42 & degrees of $f$ & & & & \\
\hline Residual deviance: 473.85 o & 18 degrees & eedom & & & \\
\hline AIC: 4820.7 & & & & & \\
\hline Number of Fisher Scoring ite & tions: 1 & & & & \\
\hline Theta: 1.651 & & & & & \\
\hline Std. Err.: 0.109 & & & & & \\
\hline $2 \times$ log-likelihood: -4810.74 & & & & & \\
\hline
\end{tabular}




\begin{tabular}{|c|c|c|c|c|c|}
\hline $\begin{array}{l}\text { L-D-III } \\
\text { Call: } \\
\text { glm.nb(formula = Collisions " } \\
\text { Trips-Attracted + Density- Tr }\end{array}$ & $\begin{array}{l}\text { Oo Bus stops } \\
\text {-Enter-Cent }\end{array}$ & $\begin{array}{l}. K m / h+5 C \\
\text { TLKM, init. }\end{array}$ & $\begin{array}{l}h+60 . \mathrm{km} \\
=0.8274\end{array}$ & $\begin{array}{l}\text { O. } \mathrm{Km} / \mathrm{h}+1 \\
\text { 4, link = log }\end{array}$ & $/ \mathrm{h}+$ \\
\hline Deviance Residuals: & & & & & \\
\hline Min & 10 & Median & $3 Q$ & Max & \\
\hline-2.9051 & -0.841 & -0.2311 & 0.2525 & 2.9891 & \\
\hline & Estimate & Std.Error & $z$ value & $\operatorname{Pr}(>|z|)$ & \\
\hline (Intercept) & $4.28 \mathrm{E}+00$ & $1.15 \mathrm{E}-01$ & 37.302 & $<2 \mathrm{E}-16$ & $* * *$ \\
\hline No Bus stops & $2.47 \mathrm{E}-02$ & $4.55 \mathrm{E}-03$ & 5.422 & $5.90 \mathrm{E}-08$ & $* * *$ \\
\hline $40 . \mathrm{Km} / \mathrm{h}$ & $3.52 \mathrm{E}-01$ & $1.17 \mathrm{E}-01$ & 3.026 & 0.00248 & $* *$ \\
\hline $50 . \mathrm{Km} / \mathrm{h}$ & $3.25 \mathrm{E}-01$ & $1.15 \mathrm{E}-01$ & 2.834 & 0.0046 & $* *$ \\
\hline $60 . \mathrm{Km} / \mathrm{h}$ & $2.65 \mathrm{E}-01$ & $1.18 \mathrm{E}-01$ & 2.247 & 0.02463 & $*$ \\
\hline $80 . \mathrm{Km} / \mathrm{h}$ & $3.14 \mathrm{E}-01$ & $1.31 \mathrm{E}-01$ & 2.395 & 0.0166 & $*$ \\
\hline $100 . \mathrm{Km} / \mathrm{h}$ & $1.04 \mathrm{E}+03$ & $4.95 \mathrm{E}+02$ & 2.09 & 0.0366 & $*$ \\
\hline Trips-Attracted & $4.19 \mathrm{E}-05$ & $8.31 \mathrm{E}-06$ & 5.043 & $4.57 \mathrm{E}-07$ & $* * *$ \\
\hline Density- Trips-Enter-Center & $-8.18 \mathrm{E}-05$ & $1.10 \mathrm{E}-05$ & -7.443 & $9.86 \mathrm{E}-14$ & $* * *$ \\
\hline TLKM & $-2.99 \mathrm{E}-01$ & $1.17 \mathrm{E}-01$ & -2.565 & 0.01031 & $*$ \\
\hline $\begin{array}{l}\text { Signif.codes: }\left(0^{` * * *}\right)(0.001 \\
\text { (Dispersion parameter for } \mathrm{N}\end{array}$ & $\begin{array}{l}\left.{ }^{\prime \prime}\right)\left(0.01^{\prime * \prime}\right)( \\
\text { ative Binom }\end{array}$ & $\begin{array}{l}\text { ') }\left(0.1^{\prime \prime}\right) \\
\text { 148) famil }\end{array}$ & n to be 1 & & \\
\hline Null deviance: 644.93 on 42 & degrees of $\mathrm{fr}$ & & & & \\
\hline Residual deviance: 508.24 o & 12 degrees & eedom & & & \\
\hline AIC: 4712.2 & & & & & \\
\hline Number of Fisher Scoring ite & tions: 1 & & & & \\
\hline Theta: 0.8275 & & & & & \\
\hline Std. Err.: 0.0559 & & & & & \\
\hline 2 x log-likelihood: -4690.21 & & & & & \\
\hline
\end{tabular}


L-D-V

Call:

glm.nb(formula $=$ Collisions $~$ Area + MS-Driver + MS-Passenger + MS-Transit + MS-Walk + Employment + Age.5-24 + Age.25-64 + Age > 65 + VKT + TLKM, init.theta $=1.062934041$, link = log)

Deviance Residuals:

\begin{tabular}{|c|c|c|c|c|c|}
\hline Min & $1 Q$ & Median & $3 Q$ & Max & \\
\hline-3.215 & -0.8209 & -0.174 & 0.3076 & 3.4893 & \\
\hline & Estimate & Std.Error & $z$ value & $\operatorname{Pr}(>|z|)$ & \\
\hline (Intercept) & $2.08 \mathrm{E}-02$ & $3.86 \mathrm{E}-01$ & 0.054 & 0.957043 & \\
\hline Area & $9.14 \mathrm{E}-03$ & 1.79E-03 & 5.111 & $3.21 \mathrm{E}-07$ & $* * *$ \\
\hline MS-Driver & $2.20 \mathrm{E}-02$ & $4.34 \mathrm{E}-03$ & 5.07 & $3.98 \mathrm{E}-07$ & $* * *$ \\
\hline MS-Passenger & $4.86 \mathrm{E}-02$ & $8.24 \mathrm{E}-03$ & 5.895 & 3.75E-09 & $* * *$ \\
\hline MS-Transit & $4.28 \mathrm{E}-02$ & $7.47 \mathrm{E}-03$ & 5.734 & 9.79E-09 & $* * *$ \\
\hline MS-Walk & $2.26 \mathrm{E}-02$ & 7.76E-03 & 2.906 & 0.003657 & $* *$ \\
\hline Employment & $-1.13 \mathrm{E}-05$ & $2.59 \mathrm{E}-06$ & -4.356 & $1.33 \mathrm{E}-05$ & $* * *$ \\
\hline Age.5-24 & $1.71 \mathrm{E}-02$ & $4.48 \mathrm{E}-03$ & 3.816 & 0.000136 & $* * *$ \\
\hline Age.25-64 & $1.79 \mathrm{E}-02$ & $2.93 \mathrm{E}-03$ & 6.113 & $9.80 \mathrm{E}-10$ & $* * *$ \\
\hline Age $>65$ & $2.58 \mathrm{E}-02$ & 7.67E-03 & 3.361 & 0.000776 & $* * *$ \\
\hline VKT & $4.86 \mathrm{E}-06$ & $2.77 \mathrm{E}-06$ & 1.753 & 0.079563 & . \\
\hline TLKM & $2.82 \mathrm{E}-02$ & $1.17 \mathrm{E}-02$ & 2.401 & 0.016355 & $*$ \\
\hline
\end{tabular}

Signif.codes: $\left(0^{\prime * * * \prime}\right)\left(0.001^{\prime * * \prime}\right)\left(0.01^{\prime * \prime}\right)\left(0.05^{\prime \prime}.\right)\left(0.1^{\prime \prime}\right)$ (1)

(Dispersion parameter for Negative Binomial (2.148) family taken to be 1 )

Null deviance: 804.91 on 421 degrees of freedom

Residual deviance: 510.21 on 410 degrees of freedom

AIC: 4609.7

Number of Fisher Scoring iterations: 1

Theta: 1.0629

Std. Err.: 0.0752

2 x log-likelihood: -4583.73 


\begin{tabular}{|c|c|c|c|c|c|}
\hline \multicolumn{6}{|c|}{$\begin{array}{l}\text { Call: } \\
\text { glm.nb(formula = Collisions } \sim \text { Trips-Attracted + Density- Trips-Enter-Center + TLKM, init.theta }= \\
0.7553840588, \text { link }=\log )\end{array}$} \\
\hline \multicolumn{6}{|l|}{ Deviance Residuals: } \\
\hline Min & $1 Q$ & Median & $3 Q$ & Max & \\
\hline-2.7547 & -0.8679 & -0.2384 & 0.2702 & 2.876 & \\
\hline & Estimate & Std.Error & $z$ value & $\operatorname{Pr}(>|z|)$ & \\
\hline (Intercept) & $4.25 \mathrm{E}+00$ & $9.22 \mathrm{E}-02$ & 46.056 & $<2 \mathrm{E}-16$ & $* * *$ \\
\hline Trips-Attracted & $4.43 \mathrm{E}-05$ & $7.94 \mathrm{E}-06$ & 5.581 & $2.39 \mathrm{E}-08$ & $* * *$ \\
\hline Density- Trips-Enter-Center & $-8.51 E-05$ & $1.01 \mathrm{E}-05$ & -8.4 & $<2 \mathrm{E}-16$ & $* * *$ \\
\hline TLKM & $7.00 \mathrm{E}-02$ & $8.63 \mathrm{E}-03$ & 8.116 & $4.84 \mathrm{E}-16$ & $* * *$ \\
\hline \multicolumn{6}{|c|}{$\begin{array}{l}\text { Signif.codes: }\left(0^{\prime * * * \prime}\right)\left(0.001^{* * \prime}\right)\left(0.01^{\prime * \prime}\right)\left(0.05^{\prime} .^{\prime}\right)\left(0.1^{\prime \prime}\right)(1) \\
\text { (Dispersion parameter for Negative Binomial }(2.148) \text { family taken to be } 1)\end{array}$} \\
\hline \multicolumn{6}{|c|}{$\begin{array}{l}\text { Null deviance: } 594.75 \text { on } 421 \text { degrees of freedom } \\
\text { Residual deviance: } 509.73 \text { on } 418 \text { degrees of freedom } \\
\text { AIC: } 4742.2\end{array}$} \\
\hline \multicolumn{6}{|c|}{ Number of Fisher Scoring iterations: 1} \\
\hline \multicolumn{6}{|l|}{ Theta: 0.7554} \\
\hline $2 \times$ log-likelihood: -4732.192 & & & & & \\
\hline
\end{tabular}




\begin{tabular}{|c|c|c|c|c|c|}
\hline \multicolumn{6}{|l|}{$\begin{array}{l}\text { Call: } \\
\text { glm.nb(formula }=\text { Collision } \\
1.077610252 \text {, link }=\log \text { ) }\end{array}$} \\
\hline \multicolumn{6}{|l|}{ Deviance Residuals: } \\
\hline Min & 10 & Median & $3 Q$ & Max & \\
\hline \multirow[t]{2}{*}{-3.2611} & -0.7901 & -0.2174 & 0.3757 & 3.0925 & \\
\hline & Estimate & Std.Error & $z$ value & $\operatorname{Pr}(>|z|)$ & \\
\hline (Intercept) & $4.41 \mathrm{E}+00$ & $7.47 \mathrm{E}-02$ & 59.046 & $<2 \mathrm{E}-16$ & $* * *$ \\
\hline Tot-Int & 8.00E-03 & $1.62 \mathrm{E}-03$ & 4.929 & $8.26 \mathrm{E}-07$ & $* * *$ \\
\hline Bike-Path & $2.03 \mathrm{E}-02$ & $1.11 \mathrm{E}-02$ & 1.831 & 0.06711 & . \\
\hline VKT & $6.31 \mathrm{E}-06$ & $2.09 \mathrm{E}-06$ & 3.02 & 0.00253 & $* *$ \\
\hline Density- Trips-Enter-Center & $-1.72 \mathrm{E}-05$ & $3.42 \mathrm{E}-06$ & -5.024 & 5.07E-07 & $* * *$ \\
\hline \multicolumn{6}{|c|}{$\begin{array}{l}\text { Signif.codes: }\left(0^{\prime * * * \prime}\right)\left(0.001^{\prime * * \prime}\right)\left(0.01^{\prime * \prime}\right)\left(0.05^{\prime \prime}\right)\left(0.1^{\prime \prime}\right)(1) \\
\text { (Dispersion parameter for Negative Binomial }(2.148) \text { family taken to be } 1)\end{array}$} \\
\hline \multicolumn{6}{|c|}{ Null deviance: 551.18 on 421 degrees of freedom } \\
\hline \multicolumn{6}{|c|}{ Residual deviance: 498.57 on 417 degrees of freedom } \\
\hline \multicolumn{6}{|l|}{ AIC: 4851.7} \\
\hline \multicolumn{6}{|c|}{ Number of Fisher Scoring iterations: 1} \\
\hline \multicolumn{6}{|l|}{ Theta: 1.0776} \\
\hline \multicolumn{6}{|l|}{ Std. Err.: 0.0715} \\
\hline $2 \times$ log-likelihood: -4839.73 & & & & & \\
\hline
\end{tabular}




\begin{tabular}{|c|c|c|c|c|c|}
\hline $\begin{array}{l}\text { M-D-III } \\
\text { Call: } \\
\text { glm.nb(formula = Collisions } \\
\text { Center + Density- Trips-Ente }\end{array}$ & $\begin{array}{l}\text { ot-Int }+40.1 \\
\text { enter }+ \text { TLK }\end{array}$ & $\begin{array}{l}+50 . \mathrm{Km} / \mathrm{h} \\
\text { it.theta }=1\end{array}$ & $\begin{array}{l}\mathrm{Km} / \mathrm{h}+ \\
37935, \mathrm{li}\end{array}$ & $\begin{array}{l}\text { Density- Tr } \\
\text { g) }\end{array}$ & \\
\hline Deviance Residuals: & & & & & \\
\hline Min & 10 & Median & 30 & Max & \\
\hline-3.2402 & -0.7773 & -0.1939 & 0.3267 & 3.1613 & \\
\hline & Estimate & Std.Error & $z$ value & $\operatorname{Pr}(>|z|)$ & \\
\hline (Intercept) & $4.48 \mathrm{E}+00$ & $9.10 \mathrm{E}-02$ & 49.279 & $<2 \mathrm{E}-16$ & $* * *$ \\
\hline Tot-Int & $8.62 \mathrm{E}-03$ & $2.67 \mathrm{E}-03$ & 3.234 & 0.001219 & $* *$ \\
\hline $40 . \mathrm{Km} / \mathrm{h}$ & $1.16 \mathrm{E}-01$ & $3.71 E-02$ & 3.118 & 0.00182 & $* *$ \\
\hline $50 . \mathrm{Km} / \mathrm{h}$ & $7.36 \mathrm{E}-02$ & $2.84 \mathrm{E}-02$ & 2.591 & 0.009567 & $* *$ \\
\hline $100 . \mathrm{Km} / \mathrm{h}$ & $9.59 \mathrm{E}+02$ & $4.25 \mathrm{E}+02$ & 2.255 & 0.024103 & $*$ \\
\hline VKT & $1.26 \mathrm{E}-05$ & $3.31 \mathrm{E}-06$ & 3.815 & 0.000136 & $* * *$ \\
\hline Density- Trips-Out-Center & $9.15 \mathrm{E}-05$ & $3.62 \mathrm{E}-05$ & 2.531 & 0.011381 & $*$ \\
\hline Density- Trips-Enter-Center & $-2.39 \mathrm{E}-05$ & 4.97E-06 & -4.819 & $1.44 \mathrm{E}-06$ & $* * *$ \\
\hline TLKM & $-1.07 E-01$ & $3.52 \mathrm{E}-02$ & -3.031 & 0.002436 & $* *$ \\
\hline $\begin{array}{l}\text { Signif.codes: }\left(0^{* * * *}\right)(0.001 \\
\text { (Dispersion parameter for } \mathrm{N}\end{array}$ & $\begin{array}{l}\text { s' }^{\prime}\left(0.01^{* \prime}\right)( \\
\text { ative Binom }\end{array}$ & $\begin{array}{l}\left..^{\prime}\right)\left(0.1^{\prime \prime}\right) \\
.148) \text { famil }\end{array}$ & n to be 1 & & \\
\hline $\begin{array}{l}\text { Null deviance: } 571.68 \text { on } 42 \\
\text { Residual deviance: } 497.06 \text { ol } \\
\text { AIC: } 4840.2\end{array}$ & $\begin{array}{l}\text { degrees of } \mathrm{fr} \\
13 \text { degrees }\end{array}$ & $\begin{array}{l}\text { om } \\
\text { eedom }\end{array}$ & & & \\
\hline Number of Fisher Scoring ite & tions: 1 & & & & \\
\hline $\begin{array}{l}\text { Theta: } 1.1218 \\
\text { Std. Err.: } 0.0748\end{array}$ & & & & & \\
\hline 2 x log-likelihood: -4820.2 & & & & & \\
\hline
\end{tabular}




\begin{tabular}{|c|c|c|c|c|c|}
\hline $\begin{array}{l}\text { M-D-IV } \\
\text { Call: } \\
\text { glm.nb(formula = Collisions " } \\
\text { Density- Trips-Out-Center + }\end{array}$ & $\begin{array}{l}\text { AS-Driver + I } \\
\text { nsity- Trips- }\end{array}$ & $\begin{array}{l}\text { assenger + } \\
\text { r-Center, i }\end{array}$ & $\begin{array}{l}\text { ransit }+ \\
\text { ta }=1.1\end{array}$ & $\begin{array}{l}\text { alk + MS-B } \\
57, \text { link = I }\end{array}$ & \\
\hline Deviance Residuals: & & & & & \\
\hline Min & 10 & Median & $3 Q$ & Max & \\
\hline-3.3083 & -0.812 & -0.2416 & 0.3582 & 3.2844 & \\
\hline & Estimate & Std.Error & $z$ value & $\operatorname{Pr}(>|z|)$ & \\
\hline (Intercept) & $1.69 \mathrm{E}+00$ & $3.13 \mathrm{E}-01$ & 5.394 & $6.91 \mathrm{E}-08$ & $* * *$ \\
\hline MS-Driver & $2.33 \mathrm{E}-02$ & $3.71 \mathrm{E}-03$ & 6.276 & $3.48 \mathrm{E}-10$ & $* * *$ \\
\hline MS-Passenger & $5.45 \mathrm{E}-02$ & 7.67E-03 & 7.108 & $1.18 \mathrm{E}-12$ & $* * *$ \\
\hline MS-Transit & $4.06 \mathrm{E}-02$ & $6.56 \mathrm{E}-03$ & 6.185 & $6.23 \mathrm{E}-10$ & $* * *$ \\
\hline MS-Walk & $2.85 \mathrm{E}-02$ & $6.56 \mathrm{E}-03$ & 4.345 & $1.39 \mathrm{E}-05$ & $* * *$ \\
\hline MS-Bike & $4.33 \mathrm{E}-02$ & $2.26 \mathrm{E}-02$ & 1.913 & 0.05574 & . \\
\hline VKT & $3.63 \mathrm{E}-06$ & $2.23 \mathrm{E}-06$ & 1.626 & 0.10405 & \\
\hline Density- Trips-Out-Center & $9.70 \mathrm{E}-05$ & $3.21 \mathrm{E}-05$ & 3.024 & 0.00249 & $* *$ \\
\hline Density- Trips-Enter-Center & $-3.06 \mathrm{E}-05$ & $5.05 E-06$ & -6.055 & $1.40 \mathrm{E}-09$ & $* * *$ \\
\hline $\begin{array}{l}\text { Signif.codes: }\left(0^{\prime * * * \prime)}(0.001\right. \\
\text { (Dispersion parameter for } \mathrm{N}\end{array}$ & $\begin{array}{l}\left.k^{\prime}\right)\left(0.01^{\prime * \prime}\right) \\
\text { ative Binom }\end{array}$ & $\begin{array}{l}\text {.') }\left(0.1^{\prime \prime}\right) \\
.148) \text { famil }\end{array}$ & $\mathrm{n}$ to be 1 & & \\
\hline $\begin{array}{l}\text { Null deviance: } 587.61 \text { on } 42 \\
\text { Residual deviance: } 494.84 \text { ol } \\
\text { AIC: } 4824.5\end{array}$ & $\begin{array}{l}\text { degrees of } \mathrm{fr} \\
13 \text { degrees }\end{array}$ & $\begin{array}{l}\text { om } \\
\text { eedom }\end{array}$ & & & \\
\hline Number of Fisher Scoring ite & tions: 1 & & & & \\
\hline $\begin{array}{l}\text { Theta: } 1.1564 \\
\text { Std. Err.: } 0.0772\end{array}$ & & & & & \\
\hline 2 x log-likelihood: -4804.473 & & & & & \\
\hline
\end{tabular}




\begin{tabular}{|c|c|c|c|c|c|}
\hline $\begin{array}{l}\text { M-D-V } \\
\text { Call: } \\
\text { glm.nb(formula = Coll } \\
\text { Employment + Age.5- }\end{array}$ & $\begin{array}{l}\text { rea + MS-D } \\
25-64+A g\end{array}$ & $\begin{array}{l}+ \text { MS-Passe } \\
+ \text { VKT, init }\end{array}$ & $\begin{array}{l}+ \\
+ \text { MS-Tr } \\
=1.293\end{array}$ & $\begin{array}{l}\text { MS-Walk + } \\
2, \text { link = log }\end{array}$ & ike + \\
\hline Deviance Residuals: & & & & & \\
\hline Min & 10 & Median & $3 Q$ & Max & \\
\hline-3.5283 & -0.7553 & -0.1361 & 0.3892 & 2.8889 & \\
\hline & Estimate & Std.Error & $z$ value & $\operatorname{Pr}(>|z|)$ & \\
\hline (Intercept) & $1.09 \mathrm{E}+00$ & $3.18 \mathrm{E}-01$ & 3.433 & 0.000597 & $* * *$ \\
\hline Area & $8.47 \mathrm{E}-03$ & $1.60 \mathrm{E}-03$ & 5.281 & $1.28 \mathrm{E}-07$ & $* * *$ \\
\hline MS-Driver & $2.46 \mathrm{E}-02$ & $3.63 \mathrm{E}-03$ & 6.758 & $1.40 \mathrm{E}-11$ & $* * *$ \\
\hline MS-Passenger & $3.68 \mathrm{E}-02$ & $7.29 E-03$ & 5.047 & $4.49 \mathrm{E}-07$ & $* * *$ \\
\hline MS-Transit & 4.09E-02 & $6.63 \mathrm{E}-03$ & 6.178 & $6.49 \mathrm{E}-10$ & $* * *$ \\
\hline MS-Walk & $2.73 \mathrm{E}-02$ & $6.41 \mathrm{E}-03$ & 4.264 & $2.01 \mathrm{E}-05$ & $* * *$ \\
\hline MS-Bike & $4.60 \mathrm{E}-02$ & $2.15 \mathrm{E}-02$ & 2.136 & 0.03266 & $*$ \\
\hline Employment & $-6.42 E-06$ & $2.30 \mathrm{E}-06$ & -2.791 & 0.00525 & $* *$ \\
\hline Age.5-24 & $8.52 \mathrm{E}-03$ & $4.08 \mathrm{E}-03$ & 2.09 & 0.036609 & $*$ \\
\hline Age.25-64 & 7.06E-03 & $2.62 \mathrm{E}-03$ & 2.693 & 0.007074 & $* *$ \\
\hline Age $>65$ & $1.42 \mathrm{E}-02$ & $6.92 \mathrm{E}-03$ & 2.056 & 0.039816 & $*$ \\
\hline VKT & $8.08 \mathrm{E}-06$ & $1.94 \mathrm{E}-06$ & 4.17 & $3.04 \mathrm{E}-05$ & $* * *$ \\
\hline $\begin{array}{l}\text { Signif.codes: }\left(0^{* * * * \prime}\right) \\
\text { (Dispersion paramete }\end{array}$ & $\begin{array}{l}\left.k^{\prime}\right)\left(0.01^{*^{\prime}}\right) \\
\text { ative Binom }\end{array}$ & $\begin{array}{l}\left..^{\prime}\right)\left(0.1^{\prime \prime}\right) \\
.148) \text { famil }\end{array}$ & n to be 1 & & \\
\hline $\begin{array}{l}\text { Null deviance: } 650.44 \\
\text { Residual deviance: } 49 \\
\text { AIC: } 4782.4\end{array}$ & $\begin{array}{l}\text { degrees of } f \\
10 \text { degrees }\end{array}$ & $\begin{array}{l}\text { om } \\
\text { eedom }\end{array}$ & & & \\
\hline Number of Fisher Scor & tions: 1 & & & & \\
\hline $\begin{array}{l}\text { Theta: } 1.2936 \\
\text { Std. Err.: } 0.0882\end{array}$ & & & & & \\
\hline 2 x log-likelihood: -47 & & & & & \\
\hline
\end{tabular}




\begin{tabular}{|c|c|c|c|c|c|}
\hline \multicolumn{6}{|c|}{$\begin{array}{l}\text { Call: } \\
\text { gIm.nb(formula }=\text { Collisions } \sim \text { VKT }+ \text { Trips-Attracted }+ \text { Density- Trips-Enter-Center }+ \text { TLKM, init.theta }= \\
\text { 1.086709632, link }=\text { log) }\end{array}$} \\
\hline \multicolumn{6}{|l|}{ Deviance Residuals: } \\
\hline Min & 10 & Median & $3 Q$ & Max & \\
\hline \multirow[t]{2}{*}{-3.3627} & -0.8386 & -0.2433 & 0.3215 & 2.9863 & \\
\hline & Estimate & Std.Error & $z$ value & $\operatorname{Pr}(>|z|)$ & \\
\hline (Intercept) & $4.44 \mathrm{E}+00$ & $7.72 \mathrm{E}-02$ & 57.471 & $<2 \mathrm{E}-16$ & $* * *$ \\
\hline VKT & $5.89 \mathrm{E}-06$ & 2.67E-06 & 2.206 & 0.0274 & $*$ \\
\hline Trips-Attracted & 3.19E-05 & $6.39 \mathrm{E}-06$ & 4.987 & $6.13 \mathrm{E}-07$ & $* * *$ \\
\hline Density- Trips-Enter-Center & $-6.01 E-05$ & $8.48 \mathrm{E}-06$ & -7.085 & $1.39 \mathrm{E}-12$ & $* * *$ \\
\hline TLKM & $3.62 \mathrm{E}-02$ & $8.75 \mathrm{E}-03$ & 4.14 & $3.47 \mathrm{E}-05$ & $* * *$ \\
\hline \multicolumn{6}{|c|}{$\begin{array}{l}\text { Signif.codes: }\left(0^{\prime * * * \prime}\right)\left(0.001^{\prime * * \prime}\right)\left(0.01^{\prime * \prime}\right)\left(0.05^{\prime \prime}\right)\left(0.1^{\prime \prime}\right)(1) \\
\text { (Dispersion parameter for Negative Binomial }(2.148) \text { family taken to be } 1)\end{array}$} \\
\hline \multicolumn{6}{|c|}{ Null deviance: 555.4 on 421 degrees of freedom } \\
\hline \multicolumn{6}{|c|}{ Residual deviance: 496.31 on 417 degrees of freedom } \\
\hline \multicolumn{6}{|l|}{ AIC: 4845.7} \\
\hline \multicolumn{6}{|c|}{ Number of Fisher Scoring iterations: 1} \\
\hline \multicolumn{6}{|l|}{ Theta: 1.0867} \\
\hline \multicolumn{6}{|l|}{ Std. Err.: 0.072} \\
\hline $2 \times$ log-likelihood: -4833.69 & & & & & \\
\hline
\end{tabular}




\section{D.3 Non-Fatal Injury Collisions Models}

\begin{tabular}{|c|c|c|c|c|c|}
\hline \multicolumn{6}{|c|}{$\begin{array}{l}\text { A-D-II } \\
\text { Call: } \\
\text { glm.nb(formula = Collisions } \sim \text { Sig-Int }+ \text { Loc }+ \text { Density- Trips-Enter-Center, init.theta }=1.607057741, \text { link }= \\
\text { log) }\end{array}$} \\
\hline \multicolumn{6}{|l|}{ Deviance Residuals: } \\
\hline Min & 10 & Median & 30 & Max & \\
\hline \multirow[t]{2}{*}{-3.0267} & -0.8991 & -0.2054 & 0.3816 & 3.078 & \\
\hline & Estimate & Std.Error & $z$ value & $\operatorname{Pr}(>|z|)$ & \\
\hline (Intercept) & $3.25 \mathrm{E}+00$ & $5.92 \mathrm{E}-02$ & 54.876 & $<2 \mathrm{E}-16$ & $* * *$ \\
\hline Sig-Int & $1.06 \mathrm{E}-02$ & $4.37 \mathrm{E}-03$ & 2.435 & 0.014892 & * \\
\hline Loc & $2.82 \mathrm{E}-02$ & $1.02 \mathrm{E}-02$ & 2.767 & 0.005663 & $* *$ \\
\hline Density- Trips-Enter-Center & $-1.25 \mathrm{E}-05$ & $3.56 \mathrm{E}-06$ & -3.501 & 0.000463 & $* * *$ \\
\hline \multicolumn{6}{|c|}{$\begin{array}{l}\text { Signif.codes: }\left(0^{\prime * * * \prime}\right)\left(0.001^{\prime * * \prime}\right)\left(0.01^{\prime * \prime}\right)\left(0.05^{\prime \prime} .\right)\left(0.1^{\prime \prime}\right)(1) \\
\text { (Dispersion parameter for Negative Binomial }(2.148) \text { family taken to be } 1)\end{array}$} \\
\hline \multicolumn{6}{|c|}{ Null deviance: 501.37 on 421 degrees of freedom } \\
\hline \multicolumn{6}{|c|}{ Residual deviance: 474.23 on 418 degrees of freedom } \\
\hline \multicolumn{6}{|l|}{ AIC: 3665.3} \\
\hline \multicolumn{6}{|c|}{ Number of Fisher Scoring iterations: 1} \\
\hline \multicolumn{6}{|l|}{ Theta: 1.607} \\
\hline \multicolumn{6}{|l|}{ Std. Err.: 0.114} \\
\hline \multicolumn{6}{|l|}{2 x log-likelihood: -3655.308} \\
\hline
\end{tabular}




\begin{tabular}{|c|c|c|c|c|c|}
\hline $\begin{array}{l}\text { A-D-III } \\
\text { Call: } \\
\text { glm.nb(formula = Collisions } \\
\text { Trips-Attracted + Density- Tr }\end{array}$ & $\begin{array}{l}\text { ot-Int }+40 \text {. } \\
\text {-Enter-Cen }\end{array}$ & $\begin{array}{l}\text { +50.Km/r } \\
\text { TLKM, init. }\end{array}$ & $\begin{array}{l}. \mathrm{Km} / \mathrm{h}+1 \\
=1.69350\end{array}$ & $\begin{array}{l}\text { n/h }+ \text { Bike- } \\
\text { link = log) }\end{array}$ & VKT + \\
\hline Deviance Residuals: & & & & & \\
\hline Min & 10 & Median & $3 Q$ & Max & \\
\hline-3.1794 & -0.898 & -0.189 & 0.3789 & 3.3691 & \\
\hline & Estimate & Std.Error & $z$ value & $\operatorname{Pr}(>|z|)$ & \\
\hline (Intercept) & $3.06 \mathrm{E}+00$ & $7.69 \mathrm{E}-02$ & 39.818 & $<2 \mathrm{E}-16$ & $* * *$ \\
\hline Tot-Int & $6.31 \mathrm{E}-03$ & $1.63 \mathrm{E}-03$ & 3.875 & 0.000107 & $* * *$ \\
\hline $40 . \mathrm{Km} / \mathrm{h}$ & $-1.38 \mathrm{E}-01$ & $3.99 \mathrm{E}-02$ & -3.457 & 0.000545 & $* * *$ \\
\hline $50 . \mathrm{Km} / \mathrm{h}$ & $-1.46 \mathrm{E}-01$ & $3.59 \mathrm{E}-02$ & -4.064 & $4.82 \mathrm{E}-05$ & $* * *$ \\
\hline $70 . \mathrm{Km} / \mathrm{h}$ & $-2.59 \mathrm{E}-01$ & $1.35 \mathrm{E}-01$ & -1.922 & 0.054633 & . \\
\hline $100 . \mathrm{Km} / \mathrm{h}$ & $1.40 \mathrm{E}+03$ & $5.52 E+02$ & 2.541 & 0.011064 & $*$ \\
\hline Bike-Path & $2.56 \mathrm{E}-02$ & $1.11 \mathrm{E}-02$ & 2.313 & 0.020731 & $*$ \\
\hline VKT & $-1.71 \mathrm{E}-06$ & $6.12 \mathrm{E}-07$ & -2.79 & 0.005277 & $* *$ \\
\hline Trips-Attracted & $7.83 \mathrm{E}-06$ & $3.81 \mathrm{E}-06$ & 2.057 & 0.039639 & * \\
\hline Density- Trips-Enter-Center & $-1.54 \mathrm{E}-05$ & 3.97E-06 & -3.873 & 0.000107 & $* * *$ \\
\hline TLKM & $1.45 \mathrm{E}-01$ & $3.60 \mathrm{E}-02$ & 4.032 & $5.54 \mathrm{E}-05$ & $* * *$ \\
\hline $\begin{array}{l}\text { Signif.codes: }\left(0^{* * * * \prime}\right)(0.001 \\
\text { (Dispersion parameter for } \mathrm{N}\end{array}$ & $\begin{array}{l}\text { ') }\left(0.01^{* \prime}\right) \\
\text { tive Binom }\end{array}$ & $\begin{array}{l}\because \prime)\left(0.1^{\prime \prime}\right) \\
.148) \text { famil }\end{array}$ & $\mathrm{n}$ to be 1 & & \\
\hline $\begin{array}{l}\text { Null deviance: } 524.86 \text { on } 42 \\
\text { Residual deviance: } 473 \text { on } 4 \\
\text { AIC: } 3656.5\end{array}$ & $\begin{array}{l}\text { legrees of } f \\
\text { degrees of }\end{array}$ & om & & & \\
\hline Number of Fisher Scoring ite & ions: 1 & & & & \\
\hline $\begin{array}{l}\text { Theta: } 1.694 \\
\text { Std. Err.: } 0.121\end{array}$ & & & & & \\
\hline $2 \times$ log-likelihood: -3632.52 & & & & & \\
\hline
\end{tabular}


A-C-IV

Call:

glm.nb(formula = Collisions MS-Driver + MS-Passenger + MS-Transit + MS-Walk + MS-Bike + VKT + Trips-Attracted + Density- Trips-Out-Center + Density- Trips-Enter-Center + TLKM, init.theta =

2.223295897, link = log)

Deviance Residuals:

\begin{tabular}{|c|c|c|c|c|c|}
\hline Min & $1 Q$ & Median & $3 Q$ & Max & \\
\hline-4.1714 & -0.8363 & -0.1473 & 0.4411 & 2.7079 & \\
\hline \multicolumn{7}{|l|}{} \\
\hline \multicolumn{7}{|c|}{ Estimate } & Std.Error & z value & $\operatorname{Pr}(>|z|)$ & \\
\hline (Intercept) & $1.02 \mathrm{E}+00$ & $2.71 \mathrm{E}-01$ & 3.76 & 0.00017 & $* * *$ \\
\hline MS-Driver & $1.45 \mathrm{E}-02$ & $3.09 \mathrm{E}-03$ & 4.711 & $2.47 \mathrm{E}-06$ & $* * *$ \\
\hline MS-Passenger & $3.40 \mathrm{E}-02$ & $5.97 \mathrm{E}-03$ & 5.694 & $1.24 \mathrm{E}-08$ & $* * *$ \\
\hline MS-Transit & $3.21 \mathrm{E}-02$ & $5.36 \mathrm{E}-03$ & 5.989 & $2.12 \mathrm{E}-09$ & $* * *$ \\
\hline MS-Walk & $1.98 \mathrm{E}-02$ & $5.10 \mathrm{E}-03$ & 3.889 & 0.000101 & $* * *$ \\
\hline MS-Bike & $3.31 \mathrm{E}-02$ & $1.69 \mathrm{E}-02$ & 1.961 & 0.049871 & $*$ \\
\hline VKT & $3.57 \mathrm{E}-06$ & $8.03 \mathrm{E}-07$ & 4.45 & $8.60 \mathrm{E}-06$ & $* * *$ \\
\hline Trips-Attracted & $1.48 \mathrm{E}-04$ & $2.89 \mathrm{E}-05$ & 5.125 & $2.98 \mathrm{E}-07$ & $* * *$ \\
\hline Density- Trips-Out-Center & $5.57 \mathrm{E}-05$ & $2.33 \mathrm{E}-05$ & 2.385 & 0.017063 & $*$ \\
\hline Density- Trips-Enter-Center & $-1.86 \mathrm{E}-05$ & $3.66 \mathrm{E}-06$ & -5.075 & $3.87 \mathrm{E}-07$ & $* * *$ \\
\hline TLKM & $7.57 \mathrm{E}-03$ & $1.50 \mathrm{E}-03$ & 5.055 & $4.30 \mathrm{E}-07$ & $* * *$ \\
\hline Signif.codes:(0 &
\end{tabular}

Signif.codes: $\left(0^{\prime * * * \prime}\right)\left(0.001^{\left(* *^{\prime}\right.}\right)\left(0.01^{\prime * \prime}\right)\left(0.05^{\prime \prime}.\right)\left(0.1^{\prime \prime}\right)$ (1)

(Dispersion parameter for Negative Binomial (2.148) family taken to be 1 )

Null deviance: 663.65 on 421 degrees of freedom

Residual deviance: 473.87 on 411 degrees of freedom

AIC: 3548.5

Number of Fisher Scoring iterations: 1

Theta: 2.223

Std. Err.: 0.167

2 x log-likelihood: -3524.512 


\begin{tabular}{|c|c|c|c|c|c|}
\hline $\begin{array}{l}\text { A-C-V } \\
\text { Call: } \\
\text { glm.nb(formula = Collisions } \\
\text { Bike + Employment + Age.0- } \\
\text { TLKM, init.theta }=2.450535\end{array}$ & $\begin{array}{l}\text { o.Schools + } \\
\text { Age.25-64 } \\
\text {, link = log) }\end{array}$ & $\begin{array}{l}\text { Driver + M } \\
T+\text { Trips-O }\end{array}$ & $\begin{array}{l}\text { enger + } \\
\text { ter + Der }\end{array}$ & $\begin{array}{l}\text { ansit + MS- } \\
\text { Trips-Enter- }\end{array}$ & MS- \\
\hline Deviance Residuals: & & & & & \\
\hline Min & $1 Q$ & Median & $3 Q$ & Max & \\
\hline-4.083 & -0.8592 & -0.1517 & 0.406 & 3.5602 & \\
\hline & Estimate & Std.Error & z value & $\operatorname{Pr}(>|z|)$ & \\
\hline (Intercept) & $1.09 \mathrm{E}+00$ & $2.68 \mathrm{E}-01$ & 4.081 & $4.49 \mathrm{E}-05$ & $* * *$ \\
\hline No.Schools & $7.84 \mathrm{E}-02$ & $3.35 \mathrm{E}-02$ & 2.342 & 0.019163 & $*$ \\
\hline MS-Driver & $1.07 \mathrm{E}-02$ & $3.06 \mathrm{E}-03$ & 3.486 & 0.00049 & $* * *$ \\
\hline MS-Passenger & $2.86 \mathrm{E}-02$ & 5.99E-03 & 4.767 & $1.87 \mathrm{E}-06$ & $* * *$ \\
\hline MS-Transit & $3.12 \mathrm{E}-02$ & 5.02E-03 & 6.205 & $5.46 \mathrm{E}-10$ & $* * *$ \\
\hline MS-Walk & $1.47 \mathrm{E}-02$ & 5.00E-03 & 2.948 & 0.003201 & $* *$ \\
\hline MS-Bike & $3.18 \mathrm{E}-02$ & 1.64E-02 & 1.937 & 0.052733 & . \\
\hline Employment & $1.46 \mathrm{E}-04$ & $2.18 \mathrm{E}-05$ & 6.718 & $1.85 \mathrm{E}-11$ & $* * *$ \\
\hline Age.0-4 & $-3.95 \mathrm{E}-02$ & $1.63 \mathrm{E}-02$ & -2.43 & 0.015092 & $*$ \\
\hline Age.25-64 & 6.91E-03 & 1.99E-03 & 3.474 & 0.000512 & $* * *$ \\
\hline VKT & $3.38 \mathrm{E}-06$ & $7.69 \mathrm{E}-07$ & 4.403 & $1.07 \mathrm{E}-05$ & $* * *$ \\
\hline Trips-Out-Center & $1.04 \mathrm{E}-04$ & $3.71 \mathrm{E}-05$ & 2.811 & 0.004938 & $* *$ \\
\hline Density- Trips-Enter-Center & $-1.44 \mathrm{E}-05$ & $3.05 \mathrm{E}-06$ & -4.733 & $2.22 \mathrm{E}-06$ & $* * *$ \\
\hline TLKM & $4.76 \mathrm{E}-03$ & 1.57E-03 & 3.034 & 0.002416 & $* *$ \\
\hline $\begin{array}{l}\text { Signif.codes: }\left(0^{* * * * \prime}\right)(0.001 \\
\text { (Dispersion parameter for } \mathrm{N}\end{array}$ & $\begin{array}{l}\text { ) }\left(0.01^{* \prime}\right)( \\
\text { ative Binom }\end{array}$ & $\begin{array}{l}\left.\because^{\prime}\right)\left(0.1^{\prime \prime}\right) \\
.148) \text { famil }\end{array}$ & n to be 1 & & \\
\hline $\begin{array}{l}\text { Null deviance: } 720.68 \text { on } 42 \\
\text { Residual deviance: } 471.64 \text { o } \\
\text { AIC: } 3514.6\end{array}$ & $\begin{array}{l}\text { legrees of } \mathrm{fr} \\
08 \text { degrees }\end{array}$ & $\begin{array}{l}\text { om } \\
\text { eedom }\end{array}$ & & & \\
\hline Number of Fisher Scoring ite & ions: 1 & & & & \\
\hline $\begin{array}{l}\text { Theta: } 2.451 \\
\text { Std. Err.: } 0.188\end{array}$ & & & & & \\
\hline 2 x log-likelihood: -3484.60 & & & & & \\
\hline
\end{tabular}




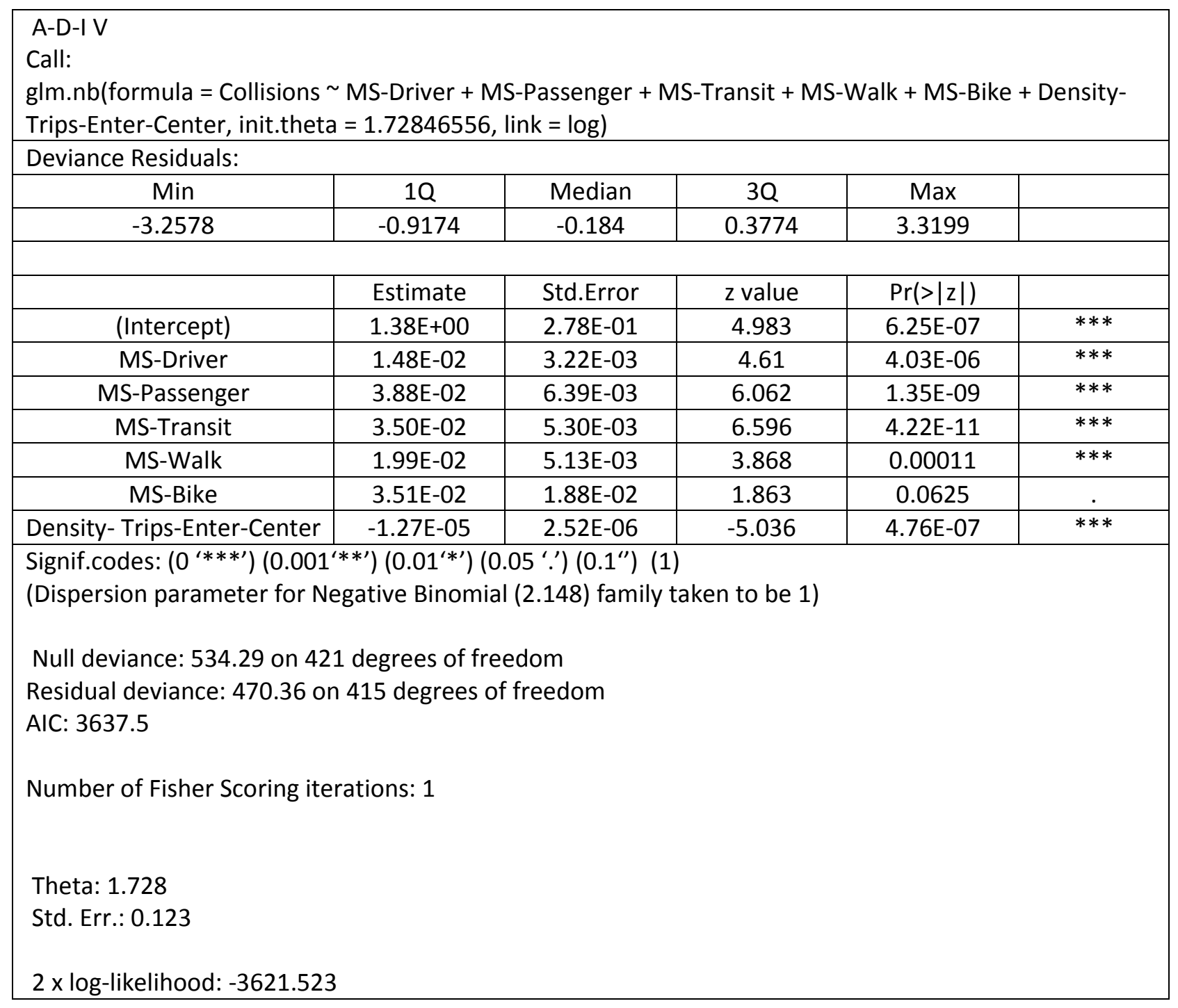




\begin{tabular}{|c|c|c|c|c|c|}
\hline \multicolumn{6}{|c|}{$\begin{array}{l}\text { Call: } \\
\text { glm.nb(formula }=\text { Collisions } ~ \text { Area }+ \text { MS-Driver + MS-Passenger + MS-Transit + MS-Walk + MS-Bike }+ \\
\text { Age.0-4 + Age.25-64 + Age }>65+\text { Density- Trips-Enter-Center, init.theta }=2.006358054, \text { link }=\text { log })\end{array}$} \\
\hline \multicolumn{6}{|l|}{ Deviance Residuals: } \\
\hline Min & 10 & Median & $3 Q$ & Max & \\
\hline \multirow[t]{2}{*}{-3.2161} & -0.8794 & -0.1137 & 0.3664 & 2.6628 & \\
\hline & Estimate & Std.Error & $z$ value & $\operatorname{Pr}(>|z|)$ & \\
\hline (Intercept) & $1.05 \mathrm{E}+00$ & $2.84 \mathrm{E}-01$ & 3.708 & 0.000209 & $* * *$ \\
\hline Area & $7.29 \mathrm{E}-03$ & $1.30 \mathrm{E}-03$ & 5.631 & $1.80 \mathrm{E}-08$ & $* * *$ \\
\hline MS-Driver & $1.58 \mathrm{E}-02$ & 3.19E-03 & 4.952 & $7.35 \mathrm{E}-07$ & $* * *$ \\
\hline MS-Passenger & $3.31 \mathrm{E}-02$ & $6.26 \mathrm{E}-03$ & 5.278 & $1.31 \mathrm{E}-07$ & $* * *$ \\
\hline MS-Transit & $4.03 \mathrm{E}-02$ & $5.13 \mathrm{E}-03$ & 7.85 & $4.16 \mathrm{E}-15$ & $* * *$ \\
\hline MS-Walk & $1.83 \mathrm{E}-02$ & $5.35 \mathrm{E}-03$ & 3.429 & 0.000605 & $* * *$ \\
\hline MS-Bike & $2.96 \mathrm{E}-02$ & $1.78 \mathrm{E}-02$ & 1.667 & 0.095568 & . \\
\hline Age.0-4 & $-2.70 \mathrm{E}-02$ & $1.69 \mathrm{E}-02$ & -1.593 & 0.111159 & \\
\hline Age.25-64 & $5.01 \mathrm{E}-03$ & $2.49 \mathrm{E}-03$ & 2.01 & 0.044387 & $*$ \\
\hline Age $>65$ & $1.05 \mathrm{E}-02$ & 5.77E-03 & 1.819 & 0.068949 & . \\
\hline Density- Trips-Enter-Center & $-1.09 \mathrm{E}-05$ & $2.56 \mathrm{E}-06$ & -4.261 & $2.03 E-05$ & $* * *$ \\
\hline \multicolumn{6}{|c|}{$\begin{array}{l}\text { Signif.codes: }\left(0^{\prime * * * \prime}\right)\left(0.001^{\prime * * \prime}\right)\left(0.01^{\prime * \prime}\right)\left(0.05^{\prime} . \prime\right)\left(0.1^{\prime \prime}\right)(1) \\
\text { (Dispersion parameter for Negative Binomial }(2.148) \text { family taken to be } 1)\end{array}$} \\
\hline \multicolumn{6}{|c|}{$\begin{array}{l}\text { Null deviance: } 607.85 \text { on } 421 \text { degrees of freedom } \\
\text { Residual deviance: } 469.89 \text { on } 411 \text { degrees of freedom } \\
\text { AIC: } 3585\end{array}$} \\
\hline \multicolumn{6}{|c|}{ Number of Fisher Scoring iterations: 1} \\
\hline \multicolumn{2}{|l|}{ Std. Err.: 0.148} & & & & \\
\hline 2 x log-likelihood: -3560.99 & & & & & \\
\hline
\end{tabular}




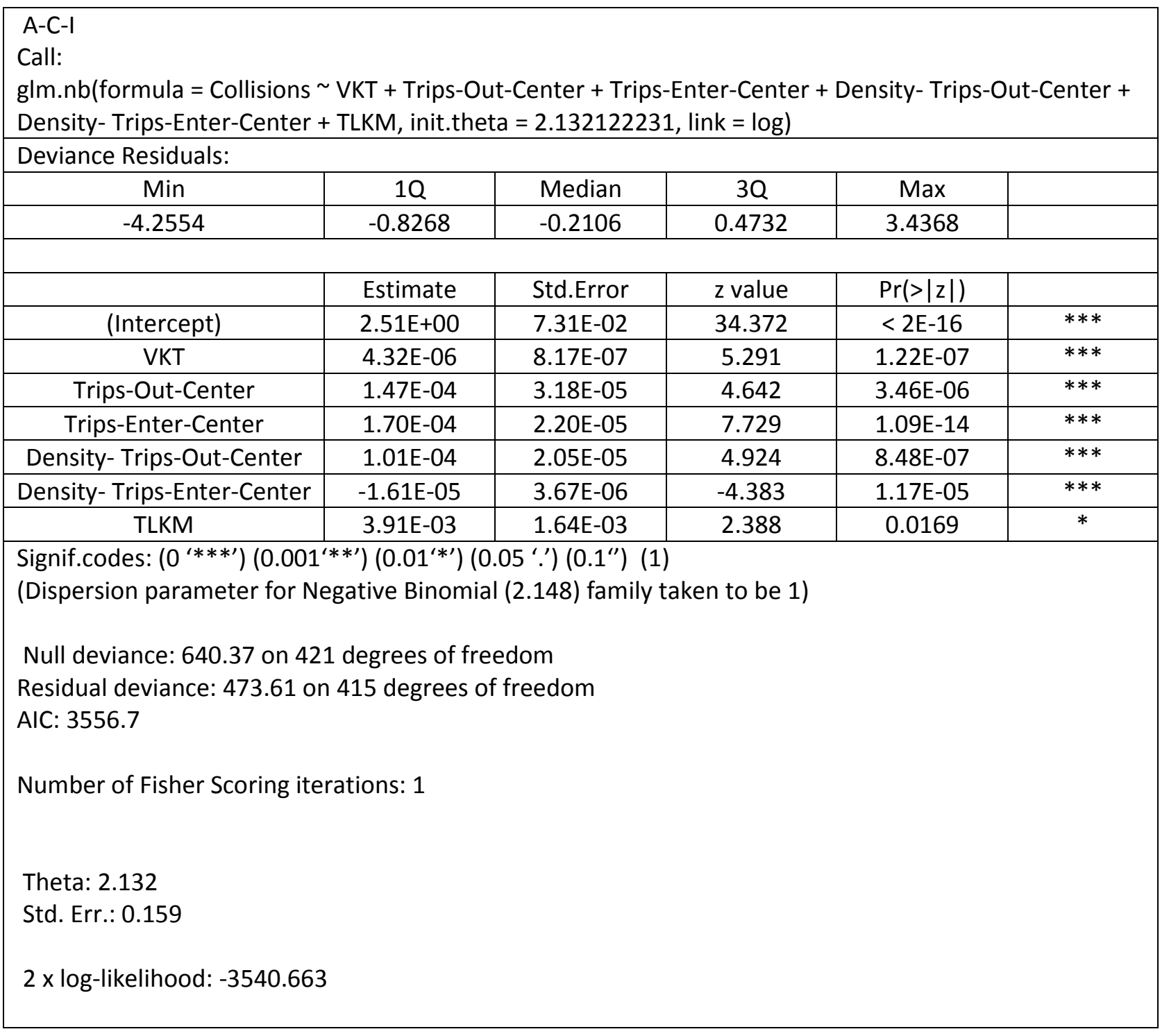




\begin{tabular}{|c|c|c|c|c|c|}
\hline \multicolumn{6}{|c|}{$\begin{array}{l}\text { Call: } \\
\text { glm.nb(formula }=\text { Collisions } \sim \text { Density- Trips-Out-Center + Density- Trips-Enter-Center, init.theta }= \\
\text { 1.576935911, link }=\log )\end{array}$} \\
\hline \multicolumn{6}{|l|}{ Deviance Residuals: } \\
\hline Min & 10 & Median & $3 Q$ & Max & \\
\hline-3.149 & -0.8874 & -0.2031 & 0.3735 & 2.915 & \\
\hline & Estimate & Std.Error & $z$ value & $\operatorname{Pr}(>|z|)$ & \\
\hline (Intercept) & $3.31 \mathrm{E}+00$ & $5.26 \mathrm{E}-02$ & 63.013 & $<2 \mathrm{E}-16$ & $* * *$ \\
\hline Density- Trips-Out-Center & $6.75 \mathrm{E}-05$ & $2.05 \mathrm{E}-05$ & 3.293 & 0.000992 & $* * *$ \\
\hline Density- Trips-Enter-Center & $-1.30 \mathrm{E}-05$ & $3.24 \mathrm{E}-06$ & -4.013 & $6.00 \mathrm{E}-05$ & $* * *$ \\
\hline \multicolumn{6}{|c|}{$\begin{array}{l}\text { Signif.codes: }\left(0^{* * * \prime}\right)\left(0.001^{* * \prime}\right)\left(0.01^{\prime * \prime}\right)\left(0.05^{\prime} .^{\prime}\right)\left(0.1^{\prime \prime}\right)(1) \\
\text { (Dispersion parameter for Negative Binomial }(2.148) \text { family taken to be } 1)\end{array}$} \\
\hline \multicolumn{6}{|c|}{ Null deviance: 493.13 on 421 degrees of freedom } \\
\hline \multicolumn{6}{|c|}{ Residual deviance: 474.64 on 419 degrees of freedom } \\
\hline \multicolumn{6}{|l|}{ AIC: 3671.6} \\
\hline \multicolumn{6}{|c|}{ Number of Fisher Scoring iterations: 1} \\
\hline \multicolumn{6}{|l|}{ Theta: 1.577} \\
\hline \multicolumn{6}{|l|}{ Std. Err.: 0.111} \\
\hline $2 \times$ log-likelihood: -3663.55 & & & & & \\
\hline
\end{tabular}




\begin{tabular}{|c|c|c|c|c|c|}
\hline \multicolumn{6}{|c|}{$\begin{array}{l}\text { Call: } \\
\text { glm.nb(formula = Collisions } \sim \text { No.Schools + Area + MS-Driver + MS-Passenger + MS-Transit + MS-Walk + } \\
\text { Employment + Age. } 25-64+\text { Age }>65+\text { VKT + Trips-Out-Center + Density- Trips-Out-Center + Density- } \\
\text { Trips-Enter-Center, init.theta }=1.619422712, \text { link }=\text { log) }\end{array}$} \\
\hline \multicolumn{6}{|l|}{ Deviance Residuals: } \\
\hline Min & 10 & Median & $3 Q$ & Max & \\
\hline \multirow[t]{2}{*}{-2.801} & -0.959 & -0.2439 & 0.3736 & 4.2574 & \\
\hline & Estimate & Std.Error & $z$ value & $\operatorname{Pr}(>|z|)$ & \\
\hline (Intercept) & $-8.02 \mathrm{E}-01$ & 4.27E-01 & -1.878 & 0.060405 & . \\
\hline No.Schools & $8.38 \mathrm{E}-02$ & $4.27 \mathrm{E}-02$ & 1.964 & 0.049539 & $*$ \\
\hline Area & $6.01 \mathrm{E}-03$ & $1.57 \mathrm{E}-03$ & 3.824 & 0.000132 & $* * *$ \\
\hline MS-Driver & $1.34 \mathrm{E}-02$ & $4.73 \mathrm{E}-03$ & 2.833 & 0.004605 & $* *$ \\
\hline MS-Passenger & 3.69E-02 & $8.24 \mathrm{E}-03$ & 4.472 & 7.74E-06 & $* * *$ \\
\hline MS-Transit & $4.89 \mathrm{E}-02$ & 7.01E-03 & 6.982 & $2.92 \mathrm{E}-12$ & $* * *$ \\
\hline MS-Walk & $1.85 \mathrm{E}-02$ & $6.93 \mathrm{E}-03$ & 2.67 & 0.007586 & $* *$ \\
\hline Employment & $2.14 \mathrm{E}-04$ & $2.87 \mathrm{E}-05$ & 7.44 & $1.01 \mathrm{E}-13$ & $* * *$ \\
\hline Age.25-64 & $1.78 \mathrm{E}-02$ & $2.43 \mathrm{E}-03$ & 7.319 & $2.50 \mathrm{E}-13$ & $* * *$ \\
\hline Age $>65$ & $2.05 \mathrm{E}-02$ & $6.61 \mathrm{E}-03$ & 3.099 & 0.001941 & $* *$ \\
\hline VKT & $2.43 \mathrm{E}-06$ & $6.77 \mathrm{E}-07$ & 3.592 & 0.000328 & $* * *$ \\
\hline Trips-Out-Center & $2.10 \mathrm{E}-04$ & $4.43 \mathrm{E}-05$ & 4.736 & $2.18 \mathrm{E}-06$ & $* * *$ \\
\hline Density- Trips-Out-Center & 1.09E-04 & $3.36 \mathrm{E}-05$ & 3.242 & 0.001188 & $* *$ \\
\hline Density- Trips-Enter-Center & $-4.51 \mathrm{E}-05$ & $6.62 \mathrm{E}-06$ & -6.81 & $9.78 \mathrm{E}-12$ & $* * *$ \\
\hline \multicolumn{6}{|c|}{$\begin{array}{l}\text { Signif.codes: }\left(0^{\prime * * * \prime}\right)\left(0.001^{\prime * *^{\prime}}\right)\left(0.01^{\prime * \prime}\right)\left(0.05^{\prime \prime}\right)\left(0.1^{\prime \prime}\right)(1) \\
\text { (Dispersion parameter for Negative Binomial }(2.148) \text { family taken to be } 1)\end{array}$} \\
\hline \multicolumn{6}{|c|}{ Null deviance: 1004.71 on 421 degrees of freedom } \\
\hline \multicolumn{6}{|c|}{ Residual deviance: 498.91 on 408 degrees of freedom } \\
\hline \multicolumn{6}{|l|}{ AIC: 3346.5} \\
\hline \multicolumn{6}{|c|}{ Number of Fisher Scoring iterations: 1} \\
\hline \multicolumn{6}{|l|}{ Theta: 1.619} \\
\hline \multicolumn{6}{|l|}{ Std. Err.: 0.131} \\
\hline $2 \times$ log-likelihood: -3316.49 & & & & & \\
\hline
\end{tabular}




\begin{tabular}{|c|c|c|c|c|c|}
\hline \multicolumn{6}{|c|}{$\begin{array}{l}\text { Call: } \\
\text { glm.nb(formula }=\text { Collisions } \sim \text { VKT + Trips-Out-Center + Trips-Enter-Center + Density- Trips-Out-Center + } \\
\text { Density- Trips-Enter-Center + TLKM, init.theta }=1.075269442, \text { link }=\text { log) }\end{array}$} \\
\hline \multicolumn{6}{|l|}{ Deviance Residuals: } \\
\hline Min & 10 & Median & $3 Q$ & Max & \\
\hline \multirow[t]{2}{*}{-2.9033} & -0.9757 & -0.2982 & 0.3638 & 2.8028 & \\
\hline & Estimate & Std.Error & $z$ value & $\operatorname{Pr}(>|z|)$ & \\
\hline (Intercept) & $1.88 \mathrm{E}+00$ & $1.02 \mathrm{E}-01$ & 18.354 & $<2 \mathrm{E}-16$ & $* * *$ \\
\hline VKT & $3.23 \mathrm{E}-06$ & $8.30 \mathrm{E}-07$ & 3.895 & $9.81 \mathrm{E}-05$ & $* * *$ \\
\hline Trips-Out-Center & $3.15 E-04$ & 4.64E-05 & 6.788 & $1.14 \mathrm{E}-11$ & $* * *$ \\
\hline Trips-Enter-Center & $1.30 \mathrm{E}-04$ & $3.14 \mathrm{E}-05$ & 4.136 & $3.53 \mathrm{E}-05$ & $* * *$ \\
\hline Density- Trips-Out-Center & $2.82 \mathrm{E}-04$ & $3.33 \mathrm{E}-05$ & 8.482 & $<2 \mathrm{E}-16$ & $* * *$ \\
\hline Density- Trips-Enter-Center & $-4.32 \mathrm{E}-05$ & $6.61 \mathrm{E}-06$ & -6.532 & $6.51 \mathrm{E}-11$ & $* * *$ \\
\hline TLKM & $7.72 \mathrm{E}-03$ & $2.18 \mathrm{E}-03$ & 3.54 & 0.000399 & $* * *$ \\
\hline \multicolumn{6}{|c|}{$\begin{array}{l}\text { Signif.codes: }\left(0^{* * * \prime \prime}\right)\left(0.001^{\prime * *^{\prime}}\right)\left(0.01^{\prime * \prime}\right)\left(0.05^{\prime \prime}\right)\left(0.1^{\prime \prime}\right)(1) \\
\text { (Dispersion parameter for Negative Binomial }(2.148) \text { family taken to be } 1)\end{array}$} \\
\hline \multicolumn{6}{|c|}{ Null deviance: 720.75 on 421 degrees of freedom } \\
\hline \multicolumn{6}{|c|}{ Residual deviance: 503.84 on 415 degrees of freedom } \\
\hline \multicolumn{6}{|l|}{ AIC: 3492.3} \\
\hline \multicolumn{6}{|c|}{ Number of Fisher Scoring iterations: 1} \\
\hline \multicolumn{6}{|l|}{ Theta: 1.0753} \\
\hline \multicolumn{6}{|l|}{ Std. Err.: 0.0812} \\
\hline \multicolumn{6}{|c|}{ Warning while fitting theta: alternation limit reached } \\
\hline \multicolumn{6}{|l|}{2 x log-likelihood: -3476.277} \\
\hline
\end{tabular}




\begin{tabular}{|c|c|c|c|c|c|}
\hline \multicolumn{6}{|c|}{$\begin{array}{l}\text { Call: } \\
\text { glm.nb(formula }=\text { Collisions } \sim \text { No Bus stops }+ \text { Tot-Int }+40 . K m / h+50 . K m / h+60 . K m / h+70 . K m / h+ \\
80 . K m / h+100 . K m / h+V K T+\text { Trips-Attracted }+ \text { Density- Trips-Enter-Center }+ \text { TLKM, init.theta }= \\
1.380845067, \text { link }=\log )\end{array}$} \\
\hline \multicolumn{6}{|l|}{ Deviance Residuals: } \\
\hline Min & $1 Q$ & Median & $3 Q$ & Max & \\
\hline \multirow[t]{2}{*}{-3.0113} & -0.9339 & -0.2392 & 0.347 & 3.7124 & \\
\hline & Estimate & Std.Error & $z$ value & $\operatorname{Pr}(>|z|)$ & \\
\hline (Intercept) & $2.58 \mathrm{E}+00$ & $8.61 \mathrm{E}-02$ & 29.918 & $<2 \mathrm{E}-16$ & $* * *$ \\
\hline No Bus stops & $1.54 \mathrm{E}-02$ & $3.90 \mathrm{E}-03$ & 3.95 & $7.81 \mathrm{E}-05$ & $* * *$ \\
\hline Tot-Int & $7.79 \mathrm{E}-03$ & $2.45 \mathrm{E}-03$ & 3.176 & 0.00149 & $* *$ \\
\hline $40 . \mathrm{Km} / \mathrm{h}$ & $-4.99 \mathrm{E}+00$ & $2.56 \mathrm{E}+00$ & -1.947 & 0.05159 &. \\
\hline $50 . \mathrm{Km} / \mathrm{h}$ & $-5.00 \mathrm{E}+00$ & $2.56 \mathrm{E}+00$ & -1.952 & 0.05098 & . \\
\hline $60 . \mathrm{Km} / \mathrm{h}$ & $-4.82 \mathrm{E}+00$ & $2.56 \mathrm{E}+00$ & -1.88 & 0.06007 & . \\
\hline $70 . \mathrm{Km} / \mathrm{h}$ & $-4.68 \mathrm{E}+00$ & $2.56 \mathrm{E}+00$ & -1.827 & 0.06771 &. \\
\hline $80 . \mathrm{Km} / \mathrm{h}$ & $-4.52 \mathrm{E}+00$ & $2.56 \mathrm{E}+00$ & -1.765 & 0.07752 &. \\
\hline $100 . \mathrm{Km} / \mathrm{h}$ & $1.65 \mathrm{E}+03$ & $6.11 \mathrm{E}+02$ & 2.696 & 0.00702 & $* *$ \\
\hline VKT & $3.11 \mathrm{E}-06$ & $1.38 \mathrm{E}-06$ & 2.263 & 0.02362 & $*$ \\
\hline Trips-Attracted & $3.16 \mathrm{E}-05$ & $6.54 \mathrm{E}-06$ & 4.833 & $1.34 \mathrm{E}-06$ & $* * *$ \\
\hline Density- Trips-Enter-Center & $-5.78 \mathrm{E}-05$ & $8.38 \mathrm{E}-06$ & -6.895 & $5.38 \mathrm{E}-12$ & $* * *$ \\
\hline TLKM & $5.01 \mathrm{E}+00$ & $2.56 \mathrm{E}+00$ & 1.954 & 0.0507 & . \\
\hline \multicolumn{6}{|c|}{$\begin{array}{l}\text { Signif.codes: }\left(0^{\prime * * * \prime}\right)\left(0.001^{\prime * * \prime}\right)\left(0.01^{\prime * \prime}\right)\left(0.05^{\prime \prime}{ }^{\prime}\right)\left(0.1^{\prime \prime}\right)(1) \\
\text { (Dispersion parameter for Negative Binomial }(2.148) \text { family taken to be } 1)\end{array}$} \\
\hline \multicolumn{6}{|c|}{ Null deviance: 626.38 on 421 degrees of freedom } \\
\hline \multicolumn{6}{|c|}{ Residual deviance: 490.79 on 409 degrees of freedom } \\
\hline \multicolumn{6}{|l|}{ AIC: 3618.9} \\
\hline \multicolumn{6}{|c|}{ Number of Fisher Scoring iterations: 1} \\
\hline \multicolumn{6}{|l|}{ Theta: 1.381} \\
\hline \multicolumn{6}{|l|}{ Std. Err.: 0.101} \\
\hline 2 x log-likelihood: -3590.922 & & & & & \\
\hline
\end{tabular}




\begin{tabular}{|c|c|c|c|c|c|}
\hline \multicolumn{6}{|c|}{$\begin{array}{l}\text { Call: } \\
\text { glm.nb(formula = Collisions } \sim \text { VKT + Trips-Out-Center + Trips-Enter-Center + Density- Trips-Out-Center + } \\
\text { Density- Trips-Enter-Center + TLKM, init.theta }=1.818017222, \text { link }=\log )\end{array}$} \\
\hline \multicolumn{6}{|l|}{ Deviance Residuals: } \\
\hline Min & 10 & Median & $3 Q$ & Max & \\
\hline \multirow[t]{2}{*}{-2.9076} & -0.8937 & -0.2749 & 0.4451 & 2.5361 & \\
\hline & Estimate & Std.Error & $z$ value & $\operatorname{Pr}(>|z|)$ & \\
\hline (Intercept) & $1.92 \mathrm{E}+00$ & 8.03E-02 & 23.857 & $<2 \mathrm{E}-16$ & $* * *$ \\
\hline VKT & $3.07 E-06$ & $7.78 \mathrm{E}-07$ & 3.945 & 7.99E-05 & $* * *$ \\
\hline Trips-Out-Center & $2.59 \mathrm{E}-04$ & 3.59E-05 & 7.214 & $5.42 \mathrm{E}-13$ & $* * *$ \\
\hline Trips-Enter-Center & $2.77 \mathrm{E}-04$ & $2.41 \mathrm{E}-05$ & 11.509 & $<2 \mathrm{E}-16$ & $* * *$ \\
\hline Density- Trips-Out-Center & $2.02 \mathrm{E}-04$ & $2.54 \mathrm{E}-05$ & 7.951 & $1.85 \mathrm{E}-15$ & $* * *$ \\
\hline Density- Trips-Enter-Center & $-3.24 \mathrm{E}-05$ & $4.98 \mathrm{E}-06$ & -6.504 & $7.81 \mathrm{E}-11$ & $* * *$ \\
\hline TLKM & $6.41 \mathrm{E}-03$ & $1.73 \mathrm{E}-03$ & 3.714 & 0.000204 & $* * *$ \\
\hline \multicolumn{6}{|c|}{$\begin{array}{l}\text { Signif.codes: }\left(0^{* * * \prime}\right)\left(0.001^{\prime * * \prime}\right)\left(0.01^{\prime * \prime}\right)\left(0.05^{\prime \prime}\right)\left(0.1^{\prime \prime}\right)(1) \\
\text { (Dispersion parameter for Negative Binomial }(2.148) \text { family taken to be } 1)\end{array}$} \\
\hline $\begin{array}{l}\text { Null deviance: } 786.42 \text { on } 42 \\
\text { Residual deviance: } 495.76 \text { o } \\
\text { AIC: } 3503.7\end{array}$ & $\begin{array}{l}\text { degrees of } f \\
+15 \text { degrees }\end{array}$ & $\begin{array}{l}\text { om } \\
\text { eedom }\end{array}$ & & & \\
\hline \multicolumn{6}{|c|}{ Number of Fisher Scoring iterations: 1} \\
\hline \multicolumn{6}{|l|}{ Theta: 1.818} \\
\hline $2 \times$ log-likelihood: -3487.70 & & & & & \\
\hline
\end{tabular}


F-D-III

Call:

glm.nb(formula $=$ Collisions $\sim$ No Bus stops + Tot-Int $+40 . \mathrm{Km} / \mathrm{h}+50 . \mathrm{Km} / \mathrm{h}+60 . \mathrm{Km} / \mathrm{h}+70 . \mathrm{Km} / \mathrm{h}+$ $80 . \mathrm{Km} / \mathrm{h}+100 . \mathrm{Km} / \mathrm{h}+\mathrm{VKT}+$ Trips-Attracted + Density- Trips-Enter-Center + TLKM, init.theta $=$

1.151887319, link = log)

Deviance Residuals:

\begin{tabular}{|c|c|c|c|c|c|}
\hline Min & 10 & Median & $3 Q$ & Max & \\
\hline-2.8407 & -0.9836 & -0.3023 & 0.3614 & 3.5131 & \\
\hline & Estimate & Std.Error & $z$ value & $\operatorname{Pr}(>|z|)$ & \\
\hline (Intercept) & $2.55 \mathrm{E}+00$ & $9.29 \mathrm{E}-02$ & 27.496 & $<2 \mathrm{E}-16$ & *** \\
\hline No Bus stops & $1.60 \mathrm{E}-02$ & 4.33E-03 & 3.697 & 0.000219 & $* * *$ \\
\hline Tot-Int & $9.92 \mathrm{E}-03$ & $2.72 \mathrm{E}-03$ & 3.647 & 0.000265 & $* * *$ \\
\hline $40 . \mathrm{Km} / \mathrm{h}$ & $-4.82 E+00$ & $2.80 \mathrm{E}+00$ & -1.721 & 0.085211 & $\cdot$ \\
\hline $50 . \mathrm{Km} / \mathrm{h}$ & $-4.84 \mathrm{E}+00$ & $2.80 \mathrm{E}+00$ & -1.727 & 0.084088 & . \\
\hline $60 . \mathrm{Km} / \mathrm{h}$ & $-4.69 E+00$ & $2.80 \mathrm{E}+00$ & -1.674 & 0.094106 & . \\
\hline $70 . \mathrm{Km} / \mathrm{h}$ & $-4.54 \mathrm{E}+00$ & $2.80 E+00$ & -1.62 & 0.105318 & \\
\hline $80 . \mathrm{Km} / \mathrm{h}$ & $-4.33 E+00$ & $2.80 \mathrm{E}+00$ & -1.547 & 0.121937 & \\
\hline $100 . \mathrm{km} / \mathrm{h}$ & $1.79 \mathrm{E}+03$ & $6.67 E+02$ & 2.677 & 0.007423 & $* *$ \\
\hline VKT & 4.17E-06 & $1.51 E-06$ & 2.761 & 0.005755 & $* *$ \\
\hline Trips-Attracted & $4.72 \mathrm{E}-05$ & $7.68 \mathrm{E}-06$ & 6.145 & 7.99E-10 & $* * *$ \\
\hline Density-Trips-Enter-Center & $-7.89 \mathrm{E}-05$ & 9.97E-06 & -7.919 & $2.40 \mathrm{E}-15$ & $* * *$ \\
\hline TLKM & $4.84 \mathrm{E}+00$ & $2.80 \mathrm{E}+00$ & 1.728 & 0.083918 & . \\
\hline
\end{tabular}

Signif.codes: $\left(0^{\prime * * * \prime}\right)\left(0.001^{* * \prime}\right)\left(0.01^{\prime * \prime}\right)\left(0.05^{\prime \prime}.\right)\left(0.1^{\prime \prime}\right)(1)$

(Dispersion parameter for Negative Binomial (2.148) family taken to be 1 )

Null deviance: 649.95 on 421 degrees of freedom

Residual deviance: 494.18 on 409 degrees of freedom

AIC: 3597.5

Number of Fisher Scoring iterations: 1

Theta: 1.1519

Std. Err.: 0.0838

2 x log-likelihood: -3569.548 


\begin{tabular}{|c|c|c|c|c|c|}
\hline $\begin{array}{l}\text { D-D-V } \\
\text { Call: } \\
\text { glm.nb(formula = Collisions } \\
\text { Age. } 25-64+\text { Age }>65+\text { Den }\end{array}$ & $\begin{array}{l}\text { rea + MS-Di } \\
\text { - Trips-Ente }\end{array}$ & $\begin{array}{l}\text { + MS-Pass } \\
\text { nter, init.th }\end{array}$ & $\begin{array}{r}\text { MS-Tra } \\
+.229853 \\
\end{array}$ & $\begin{array}{l}\text { MS-Walk + } \\
\text { ink = log) }\end{array}$ & ke + \\
\hline Deviance Residuals: & & & & & \\
\hline Min & 10 & Median & $3 Q$ & Max & \\
\hline-2.7761 & -0.9881 & -0.2417 & 0.3515 & 2.8861 & \\
\hline & Estimate & Std.Error & $z$ value & $\operatorname{Pr}(>|z|)$ & \\
\hline (Intercept) & $2.18 \mathrm{E}-01$ & $3.77 \mathrm{E}-01$ & 0.579 & 0.5629 & \\
\hline Area & 9.07E-03 & $1.65 \mathrm{E}-03$ & 5.515 & $3.48 \mathrm{E}-08$ & $* * *$ \\
\hline MS-Driver & $2.03 \mathrm{E}-02$ & $4.20 \mathrm{E}-03$ & 4.829 & 1.37E-06 & $* * *$ \\
\hline MS-Passenger & $3.93 \mathrm{E}-02$ & 8.07E-03 & 4.864 & $1.15 \mathrm{E}-06$ & $* * *$ \\
\hline MS-Transit & $5.72 \mathrm{E}-02$ & $6.77 \mathrm{E}-03$ & 8.447 & $<2 \mathrm{E}-16$ & $* * *$ \\
\hline MS-Walk & $2.70 \mathrm{E}-02$ & $6.78 \mathrm{E}-03$ & 3.979 & $6.91 \mathrm{E}-05$ & $* * *$ \\
\hline MS-Bike & $3.88 \mathrm{E}-02$ & $2.23 \mathrm{E}-02$ & 1.743 & 0.0813 &. \\
\hline Age.25-64 & $4.81 \mathrm{E}-03$ & $2.47 \mathrm{E}-03$ & 1.944 & 0.052 & . \\
\hline Age $>65$ & $1.68 \mathrm{E}-02$ & $7.21 \mathrm{E}-03$ & 2.332 & 0.0197 & $*$ \\
\hline Density- Trips-Enter-Center & $-1.72 \mathrm{E}-05$ & $3.68 \mathrm{E}-06$ & -4.672 & $2.98 \mathrm{E}-06$ & $* * *$ \\
\hline $\begin{array}{l}\text { Signif.codes: }\left(0^{\prime * * * \prime}\right)(0.001 \\
\text { (Dispersion parameter for } \mathrm{N}\end{array}$ & $\begin{array}{l}\text { (') }\left(0.01^{\prime * \prime}\right) \\
\text { ative Binom }\end{array}$ & $\begin{array}{l}\text {.') }\left(0.1^{\prime \prime}\right) \\
.148) \text { famil }\end{array}$ & $\mathrm{n}$ to be 1 & & \\
\hline Null deviance: 637.25 on 42 & degrees of $\mathrm{fr}$ & & & & \\
\hline Residual deviance: 488.46 o & 12 degrees & eedom & & & \\
\hline AIC: 3602.8 & & & & & \\
\hline Number of Fisher Scoring it & tions: 1 & & & & \\
\hline Theta: 1.2299 & & & & & \\
\hline Std. Err.: 0.0882 & & & & & \\
\hline $2 \times$ log-likelihood: -3580.82 & & & & & \\
\hline
\end{tabular}


D-D-I

Call:

glm.nb(formula $=$ Collisions $\sim$ Density- Trips-Out-Center + Density- Trips-Enter-Center, init.theta $=$ 0.9725711166, link $=\log$ )

Deviance Residuals:

\begin{tabular}{|c|c|c|c|c|c|}
\hline Min & $1 \mathrm{Q}$ & Median & $3 \mathrm{Q}$ & Max & \\
\hline-2.6836 & -0.9558 & -0.225 & 0.3959 & 2.7879 & \\
\hline \multicolumn{5}{|l|}{} \\
\hline & Estimate & Std.Error & z value & $\operatorname{Pr}(>|\mathrm{z}|)$ & \\
\hline (Intercept) & $3.26 \mathrm{E}+00$ & $6.71 \mathrm{E}-02$ & 48.648 & $<2 \mathrm{E}-16$ & $* * *$ \\
\hline Density- Trips-Out-Center & $1.09 \mathrm{E}-04$ & $2.89 \mathrm{E}-05$ & 3.783 & 0.000155 & $* * *$ \\
\hline Density- Trips-Enter-Center & $-2.29 \mathrm{E}-05$ & $5.10 \mathrm{E}-06$ & -4.498 & $6.85 \mathrm{E}-06$ & $* * *$ \\
\hline
\end{tabular}

Signif.codes: $\left(0^{(* * * \prime}\right)\left(0.001^{\prime * * \prime}\right)\left(0.01^{\prime * \prime}\right)\left(0.05^{\prime \prime}.\right)\left(0.1^{\prime \prime}\right)$ (1)

(Dispersion parameter for Negative Binomial (2.148) family taken to be 1 )

Null deviance: 520.46 on 421 degrees of freedom

Residual deviance: 495.32 on 419 degrees of freedom

AIC: 3693.5

Number of Fisher Scoring iterations: 1

Theta: 0.9726

Std. Err.: 0.0668

2 x log-likelihood: -3685.53 


\begin{tabular}{|c|c|c|c|c|c|}
\hline \multicolumn{6}{|c|}{$\begin{array}{l}\text { E-C-I } \\
\text { Call: } \\
\text { glm.nb(formula = Collisions } \sim \text { VKT + Trips-Pr } \\
\text { Center + TLKM, init.theta }=0.8913608795, \text { I }\end{array}$} \\
\hline \multicolumn{6}{|l|}{ Deviance Residuals: } \\
\hline Min & 10 & Median & $3 Q$ & Max & \\
\hline \multirow[t]{2}{*}{-3.1056} & -0.9056 & -0.2486 & 0.3732 & 2.8773 & \\
\hline & Estimate & Std.Error & $z$ value & $\operatorname{Pr}(>|z|)$ & \\
\hline (Intercept) & $3.01 E+00$ & $7.44 \mathrm{E}-02$ & 40.508 & $<2 \mathrm{E}-16$ & $* * *$ \\
\hline VKT & $1.22 \mathrm{E}-05$ & $2.06 \mathrm{E}-06$ & 5.944 & $2.78 \mathrm{E}-09$ & $* * *$ \\
\hline Trips-Produced & $-8.19 E-05$ & $4.44 \mathrm{E}-05$ & -1.846 & 0.06493 & . \\
\hline Density- Trips-Out-Center & $1.11 \mathrm{E}-04$ & $4.03 E-05$ & 2.749 & 0.00598 & $* *$ \\
\hline Density- Trips-Enter-Center & $-2.52 \mathrm{E}-05$ & $5.82 \mathrm{E}-06$ & -4.325 & $1.52 \mathrm{E}-05$ & $* * *$ \\
\hline TLKM & $3.05 \mathrm{E}-02$ & $7.58 \mathrm{E}-03$ & 4.026 & $5.68 \mathrm{E}-05$ & $* * *$ \\
\hline \multicolumn{6}{|c|}{$\begin{array}{l}\text { Signif.codes: }\left(0^{\prime * * * \prime}\right)\left(0.001^{\prime * *^{\prime}}\right)\left(0.01^{\prime * \prime}\right)\left(0.05^{\prime \prime}\right)\left(0.1^{\prime \prime}\right)(1) \\
\text { (Dispersion parameter for Negative Binomial }(2.148) \text { family taken to be } 1)\end{array}$} \\
\hline \multicolumn{6}{|c|}{ Null deviance: 558.19 on 421 degrees of freedom } \\
\hline \multicolumn{6}{|c|}{ Residual deviance: 503.87 on 416 degrees of freedom } \\
\hline \multicolumn{6}{|c|}{ AIC: 3661.6} \\
\hline \multicolumn{6}{|c|}{ Number of Fisher Scoring iterations: 1} \\
\hline \multicolumn{6}{|l|}{ Theta: 0.8914} \\
\hline \multicolumn{6}{|l|}{ Std. Err.: 0.0632} \\
\hline \multicolumn{6}{|l|}{2 x log-likelihood: -3647.594} \\
\hline
\end{tabular}




\begin{tabular}{|c|c|c|c|c|c|}
\hline $\begin{array}{l}\text { K-D-II } \\
\text { Call: } \\
\text { glm.nb(formula = Collisions } \\
\text { TLKM, init.theta = } 1.709961 \mathrm{~s}\end{array}$ & $\begin{array}{l}\text { oc + Bike-Pa } \\
\text {, link = log) }\end{array}$ & VKT + Trips & cted + D & - Trips-Ente & er + \\
\hline Deviance Residuals: & & & & & \\
\hline Min & 10 & Median & $3 Q$ & Max & \\
\hline-3.1158 & -0.8871 & -0.1877 & 0.385 & 3.4034 & \\
\hline & Estimate & Std.Error & $z$ value & $\operatorname{Pr}(>|z|)$ & \\
\hline (Intercept) & $3.19 \mathrm{E}+00$ & $6.43 \mathrm{E}-02$ & 49.592 & $<2 \mathrm{E}-16$ & $* * *$ \\
\hline LoC & $8.65 \mathrm{E}-02$ & $2.32 \mathrm{E}-02$ & 3.727 & 0.000194 & $* * *$ \\
\hline Bike-Path & $2.01 \mathrm{E}-02$ & $1.08 \mathrm{E}-02$ & 1.871 & 0.061316 & . \\
\hline VKT & $1.47 \mathrm{E}-05$ & $2.69 \mathrm{E}-06$ & 5.446 & $5.16 \mathrm{E}-08$ & $* * *$ \\
\hline Trips-Attracted & $1.18 \mathrm{E}-05$ & $4.26 \mathrm{E}-06$ & 2.77 & 0.005613 & $* *$ \\
\hline Density- Trips-Enter-Center & $-1.81 \mathrm{E}-05$ & $4.38 \mathrm{E}-06$ & -4.139 & $3.49 \mathrm{E}-05$ & $* * *$ \\
\hline TLKM & $-6.35 E-02$ & $1.94 \mathrm{E}-02$ & -3.269 & 0.00108 & $* *$ \\
\hline $\begin{array}{l}\text { Signif.codes: }\left(0^{* * * \prime}\right)(0.001 \\
\text { (Dispersion parameter for } \mathrm{N}\end{array}$ & $\begin{array}{l}\left.{ }^{\prime \prime}\right)\left(0.01^{\prime * \prime}\right) \\
\text { ative Binom }\end{array}$ & $\begin{array}{l}\text {.') }\left(0.1^{\prime \prime}\right) \\
.148) \text { famil }\end{array}$ & $\mathrm{n}$ to be 1 & & \\
\hline $\begin{array}{l}\text { Null deviance: } 529.3 \text { on } 421 \\
\text { Residual deviance: } 474 \text { on } 4 \\
\text { AIC: } 3645.6\end{array}$ & $\begin{array}{l}\text { egrees of fre } \\
\text { degrees of } f\end{array}$ & $\begin{array}{l}\mathrm{m} \\
\mathrm{lom}\end{array}$ & & & \\
\hline Number of Fisher Scoring ite & tions: 1 & & & & \\
\hline $\begin{array}{l}\text { Theta: } 1.71 \\
\text { Std. Err.: } 0.122\end{array}$ & & & & & \\
\hline $2 \times$ log-likelihood: -3629.565 & & & & & \\
\hline
\end{tabular}




\begin{tabular}{|c|c|c|c|c|c|}
\hline $\begin{array}{l}\text { K-D-III } \\
\text { Call: } \\
\text { glm.nb(formula = Collisions } \\
\text { Trips-Enter-Center + TLKM, }\end{array}$ & $\begin{array}{l}0 . \mathrm{Km} / \mathrm{h}+5 \mathrm{c} \\
\text { theta }=1.7\end{array}$ & $\begin{array}{l}/ \mathrm{h}+60 . \mathrm{Km} \\
15, \text { link }=1\end{array}$ & $00 . \mathrm{Km} / \mathrm{h}$ & -Path + VK & sity- \\
\hline Deviance Residuals: & & & & & \\
\hline Min & 10 & Median & $3 Q$ & Max & \\
\hline-3.1644 & -0.8612 & -0.1767 & 0.3913 & 3.4613 & \\
\hline & Estimate & Std.Error & $z$ value & $\operatorname{Pr}(>|z|)$ & \\
\hline (Intercept) & $3.25 \mathrm{E}+00$ & $8.01 \mathrm{E}-02$ & 40.535 & $<2 \mathrm{E}-16$ & $* * *$ \\
\hline $40 . \mathrm{Km} / \mathrm{h}$ & $1.70 \mathrm{E}-01$ & $4.54 \mathrm{E}-02$ & 3.75 & 0.000177 & $* * *$ \\
\hline $50 . \mathrm{Km} / \mathrm{h}$ & 1.39E-01 & $4.24 \mathrm{E}-02$ & 3.279 & 0.001042 & $* *$ \\
\hline $60 . \mathrm{Km} / \mathrm{h}$ & $9.81 \mathrm{E}-02$ & $4.57 \mathrm{E}-02$ & 2.146 & 0.0319 & $*$ \\
\hline $100 . \mathrm{Km} / \mathrm{h}$ & $9.48 \mathrm{E}+02$ & $3.43 E+02$ & 2.759 & 0.005793 & $* *$ \\
\hline Bike-Path & $1.75 \mathrm{E}-02$ & $1.06 \mathrm{E}-02$ & 1.644 & 0.100202 & \\
\hline VKT & $1.33 \mathrm{E}-05$ & $2.59 \mathrm{E}-06$ & 5.149 & $2.62 \mathrm{E}-07$ & $* * *$ \\
\hline Density- Trips-Enter-Center & $-1.81 \mathrm{E}-05$ & $3.15 \mathrm{E}-06$ & -5.734 & $9.80 \mathrm{E}-09$ & $* * *$ \\
\hline TLKM & $-1.49 \mathrm{E}-01$ & $4.57 \mathrm{E}-02$ & -3.257 & 0.001127 & $* *$ \\
\hline $\begin{array}{l}\text { Signif.codes: }\left(0^{* * * *}\right)(0.001 \\
\text { (Dispersion parameter for } N\end{array}$ & $\begin{array}{l}\text { k' }^{\prime}\left(0.01^{\prime * \prime}\right)( \\
\text { ative Binom }\end{array}$ & $\begin{array}{l}\left..^{\prime}\right)\left(0.1^{\prime \prime}\right) \\
.148) \text { famil }\end{array}$ & n to be 1 & & \\
\hline $\begin{array}{l}\text { Null deviance: } 537.8 \text { on } 421 \\
\text { Residual deviance: } 473.28 \text { o } \\
\text { AIC: } 3641.4\end{array}$ & $\begin{array}{l}\text { egrees of fre } \\
13 \text { degrees }\end{array}$ & eedom & & & \\
\hline Number of Fisher Scoring it & tions: 1 & & & & \\
\hline $\begin{array}{l}\text { Theta: } 1.742 \\
\text { Std. Err.: } 0.125\end{array}$ & & & & & \\
\hline $2 \times$ log-likelihood: -3621.37 & & & & & \\
\hline
\end{tabular}




\begin{tabular}{|c|c|c|c|c|c|}
\hline $\begin{array}{l}\text { K-D-IV } \\
\text { Call: } \\
\text { glm.nb(formula = Collisions } \\
\text { Enter-Center, init.theta }=1 .\end{array}$ & $\begin{array}{l}\text { IS-Driver + } \\
878375, \text { lin }\end{array}$ & assenger + & ransit + 1 & alk + VKT + & - Trips- \\
\hline Deviance Residuals: & & & & & \\
\hline Min & 10 & Median & $3 Q$ & Max & \\
\hline-3.2835 & -0.9019 & -0.1833 & 0.3761 & 3.584 & \\
\hline & Estimate & Std.Error & $z$ value & $\operatorname{Pr}(>|z|)$ & \\
\hline (Intercept) & $1.48 \mathrm{E}+00$ & 2.67E-01 & 5.556 & $2.76 \mathrm{E}-08$ & $* * *$ \\
\hline MS-Driver & $1.21 \mathrm{E}-02$ & $3.13 \mathrm{E}-03$ & 3.874 & 0.000107 & $* * *$ \\
\hline MS-Passenger & $4.00 \mathrm{E}-02$ & $6.29 \mathrm{E}-03$ & 6.359 & $2.03 \mathrm{E}-10$ & $* * *$ \\
\hline MS-Transit & $3.10 \mathrm{E}-02$ & $5.26 \mathrm{E}-03$ & 5.894 & $3.76 \mathrm{E}-09$ & $* * *$ \\
\hline MS-Walk & $1.46 \mathrm{E}-02$ & $5.01 \mathrm{E}-03$ & 2.901 & 0.003716 & $* *$ \\
\hline VKT & $8.23 \mathrm{E}-06$ & $1.74 \mathrm{E}-06$ & 4.736 & $2.18 \mathrm{E}-06$ & $* * *$ \\
\hline Density- Trips-Enter-Center & $-1.98 \mathrm{E}-05$ & $3.17 \mathrm{E}-06$ & -6.239 & $4.40 \mathrm{E}-10$ & $* * *$ \\
\hline $\begin{array}{l}\text { Signif.codes: }\left(0^{* * * * \prime}\right)(0.001 \\
\text { (Dispersion parameter for } N\end{array}$ & $\begin{array}{l}\text { ') }\left(0.01^{(* \prime}\right) \\
\text { tive Binom }\end{array}$ & $\begin{array}{l}\left..^{\prime}\right)\left(0.1^{\prime \prime}\right) \\
.148) \text { famil }\end{array}$ & $n$ to be 1 & & \\
\hline $\begin{array}{l}\text { Null deviance: } 553.16 \text { on } 42 \\
\text { Residual deviance: } 471.19 \text { o } \\
\text { AIC: } 3622.1\end{array}$ & $\begin{array}{l}\text { legrees of } f \\
15 \text { degrees }\end{array}$ & $\begin{array}{l}\text { om } \\
\text { eedom }\end{array}$ & & & \\
\hline Number of Fisher Scoring it & ions: 1 & & & & \\
\hline $\begin{array}{l}\text { Theta: } 1.799 \\
\text { Std. Err.: } 0.13\end{array}$ & & & & & \\
\hline 2 x log-likelihood: -3606.11 & & & & & \\
\hline
\end{tabular}




\begin{tabular}{|c|c|c|c|c|c|}
\hline \multicolumn{6}{|l|}{$\begin{array}{l}\text { K-D-V } \\
\text { Call: } \\
\text { glm.nb(formula = Collisions } \\
\text { Age }>65+\text { VKT + Density- Trip }\end{array}$} \\
\hline \multicolumn{6}{|l|}{ Deviance Residuals: } \\
\hline Min & 10 & Median & $3 Q$ & Max & \\
\hline \multirow[t]{2}{*}{-3.3463} & -0.8871 & -0.1932 & 0.4656 & 2.7133 & \\
\hline & Estimate & Std.Error & $z$ value & $\operatorname{Pr}(>|z|)$ & \\
\hline (Intercept) & $1.07 \mathrm{E}+00$ & $2.31 \mathrm{E}-01$ & 4.644 & $3.42 \mathrm{E}-06$ & $* * *$ \\
\hline Area & $8.64 \mathrm{E}-03$ & $1.22 \mathrm{E}-03$ & 7.101 & $1.24 \mathrm{E}-12$ & $* * *$ \\
\hline MS-Driver & $1.13 \mathrm{E}-02$ & 2.57E-03 & 4.377 & $1.20 \mathrm{E}-05$ & $* * *$ \\
\hline MS-Passenger & $3.03 \mathrm{E}-02$ & $5.76 \mathrm{E}-03$ & 5.255 & $1.48 \mathrm{E}-07$ & $* * *$ \\
\hline MS-Transit & $3.46 \mathrm{E}-02$ & 4.89E-03 & 7.08 & $1.44 \mathrm{E}-12$ & $* * *$ \\
\hline MS-Bike & $3.51 \mathrm{E}-02$ & $1.64 \mathrm{E}-02$ & 2.136 & 0.03268 & $*$ \\
\hline Age.25-64 & $5.55 \mathrm{E}-03$ & $1.79 \mathrm{E}-03$ & 3.093 & 0.00198 & $* *$ \\
\hline Age $>65$ & $1.38 \mathrm{E}-02$ & $5.38 \mathrm{E}-03$ & 2.567 & 0.01025 & $*$ \\
\hline VKT & $1.36 \mathrm{E}-05$ & $1.56 \mathrm{E}-06$ & 8.679 & $<2 \mathrm{E}-16$ & $* * *$ \\
\hline Density- Trips-Enter-Center & $-2.04 \mathrm{E}-05$ & $3.12 \mathrm{E}-06$ & -6.525 & $6.82 \mathrm{E}-11$ & $* * *$ \\
\hline \multicolumn{6}{|c|}{$\begin{array}{l}\text { Signif.codes: }\left(0^{\prime * * * \prime}\right)\left(0.001^{\prime * * \prime}\right)\left(0.01^{\prime * \prime}\right)\left(0.05^{\prime \prime}\right)\left(0.1^{\prime \prime}\right)(1) \\
\text { (Dispersion parameter for Negative Binomial }(2.148) \text { family taken to be } 1)\end{array}$} \\
\hline \multicolumn{6}{|c|}{$\begin{array}{l}\text { Null deviance: } 674.09 \text { on } 421 \text { degrees of freedom } \\
\text { Residual deviance: } 470.34 \text { on } 412 \text { degrees of freedom } \\
\text { AIC: } 3535.8\end{array}$} \\
\hline \multicolumn{6}{|c|}{ Number of Fisher Scoring iterations: 1} \\
\hline \multicolumn{5}{|l|}{ Theta: 2.264} & Std. Err.: 0.17 \\
\hline $2 \times$ log-likelihood: -3513.82 & & & & & \\
\hline
\end{tabular}


K-C-I

Call:

glm.nb(formula $=$ Collisions $\sim$ VKT + Trips-Out-Center + Trips-Enter-Center + Density- Trips-Out-Center + Density- Trips-Enter-Center + TLKM, init.theta $=2.757187944$, link $=\log$ )

\begin{tabular}{|c|c|c|c|c|c|}
\hline Deviance Residuals: \\
\hline Min & $1 \mathrm{Q}$ & Median & $3 \mathrm{Q}$ & Max & \\
\hline-3.3072 & -0.8212 & -0.1538 & 0.4976 & 2.8649 & \\
\hline \multicolumn{7}{|l|}{} \\
\hline & Estimate & Std.Error & z value & $\operatorname{Pr}(>|\mathrm{z}|)$ & \\
\hline \multicolumn{7}{|c|}{} & $2.20 \mathrm{E}+00$ & $6.74 \mathrm{E}-02$ & 32.566 & $<2 \mathrm{E}-16$ & $* * *$ \\
\hline (Intercept) & $1.66 \mathrm{E}-05$ & $1.44 \mathrm{E}-06$ & 11.534 & $<2 \mathrm{E}-16$ & $* * *$ \\
\hline VKT & $1.53 \mathrm{E}-04$ & $2.86 \mathrm{E}-05$ & 5.326 & $1.00 \mathrm{E}-07$ & $* * *$ \\
\hline Trips-Out-Center & $1.41 \mathrm{E}-04$ & $2.00 \mathrm{E}-05$ & 7.049 & $1.80 \mathrm{E}-12$ & $* * *$ \\
\hline Trips-Enter-Center & $1.44 \mathrm{E}-04$ & $1.92 \mathrm{E}-05$ & 7.503 & $6.24 \mathrm{E}-14$ & $* * *$ \\
\hline Density- Trips-Out-Center & $-1.62 \mathrm{E}-05$ & $3.57 \mathrm{E}-06$ & -4.553 & $5.29 \mathrm{E}-06$ & $* * *$ \\
\hline Density- Trips-Enter-Center & $-4.32 \mathrm{E}-03$ & $1.79 \mathrm{E}-03$ & -2.41 & 0.016 & $*$ \\
\hline TLKM &
\end{tabular}

Signif.codes: $\left(0^{(* * * \prime}\right)\left(0.001^{(* * \prime}\right)\left(0.01^{\prime * \prime}\right)\left(0.05^{\prime \prime}.\right)\left(0.1^{\prime \prime}\right)$ (1)

(Dispersion parameter for Negative Binomial (2.148) family taken to be 1 )

Null deviance: 795.48 on 421 degrees of freedom

Residual deviance: 473.76 on 415 degrees of freedom

AIC: 3458

Number of Fisher Scoring iterations: 1

Theta: 2.757

Std. Err.: 0.216

2 x log-likelihood: -3441.956 


\begin{tabular}{|c|c|c|c|c|c|}
\hline $\begin{array}{l}\text { K-D-I } \\
\text { Call: } \\
\text { glm.nb(formula = Collisions }\end{array}$ & KT + Densi & ips-Enter-C & init.thet & 46834808 & \\
\hline Deviance Residuals: & & & & & \\
\hline Min & 10 & Median & $3 Q$ & Max & \\
\hline-3.0598 & -0.8741 & -0.1978 & 0.4182 & 3.227 & \\
\hline & Estimate & Std.Error & $z$ value & $\operatorname{Pr}(>|z|)$ & \\
\hline (Intercept) & $3.20 \mathrm{E}+00$ & $5.37 \mathrm{E}-02$ & 59.509 & $<2 \mathrm{E}-16$ & $* * *$ \\
\hline VKT & $1.02 \mathrm{E}-05$ & $1.69 \mathrm{E}-06$ & 5.998 & 1.99E-09 & $* * *$ \\
\hline Density- Trips-Enter-Center & $-1.71 E-05$ & $3.09 E-06$ & -5.521 & $3.36 \mathrm{E}-08$ & $* * *$ \\
\hline $\begin{array}{l}\text { Signif.codes: }\left(0^{* * * *}\right)(0.001 \\
\text { (Dispersion parameter for } N\end{array}$ & $\begin{array}{l}\left.k^{\prime}\right)\left(0.01^{\prime * \prime}\right) \\
\text { ative Binom }\end{array}$ & $\begin{array}{l}\left.y^{\prime}\right)\left(0.1^{\prime \prime}\right) \\
.148) \text { famil }\end{array}$ & n to be 1 & & \\
\hline Null deviance: 512.21 on 42 & degrees of $t$ & & & & \\
\hline Residual deviance: 474.04 o & 19 degrees & eedom & & & \\
\hline AIC: 3653 & & & & & \\
\hline Number of Fisher Scoring ite & tions: 1 & & & & \\
\hline Theta: 1.647 & & & & & \\
\hline Std. Err.: 0.117 & & & & & \\
\hline $2 \times$ log-likelihood: -3645.02 & & & & & \\
\hline
\end{tabular}




\begin{tabular}{|c|c|c|c|c|c|}
\hline $\begin{array}{l}\text { L-D-II } \\
\text { Call: } \\
\text { glm.nb(formula = Collisions } \\
\text { Enter-Center, init.theta }=0.9\end{array}$ & $\begin{array}{l}\text { Jo Bus stops } \\
9561749, \text { । }\end{array}$ & $\begin{array}{l}\text { t-Int + Maj } \\
\log )\end{array}$ & le + Trip & acted + Den & ips- \\
\hline Deviance Residuals: & & & & & \\
\hline Min & 10 & Median & 30 & Max & \\
\hline-2.5796 & -0.9573 & -0.32 & 0.2377 & 3.1312 & \\
\hline & Estimate & Std.Error & $z$ value & $\operatorname{Pr}(>|z|)$ & \\
\hline (Intercept) & $2.91 \mathrm{E}+00$ & $7.82 \mathrm{E}-02$ & 37.243 & $<2 \mathrm{E}-16$ & $* * *$ \\
\hline No Bus stops & $1.73 \mathrm{E}-02$ & $5.17 \mathrm{E}-03$ & 3.355 & 0.000793 & $* * *$ \\
\hline Tot-Int & $1.11 \mathrm{E}-02$ & 2.67E-03 & 4.149 & $3.35 \mathrm{E}-05$ & $* * *$ \\
\hline Major-Colle & $-1.42 \mathrm{E}-01$ & $6.26 \mathrm{E}-02$ & -2.27 & 0.023199 & $*$ \\
\hline Trips-Attracted & $4.98 \mathrm{E}-05$ & $8.45 \mathrm{E}-06$ & 5.892 & $3.82 \mathrm{E}-09$ & $* * *$ \\
\hline Density- Trips-Enter-Center & $-8.78 \mathrm{E}-05$ & $1.06 \mathrm{E}-05$ & -8.309 & $<2 \mathrm{E}-16$ & $* * *$ \\
\hline $\begin{array}{l}\text { Signif.codes: }\left(0^{` * * * \prime)}(0.001\right. \\
\text { (Dispersion parameter for } \mathrm{N}\end{array}$ & $\begin{array}{l}\left.{ }^{\prime \prime}\right)\left(0.01^{\prime * \prime}\right) \\
\text { ative Binom }\end{array}$ & $\begin{array}{l}\because \prime)\left(0.1^{\prime \prime}\right) \\
.148) \text { famil }\end{array}$ & $\mathrm{n}$ to be 1 & & \\
\hline Null deviance: 638.66 on 42 & degrees of $\mathrm{fr}$ & & & & \\
\hline Residual deviance: 498.63 or & 16 degrees & eedom & & & \\
\hline AIC: 3543.3 & & & & & \\
\hline Number of Fisher Scoring ite & tions: 1 & & & & \\
\hline Theta: 0.929 & & & & & \\
\hline Std. Err.: 0.0674 & & & & & \\
\hline 2 x log-likelihood: -3529.30 & & & & & \\
\hline
\end{tabular}




\begin{tabular}{|c|c|c|c|c|c|}
\hline \multicolumn{6}{|l|}{$\begin{array}{l}\text { L-D-III } \\
\text { Call: } \\
\text { glm.nb(formula = Collisior } \\
\text { Bike-Path + VKT + Trips-At } \\
\text { log) }\end{array}$} \\
\hline \multicolumn{6}{|l|}{ Deviance Residuals: } \\
\hline Min & 10 & Median & $3 Q$ & Max & \\
\hline \multirow[t]{2}{*}{-2.6306} & -0.9688 & -0.2723 & 0.2534 & 3.2513 & \\
\hline & Estimate & Std.Error & $z$ value & $\operatorname{Pr}(>|z|)$ & \\
\hline (Intercept) & $2.88 \mathrm{E}+00$ & $1.12 \mathrm{E}-01$ & 25.83 & $<2 \mathrm{E}-16$ & $* * *$ \\
\hline No Bus stops & $2.66 \mathrm{E}-02$ & 4.49E-03 & 5.929 & $3.05 \mathrm{E}-09$ & $* * *$ \\
\hline $40 . \mathrm{Km} / \mathrm{h}$ & $3.29 \mathrm{E}-01$ & $1.18 \mathrm{E}-01$ & 2.783 & 0.00539 & $* *$ \\
\hline $50 . \mathrm{Km} / \mathrm{h}$ & $2.92 \mathrm{E}-01$ & $1.16 \mathrm{E}-01$ & 2.519 & 0.01176 & $*$ \\
\hline $60 . \mathrm{Km} / \mathrm{h}$ & $2.16 \mathrm{E}-01$ & $1.18 \mathrm{E}-01$ & 1.831 & 0.06708 & . \\
\hline $80 . \mathrm{Km} / \mathrm{h}$ & $2.80 \mathrm{E}-01$ & $1.30 \mathrm{E}-01$ & 2.158 & 0.03096 & $*$ \\
\hline $100 . \mathrm{Km} / \mathrm{h}$ & $1.17 \mathrm{E}+03$ & $4.65 \mathrm{E}+02$ & 2.518 & 0.0118 & $*$ \\
\hline Bike-Path & $3.38 \mathrm{E}-02$ & $2.00 \mathrm{E}-02$ & 1.69 & 0.09104 & . \\
\hline VKT & $6.70 \mathrm{E}-06$ & $3.79 E-06$ & 1.767 & 0.07717 & . \\
\hline Trips-Attracted & $4.86 \mathrm{E}-05$ & $8.85 \mathrm{E}-06$ & 5.489 & 4.04E-08 & $* * *$ \\
\hline Density- Trips-Enter-Center & $-9.20 \mathrm{E}-05$ & $1.26 \mathrm{E}-05$ & -7.302 & $2.83 \mathrm{E}-13$ & $* * *$ \\
\hline TLKM & $-2.91 \mathrm{E}-01$ & $1.19 \mathrm{E}-01$ & -2.449 & 0.01432 & $*$ \\
\hline \multicolumn{6}{|c|}{$\begin{array}{l}\text { Signif.codes: }\left(0^{(* * * \prime}\right)\left(0.001^{(* * \prime}\right)\left(0.01^{(* \prime}\right)\left(0.05^{\prime}{ }^{\prime \prime}\right)\left(0.1^{\prime \prime}\right)(1) \\
\text { (Dispersion parameter for Negative Binomial }(2.148) \text { family taken to be } 1)\end{array}$} \\
\hline \multicolumn{6}{|c|}{$\begin{array}{l}\text { Null deviance: } 647.99 \text { on } 421 \text { degrees of freedom } \\
\text { Residual deviance: } 499.86 \text { on } 410 \text { degrees of freedom } \\
\text { AIC: } 3549.5\end{array}$} \\
\hline \multicolumn{6}{|c|}{ Number of Fisher Scoring iterations: 1} \\
\hline \multicolumn{6}{|l|}{ Theta: 0.9453} \\
\hline $2 \times$ log-likelihood: -3523.499 & & & & & \\
\hline
\end{tabular}




\begin{tabular}{|c|c|c|c|c|c|}
\hline \multicolumn{6}{|c|}{$\begin{array}{l}\text { Call: } \\
\text { glm.nb(formula }=\text { Collisions } ~ \text { Area }+ \text { MS-Driver + MS-Passenger }+ \text { MS-Transit }+ \text { MS-Walk + Employment } \\
+ \text { Age.5-24 + Age. } 25-64+\text { Age }>65+\text { VKT, init.theta }=1.24616982, \text { link }=\text { log })\end{array}$} \\
\hline \multicolumn{6}{|l|}{ Deviance Residuals: } \\
\hline Min & 10 & Median & $3 Q$ & Max & \\
\hline \multirow[t]{2}{*}{-2.9019} & -0.9166 & -0.2363 & 0.3856 & 4.0106 & \\
\hline & Estimate & Std.Error & $z$ value & $\operatorname{Pr}(>|z|)$ & \\
\hline (Intercept) & $-1.01 E+00$ & 4.55E-01 & -2.225 & 0.026068 & $*$ \\
\hline Area & $8.69 \mathrm{E}-03$ & $1.65 \mathrm{E}-03$ & 5.26 & $1.44 \mathrm{E}-07$ & $* * *$ \\
\hline MS-Driver & $1.72 \mathrm{E}-02$ & 4.94E-03 & 3.479 & 0.000503 & $* * *$ \\
\hline MS-Passenger & $4.73 \mathrm{E}-02$ & $8.42 \mathrm{E}-03$ & 5.614 & $1.98 \mathrm{E}-08$ & $* * *$ \\
\hline MS-Transit & 4.87E-02 & $7.62 \mathrm{E}-03$ & 6.385 & $1.72 \mathrm{E}-10$ & $* * *$ \\
\hline MS-Walk & $1.78 \mathrm{E}-02$ & $7.33 \mathrm{E}-03$ & 2.429 & 0.015149 & $*$ \\
\hline Employment & $-1.13 \mathrm{E}-05$ & $2.48 \mathrm{E}-06$ & -4.561 & $5.09 \mathrm{E}-06$ & $* * *$ \\
\hline Age.5-24 & $1.38 \mathrm{E}-02$ & $4.23 \mathrm{E}-03$ & 3.272 & 0.001068 & $* *$ \\
\hline Age.25-64 & $1.95 \mathrm{E}-02$ & $2.76 \mathrm{E}-03$ & 7.086 & $1.38 \mathrm{E}-12$ & $* * *$ \\
\hline Age $>65$ & $3.11 \mathrm{E}-02$ & $7.15 \mathrm{E}-03$ & 4.344 & $1.40 \mathrm{E}-05$ & $* * *$ \\
\hline VKT & $9.13 \mathrm{E}-06$ & $2.06 \mathrm{E}-06$ & 4.438 & $9.07 \mathrm{E}-06$ & $* * *$ \\
\hline \multicolumn{6}{|c|}{$\begin{array}{l}\text { Signif.codes: }\left(0^{\prime * * * \prime}\right)\left(0.001^{\prime * * \prime}\right)\left(0.01^{\prime * \prime}\right)\left(0.05^{\prime \prime} .\right)\left(0.1^{\prime \prime}\right)(1) \\
\text { (Dispersion parameter for Negative Binomial }(2.148) \text { family taken to be } 1)\end{array}$} \\
\hline \multicolumn{6}{|c|}{ Null deviance: 813.29 on 421 degrees of freedom } \\
\hline \multicolumn{6}{|c|}{ Residual deviance: 501.37 on 411 degrees of freedom } \\
\hline \multicolumn{6}{|l|}{ AIC: 3440.7} \\
\hline \multicolumn{6}{|c|}{ Number of Fisher Scoring iterations: 1} \\
\hline \multicolumn{6}{|l|}{ Theta: 1.2462} \\
\hline \multicolumn{6}{|l|}{ Std. Err.: 0.0966} \\
\hline \multicolumn{6}{|c|}{2 x log-likelihood: -3416.665} \\
\hline
\end{tabular}




\begin{tabular}{|c|c|c|c|c|c|}
\hline \multicolumn{6}{|l|}{$\begin{array}{l}\text { Call: } \\
\text { glm.nb(formula = Collisions } \\
0.8388239309 \text {, link }=\log )\end{array}$} \\
\hline \multicolumn{6}{|l|}{ Deviance Residuals: } \\
\hline Min & 10 & Median & $3 Q$ & Max & \\
\hline \multirow[t]{2}{*}{-2.5032} & -0.9702 & -0.314 & 0.2688 & 2.8924 & \\
\hline & Estimate & Std.Error & $z$ value & $\operatorname{Pr}(>|z|)$ & \\
\hline (Intercept) & $2.92 \mathrm{E}+00$ & $8.91 \mathrm{E}-02$ & 32.776 & $<2 \mathrm{E}-16$ & $* * *$ \\
\hline Trips-Attracted & $4.66 \mathrm{E}-05$ & $8.36 \mathrm{E}-06$ & 5.572 & $2.52 \mathrm{E}-08$ & $* * *$ \\
\hline Density- Trips-Enter-Center & $-8.81 E-05$ & $1.10 \mathrm{E}-05$ & -7.982 & $1.44 \mathrm{E}-15$ & $* * *$ \\
\hline TLKM & $6.24 \mathrm{E}-02$ & $8.46 \mathrm{E}-03$ & 7.381 & $1.57 \mathrm{E}-13$ & $* * *$ \\
\hline \multicolumn{6}{|c|}{$\begin{array}{l}\text { Signif.codes: }\left(0^{\prime * * * \prime}\right)\left(0.001^{\prime * * \prime}\right)\left(0.01^{\prime * \prime}\right)\left(0.05^{\prime \prime}\right)\left(0.1^{\prime \prime}\right)(1) \\
\text { (Dispersion parameter for Negative Binomial }(2.148) \text { family taken to be } 1)\end{array}$} \\
\hline \multicolumn{6}{|c|}{ Null deviance: 586.62 on 421 degrees of freedom } \\
\hline \multicolumn{6}{|c|}{ Residual deviance: 501.69 on 418 degrees of freedom } \\
\hline \multicolumn{6}{|c|}{ AIC: 3584} \\
\hline \multicolumn{6}{|c|}{ Number of Fisher Scoring iterations: 1} \\
\hline \multicolumn{6}{|l|}{ Theta: 0.8388} \\
\hline \multicolumn{6}{|l|}{ Std. Err.: 0.0599} \\
\hline \multicolumn{6}{|l|}{2 x log-likelihood: -3573.977} \\
\hline
\end{tabular}




\begin{tabular}{|c|c|c|c|c|c|}
\hline \multicolumn{6}{|l|}{$\begin{array}{l}\text { M-D-II } \\
\text { Call: } \\
\text { glm.nb(formula = Collisions } \\
\text { init.theta = 1.168212351, lin }\end{array}$} \\
\hline \multicolumn{6}{|l|}{ Deviance Residuals: } \\
\hline Min & 10 & Median & 30 & Max & \\
\hline \multirow[t]{2}{*}{-2.9446} & -0.832 & -0.2141 & 0.3899 & 3.1238 & \\
\hline & Estimate & Std.Error & $z$ value & $\operatorname{Pr}(>|z|)$ & \\
\hline (Intercept) & $3.07 \mathrm{E}+00$ & $7.32 \mathrm{E}-02$ & 41.886 & $<2 \mathrm{E}-16$ & $* * *$ \\
\hline No Bus stops & $1.47 \mathrm{E}-02$ & $3.07 E-03$ & 4.801 & $1.58 \mathrm{E}-06$ & $* * *$ \\
\hline Maj-Colle & $-1.20 \mathrm{E}-01$ & $5.34 \mathrm{E}-02$ & -2.248 & 0.02459 & $*$ \\
\hline Bike-Path & $2.10 \mathrm{E}-02$ & 1.07E-02 & 1.963 & 0.04965 & $*$ \\
\hline VKT & $5.77 \mathrm{E}-06$ & $2.13 \mathrm{E}-06$ & 2.707 & 0.00678 & $* *$ \\
\hline Density- Trips-Enter-Center & $-1.85 \mathrm{E}-05$ & $3.86 \mathrm{E}-06$ & -4.791 & $1.66 \mathrm{E}-06$ & $* * *$ \\
\hline \multicolumn{6}{|c|}{$\begin{array}{l}\text { Signif.codes: }\left(0^{\prime * * * \prime}\right)\left(0.001^{\prime * * \prime}\right)\left(0.01^{\prime * \prime}\right)\left(0.05^{\prime \prime}\right)\left(0.1^{\prime \prime}\right)(1) \\
\text { (Dispersion parameter for Negative Binomial }(2.148) \text { family taken to be } 1)\end{array}$} \\
\hline \multicolumn{6}{|c|}{ Null deviance: 544.22 on 421 degrees of freedom } \\
\hline \multicolumn{6}{|c|}{ Residual deviance: 495.7 on 416 degrees of freedom } \\
\hline \multicolumn{6}{|l|}{ AIC: 3678.2} \\
\hline \multicolumn{6}{|c|}{ Number of Fisher Scoring iterations: 1} \\
\hline \multicolumn{6}{|l|}{ Theta: 1.1682} \\
\hline \multicolumn{6}{|l|}{ Std. Err.: 0.0832} \\
\hline \multicolumn{6}{|l|}{2 x log-likelihood: -3664.218} \\
\hline
\end{tabular}




\begin{tabular}{|c|c|c|c|c|c|}
\hline \multicolumn{6}{|c|}{$\begin{array}{l}\text { Call: } \\
\text { glm.nb(formula }=\text { Collisions } \sim \text { No Bus stops }+ \text { Non-Sig-Int }+40 . \mathrm{Km} / \mathrm{h}+50 . \mathrm{Km} / \mathrm{h}+100 . \mathrm{Km} / \mathrm{h}+\text { Bike-Path }+ \\
\text { VKT + Density- Trips-Enter-Center }+ \text { TLKM, init.theta }=1.218084543, \operatorname{link}=\log )\end{array}$} \\
\hline \multicolumn{6}{|l|}{ Deviance Residuals: } \\
\hline Min & 10 & Median & $3 Q$ & Max & \\
\hline \multirow[t]{2}{*}{-2.9679} & -0.859 & -0.2061 & 0.3457 & 3.3606 & \\
\hline & Estimate & Std.Error & $z$ value & $\operatorname{Pr}(>|z|)$ & \\
\hline (Intercept) & $3.06 \mathrm{E}+00$ & $9.10 \mathrm{E}-02$ & 33.63 & $<2 \mathrm{E}-16$ & $* * *$ \\
\hline No Bus stops & $1.19 \mathrm{E}-02$ & $3.84 \mathrm{E}-03$ & 3.089 & 0.002007 & $* *$ \\
\hline Non-Sig-Int & 5.77E-03 & $3.11 \mathrm{E}-03$ & 1.852 & 0.063985 & . \\
\hline $40 . \mathrm{Km} / \mathrm{h}$ & $1.12 \mathrm{E}-01$ & $3.61 \mathrm{E}-02$ & 3.109 & 0.001877 & $* *$ \\
\hline $50 . \mathrm{Km} / \mathrm{h}$ & 6.69E-02 & $2.76 \mathrm{E}-02$ & 2.427 & 0.015242 & $*$ \\
\hline $100 . \mathrm{Km} / \mathrm{h}$ & $1.09 E+03$ & $4.10 \mathrm{E}+02$ & 2.667 & 0.007655 & $* *$ \\
\hline Bike-Path & $2.10 \mathrm{E}-02$ & $1.06 \mathrm{E}-02$ & 1.98 & 0.047736 & $*$ \\
\hline VKT & $1.39 \mathrm{E}-05$ & $3.38 \mathrm{E}-06$ & 4.103 & $4.08 \mathrm{E}-05$ & $* * *$ \\
\hline Density-Trips-Enter-Center & $-1.42 E-05$ & $3.85 \mathrm{E}-06$ & -3.69 & 0.000224 & $* * *$ \\
\hline TLKM & $-1.02 \mathrm{E}-01$ & $3.55 \mathrm{E}-02$ & -2.863 & 0.004203 & $* *$ \\
\hline (Intercept) & $3.06 \mathrm{E}+00$ & $9.10 \mathrm{E}-02$ & 33.63 & $<2 \mathrm{E}-16$ & $* * *$ \\
\hline No Bus stops & 1.19E-02 & $3.84 \mathrm{E}-03$ & 3.089 & 0.002007 & $* *$ \\
\hline \multicolumn{6}{|c|}{$\begin{array}{l}\text { Signif.codes: }\left(0^{\prime * * * \prime}\right)\left(0.001^{\prime * * \prime}\right)\left(0.01^{\prime * \prime}\right)\left(0.05^{\prime} . \prime\right)\left(0.1^{\prime \prime}\right)(1) \\
\text { (Dispersion parameter for Negative Binomial }(2.148) \text { family taken to be } 1)\end{array}$} \\
\hline \multicolumn{6}{|c|}{ Null deviance: 563.77 on 421 degrees of freedom } \\
\hline \multicolumn{6}{|c|}{ Residual deviance: 496.53 on 412 degrees of freedom } \\
\hline \multicolumn{6}{|l|}{ AIC: 3669.8} \\
\hline \multicolumn{6}{|c|}{ Number of Fisher Scoring iterations: 1} \\
\hline \multicolumn{6}{|l|}{ Theta: 1.2181} \\
\hline \multicolumn{6}{|l|}{ Std. Err.: 0.0877} \\
\hline 2 x log-likelihood: -3647.76 & & & & & \\
\hline
\end{tabular}




\begin{tabular}{|c|c|c|c|c|c|}
\hline \multicolumn{6}{|c|}{$\begin{array}{l}\text { M-D-V } \\
\text { Call: } \\
\text { glm.nb(formula }=\text { Collisions } \sim \text { Area }+ \text { MS-Driver }+ \text { MS-Passenger }+ \\
\text { Age. } 25-64+\text { Age }>65+\text { VKT, init.theta }=1.440250803, \text { link }=\text { log })\end{array}$} \\
\hline \multicolumn{6}{|l|}{ Deviance Residuals: } \\
\hline Min & $1 Q$ & Median & $3 Q$ & Max & \\
\hline \multirow[t]{2}{*}{-3.3431} & -0.8413 & -0.1858 & 0.4381 & 2.8616 & \\
\hline & Estimate & Std.Error & $z$ value & $\operatorname{Pr}(>|z|)$ & \\
\hline (Intercept) & $-3.02 E-01$ & 3.89E-01 & -0.776 & 0.437989 & \\
\hline Area & 8.97E-03 & $1.53 \mathrm{E}-03$ & 5.848 & 4.97E-09 & $* * *$ \\
\hline MS-Driver & $2.52 \mathrm{E}-02$ & $4.27 \mathrm{E}-03$ & 5.896 & $3.72 \mathrm{E}-09$ & $* * *$ \\
\hline MS-Passenger & $3.56 \mathrm{E}-02$ & $7.53 \mathrm{E}-03$ & 4.721 & $2.35 \mathrm{E}-06$ & $* * *$ \\
\hline MS-Transit & $5.18 \mathrm{E}-02$ & $6.57 \mathrm{E}-03$ & 7.875 & $3.41 \mathrm{E}-15$ & $* * *$ \\
\hline MS-Walk & $2.09 \mathrm{E}-02$ & $6.61 \mathrm{E}-03$ & 3.167 & 0.001542 & $* *$ \\
\hline Employment & $-8.11 E-06$ & $2.19 \mathrm{E}-06$ & -3.709 & 0.000208 & $* * *$ \\
\hline Age.25-64 & $9.25 \mathrm{E}-03$ & $2.29 \mathrm{E}-03$ & 4.043 & $5.28 \mathrm{E}-05$ & $* * *$ \\
\hline Age $>65$ & $1.74 \mathrm{E}-02$ & $6.67 \mathrm{E}-03$ & 2.603 & 0.009252 & $* *$ \\
\hline VKT & $8.96 \mathrm{E}-06$ & $1.83 \mathrm{E}-06$ & 4.888 & $1.02 \mathrm{E}-06$ & $* * *$ \\
\hline \multicolumn{6}{|c|}{$\begin{array}{l}\text { Signif.codes: }\left(0^{* * * \prime}\right)\left(0.001^{\prime * * \prime}\right)\left(0.01^{\prime * \prime}\right)\left(0.05^{\prime \prime}\right)\left(0.1^{\prime \prime}\right)(1) \\
\text { (Dispersion parameter for Negative Binomial }(2.148) \text { family taken to be } 1)\end{array}$} \\
\hline \multicolumn{6}{|c|}{ Null deviance: 648.8 on 421 degrees of freedom } \\
\hline \multicolumn{6}{|c|}{ Residual deviance: 491.93 on 412 degrees of freedom } \\
\hline \multicolumn{6}{|l|}{ AIC: 3597.2} \\
\hline \multicolumn{6}{|c|}{ Number of Fisher Scoring iterations: 1} \\
\hline \multicolumn{6}{|l|}{ Theta: 1.44} \\
\hline \multicolumn{6}{|l|}{ Std. Err.: 0.106} \\
\hline \multicolumn{6}{|c|}{$2 \times$ log-likelihood: -3575.156} \\
\hline
\end{tabular}




\begin{tabular}{|c|c|c|c|c|c|}
\hline \multicolumn{6}{|c|}{$\begin{array}{l}\text { Call: } \\
\text { gIm.nb(formula }=\text { Collisions } \sim \text { VKT }+ \text { Trips-Attracted }+ \text { Density- Trips-Enter-Center }+ \text { TLKM, init.theta }= \\
\text { 1.169453792, link }=\text { log) }\end{array}$} \\
\hline \multicolumn{6}{|l|}{ Deviance Residuals: } \\
\hline Min & 10 & Median & $3 Q$ & Max & \\
\hline \multirow[t]{2}{*}{-3.0114} & -0.8763 & -0.2092 & 0.3515 & 2.9881 & \\
\hline & Estimate & Std.Error & $z$ value & $\operatorname{Pr}(>|z|)$ & \\
\hline (Intercept) & $3.11 \mathrm{E}+00$ & $7.58 \mathrm{E}-02$ & 40.967 & $<2 \mathrm{E}-16$ & $* * *$ \\
\hline VKT & 8.19E-06 & $2.63 \mathrm{E}-06$ & 3.112 & 0.00186 & $* *$ \\
\hline Trips-Attracted & $3.14 \mathrm{E}-05$ & $6.60 \mathrm{E}-06$ & 4.756 & $1.98 \mathrm{E}-06$ & $* * *$ \\
\hline Density- Trips-Enter-Center & $-5.81 E-05$ & $8.95 \mathrm{E}-06$ & -6.498 & $8.15 E-11$ & $* * *$ \\
\hline TLKM & $2.20 \mathrm{E}-02$ & $8.57 \mathrm{E}-03$ & 2.566 & 0.01028 & $*$ \\
\hline \multicolumn{6}{|c|}{$\begin{array}{l}\text { Signif.codes: }\left(0^{\prime * * * \prime}\right)\left(0.001^{\prime * * \prime}\right)\left(0.01^{\prime * \prime}\right)\left(0.05^{\prime} . \prime\right)\left(0.1^{\prime \prime}\right)(1) \\
\text { (Dispersion parameter for Negative Binomial }(2.148) \text { family taken to be } 1)\end{array}$} \\
\hline \multicolumn{6}{|c|}{ Null deviance: 544.71 on 421 degrees of freedom } \\
\hline \multicolumn{6}{|c|}{ Residual deviance: 493.56 on 417 degrees of freedom } \\
\hline \multicolumn{6}{|l|}{ AIC: 3673.6} \\
\hline \multicolumn{6}{|c|}{ Number of Fisher Scoring iterations: 1} \\
\hline \multicolumn{6}{|l|}{ Theta: 1.1695} \\
\hline \multicolumn{6}{|l|}{ Std. Err.: 0.0831} \\
\hline $2 \times$ log-likelihood: -3661.638 & & & & & \\
\hline
\end{tabular}




\section{D.4 Bike-Involved Collisions Models}

\begin{tabular}{|c|c|c|c|c|c|}
\hline \multicolumn{6}{|c|}{$\begin{array}{l}\text { A:Cll summary (S3) } \\
\text { Call: } \\
\text { glm.nb(formula }=\text { Collisions } \sim \text { No Bus stops }+ \text { Sig-Int }+50 . \mathrm{Km} / \mathrm{h}+70 . \mathrm{Km} / \mathrm{h}+90 \\
\text { Out-Center + Density- Trips-Enter-Center, init.theta }=8.229084844, \operatorname{link}=\log \text { ) }\end{array}$} \\
\hline \multicolumn{6}{|l|}{ Deviance Residuals: } \\
\hline Min & $1 Q$ & Median & $3 Q$ & Max & \\
\hline \multirow[t]{2}{*}{-2.4482} & -1.1216 & -0.2482 & 0.4276 & 3.0755 & \\
\hline & Estimate & Std.Error & $z$ value & $\operatorname{Pr}(>|z|)$ & \\
\hline (Intercept) & $-4.59 \mathrm{E}-01$ & $9.36 \mathrm{E}-02$ & -4.9 & $9.57 \mathrm{E}-07$ & $* * *$ \\
\hline No Bus stops & $1.12 \mathrm{E}-02$ & 3.99E-03 & 2.796 & 0.00517 & $* *$ \\
\hline Sig-Int & $2.39 \mathrm{E}-01$ & $1.96 \mathrm{E}-02$ & 12.197 & $<2 \mathrm{E}-16$ & $* * *$ \\
\hline $50 . \mathrm{Km} / \mathrm{h}$ & $1.26 \mathrm{E}-02$ & $8.35 \mathrm{E}-03$ & 1.513 & 0.13025 & \\
\hline $70 . \mathrm{Km} / \mathrm{h}$ & $-4.15 E-01$ & $8.13 \mathrm{E}-02$ & -5.109 & $3.24 \mathrm{E}-07$ & $* * *$ \\
\hline $90 . \mathrm{Km} / \mathrm{h}$ & $-8.29 E-01$ & $1.13 \mathrm{E}+00$ & -0.735 & 0.46237 & \\
\hline Density- Trips-Out-Center & $1.22 \mathrm{E}-04$ & $2.23 \mathrm{E}-05$ & 5.486 & $4.10 \mathrm{E}-08$ & $* * *$ \\
\hline Density- Trips-Enter-Center & $-1.24 \mathrm{E}-05$ & $4.68 \mathrm{E}-06$ & -2.659 & 0.00783 & $* *$ \\
\hline \multicolumn{6}{|c|}{$\begin{array}{l}\text { Signif.codes: }\left(0^{\prime * * * \prime}\right)\left(0.001^{\prime * * \prime}\right)\left(0.01^{\prime * \prime}\right)\left(0.05^{\prime \prime} .\right)\left(0.1^{\prime \prime}\right)(1) \\
\text { (Dispersion parameter for Negative Binomial }(2.148) \text { family taken to be } 1)\end{array}$} \\
\hline \multirow{3}{*}{\multicolumn{6}{|c|}{$\begin{array}{l}\text { Null deviance: } 768.03 \text { on } 421 \text { degrees of freedom } \\
\text { Residual deviance: } 444.92 \text { on } 414 \text { degrees of freedom } \\
\text { AIC: } 1400.3\end{array}$}} \\
\hline & & & & & \\
\hline & & & & & \\
\hline \multicolumn{6}{|c|}{ Number of Fisher Scoring iterations: 1} \\
\hline \multicolumn{6}{|l|}{ Theta: 8.23} \\
\hline \multicolumn{6}{|l|}{ Std. Err.: 2.55} \\
\hline \multicolumn{6}{|l|}{2 x log-likelihood: -1382.287} \\
\hline
\end{tabular}




\begin{tabular}{|c|c|c|c|c|c|}
\hline $\begin{array}{l}\text { A:D-III } \\
\text { Call: } \\
\text { glm.nb(formula = Collisions } \\
\text { Path + Trips-Attracted + Den }\end{array}$ & $\begin{array}{l}\text { Jo Bus stop } \\
\text { y- Trips-Ent }\end{array}$ & $\begin{array}{l}\text { t-Int }+40 . \\
\text { enter }+ \text { TLK }\end{array}$ & $\begin{array}{l}50 . \mathrm{Km} / \\
\text { theta }=\end{array}$ & $\begin{array}{l}\mathrm{Km} / \mathrm{h}+80 \\
193502, \text { lin }\end{array}$ & + Bike- \\
\hline Deviance Residuals: & & & & & \\
\hline Min & 10 & Median & $3 Q$ & Max & \\
\hline-2.972 & -1.2205 & -0.2522 & 0.3687 & 2.7912 & \\
\hline & Estimate & Std.Error & $z$ value & $\operatorname{Pr}(>|z|)$ & \\
\hline (Intercept) & $-6.17 \mathrm{E}-04$ & $1.08 \mathrm{E}-01$ & -0.006 & 0.995418 & \\
\hline No Bus stops & $1.11 \mathrm{E}-02$ & $3.66 \mathrm{E}-03$ & 3.04 & 0.002365 & $* *$ \\
\hline Tot-Int & $1.41 \mathrm{E}-02$ & $2.11 \mathrm{E}-03$ & 6.674 & $2.49 \mathrm{E}-11$ & $* * *$ \\
\hline $40 . \mathrm{Km} / \mathrm{h}$ & $-7.66 \mathrm{E}-02$ & $4.46 \mathrm{E}-02$ & -1.719 & 0.085673 & . \\
\hline $50 . \mathrm{Km} / \mathrm{h}$ & $-8.19 E-02$ & 4.05E-02 & -2.023 & 0.043119 & $*$ \\
\hline $70 . \mathrm{Km} / \mathrm{h}$ & $-2.89 \mathrm{E}-01$ & $2.04 \mathrm{E}-01$ & -1.419 & 0.155949 & \\
\hline $80 . \mathrm{Km} / \mathrm{h}$ & $-2.80 \mathrm{E}-01$ & $1.20 \mathrm{E}-01$ & -2.335 & 0.019547 & $*$ \\
\hline Bike-Path & $2.81 \mathrm{E}-02$ & 8.87E-03 & 3.167 & 0.001541 & $* *$ \\
\hline Trips-Attracted & $2.34 \mathrm{E}-05$ & $6.47 \mathrm{E}-06$ & 3.611 & 0.000305 & $* * *$ \\
\hline Density- Trips-Enter-Center & $-4.14 \mathrm{E}-05$ & $9.21 \mathrm{E}-06$ & -4.495 & $6.97 \mathrm{E}-06$ & $* * *$ \\
\hline TLKM & $8.30 \mathrm{E}-02$ & 4.07E-02 & 2.04 & 0.041358 & $*$ \\
\hline $\begin{array}{l}\text { Signif.codes: }\left(0^{\prime * * * \prime}\right)(0.001 \\
\text { (Dispersion parameter for } \mathrm{N}\end{array}$ & $\begin{array}{l}\text { 'l) }^{\prime}\left(0.01^{\prime * \prime}\right) \\
\text { ative Binom }\end{array}$ & $\begin{array}{l}\left..^{\prime}\right)\left(0.1^{\prime \prime}\right) \\
.148) \text { famil }\end{array}$ & $n$ to be 1 & & \\
\hline Null deviance: 649.4 on 421 & egrees of $\mathrm{fr}$ & & & & \\
\hline Residual deviance: 463.79 o & 11 degrees & eedom & & & \\
\hline AIC: 1498.4 & & & & & \\
\hline Number of Fisher Scoring ite & tions: 1 & & & & \\
\hline Theta: 3.915 & & & & & \\
\hline Std. Err.: 0.818 & & & & & \\
\hline $2 \times$ log-likelihood: -1474.395 & & & & & \\
\hline
\end{tabular}




\begin{tabular}{|c|c|c|c|c|c|}
\hline \multicolumn{6}{|c|}{$\begin{array}{l}\text { Call: } \\
\text { glm.nb(formula = Collisions No.Schools + MS-Driver + MS-Passenger + MS-Transit + MS-Walk + MS- } \\
\text { Bike + Population + Age.0-4 + Age.5-24 + Age. } 25-64+\text { Trips-Enter-Center + Density- Trips-Out-Center + } \\
\text { Density- Trips-Enter-Center, init.theta }=5.53518999, \text { link }=\text { log) }\end{array}$} \\
\hline \multicolumn{6}{|l|}{ Deviance Residuals: } \\
\hline Min & 10 & Median & $3 Q$ & Max & \\
\hline \multirow[t]{2}{*}{-2.596} & -1.0742 & -0.2821 & 0.4657 & 3.1571 & \\
\hline & Estimate & Std.Error & $z$ value & $\operatorname{Pr}(>|z|)$ & \\
\hline (Intercept) & $-2.78 \mathrm{E}+00$ & $8.43 \mathrm{E}-01$ & -3.298 & 0.000973 & $* * *$ \\
\hline No.Schools & $1.32 \mathrm{E}-01$ & $3.89 \mathrm{E}-02$ & 3.391 & 0.000696 & $* * *$ \\
\hline MS-Driver & $1.93 \mathrm{E}-02$ & 8.91E-03 & 2.16 & 0.030784 & $*$ \\
\hline MS-Passenger & $4.23 \mathrm{E}-02$ & $1.18 \mathrm{E}-02$ & 3.594 & 0.000325 & $* * *$ \\
\hline MS-Transit & $5.36 \mathrm{E}-02$ & $1.01 \mathrm{E}-02$ & 5.297 & $1.18 \mathrm{E}-07$ & $* * *$ \\
\hline MS-Walk & $3.45 \mathrm{E}-02$ & $9.75 \mathrm{E}-03$ & 3.542 & 0.000397 & $* * *$ \\
\hline MS-Bike & $6.21 \mathrm{E}-02$ & $1.92 \mathrm{E}-02$ & 3.232 & 0.001228 & $* *$ \\
\hline Population & $6.19 \mathrm{E}-05$ & 3.00E-05 & 2.066 & 0.038782 & $*$ \\
\hline Age.0-4 & $-5.08 \mathrm{E}-02$ & $2.32 \mathrm{E}-02$ & -2.189 & 0.028616 & $*$ \\
\hline Age.5-24 & $-7.91 E-03$ & 3.95E-03 & -2.004 & 0.045056 & $*$ \\
\hline Age.25-64 & $9.42 \mathrm{E}-03$ & $2.53 \mathrm{E}-03$ & 3.725 & 0.000195 & $* * *$ \\
\hline Trips-Enter-Center & $1.18 \mathrm{E}-04$ & $2.64 \mathrm{E}-05$ & 4.485 & 7.31E-06 & $* * *$ \\
\hline Density- Trips-Out-Center & $8.40 \mathrm{E}-05$ & 2.87E-05 & 2.931 & 0.003381 & $* *$ \\
\hline Density- Trips-Enter-Center & $-2.39 \mathrm{E}-05$ & 6.07E-06 & -3.944 & $8.00 \mathrm{E}-05$ & $* * *$ \\
\hline \multicolumn{6}{|c|}{$\begin{array}{l}\text { Signif.codes: }\left(0^{\prime * * * \prime}\right)\left(0.001^{\prime * * \prime}\right)\left(0.01^{\prime * \prime}\right)\left(0.05^{\prime \prime}\right)\left(0.1^{\prime \prime}\right)(1) \\
\text { (Dispersion parameter for Negative Binomial }(2.148) \text { family taken to be } 1)\end{array}$} \\
\hline $\begin{array}{l}\text { Null deviance: } 709.21 \text { on } 42 \\
\text { Residual deviance: } 447.89 \text { o } \\
\text { AIC: } 1449.8\end{array}$ & $\begin{array}{l}\text { degrees of } \mathrm{fr} \\
08 \text { degrees }\end{array}$ & $\begin{array}{l}\text { om } \\
\text { eedom }\end{array}$ & & & \\
\hline \multicolumn{6}{|c|}{ Number of Fisher Scoring iterations: 1} \\
\hline \multicolumn{6}{|l|}{ Theta: 5.54} \\
\hline 2 x log-likelihood: -1419.8 & & & & & \\
\hline
\end{tabular}




\begin{tabular}{|c|c|c|c|c|c|}
\hline \multicolumn{6}{|c|}{$\begin{array}{l}\text { A-C-I } \\
\text { Call: } \\
\text { glm.nb(formula = Collisions } \sim \text { VKT + Trips-Out-Center + Trips- } \\
\text { Density- Trips-Enter-Center + TLKM, init.theta }=2.451825642\end{array}$} \\
\hline \multicolumn{6}{|l|}{ Deviance Residuals: } \\
\hline Min & 10 & Median & $3 Q$ & Max & \\
\hline \multirow[t]{2}{*}{-3.1607} & -1.2575 & -0.2796 & 0.3403 & 2.7846 & \\
\hline & Estimate & Std.Error & $z$ value & $\operatorname{Pr}(>|z|)$ & \\
\hline (Intercept) & $-7.13 \mathrm{E}-02$ & 1.07E-01 & -0.667 & 0.504754 & \\
\hline VKT & $1.66 \mathrm{E}-06$ & $1.03 \mathrm{E}-06$ & 1.613 & 0.106662 & \\
\hline Trips-Out-Center & $1.22 \mathrm{E}-04$ & $4.34 \mathrm{E}-05$ & 2.801 & 0.005093 & $* *$ \\
\hline Trips-Enter-Center & $1.08 \mathrm{E}-04$ & $2.90 \mathrm{E}-05$ & 3.74 & 0.000184 & $* * *$ \\
\hline Density- Trips-Out-Center & $2.50 \mathrm{E}-04$ & $3.00 \mathrm{E}-05$ & 8.354 & $<2 \mathrm{E}-16$ & $* * *$ \\
\hline Density- Trips-Enter-Center & $-3.18 \mathrm{E}-05$ & $6.52 \mathrm{E}-06$ & -4.871 & $1.11 \mathrm{E}-06$ & $* * *$ \\
\hline TLKM & $-4.42 \mathrm{E}-03$ & $2.66 \mathrm{E}-03$ & -1.665 & 0.09582 & . \\
\hline \multicolumn{6}{|c|}{$\begin{array}{l}\text { Signif.codes: }\left(0^{(* * * \prime)}\left(0.001^{(* * \prime}\right)\left(0.01^{\prime * \prime}\right)\left(0.05^{\prime \prime}\right)\left(0.1^{\prime \prime}\right)(1)\right. \\
\text { (Dispersion parameter for Negative Binomial }(2.148) \text { family taken to be } 1)\end{array}$} \\
\hline \multicolumn{6}{|c|}{ Null deviance: 559.17 on 421 degrees of freedom } \\
\hline \multicolumn{6}{|c|}{ Residual deviance: 453.76 on 415 degrees of freedom } \\
\hline \multicolumn{6}{|c|}{ Number of Fisher Scoring iterations: 1} \\
\hline \multicolumn{6}{|l|}{ Theta: 2.452} \\
\hline \multicolumn{6}{|l|}{ Std. Err.: 0.385} \\
\hline 2 x log-likelihood: -1531.45 & & & & & \\
\hline
\end{tabular}




\begin{tabular}{|c|c|c|c|c|c|}
\hline \multicolumn{6}{|c|}{$\begin{array}{l}\text { Call: } \\
\text { glm.nb(formula }=\text { Collisions } \sim \text { Density- Trips-Out-Center + Density- Trips-Enter-Center, init.theta }= \\
2.213645231, \text { link }=\log )\end{array}$} \\
\hline \multicolumn{6}{|l|}{ Deviance Residuals: } \\
\hline Min & 10 & Median & 30 & Max & \\
\hline-3.1591 & -1.4061 & -0.2698 & 0.4199 & 2.525 & \\
\hline & Estimate & Std.Error & $z$ value & $\operatorname{Pr}(>|z|)$ & \\
\hline (Intercept) & $2.76 \mathrm{E}-01$ & $6.64 \mathrm{E}-02$ & 4.147 & 3.37E-05 & $* * *$ \\
\hline Density- Trips-Out-Center & $2.68 \mathrm{E}-04$ & $2.74 \mathrm{E}-05$ & 9.771 & $<2 \mathrm{E}-16$ & $* * *$ \\
\hline Density- Trips-Enter-Center & $-3.15 \mathrm{E}-05$ & $5.54 \mathrm{E}-06$ & -5.686 & $1.30 \mathrm{E}-08$ & $* * *$ \\
\hline \multicolumn{6}{|c|}{$\begin{array}{l}\text { Signif.codes: }\left(0^{\prime * * * \prime}\right)\left(0.001^{\prime * * \prime}\right)\left(0.01^{\prime * \prime}\right)\left(0.05^{\prime \prime} .\right)\left(0.1^{\prime \prime}\right)(1) \\
\text { (Dispersion parameter for Negative Binomial }(2.148) \text { family taken to be } 1)\end{array}$} \\
\hline \multicolumn{6}{|c|}{ Null deviance: 538.57 on 421 degrees of freedom } \\
\hline \multicolumn{6}{|c|}{ Residual deviance: 458.57 on 419 degrees of freedom } \\
\hline \multicolumn{6}{|l|}{ AIC: 1561.4} \\
\hline \multicolumn{6}{|c|}{ Number of Fisher Scoring iterations: 1} \\
\hline \multicolumn{6}{|l|}{ Theta: 2.214} \\
\hline \multicolumn{6}{|l|}{ Std. Err.: 0.334} \\
\hline 2 x log-likelihood: -1553.401 & & & & & \\
\hline
\end{tabular}




\begin{tabular}{|c|c|c|c|c|c|}
\hline \multicolumn{6}{|c|}{$\begin{array}{l}\text { Call: } \\
\text { glm.nb(formula }=\text { Collisions } \sim \text { No Bus stops }+ \text { Tot-Int }+ \text { Sig-Int }+70 . \mathrm{Km} / \mathrm{h}+90 . \mathrm{Km} / \mathrm{h}+\text { Bike-Path }+ \text { VKT }+ \\
\text { Trips-Attracted + Density- Trips-Out-Center + Density- Trips-Enter-Center, init.theta }=3.513076428, \text { link } \\
=\text { log) }\end{array}$} \\
\hline \multicolumn{6}{|l|}{ Deviance Residuals: } \\
\hline Min & 10 & Median & $3 Q$ & Max & \\
\hline \multirow[t]{2}{*}{-2.0881} & -0.9868 & -0.5128 & 0.3104 & 2.8208 & \\
\hline & Estimate & Std.Error & $z$ value & $\operatorname{Pr}(>|z|)$ & \\
\hline (Intercept) & $-8.81 E-01$ & $1.13 \mathrm{E}-01$ & -7.772 & $7.75 \mathrm{E}-15$ & $* * *$ \\
\hline No Bus stops & $8.04 \mathrm{E}-03$ & $5.31 \mathrm{E}-03$ & 1.515 & 0.1298 & \\
\hline Tot-Int & $4.43 \mathrm{E}-03$ & $2.19 \mathrm{E}-03$ & 2.025 & 0.0429 & $*$ \\
\hline Sig-Int & $2.33 \mathrm{E}-01$ & $2.12 \mathrm{E}-02$ & 10.986 & $<2 \mathrm{E}-16$ & $* * *$ \\
\hline $70 . \mathrm{Km} / \mathrm{h}$ & $-3.95 E-01$ & 8.89E-02 & -4.447 & $8.73 E-06$ & $* * *$ \\
\hline $90 . \mathrm{Km} / \mathrm{h}$ & $-1.43 E+00$ & 9.95E-01 & -1.437 & 0.1507 & \\
\hline Bike-Path & $2.05 \mathrm{E}-02$ & $1.34 \mathrm{E}-02$ & 1.529 & 0.1262 & \\
\hline VKT & $-2.29 \mathrm{E}-06$ & $1.34 \mathrm{E}-06$ & -1.718 & 0.0857 & . \\
\hline Trips-Attracted & $7.80 \mathrm{E}-05$ & 3.87E-05 & 2.016 & 0.0438 & $*$ \\
\hline Density- Trips-Out-Center & $2.00 \mathrm{E}-04$ & $3.15 E-05$ & 6.334 & $2.38 \mathrm{E}-10$ & $* * *$ \\
\hline Density- Trips-Enter-Center & $-3.16 \mathrm{E}-05$ & $7.86 \mathrm{E}-06$ & -4.022 & $5.78 \mathrm{E}-05$ & $* * *$ \\
\hline \multicolumn{6}{|c|}{$\begin{array}{l}\text { Signif.codes: }\left(0^{\prime * * * \prime}\right)\left(0.001^{\prime * * \prime}\right)\left(0.01^{\prime * \prime}\right)\left(0.05^{\prime \prime}\right)\left(0.1^{\prime \prime}\right)(1) \\
\text { (Dispersion parameter for Negative Binomial }(2.148) \text { family taken to be } 1)\end{array}$} \\
\hline \multicolumn{6}{|c|}{ Null deviance: 827.48 on 421 degrees of freedom } \\
\hline \multicolumn{6}{|c|}{ Residual deviance: 419.65 on 411 degrees of freedom } \\
\hline \multicolumn{6}{|l|}{ AIC: 1321.9} \\
\hline \multicolumn{6}{|c|}{ Number of Fisher Scoring iterations: 1} \\
\hline \multicolumn{6}{|l|}{ Theta: 3.513} \\
\hline \multicolumn{6}{|l|}{ Std. Err.: 0.704} \\
\hline \multicolumn{6}{|l|}{2 x log-likelihood: -1297.868} \\
\hline
\end{tabular}


Call: B-C-V

glm.nb(formula $=$ Collisions No.Schools +Area + MS-Passenger + MS-Transit + MS-Walk + MS-Bike + Employment + Population + Age.0-4 + Age.25-64 + Age > 65 + Density-Trips-Out-Center + Density-

Trips-Enter-Center, init.theta $=3.74565843$, link $=\log$ )

Deviance Residuals:

\begin{tabular}{|c|c|c|c|c|c|}
\hline Min & $1 Q$ & Median & $3 Q$ & Max & \\
\hline-2.7437 & -0.9195 & -0.5054 & 0.4293 & 2.8341 & \\
\hline & Estimate & Std.Error & $z$ value & $\operatorname{Pr}(>|z|)$ & \\
\hline (Intercept) & $-2.50 \mathrm{E}+00$ & $3.23 \mathrm{E}-01$ & -7.748 & $9.34 \mathrm{E}-15$ & $* * *$ \\
\hline No.Schools & 1.01E-01 & $4.26 \mathrm{E}-02$ & 2.371 & 0.017746 & $*$ \\
\hline Area & $-3.31 E-03$ & $2.48 \mathrm{E}-03$ & -1.335 & 0.181947 & \\
\hline MS-Passenger & $3.43 \mathrm{E}-02$ & $1.24 \mathrm{E}-02$ & 2.77 & 0.005603 & $* *$ \\
\hline MS-Transit & $3.99 E-02$ & $7.13 \mathrm{E}-03$ & 5.6 & $2.14 \mathrm{E}-08$ & $* * *$ \\
\hline MS-Walk & $2.11 \mathrm{E}-02$ & $6.37 \mathrm{E}-03$ & 3.315 & 0.000917 & $* * *$ \\
\hline MS-Bike & $5.83 \mathrm{E}-02$ & $2.08 \mathrm{E}-02$ & 2.797 & 0.005156 & $* *$ \\
\hline Employment & $1.13 \mathrm{E}-04$ & $3.69 \mathrm{E}-05$ & 3.059 & 0.002218 & $* *$ \\
\hline Population & $1.53 \mathrm{E}-04$ & $3.36 \mathrm{E}-05$ & 4.549 & $5.40 \mathrm{E}-06$ & $* * *$ \\
\hline Age.0-4 & $-6.15 E-02$ & $2.60 \mathrm{E}-02$ & -2.371 & 0.017757 & $*$ \\
\hline Age.25-64 & $2.07 \mathrm{E}-02$ & $3.54 \mathrm{E}-03$ & 5.839 & $5.26 \mathrm{E}-09$ & $* * *$ \\
\hline Age $>65$ & $2.09 \mathrm{E}-02$ & $7.18 \mathrm{E}-03$ & 2.91 & 0.003618 & $* *$ \\
\hline Density- Trips-Out-Center & $7.07 \mathrm{E}-05$ & 3.69E-05 & 1.915 & 0.0555 & . \\
\hline Density- Trips-Enter-Center & $-2.85 \mathrm{E}-05$ & $8.91 \mathrm{E}-06$ & -3.197 & 0.001388 & ** \\
\hline
\end{tabular}

Signif.codes: $\left(0^{\prime * * * \prime}\right)\left(0.001^{\prime * * \prime}\right)\left(0.01^{\prime * \prime}\right)\left(0.05^{\prime \prime}.\right)\left(0.1^{\prime \prime}\right)(1)$

(Dispersion parameter for Negative Binomial (2.148) family taken to be 1 )

Null deviance: 842.61 on 421 degrees of freedom

Residual deviance: 421.78 on 408 degrees of freedom

AIC: 1322.8

Number of Fisher Scoring iterations: 1

Theta: 3.746

Std. Err.: 0.805

2 x log-likelihood: -1292.782 


\begin{tabular}{|c|c|c|c|c|c|}
\hline \multicolumn{6}{|c|}{$\begin{array}{l}\text { C:C-III } \\
\text { Call: } \\
\text { glm.nb(formula = Collisions } \sim \text { Sig-Int + 50.Kr } \\
\text { Out-Center + Density- Trips-Enter-Center, i }\end{array}$} \\
\hline \multicolumn{6}{|l|}{ Deviance Residuals: } \\
\hline Min & 10 & Median & $3 Q$ & Max & \\
\hline \multirow[t]{2}{*}{-2.636} & -1.0952 & -0.2768 & 0.4198 & 2.8541 & \\
\hline & Estimate & Std.Error & $z$ value & $\operatorname{Pr}(>|z|)$ & \\
\hline (Intercept) & $-4.99 \mathrm{E}-01$ & $8.86 \mathrm{E}-02$ & -5.633 & $1.77 \mathrm{E}-08$ & $* * *$ \\
\hline Sig-Int & $2.41 \mathrm{E}-01$ & $1.96 \mathrm{E}-02$ & 12.311 & $<2 \mathrm{E}-16$ & $* * *$ \\
\hline $50 . \mathrm{Km} / \mathrm{h}$ & $2.98 \mathrm{E}-02$ & 8.47E-03 & 3.513 & 0.000443 & $* * *$ \\
\hline $70 . \mathrm{Km} / \mathrm{h}$ & $-3.15 E-01$ & $7.78 \mathrm{E}-02$ & -4.044 & $5.26 \mathrm{E}-05$ & $* * *$ \\
\hline $90 . \mathrm{Km} / \mathrm{h}$ & $-1.51 \mathrm{E}+00$ & $8.98 \mathrm{E}-01$ & -1.687 & 0.09163 & . \\
\hline Bike-Path & $2.65 \mathrm{E}-02$ & $1.25 \mathrm{E}-02$ & 2.126 & 0.03353 & $*$ \\
\hline VKT & $-2.97 \mathrm{E}-06$ & $1.48 \mathrm{E}-06$ & -2.005 & 0.044921 & $*$ \\
\hline Density- Trips-Out-Center & $1.34 \mathrm{E}-04$ & $2.49 \mathrm{E}-05$ & 5.395 & $6.83 \mathrm{E}-08$ & $* * *$ \\
\hline Density- Trips-Enter-Center & $-1.28 \mathrm{E}-05$ & $4.92 \mathrm{E}-06$ & -2.611 & 0.009037 & $* *$ \\
\hline \multicolumn{6}{|c|}{$\begin{array}{l}\text { Signif.codes: }\left(0^{\prime * * * \prime}\right)\left(0.001^{(* * \prime}\right)\left(0.01^{\prime * \prime}\right)\left(0.05^{\prime} .^{\prime}\right)\left(0.1^{\prime \prime}\right)(1) \\
\text { (Dispersion parameter for Negative Binomial }(2.148) \text { family taken to be } 1)\end{array}$} \\
\hline $\begin{array}{l}\text { Null deviance: } 774.67 \text { on } 42 \\
\text { Residual deviance: } 439.74 \text { o } \\
\text { AIC: } 1384.6\end{array}$ & $\begin{array}{l}\text { degrees of } \mathrm{fr} \\
13 \text { degrees }\end{array}$ & eedom & & & \\
\hline \multicolumn{6}{|c|}{ Number of Fisher Scoring iterations: 1} \\
\hline \multicolumn{6}{|l|}{ Theta: 4.95} \\
\hline \multicolumn{6}{|l|}{$2 \times$ log-likelihood: -1364.556} \\
\hline
\end{tabular}




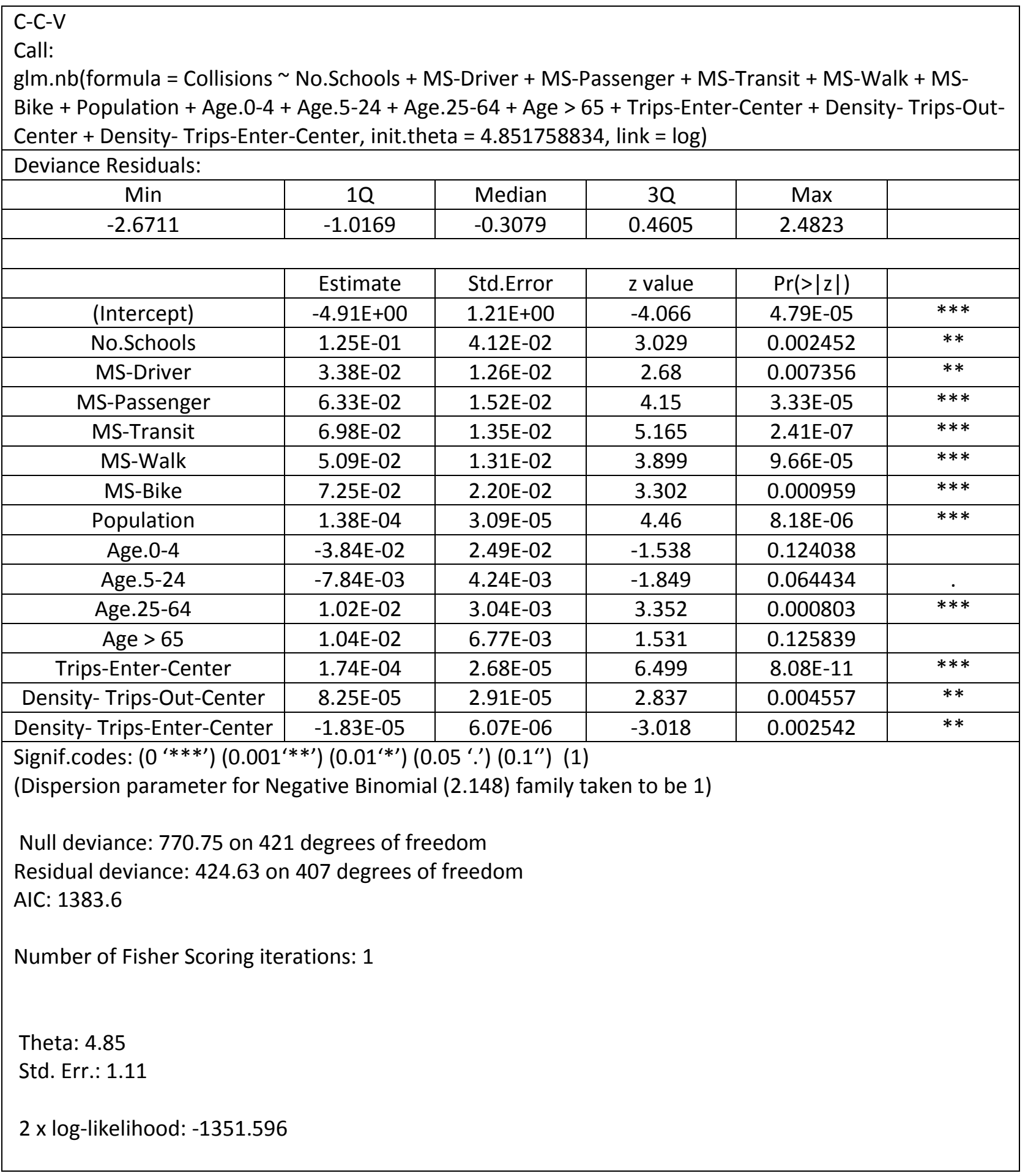




\begin{tabular}{|c|c|c|c|c|c|}
\hline $\begin{array}{l}\text { G:C-II } \\
\text { Call: } \\
\text { glm.nb(formula = Collisions } \\
\text { Density- Trips-Out-Center + }\end{array}$ & $\begin{array}{l}\text { o Bus stops } \\
\text { nsity- Trips- }\end{array}$ & $\begin{array}{l}\text { g-Int + Art } \\
\text { r-Center, ir }\end{array}$ & $\begin{array}{l}\text { le }+ \text { Maj- } \\
\mathrm{ta}=2.55\end{array}$ & $\begin{array}{l}+ \text { VKT + Tri } \\
59, \text { link }=1\end{array}$ & acted + \\
\hline Deviance Residuals: & & & & & \\
\hline Min & 10 & Median & $3 Q$ & Max & \\
\hline-3.7941 & -0.9024 & -0.124 & 0.4456 & 2.8507 & \\
\hline & Estimate & Std.Error & $z$ value & $\operatorname{Pr}(>|z|)$ & \\
\hline (Intercept) & $1.90 \mathrm{E}+00$ & $6.35 \mathrm{E}-02$ & 29.864 & $<2 \mathrm{E}-16$ & $* * *$ \\
\hline No Bus stops & $1.74 \mathrm{E}-02$ & $3.24 \mathrm{E}-03$ & 5.37 & $7.88 \mathrm{E}-08$ & $* * *$ \\
\hline Sig-Int & $2.66 \mathrm{E}-01$ & $1.72 \mathrm{E}-02$ & 15.451 & $<2 \mathrm{E}-16$ & $* * *$ \\
\hline Artl & $2.84 \mathrm{E}-02$ & $9.23 \mathrm{E}-03$ & 3.082 & 0.002053 & $* *$ \\
\hline Colle & $1.04 \mathrm{E}-02$ & $5.11 \mathrm{E}-03$ & 2.039 & 0.041481 & $*$ \\
\hline Maj-Colle & $-1.65 E-01$ & $7.95 \mathrm{E}-02$ & -2.081 & 0.037455 & $*$ \\
\hline VKT & $-2.20 \mathrm{E}-06$ & $8.82 \mathrm{E}-07$ & -2.487 & 0.012867 & $*$ \\
\hline Trips-Attracted & 1.05E-04 & $2.79 \mathrm{E}-05$ & 3.74 & 0.000184 & $* * *$ \\
\hline Density- Trips-Out-Center & $5.64 \mathrm{E}-05$ & $2.01 \mathrm{E}-05$ & 2.803 & 0.005059 & $* *$ \\
\hline Density- Trips-Enter-Center & $-1.36 \mathrm{E}-05$ & $3.77 \mathrm{E}-06$ & -3.604 & 0.000314 & $* * *$ \\
\hline $\begin{array}{l}\text { Signif.codes: }\left(0^{* * * *)}(0.001\right. \\
\text { (Dispersion parameter for } N\end{array}$ & $\begin{array}{l}\text { k') }\left(0.01^{\prime * \prime}\right)( \\
\text { ative Binom }\end{array}$ & $\begin{array}{l}\left.y^{\prime}\right)\left(0.1^{\prime \prime}\right) \\
.148) \text { famil }\end{array}$ & n to be 1 & & \\
\hline Null deviance: 1100.19 on $\angle$ & degrees of & dom & & & \\
\hline Residual deviance: 495.05 o & 12 degrees & eedom & & & \\
\hline AIC: 3360.8 & & & & & \\
\hline Number of Fisher Scoring it & tions: 1 & & & & \\
\hline Theta: 2.556 & & & & & \\
\hline Std. Err.: 0.213 & & & & & \\
\hline 2 x log-likelihood: -3338.77 & & & & & \\
\hline
\end{tabular}


D-C-I

Call:

glm.nb(formula $=$ Collisions $\sim$ VKT + Trips-Out-Center + Trips-Enter-Center + Density- Trips-Out-Center + Density- Trips-Enter-Center, init.theta $=1.521083574$, link $=\log$ )

Deviance Residuals:

\begin{tabular}{|c|c|c|c|c|c|}
\hline Min & $1 \mathrm{Q}$ & Median & $3 Q$ & Max & \\
\hline-3.0646 & -1.1289 & -0.3649 & 0.294 & 2.9616 & \\
\hline \multicolumn{7}{|l|}{} \\
\hline & Estimate & Std.Error & z value & $\operatorname{Pr}(>|\mathrm{z}|)$ & \\
\hline (Intercept) & $-4.09 \mathrm{E}-01$ & $1.27 \mathrm{E}-01$ & -3.235 & 0.00122 & $* *$ \\
\hline VKT & $1.80 \mathrm{E}-06$ & $1.10 \mathrm{E}-06$ & 1.64 & 0.10101 & \\
\hline Trips-Out-Center & $1.95 \mathrm{E}-04$ & $4.60 \mathrm{E}-05$ & 4.252 & $2.12 \mathrm{E}-05$ & $* * *$ \\
\hline Trips-Enter-Center & $8.89 \mathrm{E}-05$ & $3.54 \mathrm{E}-05$ & 2.511 & 0.01205 & $*$ \\
\hline Density- Trips-Out-Center & $3.13 \mathrm{E}-04$ & $3.51 \mathrm{E}-05$ & 8.926 & $<2 \mathrm{E}-16$ & $* * *$ \\
\hline Density- Trips-Enter-Center & $-3.93 \mathrm{E}-05$ & $7.77 \mathrm{E}-06$ & -5.066 & $4.06 \mathrm{E}-07$ & $* * *$ \\
\hline Signiffodes: &
\end{tabular}

Signif.codes: $\left(0^{\prime * * * \prime}\right)\left(0.001^{\prime * * \prime}\right)\left(0.01^{\prime * \prime}\right)\left(0.05^{\prime \prime}\right)\left(0.1^{\prime \prime}\right)(1)$

(Dispersion parameter for Negative Binomial (2.148) family taken to be 1)

Null deviance: 562.54 on 421 degrees of freedom

Residual deviance: 445.47 on 416 degrees of freedom

AIC: 1515.7

Number of Fisher Scoring iterations: 1

Theta: 1.521

Std. Err.: 0.21

2 x log-likelihood: -1501.658 


\begin{tabular}{|c|c|c|c|c|c|}
\hline $\begin{array}{l}\text { G-C-I } \\
\text { Call: } \\
\text { glm.nb(formula = Collisions } \\
\text { Density- Trips-Enter-Center, }\end{array}$ & $\begin{array}{l}\text { KT + Trips- } \\
\text { t.theta }=1\end{array}$ & $\begin{array}{l}\text { Center + Tri } \\
15872 \text {, link }\end{array}$ & er-Cente & nsity- Trips & enter + \\
\hline Deviance Residuals: & & & & & \\
\hline Min & 10 & Median & $3 Q$ & Max & \\
\hline-4.5149 & -0.9062 & -0.2235 & 0.3759 & 3.7738 & \\
\hline & Estimate & Std.Error & $z$ value & $\operatorname{Pr}(>|z|)$ & \\
\hline (Intercept) & $2.30 \mathrm{E}+00$ & $9.05 \mathrm{E}-02$ & 25.449 & $<2 \mathrm{E}-16$ & $* * *$ \\
\hline VKT & $1.05 \mathrm{E}-05$ & $9.27 \mathrm{E}-07$ & 11.347 & $<2 \mathrm{E}-16$ & $* * *$ \\
\hline Trips-Out-Center & $1.72 \mathrm{E}-04$ & 3.71E-05 & 4.628 & 3.69E-06 & $* * *$ \\
\hline Trips-Enter-Center & $1.92 \mathrm{E}-04$ & $2.75 \mathrm{E}-05$ & 6.969 & $3.18 \mathrm{E}-12$ & $* * *$ \\
\hline Density- Trips-Out-Center & 1.37E-04 & $2.73 \mathrm{E}-05$ & 5.03 & $4.91 \mathrm{E}-07$ & $* * *$ \\
\hline Density- Trips-Enter-Center & $-2.45 \mathrm{E}-05$ & $5.28 \mathrm{E}-06$ & -4.633 & $3.61 \mathrm{E}-06$ & $* * *$ \\
\hline $\begin{array}{l}\text { Signif.codes: }\left(0^{\prime * * * \prime}\right)(0.001 \\
\text { (Dispersion parameter for } \mathrm{N}\end{array}$ & $\begin{array}{l}\left.{ }^{\prime \prime}\right)\left(0.01^{\prime * \prime}\right) \\
\text { ative Binom }\end{array}$ & $\begin{array}{l}\left..^{\prime}\right)\left(0.1^{\prime \prime}\right) \\
.148) \text { fami }\end{array}$ & $\mathrm{n}$ to be 1 & & \\
\hline Null deviance: 647.06 on 42 & degrees of $t$ & & & & \\
\hline Residual deviance: 488.81 o & 16 degrees & eedom & & & \\
\hline AIC: 3589.7 & & & & & \\
\hline Number of Fisher Scoring ite & tions: 1 & & & & \\
\hline Theta: 1.3525 & & & & & \\
\hline Std. Err.: 0.0979 & & & & & \\
\hline $2 \times$ log-likelihood: -3575.66 & & & & & \\
\hline
\end{tabular}




\begin{tabular}{|c|c|c|c|c|c|}
\hline \multicolumn{6}{|c|}{$\begin{array}{l}\text { Call: } \\
\text { glm.nb(formula }=\text { Collisions } \sim \text { VKT }+ \text { Trips-Out-Center }+ \text { Trips-Enter-Center }+ \text { Density- Trips-Out-Center + } \\
\text { Density- Trips-Enter-Center, init.theta }=1.505933911, \text { link }=\log )\end{array}$} \\
\hline \multicolumn{6}{|l|}{ Deviance Residuals: } \\
\hline Min & 10 & Median & $3 Q$ & Max & \\
\hline \multirow[t]{2}{*}{-2.8668} & -1.1105 & -0.3962 & 0.3312 & 3.1076 & \\
\hline & Estimate & Std.Error & $z$ value & $\operatorname{Pr}(>|z|)$ & \\
\hline (Intercept) & $-3.97 E-01$ & $1.25 \mathrm{E}-01$ & -3.179 & 0.00148 & $* *$ \\
\hline VKT & $2.11 \mathrm{E}-06$ & $9.06 \mathrm{E}-07$ & 2.329 & 0.01986 & $*$ \\
\hline Trips-Out-Center & $1.48 \mathrm{E}-04$ & $4.68 \mathrm{E}-05$ & 3.164 & 0.00156 & $* *$ \\
\hline Trips-Enter-Center & $1.41 \mathrm{E}-04$ & $3.35 \mathrm{E}-05$ & 4.221 & $2.44 \mathrm{E}-05$ & $* * *$ \\
\hline Density- Trips-Out-Center & $3.02 \mathrm{E}-04$ & $3.46 \mathrm{E}-05$ & 8.72 & $<2 \mathrm{E}-16$ & $* * *$ \\
\hline Density- Trips-Enter-Center & $-3.87 E-05$ & $7.58 \mathrm{E}-06$ & -5.105 & $3.31 \mathrm{E}-07$ & $* * *$ \\
\hline \multicolumn{6}{|c|}{$\begin{array}{l}\text { Signif.codes: }\left(0^{\prime * * * \prime}\right)\left(0.001^{\prime * * \prime}\right)\left(0.01^{\prime * \prime}\right)\left(0.05^{\prime \prime}\right)\left(0.1^{\prime \prime}\right)(1) \\
\text { (Dispersion parameter for Negative Binomial }(2.148) \text { family taken to be } 1)\end{array}$} \\
\hline \multicolumn{6}{|c|}{ Null deviance: 557.11 on 421 degrees of freedom } \\
\hline \multicolumn{6}{|c|}{ Residual deviance: 447.37 on 416 degrees of freedom } \\
\hline \multicolumn{6}{|l|}{ AIC: 1522.5} \\
\hline \multicolumn{6}{|c|}{ Number of Fisher Scoring iterations: 1} \\
\hline \multicolumn{6}{|l|}{ Theta: 1.506} \\
\hline \multicolumn{6}{|l|}{ Std. Err.: 0.208} \\
\hline \multicolumn{6}{|l|}{$2 \times$ log-likelihood: -1508.486} \\
\hline
\end{tabular}




\begin{tabular}{|c|c|c|c|c|c|}
\hline $\begin{array}{l}\text { J-D-I } \\
\text { Call: } \\
\text { glm.nb(formula = Collisions } \\
\text { TLKM, init.theta }=1.928752\end{array}$ & $\begin{array}{l}\text { (KT + Trips-P } \\
\text { 5, link = log) }\end{array}$ & ced + Trips & cted + D & - Trips-Ente & er + \\
\hline Deviance Residuals: & & & & & \\
\hline Min & 10 & Median & $3 Q$ & Max & \\
\hline-3.6402 & -1.1934 & -0.3055 & 0.3804 & 2.8266 & \\
\hline & Estimate & Std.Error & $z$ value & $\operatorname{Pr}(>|z|)$ & \\
\hline (Intercept) & $2.20 \mathrm{E}-01$ & $8.21 \mathrm{E}-02$ & 2.683 & 0.007304 & $* *$ \\
\hline VKT & $7.53 \mathrm{E}-06$ & $2.50 \mathrm{E}-06$ & 3.015 & 0.002566 & $* *$ \\
\hline Trips-Produced & $-5.08 \mathrm{E}-05$ & $2.74 \mathrm{E}-05$ & -1.856 & 0.063499 & . \\
\hline Trips-Attracted & $2.60 \mathrm{E}-05$ & $7.26 \mathrm{E}-06$ & 3.585 & 0.000337 & $* * *$ \\
\hline Density- Trips-Enter-Center & $-5.96 \mathrm{E}-05$ & $1.06 \mathrm{E}-05$ & -5.605 & $2.09 \mathrm{E}-08$ & $* * *$ \\
\hline TLKM & $9.60 \mathrm{E}-02$ & $1.03 \mathrm{E}-02$ & 9.365 & $<2 \mathrm{E}-16$ & $* * *$ \\
\hline $\begin{array}{l}\text { Signif.codes: }\left(0^{\prime * * * \prime}\right)(0.001 \\
\text { (Dispersion parameter for } \mathrm{N}\end{array}$ & $\begin{array}{l}\left.k^{\prime}\right)\left(0.01^{\prime * \prime}\right) \\
\text { ative Binom }\end{array}$ & $\begin{array}{l}\because \prime)\left(0.1^{\prime \prime}\right) \\
.148) \text { famil }\end{array}$ & $\mathrm{n}$ to be & & \\
\hline Null deviance: 680.82 on 42 & degrees of $\mathrm{fr}$ & & & & \\
\hline Residual deviance: 473.69 o & 16 degrees & eedom & & & \\
\hline AIC: 1866.7 & & & & & \\
\hline Number of Fisher Scoring ite & tions: 1 & & & & \\
\hline Theta: 1.929 & & & & & \\
\hline Std. Err.: 0.235 & & & & & \\
\hline $2 \times$ log-likelihood: -1852.68 & & & & & \\
\hline
\end{tabular}


$\mathrm{K}: \mathrm{C}-\mathrm{III}$

Call:

gIm.nb(formula $=$ Collisions $~$ Sig-Int $+40 . \mathrm{Km} / \mathrm{h}+50 \cdot \mathrm{Km} / \mathrm{h}+60 . \mathrm{Km} / \mathrm{h}+70 . \mathrm{Km} / \mathrm{h}+80 . \mathrm{Km} / \mathrm{h}+90 . \mathrm{Km} / \mathrm{h}+$ Trips-Out-Center + Density- Trips-Out-Center + Density- Trips-Enter-Center, init.theta $=9.3987493$, link = log)

Deviance Residuals:

\begin{tabular}{|c|c|c|c|c|c|}
\hline Min & $1 \mathrm{Q}$ & Median & $3 \mathrm{Q}$ & Max & \\
\hline-2.5517 & -1.1254 & -0.2562 & 0.423 & 3.1629 & \\
\hline \multicolumn{7}{|l|}{} \\
\hline \multicolumn{7}{|c|}{} & Estimate & Std.Error & z value & $\operatorname{Pr}(>|\mathrm{z}|)$ & \\
\hline (Intercept) & $-4.02 \mathrm{E}-01$ & $9.49 \mathrm{E}-02$ & -4.233 & $2.31 \mathrm{E}-05$ & $* * *$ \\
\hline Sig-Int & $2.43 \mathrm{E}-01$ & $1.84 \mathrm{E}-02$ & 13.202 & $<2 \mathrm{E}-16$ & $* * *$ \\
\hline $40 . \mathrm{Km} / \mathrm{h}$ & $3.07 \mathrm{E}-02$ & $1.14 \mathrm{E}-02$ & 2.706 & 0.0068 & $* *$ \\
\hline $50 . \mathrm{Km} / \mathrm{h}$ & $4.56 \mathrm{E}-02$ & $9.70 \mathrm{E}-03$ & 4.698 & $2.63 \mathrm{E}-06$ & $* * *$ \\
\hline $60 . \mathrm{Km} / \mathrm{h}$ & $3.46 \mathrm{E}-02$ & $2.05 \mathrm{E}-02$ & 1.684 & 0.09213 &. \\
\hline $70 . \mathrm{Km} / \mathrm{h}$ & $-2.30 \mathrm{E}-01$ & $5.63 \mathrm{E}-02$ & -4.078 & $4.55 \mathrm{E}-05$ & $* * *$ \\
\hline $80 . \mathrm{Km} / \mathrm{h}$ & $-5.91 \mathrm{E}-03$ & $3.41 \mathrm{E}-03$ & -1.733 & 0.08306 &. \\
\hline $90 . \mathrm{Km} / \mathrm{h}$ & $-5.28 \mathrm{E}-01$ & $4.00 \mathrm{E}-01$ & -1.319 & 0.18706 & \\
\hline Trips-Out-Center & $-1.14 \mathrm{E}-04$ & $5.27 \mathrm{E}-05$ & -2.161 & 0.03071 & $*$ \\
\hline Density- Trips-Out-Center & $1.33 \mathrm{E}-04$ & $2.37 \mathrm{E}-05$ & 5.612 & $2.00 \mathrm{E}-08$ & $* * *$ \\
\hline Density- Trips-Enter-Center & $-1.48 \mathrm{E}-05$ & $4.96 \mathrm{E}-06$ & -2.987 & 0.00282 & $* *$ \\
\hline
\end{tabular}

Signif.codes: $\left(0^{(* * * \prime)}\left(0.001^{\left(* *^{\prime}\right.}\right)\left(0.01^{\prime * \prime}\right)\left(0.05^{\prime \prime}\right)\left(0.1^{\prime \prime}\right)\right.$ (1)

(Dispersion parameter for Negative Binomial (2.148) family taken to be 1 )

Null deviance: 785.24 on 421 degrees of freedom

Residual deviance: 446.73 on 411 degrees of freedom

AIC: 1398.6

Number of Fisher Scoring iterations: 1

Theta: 9.4

Std. Err.: 3.2

2 x log-likelihood: -1374.554 


\begin{tabular}{|c|c|c|c|c|c|}
\hline $\begin{array}{l}\text { K:D-III } \\
\text { Call: } \\
\text { glm.nb(formula = Collisions } \\
\text { Produced + Trips-Attracted }\end{array}$ & $\begin{array}{l}\text { o Bus stops } \\
\text { ensity- Trip }\end{array}$ & $\begin{array}{l}\mathrm{Km} / \mathrm{h}+50 \\
\text { er-Center }\end{array}$ & $\begin{array}{l}+60 . \mathrm{Kr} \\
\text { h, init.the }\end{array}$ & $\begin{array}{l}\text { Bike-Path + } \\
.53607006\end{array}$ & $\begin{array}{l}\text { Trips- } \\
=\log )\end{array}$ \\
\hline Deviance Residuals: & & & & & \\
\hline Min & $1 Q$ & Median & $3 Q$ & Max & \\
\hline-3.247 & -1.1919 & -0.222 & 0.4118 & 2.7171 & \\
\hline & Estimate & Std.Error & $z$ value & $\operatorname{Pr}(>|z|)$ & \\
\hline (Intercept) & $9.64 \mathrm{E}-02$ & $1.04 \mathrm{E}-01$ & 0.926 & 0.354433 & \\
\hline No Bus stops & $7.45 \mathrm{E}-03$ & $3.67 \mathrm{E}-03$ & 2.028 & 0.042534 & $*$ \\
\hline $40 . \mathrm{Km} / \mathrm{h}$ & $2.74 \mathrm{E}-01$ & $6.97 \mathrm{E}-02$ & 3.927 & $8.62 \mathrm{E}-05$ & $* * *$ \\
\hline $50 . \mathrm{Km} / \mathrm{h}$ & $2.69 \mathrm{E}-01$ & $6.75 \mathrm{E}-02$ & 3.981 & 6.87E-05 & $* * *$ \\
\hline $60 . \mathrm{Km} / \mathrm{h}$ & $2.44 \mathrm{E}-01$ & $6.90 \mathrm{E}-02$ & 3.531 & 0.000414 & $* * *$ \\
\hline Bike-Path & $2.73 \mathrm{E}-02$ & $8.56 \mathrm{E}-03$ & 3.19 & 0.001423 & $* *$ \\
\hline VKT & $7.27 \mathrm{E}-06$ & $2.83 \mathrm{E}-06$ & 2.572 & 0.010113 & * \\
\hline Trips-Produced & $-4.04 \mathrm{E}-05$ & $2.41 \mathrm{E}-05$ & -1.675 & 0.094012 & . \\
\hline Trips-Attracted & $3.16 \mathrm{E}-05$ & $6.82 \mathrm{E}-06$ & 4.633 & $3.61 E-06$ & $* * *$ \\
\hline Density- Trips-Enter-Center & $-6.76 \mathrm{E}-05$ & $1.08 \mathrm{E}-05$ & -6.282 & $3.34 \mathrm{E}-10$ & $* * *$ \\
\hline TLKM & $-2.13 \mathrm{E}-01$ & $7.08 \mathrm{E}-02$ & -3.003 & 0.002671 & $* *$ \\
\hline $\begin{array}{l}\text { Signif.codes: }\left(0^{* * * * \prime}\right)(0.001 \\
\text { (Dispersion parameter for } \mathrm{N}\end{array}$ & $\begin{array}{l}\text { ) }\left(0.01^{* \prime}\right) \\
\text { tive Binom }\end{array}$ & $\begin{array}{l}\because \prime)\left(0.1^{\prime \prime}\right) \\
.148) \text { famil }\end{array}$ & $\mathrm{n}$ to be 1 & & \\
\hline Null deviance: 675.7 on 421 & grees of fre & & & & \\
\hline Residual deviance: 457.18 o & 11 degrees & eedom & & & \\
\hline AIC: 1474.3 & & & & & \\
\hline Number of Fisher Scoring ite & ions: 1 & & & & \\
\hline Theta: 4.54 & & & & & \\
\hline Std. Err.: 1.01 & & & & & \\
\hline 2 x log-likelihood: -1450.28 & & & & & \\
\hline
\end{tabular}




\begin{tabular}{|c|c|c|c|c|c|}
\hline $\begin{array}{l}\text { K-C-V } \\
\text { Call: } \\
\text { glm.nb(formula = Collisions } \\
\text { Bike + Population + Age.0-4 } \\
\text { Center + Density- Trips-Ente }\end{array}$ & $\begin{array}{l}\text { No.Schools + } \\
\text { Age.5-24 + A } \\
\text { Center + TLK }\end{array}$ & $\begin{array}{l}\text { Driver + M } \\
5-64+\text { VKT } \\
\text { it.theta }=5\end{array}$ & $\begin{array}{l}\text { enger + } \\
\text { s-Enter- } C \\
11562, \text { I }\end{array}$ & $\begin{array}{l}\text { ansit + MS-V } \\
+ \text { Density- } \\
\text { g) }\end{array}$ & MS- \\
\hline Deviance Residuals: & & & & & \\
\hline Min & 10 & Median & 30 & Max & \\
\hline-2.5038 & -0.9962 & -0.2883 & 0.5113 & 2.9213 & \\
\hline & Estimate & Std.Error & $z$ value & $\operatorname{Pr}(>|z|)$ & \\
\hline (Intercept) & $-2.50 \mathrm{E}+00$ & 7.94E-01 & -3.141 & 0.001683 & $* *$ \\
\hline No.Schools & 1.17E-01 & $3.88 \mathrm{E}-02$ & 3.002 & 0.00268 & $* *$ \\
\hline MS-Driver & $1.53 \mathrm{E}-02$ & $8.43 E-03$ & 1.811 & 0.070207 & . \\
\hline MS-Passenger & 4.04E-02 & $1.14 \mathrm{E}-02$ & 3.536 & 0.000406 & $* * *$ \\
\hline MS-Transit & $4.61 \mathrm{E}-02$ & $1.00 \mathrm{E}-02$ & 4.595 & $4.32 \mathrm{E}-06$ & $* * *$ \\
\hline MS-Walk & $3.25 \mathrm{E}-02$ & $9.37 \mathrm{E}-03$ & 3.462 & 0.000536 & $* * *$ \\
\hline MS-Bike & $5.90 \mathrm{E}-02$ & $1.89 \mathrm{E}-02$ & 3.118 & 0.001821 & $* *$ \\
\hline Population & $7.04 \mathrm{E}-05$ & 3.07E-05 & 2.29 & 0.022003 & $*$ \\
\hline Age.0-4 & $-5.17 E-02$ & $2.31 \mathrm{E}-02$ & -2.243 & 0.024894 & $*$ \\
\hline Age.5-24 & $-6.38 \mathrm{E}-03$ & $3.96 \mathrm{E}-03$ & -1.613 & 0.106703 & \\
\hline Age.25-64 & $9.26 \mathrm{E}-03$ & $2.51 \mathrm{E}-03$ & 3.682 & 0.000231 & $* * *$ \\
\hline VKT & 7.67E-06 & $1.97 \mathrm{E}-06$ & 3.889 & 0.000101 & $* * *$ \\
\hline Trips-Enter-Center & $1.04 \mathrm{E}-04$ & $2.68 \mathrm{E}-05$ & 3.889 & 0.000101 & $* * *$ \\
\hline Density- Trips-Out-Center & $8.72 \mathrm{E}-05$ & $2.87 \mathrm{E}-05$ & 3.032 & 0.002427 & $* *$ \\
\hline Density-Trips-Enter-Center & $-2.15 \mathrm{E}-05$ & $6.14 \mathrm{E}-06$ & -3.504 & 0.000458 & $* * *$ \\
\hline TLKM & $-9.54 \mathrm{E}-03$ & $3.34 \mathrm{E}-03$ & -2.856 & 0.004293 & $* *$ \\
\hline $\begin{array}{l}\text { Signif.codes: }\left(0^{* * * *}\right)(0.001 \\
\text { (Dispersion parameter for } \mathrm{N}\end{array}$ & $\begin{array}{l}\left.k^{\prime}\right)\left(0.01^{* \prime \prime}\right)( \\
\text { ative Binom }\end{array}$ & $\begin{array}{l}\text {.') }\left(0.1^{\prime \prime}\right) \\
\text { 148) famil }\end{array}$ & $\mathrm{n}$ to be 1 & & \\
\hline $\begin{array}{l}\text { Null deviance: } 718.61 \text { on } 42 \\
\text { Residual deviance: } 440.12 \text { or } \\
\text { AIC: } 1440.3\end{array}$ & $\begin{array}{l}\text { degrees of } \mathrm{fr} \\
06 \text { degrees }\end{array}$ & om & & & \\
\hline Number of Fisher Scoring ite & tions: 1 & & & & \\
\hline $\begin{array}{l}\text { Theta: } 5.87 \\
\text { Std. Err.: } 1.48\end{array}$ & & & & & \\
\hline 2 x log-likelihood: -1406.296 & & & & & \\
\hline
\end{tabular}


K-D-IV

Call:

glm.nb(formula = Collisions $\sim$ MS-Driver + MS-Passenger + MS-Transit + MS-Walk + MS-Bike + VKT + Trips-Produced + Trips-Attracted + Density- Trips-Enter-Center + TLKM, init.theta $=5.453450858$, link $=$ $\log )$

\begin{tabular}{|c|c|c|c|c|c|}
\hline \multicolumn{6}{|l|}{ Deviance Residuals: } \\
\hline Min & $1 Q$ & Median & $3 Q$ & Max & \\
\hline-2.2904 & -1.0872 & -0.2199 & 0.4184 & 2.6715 & \\
\hline & Estimate & Std.Error & $\mathrm{z}$ value & $\operatorname{Pr}(>|z|)$ & \\
\hline (Intercept) & $-2.47 \mathrm{E}+00$ & $7.18 \mathrm{E}-01$ & -3.438 & 0.000586 & $* * *$ \\
\hline MS-Driver & $1.74 \mathrm{E}-02$ & 7.67E-03 & 2.271 & 0.023152 & $*$ \\
\hline MS-Passenger & $4.45 \mathrm{E}-02$ & $1.08 \mathrm{E}-02$ & 4.112 & 3.92E-05 & $* * *$ \\
\hline MS-Transit & $4.96 \mathrm{E}-02$ & $8.88 \mathrm{E}-03$ & 5.589 & $2.28 \mathrm{E}-08$ & $* * *$ \\
\hline MS-Walk & $3.22 \mathrm{E}-02$ & $9.14 \mathrm{E}-03$ & 3.529 & 0.000418 & $* * *$ \\
\hline MS-Bike & $4.44 \mathrm{E}-02$ & $1.86 \mathrm{E}-02$ & 2.386 & 0.017048 & $*$ \\
\hline VKT & $5.39 \mathrm{E}-06$ & $2.33 \mathrm{E}-06$ & 2.314 & 0.020688 & $*$ \\
\hline Trips-Produced & $-5.72 E-05$ & 2.57E-05 & -2.227 & 0.025958 & $*$ \\
\hline Trips-Attracted & $2.26 \mathrm{E}-05$ & $6.78 \mathrm{E}-06$ & 3.333 & 0.000861 & $* * *$ \\
\hline Density-Trips-Enter-Center & $-5.88 \mathrm{E}-05$ & 9.97E-06 & -5.893 & 3.79E-09 & $* * *$ \\
\hline TLKM & $6.00 \mathrm{E}-02$ & $1.02 \mathrm{E}-02$ & 5.91 & 3.42E-09 & $* * *$ \\
\hline
\end{tabular}

Signif.codes: $\left(0^{(* * * \prime)}\left(0.001^{\left(* *^{\prime}\right)}\left(0.01^{\prime * \prime}\right)\left(0.05^{\prime \prime}\right)\left(0.1^{\prime \prime}\right)\right.\right.$ (1)

(Dispersion parameter for Negative Binomial (2.148) family taken to be 1 )

Null deviance: 706.8 on 421 degrees of freedom

Residual deviance: 452.09 on 411 degrees of freedom

AIC: 1449.5

Number of Fisher Scoring iterations: 1

Theta: 5.45

Std. Err.: 1.37

$2 \times$ log-likelihood: -1425.49 


\begin{tabular}{|c|c|c|c|c|c|}
\hline \multicolumn{6}{|c|}{$\begin{array}{l}\text { Call: } \\
\text { glm.nb(formula = Collisions } \sim \text { MS-Driver + MS-Passenger + MS-Transit + MS-Walk + MS-Bike + } \\
\text { No.Households }+ \text { Age }>65+\text { VKT + Trips-Attracted + Density- Trips-Enter-Center + TLKM, init.theta }= \\
5.738431207, \text { link }=\log )\end{array}$} \\
\hline \multicolumn{6}{|l|}{ Deviance Residuals: } \\
\hline Min & $1 Q$ & Median & $3 Q$ & Max & \\
\hline \multirow[t]{2}{*}{-2.3223} & -1.0835 & -0.2124 & 0.4135 & 2.7086 & \\
\hline & Estimate & Std.Error & $z$ value & $\operatorname{Pr}(>|z|)$ & \\
\hline (Intercept) & $-2.61 E+00$ & 7.39E-01 & -3.531 & 0.000414 & $* * *$ \\
\hline MS-Driver & $1.76 \mathrm{E}-02$ & 7.87E-03 & 2.241 & 0.025054 & $*$ \\
\hline MS-Passenger & $4.32 \mathrm{E}-02$ & $1.11 \mathrm{E}-02$ & 3.894 & $9.85 \mathrm{E}-05$ & $* * *$ \\
\hline MS-Transit & 5.07E-02 & $9.05 \mathrm{E}-03$ & 5.601 & $2.13 \mathrm{E}-08$ & $* * *$ \\
\hline MS-Walk & $3.18 \mathrm{E}-02$ & $9.43 \mathrm{E}-03$ & 3.371 & 0.00075 & $* * *$ \\
\hline MS-Bike & $4.54 \mathrm{E}-02$ & $1.87 \mathrm{E}-02$ & 2.432 & 0.014998 & $*$ \\
\hline No.Households & $-4.44 \mathrm{E}-05$ & $2.25 \mathrm{E}-05$ & -1.972 & 0.048653 & $*$ \\
\hline Age $>65$ & $1.41 \mathrm{E}-02$ & $5.68 \mathrm{E}-03$ & 2.478 & 0.013208 & $*$ \\
\hline VKT & $6.10 \mathrm{E}-06$ & $2.36 \mathrm{E}-06$ & 2.588 & 0.009662 & $* *$ \\
\hline Trips-Attracted & $1.65 \mathrm{E}-05$ & $6.44 \mathrm{E}-06$ & 2.569 & 0.010192 & $*$ \\
\hline Density- Trips-Enter-Center & $-5.12 \mathrm{E}-05$ & $9.80 \mathrm{E}-06$ & -5.227 & $1.73 \mathrm{E}-07$ & $* * *$ \\
\hline TLKM & $5.17 \mathrm{E}-02$ & $1.02 \mathrm{E}-02$ & 5.051 & 4.40E-07 & $* * *$ \\
\hline \multicolumn{6}{|c|}{$\begin{array}{l}\left.\text { Signif.codes: }\left(0^{\prime * * * \prime}\right)\left(0.001^{\prime * *^{\prime}}\right)\left(0.01^{\prime * \prime}\right)\left(0.05^{\prime \prime}\right)^{\prime}\right)\left(0.1^{\prime \prime}\right)(1) \\
\text { (Dispersion parameter for Negative Binomial }(2.148) \text { family taken to be } 1)\end{array}$} \\
\hline \multicolumn{6}{|c|}{ Null deviance: 715.01 on 421 degrees of freedom } \\
\hline \multicolumn{6}{|c|}{ Residual deviance: 452.39 on 410 degrees of freedom } \\
\hline \multicolumn{6}{|l|}{ AIC: 1446.7} \\
\hline \multicolumn{6}{|c|}{ Number of Fisher Scoring iterations: 1} \\
\hline \multicolumn{6}{|l|}{ Theta: 5.74} \\
\hline \multicolumn{6}{|l|}{ Std. Err.: 1.5} \\
\hline \multicolumn{6}{|l|}{2 x log-likelihood: -1420.748} \\
\hline
\end{tabular}




\begin{tabular}{|c|c|c|c|c|c|}
\hline $\begin{array}{l}\text { K-C-I } \\
\text { Call: } \\
\text { glm.nb(formula = Collisions } \\
\text { Density- Trips-Enter-Center }\end{array}$ & $\begin{array}{l}\text { KT + Trips-C } \\
\text { LKM, init.th }\end{array}$ & $\begin{array}{l}\text { enter + Tri } \\
=2.667658\end{array}$ & $\begin{array}{l}\text { er-Cente } \\
\text { ink = log) }\end{array}$ & nsity- Trips & enter + \\
\hline Deviance Residuals: & & & & & \\
\hline Min & 10 & Median & $3 Q$ & Max & \\
\hline-3.4665 & -1.1737 & -0.2992 & 0.3446 & 2.6845 & \\
\hline & Estimate & Std.Error & $z$ value & $\operatorname{Pr}(>|z|)$ & \\
\hline (Intercept) & $-1.68 \mathrm{E}-01$ & 1.07E-01 & -1.565 & 0.117695 & \\
\hline VKT & $1.02 \mathrm{E}-05$ & $2.23 \mathrm{E}-06$ & 4.58 & $4.66 \mathrm{E}-06$ & $* * *$ \\
\hline Trips-Out-Center & $1.21 \mathrm{E}-04$ & 4.27E-05 & 2.834 & 0.004594 & $* *$ \\
\hline Trips-Enter-Center & $8.44 \mathrm{E}-05$ & $2.94 \mathrm{E}-05$ & 2.87 & 0.00411 & $* *$ \\
\hline Density- Trips-Out-Center & $2.64 \mathrm{E}-04$ & $2.94 \mathrm{E}-05$ & 8.979 & $<2 \mathrm{E}-16$ & $* * *$ \\
\hline Density- Trips-Enter-Center & $-3.14 \mathrm{E}-05$ & $6.42 \mathrm{E}-06$ & -4.888 & $1.02 \mathrm{E}-06$ & $* * *$ \\
\hline TLKM & $-1.29 \mathrm{E}-02$ & $3.58 \mathrm{E}-03$ & -3.608 & 0.000309 & $* * *$ \\
\hline $\begin{array}{l}\text { Signif.codes: }\left(0^{\prime * * * \prime}\right)(0.001 \\
\text { (Dispersion parameter for } \mathrm{N}\end{array}$ & $\begin{array}{l}\left.{ }^{\prime \prime}\right)\left(0.01^{\prime * \prime}\right)( \\
\text { ative Binom }\end{array}$ & $\begin{array}{l}\left..^{\prime}\right)\left(0.1^{\prime \prime}\right) \\
.148) \text { famil }\end{array}$ & $\mathrm{n}$ to be 1 & & \\
\hline $\begin{array}{l}\text { Null deviance: } 576 \text { on } 421 \text { c } \\
\text { Residual deviance: } 450.07 \text { o } \\
\text { AIC: } 1530.3\end{array}$ & $\begin{array}{l}\text { rees of free } \\
15 \text { degrees }\end{array}$ & eedom & & & \\
\hline Number of Fisher Scoring it & tions: 1 & & & & \\
\hline $\begin{array}{l}\text { Theta: } 2.668 \\
\text { Std. Err.: } 0.428\end{array}$ & & & & & \\
\hline 2 x log-likelihood: -1514.30 & & & & & \\
\hline
\end{tabular}




\begin{tabular}{|c|c|c|c|c|c|}
\hline \multicolumn{6}{|c|}{$\begin{array}{l}\text { Call: } \\
\text { glm.nb }(\text { formula }=\text { Collisions } \sim \text { Trips-Produced }+ \text { Density- Trips-Out-Center + Density- Trips-Enter-Center + } \\
\text { TLKM, init.theta }=3.124137195, \text { link }=\log )\end{array}$} \\
\hline \multicolumn{6}{|l|}{ Deviance Residuals: } \\
\hline Min & 10 & Median & $3 Q$ & Max & \\
\hline \multirow[t]{2}{*}{-3.5353} & -1.3112 & -0.2241 & 0.3648 & 2.4837 & \\
\hline & Estimate & Std.Error & $z$ value & $\operatorname{Pr}(>|z|)$ & \\
\hline (Intercept) & $-7.71 \mathrm{E}-02$ & $8.15 \mathrm{E}-02$ & -0.947 & 0.3437 & \\
\hline Trips-Produced & $-4.77 \mathrm{E}-05$ & 2.77E-05 & -1.72 & 0.0854 & . \\
\hline Density- Trips-Out-Center & 7.99E-05 & $3.62 \mathrm{E}-05$ & 2.207 & 0.0273 & $*$ \\
\hline Density- Trips-Enter-Center & $-2.95 \mathrm{E}-05$ & $5.32 \mathrm{E}-06$ & -5.546 & $2.92 \mathrm{E}-08$ & $* * *$ \\
\hline TLKM & $7.59 \mathrm{E}-02$ & $8.92 \mathrm{E}-03$ & 8.511 & $<2 \mathrm{E}-16$ & $* * *$ \\
\hline \multicolumn{6}{|c|}{$\begin{array}{l}\text { Signif.codes: }\left(0^{\prime * * * \prime}\right)\left(0.001^{\prime * * \prime}\right)\left(0.01^{\prime * \prime}\right)\left(0.05^{\prime} .\right)\left(0.1^{\prime \prime}\right)(1) \\
\text { (Dispersion parameter for Negative Binomial }(2.148) \text { family taken to be } 1)\end{array}$} \\
\hline $\begin{array}{l}\text { Null deviance: } 606.94 \text { on } 42 \\
\text { Residual deviance: } 457.38 \text { ol } \\
\text { AIC: } 1510.1\end{array}$ & $\begin{array}{l}\text { degrees of } \mathrm{ft} \\
17 \text { degrees }\end{array}$ & $\begin{array}{l}\text { om } \\
\text { eedom }\end{array}$ & & & \\
\hline \multicolumn{6}{|c|}{ Number of Fisher Scoring iterations: 1} \\
\hline \multicolumn{5}{|l|}{ Theta: 3.124} & Std. Err.: 0.563 \\
\hline 2 x log-likelihood: -1498.086 & & & & & \\
\hline
\end{tabular}




\begin{tabular}{|c|c|c|c|c|c|}
\hline \multicolumn{6}{|c|}{$\begin{array}{l}\text { Call: } \\
\text { glm.nb(formula }=\text { Collisions } \sim \text { Sig-Int }+40 . \mathrm{Km} / \mathrm{h}+50 . \mathrm{Km} / \mathrm{h}+70 . \mathrm{Km} / \mathrm{h}+90 . \mathrm{Km} / \mathrm{h}+\text { Trips-Attracted }+ \\
\text { Density- Trips-Out-Center }+ \text { Density- Trips-Enter-Center, init.theta }=3.684446254, \text { link }=\text { log })\end{array}$} \\
\hline \multicolumn{6}{|l|}{ Deviance Residuals: } \\
\hline Min & $1 Q$ & Median & $3 Q$ & Max & \\
\hline \multirow[t]{2}{*}{-2.2625} & -0.9786 & -0.5468 & 0.3252 & 2.8795 & \\
\hline & Estimate & Std.Error & $z$ value & $\operatorname{Pr}(>|z|)$ & \\
\hline (Intercept) & $-8.65 \mathrm{E}-01$ & $1.11 \mathrm{E}-01$ & -7.79 & $6.70 \mathrm{E}-15$ & $* * *$ \\
\hline Sig-Int & $2.34 \mathrm{E}-01$ & $1.93 \mathrm{E}-02$ & 12.116 & $<2 \mathrm{E}-16$ & $* * *$ \\
\hline $40 . \mathrm{Km} / \mathrm{h}$ & $2.23 \mathrm{E}-02$ & $9.83 \mathrm{E}-03$ & 2.264 & 0.0236 & $*$ \\
\hline $50 . \mathrm{Km} / \mathrm{h}$ & 4.09E-02 & $8.92 \mathrm{E}-03$ & 4.59 & $4.43 E-06$ & $* * *$ \\
\hline $70 . \mathrm{Km} / \mathrm{h}$ & $-2.92 \mathrm{E}-01$ & $7.10 \mathrm{E}-02$ & -4.106 & $4.03 \mathrm{E}-05$ & $* * *$ \\
\hline $90 . \mathrm{Km} / \mathrm{h}$ & $-3.11 \mathrm{E}-01$ & $2.21 \mathrm{E}-01$ & -1.407 & 0.1595 & \\
\hline Trips-Attracted & $8.29 \mathrm{E}-05$ & $3.74 \mathrm{E}-05$ & 2.218 & 0.0266 & $*$ \\
\hline Density- Trips-Out-Center & $1.99 \mathrm{E}-04$ & $3.11 \mathrm{E}-05$ & 6.39 & $1.66 \mathrm{E}-10$ & $* * *$ \\
\hline Density- Trips-Enter-Center & $-3.32 \mathrm{E}-05$ & $7.62 \mathrm{E}-06$ & -4.352 & $1.35 \mathrm{E}-05$ & $* * *$ \\
\hline \multicolumn{6}{|c|}{$\begin{array}{l}\text { Signif.codes: }\left(0^{\prime * * * \prime}\right)\left(0.001^{\prime * * \prime}\right)\left(0.01^{\prime * \prime}\right)\left(0.05^{\prime}{ }^{\prime \prime}\right)\left(0.1^{\prime \prime}\right)(1) \\
\text { (Dispersion parameter for Negative Binomial }(2.148) \text { family taken to be } 1)\end{array}$} \\
\hline \multicolumn{6}{|c|}{$\begin{array}{l}\text { Null deviance: } 838.75 \text { on } 421 \text { degrees of freedom } \\
\text { Residual deviance: } 421.85 \text { on } 413 \text { degrees of freedom } \\
\text { AIC: } 1314.7\end{array}$} \\
\hline \multicolumn{6}{|c|}{ Number of Fisher Scoring iterations: 1} \\
\hline \multicolumn{6}{|l|}{ Theta: 3.684} \\
\hline $2 \times$ log-likelihood: -1294.682 & & & & & \\
\hline
\end{tabular}


L-C-V

Call:

glm.nb(formula $=$ Collisions $\sim$ Area + MS-Passenger + MS-Transit + MS-Walk + MS-Bike + Population + Age.0-4 + Age.25-64 + Age > 65 + VKT + Trips-Attracted + Density- Trips-Out-Center + Density- Trips-

Enter-Center, init.theta $=4.019065691$, link $=\log$ )

Deviance Residuals:

\begin{tabular}{|c|c|c|c|c|c|}
\hline Min & $1 Q$ & Median & $3 Q$ & Max & \\
\hline-2.5655 & -0.9054 & -0.4942 & 0.4362 & 3.0348 & \\
\hline & Estimate & Std.Error & $z$ value & $\operatorname{Pr}(>|z|)$ & \\
\hline (Intercept) & $-2.58 \mathrm{E}+00$ & $3.23 \mathrm{E}-01$ & -7.988 & $1.37 \mathrm{E}-15$ & $* * *$ \\
\hline Area & $-8.83 \mathrm{E}-03$ & $3.60 \mathrm{E}-03$ & -2.449 & 0.014331 & $*$ \\
\hline MS-Passenger & $3.36 \mathrm{E}-02$ & $1.23 \mathrm{E}-02$ & 2.73 & 0.006326 & $* *$ \\
\hline MS-Transit & 3.39E-02 & $7.08 \mathrm{E}-03$ & 4.792 & $1.65 \mathrm{E}-06$ & $* * *$ \\
\hline MS-Walk & $2.17 E-02$ & $6.16 \mathrm{E}-03$ & 3.525 & 0.000423 & $* * *$ \\
\hline MS-Bike & 5.94E-02 & $2.06 \mathrm{E}-02$ & 2.882 & 0.003954 & $* *$ \\
\hline Population & $1.45 \mathrm{E}-04$ & 2.93E-05 & 4.953 & $7.32 \mathrm{E}-07$ & $* * *$ \\
\hline Age.0-4 & $-6.38 \mathrm{E}-02$ & $2.57 \mathrm{E}-02$ & -2.488 & 0.012858 & $*$ \\
\hline Age.25-64 & $2.20 \mathrm{E}-02$ & $3.58 \mathrm{E}-03$ & 6.159 & $7.31 \mathrm{E}-10$ & $* * *$ \\
\hline Age $>65$ & $2.22 \mathrm{E}-02$ & $6.94 \mathrm{E}-03$ & 3.199 & 0.001379 & $* *$ \\
\hline VKT & 5.65E-06 & $2.29 \mathrm{E}-06$ & 2.471 & 0.013472 & $*$ \\
\hline Trips-Attracted & $1.50 \mathrm{E}-04$ & $3.75 \mathrm{E}-05$ & 4 & $6.33 \mathrm{E}-05$ & $* * *$ \\
\hline Density- Trips-Out-Center & $8.16 \mathrm{E}-05$ & $3.64 \mathrm{E}-05$ & 2.242 & 0.024944 & $*$ \\
\hline Density- Trips-Enter-Center & $-2.63 \mathrm{E}-05$ & $8.42 \mathrm{E}-06$ & -3.127 & 0.001769 & $* *$ \\
\hline
\end{tabular}

Signif.codes: $\left(0^{\prime * * * \prime}\right)\left(0.001^{\prime * * \prime}\right)\left(0.01^{\prime * \prime}\right)\left(0.05^{\prime \prime}.\right)\left(0.1^{\prime \prime}\right)(1)$

(Dispersion parameter for Negative Binomial (2.148) family taken to be 1 )

Null deviance: 858.94 on 421 degrees of freedom

Residual deviance: 422.65 on 408 degrees of freedom

AIC: 1316

Number of Fisher Scoring iterations: 1

Theta: 4.019

Std. Err.: 0.893

$2 \times$ log-likelihood: -1286.015 
L-D-V

Call:

glm.nb(formula = Collisions MS-Passenger + MS-Transit + MS-Bike + Employment + Age.5-24 + Age.25$64+$ Age $>65+$ TLKM, init.theta $=2.992251357$, link $=$ log)

Deviance Residuals:

\begin{tabular}{|c|c|c|c|c|c|}
\hline Min & 10 & Median & $3 Q$ & Max & \\
\hline-2.2315 & -0.9462 & -0.4622 & 0.4158 & 3.0752 & \\
\hline & Estimate & Std.Error & $z$ value & $\operatorname{Pr}(>|z|)$ & \\
\hline (Intercept) & $-2.85 E+00$ & $3.06 \mathrm{E}-01$ & -9.323 & $<2 \mathrm{E}-16$ & $* * *$ \\
\hline MS-Passenger & $3.91 \mathrm{E}-02$ & $1.07 \mathrm{E}-02$ & 3.652 & 0.00026 & $* * *$ \\
\hline MS-Transit & $4.52 \mathrm{E}-02$ & $6.95 \mathrm{E}-03$ & 6.501 & $7.99 \mathrm{E}-11$ & $* * *$ \\
\hline MS-Bike & $4.22 \mathrm{E}-02$ & $2.20 \mathrm{E}-02$ & 1.918 & 0.05515 & . \\
\hline Employment & $-1.46 \mathrm{E}-05$ & $2.88 \mathrm{E}-06$ & -5.061 & 4.17E-07 & $* * *$ \\
\hline Age.5-24 & $1.01 \mathrm{E}-02$ & $4.44 \mathrm{E}-03$ & 2.276 & 0.02287 & $*$ \\
\hline Age.25-64 & $1.86 \mathrm{E}-02$ & $3.15 E-03$ & 5.923 & 3.17E-09 & $* * *$ \\
\hline Age $>65$ & $3.36 \mathrm{E}-02$ & $7.13 \mathrm{E}-03$ & 4.716 & $2.40 \mathrm{E}-06$ & $* * *$ \\
\hline TLKM & $6.20 \mathrm{E}-02$ & $7.62 \mathrm{E}-03$ & 8.132 & $4.23 \mathrm{E}-16$ & $* * *$ \\
\hline
\end{tabular}

Signif.codes: $\left(0^{\prime * * * \prime}\right)\left(0.001^{\prime * * \prime}\right)\left(0.01^{\prime * \prime}\right)\left(0.05^{\prime \prime}.\right)\left(0.1^{\prime \prime}\right)(1)$

(Dispersion parameter for Negative Binomial (2.148) family taken to be 1 )

Null deviance: 788.53 on 421 degrees of freedom

Residual deviance: 427.07 on 413 degrees of freedom

AIC: 1344.6

Number of Fisher Scoring iterations: 1

Theta: 2.992

Std. Err.: 0.583

$2 \times$ log-likelihood: -1324.572 
L-D-I

Call:

glm.nb(formula $=$ Collisions $\sim$ Trips-Attracted + Density- Trips-Enter-Center + TLKM, init.theta $=$ 1.796777081, link = log)

\section{Deviance Residuals:}

\begin{tabular}{|c|c|c|c|c|c|}
\hline Min & $1 \mathrm{Q}$ & Median & $3 \mathrm{Q}$ & Max & \\
\hline-2.2009 & -1.1094 & -0.418 & 0.2945 & 2.6429 & \\
\hline \multicolumn{5}{|l|}{} \\
\hline & Estimate & Std.Error & z value & $\operatorname{Pr}(>|\mathrm{z}|)$ & \\
\hline (Intercept) & $-5.25 \mathrm{E}-01$ & $1.01 \mathrm{E}-01$ & -5.173 & $2.30 \mathrm{E}-07$ & $* * *$ \\
\hline Trips-Attracted & $5.37 \mathrm{E}-05$ & $8.93 \mathrm{E}-06$ & 6.017 & $1.77 \mathrm{E}-09$ & $* * *$ \\
\hline Density- Trips-Enter-Center & $-1.14 \mathrm{E}-04$ & $1.32 \mathrm{E}-05$ & -8.587 & $<2 \mathrm{E}-16$ & $* * *$ \\
\hline TLKM & $1.30 \mathrm{E}-01$ & $8.47 \mathrm{E}-03$ & 15.303 & $<2 \mathrm{E}-16$ & $* * *$ \\
\hline
\end{tabular}

Signif.codes: $\left(0^{\prime * * * \prime}\right)\left(0.001^{(* * \prime}\right)\left(0.01^{\prime * \prime}\right)\left(0.05^{\prime \prime}.\right)\left(0.1^{\prime \prime}\right)$ (1)

(Dispersion parameter for Negative Binomial (2.148) family taken to be 1 )

Null deviance: 658.06 on 421 degrees of freedom

Residual deviance: 428.46 on 418 degrees of freedom

AIC: 1409.3

Number of Fisher Scoring iterations: 1

Theta: 1.797

Std. Err.: 0.273

2 x log-likelihood: -1399.343 
$\mathrm{M}: \mathrm{C}-\mathrm{III}$

Call:

glm.nb(formula $=$ Collisions $\sim$ No Bus stops + Non-Sig-Int $+40 . \mathrm{Km} / \mathrm{h}+50 . \mathrm{Km} / \mathrm{h}+60 . \mathrm{Km} / \mathrm{h}+$ Bike-Path + VKT + Density- Trips-Enter-Center + TLKM, init.theta $=3.05855976$, link $=\log$ )

Deviance Residuals:

\begin{tabular}{|c|c|c|c|c|c|}
\hline Min & $1 \mathrm{Q}$ & Median & $3 \mathrm{Q}$ & Max & \\
\hline-2.622 & -1.1364 & -0.2995 & 0.3386 & 2.855 & \\
\hline \multicolumn{7}{|l|}{} \\
\hline \multicolumn{7}{|c|}{} & Estimate & Std.Error & z value & $\operatorname{Pr}(>|\mathrm{z}|)$ & \\
\hline (Intercept) & $-1.47 \mathrm{E}-01$ & $1.17 \mathrm{E}-01$ & -1.25 & 0.211118 & \\
\hline No Bus stops & $1.30 \mathrm{E}-02$ & $3.51 \mathrm{E}-03$ & 3.693 & 0.000222 & $* * *$ \\
\hline Non-Sig-Int & $1.13 \mathrm{E}-02$ & $3.08 \mathrm{E}-03$ & 3.679 & 0.000234 & $* * *$ \\
\hline $40 . \mathrm{Km} / \mathrm{h}$ & $2.81 \mathrm{E}-01$ & $7.72 \mathrm{E}-02$ & 3.643 & 0.00027 & $* * *$ \\
\hline $50 . \mathrm{Km} / \mathrm{h}$ & $2.57 \mathrm{E}-01$ & $7.48 \mathrm{E}-02$ & 3.431 & 0.000602 & $* * *$ \\
\hline $60 . \mathrm{Km} / \mathrm{h}$ & $2.42 \mathrm{E}-01$ & $7.60 \mathrm{E}-02$ & 3.181 & 0.001468 & $* *$ \\
\hline Bike-Path & $2.82 \mathrm{E}-02$ & $8.01 \mathrm{E}-03$ & 3.516 & 0.000438 & $* * *$ \\
\hline VKT & $8.09 \mathrm{E}-06$ & $3.41 \mathrm{E}-06$ & 2.373 & 0.017639 & $*$ \\
\hline Density- Trips-Enter-Center & $-9.22 \mathrm{E}-06$ & $4.60 \mathrm{E}-06$ & -2.005 & 0.04492 & $*$ \\
\hline TLKM & $-2.51 \mathrm{E}-01$ & $7.84 \mathrm{E}-02$ & -3.199 & 0.001381 & $*$ \\
\hline
\end{tabular}

Signif.codes: $\left(0^{\prime * * * \prime}\right)\left(0.001^{\prime * * \prime}\right)\left(0.01^{\prime * \prime}\right)\left(0.05^{\prime \prime}.\right)\left(0.1^{\prime \prime}\right)$ (1)

(Dispersion parameter for Negative Binomial (2.148) family taken to be 1)

Null deviance: 677.84 on 421 degrees of freedom

Residual deviance: 450.54 on 412 degrees of freedom

AIC: 1454.3

Number of Fisher Scoring iterations: 1

Theta: 3.059

Std. Err.: 0.571

2 x log-likelihood: -1432.321 
M:D-III

Call:

glm.nb(formula $=$ Collisions $\sim$ No Bus stops + Non-Sig-Int $+40 . \mathrm{Km} / \mathrm{h}+50 . \mathrm{Km} / \mathrm{h}+60 . \mathrm{Km} / \mathrm{h}+$ Bike-Path + VKT + Trips-Produced + Trips-Attracted + Density- Trips-Enter-Center + TLKM, init.theta $=3.286923869$, link $=\log$ )

Deviance Residuals:

\begin{tabular}{|c|c|c|c|c|c|}
\hline Min & 10 & Median & $3 Q$ & Max & \\
\hline-2.5551 & -1.1433 & -0.2629 & 0.3369 & 2.8824 & \\
\hline & Estimate & Std.Error & $z$ value & $\operatorname{Pr}(>|z|)$ & \\
\hline (Intercept) & $-1.53 \mathrm{E}-01$ & 1.17E-01 & -1.311 & 0.189846 & \\
\hline No Bus stops & $1.34 \mathrm{E}-02$ & $3.62 \mathrm{E}-03$ & 3.703 & 0.000213 & $* * *$ \\
\hline Non-Sig-Int & $9.03 \mathrm{E}-03$ & 3.37E-03 & 2.68 & 0.007364 & $* *$ \\
\hline $40 . \mathrm{Km} / \mathrm{h}$ & $2.79 \mathrm{E}-01$ & 7.75E-02 & 3.594 & 0.000325 & $* * *$ \\
\hline $50 . \mathrm{Km} / \mathrm{h}$ & $2.61 \mathrm{E}-01$ & $7.52 \mathrm{E}-02$ & 3.468 & 0.000523 & $* * *$ \\
\hline $60 . \mathrm{Km} / \mathrm{h}$ & $2.56 \mathrm{E}-01$ & 7.65E-02 & 3.347 & 0.000816 & $* * *$ \\
\hline Bike-Path & 2.67E-02 & 7.97E-03 & 3.349 & 0.000811 & $* * *$ \\
\hline VKT & 8.17E-06 & $3.41 \mathrm{E}-06$ & 2.398 & 0.016505 & $*$ \\
\hline Trips-Produced & $-4.24 \mathrm{E}-05$ & $2.74 \mathrm{E}-05$ & -1.546 & 0.122057 & \\
\hline Trips-Attracted & $2.32 \mathrm{E}-05$ & $7.12 \mathrm{E}-06$ & 3.261 & 0.00111 & $* *$ \\
\hline Density- Trips-Enter-Center & $-4.02 \mathrm{E}-05$ & $1.19 \mathrm{E}-05$ & -3.383 & 0.000716 & $* * *$ \\
\hline TLKM & $-2.37 \mathrm{E}-01$ & $7.90 \mathrm{E}-02$ & -2.995 & 0.002748 & $* *$ \\
\hline
\end{tabular}

Signif.codes: $\left(0^{\prime * * * \prime}\right)\left(0.001^{* * \prime}\right)\left(0.01^{\prime * \prime}\right)\left(0.05^{\prime \prime}.\right)\left(0.1^{\prime \prime}\right)$ (1)

(Dispersion parameter for Negative Binomial (2.148) family taken to be 1 )

Null deviance: 693.1 on 421 degrees of freedom

Residual deviance: 449.51 on 410 degrees of freedom

AIC: 1447.7

Number of Fisher Scoring iterations: 1

Theta: 3.287

Std. Err.: 0.634

2 x log-likelihood: -1421.716 


\begin{tabular}{|c|c|c|c|c|c|}
\hline $\begin{array}{l}\text { M-C-V } \\
\text { Call: } \\
\text { glm.nb(formula = Collisions } \\
\text { Bike + Population + Age.0-4 } \\
\text { Center + Density- Trips-Ente }\end{array}$ & $\begin{array}{l}\text { Jo.Schools + } \\
\text { ge.5-24 + A } \\
\text { Center, init. }\end{array}$ & $\begin{array}{l}\text { Driver + M } \\
5-64+\text { Age } \\
=4.84315\end{array}$ & $\begin{array}{l}\text { enger }+ \\
\text { Trips-Er } \\
\text { link }=\text { log }\end{array}$ & $\begin{array}{l}\text { ansit + MS- } \\
\text { anter + Den }\end{array}$ & $\begin{array}{l}\text { MS- } \\
\text { ips-Out- }\end{array}$ \\
\hline Deviance Residuals: & & & & & \\
\hline Min & 10 & Median & $3 Q$ & Max & \\
\hline-2.6728 & -1.0164 & -0.3072 & 0.4648 & 2.4896 & \\
\hline & Estimate & Std.Error & $z$ value & $\operatorname{Pr}(>|z|)$ & \\
\hline (Intercept) & $-4.90 \mathrm{E}+00$ & $1.21 \mathrm{E}+00$ & -4.057 & 4.97E-05 & $* * *$ \\
\hline No.Schools & $1.22 \mathrm{E}-01$ & $4.12 \mathrm{E}-02$ & 2.966 & 0.00302 & $* *$ \\
\hline MS-Driver & $3.36 \mathrm{E}-02$ & $1.26 \mathrm{E}-02$ & 2.669 & 0.007614 & $* *$ \\
\hline MS-Passenger & $6.32 \mathrm{E}-02$ & $1.52 \mathrm{E}-02$ & 4.151 & $3.31 \mathrm{E}-05$ & $* * *$ \\
\hline MS-Transit & $6.97 \mathrm{E}-02$ & $1.35 \mathrm{E}-02$ & 5.158 & $2.49 \mathrm{E}-07$ & $* * *$ \\
\hline MS-Walk & $5.08 \mathrm{E}-02$ & $1.31 \mathrm{E}-02$ & 3.893 & $9.90 \mathrm{E}-05$ & $* * *$ \\
\hline MS-Bike & $7.23 \mathrm{E}-02$ & $2.20 \mathrm{E}-02$ & 3.295 & 0.000985 & $* * *$ \\
\hline Population & $1.39 \mathrm{E}-04$ & 3.09E-05 & 4.478 & 7.54E-06 & $* * *$ \\
\hline Age.0-4 & $-3.84 \mathrm{E}-02$ & $2.50 \mathrm{E}-02$ & -1.541 & 0.123402 & \\
\hline Age.5-24 & $-7.85 \mathrm{E}-03$ & $4.24 \mathrm{E}-03$ & -1.851 & 0.06414 & . \\
\hline Age.25-64 & $1.02 \mathrm{E}-02$ & $3.04 \mathrm{E}-03$ & 3.342 & 0.000832 & $* * *$ \\
\hline Age $>65$ & $1.04 \mathrm{E}-02$ & $6.77 \mathrm{E}-03$ & 1.541 & 0.123422 & \\
\hline Trips-Enter-Center & $1.74 \mathrm{E}-04$ & $2.68 \mathrm{E}-05$ & 6.506 & $7.73 \mathrm{E}-11$ & $* * *$ \\
\hline Density- Trips-Out-Center & $8.22 \mathrm{E}-05$ & $2.91 \mathrm{E}-05$ & 2.825 & 0.004722 & $* *$ \\
\hline Density- Trips-Enter-Center & $-1.83 \mathrm{E}-05$ & 6.07E-06 & -3.016 & 0.002557 & $* *$ \\
\hline $\begin{array}{l}\text { Signif.codes: }\left(0^{` * * * \prime}\right)(0.001 \\
\text { (Dispersion parameter for } \mathrm{N}\end{array}$ & $\begin{array}{l}\left.{ }^{\prime}\right)\left(0.01^{* \prime}\right) \\
\text { ative Binom }\end{array}$ & $\begin{array}{l}\text {.') }\left(0.1^{\prime \prime}\right) \\
.148) \text { famil }\end{array}$ & $\mathrm{n}$ to be 1 & & \\
\hline Null deviance: 770.41 on 42 & degrees of $f$ & & & & \\
\hline Residual deviance: 424.8 on & 7 degrees 0 & edom & & & \\
\hline AIC: 1383.9 & & & & & \\
\hline Number of Fisher Scoring ite & tions: 1 & & & & \\
\hline Theta: 4.84 & & & & & \\
\hline Std. Err.: 1.11 & & & & & \\
\hline 2 x log-likelihood: -1351.93 & & & & & \\
\hline
\end{tabular}




\begin{tabular}{|c|c|c|c|c|c|}
\hline $\begin{array}{l}\text { M-D-V } \\
\text { Call: } \\
\text { glm.nb(formula = Collisions } \\
\text { No.Households + Age.25-64 } \\
=3.942897227, \text { link }=\text { log) }\end{array}$ & $\begin{array}{l}\text { MS-Driver + } \\
\text { Age }>65+T\end{array}$ & S-Passenger & $\begin{array}{l}\text { S-Transit + } \\
\text { ensity- Trips- }\end{array}$ & $\begin{array}{l}\text { Walk + MS-B } \\
\text { er-Center + T }\end{array}$ & nit.theta \\
\hline Deviance Residuals: & & & & & \\
\hline Min & 10 & Median & $3 Q$ & Max & \\
\hline-2.6959 & -1.0512 & -0.2509 & 0.3708 & 2.7084 & \\
\hline & Estimate & Std.Error & $z$ value & $\operatorname{Pr}(>|z|)$ & \\
\hline (Intercept) & $-4.28 \mathrm{E}+00$ & $1.05 \mathrm{E}+00$ & -4.099 & $4.16 \mathrm{E}-05$ & $* * *$ \\
\hline MS-Driver & 3.01E-02 & $1.08 \mathrm{E}-02$ & 2.785 & 0.005352 & $* *$ \\
\hline MS-Passenger & 5.89E-02 & $1.40 \mathrm{E}-02$ & 4.201 & $2.65 \mathrm{E}-05$ & $* * *$ \\
\hline MS-Transit & $7.05 E-02$ & $1.20 \mathrm{E}-02$ & 5.858 & 4.67E-09 & $* * *$ \\
\hline MS-Walk & $4.18 \mathrm{E}-02$ & $1.22 \mathrm{E}-02$ & 3.435 & 0.000593 & $* * *$ \\
\hline MS-Bike & $4.10 \mathrm{E}-02$ & $2.16 \mathrm{E}-02$ & 1.9 & 0.057427 & . \\
\hline No.Households & $-5.52 \mathrm{E}-05$ & $2.50 \mathrm{E}-05$ & -2.206 & 0.027414 & $*$ \\
\hline Age.25-64 & $5.06 \mathrm{E}-03$ & 2.69E-03 & 1.885 & 0.059435 & . \\
\hline Age $>65$ & $1.71 \mathrm{E}-02$ & $6.75 \mathrm{E}-03$ & 2.538 & 0.011144 & $*$ \\
\hline Trips-Attracted & $1.09 \mathrm{E}-05$ & $6.62 \mathrm{E}-06$ & 1.642 & 0.100603 & \\
\hline Density- Trips-Enter-Center & $-4.00 \mathrm{E}-05$ & $9.72 \mathrm{E}-06$ & -4.117 & $3.84 \mathrm{E}-05$ & $* * *$ \\
\hline TLKM & $7.08 \mathrm{E}-02$ & $9.75 \mathrm{E}-03$ & 7.26 & $3.88 \mathrm{E}-13$ & $* * *$ \\
\hline $\begin{array}{l}\text { Signif.codes: }\left(0^{* * * \prime}\right)(0.001 \\
\text { (Dispersion parameter for } \mathrm{N}\end{array}$ & $\begin{array}{l}\left.* *^{\prime}\right)\left(0.01^{\prime * \prime}\right)( \\
\text { gative Binom }\end{array}$ & $\begin{array}{l}\left.05^{\prime \prime}\right)\left(0.1^{\prime \prime}\right) \\
(2.148) \text { fami }\end{array}$ & aken to be 1 & & \\
\hline Null deviance: 730.5 on 421 & degrees of fre & dom & & & \\
\hline Residual deviance: $443.21 \mathrm{o}$ & 410 degrees & freedom & & & \\
\hline AIC: 1418.9 & & & & & \\
\hline Number of Fisher Scoring ite & rations: 1 & & & & \\
\hline Theta: 3.943 & & & & & \\
\hline Std. Err.: 0.849 & & & & & \\
\hline $2 \times$ log-likelihood: -1392.93 & & & & & \\
\hline
\end{tabular}




\begin{tabular}{|c|c|c|c|c|c|}
\hline \multicolumn{6}{|l|}{$\begin{array}{l}\text { Call: } \\
\text { glm.nb(formula = Collisions } \\
2.339734593, \text { link }=\log \text { ) }\end{array}$} \\
\hline \multicolumn{6}{|l|}{ Deviance Residuals: } \\
\hline Min & 10 & Median & $3 Q$ & Max & \\
\hline \multirow[t]{2}{*}{-2.8567} & -1.2404 & -0.2662 & 0.3046 & 2.6117 & \\
\hline & Estimate & Std.Error & $z$ value & $\operatorname{Pr}(>|z|)$ & \\
\hline (Intercept) & $-2.26 \mathrm{E}-01$ & 8.87E-02 & -2.545 & 0.0109 & $*$ \\
\hline Trips-Attracted & $2.79 \mathrm{E}-05$ & $7.05 E-06$ & 3.965 & $7.35 \mathrm{E}-05$ & $* * *$ \\
\hline Density- Trips-Enter-Center & $-5.67 \mathrm{E}-05$ & $1.01 \mathrm{E}-05$ & -5.644 & $1.66 \mathrm{E}-08$ & $* * *$ \\
\hline TLKM & $9.56 \mathrm{E}-02$ & $7.35 \mathrm{E}-03$ & 13.009 & $<2 \mathrm{E}-16$ & $* * *$ \\
\hline \multicolumn{6}{|c|}{$\begin{array}{l}\text { Signif.codes: }\left(0^{\prime * * * \prime}\right)\left(0.001^{\prime * * \prime}\right)\left(0.01^{\prime * \prime}\right)\left(0.05^{\prime \prime}\right)\left(0.1^{\prime \prime}\right)(1) \\
\text { (Dispersion parameter for Negative Binomial }(2.148) \text { family taken to be } 1)\end{array}$} \\
\hline \multicolumn{6}{|c|}{ Null deviance: 619.31 on 421 degrees of freedom } \\
\hline \multicolumn{6}{|c|}{ Residual deviance: 451.51 on 418 degrees of freedom } \\
\hline \multicolumn{6}{|l|}{ AIC: 1482.5} \\
\hline \multicolumn{6}{|c|}{ Number of Fisher Scoring iterations: 1} \\
\hline \multicolumn{6}{|l|}{ Theta: 2.34} \\
\hline \multicolumn{6}{|l|}{ Std. Err.: 0.381} \\
\hline $2 \times$ log-likelihood: $-1472.48 \mathrm{~s}$ & & & & & \\
\hline
\end{tabular}




\section{D.5 Pedestrian-Involved Collisions Models}

\begin{tabular}{|c|c|c|c|c|c|}
\hline $\begin{array}{l}\text { A-D-II } \\
\text { Call: } \\
\text { glm.nb(formula = Collisions } \\
\text { Density- Trips-Enter-Center, }\end{array}$ & $\begin{array}{l}\text { Jo Bus stop } \\
\text { t.theta }=1\end{array}$ & $\begin{array}{l}\text { C + Bike-Pa } \\
99416, \text { link }\end{array}$ & rips-Proc & + Trips-Attr & \\
\hline Deviance Residuals: & & & & & \\
\hline Min & 10 & Median & $3 Q$ & Max & \\
\hline-2.4471 & -1.2325 & -0.3217 & 0.3817 & 3.0248 & \\
\hline & Estimate & Std.Error & $z$ value & $\operatorname{Pr}(>|z|)$ & \\
\hline (Intercept) & $1.82 \mathrm{E}-01$ & $8.20 \mathrm{E}-02$ & 2.222 & 0.026267 & $*$ \\
\hline No Bus stops & $1.90 \mathrm{E}-02$ & $3.26 \mathrm{E}-03$ & 5.808 & $6.30 \mathrm{E}-09$ & $* * *$ \\
\hline LoC & $1.12 \mathrm{E}-01$ & $1.36 \mathrm{E}-02$ & 8.236 & $<2 \mathrm{E}-16$ & $* * *$ \\
\hline Bike-Path & $3.66 \mathrm{E}-02$ & $1.04 \mathrm{E}-02$ & 3.525 & 0.000423 & $* * *$ \\
\hline Trips-Produced & $4.31 \mathrm{E}-05$ & $2.31 \mathrm{E}-05$ & 1.869 & 0.061676 & . \\
\hline Trips-Attracted & $1.03 \mathrm{E}-05$ & $3.92 \mathrm{E}-06$ & 2.633 & 0.008454 & $* *$ \\
\hline Density- Trips-Enter-Center & $-8.85 E-06$ & 4.53E-06 & -1.955 & 0.050563 & . \\
\hline $\begin{array}{l}\text { Signif.codes: }\left(0^{* * * *}\right)(0.001 \\
\text { (Dispersion parameter for } \mathrm{N}\end{array}$ & $\begin{array}{l}\left.k^{\prime}\right)\left(0.01^{(* \prime}\right) \\
\text { ative Binom }\end{array}$ & $\begin{array}{l}\left.y^{\prime}\right)\left(0.1^{\prime \prime}\right) \\
.148) \text { fami }\end{array}$ & en to be 1 & & \\
\hline Null deviance: 780.08 on 42 & degrees of $t$ & om & & & \\
\hline Residual deviance: 471.46 o & 15 degrees & eedom & & & \\
\hline AIC: 1930.2 & & & & & \\
\hline Number of Fisher Scoring ite & tions: 1 & & & & \\
\hline Theta: 1.983 & & & & & \\
\hline Std. Err.: 0.235 & & & & & \\
\hline 2 x log-likelihood: -1914.218 & & & & & \\
\hline
\end{tabular}




\begin{tabular}{|c|c|c|c|c|c|}
\hline \multicolumn{6}{|c|}{$\begin{array}{l}\text { Call: } \\
\text { glm.nb(formula }=\text { Collisions } \sim \text { No Bus stops }+ \text { Tot-Int }+40 . \mathrm{Km} / \mathrm{h}+50 . \mathrm{Km} / \mathrm{h}+60 . \mathrm{Km} / \mathrm{h}+70 . \mathrm{Km} / \mathrm{h}+\text { Bike- } \\
\text { Path }+ \text { Trips-Produced }+ \text { Trips-Attracted }+ \text { Density- Trips-Enter-Center }+ \text { TLKM, init.theta }=2.14480156 \text {, } \\
\text { link }=\log )\end{array}$} \\
\hline \multicolumn{6}{|l|}{ Deviance Residuals: } \\
\hline Min & 10 & Median & $3 Q$ & Max & \\
\hline \multirow[t]{2}{*}{-2.5504} & -1.1815 & -0.3251 & 0.4162 & 3.4592 & \\
\hline & Estimate & Std.Error & $z$ value & $\operatorname{Pr}(>|z|)$ & \\
\hline (Intercept) & 3.73E-01 & $1.07 \mathrm{E}-01$ & 3.495 & 0.000474 & $* * *$ \\
\hline No Bus stops & $1.19 \mathrm{E}-02$ & $3.52 \mathrm{E}-03$ & 3.38 & 0.000725 & $* * *$ \\
\hline Tot-Int & $1.60 \mathrm{E}-02$ & $2.32 \mathrm{E}-03$ & 6.912 & $4.79 \mathrm{E}-12$ & $* * *$ \\
\hline $40 . \mathrm{Km} / \mathrm{h}$ & 4.37E-01 & $1.24 \mathrm{E}-01$ & 3.535 & 0.000407 & $* * *$ \\
\hline $50 . \mathrm{Km} / \mathrm{h}$ & 4.19E-01 & $1.23 \mathrm{E}-01$ & 3.396 & 0.000684 & $* * *$ \\
\hline $60 . \mathrm{Km} / \mathrm{h}$ & 4.73E-01 & $1.25 \mathrm{E}-01$ & 3.782 & 0.000156 & $* * *$ \\
\hline $70 . \mathrm{Km} / \mathrm{h}$ & $2.75 \mathrm{E}-01$ & $2.04 \mathrm{E}-01$ & 1.347 & 0.177961 & \\
\hline Bike-Path & $3.15 \mathrm{E}-02$ & $1.01 \mathrm{E}-02$ & 3.106 & 0.001893 & $* *$ \\
\hline Trips-Produced & 6.07E-05 & $2.33 \mathrm{E}-05$ & 2.611 & 0.009025 & $* *$ \\
\hline Trips-Attracted & $6.52 \mathrm{E}-06$ & $4.41 \mathrm{E}-06$ & 1.478 & 0.139319 & \\
\hline Density- Trips-Enter-Center & $-1.23 \mathrm{E}-05$ & $5.40 \mathrm{E}-06$ & -2.282 & 0.022498 & $*$ \\
\hline TLKM & $-4.21 \mathrm{E}-01$ & $1.23 \mathrm{E}-01$ & -3.414 & 0.000639 & $* * *$ \\
\hline \multicolumn{6}{|c|}{$\begin{array}{l}\text { Signif.codes: }\left(0^{\prime * * * \prime}\right)\left(0.001^{\prime * * \prime}\right)\left(0.01^{\prime * \prime}\right)\left(0.05^{\prime \prime}\right)\left(0.1^{\prime \prime}\right)(1) \\
\text { (Dispersion parameter for Negative Binomial }(2.148) \text { family taken to be } 1)\end{array}$} \\
\hline \multicolumn{6}{|c|}{ Null deviance: 813.22 on 421 degrees of freedom } \\
\hline \multicolumn{6}{|c|}{ Residual deviance: 476.67 on 410 degrees of freedom } \\
\hline \multicolumn{6}{|l|}{ AIC: 1927.8} \\
\hline \multicolumn{6}{|c|}{ Number of Fisher Scoring iterations: 1} \\
\hline \multicolumn{6}{|l|}{ Theta: 2.145} \\
\hline \multicolumn{6}{|l|}{ Std. Err.: 0.264} \\
\hline \multicolumn{6}{|l|}{2 x log-likelihood: -1901.776 } \\
\hline
\end{tabular}




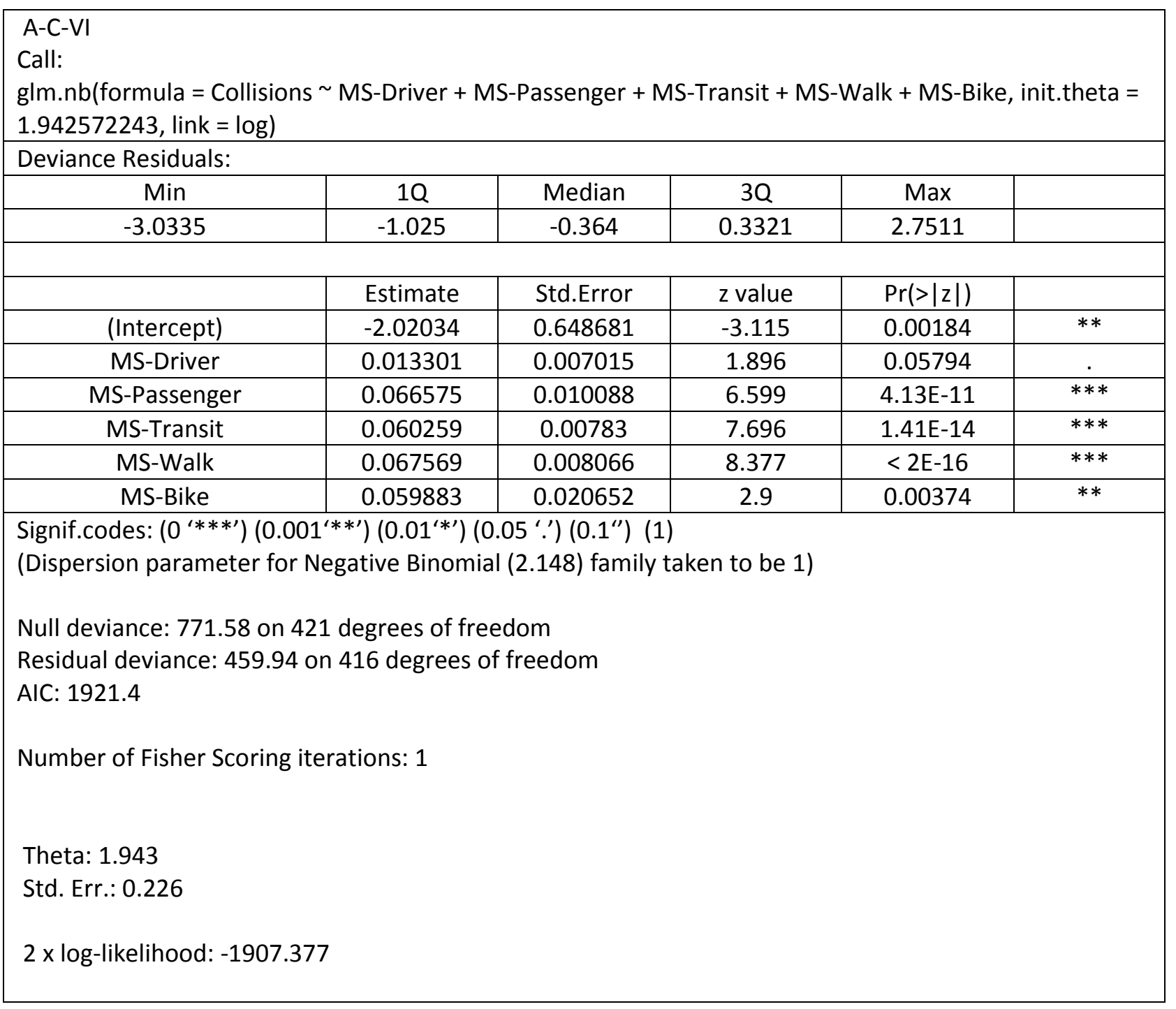




\begin{tabular}{|c|c|c|c|c|c|}
\hline \multicolumn{6}{|c|}{$\begin{array}{l}\text { Call: } \\
\text { glm.nb(formula }=\text { Collisions } \sim \text { Trips-Out-Center }+ \text { Trips-Enter-Center }+ \text { Density- Trips-Out-Center + } \\
\text { Density- Trips-Enter-Center }+ \text { TLKM, init.theta }=1.605453902, \text { link }=\log )\end{array}$} \\
\hline \multicolumn{6}{|l|}{ Deviance Residuals: } \\
\hline Min & 10 & Median & $3 Q$ & Max & \\
\hline \multirow[t]{2}{*}{-3.7697} & -1.1629 & -0.3729 & 0.4218 & 2.7629 & \\
\hline & Estimate & Std.Error & $z$ value & $\operatorname{Pr}(>|z|)$ & \\
\hline (Intercept) & $1.72 \mathrm{E}-01$ & $1.08 \mathrm{E}-01$ & 1.596 & 0.11057 & \\
\hline Trips-Out-Center & $2.01 \mathrm{E}-04$ & 4.47E-05 & 4.505 & $6.64 \mathrm{E}-06$ & $* * *$ \\
\hline Trips-Enter-Center & $1.31 \mathrm{E}-04$ & $2.96 \mathrm{E}-05$ & 4.441 & 8.97E-06 & $* * *$ \\
\hline Density- Trips-Out-Center & $3.35 \mathrm{E}-04$ & $3.02 \mathrm{E}-05$ & 11.103 & $<2 \mathrm{E}-16$ & $* * *$ \\
\hline Density- Trips-Enter-Center & $-2.36 \mathrm{E}-05$ & $5.86 \mathrm{E}-06$ & -4.016 & 5.91E-05 & $* * *$ \\
\hline TLKM & $-9.06 \mathrm{E}-03$ & $3.05 \mathrm{E}-03$ & -2.972 & 0.00295 & $* *$ \\
\hline \multicolumn{6}{|c|}{$\begin{array}{l}\text { Signif.codes: }\left(0^{\prime * * * \prime}\right)\left(0.001^{\prime * * \prime}\right)\left(0.01^{\prime * \prime}\right)\left(0.05^{\prime . \prime}\right)\left(0.1^{\prime \prime}\right)(1) \\
\text { (Dispersion parameter for Negative Binomial }(2.148) \text { family taken to be } 1)\end{array}$} \\
\hline \multicolumn{6}{|c|}{$\begin{array}{l}\text { Null deviance: } 694.35 \text { on } 421 \text { degrees of freedom } \\
\text { Residual deviance: } 466.06 \text { on } 416 \text { degrees of freedom } \\
\text { AIC: } 1973\end{array}$} \\
\hline \multicolumn{6}{|c|}{ Number of Fisher Scoring iterations: 1} \\
\hline \multicolumn{6}{|l|}{$\begin{array}{l}\text { Theta: } 1.605 \\
\text { Std. Err.: } 0.171\end{array}$} \\
\hline \multicolumn{6}{|l|}{2 x log-likelihood: -1959.001} \\
\hline
\end{tabular}




\begin{tabular}{|c|c|c|c|c|c|}
\hline \multicolumn{6}{|c|}{$\begin{array}{l}\text { A-D-I } \\
\text { Call: } \\
\text { glm.nb(formula = Collisions } \sim \text { Trips-Produced + Density- Trips-Out-Center + Density- Trips-Enter-Center, } \\
\text { init.theta }=1.460551328, \text { link }=\log \text { ) }\end{array}$} \\
\hline \multicolumn{6}{|l|}{ Deviance Residuals: } \\
\hline Min & 10 & Median & 30 & Max & \\
\hline \multirow[t]{2}{*}{-3.4223} & -1.2519 & -0.3982 & 0.4325 & 2.4802 & \\
\hline & Estimate & Std.Error & $z$ value & $\operatorname{Pr}(>|z|)$ & \\
\hline (Intercept) & 5.97E-01 & $6.80 \mathrm{E}-02$ & 8.778 & $<2 \mathrm{E}-16$ & $* * *$ \\
\hline Trips-Produced & $8.65 \mathrm{E}-05$ & $2.71 \mathrm{E}-05$ & 3.191 & 0.001419 & $* *$ \\
\hline Density- Trips-Out-Center & $2.94 \mathrm{E}-04$ & $3.50 \mathrm{E}-05$ & 8.409 & $<2 \mathrm{E}-16$ & $* * *$ \\
\hline Density- Trips-Enter-Center & $-1.99 \mathrm{E}-05$ & $5.25 \mathrm{E}-06$ & -3.793 & 0.000149 & $* * *$ \\
\hline \multicolumn{5}{|c|}{$\begin{array}{l}\text { Null deviance: } 657.61 \text { on } 421 \text { degrees of freedom } \\
\text { Residual deviance: } 475.41 \text { on } 418 \text { degrees of freedom } \\
\text { AIC: } 2002.2\end{array}$} & \\
\hline \multicolumn{6}{|c|}{ Number of Fisher Scoring iterations: 1} \\
\hline \multicolumn{6}{|l|}{$\begin{array}{l}\text { Theta: } 1.461 \\
\text { Std. Err.: } 0.154\end{array}$} \\
\hline \multicolumn{6}{|l|}{2 x log-likelihood: -1992.189 } \\
\hline
\end{tabular}




\begin{tabular}{|c|c|c|c|c|c|}
\hline \multicolumn{6}{|c|}{$\begin{array}{l}\text { Call: } \\
\text { glm.nb(formula }=\text { Collisions } \sim \text { Sig-Int }+ \text { Colle }+ \text { Loc }+ \text { VKT }+ \text { Density- Trips-Enter-Center, init.theta }= \\
\text { 1.636241559, link }=\log )\end{array}$} \\
\hline \multicolumn{6}{|l|}{ Deviance Residuals: } \\
\hline Min & $1 Q$ & Median & $3 Q$ & Max & \\
\hline \multirow[t]{2}{*}{-2.2917} & -1.3138 & -0.348 & 0.3555 & 3.2154 & \\
\hline & Estimate & Std.Error & $z$ value & $\operatorname{Pr}(>|z|)$ & \\
\hline (Intercept) & 1.19E-01 & 8.99E-02 & 1.317 & 0.18776 & \\
\hline Sig-Int & $4.56 \mathrm{E}-02$ & $6.56 \mathrm{E}-03$ & 6.954 & $3.56 \mathrm{E}-12$ & $* * *$ \\
\hline Colle & $1.73 \mathrm{E}-01$ & $5.85 \mathrm{E}-02$ & 2.95 & 0.00318 & $* *$ \\
\hline Loc & $1.34 \mathrm{E}-01$ & $1.32 \mathrm{E}-02$ & 10.134 & $<2 \mathrm{E}-16$ & $* * *$ \\
\hline VKT & $4.23 \mathrm{E}-06$ & $1.30 \mathrm{E}-06$ & 3.249 & 0.00116 & $* *$ \\
\hline Density- Trips-Enter-Center & $-2.07 \mathrm{E}-05$ & $6.59 \mathrm{E}-06$ & -3.148 & 0.00164 & $* *$ \\
\hline (Intercept) & $1.19 \mathrm{E}-01$ & 8.99E-02 & 1.317 & 0.18776 & \\
\hline Sig-Int & $4.56 \mathrm{E}-02$ & $6.56 \mathrm{E}-03$ & 6.954 & $3.56 \mathrm{E}-12$ & $* * *$ \\
\hline Colle & $1.73 \mathrm{E}-01$ & $5.85 \mathrm{E}-02$ & 2.95 & 0.00318 & $* *$ \\
\hline \multicolumn{6}{|c|}{$\begin{array}{l}\text { Signif.codes: }\left(0^{(* * * \prime}\right)\left(0.001^{\prime * * \prime}\right)\left(0.01^{* \prime \prime}\right)\left(0.05^{\prime}{ }^{\prime}\right)\left(0.1^{\prime \prime}\right)(1) \\
\text { (Dispersion parameter for Negative Binomial }(2.148) \text { family taken to be } 1)\end{array}$} \\
\hline \multicolumn{6}{|c|}{ Null deviance: 832.05 on 421 degrees of freedom } \\
\hline \multicolumn{6}{|c|}{ Residual deviance: 477.46 on 416 degrees of freedom } \\
\hline \multicolumn{6}{|l|}{ AIC: 1867.7} \\
\hline \multicolumn{6}{|c|}{ Number of Fisher Scoring iterations: 1} \\
\hline \multicolumn{6}{|l|}{ Theta: 1.636} \\
\hline \multicolumn{6}{|l|}{ Std. Err.: 0.2} \\
\hline 2 x log-likelihood: -1853.68 & & & & & \\
\hline
\end{tabular}




\begin{tabular}{|c|c|c|c|c|c|}
\hline \multicolumn{6}{|c|}{$\begin{array}{l}\text { Call: } \\
\text { glm.nb(formula }=\text { Collisions } \sim \text { MS-Driver + MS-Passenger + MS-Transit + MS-Walk + MS-Bike, init.theta }= \\
1.283380166, \text { link }=\log )\end{array}$} \\
\hline \multicolumn{6}{|l|}{ Deviance Residuals: } \\
\hline Min & 10 & Median & $3 Q$ & Max & \\
\hline \multirow[t]{2}{*}{-2.675} & -1.147 & -0.365 & 0.3 & 3.203 & \\
\hline & Fstimate & Std Frror & 7 value & $\operatorname{Pr}(>|z|)$ & \\
\hline (Intercept) & -2.76114 & 0.828887 & -3.331 & 0.000865 & (Intercept) \\
\hline MS-Driver & 0.01836 & 0.00888 & 2.068 & 0.038672 & MS-Driver \\
\hline MS-Passenger & 0.07569 & 0.012408 & 6.1 & $1.06 \mathrm{E}-09$ & $\begin{array}{c}\text { MS- } \\
\text { Passenger }\end{array}$ \\
\hline MS-Transit & 0.072091 & 0.009791 & 7.363 & $1.80 \mathrm{E}-13$ & MS-Transit \\
\hline MS-Walk & 0.076304 & 0.010142 & 7.524 & $5.32 \mathrm{E}-14$ & MS-Walk \\
\hline MS-Bike & 0.074222 & 0.024539 & 3.025 & 0.002489 & MS-Bike \\
\hline \multicolumn{6}{|c|}{$\begin{array}{l}\text { Signif.codes: }\left(0^{\prime * * * \prime}\right)\left(0.001^{\prime * * \prime}\right)\left(0.01^{\prime * \prime}\right)\left(0.05^{\prime} .^{\prime}\right)\left(0.1^{\prime \prime}\right)(1) \\
\text { (Dispersion parameter for Negative Binomial }(2.148) \text { family taken to be } 1)\end{array}$} \\
\hline $\begin{array}{l}\text { Null deviance: } 725.6 \\
\text { Residual deviance: } 45 \\
\text { AIC: } 1905.4\end{array}$ & $\begin{array}{l}\text { degrees of } \\
416 \text { degree }\end{array}$ & freedom & & & \\
\hline \multicolumn{6}{|c|}{ Number of Fisher Scoring iterations: 1} \\
\hline \multicolumn{6}{|l|}{ Theta: 1.283} \\
\hline 2 x log-likelihood: -1 & & & & & \\
\hline
\end{tabular}




\begin{tabular}{|c|c|c|c|c|c|}
\hline \multicolumn{6}{|c|}{$\begin{array}{l}\text { Call: } \\
\text { glm.nb(formula }=\text { Collisions } \sim \text { Trips-Out-Center }+ \text { Trips-Enter-Center }+ \text { Density- Trips-Out-Center }+ \\
\text { Density- Trips-Enter-Center }+ \text { TLKM, init.theta }=1.281914404, \text { link }=\log \text { ) }\end{array}$} \\
\hline \multicolumn{6}{|l|}{ Deviance Residuals: } \\
\hline Min & 10 & Median & $3 Q$ & Max & \\
\hline \multirow[t]{2}{*}{-3.8353} & -1.0604 & -0.5091 & 0.3397 & 2.4162 & \\
\hline & Estimate & Std.Error & $z$ value & $\operatorname{Pr}(>|z|)$ & \\
\hline (Intercept) & $-4.35 \mathrm{E}-01$ & $1.26 \mathrm{E}-01$ & -3.464 & 0.000532 & $* * *$ \\
\hline Trips-Out-Center & $2.97 \mathrm{E}-04$ & $5.00 \mathrm{E}-05$ & 5.945 & 2.77E-09 & $* * *$ \\
\hline Trips-Enter-Center & $2.18 \mathrm{E}-04$ & $3.25 \mathrm{E}-05$ & 6.709 & $1.96 \mathrm{E}-11$ & $* * *$ \\
\hline Density- Trips-Out-Center & $4.49 \mathrm{E}-04$ & $3.45 \mathrm{E}-05$ & 13.005 & $<2 \mathrm{E}-16^{* * *}$ & $* * *$ \\
\hline Density- Trips-Enter-Center & $-3.91 \mathrm{E}-05$ & $6.78 \mathrm{E}-06$ & -5.775 & 7.71E-09 & $* * *$ \\
\hline TLKM & $-8.38 \mathrm{E}-03$ & $3.24 \mathrm{E}-03$ & -2.586 & 0.009712 & $* *$ \\
\hline \multicolumn{6}{|c|}{$\begin{array}{l}\text { Signif.codes: }\left(0^{* * * \prime}\right)\left(0.001^{\prime * * \prime}\right)\left(0.01^{* \prime \prime}\right)\left(0.05^{\prime \prime}\right)\left(0.1^{\prime \prime}\right)(1) \\
\text { (Dispersion parameter for Negative Binomial }(2.148) \text { family taken to be } 1)\end{array}$} \\
\hline \multicolumn{6}{|c|}{ Null deviance: 725.12 on 421 degrees of freedom } \\
\hline \multicolumn{6}{|c|}{ Residual deviance: 444.23 on 416 degrees of freedom } \\
\hline \multicolumn{6}{|l|}{ AIC: 1892} \\
\hline \multicolumn{6}{|c|}{ Number of Fisher Scoring iterations: 1} \\
\hline \multicolumn{6}{|l|}{ Theta: 1.282} \\
\hline \multicolumn{6}{|l|}{ Std. Err.: 0.134} \\
\hline 2 xlog-likelihood: -1878.007 & & & & & \\
\hline
\end{tabular}




\begin{tabular}{|c|c|c|c|c|c|}
\hline \multicolumn{6}{|c|}{$\begin{array}{l}\text { D-D-II } \\
\text { Call: } \\
\text { glm.nb(formula }=\text { Collisions } ~ \text { No Bus stops }+ \text { Loc }+ \text { Bike-Path }+ \text { Trips-Attracted + Density- Trips-Enter- } \\
\text { Center, init.theta }=1.393659775, \text { link }=\text { log) }\end{array}$} \\
\hline \multicolumn{6}{|l|}{ Deviance Residuals: } \\
\hline Min & 10 & Median & $3 Q$ & Max & \\
\hline-2.7441 & -1.1877 & -0.3778 & 0.3027 & 3.3948 & \\
\hline & Estimate & Std.Error & $z$ value & $\operatorname{Pr}(>|z|)$ & \\
\hline (Intercept) & $-1.20 \mathrm{E}-01$ & $9.30 \mathrm{E}-02$ & -1.291 & 0.196872 & \\
\hline No Bus stops & 2.77E-02 & $4.00 \mathrm{E}-03$ & 6.909 & $4.88 \mathrm{E}-12$ & $* * *$ \\
\hline Loc & $1.46 \mathrm{E}-01$ & $1.51 \mathrm{E}-02$ & 9.669 & $<2 \mathrm{E}-16$ & $* * *$ \\
\hline Bike-Path & 4.07E-02 & $1.22 \mathrm{E}-02$ & 3.328 & 0.000874 & $* * *$ \\
\hline Trips-Attracted & $2.64 \mathrm{E}-05$ & $7.38 \mathrm{E}-06$ & 3.577 & 0.000347 & $* * *$ \\
\hline Density- Trips-Enter-Center & $-3.11 \mathrm{E}-05$ & 9.77E-06 & -3.186 & 0.001444 & $* *$ \\
\hline \multicolumn{6}{|c|}{$\begin{array}{l}\text { Signif.codes: }\left(0^{* * * \prime}\right)\left(0.001^{\prime * *^{\prime}}\right)\left(0.01^{\prime * \prime}\right)\left(0.05^{\prime \prime}\right)\left(0.1^{\prime \prime}\right)(1) \\
\text { (Dispersion parameter for Negative Binomial }(2.148) \text { family taken to be } 1)\end{array}$} \\
\hline \multicolumn{6}{|c|}{ Null deviance: 813.05 on 421 degrees of freedom } \\
\hline \multicolumn{6}{|c|}{ Residual deviance: 463.15 on 416 degrees of freedom } \\
\hline \multicolumn{6}{|l|}{ AIC: 1850.2} \\
\hline \multicolumn{6}{|c|}{ Number of Fisher Scoring iterations: 1} \\
\hline \multicolumn{6}{|l|}{ Theta: 1.394} \\
\hline 2 x log-likelihood: -1836.16 & & & & & \\
\hline
\end{tabular}




\begin{tabular}{|c|c|c|c|c|c|}
\hline \multicolumn{6}{|c|}{$\begin{array}{l}\text { Call: } \\
\text { glm.nb(formula }=\text { Collisions } \sim \text { No Bus stops }+ \text { Tot-Int }+ \text { Colle }+ \text { Major-Colle }+ \text { Bike-Path }+ \text { Density- Trips- } \\
\text { Out-Center + Density- Trips-Enter-Center, init.theta }=1.531041038, \text { link }=\log )\end{array}$} \\
\hline \multicolumn{6}{|l|}{ Deviance Residuals: } \\
\hline Min & 10 & Median & $3 Q$ & Max & \\
\hline \multirow[t]{2}{*}{-2.7178} & -1.2312 & -0.3741 & 0.3119 & 3.7042 & \\
\hline & Estimate & Std.Error & z value & $\operatorname{Pr}(>|z|)$ & \\
\hline (Intercept) & $-7.01 \mathrm{E}-02$ & $9.73 \mathrm{E}-02$ & -0.721 & 0.4712 & \\
\hline No Bus stops & $2.39 \mathrm{E}-02$ & $4.23 \mathrm{E}-03$ & 5.64 & 1.70E-08 & $* * *$ \\
\hline Tot-Int & $1.93 \mathrm{E}-02$ & $2.74 \mathrm{E}-03$ & 7.042 & $1.90 \mathrm{E}-12$ & $* * *$ \\
\hline Colle & $9.62 \mathrm{E}-02$ & $5.85 \mathrm{E}-02$ & 1.645 & 0.10002 & \\
\hline Major-Colle & $-2.26 \mathrm{E}-01$ & $1.13 \mathrm{E}-01$ & -1.999 & 0.04564 & $*$ \\
\hline Bike-Path & $7.82 \mathrm{E}-03$ & $4.82 \mathrm{E}-03$ & 1.623 & 0.1046 & \\
\hline Density- Trips-Out-Center & $6.79 \mathrm{E}-05$ & 3.74E-05 & 1.815 & 0.06947 & . \\
\hline Density-Trips-Enter-Center & $-1.74 \mathrm{E}-05$ & $6.23 \mathrm{E}-06$ & -2.79 & 0.00527 & $* *$ \\
\hline \multicolumn{6}{|c|}{$\begin{array}{l}\text { Signif.codes: }\left(0^{* * * \prime}\right)\left(0.001^{\prime * *^{\prime}}\right)\left(0.01^{\prime * \prime}\right)\left(0.05^{\prime \prime}\right)\left(0.1^{\prime \prime}\right)(1) \\
\text { (Dispersion parameter for Negative Binomial }(2.148) \text { family taken to be } 1)\end{array}$} \\
\hline \multicolumn{6}{|c|}{ Null deviance: 809.72 on 421 degrees of freedom } \\
\hline \multicolumn{6}{|c|}{ Residual deviance: 471.28 on 414 degrees of freedom } \\
\hline \multicolumn{6}{|l|}{ AIC: 1875} \\
\hline \multicolumn{6}{|c|}{ Number of Fisher Scoring iterations: 1} \\
\hline \multicolumn{6}{|l|}{ Theta: 1.531} \\
\hline \multicolumn{6}{|l|}{ Std. Err.: 0.181} \\
\hline 2 x log-likelihood: -1856.971 & & & & & \\
\hline
\end{tabular}




\begin{tabular}{|c|c|c|c|c|c|}
\hline $\begin{array}{l}\text { G-D-III } \\
\text { Call: } \\
\text { glm.nb(formula = Collisions } \\
\text { + Density- Trips-Out-Center }\end{array}$ & $\begin{array}{l}\text { Jo Bus stops } \\
\text { Density- Trip }\end{array}$ & $\begin{array}{l}\text { t-Int }+40.1 \\
\text { ter-Center }\end{array}$ & $\begin{array}{l}50 . \mathrm{Km} \\
\mathrm{n}, \text { init.th }\end{array}$ & $\begin{array}{l}\mathrm{Km} / \mathrm{h}+\mathrm{B} \\
.5551965\end{array}$ & $\begin{array}{l} \\
+ \text { + VKT } \\
=\log )\end{array}$ \\
\hline Deviance Residuals: & & & & & \\
\hline Min & 10 & Median & $3 Q$ & Max & \\
\hline-2.6669 & -1.2031 & -0.3806 & 0.3238 & 3.7228 & \\
\hline & Estimate & Std.Error & $z$ value & $\operatorname{Pr}(>|z|)$ & \\
\hline (Intercept) & $-5.02 \mathrm{E}-02$ & $1.08 \mathrm{E}-01$ & -0.463 & 0.64342 & \\
\hline No Bus stops & $2.03 \mathrm{E}-02$ & $4.31 E-03$ & 4.717 & $2.39 \mathrm{E}-06$ & $* * *$ \\
\hline Tot-Int & $1.69 \mathrm{E}-02$ & $3.06 \mathrm{E}-03$ & 5.515 & $3.49 \mathrm{E}-08$ & $* * *$ \\
\hline $40 . \mathrm{Km} / \mathrm{h}$ & $-8.17 \mathrm{E}-02$ & $5.32 \mathrm{E}-02$ & -1.538 & 0.12411 & \\
\hline $50 . \mathrm{Km} / \mathrm{h}$ & $-1.03 \mathrm{E}-01$ & $4.82 \mathrm{E}-02$ & -2.138 & 0.03252 & $*$ \\
\hline $80 . \mathrm{Km} / \mathrm{h}$ & $-2.60 \mathrm{E}-01$ & $1.43 \mathrm{E}-01$ & -1.813 & 0.06985 & . \\
\hline Bike-Path & $1.03 \mathrm{E}-02$ & $5.15 \mathrm{E}-03$ & 1.99 & 0.04661 & $*$ \\
\hline VKT & $-1.49 \mathrm{E}-06$ & $1.04 \mathrm{E}-06$ & -1.436 & 0.15099 & \\
\hline Density- Trips-Out-Center & $8.17 \mathrm{E}-05$ & $3.71 \mathrm{E}-05$ & 2.2 & 0.02782 & $*$ \\
\hline Density- Trips-Enter-Center & $-1.67 \mathrm{E}-05$ & 5.99E-06 & -2.792 & 0.00523 & $* *$ \\
\hline TLKM & $1.15 \mathrm{E}-01$ & 4.87E-02 & 2.357 & 0.01844 & $*$ \\
\hline $\begin{array}{l}\text { Signif.codes: }\left(0^{\star * * * \prime}\right)(0.001 \\
\text { (Dispersion parameter for } N \\
\text { Null deviance: } 816.79 \text { on } 42 \\
\text { Residual deviance: } 471.03 \text { or } \\
\text { AIC: } 1877.1\end{array}$ & $\begin{array}{l}\left.\mathrm{k}^{\prime}\right)\left(0.01^{\prime * \prime}\right) \\
\text { ative Binom } \\
\text { degrees of } \mathrm{fr} \\
11 \text { degrees }\end{array}$ & $\begin{array}{l}\text { "') }\left(0.1^{\prime \prime}\right) \\
.148) \text { famil } \\
\text { om } \\
\text { eedom }\end{array}$ & $\mathrm{n}$ to be & & \\
\hline Number of Fisher Scoring ite & tions: 1 & & & & \\
\hline $\begin{array}{l}\text { Theta: } 1.555 \\
\text { Std. Err.: } 0.185\end{array}$ & & & & & \\
\hline 2 x log-likelihood: -1853.122 & & & & & \\
\hline
\end{tabular}




\begin{tabular}{|c|c|c|c|c|c|}
\hline $\begin{array}{l}\text { H-D-II } \\
\text { Call: } \\
\text { glm.nb(formula = Collisions } \\
\text { Center + Density- Trips-Ente }\end{array}$ & $\begin{array}{l}\text { lo Bus stops } \\
\text { enter, init.t }\end{array}$ & $\begin{array}{l}t-I n t+\text { Coll } \\
=1.44723\end{array}$ & $\begin{array}{l}\text { ajor-Colle } \\
\text { link }=\text { log }\end{array}$ & + Density & Out- \\
\hline Deviance Residuals: & & & & & \\
\hline Min & 10 & Median & $3 Q$ & Max & \\
\hline-2.6238 & -1.1925 & -0.4245 & 0.3321 & 3.7679 & \\
\hline & Estimate & Std.Error & $z$ value & $\operatorname{Pr}(>|z|)$ & \\
\hline (Intercept) & $-1.75 \mathrm{E}-01$ & $9.88 \mathrm{E}-02$ & -1.769 & 0.07696 &. \\
\hline No Bus stops & $2.25 \mathrm{E}-02$ & $4.51 \mathrm{E}-03$ & 4.995 & $5.88 \mathrm{E}-07$ & $* * *$ \\
\hline Tot-Int & $1.98 \mathrm{E}-02$ & $2.78 \mathrm{E}-03$ & 7.112 & $1.14 \mathrm{E}-12$ & $* * *$ \\
\hline Colle & 1.09E-01 & $6.06 \mathrm{E}-02$ & 1.794 & 0.07289 & . \\
\hline Major-Colle & $-2.37 E-01$ & $1.18 \mathrm{E}-01$ & -2.004 & 0.04512 & $*$ \\
\hline VKT & $5.31 \mathrm{E}-06$ & $1.70 \mathrm{E}-06$ & 3.123 & 0.00179 & $* *$ \\
\hline Density- Trips-Out-Center & 8.77E-05 & $3.83 E-05$ & 2.292 & 0.02192 & $*$ \\
\hline Density- Trips-Enter-Center & $-1.89 \mathrm{E}-05$ & $6.55 \mathrm{E}-06$ & -2.885 & 0.00392 & $* *$ \\
\hline $\begin{array}{l}\text { Signif.codes: }\left(0^{* * * * \prime}\right)(0.001 \\
\text { (Dispersion parameter for } N\end{array}$ & $\begin{array}{l}\text { (6) }\left(0.01^{\prime * \prime}\right)( \\
\text { ative Binom }\end{array}$ & $\begin{array}{l}\left.y^{\prime}\right)\left(0.1^{\prime \prime}\right) \\
.148) \text { famil }\end{array}$ & n to be 1 & & \\
\hline $\begin{array}{l}\text { Null deviance: } 837.28 \text { on } 42 \\
\text { Residual deviance: } 466.71 \text { o } \\
\text { AIC: } 1845.7\end{array}$ & $\begin{array}{l}\text { degrees of } \mathrm{fr} \\
14 \text { degrees }\end{array}$ & $\begin{array}{l}\text { om } \\
\text { eedom }\end{array}$ & & & \\
\hline Number of Fisher Scoring it & tions: 1 & & & & \\
\hline $\begin{array}{l}\text { Theta: } 1.447 \\
\text { Std. Err.: } 0.172\end{array}$ & & & & & \\
\hline $2 \times$ log-likelihood: -1827.75 & & & & & \\
\hline
\end{tabular}




\begin{tabular}{|c|c|c|c|c|c|}
\hline \multicolumn{6}{|c|}{$\begin{array}{l}\text { Call: } \\
\text { glm.nb(formula }=\text { Collisions } \sim \text { No Bus stops }+ \text { Tot-Int }+40 . \mathrm{Km} / \mathrm{h}+50 . \mathrm{Km} / \mathrm{h}+60 . \mathrm{Km} / \mathrm{h}+70 . \mathrm{Km} / \mathrm{h}+ \\
80 . \mathrm{Km} / \mathrm{h}+90 . \mathrm{Km} / \mathrm{h}+\text { VKT }+ \text { Density- Trips-Out-Center + Density- Trips-Enter-Center }+ \text { TLKM, init.theta }= \\
\text { 1.48228, link }=\log )\end{array}$} \\
\hline \multicolumn{6}{|l|}{ Deviance Residuals: } \\
\hline Min & $1 Q$ & Median & $3 Q$ & Max & \\
\hline \multirow[t]{2}{*}{-2.3924} & -1.1614 & -0.4111 & 0.3629 & 3.757 & \\
\hline & Estimate & Std.Error & $z$ value & $\operatorname{Pr}(>|z|)$ & \\
\hline (Intercept) & $-1.90 \mathrm{E}-01$ & $1.11 \mathrm{E}-01$ & -1.715 & 0.0864 & . \\
\hline No Bus stops & $2.09 \mathrm{E}-02$ & $4.55 \mathrm{E}-03$ & 4.592 & $4.40 \mathrm{E}-06$ & $* * *$ \\
\hline Tot-Int & $1.81 \mathrm{E}-02$ & $3.14 \mathrm{E}-03$ & 5.761 & $8.34 \mathrm{E}-09$ & $* * *$ \\
\hline $40 . \mathrm{Km} / \mathrm{h}$ & $4.14 \mathrm{E}+01$ & $2.41 \mathrm{E}+01$ & 1.717 & 0.08598 & . \\
\hline $50 . \mathrm{Km} / \mathrm{h}$ & $4.14 \mathrm{E}+01$ & $2.41 \mathrm{E}+01$ & 1.716 & 0.0862 & . \\
\hline $60 . \mathrm{Km} / \mathrm{h}$ & $4.15 \mathrm{E}+01$ & $2.41 \mathrm{E}+01$ & 1.72 & 0.08536 & . \\
\hline $70 . \mathrm{Km} / \mathrm{h}$ & $4.15 \mathrm{E}+01$ & $2.41 \mathrm{E}+01$ & 1.721 & 0.08527 & . \\
\hline $80 . \mathrm{Km} / \mathrm{h}$ & $4.13 \mathrm{E}+01$ & $2.41 \mathrm{E}+01$ & 1.711 & 0.08707 & . \\
\hline $90 . \mathrm{Km} / \mathrm{h}$ & $4.12 \mathrm{E}+01$ & $2.42 \mathrm{E}+01$ & 1.705 & 0.08822 & . \\
\hline VKT & $4.52 \mathrm{E}-06$ & $1.72 \mathrm{E}-06$ & 2.631 & 0.0085 & $* *$ \\
\hline Density- Trips-Out-Center & $9.11 \mathrm{E}-05$ & $3.82 \mathrm{E}-05$ & 2.382 & 0.01722 & $*$ \\
\hline Density-Trips-Enter-Center & $-1.96 \mathrm{E}-05$ & $6.42 \mathrm{E}-06$ & -3.054 & 0.00226 & $* *$ \\
\hline TLKM & $-4.14 \mathrm{E}+01$ & $2.41 \mathrm{E}+01$ & -1.715 & 0.08628 & . \\
\hline \multicolumn{6}{|c|}{$\begin{array}{l}\text { Signif.codes: }\left(0^{* * * \prime}\right)\left(0.001^{\prime * * \prime}\right)\left(0.01^{\prime * \prime}\right)\left(0.05^{\prime} . \prime\right)\left(0.1^{\prime \prime}\right)(1) \\
\text { (Dispersion parameter for Negative Binomial }(2.148) \text { family taken to be } 1)\end{array}$} \\
\hline \multicolumn{6}{|c|}{$\begin{array}{l}\text { Null deviance: } 848.56 \text { on } 421 \text { degrees of freedom } \\
\text { Residual deviance: } 466.73 \text { on } 409 \text { degrees of freedom } \\
\text { AIC: } 1850.4\end{array}$} \\
\hline \multicolumn{6}{|c|}{ Number of Fisher Scoring iterations: 1} \\
\hline \multicolumn{6}{|l|}{ Theta: 1.482} \\
\hline 2 x log-likelihood: -1822.351 & & & & & \\
\hline
\end{tabular}




\begin{tabular}{|c|c|c|c|c|c|}
\hline \multicolumn{6}{|c|}{$\begin{array}{l}\text { Call: } \\
\text { glm.nb(formula }=\text { Collisions } \sim \text { MS-Driver + MS-Passenger + MS-Transit + MS-Walk + MS-Bike, init.theta }= \\
\text { 1.164911258, link }=\text { log) }\end{array}$} \\
\hline \multicolumn{6}{|l|}{ Deviance Residuals: } \\
\hline Min & 10 & Median & $3 Q$ & Max & \\
\hline \multirow[t]{2}{*}{-2.599} & -1.1826 & -0.4279 & 0.3175 & 3.1504 & \\
\hline & Estimate & Std.Error & $z$ value & $\operatorname{Pr}(>|z|)$ & \\
\hline (Intercept) & -2.05049 & 0.712585 & -2.878 & 0.00401 & $* *$ \\
\hline MS-Driver & 0.011707 & 0.007754 & 1.51 & 0.13109 & \\
\hline MS-Passenger & 0.068839 & 0.011582 & 5.943 & 2.79E-09 & $* * *$ \\
\hline MS-Transit & 0.061468 & 0.008931 & 6.882 & $5.89 \mathrm{E}-12$ & $* * *$ \\
\hline MS-Walk & 0.069726 & 0.009229 & 7.555 & 4.19E-14 & $* * *$ \\
\hline MS-Bike & 0.062555 & 0.025216 & 2.481 & 0.01311 & $*$ \\
\hline \multicolumn{6}{|c|}{$\begin{array}{l}\text { Signif.codes: }\left(0^{\prime * * * \prime}\right)\left(0.001^{\prime * * \prime}\right)\left(0.01^{\prime * \prime}\right)\left(0.05^{\prime \prime}\right)\left(0.1^{\prime \prime}\right)(1) \\
\text { (Dispersion parameter for Negative Binomial }(2.148) \text { family taken to be } 1)\end{array}$} \\
\hline \multicolumn{6}{|c|}{ Null deviance: 691.84 on 421 degrees of freedom } \\
\hline \multicolumn{6}{|c|}{ Residual deviance: 461.32 on 416 degrees of freedom } \\
\hline \multicolumn{6}{|l|}{ AIC: 1927.1} \\
\hline \multicolumn{6}{|c|}{ Number of Fisher Scoring iterations: 1} \\
\hline \multicolumn{6}{|l|}{ Theta: 1.165} \\
\hline \multicolumn{6}{|l|}{ Std. Err.: 0.125} \\
\hline \multicolumn{6}{|c|}{2 x log-likelihood: -1913.058} \\
\hline
\end{tabular}




\begin{tabular}{|c|c|c|c|c|c|}
\hline \multicolumn{6}{|c|}{$\begin{array}{l}\text { Call: } \\
\text { glm.nb(formula }=\text { Collisions } \sim \text { VKT }+ \text { Trips-Out-Center }+ \text { Trips-Enter-Center + Density- Trips-Out-Center + } \\
\text { Density- Trips-Enter-Center + TLKM, init.theta }=1.1030107, \text { link }=\log )\end{array}$} \\
\hline \multicolumn{6}{|l|}{ Deviance Residuals: } \\
\hline Min & 10 & Median & $3 Q$ & Max & \\
\hline \multirow[t]{2}{*}{-3.4365} & -1.1376 & -0.4392 & 0.3349 & 3.4866 & \\
\hline & Estimate & Std.Error & $z$ value & $\operatorname{Pr}(>|z|)$ & \\
\hline (Intercept) & $-1.76 \mathrm{E}-01$ & $1.27 \mathrm{E}-01$ & -1.388 & 0.16522 & \\
\hline VKT & $3.55 \mathrm{E}-06$ & $1.23 \mathrm{E}-06$ & 2.88 & 0.00398 & $* *$ \\
\hline Trips-Out-Center & $2.11 \mathrm{E}-04$ & $5.18 \mathrm{E}-05$ & 4.069 & 4.73E-05 & $* * *$ \\
\hline Trips-Enter-Center & $1.81 \mathrm{E}-04$ & $3.45 \mathrm{E}-05$ & 5.237 & $1.64 \mathrm{E}-07$ & $* * *$ \\
\hline Density- Trips-Out-Center & 4.37E-04 & 3.67E-05 & 11.923 & $<2 \mathrm{E}-16$ & $* * *$ \\
\hline Density- Trips-Enter-Center & $-4.24 \mathrm{E}-05$ & 7.27E-06 & -5.829 & 5.57E-09 & $* * *$ \\
\hline TLKM & $-9.23 \mathrm{E}-03$ & $3.25 \mathrm{E}-03$ & -2.844 & 0.00446 & $* *$ \\
\hline \multicolumn{6}{|c|}{$\begin{array}{l}\text { Signif.codes: }\left(0^{\prime * * * \prime}\right)\left(0.001^{\prime * * \prime}\right)\left(0.01^{\prime * \prime}\right)\left(0.05^{\prime \prime \prime}\right)\left(0.1^{\prime \prime}\right)(1) \\
\text { (Dispersion parameter for Negative Binomial }(2.148) \text { family taken to be } 1)\end{array}$} \\
\hline \multicolumn{6}{|c|}{ Null deviance: 669.63 on 421 degrees of freedom } \\
\hline \multicolumn{6}{|c|}{ Residual deviance: 455.93 on 415 degrees of freedom } \\
\hline \multicolumn{6}{|l|}{ AIC: 1937.6} \\
\hline \multicolumn{6}{|c|}{ Number of Fisher Scoring iterations: 1} \\
\hline \multicolumn{6}{|l|}{ Theta: 1.103} \\
\hline \multicolumn{6}{|l|}{ Std. Err.: 0.113} \\
\hline $2 \times$ log-likelihood: -1921.63 & & & & & \\
\hline
\end{tabular}




\begin{tabular}{|c|c|c|c|c|c|}
\hline \multicolumn{6}{|c|}{$\begin{array}{l}\text { Call: } \\
\text { glm.nb(formula }=\text { Collisions } \sim \text { VKT }+ \text { Trips-Out-Center }+ \text { Trips-Enter-Center + Density- Trips-Out-Center + } \\
\text { Density- Trips-Enter-Center }+ \text { TLKM, init.theta }=1.094366936, \text { link }=\text { log) }\end{array}$} \\
\hline \multicolumn{6}{|l|}{ Deviance Residuals: } \\
\hline Min & 10 & Median & $3 Q$ & Max & \\
\hline \multirow[t]{2}{*}{-3.4741} & -1.0978 & -0.4722 & 0.3086 & 2.483 & \\
\hline & Estimate & Std.Error & $z$ value & $\operatorname{Pr}(>|z|)$ & \\
\hline (Intercept) & $-3.23 E-01$ & $1.29 \mathrm{E}-01$ & -2.496 & 0.01255 & $*$ \\
\hline VKT & $3.08 \mathrm{E}-06$ & $1.06 \mathrm{E}-06$ & 2.922 & 0.00348 & $* *$ \\
\hline Trips-Out-Center & $2.42 \mathrm{E}-04$ & $5.24 \mathrm{E}-05$ & 4.624 & 3.77E-06 & $* * *$ \\
\hline Trips-Enter-Center & $2.02 \mathrm{E}-04$ & $3.46 \mathrm{E}-05$ & 5.849 & 4.94E-09 & $* * *$ \\
\hline Density- Trips-Out-Center & $4.52 \mathrm{E}-04$ & 3.69E-05 & 12.249 & $<2 \mathrm{E}-16$ & $* * *$ \\
\hline Density- Trips-Enter-Center & $-4.33 \mathrm{E}-05$ & 7.31E-06 & -5.917 & $3.28 \mathrm{E}-09$ & $* * *$ \\
\hline TLKM & $-8.80 \mathrm{E}-03$ & $3.20 \mathrm{E}-03$ & -2.754 & 0.00589 & $* *$ \\
\hline \multicolumn{6}{|c|}{$\begin{array}{l}\text { Signif.codes: }\left(0^{\prime * * * \prime}\right)\left(0.001^{\prime * * \prime}\right)\left(0.01^{\prime * \prime}\right)\left(0.05^{\prime \prime \prime}\right)\left(0.1^{\prime \prime}\right)(1) \\
\text { (Dispersion parameter for Negative Binomial }(2.148) \text { family taken to be } 1)\end{array}$} \\
\hline \multicolumn{6}{|c|}{ Null deviance: 685.33 on 421 degrees of freedom } \\
\hline \multicolumn{6}{|c|}{ Residual deviance: 450.98 on 415 degrees of freedom } \\
\hline \multicolumn{6}{|l|}{ AIC: 1917.6} \\
\hline \multicolumn{6}{|c|}{ Number of Fisher Scoring iterations: 1} \\
\hline \multicolumn{6}{|l|}{ Theta: 1.094} \\
\hline \multicolumn{6}{|l|}{ Std. Err.: 0.112} \\
\hline \multicolumn{6}{|l|}{2 x log-likelihood: -1901.564} \\
\hline
\end{tabular}




\begin{tabular}{|c|c|c|c|c|c|}
\hline $\begin{array}{l}\text { J-D-I } \\
\text { Call: } \\
\text { glm.nb(formula = Collisions } \\
\text { log) }\end{array}$ & KT + Densit & ips-Enter-C & TLKM, i & eta $=1.341$ & , link = \\
\hline Deviance Residuals: & & & & & \\
\hline Min & 10 & Median & $3 Q$ & Max & \\
\hline-3.4554 & -1.1442 & -0.362 & 0.3085 & 2.6184 & \\
\hline & Estimate & Std.Error & $z$ value & $\operatorname{Pr}(>|z|)$ & \\
\hline (Intercept) & $5.85 \mathrm{E}-01$ & $8.15 \mathrm{E}-02$ & 7.181 & $6.90 \mathrm{E}-13$ & $* * *$ \\
\hline VKT & $5.92 \mathrm{E}-06$ & $2.53 \mathrm{E}-06$ & 2.342 & 0.019162 & $*$ \\
\hline Density- Trips-Enter-Center & $-1.34 \mathrm{E}-05$ & $3.79 \mathrm{E}-06$ & -3.523 & 0.000426 & $* * *$ \\
\hline TLKM & $1.03 \mathrm{E}-01$ & $8.50 \mathrm{E}-03$ & 12.118 & $<2 \mathrm{E}-16$ & $* * *$ \\
\hline $\begin{array}{l}\text { Signif.codes: }\left(0^{* * * * \prime}\right)(0.001 \\
\text { (Dispersion parameter for } \mathrm{N}\end{array}$ & $\begin{array}{l}\text { ") }\left(0.01^{\prime * \prime}\right) \\
\text { ative Binom }\end{array}$ & $\begin{array}{l}\because \prime)\left(0.1^{\prime \prime}\right) \\
.148) \text { famil }\end{array}$ & n to be 1 & & \\
\hline Null deviance: 806.52 on 42 & legrees of $f$ & & & & \\
\hline Residual deviance: 491.53 o & 18 degrees & eedom & & & \\
\hline AIC: 2354.7 & & & & & \\
\hline Number of Fisher Scoring it & tions: 1 & & & & \\
\hline Theta: 1.341 & & & & & \\
\hline Std. Err.: 0.129 & & & & & \\
\hline $2 \times$ log-likelihood: -2344.72 & & & & & \\
\hline
\end{tabular}




\begin{tabular}{|c|c|c|c|c|c|}
\hline $\begin{array}{l}\text { K-D-II } \\
\text { Call: } \\
\text { glm.nb(formula = Collisions } \\
\text { Produced + Trips-Attracted }\end{array}$ & $\begin{array}{l}\text { No Bus stops } \\
\text { ensity- Trip }\end{array}$ & $\begin{array}{l}\text { t-Int + ArtI } \\
\text { ter-Center, }\end{array}$ & $\begin{array}{l}\text { le }+ \text { Maj- } \\
\text { eta }=2.1\end{array}$ & $\begin{array}{l}+ \text { Bike-Path } \\
\text { 452, link = Ic }\end{array}$ & + Trips- \\
\hline Deviance Residuals: & & & & & \\
\hline Min & 10 & Median & $3 Q$ & Max & \\
\hline-2.9973 & -1.1847 & -0.3205 & 0.3258 & 3.2777 & \\
\hline & Estimate & Std.Error & $z$ value & $\operatorname{Pr}(>|z|)$ & \\
\hline (Intercept) & $8.77 \mathrm{E}-02$ & $8.98 \mathrm{E}-02$ & 0.977 & 0.328671 & \\
\hline No Bus stops & $1.22 \mathrm{E}-02$ & $3.73 E-03$ & 3.256 & 0.00113 & ** \\
\hline Tot-Int & $1.74 \mathrm{E}-02$ & $2.23 \mathrm{E}-03$ & 7.82 & $5.29 E-15$ & $* * *$ \\
\hline Artl & $-4.66 \mathrm{E}-02$ & $2.32 \mathrm{E}-02$ & -2.006 & 0.044885 & $*$ \\
\hline Colle & $6.50 \mathrm{E}-02$ & $3.93 \mathrm{E}-02$ & 1.654 & 0.09809 & . \\
\hline Maj-Colle & $-9.84 \mathrm{E}-02$ & $5.12 \mathrm{E}-02$ & -1.922 & 0.054631 & . \\
\hline Bike-Path & $3.83 \mathrm{E}-02$ & $1.02 \mathrm{E}-02$ & 3.766 & 0.000166 & $* * *$ \\
\hline VKT & $1.16 \mathrm{E}-05$ & $3.04 \mathrm{E}-06$ & 3.811 & 0.000139 & $* * *$ \\
\hline Trips-Produced & $4.23 \mathrm{E}-05$ & $2.36 \mathrm{E}-05$ & 1.792 & 0.073105 & . \\
\hline Trips-Attracted & $1.12 \mathrm{E}-05$ & $4.83 \mathrm{E}-06$ & 2.314 & 0.020655 & $*$ \\
\hline Density- Trips-Enter-Center & $-1.57 \mathrm{E}-05$ & $6.38 \mathrm{E}-06$ & -2.465 & 0.0137 & $*$ \\
\hline $\begin{array}{l}\text { Signif.codes: }\left(0^{* * * * \prime}\right)(0.001 \\
\text { (Dispersion parameter for } N\end{array}$ & $\begin{array}{l}\left.k^{\prime}\right)\left(0.01^{* \prime}\right) \\
\text { ative Binom }\end{array}$ & $\begin{array}{l}.^{\prime \prime}\left(0.1^{\prime \prime}\right) \\
.148) \text { famil }\end{array}$ & n to be 1 & & \\
\hline $\begin{array}{l}\text { Null deviance: } 814.14 \text { on } 42 \\
\text { Residual deviance: } 474.19 \text { o } \\
\text { AIC: } 1922.8\end{array}$ & $\begin{array}{l}\text { degrees of } \mathrm{fi} \\
11 \text { degrees }\end{array}$ & $\begin{array}{l}\text { om } \\
\text { eedom }\end{array}$ & & & \\
\hline Number of Fisher Scoring it & tions: 1 & & & & \\
\hline $\begin{array}{l}\text { Theta: } 2.149 \\
\text { Std. Err.: } 0.264\end{array}$ & & & & & \\
\hline 2 x log-likelihood: -1898.81 & & & & & \\
\hline
\end{tabular}




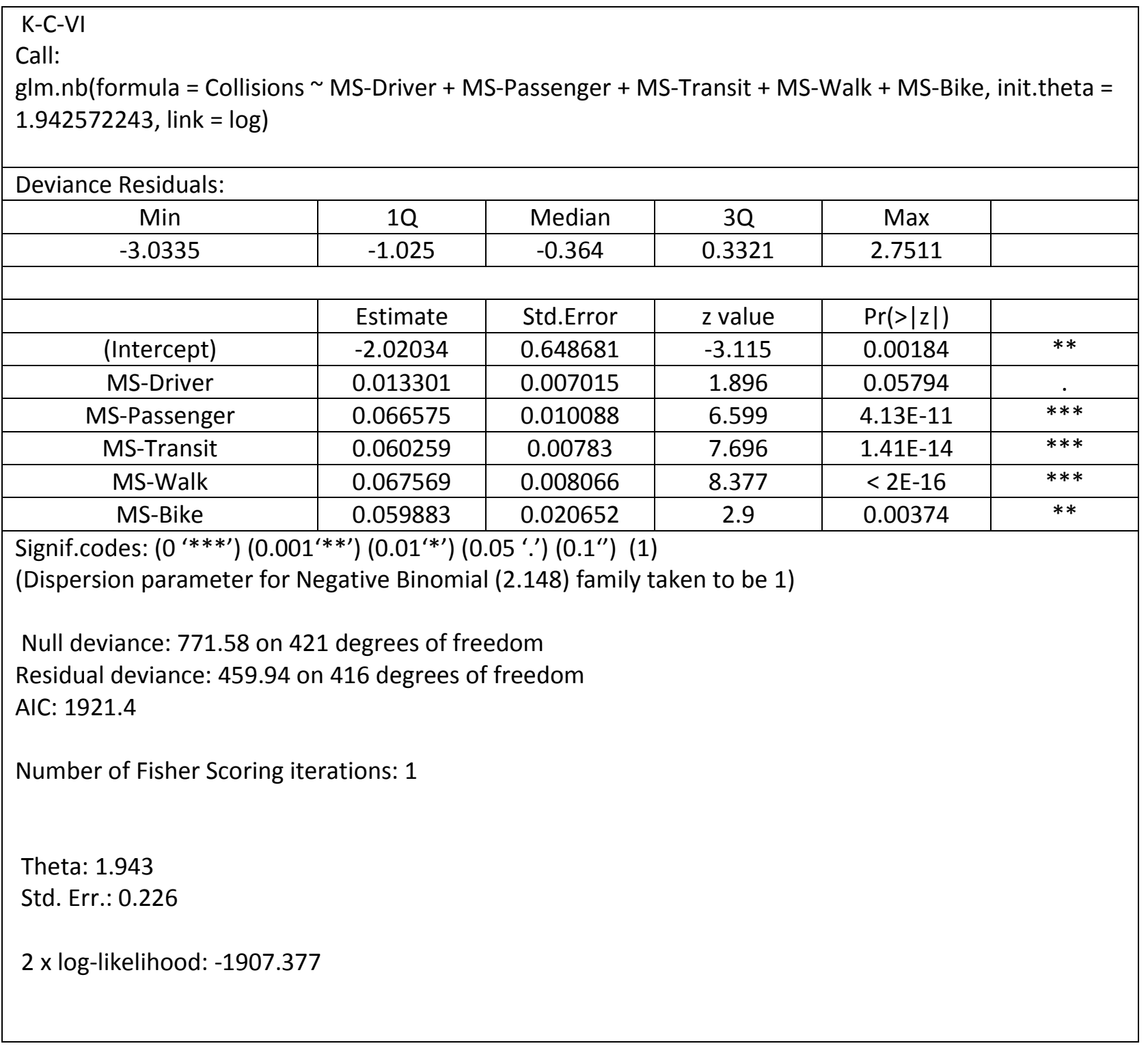




\begin{tabular}{|c|c|c|c|c|c|}
\hline $\begin{array}{l}\text { K-D-IV } \\
\text { Call: } \\
\text { glm.nb(formula = Collisions } \\
\text { + Density- Trips-Out-Center }\end{array}$ & $\begin{array}{l}\text { MS-Driver + } \\
\text { Density- Trip }\end{array}$ & $\begin{array}{l}\text { Passenger }+ \\
\text { ter-Center }\end{array}$ & $\begin{array}{l}\text { ransit + } ~ \\
\text { M, init.th }\end{array}$ & $\begin{array}{l}\text { alk + VKT + } \\
3.03811181\end{array}$ & $\begin{array}{l}\text { roduced } \\
=\log )\end{array}$ \\
\hline Deviance Residuals: & & & & & \\
\hline Min & 10 & Median & $3 Q$ & Max & \\
\hline-2.389 & -1.0698 & -0.3624 & 0.4631 & 2.9434 & \\
\hline & Estimate & Std.Error & $z$ value & $\operatorname{Pr}(>|z|)$ & \\
\hline (Intercept) & $-2.14 \mathrm{E}+00$ & $6.18 \mathrm{E}-01$ & -3.471 & 0.000518 & $* * *$ \\
\hline MS-Driver & $1.12 \mathrm{E}-02$ & $6.72 \mathrm{E}-03$ & 1.67 & 0.094886 & . \\
\hline MS-Passenger & $6.13 \mathrm{E}-02$ & $9.63 \mathrm{E}-03$ & 6.363 & $1.98 \mathrm{E}-10$ & $* * *$ \\
\hline MS-Transit & $6.01 \mathrm{E}-02$ & 8.06E-03 & 7.456 & 8.93E-14 & $* * *$ \\
\hline MS-Walk & $4.10 \mathrm{E}-02$ & $8.25 \mathrm{E}-03$ & 4.971 & $6.65 \mathrm{E}-07$ & $* * *$ \\
\hline VKT & 5.77E-06 & $2.16 \mathrm{E}-06$ & 2.671 & 0.007559 & $* *$ \\
\hline Trips-Produced & $-4.69 \mathrm{E}-05$ & 2.39E-05 & -1.961 & 0.049924 & $*$ \\
\hline Density- Trips-Out-Center & $5.67 \mathrm{E}-05$ & $3.04 \mathrm{E}-05$ & 1.867 & 0.061954 & . \\
\hline Density- Trips-Enter-Center & $-3.09 \mathrm{E}-05$ & 4.77E-06 & -6.473 & $9.61 \mathrm{E}-11$ & $* * *$ \\
\hline TLKM & $5.82 \mathrm{E}-02$ & $1.01 \mathrm{E}-02$ & 5.742 & 9.33E-09 & $* * *$ \\
\hline $\begin{array}{l}\text { Signif.codes: }\left(0^{* * * \prime}\right)(0.001 \\
\text { (Dispersion parameter for } \mathrm{N}\end{array}$ & $\begin{array}{l}\left.\mathrm{k}^{\prime}\right)\left(0.01^{(* \prime}\right) \\
\text { ative Binom }\end{array}$ & $\begin{array}{l}\left.\because^{\prime}\right)\left(0.1^{\prime \prime}\right) \\
.148) \text { fami }\end{array}$ & n to be 1 & & \\
\hline $\begin{array}{l}\text { Null deviance: } 965.82 \text { on } 42 \\
\text { Residual deviance: } 469 \text { on } 4 \\
\text { AIC: } 1844.5\end{array}$ & $\begin{array}{l}\text { degrees of } f \mathrm{f} \\
\text { degrees of }\end{array}$ & om & & & \\
\hline Number of Fisher Scoring ite & tions: 1 & & & & \\
\hline $\begin{array}{l}\text { Theta: } 3.038 \\
\text { Std. Err.: } 0.424\end{array}$ & & & & & \\
\hline $2 \times$ log-likelihood: -1822.50 & & & & & \\
\hline
\end{tabular}




\begin{tabular}{|c|c|c|c|c|c|}
\hline \multicolumn{6}{|c|}{$\begin{array}{l}\text { Call: } \\
\text { glm.nb(formula = Collisions Area + MS-Driver + MS-Passenger + MS-Transit + MS-Walk + MS-Bike + } \\
\text { Age. } 0-4+\text { Age }>65+\text { VKT + Density- Trips-Enter-Center + TLKM, init.theta }=3.269428236, \text { link }=\log \text { ) }\end{array}$} \\
\hline \multicolumn{6}{|l|}{ Deviance Residuals: } \\
\hline Min & 10 & Median & $3 Q$ & Max & \\
\hline \multirow[t]{2}{*}{-2.4102} & -1.0539 & -0.3457 & 0.4705 & 2.7478 & \\
\hline & Estimate & Std.Error & $z$ value & $\operatorname{Pr}(>|z|)$ & \\
\hline (Intercept) & $-2.63 \mathrm{E}+00$ & $7.34 \mathrm{E}-01$ & -3.584 & 0.000338 & $* * *$ \\
\hline Area & $-3.83 E-03$ & $2.70 \mathrm{E}-03$ & -1.418 & 0.156191 & \\
\hline MS-Driver & $1.38 \mathrm{E}-02$ & $7.64 \mathrm{E}-03$ & 1.799 & 0.071986 & . \\
\hline MS-Passenger & $6.16 \mathrm{E}-02$ & $1.05 \mathrm{E}-02$ & 5.883 & $4.02 \mathrm{E}-09$ & $* * *$ \\
\hline MS-Transit & $6.10 \mathrm{E}-02$ & $8.81 \mathrm{E}-03$ & 6.925 & $4.37 \mathrm{E}-12$ & $* * *$ \\
\hline MS-Walk & $3.82 \mathrm{E}-02$ & $8.63 \mathrm{E}-03$ & 4.428 & $9.50 \mathrm{E}-06$ & $* * *$ \\
\hline MS-Bike & $2.90 \mathrm{E}-02$ & $1.87 \mathrm{E}-02$ & 1.557 & 0.119566 & \\
\hline Age.0-4 & 3.77E-02 & $1.68 \mathrm{E}-02$ & 2.237 & 0.025258 & $*$ \\
\hline Age $>65$ & 1.90E-02 & $5.45 \mathrm{E}-03$ & 3.489 & 0.000485 & $* * *$ \\
\hline VKT & $8.59 \mathrm{E}-06$ & $2.25 \mathrm{E}-06$ & 3.81 & 0.000139 & $* * *$ \\
\hline Density- Trips-Enter-Center & $-2.03 \mathrm{E}-05$ & $3.72 \mathrm{E}-06$ & -5.455 & $4.88 \mathrm{E}-08$ & $* * *$ \\
\hline TLKM & $4.55 \mathrm{E}-02$ & $9.25 \mathrm{E}-03$ & 4.921 & $8.63 \mathrm{E}-07$ & $* * *$ \\
\hline \multicolumn{6}{|c|}{$\begin{array}{l}\text { Signif.codes: }\left(0^{* * * \prime}\right)\left(0.001^{\prime * *^{\prime}}\right)\left(0.01^{\prime * \prime}\right)\left(0.05^{\prime \prime}\right)\left(0.1^{\prime \prime}\right)(1) \\
\text { (Dispersion parameter for Negative Binomial }(2.148) \text { family taken to be } 1)\end{array}$} \\
\hline \multicolumn{6}{|c|}{ Null deviance: 998.84 on 421 degrees of freedom } \\
\hline \multicolumn{6}{|c|}{ Residual deviance: 464.86 on 410 degrees of freedom } \\
\hline \multicolumn{6}{|l|}{ AIC: 1830.6} \\
\hline \multicolumn{6}{|c|}{ Number of Fisher Scoring iterations: 1} \\
\hline \multicolumn{6}{|l|}{ Theta: 3.269} \\
\hline \multicolumn{6}{|l|}{ Std. Err.: 0.472} \\
\hline \multicolumn{6}{|l|}{2 x log-likelihood: -1804.607} \\
\hline
\end{tabular}




\begin{tabular}{|c|c|c|c|c|c|}
\hline \multicolumn{6}{|c|}{$\begin{array}{l}\text { Call: } \\
\text { glm.nb(formula = Collisions } \sim \text { VKT + Trips-Out-Center + Trips-Enter-Center + Density- Trips-Out-Center + } \\
\text { Density- Trips-Enter-Center + TLKM, init.theta }=1.66221822, \text { link }=\log )\end{array}$} \\
\hline \multicolumn{6}{|l|}{ Deviance Residuals: } \\
\hline Min & 10 & Median & $3 Q$ & Max & \\
\hline \multirow[t]{2}{*}{-3.9466} & -1.1216 & -0.3512 & 0.3985 & 2.8206 & \\
\hline & Estimate & Std.Error & $z$ value & $\operatorname{Pr}(>|z|)$ & \\
\hline (Intercept) & $9.20 \mathrm{E}-02$ & $1.10 \mathrm{E}-01$ & 0.837 & 0.402743 & \\
\hline VKT & $9.76 \mathrm{E}-06$ & $2.54 \mathrm{E}-06$ & 3.851 & 0.000118 & $* * *$ \\
\hline Trips-Out-Center & $2.00 \mathrm{E}-04$ & $4.46 \mathrm{E}-05$ & 4.49 & $7.12 \mathrm{E}-06$ & $* * *$ \\
\hline Trips-Enter-Center & $1.08 \mathrm{E}-04$ & $3.00 \mathrm{E}-05$ & 3.591 & 0.000329 & $* * *$ \\
\hline Density- Trips-Out-Center & $3.50 \mathrm{E}-04$ & $3.01 E-05$ & 11.645 & $<2 \mathrm{E}-16$ & $* * *$ \\
\hline Density- Trips-Enter-Center & $-2.35 \mathrm{E}-05$ & $5.82 \mathrm{E}-06$ & -4.033 & $5.50 \mathrm{E}-05$ & $* * *$ \\
\hline TLKM & $-1.91 \mathrm{E}-02$ & $4.32 \mathrm{E}-03$ & -4.414 & $1.01 \mathrm{E}-05$ & $* * *$ \\
\hline \multicolumn{6}{|c|}{$\begin{array}{l}\text { Signif.codes: }\left(0^{\prime * * * \prime}\right)\left(0.001^{\prime * * \prime}\right)\left(0.01^{\prime * \prime}\right)\left(0.05^{\prime} .\right)\left(0.1^{\prime \prime}\right)(1) \\
\text { (Dispersion parameter for Negative Binomial }(2.148) \text { family taken to be } 1)\end{array}$} \\
\hline \multicolumn{6}{|c|}{ Null deviance: 708.13 on 421 degrees of freedom } \\
\hline \multicolumn{6}{|c|}{ Residual deviance: 461.94 on 415 degrees of freedom } \\
\hline \multicolumn{6}{|l|}{ AIC: 1962.3} \\
\hline \multicolumn{6}{|c|}{ Number of Fisher Scoring iterations: 1} \\
\hline \multicolumn{6}{|l|}{ Theta: 1.662} \\
\hline \multicolumn{6}{|l|}{ Std. Err.: 0.178} \\
\hline \multicolumn{6}{|l|}{2 x log-likelihood: -1946.334 } \\
\hline
\end{tabular}




\begin{tabular}{|c|c|c|c|c|c|}
\hline $\begin{array}{l}\text { K-D-I } \\
\text { Call: } \\
\text { glm.nb(formula = Collisions } \\
\text { init.theta = 1.952459205, lin }\end{array}$ & $\begin{array}{l}\text { KT + Densit } \\
\text { (og) }\end{array}$ & ips-Out-Cer & Density- & Enter-Cent & \\
\hline Deviance Residuals: & & & & & \\
\hline Min & 10 & Median & $3 Q$ & Max & \\
\hline-3.5951 & -1.2129 & -0.3167 & 0.3912 & 2.9797 & \\
\hline & Estimate & Std.Error & $z$ value & $\operatorname{Pr}(>|z|)$ & \\
\hline (Intercept) & $2.21 \mathrm{E}-01$ & $7.91 \mathrm{E}-02$ & 2.79 & 0.00527 & $* *$ \\
\hline VKT & 4.53E-06 & $2.35 \mathrm{E}-06$ & 1.924 & 0.05435 & . \\
\hline Density- Trips-Out-Center & $1.01 \mathrm{E}-04$ & $3.32 \mathrm{E}-05$ & 3.034 & 0.00241 & $* *$ \\
\hline Density- Trips-Enter-Center & $-2.46 \mathrm{E}-05$ & $5.03 E-06$ & -4.894 & $9.87 \mathrm{E}-07$ & $* * *$ \\
\hline TLKM & 7.63E-02 & $1.00 \mathrm{E}-02$ & 7.625 & $2.44 \mathrm{E}-14$ & $* * *$ \\
\hline $\begin{array}{l}\text { Signif.codes: }\left(0^{* * * *}\right)(0.001 \\
\text { (Dispersion parameter for } \mathrm{N}\end{array}$ & $\begin{array}{l}\text { s' }^{\prime}\left(0.01^{\prime * \prime}\right)( \\
\text { ative Binom }\end{array}$ & $\begin{array}{l}\left..^{\prime}\right)\left(0.1^{\prime \prime}\right) \\
.148) \text { famil }\end{array}$ & n to be 1 & & \\
\hline Null deviance: 773.7 on 421 & grees of fre & & & & \\
\hline Residual deviance: 482.07 ol & 17 degrees & eedom & & & \\
\hline AIC: 1940.3 & & & & & \\
\hline Number of Fisher Scoring ite & tions: 1 & & & & \\
\hline Theta: 1.952 & & & & & \\
\hline Std. Err.: 0.232 & & & & & \\
\hline 2 x log-likelihood: -1928.343 & & & & & \\
\hline
\end{tabular}




\begin{tabular}{|c|c|c|c|c|c|}
\hline \multicolumn{6}{|c|}{$\begin{array}{l}\text { Call: } \\
\text { glm.nb(formula }=\text { Collisions } \sim \text { Area }+ \text { MS-Passenger + MS-Transit }+ \text { MS-Walk + Employment }+ \text { Age. } 0-4+ \\
\text { Age.5-24 + Age. } 25-64+\text { Age }>65+\text { TLKM, init.theta }=1.690862288, \text { link }=\text { log })\end{array}$} \\
\hline \multicolumn{6}{|l|}{ Deviance Residuals: } \\
\hline Min & 10 & Median & $3 Q$ & Max & \\
\hline \multirow[t]{2}{*}{-2.3933} & -1.0272 & -0.472 & 0.3368 & 4.0877 & \\
\hline & Estimate & Std.Error & $z$ value & $\operatorname{Pr}(>|z|)$ & \\
\hline (Intercept) & $-2.68 \mathrm{E}+00$ & $2.90 \mathrm{E}-01$ & -9.217 & $<2 \mathrm{E}-16$ & $* * *$ \\
\hline Area & $-4.03 E-03$ & 3.09E-03 & -1.305 & 0.191883 & \\
\hline MS-Passenger & 4.97E-02 & $1.08 \mathrm{E}-02$ & 4.59 & $4.43 \mathrm{E}-06$ & $* * *$ \\
\hline MS-Transit & $5.68 \mathrm{E}-02$ & $7.28 \mathrm{E}-03$ & 7.812 & $5.62 \mathrm{E}-15$ & $* * *$ \\
\hline MS-Walk & $2.40 \mathrm{E}-02$ & $6.73 \mathrm{E}-03$ & 3.56 & 0.000371 & $* * *$ \\
\hline Employment & $-1.14 \mathrm{E}-05$ & $2.45 \mathrm{E}-06$ & -4.667 & $3.06 \mathrm{E}-06$ & $* * *$ \\
\hline Age.0-4 & $4.86 \mathrm{E}-02$ & $2.59 \mathrm{E}-02$ & 1.88 & 0.060101 & . \\
\hline Age.5-24 & $8.01 \mathrm{E}-03$ & $4.57 \mathrm{E}-03$ & 1.752 & 0.079825 & . \\
\hline Age.25-64 & $1.08 \mathrm{E}-02$ & $3.67 \mathrm{E}-03$ & 2.951 & 0.003165 & $* *$ \\
\hline Age $>65$ & $3.40 \mathrm{E}-02$ & $7.63 \mathrm{E}-03$ & 4.457 & $8.30 \mathrm{E}-06$ & $* * *$ \\
\hline TLKM & $7.52 \mathrm{E}-02$ & $9.27 \mathrm{E}-03$ & 8.113 & $4.94 \mathrm{E}-16$ & $* * *$ \\
\hline \multicolumn{6}{|c|}{$\begin{array}{l}\text { Signif.codes: }\left(0^{* * * \prime}\right)\left(0.001^{\prime * *^{\prime}}\right)\left(0.01^{\prime * \prime}\right)\left(0.05^{\prime \prime}\right)\left(0.1^{\prime \prime}\right)(1) \\
\text { (Dispersion parameter for Negative Binomial }(2.148) \text { family taken to be } 1)\end{array}$} \\
\hline \multicolumn{6}{|c|}{$\begin{array}{l}\text { Null deviance: } 976.17 \text { on } 421 \text { degrees of } \\
\text { Residual deviance: } 459.46 \text { on } 411 \text { degrees of freedom } \\
\text { AIC: } 1751.5\end{array}$} \\
\hline \multicolumn{6}{|c|}{ Number of Fisher Scoring iterations: 1} \\
\hline \multicolumn{6}{|l|}{ Theta: 1.691} \\
\hline $2 \times$ log-likelihood: -1 & & & & & \\
\hline
\end{tabular}




\begin{tabular}{|c|c|c|c|c|c|}
\hline \multicolumn{6}{|c|}{$\begin{array}{l}\text { Call: } \\
\text { gIm.nb(formula }=\text { Collisions } \sim \text { VKT }+ \text { Trips-Attracted }+ \text { Density- Trips-Enter-Center }+ \text { TLKM, init.theta }= \\
0.984117155, \text { link }=\text { log) }\end{array}$} \\
\hline \multicolumn{6}{|l|}{ Deviance Residuals: } \\
\hline Min & 10 & Median & $3 Q$ & Max & \\
\hline \multirow[t]{2}{*}{-2.6885} & -1.2138 & -0.4321 & 0.2997 & 3.0083 & \\
\hline & Estimate & Std.Error & $z$ value & $\operatorname{Pr}(>|z|)$ & \\
\hline (Intercept) & $-1.37 \mathrm{E}-01$ & $1.05 \mathrm{E}-01$ & -1.312 & 0.189651 & \\
\hline VKT & $-6.86 E-06$ & $3.41 \mathrm{E}-06$ & -2.013 & 0.044095 & $*$ \\
\hline Trips-Attracted & $3.16 \mathrm{E}-05$ & $9.23 \mathrm{E}-06$ & 3.426 & 0.000612 & $* * *$ \\
\hline Density- Trips-Enter-Center & $-6.80 \mathrm{E}-05$ & $1.34 \mathrm{E}-05$ & -5.073 & 3.91E-07 & $* * *$ \\
\hline TLKM & $1.56 \mathrm{E}-01$ & $1.09 \mathrm{E}-02$ & 14.408 & $<2 \mathrm{E}-16$ & $* * *$ \\
\hline \multicolumn{6}{|c|}{$\begin{array}{l}\text { Signif.codes: }\left(0^{\prime * * * \prime}\right)\left(0.001^{\prime * * \prime}\right)\left(0.01^{\prime * \prime}\right)\left(0.05^{\prime . \prime}\right)\left(0.1^{\prime \prime}\right)(1) \\
\text { (Dispersion parameter for Negative Binomial }(2.148) \text { family taken to be } 1)\end{array}$} \\
\hline \multicolumn{6}{|c|}{ Null deviance: 715.52 on 421 degrees of freedom } \\
\hline \multicolumn{6}{|c|}{ Residual deviance: 455.32 on 417 degrees of freedom } \\
\hline \multicolumn{6}{|l|}{ AIC: 1857.3} \\
\hline \multicolumn{6}{|c|}{ Number of Fisher Scoring iterations: 1} \\
\hline \multicolumn{6}{|l|}{ Theta: 0.984} \\
\hline \multicolumn{6}{|l|}{ Std. Err.: 0.108} \\
\hline 2 x log-likelihood: -1845.30 & & & & & \\
\hline
\end{tabular}




\begin{tabular}{|c|c|c|c|c|c|}
\hline \multicolumn{6}{|c|}{$\begin{array}{l}\text { M-D-III } \\
\text { Call: } \\
\text { glm.nb(formula = Collisions } \sim \text { Sig-Int + Non-Sig-Int }+40 . \mathrm{Km} / \mathrm{h}+50 . \mathrm{Km} / \mathrm{h}+60 . \mathrm{Km} / \mathrm{h}+70 . \mathrm{Km} / \mathrm{h}+\text { Bike- } \\
\text { Path + VKT + Density- Trips-Enter-Center + TLKM, init.theta }=1.602487401, \operatorname{link}=\log \text { ) }\end{array}$} \\
\hline \multicolumn{6}{|l|}{ Deviance Residuals: } \\
\hline Min & 10 & Median & $3 Q$ & Max & \\
\hline \multirow[t]{3}{*}{-3.2314} & -1.21 & -0.358 & 0.3489 & 3.3918 & \\
\hline & & & & & \\
\hline & Estimate & Std.Error & $z$ value & $\operatorname{Pr}(>|z|)$ & \\
\hline (Intercept) & $3.35 \mathrm{E}-01$ & $1.17 \mathrm{E}-01$ & 2.848 & 0.004393 & $* *$ \\
\hline Sig-Int & $9.41 \mathrm{E}-03$ & $3.54 \mathrm{E}-03$ & 2.662 & 0.007778 & $* *$ \\
\hline Non-Sig-Int & $1.34 \mathrm{E}-02$ & $3.23 \mathrm{E}-03$ & 4.154 & $3.27 \mathrm{E}-05$ & $* * *$ \\
\hline $40 . \mathrm{Km} / \mathrm{h}$ & 5.09E-01 & $9.58 \mathrm{E}-02$ & 5.315 & 1.07E-07 & $* * *$ \\
\hline $50 . \mathrm{Km} / \mathrm{h}$ & $4.72 \mathrm{E}-01$ & $9.34 \mathrm{E}-02$ & 5.048 & $4.47 \mathrm{E}-07$ & $* * *$ \\
\hline $60 . \mathrm{Km} / \mathrm{h}$ & $4.06 \mathrm{E}-01$ & $9.43 \mathrm{E}-02$ & 4.302 & $1.69 \mathrm{E}-05$ & $* * *$ \\
\hline $70 . \mathrm{Km} / \mathrm{h}$ & $3.16 \mathrm{E}-01$ & $1.40 \mathrm{E}-01$ & 2.26 & 0.02382 & $*$ \\
\hline Bike-Path & $3.22 \mathrm{E}-02$ & $9.60 \mathrm{E}-03$ & 3.357 & 0.000789 & $* * *$ \\
\hline VKT & $1.14 \mathrm{E}-05$ & $3.61 \mathrm{E}-06$ & 3.169 & 0.001532 & $* *$ \\
\hline Density- Trips-Enter-Center & $-9.25 E-06$ & $4.37 \mathrm{E}-06$ & -2.115 & 0.034449 & $*$ \\
\hline TLKM & $-4.45 \mathrm{E}-01$ & $9.70 \mathrm{E}-02$ & -4.59 & $4.43 \mathrm{E}-06$ & $* * *$ \\
\hline \multicolumn{6}{|c|}{$\begin{array}{l}\text { Null deviance: } 822.6 \text { on } 421 \text { degrees of freedom } \\
\text { Residual deviance: } 475.34 \text { on } 411 \text { degrees of freedom } \\
\text { AIC: } 1880.3\end{array}$} \\
\hline \multicolumn{6}{|c|}{ Number of Fisher Scoring iterations: 1} \\
\hline \multicolumn{4}{|l|}{ Theta: 1.602} & \multicolumn{2}{|c|}{ Std. Err.: 0.194} \\
\hline 2 x log-likelihood: -1856.277 & & & & & \\
\hline
\end{tabular}




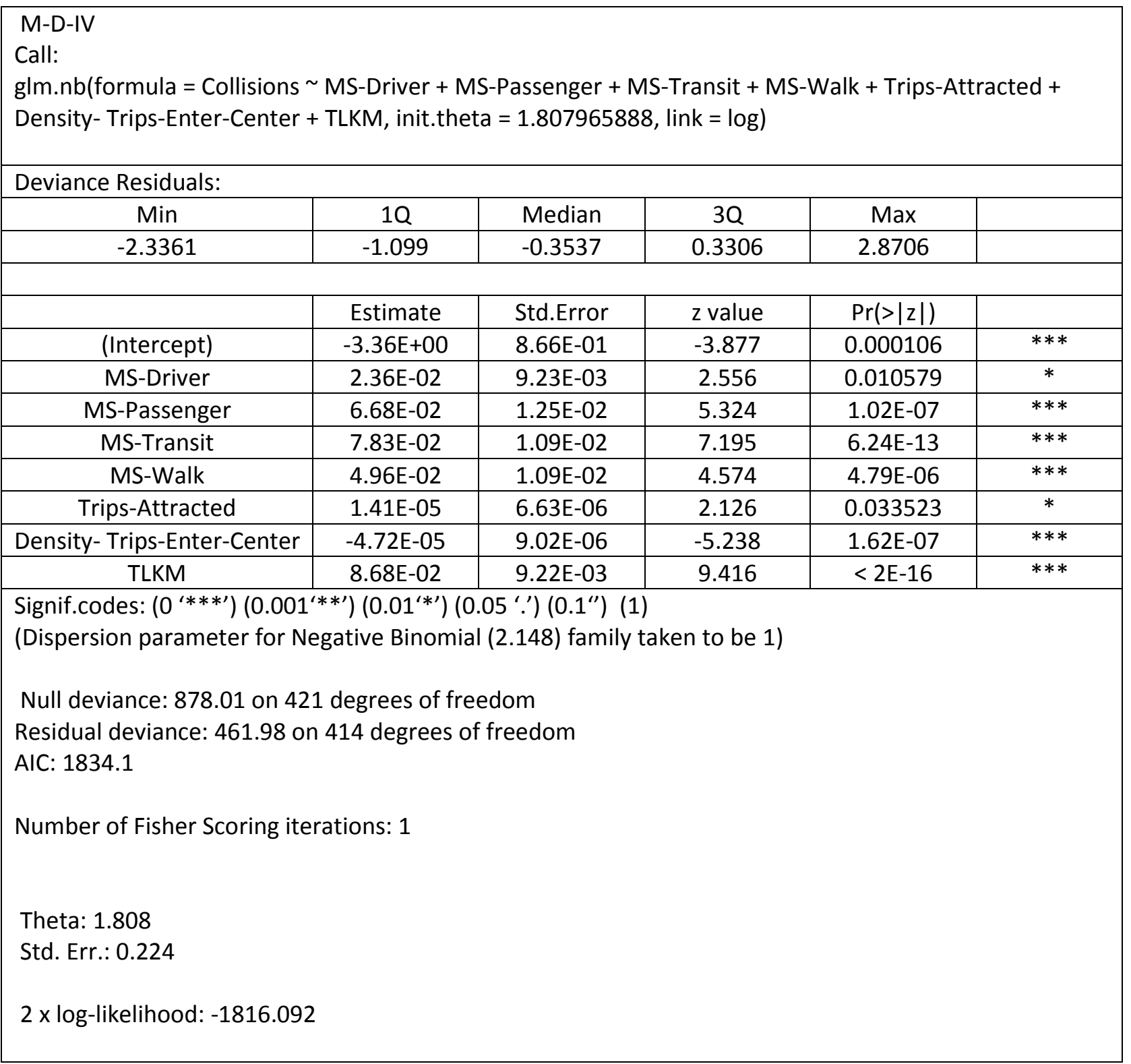




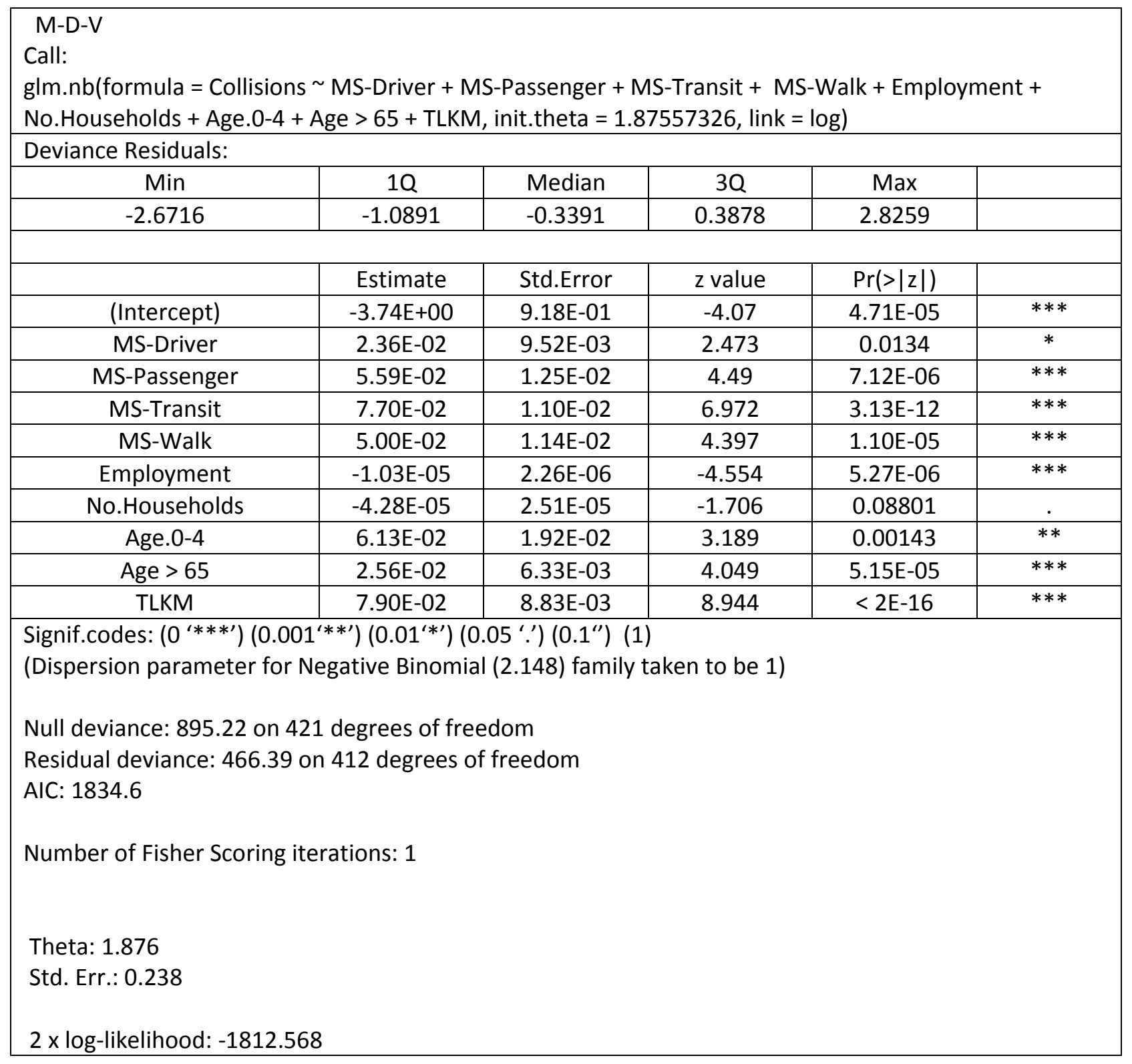




\begin{tabular}{|c|c|c|c|c|c|}
\hline \multicolumn{6}{|l|}{$\begin{array}{l}\text { Call: } \\
\text { glm.nb(formula = Collisions } \\
1.272407188, \text { link }=\log \text { ) }\end{array}$} \\
\hline \multicolumn{6}{|l|}{ Deviance Residuals: } \\
\hline Min & 10 & Median & $3 Q$ & Max & \\
\hline \multirow[t]{2}{*}{-3.2005} & -1.3274 & -0.3523 & 0.321 & 3.0352 & \\
\hline & Estimate & Std.Error & $z$ value & $\operatorname{Pr}(>|z|)$ & \\
\hline (Intercept) & $7.40 \mathrm{E}-02$ & $9.24 \mathrm{E}-02$ & 0.802 & 0.422841 & \\
\hline Trips-Attracted & $2.45 \mathrm{E}-05$ & $7.45 \mathrm{E}-06$ & 3.294 & 0.000987 & $* * *$ \\
\hline Density- Trips-Enter-Center & $-4.82 \mathrm{E}-05$ & $1.01 \mathrm{E}-05$ & -4.767 & 1.87E-06 & $* * *$ \\
\hline TLKM & $1.20 \mathrm{E}-01$ & $7.96 \mathrm{E}-03$ & 15.063 & $<2 \mathrm{E}-16$ & $* * *$ \\
\hline \multicolumn{6}{|c|}{$\begin{array}{l}\text { Signif.codes: }\left(0^{* * * \prime}\right)\left(0.001^{\prime * * \prime}\right)\left(0.01^{*^{\prime} \prime}\right)\left(0.05^{\prime \prime}\right)\left(0.1^{\prime \prime}\right)(1) \\
\text { (Dispersion parameter for Negative Binomial }(2.148) \text { family taken to be } 1)\end{array}$} \\
\hline $\begin{array}{l}\text { Null deviance: } 721.99 \text { on } 4 \\
\text { Residual deviance: } 472.99 \text { o } \\
\text { AIC: } 1918.6\end{array}$ & $\begin{array}{l}\text { legrees of } f \\
18 \text { degrees }\end{array}$ & eedom & & & \\
\hline \multicolumn{6}{|c|}{ Number of Fisher Scoring iterations: 1} \\
\hline \multicolumn{6}{|l|}{ Theta: 1.272} \\
\hline \multicolumn{6}{|l|}{ Std. Err.: 0.142} \\
\hline 2 x log-likelihood: -1908.59 & & & & & \\
\hline
\end{tabular}




\section{D.6 Fatal Collisions Models}

\begin{tabular}{|c|c|c|c|c|c|}
\hline \multicolumn{6}{|c|}{$\begin{array}{l}\text { L-C } \\
\text { Call: } \\
\text { zeroinfl(formula }=\text { Collisions } \sim A+B, \text { dist }=\text { negbin })\end{array}$} \\
\hline \multicolumn{6}{|c|}{ Pearson residuals: } \\
\hline Min & 10 & Median & $3 Q$ & Max & \\
\hline-1.174 & -0.4616 & -0.3535 & -0.185 & 6.1386 & \\
\hline \multicolumn{6}{|c|}{ Count model coefficients (negbin with log link): } \\
\hline & Estimate & Std.Error & $z$ value & $\operatorname{Pr}(>|z|)$ & \\
\hline (Intercept) & -4.1768 & 2.1557 & -1.938 & 0.05268 & . \\
\hline A & 0.1574 & 0.2343 & 0.672 & 0.50159 & \\
\hline $\mathrm{B}$ & 0.6191 & 0.16 & 3.868 & 0.00011 & $* * *$ \\
\hline Log(theta) & 6.6947 & 24.9724 & 0.268 & 0.78864 & \\
\hline
\end{tabular}

\begin{tabular}{|c|c|c|c|c|c|}
\hline \multicolumn{6}{|c|}{$\begin{array}{l}\text { M-C } \\
\text { Call: } \\
\text { zeroinfl(formula }=\text { Collisions } \sim A+B \text {, dist }=\text { negbin })\end{array}$} \\
\hline \multicolumn{6}{|c|}{ Pearson residuals: } \\
\hline Min & 10 & Median & $3 Q$ & Max & \\
\hline-1.0838 & -0.4806 & -0.3516 & -0.1878 & 6.7163 & \\
\hline \multicolumn{6}{|c|}{ Count model coefficients (negbin with log link): } \\
\hline & Estimate & Std.Error & $z$ value & $\operatorname{Pr}(>|z|)$ & \\
\hline (Intercept) & -5.7249 & 2.2093 & -2.591 & 0.00956 & $* *$ \\
\hline$A$ & 0.3434 & 0.236 & 1.455 & 0.14564 & \\
\hline$B$ & 0.4823 & 0.1545 & 3.121 & 0.0018 & $* *$ \\
\hline Log(theta) & 7.5877 & 29.0692 & 0.261 & 0.79408 & \\
\hline \multicolumn{6}{|c|}{ Signif.codes: $\left(0^{\prime * * * \prime}\right)\left(0.001^{* * \prime}\right)\left(0.01^{\prime * \prime}\right)\left(0.05^{\prime \prime}.\right)\left(0.1^{\prime \prime}\right)$} \\
\hline
\end{tabular}

\begin{tabular}{|c|c|c|c|c|c|}
\hline \multicolumn{6}{|c|}{$\begin{array}{l}\text { I-C } \\
\text { Call: } \\
\text { zeroinfl(formula }=\text { Collisions } \sim A+B \text {, dist }=\text { negbin })\end{array}$} \\
\hline \multicolumn{6}{|c|}{ Pearson residuals: } \\
\hline Min & $1 Q$ & Median & $3 Q$ & Max & \\
\hline-1.20009 & -0.46378 & -0.29526 & -0.04237 & 4.97823 & \\
\hline \multicolumn{6}{|c|}{ Count model coefficients (negbin with log link): } \\
\hline & Estimate & Std.Error & $z$ value & $\operatorname{Pr}(>|z|)$ & \\
\hline (Intercept) & -4.712 & 1.9516 & -2.414 & 0.0158 & $*$ \\
\hline A & 0.311 & 0.2175 & 1.43 & 0.1528 & \\
\hline $\mathrm{B}$ & 0.3509 & 0.161 & 2.179 & 0.0293 & $*$ \\
\hline Log(theta) & 1.909 & 2.0375 & 0.937 & 0.3488 & \\
\hline \multicolumn{6}{|c|}{ Signif.codes: $\left(0^{\prime * * * \prime}\right)\left(0.001^{\prime * * \prime}\right)\left(0.01^{\prime * \prime}\right)\left(0.05^{\prime \prime} .^{\prime}\right)\left(0.1^{\prime \prime}\right)$ (1) } \\
\hline
\end{tabular}




\begin{tabular}{|c|c|c|c|c|c|}
\hline \multicolumn{6}{|c|}{$\begin{array}{l}\text { F-C } \\
\text { Call: } \\
\text { zeroinfl(formula }=\text { Collisions } \sim A+B \text {, dist }=\text { negbin })\end{array}$} \\
\hline \multicolumn{6}{|c|}{ Pearson residuals: } \\
\hline Min & $1 Q$ & Median & $3 Q$ & Max & \\
\hline-1.1644 & -0.4731 & -0.3459 & -0.0797 & 5.6137 & \\
\hline \multicolumn{6}{|c|}{ Count model coefficients (negbin with log link): } \\
\hline & Estimate & Std.Error & $z$ value & $\operatorname{Pr}(>|z|)$ & \\
\hline (Intercept) & -3.5732 & 1.3297 & -2.687 & 0.00721 & $* *$ \\
\hline $\mathrm{A}$ & 0.1506 & 0.1496 & 1.006 & 0.31425 & \\
\hline $\mathrm{B}$ & 0.5348 & 0.1204 & 4.442 & $8.92 \mathrm{E}-06$ & $* * *$ \\
\hline Log(theta) & 9.2433 & 214.5478 & 0.043 & 0.96564 & \\
\hline \multicolumn{6}{|c|}{ Signif.codes: $\left(0^{\prime * * * \prime}\right)\left(0.001^{* * \prime}\right)\left(0.01^{\prime * \prime}\right)\left(0.05^{\prime \prime}.\right)\left(0.1^{\prime \prime}\right)$ (1) } \\
\hline
\end{tabular}

\begin{tabular}{|c|c|c|c|c|c|}
\hline \multicolumn{6}{|c|}{$\begin{array}{l}\text { D-C } \\
\text { Call: } \\
\text { zeroinfl(formula }=\text { Collisions } \sim A+B \text {, dist }=\text { negbin })\end{array}$} \\
\hline \multicolumn{6}{|c|}{ Pearson residuals: } \\
\hline Min & 10 & Median & $3 Q$ & Max & \\
\hline-1.1612 & -0.4576 & -0.351 & -0.1755 & 5.3725 & \\
\hline \multicolumn{6}{|c|}{ Count model coefficients (negbin with log link): } \\
\hline & Estimate & Std.Error & $z$ value & $\operatorname{Pr}(>|z|)$ & \\
\hline (Intercept) & -3.6394 & 1.4109 & -2.579 & 0.0099 & $* *$ \\
\hline $\mathrm{A}$ & 0.1523 & 0.1554 & 0.98 & 0.3271 & \\
\hline B & 0.552 & 0.1248 & 4.423 & $9.75 \mathrm{E}-06$ & $* * *$ \\
\hline Log(theta) & 5.1862 & 33.7084 & 0.154 & 0.8777 & \\
\hline \multicolumn{6}{|c|}{ Signif.codes: $\left(0^{\prime * * * \prime}\right)\left(0.001^{\prime * * \prime}\right)\left(0.01^{\prime * \prime}\right)\left(0.05^{\prime \prime}{ }^{\prime}\right)\left(0.1^{\prime \prime}\right)(1)$} \\
\hline
\end{tabular}

\begin{tabular}{|c|c|c|c|c|c|}
\hline \multicolumn{6}{|c|}{$\begin{array}{l}\text { C-C } \\
\text { Call: } \\
\text { zeroinfl(formula }=\text { Collisions } \sim A+B \text {, dist }=\text { negbin })\end{array}$} \\
\hline \multicolumn{6}{|c|}{ Pearson residuals: } \\
\hline Min & 10 & Median & $3 Q$ & Max & \\
\hline-1.13398 & -0.47557 & -0.343 & -0.06833 & 6.61976 & \\
\hline \multicolumn{6}{|c|}{ Count model coefficients (negbin with log link): } \\
\hline & Estimate & Std.Error & $z$ value & $\operatorname{Pr}(>|z|)$ & \\
\hline (Intercept) & -3.1736 & 1.3732 & -2.311 & 0.0208 & $*$ \\
\hline A & 0.1208 & 0.1553 & 0.778 & 0.4364 & \\
\hline $\mathrm{B}$ & 0.5021 & 0.1219 & 4.119 & $3.81 \mathrm{E}-05$ & $* * *$ \\
\hline Log(theta) & 9.213 & 72.9679 & 0.126 & 0.8995 & \\
\hline \multicolumn{6}{|c|}{ Signif.codes: $\left(0^{\prime * * * \prime}\right)\left(0.001^{\prime * * \prime}\right)\left(0.01^{\prime * \prime}\right)\left(0.05^{\prime} .^{\prime}\right)\left(0.1^{\prime \prime}\right)$ (1) } \\
\hline
\end{tabular}




\begin{tabular}{|c|c|c|c|c|c|}
\hline \multicolumn{6}{|c|}{$\begin{array}{l}\text { G-C } \\
\text { Call: } \\
\text { zeroinfl(formula }=\text { Collisions } \sim A+B, \text { dist }=\text { negbin })\end{array}$} \\
\hline \multicolumn{6}{|c|}{ Pearson residuals: } \\
\hline Min & $1 Q$ & Median & $3 Q$ & Max & \\
\hline-1.0883 & -0.456 & -0.3136 & -0.112 & 5.734 & \\
\hline \multicolumn{6}{|c|}{ Count model coefficients (negbin with log link): } \\
\hline & Estimate & Std.Error & $z$ value & $\operatorname{Pr}(>|z|)$ & \\
\hline (Intercept) & -4.6259 & 1.8953 & -2.441 & 0.0147 & $*$ \\
\hline $\mathrm{A}$ & 0.291 & 0.2106 & 1.382 & 0.167 & \\
\hline B & 0.3822 & 0.1511 & 2.529 & 0.0114 & * \\
\hline Log(theta) & 1.6123 & 1.5866 & 1.016 & 0.3095 & \\
\hline \multicolumn{6}{|c|}{ Signif.codes: $\left(0^{\prime * * * \prime}\right)\left(0.001^{* * \prime}\right)\left(0.01^{\prime * \prime}\right)\left(0.05^{\prime \prime}.\right)\left(0.1^{\prime \prime}\right)$ (1) } \\
\hline
\end{tabular}

\begin{tabular}{|c|c|c|c|c|c|}
\hline \multicolumn{6}{|c|}{$\begin{array}{l}\text { B-C } \\
\text { Call: } \\
\text { zeroinfl(formula }=\text { Collisions } \sim A+B, \text { dist }=\text { negbin })\end{array}$} \\
\hline \multicolumn{6}{|c|}{ Pearson residuals: } \\
\hline Min & 10 & Median & $3 Q$ & Max & \\
\hline-1.06297 & -0.46538 & -0.33162 & -0.00262 & 5.984531 & -1.06297 \\
\hline \multicolumn{6}{|c|}{ Count model coefficients (negbin with log link): } \\
\hline & Estimate & Std.Error & $z$ value & $\operatorname{Pr}(>|z|)$ & \\
\hline (Intercept) & -2.44339 & 1.42912 & -1.71 & 0.0873 & . \\
\hline $\mathrm{A}$ & 0.01982 & 0.16663 & 0.119 & 0.9053 & \\
\hline B & 0.59251 & 0.13603 & 4.356 & $1.33 \mathrm{E}-05$ & $* * *$ \\
\hline Log(theta) & 6.24461 & 60.8013 & 0.103 & 0.9182 & \\
\hline \multicolumn{6}{|c|}{ Signif.codes: $\left(0^{\prime * * * \prime}\right)\left(0.001^{\prime * * \prime}\right)\left(0.01^{\prime * \prime}\right)\left(0.05^{\prime \prime}{ }^{\prime}\right)\left(0.1^{\prime \prime}\right)(1)$} \\
\hline
\end{tabular}

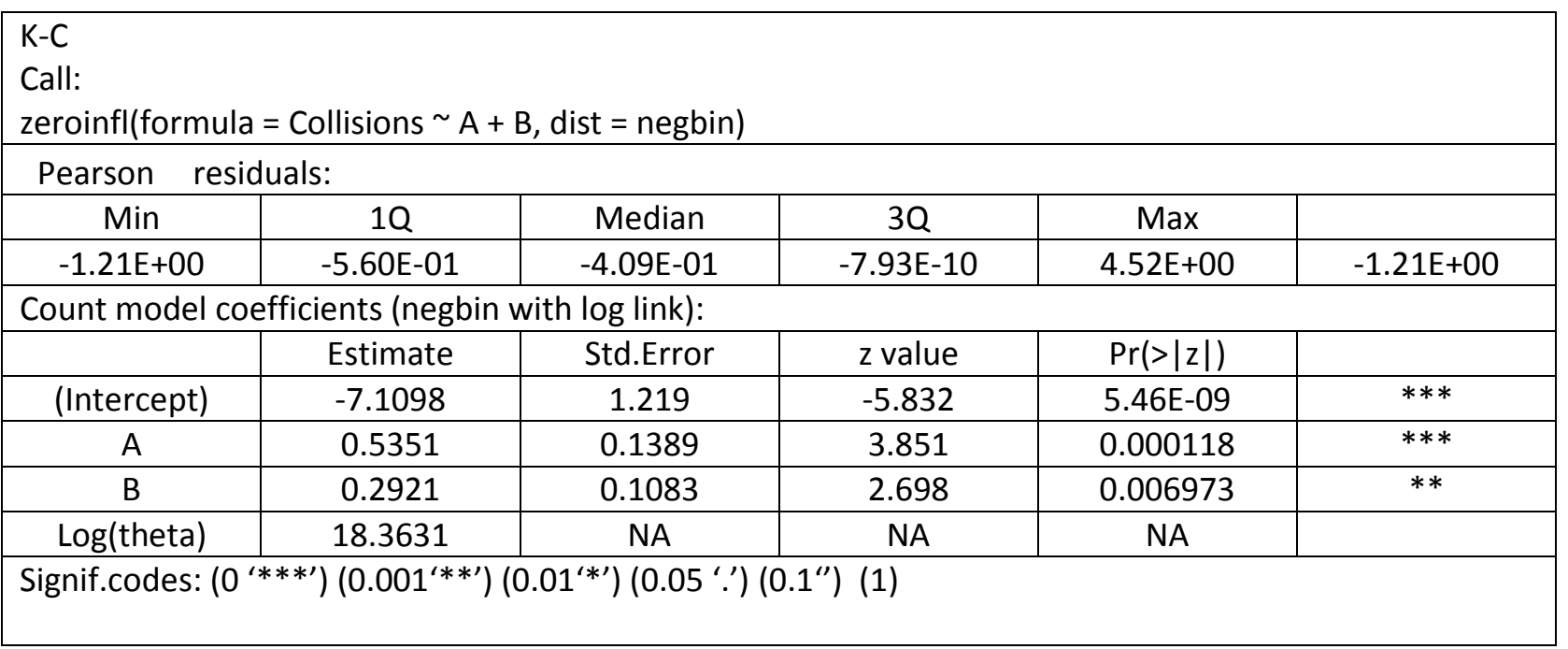




\begin{tabular}{|c|c|c|c|c|c|}
\hline \multicolumn{6}{|c|}{$\begin{array}{l}\text { E-C } \\
\text { Call: } \\
\text { zeroinfl(formula }=\text { Collisions } \sim A+B \text {, dist }=\text { negbin })\end{array}$} \\
\hline \multicolumn{6}{|c|}{ Pearson residuals: } \\
\hline Min & 10 & Median & $3 Q$ & Max & \\
\hline-0.98843 & -0.49547 & -0.36006 & -0.01357 & 6.16296 & -0.98843 \\
\hline \multicolumn{6}{|c|}{ Count model coefficients (negbin with log link): } \\
\hline & Estimate & Std.Error & $z$ value & $\operatorname{Pr}(>|z|)$ & \\
\hline (Intercept) & -8.25504 & 0.83335 & -9.906 & $<2 \mathrm{e}-16$ & $* * *$ \\
\hline A & 0.75022 & 0.08435 & 8.894 & $<2 \mathrm{e}-16$ & $* * *$ \\
\hline Log(theta) & 1.12198 & 0.83836 & 1.338 & 0.181 & \\
\hline
\end{tabular}

